\title{
Individual-, family-, and school-level interventions targeting multiple risk behaviours in young people (Review)
}

MacArthur G, Caldwell DM, Redmore J, Watkins SH, Kipping R, White J, Chittleborough C, Langford R, Er V, Lingam R, Pasch K, Gunnell D, Hickman M, Campbell R

MacArthur G, Caldwell DM, Redmore J, Watkins SH, Kipping R, White J, Chittleborough C, Langford R, Er V, Lingam R, Pasch K, Gunnell D, Hickman M, Campbell R.

Individual-, family-, and school-level interventions targeting multiple risk behaviours in young people.

Cochrane Database of Systematic Reviews 2018, Issue 10. Art. No.: CD009927.

DOI: 10.1002/14651858.CD009927.pub2.

www.cochranelibrary.com

Individual-, family-, and school-level interventions targeting multiple risk behaviours in young people (Review) 
TABLE OF CONTENTS

HEADER 1

ABSTRACT

PLAIN LANGUAGE SUMMARY

SUMMARY OF FINDINGS

BACKGROUND

Figure 1.

OBJECTIVES

METHODS

RESULTS

Figure 2.

Figure 3.

Figure 4.

Figure 5.

Figure 6.

Figure 7.

Figure 8.

Figure 9.

Figure 10.

Figure 11.

Figure 12.

Figure 13.

DISCUSSION

AUTHORS' CONCLUSIONS

ACKNOWLEDGEMENTS

REFERENCES

CHARACTERISTICS OF STUDIES

DATA AND ANALYSES

Analysis 1.1. Comparison 1 Tobacco, Outcome 1 Tobacco Use (short-term).

Analysis 1.2. Comparison 1 Tobacco, Outcome 2 Tobacco Use (long-term).

Analysis 2.1. Comparison 2 Alcohol, Outcome 1 Alcohol Use (short-term).

Analysis 2.2. Comparison 2 Alcohol, Outcome 2 Alcohol Use (long-term).

Analysis 3.1. Comparison 3 Binge drinking, Outcome 1 Drunkenness or Excess Drinking (short-term).

Analysis 3.2. Comparison 3 Binge drinking, Outcome 2 Drunkenness or Excess Drinking (long-term).

Analysis 4.1. Comparison 4 Illicit drug use, Outcome 1 Illicit drug use (short-term).

Analysis 4.2. Comparison 4 Illicit drug use, Outcome 2 Illicit drug use (long-term).

Analysis 5.1. Comparison 5 Cannabis use, Outcome 1 Cannabis Use (short-term).

Analysis 5.2. Comparison 5 Cannabis use, Outcome 2 Cannabis Use (long-term).

Analysis 6.1. Comparison 6 Alcohol, tobacco, and/or drug use, Outcome 1 Composite Substance Use (short-term).

Analysis 6.2. Comparison 6 Alcohol, tobacco, and/or drug use, Outcome 2 Composite Substance Use (long-term).

Analysis 7.1. Comparison 7 Antisocial behaviour and offending, Outcome 1 Antisocial Behaviour and Offending - Any (shortterm).

Analysis 7.2. Comparison 7 Antisocial behaviour and offending, Outcome 2 Violent Offences.

Analysis 7.3. Comparison 7 Antisocial behaviour and offending, Outcome 3 School or General Delinquency.

Analysis 7.4. Comparison 7 Antisocial behaviour and offending, Outcome 4 Antisocial Behaviour and Offending - Any (long term).

Analysis 8.1. Comparison 8 Vehicle-related risk behaviours, Outcome 1 Driving Under the Influence of Alcohol and/or Drugs. ..

Analysis 9.1. Comparison 9 Sexual risk behaviours, Outcome 1 Sexual Risk Behaviour (short-term).

Analysis 9.2. Comparison 9 Sexual risk behaviours, Outcome 2 Sexual Risk Behaviour (long-term).

Analysis 10.1. Comparison 10 Physical activity, Outcome 1 Physical Activity.

Analysis 11.1. Comparison 11 Mental health, Outcome 1 Depressive Symptoms (short-term).

Analysis 11.2. Comparison 11 Mental health, Outcome 2 Depressive Symptoms (long-term). 
Analysis 12.1. Comparison 12 Unhealthy diet, Outcome $1 \mathrm{BMI}$.

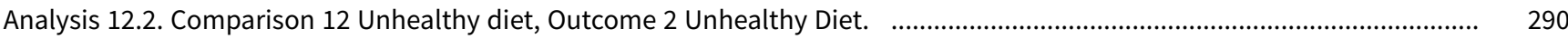

Analysis 13.1. Comparison 13 School-related outcomes, Outcome 1 Academic Performance (short-term). .............................. 291 ADDITIONAL TABLES

HISTORY 
[Intervention Review]

\title{
Individual-, family-, and school-level interventions targeting multiple risk behaviours in young people
}

\author{
Georgina MacArthur ${ }^{1}$, Deborah M Caldwell ${ }^{1}$, James Redmore ${ }^{1}$, Sarah H Watkins ${ }^{1}$, Ruth Kipping ${ }^{1}$, James White ${ }^{2}$, Catherine \\ Chittleborough $^{3}$, Rebecca Langford ${ }^{1}$, Vanessa Er ${ }^{1}$, Raghu Lingam ${ }^{4}$, Keryn Pasch ${ }^{5}$, David Gunnell ${ }^{1}$, Matthew Hickman ${ }^{1}$, Rona Campbell ${ }^{1}$ \\ 1Population Health Sciences, Bristol Medical School, University of Bristol, Bristol, UK. 2DECIPHer (Centre for the Development and \\ Evaluation of Complex Interventions for Public Health Improvement), Centre for Trials Research, School of Medicine, Cardiff University, \\ Cardiff, UK. ${ }^{3}$ School of Public Health, University of Adelaide, Adelaide, Australia. ${ }^{4}$ Institute of Health and Society, Newcastle University, \\ Newcastle Upon Tyne, UK. ${ }^{5}$ Department of Kinesiology and Health Education, University of Texas, Austin, Texas, USA
}

Contact address: Georgina MacArthur, Population Health Sciences, Bristol Medical School, University of Bristol, 39 Whatley Road, Bristol, BS8 2PS, UK. Georgie.macarthur@bristol.ac.uk.

Editorial group: Cochrane Public Health Group.

Publication status and date: New, published in Issue 10, 2018.

Citation: MacArthur G, Caldwell DM, Redmore J, Watkins SH, Kipping R, White J, Chittleborough C, Langford R, Er V, Lingam R, Pasch K, Gunnell D, Hickman M, Campbell R. Individual-, family-, and school-level interventions targeting multiple risk behaviours in young people. Cochrane Database of Systematic Reviews 2018, Issue 10. Art. No.: CD009927. DOI: 10.1002/14651858.CD009927.pub2.

Copyright @ 2018 The Cochrane Collaboration. Published by John Wiley \& Sons, Ltd.

\section{A B S T R A C T}

\section{Background}

Engagement in multiple risk behaviours can have adverse consequences for health during childhood, during adolescence, and later in life, yet little is known about the impact of different types of interventions that target multiple risk behaviours in children and young people, or the differential impact of universal versus targeted approaches. Findings from systematic reviews have been mixed, and effects of these interventions have not been quantitatively estimated.

\section{Objectives}

To examine the effects of interventions implemented up to 18 years of age for the primary or secondary prevention of multiple risk behaviours among young people.

\section{Search methods}

We searched 11 databases (Australian Education Index; British Education Index; Campbell Library; Cumulative Index to Nursing and Allied Health Literature (CINAHL); Cochrane Central Register of Controlled Trials (CENTRAL), in the Cochrane Library; Embase; Education Resource Information Center (ERIC); International Bibliography of the Social Sciences; MEDLINE; PsycINFO; and Sociological Abstracts) on three occasions (2012, 2015, and 14 November 2016)). We conducted handsearches of reference lists, contacted experts in the field, conducted citation searches, and searched websites of relevant organisations.

\section{Selection criteria}

We included randomised controlled trials (RCTs), including cluster RCTs, which aimed to address at least two risk behaviours. Participants were children and young people up to 18 years of age and/or parents, guardians, or carers, as long as the intervention aimed to address involvement in multiple risk behaviours among children and young people up to 18 years of age. However, studies could include outcome data on children $>18$ years of age at the time of follow-up. Specifically,we included studies with outcomes collected from those eight to 25 years of age. Further, we included only studies with a combined intervention and follow-up period of six months or longer. We excluded interventions aimed at individuals with clinically diagnosed disorders along with clinical interventions. We categorised interventions according to whether they were conducted at the individual level; the family level; or the school level. 


\section{Data collection and analysis}

We identified a total of 34,680 titles, screened 27,691 articles and assessed 424 full-text articles for eligibility. Two or more review authors independently assessed studies for inclusion in the review, extracted data, and assessed risk of bias.

We pooled data in meta-analyses using a random-effects (DerSimonian and Laird) model in RevMan 5.3. For each outcome, we included subgroups related to study type (individual, family, or school level, and universal or targeted approach) and examined effectiveness at up to 12 months' follow-up and over the longer term (> 12 months). We assessed the quality and certainty of evidence using the Grades of Recommendation, Assessment, Development and Evaluation (GRADE) approach.

\section{Main results}

We included in the review a total of 70 eligible studies, of which a substantial proportion were universal school-based studies ( $n=28 ; 40 \%$ ). Most studies were conducted in the USA $(n=55 ; 79 \%)$. On average, studies aimed to prevent four of the primary behaviours. Behaviours that were most frequently addressed included alcohol use $(n=55)$, drug use $(n=53)$, and/or antisocial behaviour $(n=53)$, followed by tobacco use $(n=42)$. No studies aimed to prevent self-harm or gambling alongside other behaviours.

Evidence suggests that for multiple risk behaviours, universal school-based interventions were beneficial in relation to tobacco use (odds ratio (OR) $0.77,95 \%$ confidence interval $(\mathrm{Cl}) 0.60$ to $0.97 ; \mathrm{n}=9$ studies; 15,354 participants) and alcohol use $(\mathrm{OR} 0.72,95 \% \mathrm{Cl} 0.56$ to $0.92 ; \mathrm{n}$ $=8$ studies; 8751 participants; both moderate-quality evidence) compared to a comparator, and that such interventions may be effective in preventing illicit drug use (OR $0.74,95 \% \mathrm{Cl} 0.55$ to $1.00 ; n=5$ studies; 11,058 participants; low-quality evidence) and engagement in any antisocial behaviour (OR $0.81,95 \% \mathrm{Cl} 0.66$ to $0.98 ; \mathrm{n}=13$ studies; 20,756 participants; very low-quality evidence) at up to 12 months' followup, although there was evidence of moderate to substantial heterogeneity $\left(I^{2}=49 \%\right.$ to $\left.69 \%\right)$. Moderate-quality evidence also showed that multiple risk behaviour universal school-based interventions improved the odds of physical activity $\left(\mathrm{OR} 1.32,95 \% \mathrm{Cl} 1.16\right.$ to $1.50 ; I^{2}=0 \%$; $\mathrm{n}=4$ studies; 6441 participants). We considered observed effects to be of public health importance when applied at the population level. Evidence was less certain for the effects of such multiple risk behaviour interventions for cannabis use $(\mathrm{OR} 0.79,95 \% \mathrm{Cl} 0.62$ to $1.01 ; \mathrm{P}=$ $0.06 ; n=5$ studies; 4140 participants; $I^{2}=0 \%$; moderate-quality evidence), sexual risk behaviours (OR $0.83,95 \% \mathrm{Cl} 0.61$ to $1.12 ; \mathrm{P}=0.22 ; \mathrm{n}=$ 6 studies; 12,633 participants; $I^{2}=77 \%$; low-quality evidence), and unhealthy diet (OR $0.82,95 \% \mathrm{Cl} 0.64$ to $1.06 ; \mathrm{P}=0.13 ; \mathrm{n}=3$ studies; 6441 participants; $I^{2}=49 \%$; moderate-quality evidence). It is important to note that some evidence supported the positive effects of universal school-level interventions on three or more risk behaviours.

For most outcomes of individual- and family-level targeted and universal interventions, moderate- or low-quality evidence suggests little or no effect, although caution is warranted in interpretation because few of these studies were available for comparison ( $\mathrm{n} \leq 4$ studies for each outcome).

Seven studies reported adverse effects, which involved evidence suggestive of increased involvement in a risk behaviour among participants receiving the intervention compared to participants given control interventions.

We judged the quality of evidence to be moderate or low for most outcomes, primarily owing to concerns around selection, performance, and detection bias and heterogeneity between studies.

\section{Authors' conclusions}

Available evidence is strongest for universal school-based interventions that target multiple- risk behaviours, demonstrating that they may be effective in preventing engagement in tobacco use, alcohol use, illicit drug use, and antisocial behaviour, and in improving physical activity among young people, but not in preventing other risk behaviours. Results of this review do not provide strong evidence of benefit for family- or individual-level interventions across the risk behaviours studied. However, poor reporting and concerns around the quality of evidence highlight the need for high-quality multiple- risk behaviour intervention studies to further strengthen the evidence base in this field.

\section{PLAIN LANGUAGE SUMMARY}

\section{Interventions for preventing multiple risk behaviours in young people}

\section{Background}

Health risk behaviours, such as smoking and drug use, can group together during the teenage years, and engagement in these multiple risk behaviours can lead to health problems such as injury and substance abuse during childhood and adolescence, as well as noncommunicable diseases later in life. Currently, we do not know which interventions are effective in preventing or decreasing these risky behaviours among children and young people.

\section{Search methods and selection of studies}

We carried out thorough searches of multiple scientific databases to identify studies that looked at ways of preventing or decreasing engagement in two or more risk behaviours, including tobacco use, alcohol use, illicit drug use, gambling, self-harm, sexual risk behaviour, antisocial behaviour, vehicle-risk behaviour, physical inactivity, and poor nutrition, among young people aged eight to 25 years. We 
divided these studies into groups (individual-level, family-level, and school-level studies) according to whether researchers worked with individuals, families, or children and young people in schools, respectively. We specifically looked at "gold standard" studies - randomised controlled trials that aimed to examine two or more behaviours of interest.

\section{Main results}

In total, 70 studies were eligible for inclusion in this review. Half included populations without any consideration for risk status, and half focused on higher-risk groups. Most were conducted in the USA or in high-income countries. On average, studies examined the effects of interventions on four behaviours, most commonly alcohol, tobacco use, drug use, and antisocial behaviour.

We found that for multiple risk behaviours, school-based studies for all young people are more beneficial than a comparator for preventing tobacco use, alcohol use, and physical inactivity, and that they may also be beneficial in relation to illicit drug use and antisocial behaviour. Findings were weaker for cannabis use, sexual risk behaviour, and unhealthy diet. Evidence suggests that certain school-based programmes could have a beneficial impact on more than one behaviour. In contrast, we did not find strong evidence of beneficial effects of interventions for families or individuals for the behaviours of interest, although caution must be applied in interpreting these findings because we identified fewer of these studies. Last, we found seven studies that reported increased levels of engagement in risk behaviours among those receiving the intervention compared to those given the control.

Overall, reviewers judged the quality of the evidence to be moderate or low for most behaviours examined using standardised criteria, with one behaviour found to have very low quality evidence. In part, this was due to concerns around how some studies were conducted, which could have introduced bias.

\section{Conclusions}

Our findings suggest that school-based interventions offered to all children that aim to address engagement in multiple risk behaviours may have a role to play in preventing tobacco use, alcohol use, illicit drug use, and antisocial behaviour, as well as in improving physical activity, among young people, but not in the other behaviours examined. We did not find strong evidence of benefit of interventions for families or individuals. Concerns around reporting of studies and study quality highlight the need for additional robust, high-quality studies to further strengthen the evidence base in this field. 


\section{SUMMARY OF FINDINGS}

\section{Summary of findings for the main comparison.}

Summary of findings table for the effectiveness of targeted individual-level multiple risk behaviour interventions compared to usual practice for outcomes up to 12 months post intervention

Patient or population: children and young people aged 0 to 18 years

Settings: varied settings (home, kindergarten, primary school, secondary school, clinic, community)

Intervention: multiple risk behaviour interventions

Comparison: no intervention/usual practice

\begin{tabular}{|c|c|c|c|c|c|c|}
\hline Outcomes & $\begin{array}{l}\text { Risk with usual } \\
\text { practice }\end{array}$ & $\begin{array}{l}\text { Risk with intervention } \\
(95 \% \mathrm{Cl})\end{array}$ & $\begin{array}{l}\text { Relative effect } \\
(95 \% \mathrm{CI})\end{array}$ & $\begin{array}{l}\text { No. of partici- } \\
\text { pants } \\
\text { (studies) }\end{array}$ & $\begin{array}{l}\text { Quality of the evi- } \\
\text { dence } \\
\text { (GRADE) }\end{array}$ & Comments \\
\hline Tobacco use & 156 per 1000 & $\begin{array}{l}191 \text { per } 1000 \\
\text { (122 to } 288 \text { ) }\end{array}$ & $\begin{array}{l}\text { OR } 1.28 \\
\text { (0.75 to } 2.19)\end{array}$ & $\begin{array}{l}521 \\
(2 \mathrm{RCTs})\end{array}$ & $\begin{array}{l}\oplus \oplus \oplus \ominus \\
\text { Moderate }\end{array}$ & \\
\hline Alcohol use & 613 per 1000 & $\begin{array}{l}618 \text { per } 1000 \\
\text { (559 to } 675)\end{array}$ & $\begin{array}{l}\text { OR } 1.02 \\
\text { (0.80 to } 1.31)\end{array}$ & $\begin{array}{l}1204 \\
\text { (4 RCTs) }\end{array}$ & $\begin{array}{l}\oplus \oplus \oplus \ominus \\
\text { Moderate }\end{array}$ & \\
\hline Cannabis use & 110 per 1000 & $\begin{array}{l}120 \text { per } 1000 \\
\text { (79 to } 179 \text { ) }\end{array}$ & $\begin{array}{l}\text { OR } 1.10 \\
\text { (0.69 to } 1.76)\end{array}$ & $\begin{array}{l}126 \\
\text { (2 RCTs) }\end{array}$ & $\begin{array}{l}\oplus \oplus \oplus \ominus \\
\text { Moderate }\end{array}$ & \\
\hline Illicit drug use & 32 per 1000 & $\begin{array}{l}30 \text { per } 1000 \\
(23 \text { to } 400)\end{array}$ & $\begin{array}{l}\text { OR } 0.94 \\
\text { (0.71 to } 1.25 \text { ) }\end{array}$ & $\begin{array}{l}638 \\
\text { (3 RCTs) }\end{array}$ & $\begin{array}{l}\oplus \oplus \oplus \ominus \\
\text { Moderate }^{a}\end{array}$ & \\
\hline $\begin{array}{l}\text { Vehicle-related } \\
\text { risk behaviour }\end{array}$ & 81 per 1000 & $\begin{array}{l}49 \text { per } 1000 \\
(12 \text { to } 179)\end{array}$ & $\begin{array}{l}\text { OR } 0.59 \\
\text { (0.14 to } 2.48)\end{array}$ & $\begin{array}{l}94 \\
(2 \mathrm{RCTs})\end{array}$ & $\begin{array}{l}\oplus \ominus \odot \ominus \\
\text { Very low } b\end{array}$ & \\
\hline $\begin{array}{l}\text { Sexual risk behav- } \\
\text { iour }\end{array}$ & 610 per 1000 & $\begin{array}{l}533 \text { per } 1000 \\
\text { (434 to } 628 \text { ) }\end{array}$ & $\begin{array}{l}\text { OR } 0.73 \\
\text { (0.49 to } 1.08 \text { ) }\end{array}$ & $\begin{array}{l}494 \\
(2 \mathrm{RCTs})\end{array}$ & $\begin{array}{l}\oplus \oplus \oplus \ominus \\
\text { Moderate }\end{array}$ & \\
\hline
\end{tabular}




\begin{tabular}{|c|c|c|c|c|c|c|c|}
\hline \multirow{11}{*}{ 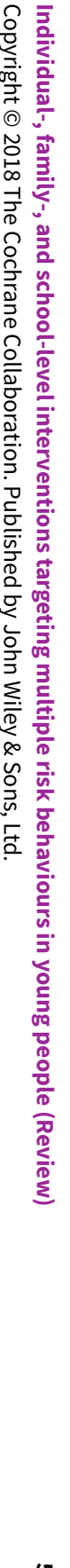 } & Physical activity & 134 per 1000 & N/A & \multicolumn{2}{|c|}{ No studies in meta-analysis } & & \\
\hline & \multicolumn{7}{|c|}{$\begin{array}{l}\text { bowngraded owing to high risk of bias on the basis of blinding and/or high or unclear risk of bias across additional domains, as well as imprecision related to width of the } \\
95 \% \text { confidence interval of the summary estimate and inconsistency between effect estimates }\left(I^{2}=81 \%\right) \text {. } \\
\text { Note that variation was evident in measures of risk with usual practice. Baseline risk measures were calculated at follow-up. When no data were reported for any study in } \\
\text { that meta-analysis, baseline measures were used. }\end{array}$} \\
\hline & \multicolumn{7}{|c|}{$\begin{array}{l}\text { GRADE Working Group grades of evidence } \\
\text { High quality: further research is very unlikely to change our confidence in the estimate of effect. } \\
\text { Moderate quality: further research is likely to have an important impact on our confidence in the estimate of effect and may change the estimate. } \\
\text { Low quality: further research is very likely to have an important impact on our confidence in the estimate of effect and is likely to change the estimate. } \\
\text { Very low quality: we are very uncertain about the estimate. }\end{array}$} \\
\hline & \multicolumn{7}{|c|}{ Summary of findings 2.} \\
\hline & \multicolumn{7}{|c|}{$\begin{array}{l}\text { Summary of findings table for the effectiveness of universal individual-level multiple risk behaviour interventions compared to usual practice for outcomes up to } \\
12 \text { months post intervention }\end{array}$} \\
\hline & \multicolumn{7}{|c|}{ Patient or population: children and young people aged 0 to 18 years } \\
\hline & $\begin{array}{l}\text { Setting: varied set } \\
\text { Intervention: mult } \\
\text { Comparison: no in }\end{array}$ & $\begin{array}{l}\text { gs (home, clinic, } \mathrm{c} \\
\text { le risk behaviour ir } \\
\text { rvention/usual pra }\end{array}$ & nunity) & & & & \\
\hline & Outcomes & $\begin{array}{l}\text { Risk with usual } \\
\text { practice }\end{array}$ & $\begin{array}{l}\text { Risk with intervention } \\
(95 \% \mathrm{Cl})\end{array}$ & $\begin{array}{l}\text { Relative effect } \\
(95 \% \mathrm{CI})\end{array}$ & $\begin{array}{l}\text { No. of partici- } \\
\text { pants } \\
\text { (studies) }\end{array}$ & $\begin{array}{l}\text { Quality of the evi- } \\
\text { dence } \\
\text { (GRADE) }\end{array}$ & Comments \\
\hline & Tobacco use & 32 per 1000 & $\begin{array}{l}33 \text { per } 1000 \\
(10 \text { to } 98)\end{array}$ & $\begin{array}{l}\text { OR } 1.03 \\
(0.32 \text { to } 3.27)\end{array}$ & $\begin{array}{l}1549 \\
(2 \mathrm{RCTS})\end{array}$ & $\begin{array}{l}\oplus \oplus \Theta \Theta \\
\text { Lowa }\end{array}$ & \\
\hline & Alcohol use & 41 per 1000 & $\begin{array}{l}33 \text { per } 1000 \\
(24 \text { to } 45)\end{array}$ & $\begin{array}{l}\text { OR } 0.80 \\
(0.58 \text { to } 1.11)\end{array}$ & $\begin{array}{l}1911 \\
(4 \mathrm{RCTs})\end{array}$ & $\begin{array}{l}\oplus \oplus \oplus \ominus \\
\text { Moderate }\end{array}$ & \\
\hline & Cannabis use & 264 per 1000 & 198 per 1000 & $\begin{array}{l}\text { OR } 0.69 \\
(0.46 \text { to } 1.04)\end{array}$ & $\begin{array}{l}362 \\
(2 \mathrm{RCTS})\end{array}$ & $\begin{array}{l}\oplus \oplus \oplus \ominus \\
\text { Moderate } b\end{array}$ & \\
\hline
\end{tabular}




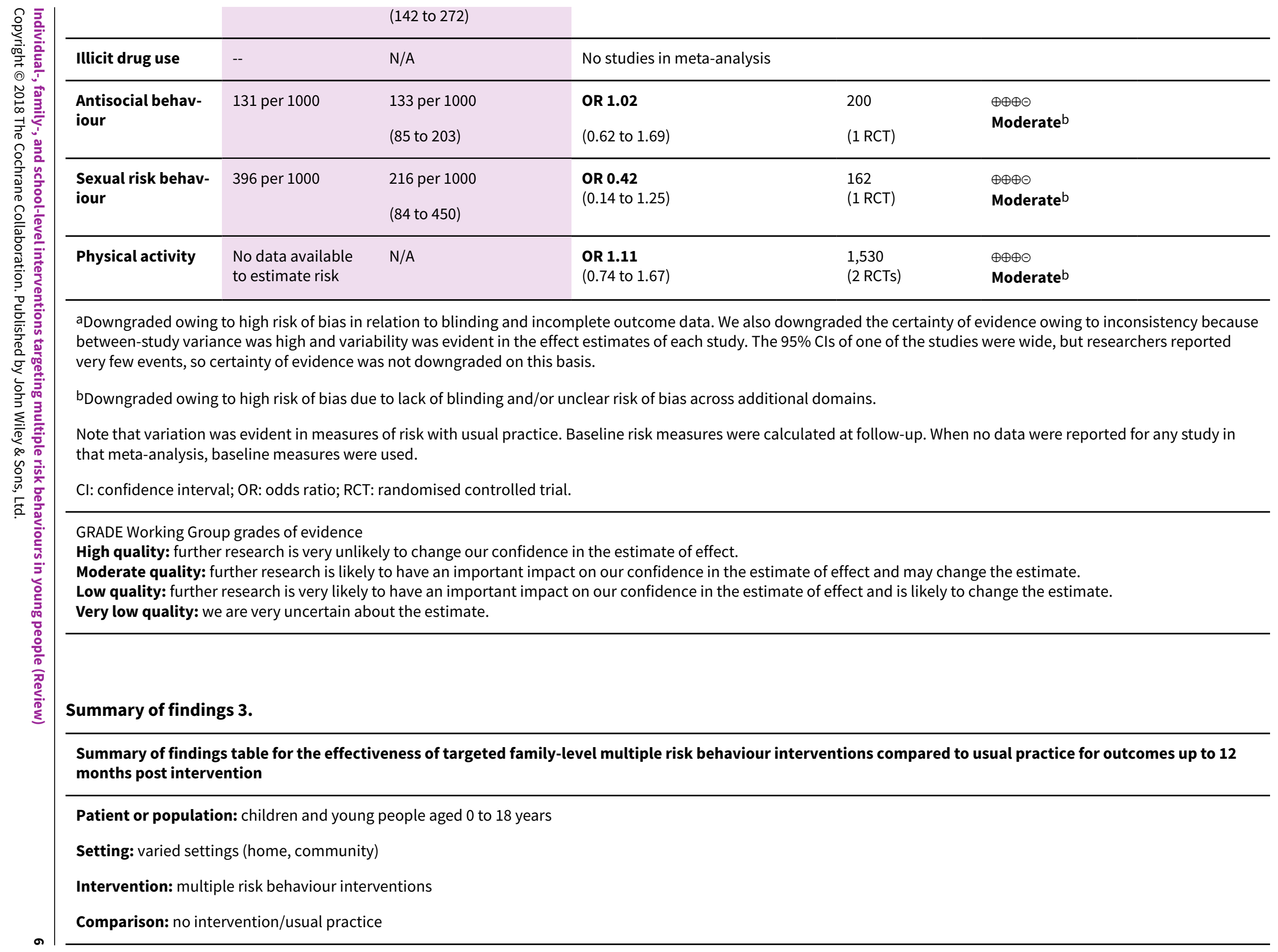

Downgraded owing to high risk of bias in relation to blinding and incomplete outcome data. We also downgraded the certainty of evidence owing to inconsistency because between-study variance was high and variability was evident in the effect estimates of each study. The $95 \%$ Cls of one of the studies were wide, but researchers reported few events, so certainty of evidence was not downgraded on this basis.

Note that variation was evident in measures of risk with usual practice. Baseline risk measures were calculated at follow-up. When no data were reported for any study in that meta-analysis, baseline measures were used.

(: confidence interval; OR: odds ratio; RCT: randomised controlled trial.

Low quality: further research is very likely to have an important impact on our confidence in the estimate of effect and is likely to change the estimate.

Very low quality: we are very uncertain about the estimate.

\section{Summary of findings 3}

Summary of findings table for the effectiveness of targeted family-level multiple risk behaviour interventions compared to usual practice for outcomes up to 12

Patient or population: children and young people aged 0 to 18 years

Comparison: no intervention/usual practice 


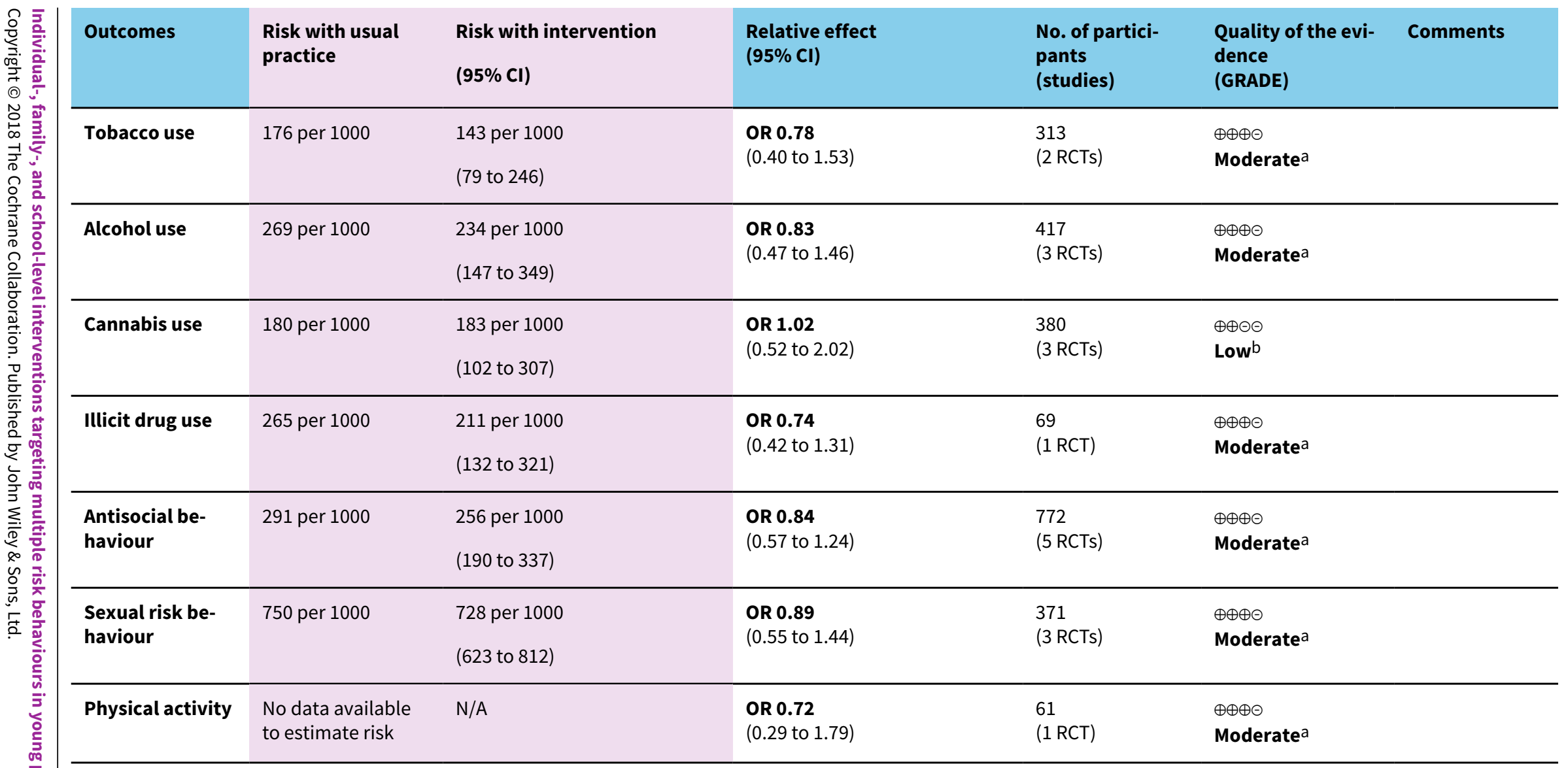

aDowngraded owing to high risk of bias on the basis of blinding and/or high or unclear risk of bias across additional domains.

bDowngraded owing to high risk of bias on the basis of blinding and/or high or unclear risk of bias across additional domains. The quality of the evidence was also downgraded on the basis of inconsistency because between-study variance was high, and although $\mathrm{I}^{2}$ was moderate, inconsistency was evident in effect estimates of individual studies, two of which had small sample sizes.

Note that variation was evident in measures of risk with usual practice. Baseline risk measures were calculated at follow-up. When no data were reported for any study in that meta-analysis, baseline measures were used.

$\mathrm{Cl}$ : confidence interval; OR: odds ratio; RCT: randomised controlled trial.

GRADE Working Group grades of evidence

High quality: further research is very unlikely to change our confidence in the estimate of effect.

Moderate quality: further research is likely to have an important impact on our confidence in the estimate of effect and may change the estimate.

Low quality: further research is very likely to have an important impact on our confidence in the estimate of effect and is likely to change the estimate. 


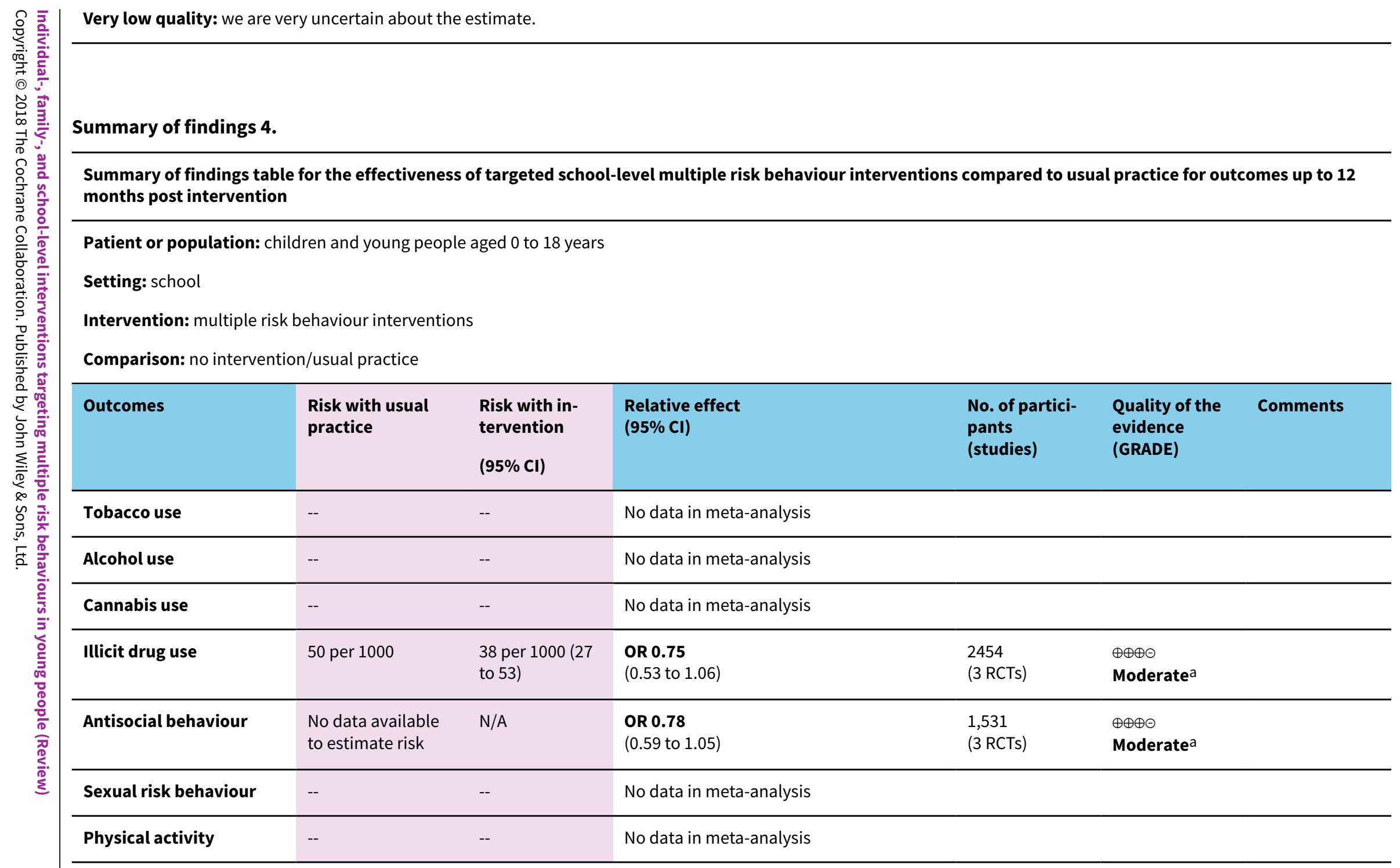

aDowngraded owing to high risk of bias on the basis of blinding and/or high or unclear risk of bias across additional domains.

Note that variation was evident in measures of risk with usual practice. Baseline risk measures were calculated at follow-up. When no data were reported for any study in that meta-analysis, baseline measures were used.

Cl: confidence interval; OR: odds ratio; RCT: randomised controlled trial. 
GRADE Working Group grades of evidence

High quality: further research is very unlikely to change our confidence in the estimate of effect.

Moderate quality: further research is likely to have an important impact on our confidence in the estimate of effect and may change the estimate.

Low quality: further research is very likely to have an important impact on our confidence in the estimate of effect and is likely to change the estimate.

Very low quality: we are very uncertain about the estimate.

Summary of findings 5.

Summary of findings table for the effectiveness of universal school-level multiple risk behaviour interventions compared to usual practice for outcomes up to 12 months post intervention

Patient or population: children and young people aged 0 to 18 years

Setting: school

Intervention: multiple risk behaviour interventions

Comparison: no intervention/usual practice

\begin{tabular}{|c|c|c|c|c|c|c|}
\hline Outcomes & $\begin{array}{l}\text { Risk with usual } \\
\text { practice }\end{array}$ & $\begin{array}{l}\text { Risk with intervention } \\
(95 \% \mathrm{Cl})\end{array}$ & $\begin{array}{l}\text { Relative effect } \\
(95 \% \mathrm{Cl})\end{array}$ & $\begin{array}{l}\text { No. of participants } \\
\text { (studies) }\end{array}$ & $\begin{array}{l}\text { Quality of the evi- } \\
\text { dence } \\
\text { (GRADE) }\end{array}$ & Comments \\
\hline Tobacco use & 54 per 1000 & $\begin{array}{l}42 \text { per } 1000 \\
(33 \text { to } 52 \text { ) }\end{array}$ & $\begin{array}{l}\text { OR } 0.77 \\
\text { (0.60 to } 0.97 \text { ) }\end{array}$ & $\begin{array}{l}15,354 \\
\text { (9 RCTs) }\end{array}$ & $\begin{array}{l}\oplus \oplus \oplus \ominus \\
\text { Moderate }^{a}\end{array}$ & \\
\hline Alcohol use & 163 per 1000 & $\begin{array}{l}123 \text { per } 1000 \\
\text { (98 to } 152 \text { ) }\end{array}$ & $\begin{array}{l}\text { OR } 0.72 \\
\text { (0.56 to } 0.92 \text { ) }\end{array}$ & $\begin{array}{l}8751 \\
\text { (8 RCTs) }\end{array}$ & $\begin{array}{l}\oplus \oplus \oplus \ominus \\
\text { Moderate }^{a}\end{array}$ & \\
\hline Cannabis use & 110 per 1000 & $\begin{array}{l}89 \text { per } 1000 \\
(71 \text { to } 111)\end{array}$ & $\begin{array}{l}\text { OR } 0.79 \\
\text { (0.62 to } 1.01)\end{array}$ & $\begin{array}{l}4140 \\
\text { (5 RCTs) }\end{array}$ & $\begin{array}{l}\oplus \oplus \oplus \ominus \\
\text { Moderate }^{a}\end{array}$ & \\
\hline Illicit drug use & 41 per 1000 & $\begin{array}{l}30 \text { per } 1000 \\
(21 \text { to } 44)\end{array}$ & $\begin{array}{l}\text { OR } 0.73 \\
\text { (0.50 to } 1.07)\end{array}$ & $\begin{array}{l}10,266 \\
\text { (5 RCTs) }\end{array}$ & $\begin{array}{l}\oplus \oplus \ominus \ominus \\
\text { Low }^{b}\end{array}$ & \\
\hline
\end{tabular}




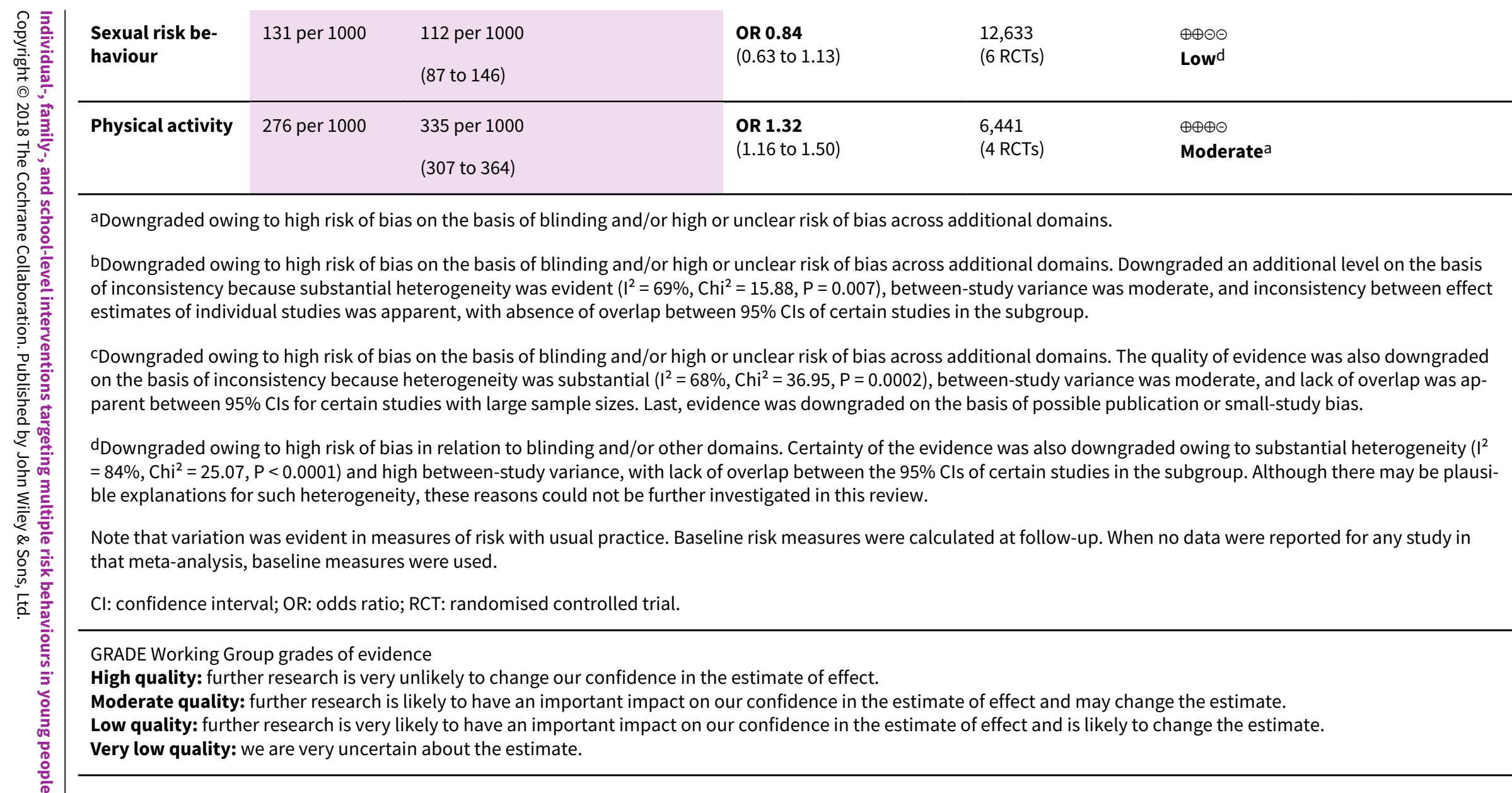




\section{B A C K G R O U N D}

\section{Description of the condition}

Adolescence and young adulthood represent critical periods in the life course in relation to current and future health and wellbeing (Lancet 2012; Lancet Commission on Adolescent Health \& Wellbeing; Patton 2012; World Health Organization 2014). Many of the health risk behaviours that give rise to chronic noncommunicable diseases (NCDs) later in life, such as tobacco use, alcohol use, consumption of calorific foods, and physical inactivity, are initiated during adolescence (Resnick 2012; Sawyer 2012), and they can continue into late adolescence and young adulthood (Mahalik 2013; McCambridge 2011; Ortega 2013; Resnick 2012; Sawyer 2012; Wanner 2006). Engagement in such behaviours can increase risks of low educational attainment, antisocial behaviour, sexually transmitted infections, injury, and substance use dependence during adolescence and young adulthood, and can influence morbidity later in life (Chen 1995; Djousse 2009; Hall 2016; Mason 2010; McCambridge 2011; Ortega 2013; Silins 2015), thus affecting health throughout the life course (World Health Organization 2014). Globally, for instance, alcohol use (7\% of disability-adjusted life-years (DALYs)), unsafe sex (4\%), and illicit drug use (2\%) are among the main risk factors for the incidence of DALYs among young people aged 10 to 24 years (Gore 2011; Mokdad 2013).

Estimates of the prevalence of concurrent tobacco smoking, drinking of alcohol, and recent illicit drug or cannabis use for adolescents in the UK, the USA, and Canada range from $6 \%$ to 13\% (Connell 2009; de Winter 2016; Fuller 2015; Leatherdale 2010; McVie 2005; NHS 2008), and a recent UK report estimated that over $20 \%$ of young people aged 16 engage in two or more substance use and delinquent behaviours (Hale and Viner 2016). Critically, risk behaviours such as smoking, antisocial behaviour, alcohol consumption, and sexual risk behaviour have been shown to cluster in adolescence (Basen-Engquist 1996; Burke 1997; de Looze 2014; Junger 2001; Mistry 2009; Pahl 2010; van Nieuwenhuijzen 2009), and engagement in one risk behaviour increases the likelihood of engagement in others. For example, both smoking and low vegetable intake at age 13.5 increased the odds of engagement in multiple health risk behaviours at age 16 by over twofold (de Winter 2016), and odds ratios for associations between use of individual substances and sexual risk behaviours range between 1.4 and 4.7 (Jackson 2012; Meader 2016).

Engagement in multiple risk behaviours therefore can be viewed as supporting the syndemic concept, whereby synergistic involvement in risk behaviours may worsen the outcomes of engagement in risk behaviours and associated comorbidities later in life (Mendenhall 2017). Given that adolescents comprise a quarter of the world's population worldwide, and often more than a fifth of a country's population, engagement in multiple health risk behaviours and the impact of such engagement represent a significant public health concern. In recognition of the importance of investment in adolescent health as the foundation of health and wellbeing across the life course, recent literature has highlighted the need for greater focus on adolescent health worldwide and the global application of preventive interventions and policies (Lancet 2012; Patton 2014; Resnick 2012; World Health Organization 2014). Evidence has also highlighted the health, economic, and social returns that could be realised from greater global investment in adolescent health (Catalano 2012; Lancet Commission on Adolescent Health \& Wellbeing; Sheehan 2017).

\section{Description of the intervention}

This review examines evidence for interventions that are universal in their approach (i.e. that address whole populations with the aim of preventing the onset or advancement of risk behaviours, as well as those that target particular groups who may be at higher risk (e.g. those identified through screening or other assessment of risk factors such as following referral from the criminal justice system)). Interventions provided at individual, family, and school levels, as well as those that encompass more than one of these domains, are considered. Thus, the interventions considered in this review are wide-ranging in design and may be implemented in a range of settings by providers such as nurses, teachers, or peers, with the goal of impacting behaviours of young people up to 18 years of age.

Interventions focused at the individual level include mentoring, coaching, Internet-level education, conditional cash transfers, development of prosocial networks, and motivational interviewing. Family-focused interventions may involve group sessions or home visits and support, and they aim to improve child-parent interactions, communication, the family environment (e.g. through conflict resolution and problem-solving), parenting skills, parental support, resilience and wellbeing, and knowledge and awareness. Such programmes may incorporate components for children or adolescents, including adolescent skills-building and decision-making curricula, goal-setting, or practice and reinforcement of skills and behaviours. Targeted family-based interventions may be targeted to adolescents at higher risk, such as those who are homeless or are experiencing parental substance abuse.

School programmes aim to target normative beliefs, bonding to school, behavioural goals, and commitments to not engage in risk behaviours and knowledge. They do so by utilising a range of diverse strategies, including formal classroom curricula, peer delivery, behaviour management practices, role-play, goalsetting, and whole-school approaches that aim to change the school climate or ethos. Such domains can be implemented either alone or alongside additional parent or community components, such as parent leaflets, parent-child homework exercises, extracurricular activities, and community engagement activities. Targeted interventions delivered at the school level may focus on particular higher-risk groups, such as those in lower socioeconomic groups, those demonstrating aggressive behaviour, and those identified as being at high risk of school dropout.

\section{How the intervention might work}

The goals of multiple risk behaviour interventions are to prevent engagement in two or more behaviours, to reduce the frequency of engagement in these behaviours, and to reduce the prevalence and impact of short- and long-term negative consequences associated with engagement in those behaviours.

Interventions at individual, family, and school levels may have distinct hypotheses regarding mechanisms of effect, as discussed below. For instance, individual-level interventions may focus more exclusively on improving motivation to act, identifying goals, obtaining normative feedback, coaching, and modelling positive behaviour, with some models such as mentoring based on the 
underlying hypothesis that providing positive role models, support, and prosocial aspirations can change behaviour and reduce risk.

Family-based interventions may focus on provision of skills, knowledge, and support; frequency and quality of parentchild communications; and reinforcement of shared values and behaviours. Grounding of several interventions in social development theory, with a focus on family as one 'unit of socialisation', suggests that sufficient engagement and involvement with family and subsequent positive reinforcement can enhance family attachment, thus helping to underpin strong bonds to school, increased likelihood of involvement with prosocial peers, and reduced likelihood of risk behaviour (Hawkins and Weis 1985). Building on cognitive models, interventions may also work by influencing perceptions of risk, behavioural intentions, and selfregulation via recognition that risk behaviours may result from a reaction to circumstances conducive to risk-taking, depending on the intention and willingness of the individual to engage in the behaviour and perceptions of risk associated with the behaviour (Gibbons 2007). In addition, interventions may act by improving parental monitoring and providing support. In this way, parents serve as a source of socialisation regarding norms and behavioural expectations, but also provide feedback that can influence attitudes and behaviour (Brody 2005; Murry 2009; Murry 2014).

School-based programmes may aim to enhance knowledge, social and emotional skills, resilience, and social competence, thereby improving self-esteem and self-control and reducing the impact of negative peer, family, and/or social influences - all of which can increase the risk of engagement in risk behaviours (Biglan 2004; Chen 1995; Hawkins 2005; Jackson 2010; Mason 2010). Alternatively, programmes may seek to reinforce engagement in healthy behaviours by providing positive role-modelling, addressing perceptions of behaviours and their consequences, and considering social influences and norms.

Theories that seek to explain why risk behaviours cluster during adolescence are relevant to consideration of how interventions might work. First, Moffitt's theory of adolescence-limited antisocial behaviour highlights two distinct categories of individuals with differing natural histories and etiologies of antisocial behaviour: the 'adolescence-limited' group, whose behaviour is limited to adolescence and whose behaviour is normative but in whom risk behaviours may be temporarily sustained via mimicry of antisocial behaviour observed in antisocial peers; and the second, smaller group - the 'life course persistent' group, whose members progress to become lifelong offenders (Moffitt 1993). Second, Jessor's problem behaviour theory (PBT) proposes that clustering of behaviours results from a complex web of interrelated predisposing and protective factors involving interaction between individual and environment (Jessor 1991; Jessor 1992). To date, studies have highlighted shared predisposing or protective factors at individual, intermediate, and structural levels, such as positive mental health, family attachment, peer relationships, socioeconomic status, social environment, and connection with school and religion (Beyers 2004; Catalano 2012; de Looze 2014; Hale and Viner 2016; Jackson 2010; Kipping 2014; Sawyer 2012; Viner 2006).

Determinants of engagement in risk behaviours during adolescence are therefore complex, and it is noteworthy that their antecedents may originate before birth or during the early years of life, and may accumulate early in the life course (Biglan 2004; Catalano 2012; Jessor 1991; Kuh 2003). Early adverse experiences and stressors such as violence, disease, and poor nutrition in infancy and early childhood can affect growth, health, and developmental milestones such as school readiness, literacy, and healthy peer relationships. Interventions that influence early determinants of risk are central to a life course approach and may have a greater impact on an individual's propensity to engage in risk behaviours during adolescence than those that focus on reducing behaviours or mitigating harms once the risk behaviours have become established, as outlined in the logic model (Figure 1). Interventions that provide support to mothers during pregnancy, for instance, may enhance maternal skills, promote healthy behaviours, and enhance emotional well-being, which may increase mother-child interaction and reduce environmental stressors (Biglan 2004; Eckenrode 2010). Interventions provided during the preschool years, which comprise training in parenting or increased preschool attendance, may prevent multiple risk behaviours later in life by reducing stressors within the family environment and by enhancing maternal and child skills (Biglan 2004; Hawkins 2005, Reid 1999; Shepard and Dickstein 2009; Tremblay 1995; Webster-Stratton and Taylor 2001). If unchecked, however, risk can continue to accumulate from early life to adolescence, increasing the likelihood of peer rejection, lack of engagement with school, low academic achievement, and a trajectory towards engagement with risk behaviours (Catalano 2012; Sawyer 2012). Thus, interventions implemented during adolescence can build on investment in the early years and target those at higher risk, or can be implemented with the aim of protecting young people from normative increases in engagement in risk behaviours (Catalano 2012; World Health Organization 2014). 
Figure 1. Logic Model: interventions to prevent multiple risk behaviours in individuals aged 8 to 25 years.

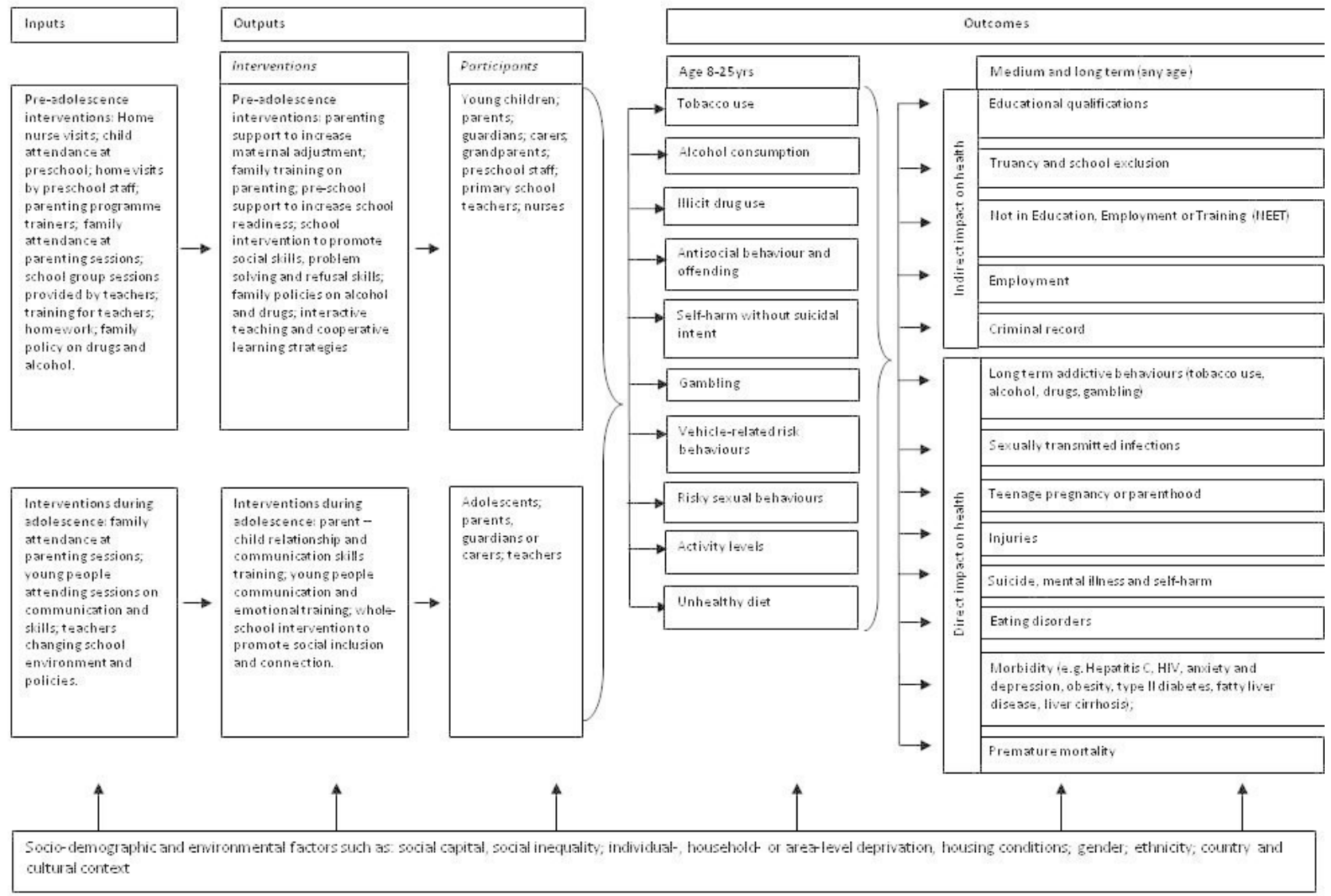

\section{Why it is important to do this review}

Whilst many health interventions aim to prevent single behaviours, and several Cochrane reviews have focused on specific types of interventions to address single behaviours (Carney 2016; Faggiano 2014; Fellmeth 2011; Foxcroft 2011; Livingstone 2010; Mytton 2006), less is known about the effectiveness of interventions that aim to simultaneously prevent a wide range of multiple risk behaviours (Biglan 2004; Jackson 2010). Given that risk behaviours cluster, and that determinants of engagement in these behaviours may overlap, it is possible that multiple-behaviour interventions may be both efficient and effective. A recent scoping review examined characteristics of interventions to prevent multiple risk behaviours but focused on adult populations (King 2015). Of the two systematic literature reviews that have focused on prevention of multiple risk behaviour in young people, one focused on the impact of interventions to target substance use and sexual risk behaviour (Jackson 2010; Jackson 2011), and the other focused on interventions that target substance use, antisocial behaviour, and sexual risk behaviour (Hale 2014), while including only interventions that reported statistically significant effects. To date, therefore, no single Cochrane review has systematically examined evidence relating to the impact of interventions that address multiple behaviours. Critically, there remains no quantitative estimate of effect to guide public health decision-making.
This review considers the effectiveness of individual-, family-, and/or school-level interventions that aim to address tobacco use, alcohol use, illicit drug use, gambling, self-harm, vehicle-risk behaviours, antisocial behaviour, sexual risk behaviour, physical inactivity, and poor nutrition. This review is therefore broader with respect to the number of behaviours, settings, and populations of focus.

Given limited opportunities and resources to prevent risk behaviours, it is important to explore whether targeting multiple behaviours may be more efficient than targeting single behaviours. Greater understanding of the effects of multiple risk behaviour interventions in the context of tightening budgets has substantial potential to influence decisions around commissioning and/or decommissioning of risk prevention interventions for children and young people.

\section{O B J E C T IVES}

\section{Primary research objective}

- To examine the effects of interventions implemented up to 18 years of age for the primary or secondary prevention of multiple risk behaviours among individuals aged eight to 25 years (see MacArthur 2012 for the protocol of this review) 


\section{Secondary research objectives}

- To explore whether effects of the intervention differ within and between population subgroups

- To examine whether effects of the intervention differ by risk behaviours and by outcomes

- To investigate the influence of the setting of the intervention on design, delivery, and outcomes of the interventions

- To investigate the relationship between numbers and/or types of component(s) of an intervention, intervention duration, and intervention effects

- To evaluate whether the impacts of interventions differ according to whether behaviours are addressed simultaneously or sequentially and/or whether behaviours are addressed in a particular order

- To explore the association between clustering of particular behaviours and effects of the interventions

- To assess the cost-effectiveness of the interventions

- To consider the implications of the findings of this review for further research, policy, and practice

In this review, we aim to examine the effects of interventions on each of the studied behaviours, in turn, and through further analyses to ascertain the effects of these interventions on multiple risk behaviours.

\section{METHODS}

\section{Criteria for considering studies for this review}

\section{Types of studies}

We included only randomised controlled trials (RCTs), including cluster RCTs, that aimed to address at least two risk behaviours of interest. We included only RCTs because studies using this design provide the most reliable type of evidence for assessing effects of interventions in that they minimise the risk that findings may have been influenced by confounding (Akobeng 2005). We included RCTs that primarily assessed effectiveness of interventions but also reported findings of a full or partial economic evaluation, and those that reported resource use or costs associated with an RCT intervention. We included only studies with a combined intervention and follow-up period of six months or longer, to enable identification of the impact of interventions over the shorter term without exclusion of studies that were not able to monitor outcomes over a longer period.

\section{Types of participants}

Participants were children and young people aged up to 18 years. Studies were also included in which participants receiving the intervention were parents, guardians, carers, peers, and/or members of a school, as long as the intervention aimed to impact involvement in multiple risk behaviours among children and young people aged up to 18 years. We included interventions targeting participants in subgroups of the population, but we excluded interventions aimed at individuals with clinically diagnosed disorders.

\section{Types of interventions}

Interventions included in this review comprised interventions that aimed to address at least two risk behaviours from among regular tobacco use; alcohol consumption; recent cannabis or other regular illicit drug use; risky sexual behaviours; antisocial behaviour and offending; vehicle-related risk behaviours; self-harm (without suicidal intent); gambling; unhealthy diet; high levels of sedentary behaviour; and low levels of physical activity. We excluded interventions that addressed just two risk behaviours including unhealthy diet, low levels of physical activity, and/ or high levels of sedentary behaviour, to avoid overlap with a previous Cochrane systematic review (Waters 2012). In addition, we excluded interventions that address two or more risk behaviours from among tobacco use, alcohol consumption, and/ or drug use; a separate review will examine these interventions (Hickman 2014). In this way, we excluded interventions that target only healthy eating and physical activity, or only tobacco use, alcohol consumption, and drug use, for example, but included interventions that target healthy eating, physical activity, and risky sexual behaviour; or alcohol use, tobacco use, and antisocial behaviour. Last, we included RCTs delivered at the individual, family, or school level; another Cochrane review will include studies conducted at the community or population level, such as media campaigns or policy, regulatory, or legislative interventions, owing to their distinct study design (Campbell 2012). We classified studies as 'individual' if they recruited participants from the general community setting (but not from the school or family), and if they delivered the bulk of the intervention component(s) in one of the following settings: criminal justice (i.e. prisons or youth offending institutions), general practice surgeries, accident and emergency departments, or community-based settings (for mentoring-only interventions delivered to individuals within a community setting). We classified studies as 'school-based' if researchers recruited participants from schools and delivered most of the intervention components in a school setting, and as 'family-level' if investigators recruited parent(s) or child(ren) from the community and delivered most of the intervention components to the family within the home or in a neutral centre-based environment.

Researchers compared those receiving the intervention versus those receiving usual practice, no intervention, or placebo or attention control. Interventions could be conducted at the individual, family, or school level and could include psychological, educational, parenting, or environmental approaches. As described above, interventions could be provided universally, without regard for the young people's level of risk, or they could be targeted to particular young people or families. Thus, for example, studies could be conducted at an individual level without regard for risk status (universal individual-level interventions), or they could target particular groups of students in schools (targeted school-level interventions). We classified studies as 'universal' in their approach if all school children within a school (or those in a particular year group), all individuals within a community/organisation, or all families within a community were eligible to participate in those studies. This contrasts with interventions classified as 'targeted', usually defined by participant characteristics (e.g. ethnicity, gender, pre-existing behavioural problems/issues). However, for studies implementing an intervention for individuals/families in an area with a high crime rate, a high percentage of social deprivation, or a high percentage of black minority ethnic individuals, or for schools specially selected to include a certain percentage of students with a specific student ethnic population, we viewed interventions as 'universal', as not all participants would be subject to these characteristics. Interventions could start before the onset of behaviours (primary prevention), or they could target those currently engaged in 
risk behaviours (secondary prevention). We excluded stand-alone clinical interventions (e.g. cognitive-behavioural therapy).

\section{Types of outcome measures}

\section{Primary outcomes}

The primary outcome was the primary or secondary prevention of two or more risk behaviours in individuals aged eight to 25 years. This age range was chosen owing to the likelihood of engagement in risk behaviours over this age range and the impact(s) and public health importance of engaging in risk behaviours during this period of the life course. Relatively few studies have examined the epidemiology of multiple risk behaviours; therefore the review includes behaviours that have an adverse impact on health, whether or not the behaviour involves an active desire for 'risk-taking' or immediate gratification. We excluded from this review risk behaviours such as lack of ultraviolet (UV) protection, disordered eating, disordered sleep, and the choking game based on available evidence regarding prevalence, adverse impact on health, or relatedness to included behaviours; or we did so to avoid overlap with, or incorporation of clinically diagnosed disorders. Consultation with the Centre for the Development and Evaluation of Complex Interventions for Public Health ImpRovement (DECIPHer) Public Involvement Advisory Group ALPHA (Advice Leading to Public Health Advancement) and the advisory group for the Avon Longitudinal Study of Parents and Children (ALSPAC) has supported inclusion of the range of behaviours outlined below.

We categorised risk behaviours as follows.

- Tobacco use: regular tobacco use.

- Alcohol consumption: binge drinking (alcohol); heavy/hazardous drinking; regular or problem drinking.

- Drug use: recent cannabis use; recent illicit drug use (other than cannabis); regular illicit drug use.

- Antisocial behaviour and offending: murder; aggravated assault; sexual assault; violence (including domestic or sexual violence); assault with or without injury; gang fights; hitting a teacher, parent, or student; racist abuse; criminal damage; robbery; burglary/breaking and entering; vehicle-related theft; prostitution; selling drugs; joy-riding; carrying a weapon; engaging in petty theft or other theft; pan-handling (begging); buying stolen goods; being noisy and rude; exhibiting disorderly conduct; being a nuisance to neighbours; graffiti (Biglan 2004; Hales 2010).

- Self-harm: self-harm without suicidal intent.

- Gambling: gambling; regular/uncontrolled gambling.

- Vehicle-related risk behaviours: cycling without a helmet; not using a car seatbelt; driving under the influence of alcohol, cannabis, or illicit substances.

- Risky sexual behaviours: unprotected sexual intercourse; early sexual debut experience.

- Activity levels: low levels of physical activity; high levels of sedentary behaviour.

- Unhealthy diet: low levels of fruit and vegetable consumption; low-fibre diet; high-fat diet; high-sugar diet.

We excluded behaviours reported as clinical disorders (e.g. substance use disorder representing a clinical diagnosis). We included studies that addressed behaviours via upstream precursors for which a hypothesis or a clear rationale for the pathway of effect from the precursor to the subsequent behaviour was reported. This was particularly relevant for studies targeting young children (e.g. in primary school settings).

\section{Secondary outcomes}

Secondary outcomes include potential medium- and longer-term outcomes that interventions are aiming to effect.

- Education and employment: educational qualifications; truancy and school exclusion; employment; not being in education, employment, or training (NEET).

- Crime: criminal record/offending; re-offending.

- Long-term addictive behaviours: smoking, alcohol, drugs, gambling.

- Health outcomes: teenage pregnancy or parenthood; sexually transmitted infections; injuries; morbidity (e.g. hepatitis C, HIV, anxiety and depression, obesity, type 2 diabetes, fatty liver disease, liver cirrhosis); suicide/self-harm; premature mortality.

- Harm associated with the process or outcomes of the intervention: for instance, if the extent of engagement in risk behaviours or adverse health outcomes increases as a result of the intervention.

- Cost-effectiveness of the intervention: measures of resource use; costs; or cost-effectiveness of the intervention (e.g. incremental cost-effectiveness ratios (ICERs), incremental cost per qualityadjusted life-year (QALY); cost-benefit ratio).

Given the longer-term adverse consequences of engagement in multiple risk behaviours and the importance of sustained outcomes, we used a primary endpoint for outcome data at the longest follow-up point post intervention, up to a period of 12 months. We grouped outcome data from interventions with longer duration of follow-up into a longer-term category, which included any outcome data collected after 12 months post intervention. When data from more than one time point were reported, we took data from the furthest time point from the end of the intervention for each group.

\section{Search methods for identification of studies}

\section{Electronic searches}

We searched the following databases in May 2012. We conducted updated searches in 2015 - beginning 6 May 2015 and ending 15 May 2015 - and a third updated search, which commenced 10 November 2016 and ended 14 November 2016.

We did not apply any date or language restrictions to our searches. We did not exclude studies on the basis of their publication status. We included abstracts, conference proceedings, and other 'grey literature' if they met the inclusion criteria.

- Australian Education Index (ProQuest) - 1979 to current.

- Bibliomap - database of health promotion research (http:// eppi.ioe.ac.uk/webdatabases/Search.aspx).

- British Education Index (ProQuest) - 1975 to current.

- Campbell Library (http://www.campbellcollaboration.org/lib/).

- Cumulative Index to Nursing and Allied Health Literature (CINAHL) (Ovid) - 1950 to present.

- Clinicaltrials.gov (https://clinicaltrials.gov/). 
- Cochrane Central Register of Controlled Trials (CENTRAL), in the Cochrane Library - 1950 to 2015.

- Dissertation Express - cutdown versions of dissertation abstracts (http://dissexpress.umi.com/dxweb/search.html).

- Database of Promoting Health Effectiveness Reviews (DoPHER) (http://eppi.ioe.ac.uk/webdatabases4/Search.aspx).

- Embase (Ovid) - 1974 to 2015, week 16.

- Education Resources Information Centre (ERIC; ProQuest) - 1966 to current.

- EThOS - British Library electronic theses online (http:// ethos.bl.uk/AdvancedSearch.do?new=1).

- International Bibliography of the Social Sciences - Politics \& Economics (ProQuest) - 1950 to current.

- MEDLINE (Ovid) - 1950 to 6 May 2015.

- PsycINFO (Ovid) - 1806 to 2015, week 17.

- Sociological Abstracts (CSA) - 1952 to current.

Several of the databases and most of the websites that we searched in May 2012 yielded no or very few studies eligible for inclusion. The few eligible studies identified via these databases or websites were also available through searches of Cochrane CENTRAL, Embase, MEDLINE, and PsycINFO. We therefore chose to exclude the following from our updated searches in 2015 and 2016: Bibliomap, Dissertation Express, Clinicaltrials.gov, DoPHER, and EThOS.

The search strategies that we used to search databases can be found in Appendix 1.

\section{Searching other resources}

We carried out handsearches of reference lists of relevant articles to identify additional relevant studies. We contacted experts in the field to identify ongoing research. We carried out citation searches for key studies identified. We also searched the following websites of organisations actively involved in prevention of risk behaviours.

- World Health Organization.

- UNICEF; United Nations.

- World Bank.

- Centers for Disease Control and Prevention.

- National Institutes of Health.

- National Youth Agency.

- Foundations: Joseph Rowntree, Nuffield Trust.

- National Criminal Justice Reference Service.

- Policy organisations - Evidence for Policy and Practice Information and Co-ordinating Centre (EPPI Centre), National Institute for Health and Clinical Excellence (NICE), Scottish Intercollegiate Guidelines Network (SIGN), Department of Health, University of York Centre for Reviews and Dissemination, The King's Fund, Institute for Public Policy Research.

\section{Data collection and analysis}

\section{Selection of studies}

Two review authors independently carried out the initial screening process for the first 500 publications retrieved to ensure quality and accuracy of the process. We selected a further $10 \%$ of studies at random and double-screened them to ensure that the screening process was consistent and accurate throughout. In May 2012 and
May 2015, we conducted this process, which yielded an overall Kappa statistic of 0.83 , reflecting high agreement between study authors. We obtained full-text articles if we required additional information to assess eligibility for inclusion.

We obtained the full texts of eligible articles and, when necessary, grouped together multiple publications arising from a single study. Two review authors screened full-text papers using a prespecified set of criteria for inclusion. We resolved disagreements by discussion; when disagreements persisted, we consulted a third review author to enable a consensus to be reached.

\section{Data extraction and management}

Two review authors independently used a data extraction form created for this review to extract data from included studies. Two review authors had piloted the data extraction form to ensure that it captured study data and could be used to assess study quality effectively. Data extracted from full text studies included the following.

- Lead author, review title, or unique identifier and date.

- Study design.

- Study location.

- Study setting.

- Year of study.

- Theoretical underpinning.

- Context.

- Equity (using PROGRESS Plus (see below for details)).

- Interventions (content and activities, numbers/types of behaviours addressed, duration of interventions, and details of any intervention offered to the control group).

- Participants in the intervention (including number randomised and number included in each intervention group; age at the start of the intervention; and demographic data when possible (e.g. ethnicity, gender, socioeconomic status).

- Scope of the interventions (universal or targeted to high-risk or vulnerable groups).

- Methods of measurement of risk behaviour (self-report or objective measure).

- Duration of follow-up(s).

- Attrition rate.

- Randomisation.

- Allocation concealment.

- Outcome measures post intervention at each stage of follow-up (including unit of measurement).

- Effect size and precision (e.g. 95\% confidence interval).

- Whether clustering was taken into account in cluster RCTs and intracluster correlation coefficient (ICC).

- Methods of analysis.

- Process evaluation (including fidelity, acceptability, reach, intensity, and context of interventions).

- Cost-effectiveness data when provided (e.g. estimates of resource use, source of resources used, estimates and sources of unit costs, price year, currency, incremental resource use and costs, point estimate and measure of uncertainty for incremental resource use, costs and cost-effectiveness, economic analytical viewpoint, time horizon for costs and effects, and discount rate). 


\section{- Any other comments.}

We used the PROGRESS Plus checklist to collect data relevant to equity. This includes place of residence, race/ethnicity, occupation, gender, religion, education, social capital, and socioeconomic status, with Plus representing the additional categories of age, disability, and sexual orientation. We collected PROGRESS Plus factors reported at baseline and follow-up when reported. We resolved disagreements between review authors around data extraction by discussion, or by consultation with a third review author when consensus was not reached by discussion alone. We contacted study authors to obtain additional information or data not available from published study reports, when necessary.

\section{Assessment of risk of bias in included studies}

We assessed risk of bias of included studies using the Cochrane 'Risk of bias' tool (Higgins 2008). For each domain, two review authors rated studies as having 'high', 'low', or 'unclear' risk of bias. We resolved disagreements by discussion and, when necessary, by referral to a third review author. Selection bias included assessment of both adequate sequence generation and allocation concealment. We assessed studies as having low risk of selection bias when study authors reported a clearly specified method of generating a random sequence; and as having low risk of bias associated with lack of allocation concealment when study authors clearly described methods of concealment, such as use of opaque envelopes. We assessed studies as having high risk of performance bias unless study authors explicitly stated that students were blinded to group allocation, although participants could rarely be blinded to the fact that they were participants in an intervention owing to the nature of the studies. When studies clearly stated that outcome assessors were blinded, we judged them as having low risk of bias. When outcomes were assessed by self-report, we rated studies as having high risk of bias when students were unlikely to have been adequately blinded. To assess attrition bias, we considered rates of attrition both overall and between groups, and we assessed whether this was likely to be related to intervention outcomes. We assessed studies as having low risk of reporting bias when a published protocol or study design paper was available and all prespecified outcomes were presented in the report; or when all expected outcomes were reported. If we had additional concerns, such as baseline imbalance between groups, we noted this in the 'other bias' domain.

\section{Measures of treatment effect}

For dichotomous (binary) data, we used odds ratios (ORs) with 95\% confidence intervals (Cls) to summarise results within each study. When ORs were not provided, we calculated ORs and their standard errors (SEs) using reported outcome data. When studies reported ORs that represented the opposite measure (e.g. wearing a condom vs not wearing a condom), we took the inverse of the value.

For continuous outcomes, we extracted or calculated mean differences (MDs) based on final value measurements, ensuring that baseline mean values were sufficiently comparable (i.e. both lay within the standard deviations (SDs) for intervention and control). When this was not the case for baseline mean values in each study arm, we excluded data from the meta-analysis and included them in a table. We calculated a pooled standard deviation from intervention and control SDs at follow-up and standardised results to a uniform scale by calculating standardised mean differences (SMDs).
When studies reported an outcome as dichotomous and others provided a continuous measure, we converted results to dichotomous data, assuming that the underlying continuous measurement had an approximate logistical distribution, using the methods described in Borenstein 2009 (see Chapter 7). We conducted sensitivity analyses to assess the impact of this on study findings.

\section{Unit of analysis issues}

Several interventions that were randomised at the school level did not appear to take clustering of participants into account, for instance, by using a multi-level model or generalised estimating equations. When clustering was not taken into account, and when study authors could not provide adjusted data, we followed the approach suggested in Chapter 16.3.5 of the Cochrane Handbook for Systematic Reviews of Interventions, to conduct an 'approximately correct analysis' (Bush 1989; Fearnow-Kenney 2003; Griffin 2009; Ialongo 1999; Ialongo 1999b; Kellam 2014; Li 2011; Lochman 2003a; Lochman 2004a; McNeal 2004; Nader 1999; O'Neill 2011; Sanchez 2007; Shek 2011). We imputed intracluster correlation coefficients (ICCS) for each outcome, which provide a measure of the relative variability within and between clusters, from other included studies that reported an ICC for the same outcome, to enable the design effect to be calculated. For all analyses, we selected the most conservative ICC for that behaviour. When no ICC was available for that behaviour, we used the largest available ICC for any behaviour to be conservative. We conducted sensitivity analyses, which utilised the lowest reported ICC for the same behaviour. When no ICC was reported, we calculated an average of available ICCS and used this value. A list of the ICCs used in the data analyses is provided in Additional Table 1.

A very small number of trials did not report the number of participants in each study arm. If it was reported that attrition was comparable between study arms, we divided the total $\mathrm{N}$ by two to yield an approximate number for each arm. When we found interventions with multiple study arms, we split the control group to avoid double-counting, as outlined in Section 16.5.4 of the Cochrane Handbook for Systematic Reviews of Interventions (Higgins 2008).

\section{Dealing with missing data}

When we encountered missing or unclear data related to participants or outcomes, we contacted study authors via email. We noted missing data on the data extraction form and took them into account when judging the risk of bias of each study. We excluded from quantitative analyses studies for which insufficient data were available (e.g. in study reports, and when missing data could not be obtained) and included data from study reports in Additional Table 2 .

\section{Assessment of heterogeneity}

We anticipated that the studies included in this review would be heterogeneous with respect to settings, participants, interventions, and outcomes, and so conducted random-effects meta-analyses. We examined heterogeneity via visual inspection of the forest plot, the $\mathrm{Chi}^{2}$ test, and the $\mathrm{I}^{2}$ statistic to assess whether observed variability was compatible with chance. For each outcome, we included subgroups for study setting and focus (individual, family, or school level and universal or targeted). Data were insufficient 
for examination of further subgroupings and/or for exploration of further reasons for heterogeneity.

\section{Assessment of reporting biases}

If we identified sufficient studies (minimum of 10), we used funnel plots to examine the study effect size against the sample size to look for publication bias or small-study effects (Sterne 2011).

\section{Data synthesis}

We applied a random-effects (DerSimonian and Laird) model for meta-analysis using RevMan 5.3, so we could allow for the assumption that different studies are estimating different, yet related, intervention effects (DerSimonian 1986). Therefore the pooled effect estimates described in the review should be interpreted as the average treatment effect. For each outcome, we grouped studies according to study type (school, family, or individual level, and whether they were universal or targeted). We obtained the overall effect estimate for each subgroup. We did not calculate a summary estimate of subgroups together owing to the distinct nature of each group. However, we presented data for each group on a single forest plot for simplicity in presentation.

When studies presented results of multi-arm trials, review authors (JR, GJM, DMC RC, MH) agreed on the extent to which additional study arms included a component with a distinct mode of delivery. When the additional study arm included an intervention component delivered in a distinct way, we treated these as separate comparisons, as described in Higgins 2008 (see Chapter 16 (16.5.4)). For studies in which the additional study arm was similar, we combined data from different study arms, as described in Higgins 2008 (see Chapter 7 (7.7.3.8)). When data were presented separately by gender or by ethnic group, we also combined them using methods described in Higgins 2008 (see Chapter 7 (7.7.3.8)). We combined measures that were highly related (e.g. overt and covert delinquency), using methods described in Borenstein 2009 (see Chapter 24). We used the value for the correlation between measures, when provided. Otherwise, we used a value of 0.5 , as recommended.

When study authors reported multiple measures of behaviours (e.g. condom use, number of sexual partners), we extracted all data and we selected as the main outcome measure the outcome that represented the behaviour leading to greatest harm for public health, as demonstrated by evidence regarding consequences for mortality or morbidity. For studies with long follow-up that reported multiple repeated measurements, or that measured the outcome at multiple points in time, we extracted data from each time point. We included two follow-up periods: up to 12 months (short-term) and over 12 months (long-term), which included follow-up periods up to our maximum of 15 years post intervention. Quantitative analyses included data related to the longest point of follow-up within the follow-up category (e.g. for short-term followup) and used the time point closest to 12 months' post intervention as the primary endpoint.

\section{Subgroup analysis and investigation of heterogeneity}

We planned to conduct subgroup analyses; however data within each subgroup for each behavioural outcome were insufficient for further analyses to examine the impact of interventions according to our prespecified characteristics of population subgroups, settings, and intervention components.

\section{Sensitivity analysis}

We conducted sensitivity analyses to examine the impact of using conservative versus low imputed ICCS when accounting for clustering in school-based RCTs. For analyses that demonstrated a beneficial effect, we also conducted sensitivity analyses to examine whether statistical transformations had an impact on the findings. Last, given that a small number of studies were conducted in middle-income countries, we conducted sensitivity analyses to test whether findings were affected by their exclusion. We did not conduct sensitivity analyses around risk of bias of included studies because exclusion of those at high or unclear risk of bias in key domains of selection and performance bias left insufficient studies in each study type subgroup.

\section{Summarising and interpreting results}

We assessed the overall quality of the body of evidence for each outcome using the Grades of Recommendation, Assessment, Development and Evaluation (GRADE) approach (Guyatt 2008), in keeping with standards for reporting of Cochrane Intervention Reviews, specifically, MECIR (Methodological Expectations of Cochrane Intervention Reviews) conduct standards 74 and 75 . GRADE is also the most widely used approach for summarising confidence in effects of interventions by outcome; it is used by over 20 organisations internationally (Higgins 2008; Higgins 2018). The GRADE approach defines the quality of the evidence, involving consideration of within-study risk of bias (methodological quality), directness of evidence, inconsistency (heterogeneity), precision of effect estimates, and risk of publication bias. We downgraded evidence depending on the presence of these factors. We summarised findings for seven key outcomes (alcohol use, tobacco use, cannabis use, illicit drug use, sexual risk behaviour, antisocial behaviour, and physical activity) in Summary of findings for the main comparison, which includes the number of participants and studies for each outcome, the intervention effect for the relevant subgroup, and a measure of the quality of the body of evidence, using the GRADE approach.

Although we note that blinding is not possible in the interventions included in this review, we downgraded all studies on the basis of high risk of bias related to lack of blinding and, in some cases, unclear risk of bias related to allocation concealment and/ or selective reporting, which were largely due to lack of clarity in reporting. We downgraded studies on the basis of inconsistency if we found evidence of substantial heterogeneity $\left(\mathrm{I}^{2}\right)$, as well as poor overlap in 95\% confidence intervals between studies and large between-study variance $\left(\operatorname{tau}^{2}\right)$. We took into account the extent of consistency in the direction of point estimates of individual studies. Further to subgroups by study type, we were unable to conduct additional subgroup analyses to explore possible explanations for observed heterogeneity, such as variation in intensity or duration of interventions, or age at implementation; these could not be examined further.

Most of the studies included in this review were conducted in highincome countries $(n=67 ; 96 \%)$, one $(1.4 \%)$ in a lower-middleincome country (Saraf 2015), and one (1.4\%) in an upper-middleincome country (Matthews 2016); one (1.4\%) was a joint study including an upper-middle-income country and a high-income country (Lana 2014). Although this fact limits generalisability to these settings, we did not downgrade the quality of the evidence on the basis of indirectness because the objective of the review 
was to examine the overall effectiveness of interventions that aimed to prevent engagement in multiple risk behaviours, rather than the impact of these interventions in particular settings or geographical regions. Furthermore, although it could be argued that generalisability for certain targeted family-level studies (e.g. the Nurse Family Partnership) could be limited owing to contextual factors related to service provision, this was relevant only to a small proportion of studies; thus we did not consider it of sufficient concern to justify downgrading the quality of the evidence on this basis. We downgraded the quality of evidence on the basis of possible small-study or publication bias if we identified at least ten studies assessing that outcome, and if asymmetry was evident in the funnel plot.

\section{RES U L T S}

\section{Description of studies}

\section{Results of the search}

During the course of this review, we conducted three database searches (in 2012, 2015, and 2016). The initial search in 2012 produced 19,220 records, yielding 18,706 reports after removal of duplicate records. We obtained a further 9302 records in May 2015, leaving 5847 reports after removal of duplicates; we identified 5944 articles in November 2016, for a total of 3138 articles after removal of duplicates. Therefore, in total we identified 34,680 titles, screened 27,691 unique articles (2012: $n=18,706 ; 2015: n=5847$; 2016: $n=3138$ ), and we obtained 424 full-text articles. From these full-text articles, we identified 70 studies for inclusion in this review (Figure 2). We have provided a description of each study in the Characteristics of included studies table. We will include ongoing studies and those awaiting classification in future updates of this review. 
Figure 2. Study flow diagram (searches conducted in 2012, 2015, and 2016).

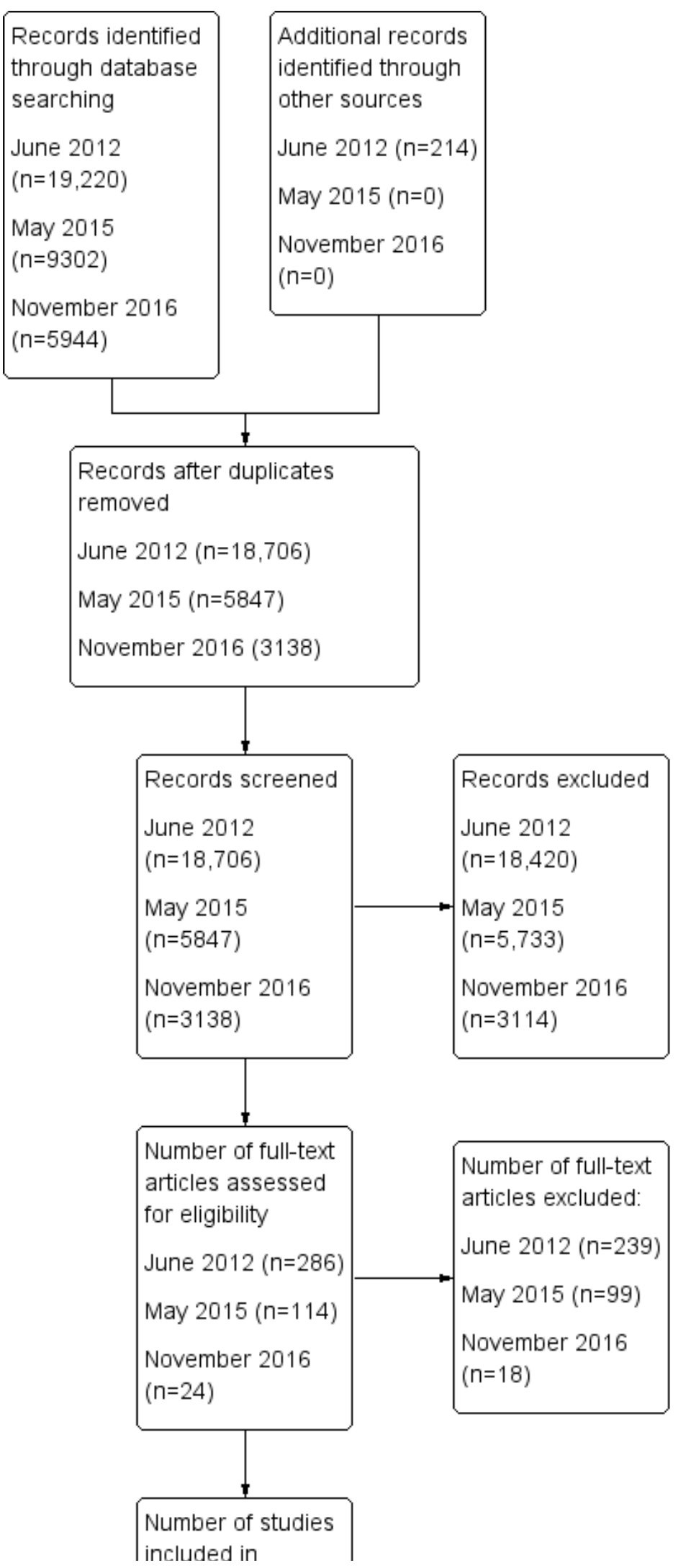


Figure 2. (Continued)

\begin{tabular}{l}
$\begin{array}{l}\text { Number of studies } \\
\text { included in } \\
\text { qualitative } \\
\text { synthesis } \\
\text { June } 2012 \text { ( } n=47) \\
\text { May } 2015 \text { ( } n=15) \\
\text { November } 2016 \\
\text { (n=6 regarding } 8 \\
\text { interventions) } \\
\text { Total studies: } \\
n=70\end{array}$ \\
$\begin{array}{l}\text { Number of studies } \\
\text { included in } \\
\text { quantitative } \\
\text { synthesis } \\
\text { (meta-analysis) } \\
n=54\end{array}$ \\
\hline
\end{tabular}

\section{Included studies}

We included 70 studies in this review. We have provided summaries of each of the 70 included studies in the Characteristics of included studies table, and in Additional Table 3, we have provided details about each study and behaviours targeted by study type (e.g. universal family-level interventions, targeted school-level interventions).

\section{Countries}

Most studies were conducted in the USA ( $n=55 ; 79 \%$ ); the remainder were conducted in Europe $(n=8)$, Canada $(n=2)$, Australia $(n=1)$, Hong Kong $(n=1)$, India $(n=1)$, Spain and Mexico $(n=1)$, and South Africa $(n=1)$. Based on the World Bank classification of countries by income, most studies were conducted in high-income countries ( $n=67 ; 96 \%)$, one $(1.4 \%)$ was conducted in a lower-middle-income country, one (1.4\%) was a joint study including an upper-middle-income country and a high-income country, and one (1.4\%) was conducted in an upper-middle-income country.

\section{Study types}

Of the 70 included studies, 12 (17\%) provided targeted individuallevel interventions, and four (6\%) used universal individual-level interventions; 17 interventions (24\%) were targeted to families, three $(4 \%)$ were universal family-level interventions, six $(9 \%)$ were targeted in school-based studies, and most $(n=28 ; 40 \%)$ explored use of universal school-based studies.

Most individual-level interventions were mentoring or motivational interventions targeting young people at risk (e.g. of antisocial or criminal behaviour, of alcohol-related injury or harm). Researchers recruited participants from community (Minnis 2014), clinic (Bernstein 2010a; Cunningham 2012; Johnson 2015; Monti 1999; Nirenberg 2013; Walker 2002), and criminal justice settings (Freidman 2002; Freudenberg 2010; Redding 2015), and via relevant service providers, agencies, or charities (Berry 2009; Dolan 2010; Tierney 1995), or through schools (Bodin 2011; Lana 2014; Wagner 2014). Family-level interventions were targeted to particular families on the basis of a variety of factors (e.g. ethnicity (AfricanAmerican (Beach 2016; Brody 2012; Murry 2014) or MexicanAmerican (Pantin 2009; Sanchez 2007)); some populations included parents who were being treated for substance misuse (Catalano 1999), others included young mothers (Kitzman 2010; Olds 1998), and others included those living in deprived communities (e.g. targeting those in public housing) (Li 2002; Schwinn 2014). Schoolbased interventions included whole-school approaches as well as those that were focused towards individuals of a particular age or age range, and some were implemented over multiple school years. School-based interventions that were targeted in their focus utilised procedures such as screening on the basis of ratings of aggressive and/or disruptive behaviours (Conduct Problems Prevention Research Group 2010; Lochman 2003a; Lochman 2004a), criteria of high-risk status (e.g. through truancy, low grade point average (GPA), disciplinary action, or referral by a teacher (Sanchez 2007; Shetgiri 2011)), or low socioeconomic status and low levels of parental education (Schweinhart 1993).

Several studies included multiple components, but additional components tended to be implemented alongside a primary component directed to a particular group (e.g. parental involvement with homework, parental leaflets in addition to a 
school-based curriculum targeted to secondary school students). Most interventions were based on education, mentoring, and/or behavioural approaches to risk reduction, but two interventions involved the provision of financial support or financial incentives (Minnis 2014; Morris 2003). One intervention offered earnings supplements to single parents who left welfare for full-time employment (Morris 2003), and the other offered small cash payments upon completion of activities such as receiving job training or education, or visiting a reproductive health clinic (Minnis 2014).

Seventeen studies were conducted and analysed as cluster RCTs (Beets 2009; Bond 2004; Conduct Problems Prevention Research Group 2010; DeGarmo 2009; Flay 2004a; Gottfredson 2010; Griffin 2006; Kellam 2014; Li 2011; Melnyk 2013; Nader 1999; O'Neill 2011; Piper 2000a; Sanchez 2007; Saraf 2015; Walter 1989; Wolfe 2012).

\section{Age range of study participants}

Interventions generally targeted children and young people across a one- or two-year age range. Most interventions were implemented when children were between 9 and 14 years of age.

Among individual-level interventions, mentoring-style interventions tended to include young people of a range of ages in mid to late adolescence, with two mentoring interventions including young people aged 10 to 16 (Dolan 2010; Tierney 1995), and another including those aged 13 to 18 (Bodin 2011). Those targeting alcohol use and related risks tended to target those in later adolescence, reflecting levels of engagement in these risk behaviours. Among family-level interventions, the age range of participants was variable. Broadly, four interventions targeted those across early childhood to mid-adolescence (Averdijk 2016; Beach 2016; Catalano 1999; Morris 2003), two targeted children early in the life course (up to two years of age) in the Nurse Family Partnership (Kitzman 2010; Olds 1998), six targeted those in early adolescence (nine to 12 years of age) (Bonds 2010; Connell 2007; Gonzales 2012; Kim 2011; Murry 2014; Schwinn 2014), and eight targeted those in mid-adolescence (13 to 16 years of age) (Brody 2012; Estrada 2015; Haggerty 2007a; Jalling 2016; Jalling 2016b; Li 2002; Milburn 2012; Pantin 2009), demonstrating a focus on primary prevention by targeting interventions at relatively earlier stages of adolescence before the rise in engagement in multiple risk behaviours.

Similarly, among school-based interventions, although variable with regard to the age of participants, most were implemented before age 16 , again demonstrating a focus on primary prevention. A small number were implemented before or during primary school, with one provided during preschool (Schweinhart 1993), three spanning kindergarten through to twelfth grade (Averdijk 2016b; Beets 2009; Conduct Problems Prevention Research Group 2010), and three targeting those aged 6 to 8 years (lalongo 1999; Kellam 2014) (note that the lalongo 1999 publications describe two interventions). Eighteen interventions targeted children aged eight to 14, six targeted those aged 13 to 16 (Bond 2004; Johnson 2015; Melnyk 2013; Sanchez 2007; Shetgiri 2011; Wolfe 2012), and two targeted those 13 to 19 years of age (D'Amico 2002a; FearnowKenney 2003).

\section{Intervention duration}

The duration of interventions was variable, both overall and by study type. Twenty-two studies were less than three months in duration (31\% of all studies), nine lasted three to six months (13\%), 15 took place over a six- to 12 -month period (21\%), and 24 continued for longer than one year (34\%). The latter were mostly school-based interventions, which were provided over successive school years, although the total time period of intervention, for instance in terms of total hours of exposure/lessons, would have been markedly less.

Individual-level interventions were generally shorter, with all 12 targeted interventions of less than six months' duration. Targeted family-level interventions, in contrast, were mixed, with seven of 17 (41\%) less than three months in duration and seven of 17 (41\%) over six months in duration, three of which took place over several years (the Nurse Family Partnership and the Self-Sufficiency Project: Kitzman 2010; Morris 2003; Olds 1998). As mentioned above, most school-based interventions were provided over a period longer than one year, with four of six targeted interventions over 12 months in duration, one of which was implemented over a 10-year period (Conduct Problems Prevention Research Group 2010), and none with duration less than six months. Sixteen of the $28(57 \%)$ universal school-based interventions took place over a period longer than 12 months, several of which were provided for a total period of at least three school years (Beets 2009; Bush 1989; Flay 2004a; Griffin 2006; Griffin 2009; Li 2011; Nader 1999; Piper 2000a; Shek 2011; Simons-Morton 2005; Walter 1989). Two studies were implemented for less than three months (D'Amico 2002a; DeGarmo 2009).

Alongside duration, the variable intensity of interventions should be noted. For instance, several individual-focused interventions, particularly those involving motivational interviewing, were short in duration and were characterised by lower intensity compared to individual-focused mentoring interventions, for instance, when participants meet their mentor weekly over the course of a year. Similarly, certain family-based interventions involved, for example, five to nine weekly parent and child-focused sessions, joint group sessions, and home visits (Beach 2016; Brody 2012; Catalano 1999; Gottfredson 2010; Kim 2011; Murry 2014; Pantin 2009), but others involved up to two years of nurse home visitation to infants up to the age of two (Kitzman 2010; Olds 1998); in contrast, another intervention involved a low-intensity singlesession consisting of video and role-play (Li 2002). In relation to school-based programmes, studies included those with multiple components (e.g. family, curriculum, school policy components) provided over one or more school years (Beets 2009; Conduct Problems Prevention Research Group 2010; Flay 2004a; Johnson 2015; Li 2011; Nader 1999), others included classroom sessions provided over one or more school years (Fearnow-Kenney 2003; O'Neill 2011; Perry 2003a; Shek 2011; Wolfe 2012), and still others applied a whole-school approach (Bond 2004), demonstrating how the intensity of different interventions can vary between studies. We have provided further details for each study in Table 3.

\section{Post-intervention follow-up}

Among all studies, the duration of follow-up was relatively evenly distributed between those that provided a post-test follow-up ( $n=17 ; 24 \%$ of studies) (i.e. at completion of the intervention), those that followed participants for up to six months following the intervention ( $n=14 ; 20 \%)$, those that followed participants for six to 12 months ( $n=16 ; 23 \%$ of studies), and those that followed participants for over one year $(n=23 ; 33 \%)$. Seventeen studies conducted follow-up after at least five years post intervention, the 
largest number of which were family-targeted studies ( $\mathrm{n}=6$ studies) (Bonds 2010; Gonzales 2012; Kitzman 2010; Murry 2014; Olds 1998; Pantin 2009) and studies providing universal school interventions ( $\mathrm{n}=7$ studies) (Averdijk 2016b; DeGarmo 2009; Griffin 2006; Ialongo 1999; Kellam 2014; Nader 1999) (note that lalongo 1999 describes two interventions).

Among the 16 individual-level interventions, none conducted follow-up over one year, although seven studies conducted sixto 12 -month follow-ups. In contrast, all studies using universal family-level interventions provided longer-term follow-up (over 12 months post intervention) (Averdijk 2016; Connell 2007; Haggerty 2007a), and eight of the 17 studies examining targeted familylevel interventions ( $47 \%$ of these studies) provided longer-term follow-up (> 12 months) (Bonds 2010; Brody 2012; Estrada 2015; Gonzales 2012; Kim 2011; Kitzman 2010; Murry 2014; Olds 1998). Two school-level intervention studies provided longer-term follow up (> 5 years) (Conduct Problems Prevention Research Group 2010; Schweinhart 1993), and three provided follow-up data at six to 12 months (Lochman 2003a; Lochman 2004a; Sanchez 2007). Lastly, a substantial proportion of studies exploring universal schoolbased interventions reported follow-up data at completion of the intervention ( $n=11$ of 28 studies; $39 \%$ ). Five studies (18\%) reported short-term follow-up data at less than six months, two reported data at up to 12 months, and 10 (36\%) reported longer-term followup data (> 12 months).

\section{Theoretical framework}

Most studies ( $n=53$ of 70 studies; $76 \%$ ) reported that theory informed development of the intervention; some used more than one theory, and $17(24 \%)$ omitted mention of a theoretical model. Eligible studies used 33 different theoretical models. Social learning theory informed 15 studies, with social-cognitive/cognitive-behavioural theories informing seven additional interventions. Life skills training, the social development model, and social field theory each informed the development of three interventions. Remaining theories informed one or two of the interventions and included the theory of triadic influence, the transtheoretical model, the Health Belief Model, theories of child development, problem behaviour theory, the Health Promoting Schools framework, and the life course model. Notably, some interventions were based on multiple theories. Nevertheless, few interventions provided detail about how programmes were informed by, or incorporated, such theories.

\section{Intervention focus}

On average, studies aimed to address four of the primary behaviours (range two to five). Five studies aimed to address five of the primary behaviours (Bond 2004; Haggerty 2007a; Li 2002; McNeal 2004; Pantin 2009; Piper 2000a; Shek 2011; Wolfe 2012). Most studies targeted alcohol use $(n=55)$, drug use $(n=53)$, and/or antisocial behaviour $(n=53)$ alongside other behaviours, followed by tobacco use $(n=42)$. In terms of combinations of behaviours addressed, most studies targeted substance use together with antisocial behaviour ( $n=49 ; 70 \%$ ). Relatively fewer studies addressed sexual risk behaviour ( $\mathrm{n}=$ 21); 20 interventions in these studies also targeted tobacco, alcohol, and/or drug use, with 16 of those (76\%) simultaneously addressing antisocial behaviour. Physical activity and nutrition were targeted by fewer studies again ( $n=9, n=11$, respectively), and no studies targeted self-harm or gambling alongside other behaviours (although we note that interventions that aimed to address only physical inactivity and nutrition were excluded from this review (see Types of interventions)). Education/attainment and mental health were targeted by 19 and 17 studies, respectively, alongside other risk behaviours. We have provided further details regarding the numbers and range of behaviours targeted by different interventions, by study type, in Table 4 and Table 5 .

\section{Process data}

A large proportion of eligible studies reported some process evaluation data ( $n=47$ of $70 ; 67 \%$ ). Many lacked detail about how the intervention was implemented. Among studies that reported process data, $47(100 \%)$ provided quantitative data and $14(30 \%)$ qualitative data, with 47 studies $(100 \%)$ providing details about fidelity or intensity of the intervention. Nevertheless, few studies conducted in-depth analyses of whether the intervention was delivered as intended, any deviations from study protocols and manuals, and/or mechanisms by which the intervention had an effect on behaviour. This is reflected in the comparative lack of qualitative data, which may complement quantitative data in evaluating fidelity and contextual factors such as barriers to delivery and/or engagement and uptake.

\section{Economic data}

Only four studies reported economic data: one targeted a schoollevel intervention with 40-year follow-up (Schweinhart 1993), two separate studies examined the family-level Nurse Family Partnership intervention (Kitzman 2010; Olds 1998), and one was a universal school-level study (Wolfe 2012).

\section{Equity}

All studies provided data regarding age of participants at baseline, and most provided a breakdown by gender (63 studies; 90\%), with one intervention provided to female participants only. Fiftyeight studies (83\%) also reported the proportions of participants in different ethnic groups, although three studies included individuals of a particular race/ethnicity. However, only 39 studies (56\%) provided data regarding socioeconomic status of participants. Those that did highlighted factors such as parental income, education, or occupation; the proportion of participants residing in public housing; or the proportion of participants receiving free school meals. Few reports presented findings according to ethnic group (two studies), gender (nine studies), or socioeconomic status (two studies). In addition, only two studies were reported from low- or middle-income countries; this limits the generalisability of our findings to these settings. Most papers reported sources of funding for studies; only six failed to provide details. Most studies were supported by government agencies or charities, with one study supported by a foundation or a private source alone or in conjunction with other sources. We have provided further details for individual studies in the Characteristics of included studies table. Further analyses have been performed by our research group to examine equity and effects of the interventions included in this review (Tinner 2018).

\section{Adverse events and outcomes}

Seven studies reported adverse events (two of which - Jalling 2016, Jalling 2016b - were reported from a three-arm trial) (Conduct Problems Prevention Research Group 2010; Jalling 2016; Jalling 2016b; Morris 2003; Nirenberg 2013; Sanchez 2007). First, in the Self- 
Sufficiency Project (SSP) (Morris 2003), which involved provision of financial support for parents who left welfare for employment, mothers in the intervention group reported lower average school achievement for their children than for children in the control group. However, results showed no significant programme control differences in the proportion of parents reporting that their children were performing below average in school. Nearly $19 \%$ of children in the programme group said they were below average in at least one subject in school, compared to about $14 \%$ in the control group $(P<0.05)$. The SSP was also associated with increased frequency of minor delinquent activity for older children (aged 15 to 18 ; $P<0.05$ ), but this was not the case for their younger peers (aged 12 to 14); and the programme led to increased use of tobacco, alcohol, and drugs by approximately four percentage points (Morris 2003).

For the Fast Track study (Conduct Problems Prevention Research Group 2010), the death rate at age 25 -year follow-up was $2.5 \%$ for the intervention group and $1.6 \%$ for the control group, and the incarceration rate for the intervention group was $6.3 \%$ compared to $5 \%$ for the control group (approximately 19 years following intervention implementation), although study authors noted that these findings were not statistically significant. Sanchez 2007 also reported that Reconnecting Youth was associated with worse outcomes in the intervention group for conventional peer bonding, high-risk peer bonding, and prosocial weekend activities compared to the control group.

Nirenberg 2013 reported that participants in the intervention arm reported more speeding and distracted driving and higher levels of hazardous drinking compared to those in the control group. Study authors also reported reduced odds of having at least one high-risk driving or alcohol police charge in the intervention arms and no differences between study arms in relation to dangerous driving or alcohol, drugs, and driving. Study authors postulated (1) that this finding may have resulted from discussion of behaviours among participants during intervention sessions, which might have led to greater sensitivity in reporting risky driving behaviours (response shift bias), or (2) that those in the intervention group might have reported the behaviour more readily.

Lastly, investigators reported that the odds of illicit drug use were higher in the two intervention arms (Comet 12-18 and ParentSteps) of a three-arm trial (odds of any drug use: Comet 12-18: OR 3.52, $95 \% \mathrm{Cl} 1.23$ to 10.10 ; ParentSteps: OR $3.23,95 \% \mathrm{Cl} 3.23$ to 9.08 ). Study authors suggested that this may be due to measurement error, in that illicit drug use (in the past six months) increased between baseline and follow-up among intervention groups but decreased in the control group, whereas evidence suggests that illicit drug use increases during adolescence in Sweden. Study authors also highlighted that the small sample size may have given rise to uncertainty in the effect estimate.

\section{Missing data}

We contacted the authors of 34 studies to obtain additional study or outcome data. We received responses from the authors of 23 studies; data from four studies were not available owing to time elapsed since the studies were conducted (D'Amico 2002a; Griffin 2009; Ialongo 1999; Ialongo 1999b); we received additional study data from 11 studies (Conduct Problems Prevention Research Group 2010; Gottfredson 2010; Li 2011; Lochman 2003a; Lochman 2004a; McNeal 2004; Milburn 2012; O'Neill 2011; Pantin 2009; Shek 2011; Shetgiri 2011).

\section{Ongoing studies}

From updated searches in November 2016, we identified nine ongoing studies that may be eligible for inclusion in this review; we have listed these in the Characteristics of ongoing studies table. Four were school-based, one was school- and community-based, one was based in a further education setting, one at a university, one in the community, and one in general practice. Two studies were conducted in the UK, two in Australia, two in the USA, one in Italy, one in Guatemala, and one in Brazil. All were universal. Six studies addressed tobacco use, alcohol use, physical inactivity, and nutrition; one addressed substance use and sexual risk behaviour; and one addressed tobacco use, alcohol use, sexual risk behaviour, and violence. One was an online intervention study, and another a video game intervention study (for details of each study, see Characteristics of ongoing studies).

\section{Excluded studies}

We have summarised in the Characteristics of excluded studies table studies that did not meet eligibility criteria, such as addressing two or more eligible behaviours or being randomised controlled trials.

\section{Risk of bias in included studies}

We have provided in Figure 3 a summary of the overall risk of bias assessment for included studies, and we have given detailed summaries of study-specific judgements by study in Figure 4 . Overall, a large proportion of studies were at high or unclear risk of bias, and many studies lacked sufficient detail to permit a judgement around risk of different forms of bias. 
Figure 3. Risk of bias graph: review authors' judgements about each risk of bias item presented as percentages across all included studies.

Random sequence generation (selection bias)

Allocation concealment (selection bias)

Blinding of participants and personnel (performance bias)

Blinding of outcome assessment (detection bias)

Incomplete outcome data (attrition bias)

Selective reporting (reporting bias)

Other bias

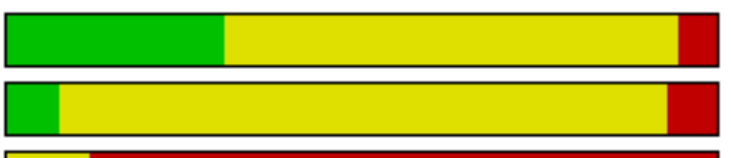

L
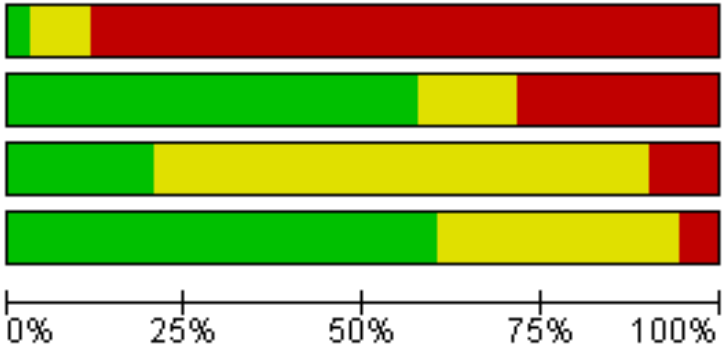

\begin{tabular}{lllll}
\hline $0 \%$ & $25 \%$ & $50 \%$ & $75 \%$ & $100 \%$
\end{tabular}

Low risk of bias

Unclear risk of bias

High risk of bias 
Figure 4. Risk of bias summary: review authors' judgements about each risk of bias item for each included study.

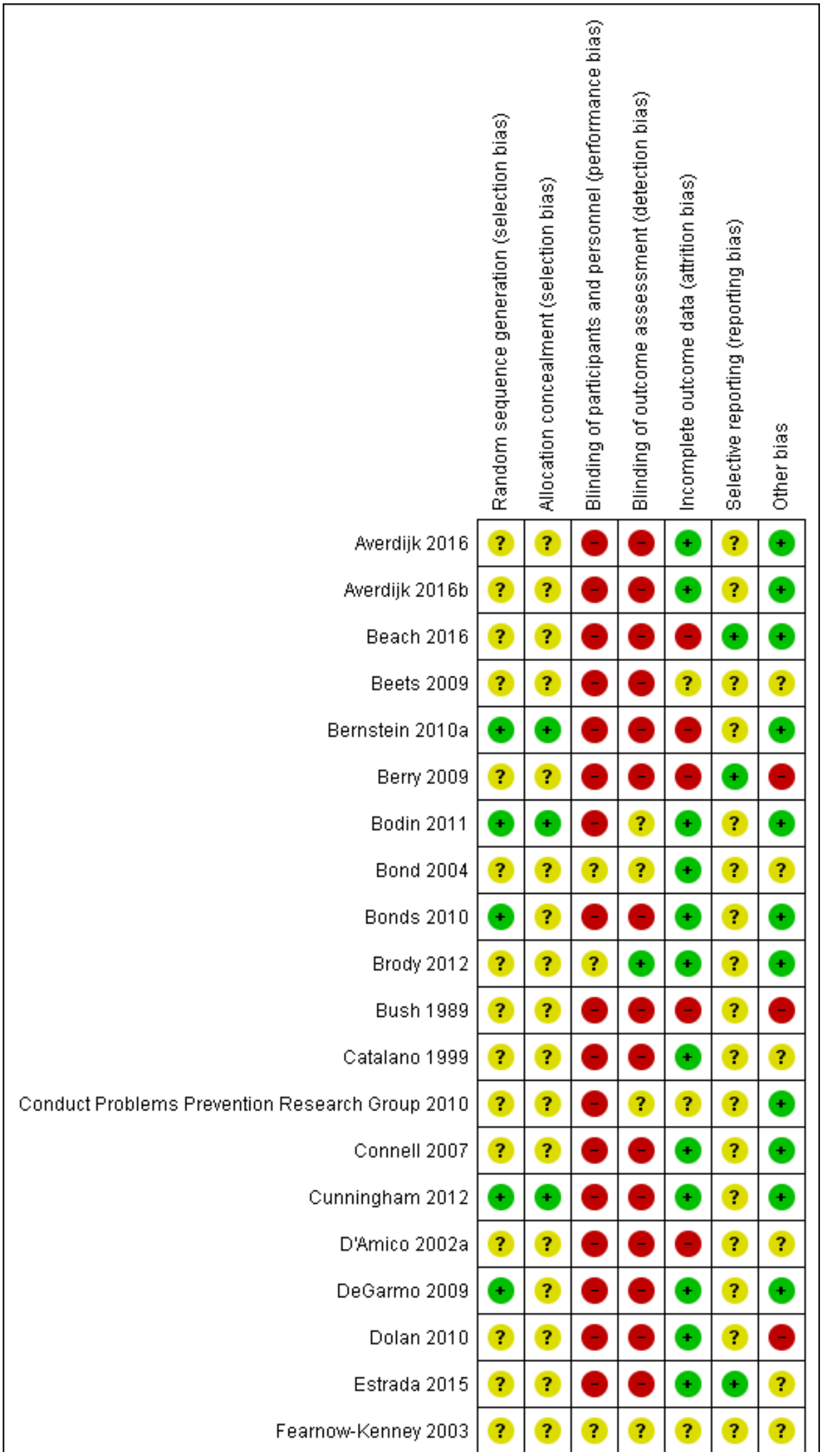


Figure 4. (Continued)

\begin{tabular}{|c|c|c|c|c|c|c|c|}
\hline Fearnow-Kenney 2003 & $?$ & $?$ & $?$ & $?$ & $?$ & $?$ & $?$ \\
\hline Flay 2004a & $?$ & $?$ & $?$ & $\odot$ & $\odot$ & $?$ & $?$ \\
\hline Freidman 2002 & $?$ & $?$ & - & $\odot$ & $\odot$ & $?$ & $?$ \\
\hline Freudenberg 2010 & $?$ & $?$ & $\odot$ & 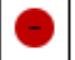 & $?$ & $?$ & + \\
\hline Gonzales 2012 & $\oplus$ & $?$ & $\odot$ & + & $\odot$ & $?$ & + \\
\hline Gottfredson 2010 & $\odot$ & $?$ & $\odot$ & $\odot$ & + & $?$ & + \\
\hline Griffin 2006 & $?$ & $?$ & $\odot$ & $\odot$ & $\odot$ & $\odot$ & $?$ \\
\hline Griffin 2009 & - & $\odot$ & - & $\odot$ & + & $?$ & $?$ \\
\hline Haggerty 2007a & $?$ & $?$ & $\odot$ & $\odot$ & + & $?$ & + \\
\hline lalongo 1999 & + & $?$ & $\odot$ & $\odot$ & + & $?$ & $?$ \\
\hline lalongo 1999b & + & $?$ & - & $\odot$ & + & $?$ & $?$ \\
\hline Jalling 2016 & $\odot$ & $\odot$ & - & $\odot$ & $\oplus$ & + & $?$ \\
\hline Jalling $201 \mathrm{bb}$ & $\odot$ & - & $\odot$ & $\odot$ & + & + & $?$ \\
\hline Johnson 2015 & + & - & $\odot$ & $\odot$ & + & + & $?$ \\
\hline Kellam 2014 & $?$ & $?$ & $\Theta$ & $\Theta$ & $?$ & $?$ & + \\
\hline Kim 2011 & + & $?$ & - & $\odot$ & + & $?$ & + \\
\hline Kittman 2010 & $?$ & $?$ & - & $\Theta$ & + & $?$ & + \\
\hline Lana 2014 & + & $?$ & 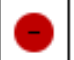 & 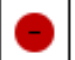 & $\odot$ & $\odot$ & + \\
\hline Li 2002 & + & $?$ & - & $\odot$ & $?$ & $?$ & + \\
\hline Li 2011 & + & $?$ & $\odot$ & $\odot$ & $?$ & $?$ & + \\
\hline Lochman 2003a & $?$ & $?$ & $\Theta$ & $\Theta$ & $\Theta$ & ? & $?$ \\
\hline Lochman 2004a & $?$ & $?$ & $\odot$ & $\odot$ & $\odot$ & $?$ & + \\
\hline LoSciuto 1999 & $?$ & $?$ & $\odot$ & $\odot$ & + & $?$ & $\odot$ \\
\hline Matthews 2016 & $\odot$ & $\odot$ & - & $\Theta$ & $\odot$ & $?$ & $?$ \\
\hline McNeal 2004 & $?$ & $?$ & $\Theta$ & $\odot$ & $\odot$ & $?$ & + \\
\hline Melnyk 2013 & $?$ & $?$ & $?$ & $\odot$ & $?$ & $\odot$ & $\odot$ \\
\hline Milburn 2012 & $\odot$ & $?$ & $\odot$ & $\odot$ & $\odot$ & $?$ & $\odot$ \\
\hline Minnis 2014 & $?$ & $?$ & $\odot$ & $\odot$ & $?$ & + & + \\
\hline Monti 1999 & $?$ & $?$ & - & $\odot$ & + & $?$ & + \\
\hline Morris 2003 & $?$ & $?$ & - & $\Theta$ & $?$ & $?$ & + \\
\hline Murry 2014 & $?$ & $?$ & 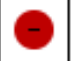 & $\Theta$ & + & $\odot$ & + \\
\hline
\end{tabular}


Figure 4. (Continued)

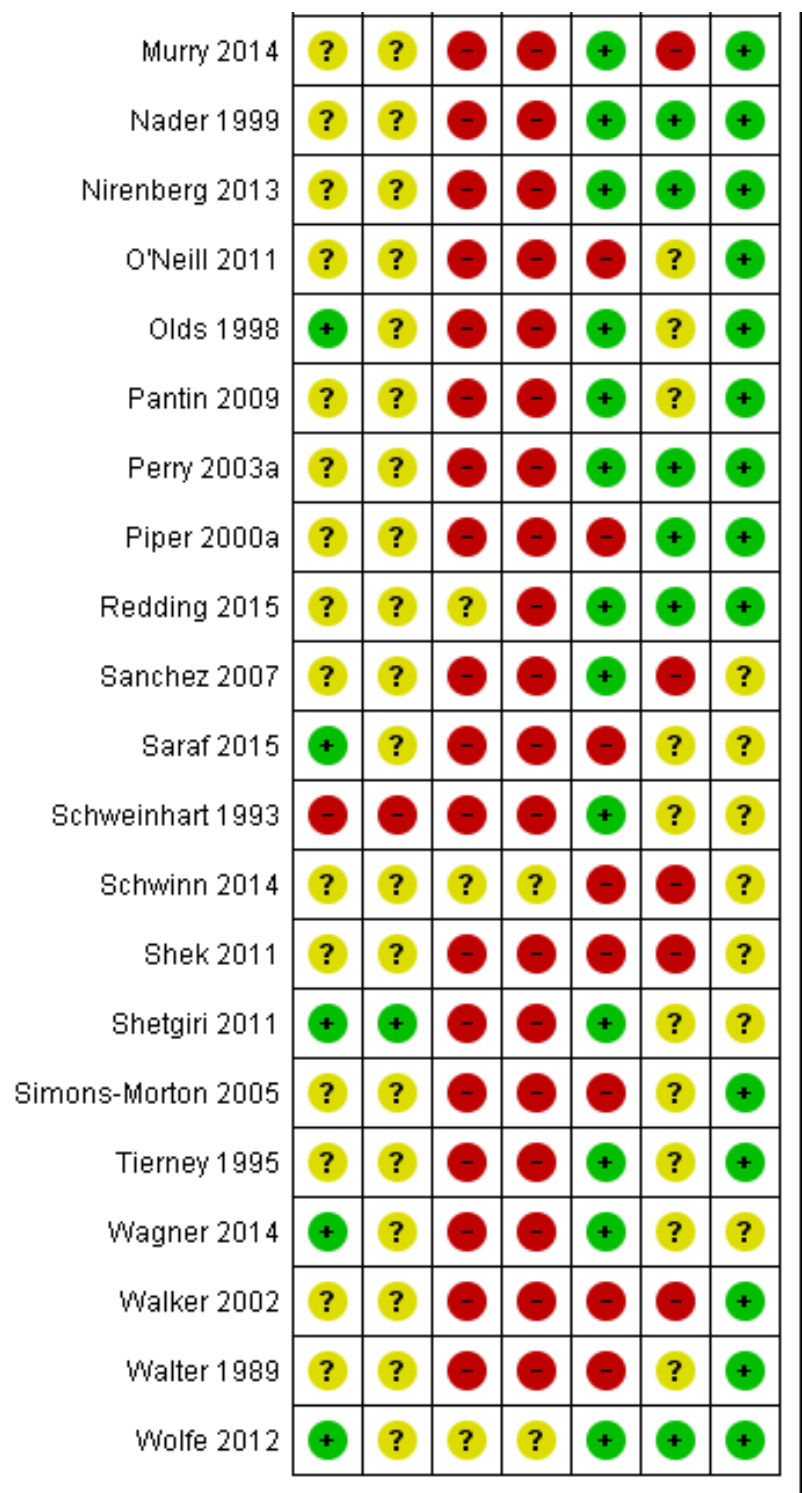

\section{Allocation}

Most studies lacked detail with regard to reporting around the method of randomisation and the use and/or method of allocation concealment; thus we classed these studies as having unclear risk of bias. In particular, researchers rarely reported concealment of allocation and/or the method of concealment. Thus, we judged only 19 studies as having low risk of selection bias on the basis of random sequence generation (Bernstein 2010a; Bodin 2011; Bonds 2010; Cunningham 2012; DeGarmo 2009; Gonzales 2012; Gottfredson 2010; lalongo 1999; lalongo 1999b; Kim 2011; Lana 2014; Li 2002; Li 2011; Milburn 2012; Olds 1998; Saraf 2015; Shetgiri 2011; Wagner 2014; Wolfe 2012).

\section{Blinding}

We classed most studies as having high risk of performance bias owing to lack of blinding of participants. We judged only two interventions to present low risk of detection bias (Brody 2012; Gonzales 2012). Without adequate blinding of participants and personnel, it is possible that outcome measurements, made mostly via self-report of the frequency or extent of engagement in risk behaviours, could have been influenced, particularly because data collection occurred in the school context for many of the included studies. It should be noted, however, that it is generally not possible to blind interventions of the kind included in this review, and so assessments of high risk of bias are somewhat inevitable.

\section{Incomplete outcome data}

Judgements around risk of attrition bias among studies were variable, with 20 studies judged to be at high risk of attrition bias (Bernstein 2010a; Berry 2009; Bush 1989; D'Amico 2002a; Flay 2004a; Freidman 2002; Griffin 2006; Lana 2014; Li 2011; Lochman 2003a; Lochman 2004a; Milburn 2012; O'Neill 2011; Piper 2000a; Saraf 2015; Schwinn 2014; Shek 2011; Simons-Morton 2005; Walker 2002; Walter 1989) owing to high levels of attrition overall (> 30\%), differential attrition, and/or lack of imputation to manage missing data. We judged 33 studies to be at low risk of attrition bias owing to low attrition in the study overall, clear descriptions related to 
differential attrition, and/or imputation (Bodin 2011; Bond 2004; Bonds 2010; Brody 2012; Catalano 1999; Connell 2007; Cunningham 2012; DeGarmo 2009; Dolan 2010; Gonzales 2012; Gottfredson 2010; Griffin 2009; Haggerty 2007a; lalongo 1999; Ialongo 1999b; Kim 2011; Kitzman 2010; LoSciuto 1999; McNeal 2004; Monti 1999; Murry 2014; Nader 1999; Nirenberg 2013; Olds 1998; Pantin 2009; Perry 2003a; Redding 2015; Sanchez 2007; Schweinhart 1993; Shetgiri 2011; Tierney 1995; Wagner 2014; Wolfe 2012). Sixteen studies conducted imputation or used an appropriate method to account for missing data (Bodin 2011; Bonds 2010; Conduct Problems Prevention Research Group 2010; Connell 2007; DeGarmo 2009; Dolan 2010; Gonzales 2012; Gottfredson 2010; Haggerty 2007a; Kellam 2014; Kim 2011; Li 2011; Murry 2014; O'Neill 2011; Redding 2015; Wagner 2014).

\section{Selective reporting}

Only two studies had a published and accessible protocol for the intervention (Lana 2014; Melnyk 2013); in many cases, the extent of selective reporting bias was unclear because we were not able to judge whether all expected outcomes had been reported according to prespecified protocols. We judged seven studies to be at high risk of selective reporting bias owing to absence of data for expected outcomes or time points (Griffin 2006; Melnyk 2013; Murry 2014; Sanchez 2007; Schwinn 2014; Shek 2011; Walker 2002), with some studies lacking clarity in the presentation of data.

\section{Other potential sources of bias}

Other sources of bias identified included the possibility of contamination between study arms, for instance, when students were randomised within schools at the level of the individual or the classroom. If this were the case, the effect of the intervention would be diluted. When we encountered other issues, such as baseline imbalance, we also noted this in this domain. Studies with long-term follow-up measured and reported multiple outcomes; thus, there remains a possibility that positive findings might have been identified by chance, particularly when such interventions had broad aims and were implemented early in the life course (e.g. Schweinhart 1993), such that the full range of outcomes could not be clearly prespecified.

\section{'Summary of findings'}

Among the outcomes listed in the 'Summary of findings' tables (Summary of findings for the main comparison), we judged that much of the evidence related to tobacco use, alcohol use, cannabis use, illicit drug use, sexual risk behaviour, and physical activity was of moderate or low quality, primary owing to risk of bias and inconsistency in findings between studies. For antisocial behaviour, we judged the evidence to be of very low quality owing to high risk of bias across domains, as well as identification of heterogeneity between studies and funnel plot asymmetry. We judged that the odds ratios observed among universal school-level interventions for tobacco, alcohol, and drug use; antisocial behaviour; and sexual risk behaviour, which ranged between 0.72 and 0.83 , and the size of the effect observed for physical activity reflected reductions in engagement in risk behaviours that could be of potential public health importance at the population level.

\section{Funnel plots}

Within each study type subgroup, we identified too few studies ( $\mathrm{n}$ $<10$ ) to examine possible publication bias or small-study effects, with the exception of antisocial behaviour (universal school-based interventions) (Figure 5). The funnel plot for universal schoolbased interventions targeting antisocial behaviour shows an under-representation of small studies reporting negative findings, suggesting possible small-study effects or possible reporting biases leading to overestimation of effectiveness (Sterne 2011). This was also evident in funnel plots that included all studies of each study type addressing antisocial behaviour (Figure 6; Figure 7; Figure 8). 
Figure 5. Funnel plot. Outcome 7: antisocial behaviour and offending (short-term): universal school-based interventions.

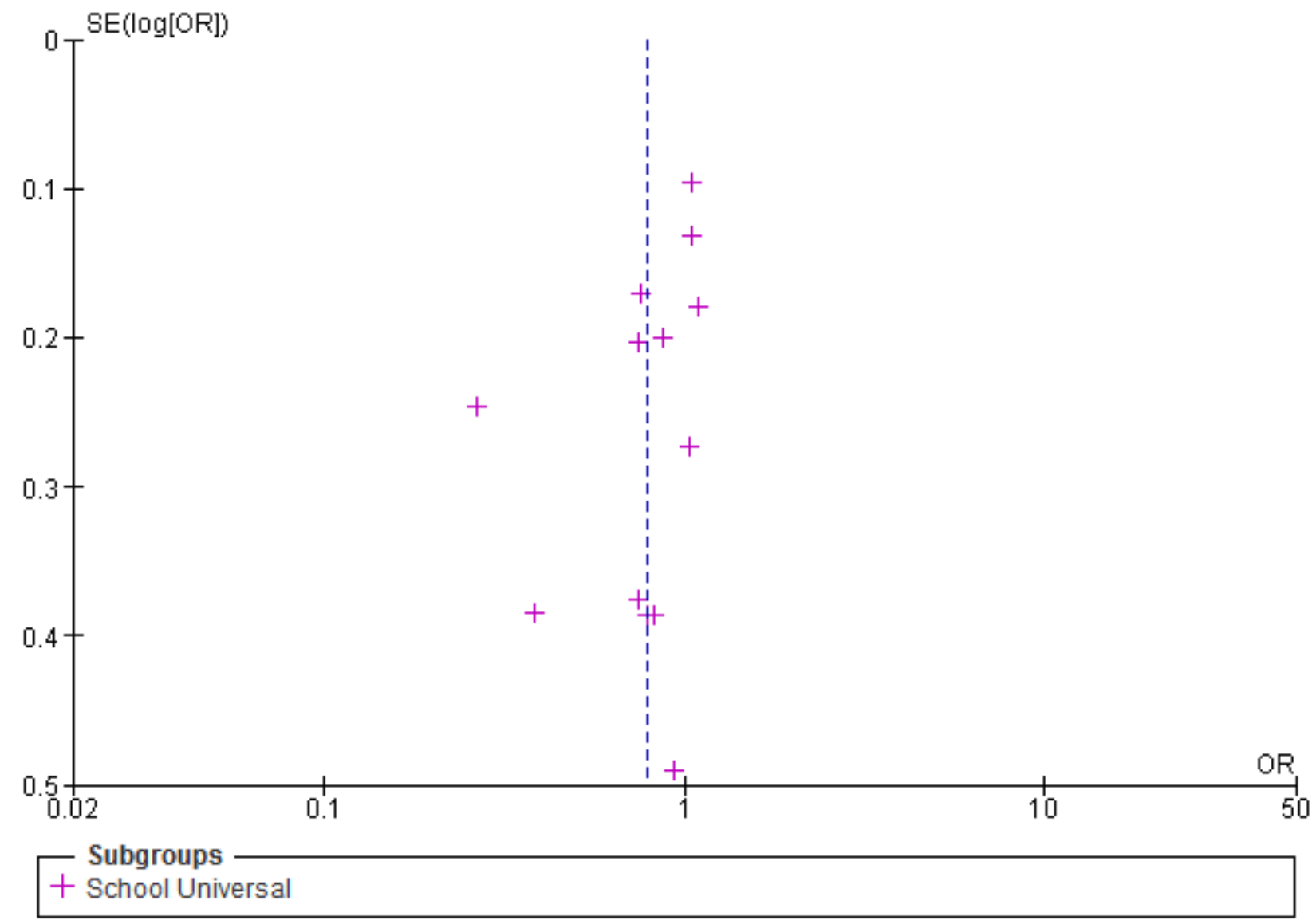


Figure 6. Funnel plot of comparison. Outcome 7: antisocial behaviour and offending. Outcome 7.1: antisocial behaviour and offending - any (short-term).

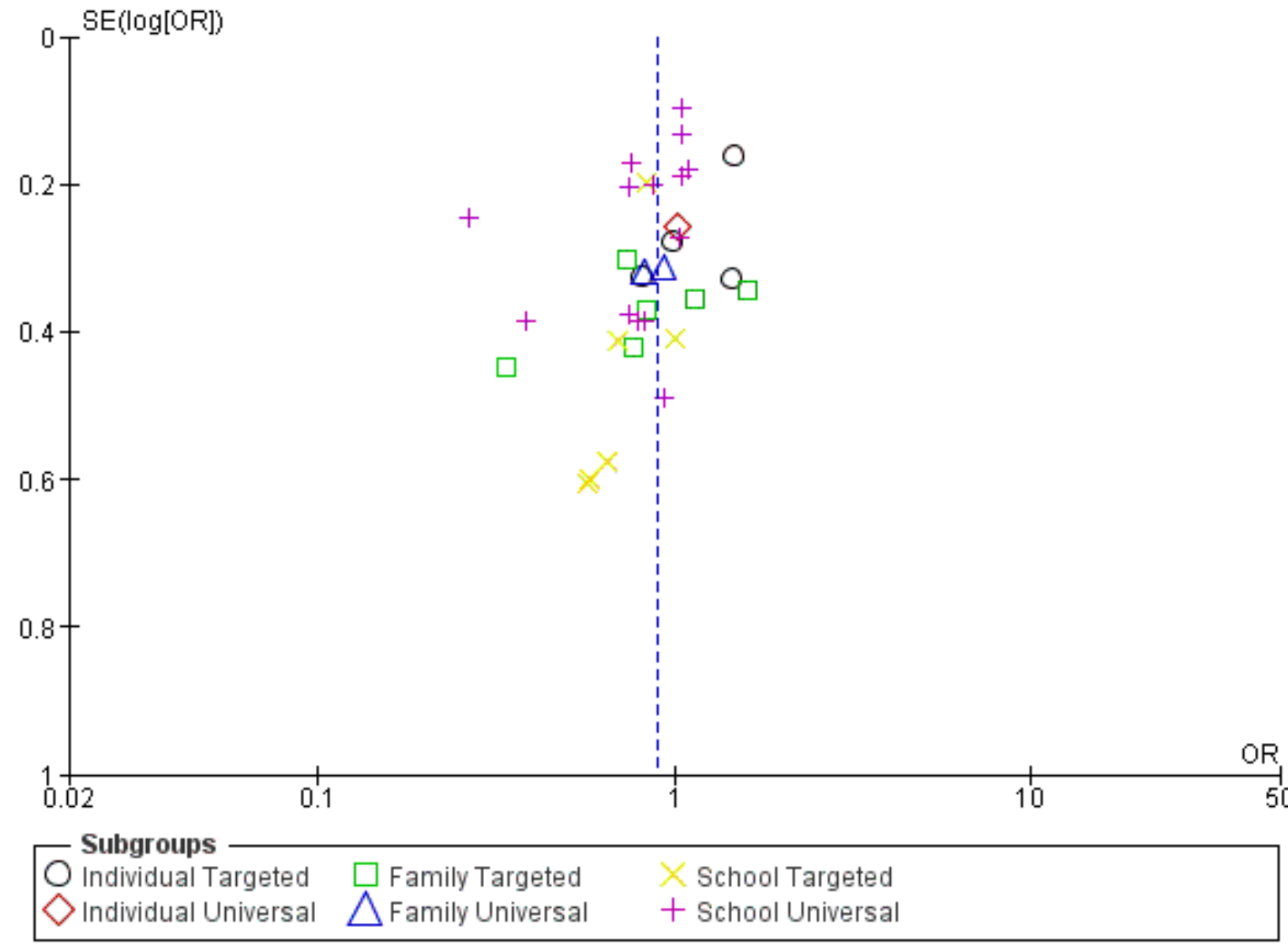


Figure 7. Funnel plot of comparison. Outcome 7: antisocial behaviour and offending. Outcome 7.2: violent offences (short-term).

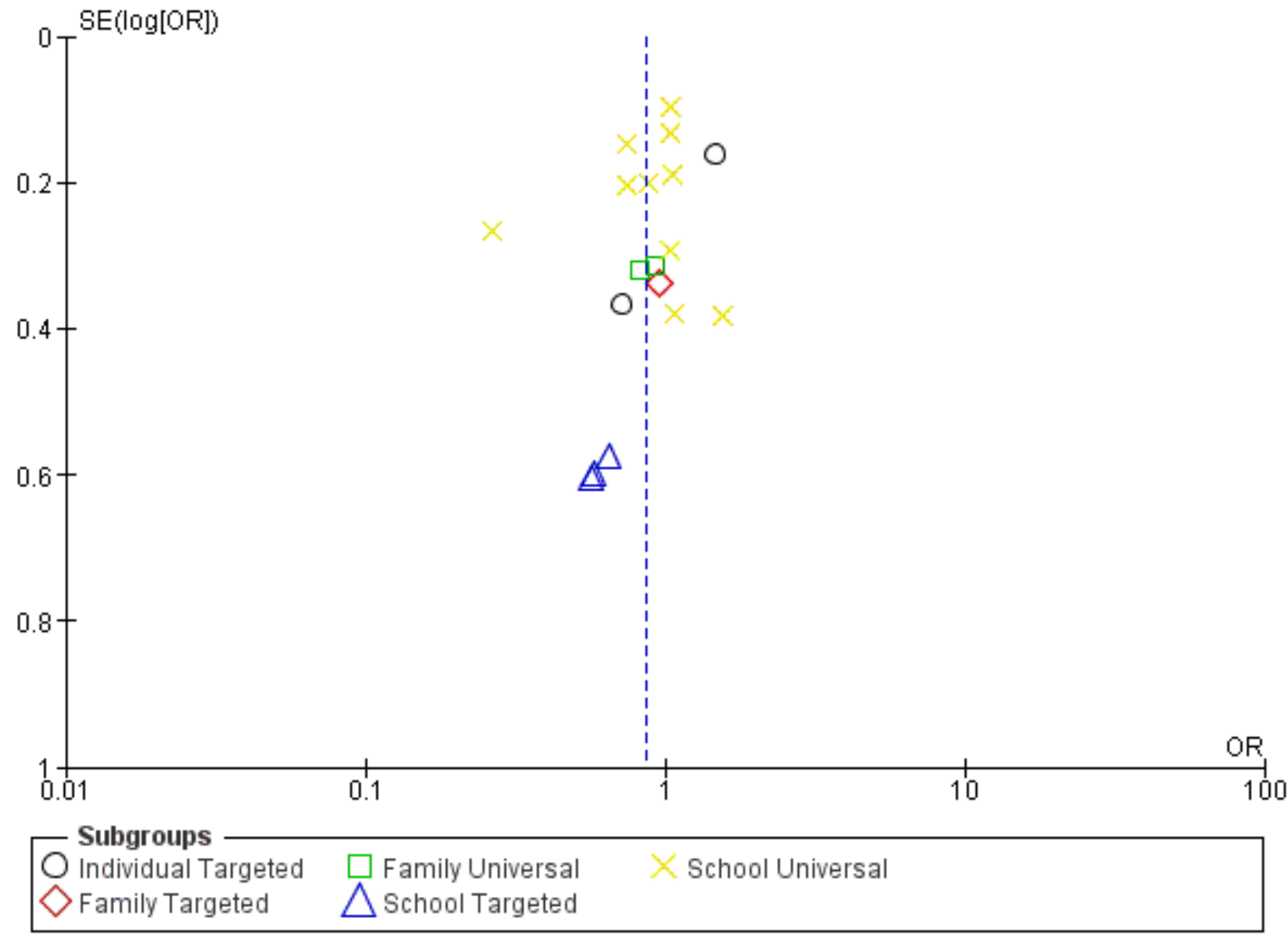


Figure 8. Funnel plot of comparison. Outcome 7: antisocial behaviour and offending. Outcome 7.3: school or general delinquency (short-term).

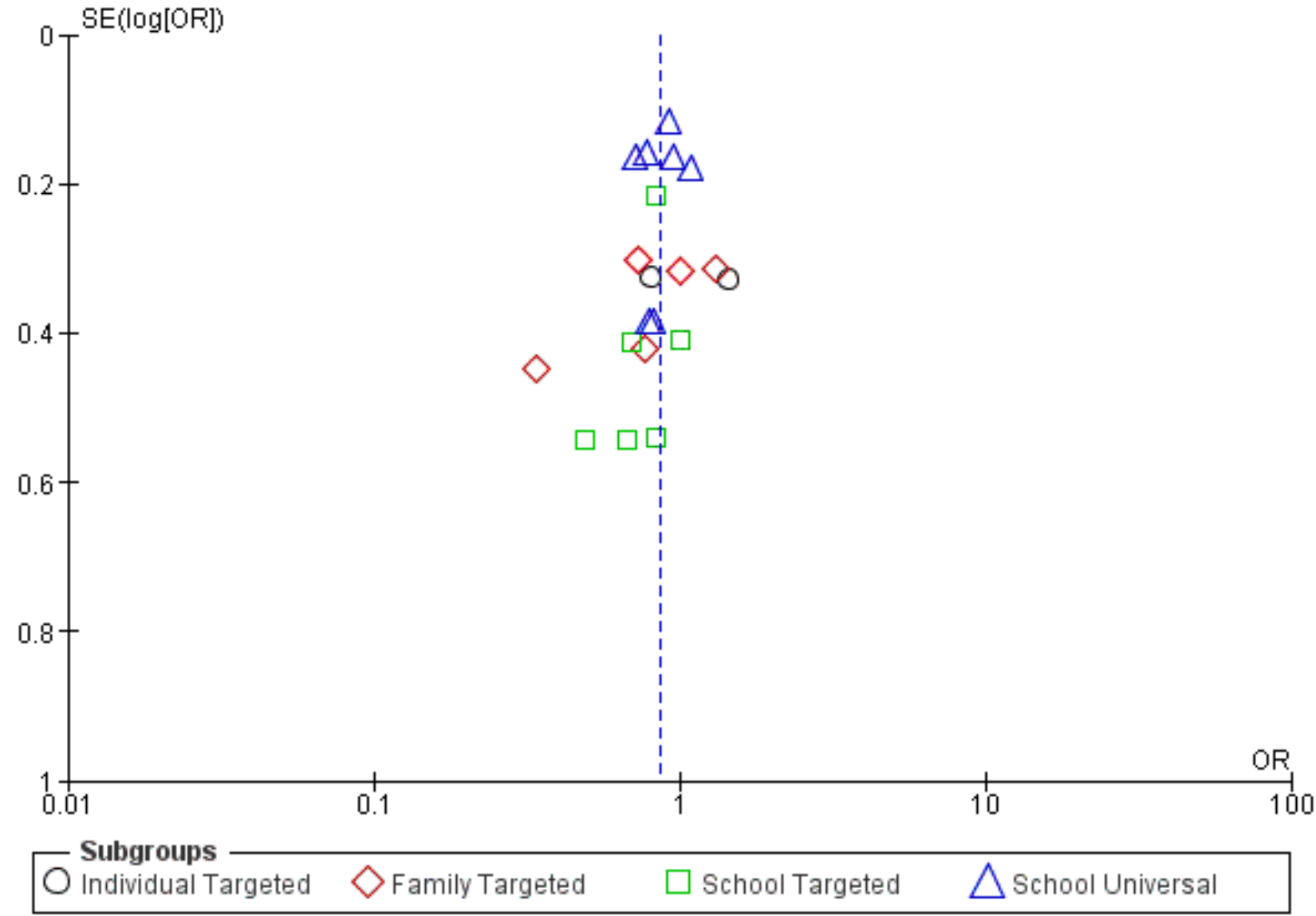

For tobacco use, alcohol use, cannabis use, illicit drug use, and sexual risk behaviour, we plotted funnel plots for each intervention type addressing these outcomes (Figure 9; Figure 10; Figure 11; Figure 12; Figure 13). The cylindrical appearance of plots for alcohol use and cannabis suggests that high levels of heterogeneity may be present, which would be expected when different study types are combined. Lastly, the funnel plots of studies targeting illicit drug use and sexual risk behaviour are asymmetrical, suggesting that small-study bias may be present, leading to the possibility of an overestimation of effect, given the under-representation of small studies with negative findings (Sterne 2011). 
Figure 9. Funnel plot of comparison. Outcome 1: tobacco. Outcome 1.1: tobacco use (short-term).

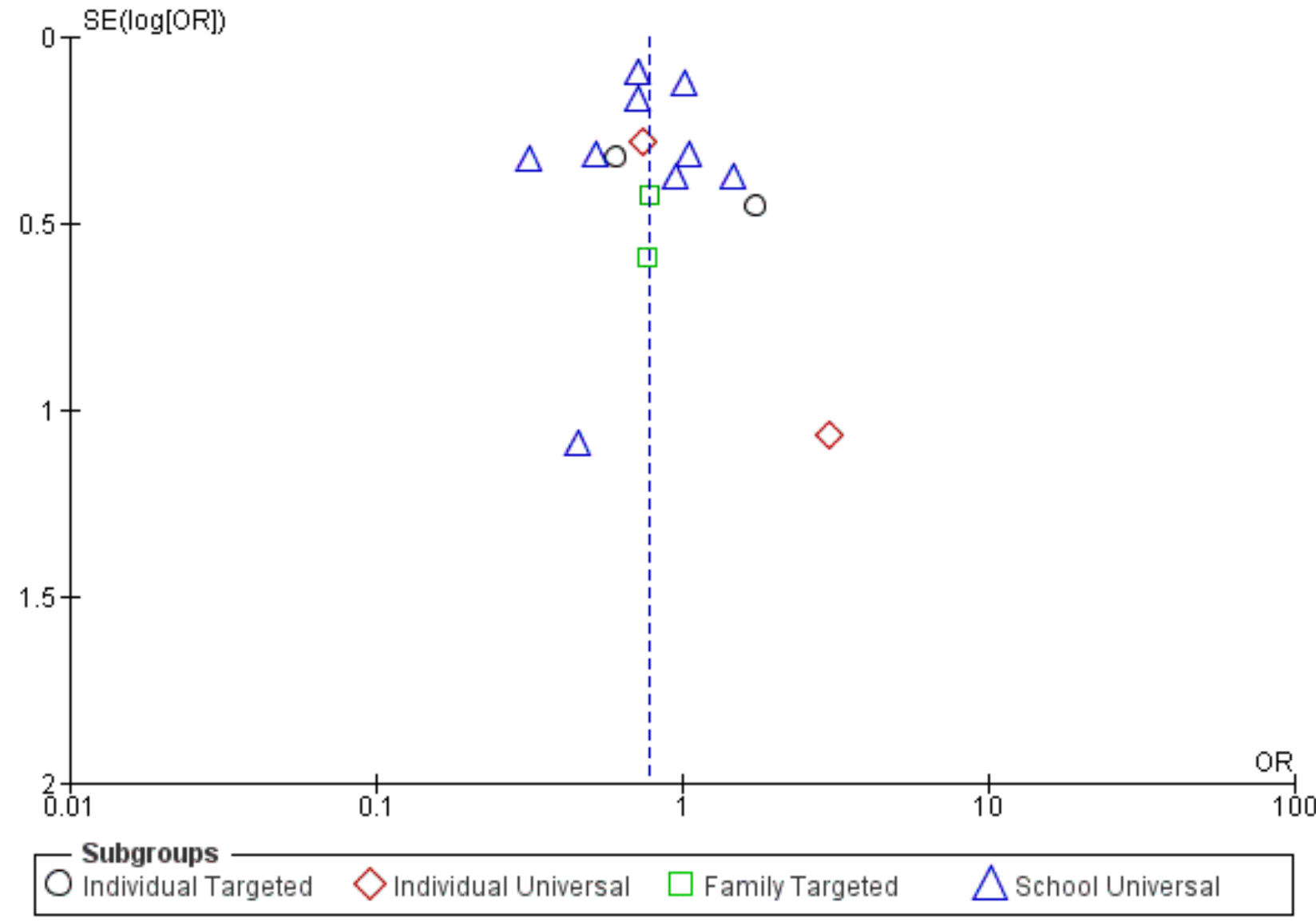


Figure 10. Funnel plot of comparison. Outcome 2: alcohol. Outcome 2.1: alcohol use (short-term).

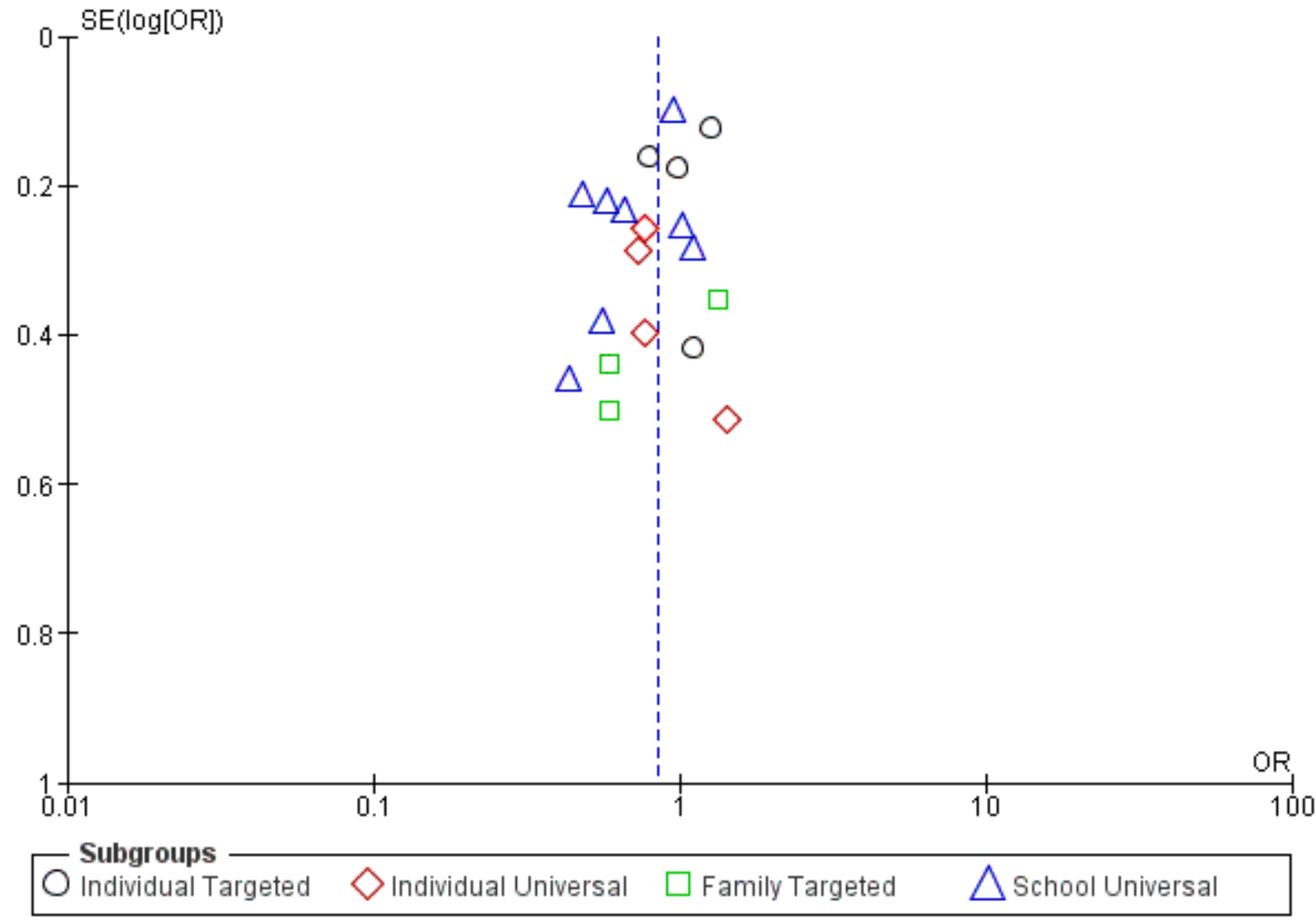


Figure 11. Funnel plot of comparison. Outcome 4: cannabis use. Outcome 4.1: cannabis use (short-term).

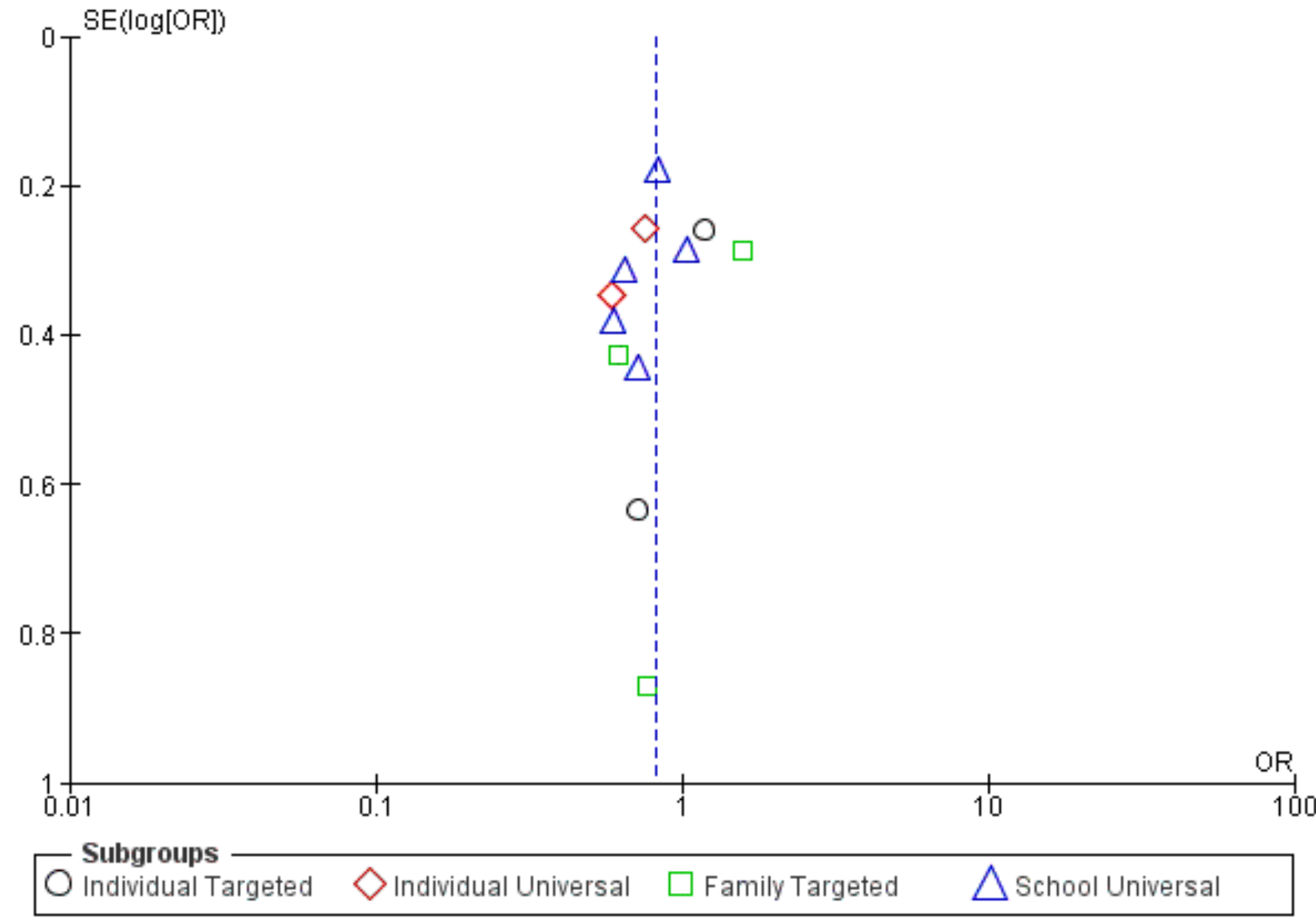


Figure 12. Funnel plot of comparison. Outcome 5: illicit drug use. Outcome 5.1: Illicit drug use (short-term).

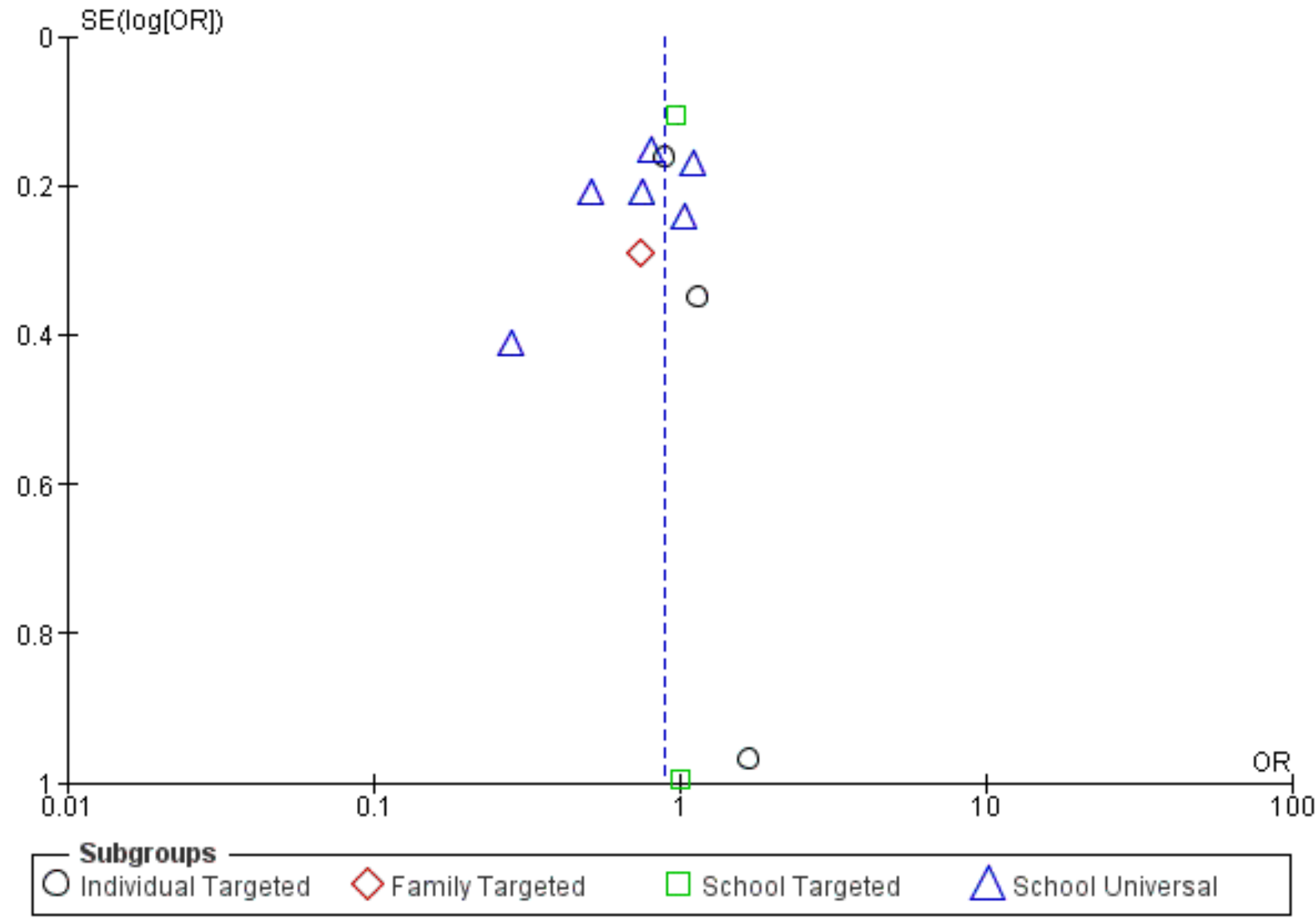


Figure 13. Funnel plot of comparison. Outcome 9: sexual risk behaviours. Outcome 9.1: sexual risk behaviour (short-term).

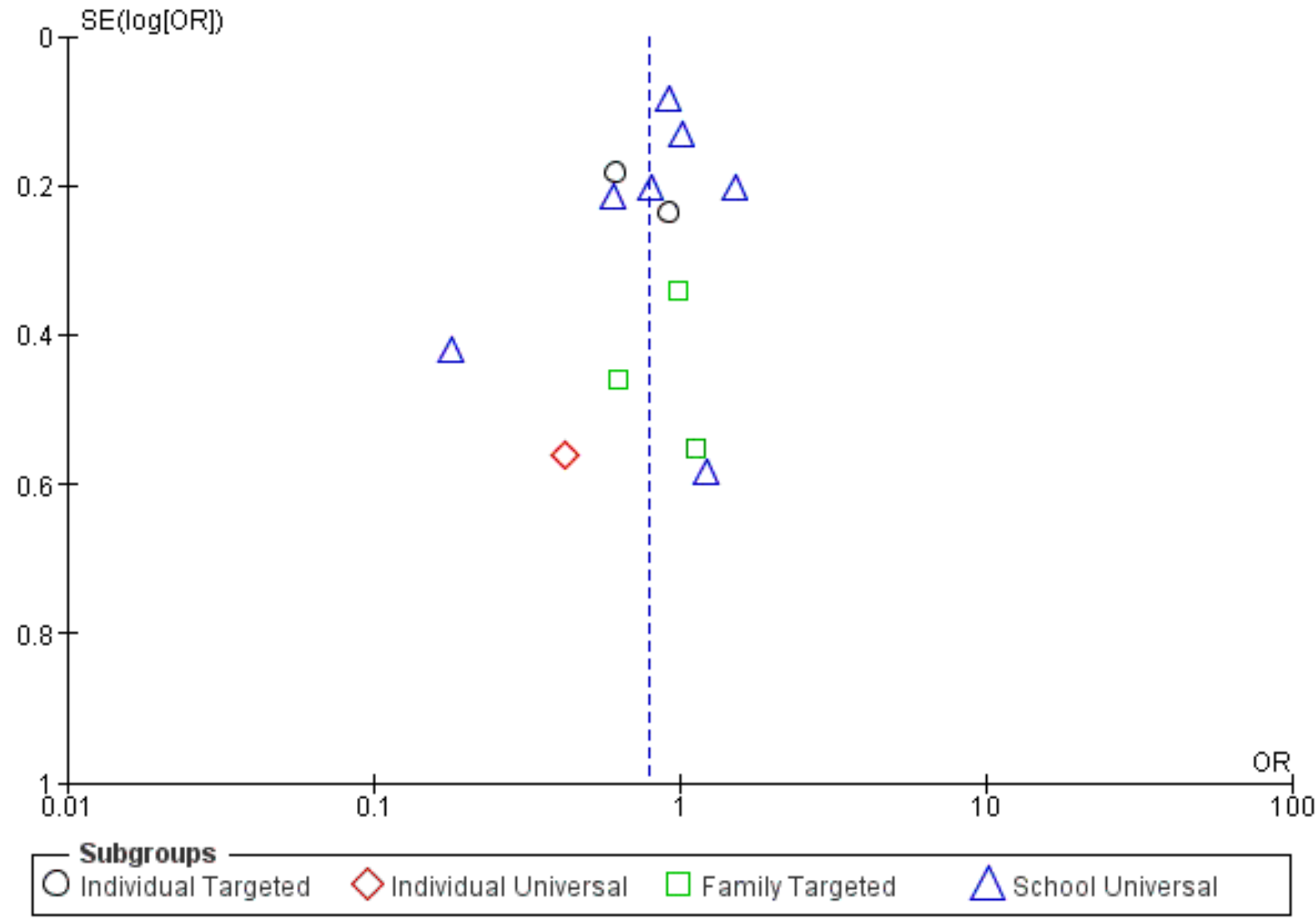

\section{Effects of interventions}

See: Summary of findings for the main comparison; Summary of findings 2; Summary of findings 3; Summary of findings 4; Summary of findings 5

Although the studies included in this review addressed two or more of the behaviours of interest, we will describe effects of the interventions on each behaviour, separately, below, because this is how they were reported in the study papers. In future research, we will explore the possibility of examining effects of interventions on multiple behaviours. We note that we were not able to address the secondary objectives of the review owing to a lack of available data.

\section{Primary outcomes}

\section{Tobacco use}

Forty-two studies (60\%) targeted tobacco use among young people; most of these studies provided universal school-based interventions $(n=23)$. We provide a further breakdown of these studies by intervention type and name in Table 4 and Table 5 . Thirty-seven of these studies concomitantly targeted use of another substance, five targeted tobacco use alongside physical activity and nutrition as a cardiovascular prevention intervention (Bush 1989; Nader 1999; O'Neill 2011; Saraf 2015; Walter 1989), and one targeted tobacco use and sexual risk behaviour (Redding 2015). Three of these 38 studies targeted tobacco use via proposed risk factors for later substance use (Ialongo 1999; lalongo 1999b; Kellam 2014), and a further four studies targeted tobacco use through hypothesised indirect mechanisms (Conduct Problems Prevention Research Group 2010; Kitzman 2010; Olds 1998; Schweinhart 1993). Of studies targeting tobacco use, one study was conducted in Sweden (Bodin 2011), one in Ireland (Dolan 2010), two in Switzerland (Averdijk 2016; Averdijk 2016b), one in India (Saraf 2015), one in Hong Kong (Shek 2011), one in Spain/Mexico (Lana 2014), one in the UK (Walker 2002), two in Canada (Morris 2003; Wolfe 2012), and one in Australia (Bond 2004); the remainder were conducted in the USA.

Fifteen studies reported data up to 12 months following completion of the intervention that could be included in the meta-analyses for tobacco use (Analysis 1.1); seven studies reported longerterm tobacco outcomes (over 12 months following the end of the intervention, extending up to 12 and 22 years of follow-up (Kellam 2014 and Schweinhart 1993, respectively)). Four studies reported tobacco outcomes as part of a substance use composite score or measure (Kitzman 2010; LoSciuto 1999; Pantin 2009; Shek 2011). We have recorded in Table 2 outcomes from the 12 studies that could not be included (outcomes not included in meta-analysis). Two studies did not report data regarding tobacco use (Dolan 2010; Wolfe 2012). 


\section{Measures}

Among those included in the meta-analysis of findings up to 12 months, studies reported outcome data in terms of frequency data (e.g. smoking in the past 30 days) or ever having smoked, depending on which was more appropriate for the age at which data were collected. Among those with longer-term followup, outcome measures included smoking initiation (which was relevant when studies were initiated with very young children, e.g. in primary or elementary school) and smoking frequency.

\section{Effectiveness over the short term}

Analysis 1.1 presents results of the meta-analysis for tobacco use by intervention type. We identified that individual-level universal or targeted interventions may have little or no effect in relation to tobacco use at up to 12 months' follow-up (universal: odds ratio (OR) $1.03,95 \%$ confidence interval $(\mathrm{Cl}) 0.32$ to $3.27 ; \mathrm{P}=0.97 ; \mathrm{n}=$ 2 studies; 1549 participants; $I^{2}=38 \%$; moderate-quality evidence; targeted: OR $0.98,95 \% \mathrm{Cl} 0.35$ to $2.73 ; \mathrm{P}=0.97 ; \mathrm{n}=2$ studies; 1549 participants; $I^{2}=72 \%$; low-quality evidence), although each metaanalysis included only two studies (universal: Lana 2014; Walker 2002; targeted: Bodin 2011; Redding 2015).

Among family-based interventions (Catalano 1999; Li 2002), data show uncertainty of the effect in relation to tobacco use (OR 0.78 , $95 \% \mathrm{Cl} 0.40$ to $1.53 ; \mathrm{P}=0.47 ; \mathrm{n}=2$ studies; 313 participants; $\mathrm{I}^{2}=0 \%$; moderate-quality evidence) (Analysis 1.1).

For universal school-based interventions (Beets 2009; Bond 2004; Fearnow-Kenney 2003; Gottfredson 2010; Griffin 2009; Li 2002; Nader 1999; O'Neill 2011; Simons-Morton 2005), moderate-quality evidence shows that such interventions, on average, reduced smoking among young people (OR $0.77,95 \% \mathrm{Cl} 0.60$ to $0.97 ; \mathrm{P}=$ $0.03 ; \mathrm{n}=9$ studies; 15,354 participants), although heterogeneity was moderate $\left(I^{2}=57 \%\right)$. We considered the size of this effect to represent a potentially important change in engagement in this risk behaviour at the population level. In particular, the Positive Action programme (Beets 2009), the Gatehouse Study (Bond 2004), the Michigan Model for Health (O'Neill 2011), and Going Places reported positive findings (Simons-Morton 2005). Sensitivity analysis using a different intracluster correlation coefficient (ICC) did not change this finding, and analysis of only those reporting dichotomous outcomes gave a similar result, although this was not the case for two studies reporting continuous data (Table 6).

Among the studies that could not be included in the meta-analysis with follow-up data up to 12 months, one study examining a targeted individual-level intervention reported positive findings (Tierney 1995). One study providing a targeted family-level intervention that could not be included in the meta-analysis reported positive findings (Gonzales 2012), and one study providing a universal family-level intervention reported null findings (Connell 2007). Five universal school-level interventions that could not be included in the meta-analysis showed beneficial effects (Bush 1989; DeGarmo 2009; McNeal 2004; Saraf 2015; Walter 1989), two universal school-level interventions showed no effect (LoSciuto 1999; Piper 2000a), and another showed a beneficial effect of one of two active intervention arms among boys only (Perry 2003a).

\section{Long-term effectiveness}

Analysis 1.2 presents results of the meta-analysis for follow-up data over 12 months. One targeted individual-level study $(\mathrm{n}=$
397 participants), which reported findings at 18 months' follow-up (Redding 2015), showed no benefit of the intervention.

Two targeted family-level intervention studies, representing 1177 participants, provided longer-term follow-up data, reporting findings at approximately 24 months in Kim 2011 and at 36 months in Morris 2003. The two studies reported contrasting results, with one caregiver training and skills-building programme favouring intervention (OR $0.48,95 \% \mathrm{Cl} 0.23$ to 0.98 ; Kim 2011), and the other income/employment support programme reporting an increase in tobacco use among those in the intervention group (OR 1.27, 95\% Cl 0.96 to 1.68; Morris 2003).

Across three school-based universal interventions that were implemented in primary school and provided longer-term followup, evidence shows that such interventions probably have a positive effect (OR $0.60,95 \% \mathrm{Cl} 0.33$ to $1.09 ; \mathrm{P}=0.09 ; \mathrm{n}=3$ studies; 879 participants; $I^{2}=0 \%$ ) (lalongo 1999; lalongo 1999b; Kellam 2014).

\section{Alcohol use}

Fifty-five studies (79\%) addressed alcohol use, three of which targeted alcohol use through early risk factors for later alcohol use (lalongo 1999; Ialongo 1999b; Kellam 2014). Two additional studies addressed alcohol use by targeting early life experiences and thus targeted indirect pathways (Conduct Problems Prevention Research Group 2010; Schweinhart 1993). Forty-two of 48 studies $(88 \%)$ also addressed another substance together with antisocial behaviour, and four addressed alcohol use and vehicle-risk behaviour (Bernstein 2010a; D'Amico 2002a; Monti 1999; Nirenberg 2013). We provide in Table 4 and Table 5 a summary of the studies that aimed to prevent alcohol use by intervention type and study name.

Most studies were conducted in the USA, and two were conducted in the UK (Berry 2009; Walker 2002), three in Sweden (Bodin 2011; Jalling 2016; Jalling 2016b), two in Switzerland (Averdijk 2016; Averdijk 2016b), one in Ireland (Dolan 2010), one in Spain/Mexico (Lana 2014), one in Australia (Bond 2004), one in Hong Kong (Shek 2011), and one in Canada (Wolfe 2012).

We could not include 17 studies in the meta-analysis (Connell 2007; Cunningham 2012; D'Amico 2002a; DeGarmo 2009; Estrada 2015; Freidman 2002; Jalling 2016; Jalling 2016b; McNeal 2004; Monti 1999; Murry 2014; Nirenberg 2013; Olds 1998; Perry 2003a; Piper 2000a; Shetgiri 2011; Tierney 1995). See Table 2 for a summary of these data. Nine studies provided composite measures of substance use (Averdijk 2016; Averdijk 2016b; Beach 2016; Kitzman 2010; Lochman 2003a; Lochman 2004a; LoSciuto 1999; Shek 2011; Wolfe 2012).

Eleven studies measured binge drinking or drunkenness (Beets 2009; Bond 2004; Conduct Problems Prevention Research Group 2010; Cunningham 2012; Dolan 2010; Fearnow-Kenney 2003; Griffin 2009; Li 2011; Bernstein 2010a; Bodin 2011; Bonds 2010), eight of which could be included in a meta-analysis of short-term effects (Analysis 3.1). One study could not be included (Cunningham 2012), and another two studies provided long-term follow-up 10 and 15 years after the intervention (Conduct Problems Prevention Research Group 2010; Bonds 2010, respectively). We have provided these data in Table 2, 


\section{Measures}

For alcohol use, measures included initiation of alcohol use, frequency of alcohol use over a recent time period (appropriate to the age of participants), and measures of days drinking per week or month. For binge drinking, measures included ever having been drunk/really drunk, being drunk in the past 30 days, and frequency of binge drinking.

\section{Effectiveness over the short term}

Analysis 2.1 presents results of the meta-analysis for alcohol use by intervention type for studies with follow-up periods up to 12 months. We identified moderate-quality evidence showing that universal or targeted interventions at the individual level probably have little or no effect in relation to alcohol use (universal individual-level interventions: $\mathrm{OR} 0.80,95 \% \mathrm{Cl} 0.58$ to $1.11 ; \mathrm{P}=$ $0.18 ; n=4$ studies; 1911 participants; $I^{2}=0 \%$; targeted individuallevel interventions: $\mathrm{OR} 1.02,95 \% \mathrm{Cl} 0.80$ to $1.31 ; \mathrm{P}=0.87$; 2044 participants), although each analysis included relatively few studies ( $\mathrm{n}=4$ studies; 1911 participants; and $\mathrm{n}=4$ studies; 2044 participants, respectively; moderate-quality evidence). However, for universal individual-level interventions, the effect estimate was in the direction of benefit.

Targeted family-level interventions also probably have little or no effect in relation to alcohol use $(\mathrm{OR} 0.83,95 \% \mathrm{Cl} 0.47$ to $1.46 ; \mathrm{P}=$ $0.52 ; n=3$ studies; 417 participants; moderate-quality evidence).

On average, across eight universal school-level interventions, evidence shows that such interventions probably reduce alcohol use (OR $0.72,95 \% \mathrm{Cl} 0.56$ to $0.92 ; \mathrm{P}=0.009 ; \mathrm{n}=8$ studies; 8751 participants; moderate-quality evidence). Heterogeneity was moderate $\left(I^{2}=58 \%\right)$. Sensitivity analysis around the ICC used did not change the findings; and meta-analysis of only those reporting dichotomous outcomes yielded a similar result, although this was not the case for two studies reporting continuous data (Table 6).

Among the studies that could not be included in the meta-analysis with follow-up data up to 12 months, one providing a targeted individual-level intervention reported beneficial effects (Tierney 1995), and three exploring targeted individual-level interventions reported no effect (Cunningham 2012; Freidman 2002; Monti 1999).

One targeted family-level intervention showed beneficial effects (Gonzales 2012); one universal family-based intervention (in Connell 2007) and three targeted family-level interventions (in Estrada 2015, Jalling 2016, and Jalling 2016b) showed null effects.

Among school-based interventions, one universal school-level intervention showed beneficial effects (Piper 2000a), and one universal school-level intervention showed benefit of one active intervention arm for boys only (Perry 2003a).

\section{Long-term effectiveness}

Analysis 2.2 presents results of the meta-analysis for alcohol use by study type for studies reporting follow-up data over the longer term (> 12 months). Each subgroup included only two studies. One targeted school-level study intervention produced a positive effect (Conduct Problems Prevention Research Group 2010). Other findings were inconclusive.

\section{Binge drinking}

\section{Effectiveness over the short term}

Analysis 3.1 presents results of the meta-analysis for binge drinking for studies with follow-up to 12 months. They show absence of evidence of an effect of targeted individual-level interventions on binge drinking (OR $0.97,95 \% \mathrm{Cl} 0.68$ to $1.37 ; \mathrm{P}=0.85 ; \mathrm{n}=3$ studies; 250 participants; $I^{2}=48 \%$ ). On average, across five universal schoollevel interventions, evidence suggests that such interventions may have a beneficial effect $(\mathrm{OR} 0.66,95 \% \mathrm{Cl} 0.41$ to 1.06 ; $\mathrm{P}=0.09$; $\mathrm{n}=5$ studies; 5494 participants; low-quality evidence), but the $95 \% \mathrm{Cl}$ was also consistent with the null hypothesis of no effect. Heterogeneity was moderate $\left(I^{2}=43 \%\right)$. Sensitivity analysis around the ICC did not change the summary effect estimate, but the $95 \% \mathrm{Cl}$ was narrower ( $95 \% \mathrm{Cl} 0.45$ to 0.99$)$, and heterogeneity was slightly increased $\left(I^{2}=49 \%\right.$ ) (Table 6$)$.

\section{Long-term effectiveness}

Analysis 3.2 presents results of the meta-analysis for excess drinking for studies reporting data over the longer term. One targeted family-level study showed an absence of effect (OR 1.30, $95 \% \mathrm{Cl} 0.79$ to 2.13 ), and one targeted school-level study reported an OR of 0.75 ( $95 \% \mathrm{Cl} 0.55$ to 1.02 ).

\section{Illicit drug use}

A total of 53 interventions (76\%) addressed illicit drug use (see Table 3 and Table 4). As above, three intervention studies aimed to prevent drug use by targeting early risk factors for later substance use (lalongo 1999; lalongo 1999b; Kellam 2014); and two additional intervention studies aimed to prevent drug use by acting on early life events and thus indirect mechanisms (Conduct Problems Prevention Research Group 2010; Schweinhart 1993). Most were conducted in the USA, with one conducted in the UK (Dolan 2010), two in Switzerland (Averdijk 2016; Averdijk 2016b), two in Sweden (Jalling 2016; Jalling 2016b), two in Canada (Morris 2003; Wolfe 2012), one in Australia (Bond 2004), and one in Hong Kong (Shek 2011).

We identified 29 studies that specifically measured cannabis use alone, all of which targeted other substances as well, some indirectly (as above). Twenty-five of these studies also targeted antisocial behaviour. One study was conducted in the UK (Dolan 2010), two in Switzerland (Averdijk 2016; Averdijk 2016b), and the others in the USA (Table 3).

\section{Measures}

Measures of cannabis use included ever use, initiation of use (appropriate to the age of participants), frequency of use (e.g. in past month, in past six months). Similarly, measures of illicit drug use focused on use of one or more illicit substances, measuring frequency in the past month or a measure of initiation of illicit drug use.

\section{Effectiveness over the short term}

Analysis 4.1 presents results of the meta-analysis for illicit drug use by study type for studies with follow-up periods up to 12 months. Evidence from three studies shows that targeted individual-level interventions probably had little or no effect in reducing illicit drug use over the short term (OR $0.94,95 \% \mathrm{Cl} 0.71$ to $1.25 ; \mathrm{P}=0.67 ; \mathrm{n}=3$ studies; 638 participants; $I^{2}=0 \%$; moderate-quality evidence). 
One targeted family-level intervention showed a null effect (OR $0.74,95 \% \mathrm{Cl} 0.42$ to $1.31 ; 69$ participants; moderate-quality evidence).

Analyses also indicated that two targeted school-based interventions probably have little or no effect in relation to illicit drug use (OR $0.96,95 \% \mathrm{Cl} 0.79$ to $1.18 ; \mathrm{P}=0.72 ; \mathrm{n}=2$ studies; 1299 participants; $I^{2}=0 \%$; moderate-quality evidence). On average, across five universal school-based interventions, evidence suggests that these interventions may have a positive effect in relation to illicit drug use (OR 0.74, $95 \% \mathrm{Cl} 0.55$ to $1.00 ; \mathrm{P}=0.05 ; \mathrm{n}=5$ studies; 11,058 participants; low-quality evidence), although heterogeneity was substantial $\left(I^{2}=69 \%\right)$. Sensitivity analyses did not markedly change these findings (Table 6 ).

\section{Long-term effectiveness}

Ten studies provided data from long-term follow-up, ranging from two years up to 10 years (Conduct Problems Prevention Research Group 2010; Schweinhart 1993), 12 years (Kellam 2014), and 15 years post intervention (Analysis 4.2) (Bonds 2010).

Analysis 4.2 presents results of meta-analyses for illicit drug use for studies with a follow-up period greater than 12 months. On average, across four studies (five active intervention arms), data suggest uncertainty around the benefit of targeted family-level interventions (OR $0.80,95 \% \mathrm{Cl} 0.52$ to $1.24 ; \mathrm{P}=0.32 ; \mathrm{n}=5$ studies; 2032 participants; $I^{2}=66 \%$ ) due to the very low quality of the evidence, although the effect estimate was in the direction of benefit. Three of these estimates favoured intervention but the $95 \%$ $\mathrm{Cls}$ were consistent with the null hypothesis of no effect. Bonds 2010 reported a beneficial effect $(\mathrm{OR} 0.54,95 \% \mathrm{Cl} 0.33$ to 0.89$)$ and Morris 2003 reported an adverse effect of the intervention (OR 1.24, $95 \% \mathrm{Cl} 1.00$ to 1.55$)$.

Evidence suggests that, on average, universal school-level interventions may be effective in reducing illicit drug use (OR 0.73 , $95 \% \mathrm{Cl} 0.56$ to $0.95 ; \mathrm{P}=0.02 ; \mathrm{n}=4$ studies; 3338 participants; $\mathrm{I}^{2}=0 \%$; low-quality evidence). Two of the interventions that were included in the meta-analysis were conducted in the same study with one control group, which we accounted for in the analysis (see Data synthesis section).

\section{Cannabis use}

\section{Effectiveness over the short term}

Analysis 5.1 presents results of the meta-analysis for cannabis use by study type. Ten studies reported data regarding cannabis use that could be synthesised in a meta-analysis of effects of interventions with up to 12 months' follow-up. Moderatequality evidence shows that targeted individual-level interventions probably have little or no beneficial effects in relation to cannabis use (OR $1.10,95 \% \mathrm{Cl} 0.69$ to $1.76, \mathrm{P}=0.39 ; \mathrm{n}=2$ studies; 126 participants; $I^{2}=0 \%$ ), whereas although a null effect was found for universal individual-level interventions, the effect estimate was in the direction of benefit (OR $0.69,95 \% \mathrm{Cl} 0.46$ to $1.04 ; \mathrm{P}=0.08$; two studies; 362 participants; $I^{2}=0 \%$; moderate-quality evidence). Analysis also suggests that targeted family-level interventions may have little or no difference in reducing cannabis use (OR 1.02, 95\% $\mathrm{Cl} 0.52$ to $2.02 ; \mathrm{P}=0.95 ; \mathrm{n}=3$ studies; 380 participants; $\left.\right|^{2}=43 \%$; lowquality evidence), with one study reporting an increase in cannabis use as a result of the intervention (mean frequency of marijuana use in intervention 10.8 compared to 6.4 among controls) (Milburn 2012).

On average, across five universal school-based interventions, evidence shows that they may be beneficial (OR 0.79, 95\% Cl 0.62 to $1.01 ; \mathrm{P}=0.06 ; \mathrm{n}=5$ studies; 4140 participants; $\mathrm{I}^{2}=0 \%$; moderate-quality evidence), and we considered the size of the effect to represent public health benefit at the population level, but the confidence interval spanned the null value. The sensitivity analysis conducted with different ICCs revealed a slightly lower effect estimate (OR $0.77,95 \% \mathrm{Cl} 0.61$ to 0.97 ) and heterogeneity that remained the same $\left(I^{2}=0 \%\right)$ (Table 6$)$.

Seven studies reported data regarding effects on cannabis or illicit drug use that could not be used in a meta-analysis (Connell 2007; D'Amico 2002a; DeGarmo 2009; Freudenberg 2010; Griffin 2006; McNeal 2004; Piper 2000a); we present reported data in Table 2. In summary, two targeted individual-level interventions showed a beneficial effect (Freudenberg 2010; Tierney 1995).

One family-based intervention study reported an effect at one time point only (Connell 2007), and another reported no positive effect (Estrada 2015). Two family-based intervention studies reported higher illicit drug use among those in the intervention arm compared to the control arm (Jalling 2016; Jalling 2016b).

One universal school-based intervention study reported a beneficial effect (Griffin 2006); one universal school-based programme reported an effect in one of the active intervention arms only (Piper 2000a); and two universal school-based studies reported no effect (DeGarmo 2009; McNeal 2004).

\section{Long-term effectiveness}

Analysis 5.2 presents results of the meta-analysis for studies that aimed to address cannabis use and conducted follow-up over the longer term. Only two studies in each study type subgroup could be included, and they provided no evidence of any effect for any study type.

Among two studies that could not be included in the meta-analysis for cannabis or illicit drug use, one reported no effect of the intervention (Bonds 2010), and the other reported a beneficial effect (Griffin 2006)

\section{Tobacco, alcohol, and/or illicit drug use (composite measures)}

Several studies reported their findings as a composite measure of substance use (e.g. tobacco, alcohol, and/or illicit drug use) or alcohol and drug use. Six studies reported short-term findings that could be included in meta-analyses (Analysis 6.1). All except one study were conducted in the USA, the other in Hong Kong (Shek 2011). We have reported in Table 2 findings of an additional eight studies that could not be included in the meta-analysis (Beach 2016; Berry 2009; Estrada 2015; Freudenberg 2010; Gonzales 2012; Griffin 2006; LoSciuto 1999; Olds 1998).

\section{Measures}

Measures included measures of substance use (tobacco, alcohol, and/or drugs; alcohol, tobacco, and/or marijuana; or alcohol and marijuana) over the past month, 6 months, or year, depending on the age of the participants. 


\section{Effectiveness over the short term}

Analysis 6.1 presents results of the meta-analysis for composite measures of substance use (i.e. tobacco, alcohol, and/or drug use). Evidence shows that targeted family-level interventions may have little or no difference in reducing substance use (OR $0.81,95 \% \mathrm{Cl}$ 0.50 to $1.33 ; \mathrm{P}=0.40 ; \mathrm{n}=2$ studies; 213 participants; $\mathrm{I}^{2}=0 \%$ ), although only two studies were included in each subgroup. We also found that universal school-based interventions may show little or no difference in reducing substance use (OR 1.03, 95\% Cl 0.77 to $1.37 ; \mathrm{P}=0.85 ; \mathrm{n}=3$ studies; 7390 participants; $\mathrm{I}^{2}=28 \%$ ). Sensitivity analysis with a changed ICC altered the finding for the two universal school-based interventions such that results of the meta-analysis favoured control (OR $1.10,95 \% \mathrm{Cl} 1.01$ to $1.20 ; \mathrm{P}=$ 0.03). Two targeted school-based studies with multiple study arms were included in the meta-analysis. Two arms of one of the studies showed benefit of the intervention (Lochman 2003a), but overall, data show uncertainty around the effects of these studies (Analysis 6.1) (Lochman 2003a; Lochman 2004a). Sensitivity analysis around the ICC did not change these findings (Table 6).

Among studies that could not be included in the meta-analysis, two individual-level intervention studies reported a beneficial effect (Berry 2009; Freudenberg 2010), two family-level intervention studies reported a beneficial effect (Beach 2016; Gonzales 2012), and one family-level intervention study reported a null effect (Estrada 2015). One school-based intervention study reported an adverse effect (LoSciuto 1999).

\section{Long-term effectiveness}

Analysis 6.2 presents the meta-analysis for composite substance use by study type for studies reporting longer-term follow-up (> 12 months). Weak evidence from four targeted family-based studies showed benefit in relation to substance use (OR $0.69,95 \% \mathrm{Cl}$ 0.47 to $1.03 ; \mathrm{P}=0.07 ; \mathrm{n}=4$ studies; 1622 participants; moderatequality evidence) along with substantial heterogeneity $\left(I^{2}=82 \%\right)$ (Averdijk 2016; Brody 2012; Kim 2011; Kitzman 2010). Three studies were conducted in the USA, and one in Switzerland. Two universal school-based intervention studies showed a null effect on drug and alcohol use approximately two years post intervention (OR 1.09, $95 \% \mathrm{Cl} 0.94$ to $1.27 ; \mathrm{P}=0.24 ; \mathrm{n}=2$ studies; 2145 participants; $\mathrm{I}^{2}=0 \%$ ) (Averdijk 2016b; Wolfe 2012).

Among two studies that could not be included in the meta-analysis, one targeting a family-level intervention reported no effect of the intervention on this outcome (Olds 1998), and one targeting a universal school-based intervention showed a beneficial effect (Griffin 2006)..

\section{Antisocial behaviour and offending}

Fifty-three studies (76\%) addressed antisocial behaviour, 11 of which were targeted to individuals, 17 to families, six to higherrisk participants at schools, and 19 to participants at schools irrespective of risk. We have provided in Table 4 and Table 5 details of studies that targeted antisocial behaviour by study type.

All but one of the studies addressing antisocial behaviour concurrently aimed to prevent use of at least one substance (two of which were provided via indirect mechanisms (Conduct Problems Prevention Research Group 2010; Schweinhart 1993)); 16 studies $(30 \%)$ concurrently aimed to reduce sexual risk behaviour. Twentyseven studies provided short-term data that could be included in the meta-analysis summarising impact of interventions on engagement in any antisocial behaviour (Analysis 7.1). Studies of each type were insufficient to show the impact of interventions on different forms of antisocial behaviour discussed in this review (e.g. property-related offences vs arrests and general offences). Eleven additional studies reported long-term data, one of which reported both short- and long-term data (Lochman 2003a). We could not include 14 studies in the meta-analysis; we have reported these data in Table 2. Most studies were conducted in the USA ( $n=38$ of 53; 72\%), with one conducted in Canada (Wolfe 2012), one in Hong Kong (Shek 2011), one in India (Saraf 2015), one in Australia (Bond 2004), two in the UK (Berry 2009; Walker 2002), two in Switzerland (Averdijk 2016; Averdijk 2016b), one in Mexico/Spain (Lana 2014), one in Ireland (Dolan 2010), three in Sweden (Bodin 2011; Jalling 2016; Jalling 2016b), and one in South Africa (Matthews 2016). Thus, most studies included here were conducted in high-income countries.

\section{Measures}

Measures for studies that provided shorter-term follow-up included aggressive or violent behaviour and school-level or general delinquency; those with longer-term follow-up included measures around arrests in addition to measures of violence, aggressive behaviour, and/or conduct problems.

\section{Effectiveness over the short term}

Analysis 7.1 presents results for the meta-analysis for antisocial behaviour by study type for studies reporting follow-up data up to 12 months. Overall, moderate-quality evidence shows that individual-level interventions probably produced little or no difference in reducing antisocial behaviour (universal individuallevel: OR 1.02, 95\% Cl 0.62 to 1.69; one study; 200 participants; targeted individual-level: $\mathrm{OR} 1.21,95 \% \mathrm{Cl} 0.92$ to $1.60 ; \mathrm{P}=0.17$; $\mathrm{I}^{2}=19 \% ; \mathrm{n}=4$ studies; 764 participants; both moderate-quality evidence).

Among family-level interventions, one universal study with two study arms showed no effect on engagement in antisocial behaviour (OR $0.87,95 \% \mathrm{Cl} 0.56$ to $1.35 ; \mathrm{P}=0.53 ; 306$ participants; $\mathrm{I}^{2}=0 \%$; moderate-quality evidence), and six targeted family-level studies showed no effect in relation to engagement in antisocial behaviour (OR $0.84,95 \% \mathrm{Cl} 0.57$ to $1.24 ; \mathrm{P}=0.39 ; \mathrm{I}^{2}=42 \% ; \mathrm{n}=6$ studies; 772 participants; moderate-quality evidence). The STRIVE programme, in particular, showed a large effect (Milburn 2012).

Studies provided weaker evidence of a probable positive effect of targeted school-based interventions in relation to antisocial behaviour (OR $0.78,95 \% \mathrm{Cl} 0.59$ to $1.05 ; \mathrm{P}=0.1 ; \mathrm{I}^{2}=0 \% ; \mathrm{n}=3$ studies; 1531 participants; moderate-quality evidence), although the effect was in the direction of benefit, and sensitivity analysis around the ICC slightly altered this finding in favour of the interventions (OR $0.82,95 \% \mathrm{Cl} 0.68$ to $0.99 ; \mathrm{P}=0.04 ; \mathrm{I}^{2}=0 \%$ ). Last, on average, across 13 universal school-based interventions, evidence suggests that such interventions may have an impact in reducing antisocial behaviour (OR $0.81,95 \% \mathrm{Cl} 0.66$ to 0.98 ; $\mathrm{P}=0.03 ; \mathrm{n}=13$ studies; 20,756 participants; very low-quality evidence), although heterogeneity was substantial $\left(I^{2}=66 \%\right)$. Sensitivity analyses around the ICC, income classification of study country, and dichotomous versus continuous outcomes yielded similar results (Table 6). 
Analysis 7.2 presents results for the meta-analysis for violent offences for studies reporting data at up to 12 months' followup. For individual- and family-based interventions, data showed uncertainty around whether there was an effect, or evidence suggested that such interventions may have little or no effect in reducing violent behaviour, although few studies could be included in each subgroup (targeted individual-level studies: OR 1.11, 95\% $\mathrm{Cl} 0.56$ to $2.17 ; \mathrm{P}=0.77 ; \mathrm{n}=2$ studies; 514 participants; $\mathrm{I}^{2}=68 \%$; targeted family studies: OR $0.95,95 \% \mathrm{Cl} 0.49$ to $1.84 ; \mathrm{n}=1$ study; 238 participants).

Meta-analyses did not show benefit of school-based targeted interventions (OR $0.60,95 \% \mathrm{Cl} 0.31$ to $1.16 ; \mathrm{n}=1$ study with three arms; 158 participants; $\left.\mathrm{P}=0.13 ; \mathrm{I}^{2}=0 \%\right)$.

Similarly, on average, across nine universal school-level interventions, evidence indicated that such interventions may have little effect (OR $0.86,95 \% \mathrm{Cl} 0.69$ to $1.07 ; \mathrm{P}=0.18 ; \mathrm{n}=9$ studies; 11,347 participants; $I^{2}=70 \%$ ), although as above, the effect estimate was in the direction of benefit, but the $95 \% \mathrm{Cl}$ was consistent with the null hypothesis. Sensitivity analyses around the ICC did not change these findings (Table 6).

Analysis 7.3 presents results for the meta-analysis for school-level or general delinquency (e.g. stealing things worth less than USD50) by study type. For targeted individual-level interventions, two studies provided evidence of a null effect (OR $1.07,95 \% \mathrm{Cl} 0.61$ to 1.89; 250 participants; $\left.P=0.81 ; I^{2}=37 \%\right)$.

For targeted family-level interventions, evidence of benefit was insufficient (OR $0.80,95 \% \mathrm{Cl} 0.54$ to $1.20 ; \mathrm{P}=0.28 ; \mathrm{n}=4$ studies; 598 participants; $I^{2}=40 \%$ ), although the effect estimate was in the direction of benefit.

For targeted school-based interventions, evidence of benefit was insufficient (OR $0.79,95 \% \mathrm{Cl} 0.59$ to $1.06 ; \mathrm{P}=0.11 ; \mathrm{n}=3$ studies; 1573 participants; $I^{2}=0 \%$ ), although the summary estimate was in the direction of benefit, and on average, evidence showed a beneficial effect from six universal school-based interventions in relation to school or general delinquency (OR $0.88,95 \% \mathrm{Cl} 0.77$ to $1.00 ; \mathrm{P}=0.05$; $\mathrm{n}=6$ studies; 10,113 participants; $\mathrm{I}^{2}=0 \%$ ).

We did not have sufficient data to analyse the effects of interventions on any other individual domains of antisocial behaviour.

Among studies that could not be included in the meta-analysis, three providing targeted individual-level interventions reported a beneficial effect (Berry 2009; Freudenberg 2010; Tierney 1995), and two reported a null effect (Cunningham 2012; Freidman 2002). Two studies examining targeted family-level interventions reported a beneficial effect (Beach 2016; Gonzales 2012), and a universal family-level programme reported a null effect (Connell 2007). One targeted school-level intervention revealed a null effect (Shetgiri 2011). Last, one universal school-level intervention showed a beneficial effect (DeGarmo 2009), one showed an effect of one active study arm for boys only (Perry 2003a), and one revealed a null effect (LoSciuto 1999).

\section{Long-term effectiveness}

Analysis 7.4 presents results of the meta-analysis for antisocial behaviour by study type from studies with follow-up over 12 months. Eleven studies reported longer-term outcome data and could be included in the meta-analysis. Evidence suggested that targeted family-level interventions representing 2486 participants may be beneficial (OR $0.74,95 \% \mathrm{Cl} 0.54$ to $1.03 ; \mathrm{P}=0.08 ; \mathrm{n}=5$ studies; 2486 participants; $\left.\right|^{2}=78 \%$; low-quality evidence) although heterogeneity was substantial, and weak evidence suggested that a universal family-based programme with two active study arms may have a beneficial effect (OR $0.67,95 \% \mathrm{Cl} 0.43$ to $1.04 ; \mathrm{n}=1$ study, 304 participants; $P=0.07$ ), although the $95 \% \mathrm{Cls}$ highlight that the data are inconclusive.

Evidence on two universal school-based interventions highlighted uncertainty in their effect over the long term (OR 0.91, 95\% Cl 0.63 to $1.31 ; \mathrm{P}=0.6 ; \mathrm{n}=2$ studies; 4146 participants; $\mathrm{I}^{2}=60 \%$ ), and the meta-analysis included only two studies.

Among studies that could not be included in the meta-analysis, one providing a targeted family intervention reported a null effect (Averdijk 2016), one providing a targeted family-level intervention showed a null effect for externalising problems and major delinquent acts but a beneficial effect for incidence of arrests (Olds 1998), and one providing a targeted family-based intervention reported a null effect for carrying a gun or knife once or more (Schweinhart 1993).

Two targeted school-based studies reported a beneficial effect (Conduct Problems Prevention Research Group 2010; Kellam 2014), and one universal school-based study reported a null effect (Averdijk 2016b).

\section{Vehicle-related risk behaviour}

Four studies addressed vehicle-related risk behaviours (Bernstein 2010a; D'Amico 2002a; Monti 1999; Nirenberg 2013); three provided targeted individual-level interventions (Bernstein 2010a; Monti 1999; Nirenberg 2013), and one provided a universal school-based intervention (D'Amico 2002a). Two studies could be included in a meta-analysis of effects of the interventions in relation to driving under the effect of alcohol and/or drugs (Bernstein 2010a; Monti 1999); we have reported in Table 2 findings from the other two studies.

\section{Measures}

Measures from these studies related to dangerous driving, alcoholand/or drug-related vehicle-risk behaviour, and driving with a drinking driver.

\section{Effectiveness over the short term}

Analysis 8.1 presents results of the meta-analysis for vehiclerelated risk behaviour. Two studies with targeted individual-level interventions reported different effects, with one accident and emergency (A\&E)-based brief motivational intervention showing no effect on alcohol-related vehicle-risk behaviour (OR 1.11, 95\% CI 0.74 to 1.69 (n of participants not provided)) (Bernstein 2010a), and a second brief intervention showing benefit for reducing drinking and driving (OR $0.26,95 \% \mathrm{Cl} 0.08$ to $0.83 ; \mathrm{n}=94$ participants), although this was a small study and the $95 \%$ confidence intervals were wide (Monti 1999).

Among the two studies that could not be included in the metaanalysis, one providing a targeted individual-level intervention (Nirenberg 2013) and one providing a universal school-based intervention (D'Amico 2002a) reported null effects. 


\section{Long-term effectiveness}

Identified studies conducted follow-up over a period of six months post intervention (D'Amico 2002a; Monti 1999; Nirenberg 2013), or over 12 months post intervention (Bernstein 2010a). No studies provided longer-term follow-up.

\section{Sexual risk behaviour}

Twenty-one studies (30\%) aimed to reduce sexual risk behaviour alongside other risk behaviours (see Table 4; and Table 5), and two studies examining additional interventions measured sexual risk behaviour at long-term follow-up (Conduct Problems Prevention Research Group 2010; Kellam 2014), giving a rationale for an indirect effect of the interventions on sexual risk behaviour (e.g. via targeting of early aggressive and disruptive behaviour, which are antecedent risk factors for later risk behaviours including sexual risk).

Most of the studies provided universal school-based interventions $(n=9 ; 43 \%)$ or targeted family-based interventions ( $n=7 ; 33 \%$ ). Most interventions concurrently addressed antisocial behaviour and prevention of use of at least one substance $(n=15)$. One study was conducted in Australia (Bond 2004), one in Hong Kong (Shek 2011), one in Canada (Wolfe 2012), and one in South Africa (Matthews 2016); the remainder were conducted in the USA ( $n=16$ studies). Further study details can be found in Table 3; and Table 5.

Eleven studies provided short-term data and could be included in the meta-analysis (Beets 2009; Bernstein 2010a; Bond 2004; Flay 2004a; Li 2002; McNeal 2004; Milburn 2012; Minnis 2014; Pantin 2009; Redding 2015; Shek 2011), and eight studies provided longer-term follow-up (Bond 2004; Bonds 2010; Conduct Problems Prevention Research Group 2010; Haggerty 2007a; Kellam 2014; Kim 2011; Redding 2015; Wolfe 2012). Five studies could not be included in the meta-analysis (Estrada 2015; Freudenberg 2010; Griffin 2006; Olds 1998; Piper 2000a); we have provided in Table 2 data from these studies.

\section{Measures}

Measures of sexual risk behaviour included condom use, unprotected sex, multiple partners, and composite measures of sexual risk. Studies that targeted primary school age or early adolescence reported early initiation of sexual intercourse.

\section{Effectiveness over the short term}

Analysis 9.1 presents results of the meta-analysis for sexual risk behaviour by study type for interventions reporting follow-up data up to 12 months. Moderate quality evidence shows that individuallevel interventions probably have little or no effect in reducing sexual risk behaviour (targeted: OR $0.73,95 \% \mathrm{Cl} 0.49$ to 1.08 ; $\mathrm{P}$ $=0.11 ; \mathrm{n}=2$ studies; 494 participants; $\mathrm{I}^{2}=45 \%$; universal: $\mathrm{OR}$ $0.42,95 \% \mathrm{Cl} 0.14$ to $1.25 ; \mathrm{n}=1$ study; 162 participants). The effect estimate for targeted individual-level interventions was in the direction of benefit. However, each subgroup of the meta-analysis included few studies (each subgroup synthesis included fewer than 500 participants); thus findings must be interpreted with caution.

Moderate-quality evidence shows that targeted family interventions also probably have little or no effect in reducing sexual risk behaviour (OR $0.89,95 \% \mathrm{Cl} 0.55$ to $1.44 ; \mathrm{P}=0.63 ; \mathrm{n}=3$ studies; 371 participants).
On average, across six universal school-based interventions, the effect was in the direction of benefit of the interventions, but the $95 \% \mathrm{Cl}$ was consistent with the null hypothesis of no effect (OR 0.83 , $95 \% \mathrm{Cl} 0.61$ to $1.12 ; \mathrm{P}=0.22 ; \mathrm{n}=6$ studies; 12,633 participants; low quality evidence) and heterogeneity was substantial $\left(I^{2}=77 \%\right)$, in part because one study showed a clear beneficial effect (OR 0.18 , $95 \% \mathrm{Cl} 0.08$ to 0.41 ) (Beets 2009). Sensitivity analysis did not change these findings (Table 6).

Among studies that could not be included in the meta-analysis, one targeted individual-level study reported null findings (Freudenberg 2010). One targeted family-based study reported beneficial findings (Murry 2014), and one reported null findings (Estrada 2015).

\section{Long-term effectiveness}

Analysis 9.2 presents results of the meta-analysis for sexual risk behaviour by study type, for studies reporting longer-term follow up. As for short-term analyses, few interventions in each study type subgroup provided long-term follow-up data. One targeted individual-level study reported a null effect (OR $0.93,95 \% \mathrm{Cl} 0.64$ to $1.35 ; \mathrm{n}=461$ participants).

Moderate-quality evidence shows benefit of targeted family-based interventions on average (OR $0.47,0.31$ to $0.71 ; \mathrm{P}=0.0004 ; \mathrm{n}=2$ studies; 318 participants; $I^{2}=0 \%$ ), and one universal family-level intervention (with two study arms) reported a null effect (OR 1.12, $95 \% \mathrm{Cl} 0.64$ to $1.96 ; 237$ participants). One targeted school-based intervention provided evidence of benefit (OR $0.62,95 \% \mathrm{Cl} 0.47$ to $0.82 ; \mathrm{P}=0.0009 ; 650$ participants). As above, the overall effect of universal school-based interventions was in the direction of benefit, but the $95 \% \mathrm{Cl}$ was consistent with the null hypothesis of no effect (OR 0.74, 95\% Cl 0.50 to 1.09; $\mathrm{P}=0.13 ; \mathrm{n}=3$ studies; 3391 participants).

Among studies that could not be included in the meta-analysis, one targeted family-based study reported beneficial findings (Bonds 2010), and one reported beneficial findings in one subgroup for one study arm only (Olds 1998). One universal school-based study reported beneficial findings for number of sexual partners but not for condom use (Griffin 2006).

\section{Physical activity}

Nine studies targeted physical inactivity (Bush 1989; Lana 2014; Melnyk 2013; Nader 1999; O'Neill 2011; Saraf 2015; Schwinn 2014; Walker 2002; Walter 1989), six of which were universal school-based interventions (Bush 1989; Melnyk 2013; Nader 1999; O'Neill 2011; Saraf 2015; Walter 1989). All concurrently aimed to prevent poor nutrition, and all simultaneously aimed to prevent use of at least one substance. One study was conducted in the UK (Walker 2002), one in Spain/Mexico (Lana 2014), and one in India (Saraf 2015); the remainder were conducted in the USA (six studies).

\section{Measures}

Studies assessed vigorous activity, fitness, and physical activity.

\section{Effectiveness over the short term}

Analysis 10.1 presents findings of the meta-analysis for vigorous physical activity by study type for studies reporting follow-up data up to 12 months. Seven studies could be included in the meta-analysis. Evidence shows that universal individual-level interventions probably have little or no effect in enhancing physical 
activity (OR 1.11, 95\% $\mathrm{Cl} 0.74$ to $1.67 ; \mathrm{P}=0.62 ; \mathrm{n}=2$ studies; 1530 participants; $I^{2}=0 \%$; moderate-quality evidence).

One targeted family-level intervention provided no benefit (OR $0.72,95 \% \mathrm{Cl} 0.29$ to $1.75 ; \mathrm{n}=61$ participants). Note here that positive odds ratios demonstrate that the intervention can increase physical activity and thus represents a benefit (i.e. favouring the intervention).

In contrast to the aforementioned studies, evidence shows that on average, across four studies, universal school-based studies improve physical activity (OR 1.32, 95\% $\mathrm{Cl} 1.16$ to 1.50 ; $\mathrm{P}<0.0001 ; \mathrm{n}$ $=4$ studies; 6441 participants; $I^{2}=0 \%$; moderate-quality evidence). Sensitivity analysis around the ICC did not change this result (OR $1.33,95 \% \mathrm{Cl} 1.18$ to $1.50 ; \mathrm{P}<0.00001 ; \mathrm{I}^{2}=0 \%$ ) (Table 6), and including only studies conducted in high-income countries slightly increased the odds ratio (Table 6).

Among four universal school-based studies that could not be included in the meta-analysis, three reported beneficial findings (Bush 1989; O'Neill 2011; Saraf 2015), and one showed a null effect (Walter 1989).

\section{Long-term effectiveness}

No studies reported longer-term follow-up.

\section{Unhealthy diet outcomes}

Eleven (16\%) of the 70 studies addressed unhealthy diet (Bush 1989; Fearnow-Kenney 2003; Lana 2014; Melnyk 2013; Nader 1999; O'Neill 2011; Piper 2000a; Saraf 2015; Schwinn 2014; Walker 2002; Walter 1989), eight of which provided universal-school based interventions (Bush 1989; Fearnow-Kenney 2003; Melnyk 2013; Nader 1999; O'Neill 2011; Piper 2000a; Saraf 2015; Walter 1989). All simultaneously addressed substance use in addition to unhealthy diet; and nine studies concurrently addressed physical activity. One study was conducted in the UK (Walker 2002), one in Spain/Mexico (Lana 2014), and one in India (Saraf 2015); the remainder were conducted in the USA. Further details can be found in Table 4 and Table 5.

\section{Measures}

Studies reported outcomes related to unhealthy diet (e.g. dietary cholesterol, dietary fat) as well as body mass index (BMI) to provide a measure of obesity. We conducted separate meta-analyses for these outcome measures.

\section{Effectiveness over the short term}

Analysis 12.1 and Analysis 12.2 present results of the meta-analyses for BMI and unhealthy diet by study type for studies reporting follow-up to 12 months.

For BMI, one universal individual-level intervention showed a null effect (OR $0.80,95 \% \mathrm{Cl} 0.48$ to $1.31 ; 579$ participants). There was no evidence that universal school-based interventions, on average, had a positive effect (OR $0.84,95 \% \mathrm{Cl} 0.60$ to $1.19 ; \mathrm{P}=0.33 ; \mathrm{n}=3$ studies; 5017 participants), and heterogeneity was substantial $\left(I^{2}=\right.$ $61 \%)$.

For unhealthy diet, the evidence for individual-level interventions was uncertain (OR $0.76,95 \% \mathrm{Cl} 0.42$ to $1.34 ; \mathrm{P}=0.34 ; \mathrm{n}=2$ studies; 1549 participants; $\left.\mathrm{I}^{2}=51 \%\right)$. On average, across three studies, the effect of universal school-based interventions was in the direction of benefit in relation to unhealthy diet, but the $95 \% \mathrm{Cl}$ was consistent with the null hypothesis of no effect (OR $0.82,95 \% \mathrm{Cl}$ 0.64 to $1.06 ; P=0.13 ; n=3$ studies; 6441 participants; $I^{2}=49 \%$ ), and few studies were included in the meta-analysis. Sensitivity analysis including only studies conducted in high-income countries $(n=2$ studies) slightly increased the odds ratio (OR $0.95,95 \% \mathrm{Cl} 0.76$ to 1.19; $P=0.68 ; I^{2}=0 \%$ (Table 6).

Among studies for which data could not be included in the metaanalyses, one universal school-based study reported a null effect for BMI and a beneficial effect for total fat intake in one of two areas included in the study (Walter 1989). A second universal schoolbased study reported beneficial effects (O'Neill 2011).

\section{Long-term effectiveness}

No studies provided longer-term follow-up data.

\section{Secondary outcomes}

We identified data regarding mental health and educational attainment that could be included in quantitative syntheses; we have reported in Table 2 data regarding teenage pregnancy. We did not identify data that could be analysed regarding costeffectiveness of the interventions.

\section{Mental health}

Seventeen (27\%) of 62 studies aimed to improve mental health (Bodin 2011; Bond 2004; Bonds 2010; Brody 2012; Conduct Problems Prevention Research Group 2010; Gonzales 2012; Ialongo 1999; Ialongo 1999b; Kellam 2014; Kim 2011; Kitzman 2010; Li 2011; Melnyk 2013; Morris 2003; Olds 1998; Sanchez 2007; Walker 2002), one through indirect long-term mechanisms (Olds 1998). Six of these (35\%) provided targeted family-based interventions, and five (29\%) universal school-based interventions (see Table 5). Fifteen of these studies addressed substance use, and fourteen also aimed to prevent antisocial behaviour. Among studies not conducted in the USA, one was conducted in Sweden (Bodin 2011), one in Ireland (Dolan 2010), one in the UK (Walker 2002), and one in Australia (Bond 2004).

Seven studies could not be included in the meta-analysis (Gonzales 2012; Ialongo 1999; Ialongo 1999b; Kellam 2014; Sanchez 2007; Conduct Problems Prevention Research Group 2010; Olds 1998), four of which reported clinical assessments (e.g. diagnosis of conduct disorder). We have reported findings from these studies in Table 2.

\section{Measures}

All studies included in the meta-analysis measured depression or internalising behaviour/problems.

\section{Effectiveness over the short term}

Analysis 11.1 presents results of meta-analyses for depressive symptoms by study type for studies reporting follow-up to 12 months. Four studies provided short-term follow-up data and could be included in the meta-analysis. One targeted individual-level intervention showed a null effect $(\mathrm{OR} 1.02,95 \% \mathrm{Cl} 0.54$ to $1.93 ; \mathrm{n}=$ 124 participants). On average, across three universal school-based studies, we found no evidence of benefit in relation to prevention of depressive symptoms (OR $0.92,95 \% \mathrm{Cl} 0.71$ to $1.20 ; \mathrm{P}=0.56 ; \mathrm{n}=$ 3 studies; 3907 participants), and heterogeneity was substantial ( $I^{2}$ $=63 \%)$. 


\section{Long-term effectiveness}

Analysis 11.2 presents results of meta-analyses for depressive symptoms by study type for studies reporting longer-term followup data ( $>12$ months). Five studies were included in the meta-analysis, four of which provided targeted family-based interventions. Overall, evidence showed benefit of targeted familybased interventions in preventing depressive symptoms over the longer term (OR $0.88,95 \% \mathrm{Cl} 0.80$ to $0.98 ; \mathrm{P}=0.02 ; \mathrm{n}=4$ studies; 2386 participants; $\left.I^{2}=0 \%\right)$. One targeted school-based study reported inconclusive evidence (OR $0.68,95 \% \mathrm{Cl} 0.42$ to $1.09 ; \mathrm{n}=721$ participants).

Two targeted family-based studies that could not be included in the meta-analysis reported beneficial effects for some outcomes and not for others or at different time points (Bonds 2010; Gonzales 2012); another reported a null effect (Olds 1998). One targeted school-based intervention showed a null effect (Kellam 2014), and one universal individual-level intervention showed benefit only among those reporting probable depression (Walker 2002).

\section{Educational attainment}

Nineteen studies (31\%) aimed to improve educational attainment alongside other risk behaviours (Berry 2009; Conduct Problems Prevention Research Group 2010; Dolan 2010; Freidman 2002; Freudenberg 2010; Gonzales 2012; lalongo 1999; lalongo 1999b; Kellam 2014; Kitzman 2010; Li 2011; LoSciuto 1999; Melnyk 2013; Minnis 2014; Morris 2003; Olds 1998; Sanchez 2007; Schweinhart 1993; Tierney 1995), five of which were targeted at the individual level and four at the family level; seven provided universal schoolbased interventions (see Table 4). One of these was conducted in the UK (Berry 2009), one in Ireland (Dolan 2010), and one in Canada (Morris 2003); the remaining 17 studies (85\%) were conducted in the USA. Sixteen studies concurrently targeted substance use, and 17 targeted antisocial behaviour.

\section{Measures}

Studies reported academic achievement in terms of grade point average (GPA) or scores for particular subjects (e.g. mathematics achievement scores).

\section{Effectiveness over the short term}

Analysis 13.1 presents data for the meta-analysis for academic achievement by study type for studies reporting follow-up data up to 12 months. Six studies could be included in meta-analyses of short-term follow-up data, one of which tested two study arms at the same time (lalongo 1999; lalongo 1999b). Findings were variable, and each subgroup included few studies. One targeted individual-level intervention reported a null effect (OR 1.34, 95\% Cl 0.71 to $2.52 ; 126$ participants). Three targeted school-based studies reported null findings $(\mathrm{OR} 0.91,95 \% \mathrm{Cl} 0.30$ to $2.73 ; \mathrm{P}=0.86 ; \mathrm{n}=$ 3 studies; 1247 participants; $I^{2}=84 \%$ ), and one universal schoolbased study with two study arms reported null findings (OR 0.94, $95 \% \mathrm{Cl} 0.62$ to $1.44 ; 579$ participants; $I^{2}=0 \%$ ).

\section{Long-term effectiveness}

We were not able to include in a meta-analysis data regarding longer-term educational outcomes. Consequently, we did not conduct a meta-analysis to examine long-term effectiveness for this outcome. Among studies that could not be included in the metaanalysis: three targeted individual-level interventions reported a beneficial effect for education or employment (Berry 2009;
Freudenberg 2010), as well as academic achievement (Tierney 1995), and one reported no effect on school problems (Freidman 2002).

One targeted family-level intervention reported an indirect effect of the intervention through school engagement (Gonzales 2012), and another reported benefit in relation to educational attainment but no effect on school suspensions (Kitzman 2010). For another targeted family-level financial support intervention, findings were uncertain (Morris 2003). One targeted school-based intervention reported benefit of preschool programme (Schweinhart 1993), and the findings of a targeted school-based study were inconclusive (Conduct Problems Prevention Research Group 2010).

Two universal school-level interventions reported a positive effect in relation to school attendance and academic competence (LoSciuto 1999; Melnyk 2013), one universal school-based intervention showed no effect on school attachment (Bond 2004), another reported no effect on school suspensions (Li 2011), and another reported possible benefit in relation to high school graduation (Kellam 2014).

We have presented these results in Table 2.

\section{Multiple behaviours}

Although some studies provided insufficient evidence of a positive effect on more than one outcome, several interventions showed benefit in preventing more than one behaviour, although the strength of the evidence for an effect varied between studies.

Among targeted individual-level interventions, the REAL MEN intervention for young males leaving jail, described in Freudenberg 2010, and the mentoring intervention, Big Brothers Big Sisters, reported by Tierney 1995, yielded effects for multiple behaviours that were in the direction of benefit, as did the individuallevel conditional cash transfer and life skills intervention, Yo Puedo (Minnis 2014). Evidence suggests that targeted familylevel interventions New Beginnings (Bonds 2010), SAAF-T (Brody 2012), Middle School Success (Kim 2011), and the Nurse Family Partnership (Kitzman 2010) produced positive effects for at least three behaviours; and evidence shows that the targeted schoollevel intervention FAST TRACK showed benefit in relation to at least three risk behaviours (Conduct Problems Prevention Research Group 2010).

Researchers provided evidence of a positive effect of six universal school-based interventions on at least three risk behaviours (Beets 2009; Bond 2004; Li 2011; Melnyk 2013; O'Neill 2011; Saraf 2015), and they provided evidence suggestive of benefit for three others (DeGarmo 2009; Griffin 2006; Kellam 2014). Positive Action (Hawaii) showed benefit in preventing tobacco use, alcohol use, drug use, antisocial behaviour, and sexual risk behaviour (Beets 2009), and Positive Action (Chicago) showed benefit in relation to alcohol use, antisocial behaviour, depressive symptoms, and illicit drug use (Lewis 2012; Lewis 2013; Li 2011). Positive Action (a socialemotional and character development model) involves more than 14015 -minute, age-appropriate lessons taught four days per week from kindergarten to grade six, and 70 lessons taught two days per week for grades seven and eight. Training for teachers, families, communities, and school climate changes is also involved. The Michigan Model for Health (MMH) (O'Neill 2011), a health education curriculum intervention for students from kindergarten to grade 
12 , showed beneficial results in relation to tobacco use, alcohol use, and antisocial behaviour, with a more recent study highlighting effects for physical activity and nutrition (see Studies awaiting classification). The MMH involves 24 lessons in grade four and 28 lessons in grade five, focusing on skills-based learning. The 15week health course, COPE, which incorporated physical activity into skills-building sessions, was effective in reducing alcohol use and BMI and increasing physical activity (Melnyk 2013). Saraf 2015 reported beneficial effects of a multi-component school-based study in relation to tobacco use, poor diet, and physical inactivity. Over the longer term, FAST TRACK, which was implemented between grades 1 and 10, and which involved parent training, tutoring, skills development curriculum, home visits, and parentchild and parent-youth groups, was effective in preventing alcohol use, illicit drug use, and sexual risk behaviour (Conduct Problems Prevention Research Group 2010). Last, the Gatehouse study (Bond 2004), a whole-school intervention involving a curriculum and school-wide changes, reduced tobacco use as well as sexual risk behaviour and antisocial behaviour over the longer term.

Investigation of the impact of interventions on combinations of behaviours will be the topic of further research to be conducted by the study team in the future.

\section{DISCUSSION}

\section{Summary of main results}

This review has examined evidence related to the effectiveness of individual-, school-, and family-level interventions that target multiple risk behaviours among young people eight to 25 years of age. We identified 70 studies, of which half were universal and half were targeted to individuals in particular ethnic or socioeconomic groups, or to those identified as being at higher risk of engagement in risk behaviours and/or consequent harms. A substantial proportion of the interventions identified were universal school-based interventions ( $n=28 ; 40 \%)$, and these made up the majority of school-based programmes. Of 35 targeted studies, 17 (49\%) provided family-level interventions, six (17\%) school-level interventions, and 12 (34\%) individual-level interventions.

The included studies focused on a broad range of outcomes, targeting, on average, four behaviours. Most studies targeted tobacco, alcohol, and/or drug use and antisocial behaviour. We conducted meta-analyses for ten primary outcomes (tobacco use, alcohol use, binge drinking, illicit drug use, cannabis use, substance use, vehicle-related risk behaviour, sexual risk behaviour, physical inactivity, and unhealthy diet) and two secondary outcomes (depressive symptoms and educational attainment).

Overall, evidence from meta-analyses showed that on average, universal school-based interventions probably have a positive effect in relation to tobacco use, alcohol use, and physical activity, and that they may also have a beneficial effect in relation to illicit drug use and antisocial behaviour at up to 12 months' followup, versus a comparator. Available data did not permit analysis of particular intervention components associated with effectiveness, and no single component was consistently associated with stronger effects. Nevertheless, our data suggest that interventions for which meta-analyses showed beneficial effects in relation to at least one behaviour included additional components, such as school policy changes, school-wide adoption of the intervention principles, or family engagement.

For instance, our meta-analyses demonstrated a beneficial effect of the Positive Action programme on tobacco, alcohol, and drug use, as well as antisocial behaviour (Beets 2009; Li 2011). This programme commenced in primary school and involved classroom curricula each year, school-wide climate changes to reinforce positive actions throughout the school, and family components organised around the core concepts of self-concept, positive actions for body and mind, getting along with others, social and emotional actions for managing oneself responsibly, being honest with yourself and others, and self-improvement. In addition to Positive Action, the meta-analysis demonstrated that interventions that were effective in relation to tobacco use included the Gatehouse Study (Bond 2004), the Michigan Model for Health (O'Neill 2011), and Going Places (Simons-Morton 2005), with the Michigan Model for Health being effective in relation to alcohol use and antisocial behaviour as well. As noted for Positive Action (Beets 2009; Li 2011), which is a kindergarten through grade 12 multi-component programme, these interventions have in common an extended duration of intervention, or a multicomponent or whole-school approach. For instance, Going Places included a social skills curriculum, parent education, and school environment enhancement and was implemented across three school years (Simons-Morton 2005); the Gatehouse Project is a whole-school intervention based on the Health-Promoting Schools Framework (Bond 2004); and the Michigan Model for Health is a kindergarten through grade 12 school curriculum programme (O'Neill 2011). Additional programmes that were effective in relation to two behaviours (COPE and BRAVE) involved family or mentoring components as well as school curricula (Griffin 2009; Melnyk 2013). Thus, it is possible that interventions that have multiple components, involve school-wide changes, and/or are extended in duration may be effective in relation to these behaviours.

In contrast to the above studies, we did not find evidence that family-level or individual-level interventions have a positive effect on the outcomes investigated, although we identified fewer of these studies. In addition, effect estimates for individual-level studies were in the direction of benefit for certain outcomes (alcohol use, cannabis use, sexual risk behaviour) but not for others.

Over the longer term, evidence showed that universal school-based interventions that took a whole-school approach - as described by Bond 2004 - or that commenced early in primary school and targeted antecedent risk factors - as examined by lalongo 1999, lalongo 1999b, and Kellam 2014 - may be beneficial in relation to illicit drug use. Evidence showed that targeted family-level interventions may be effective in reducing substance use, antisocial behaviour, sexual risk behaviour, and depressive symptoms over the longer term. However, it must be noted that comparatively few studies reported longer-term follow-up data (i.e. at least two years post intervention), so these findings should be interpreted with caution. Evidence suggests that multiple risk behaviour interventions conducted at the individual level may have little or no effect in preventing these outcomes, although, again, these studies are few. 


\section{Overall completeness and applicability of evidence}

In our review, most studies addressed tobacco, alcohol, and/or drug use alongside antisocial behaviour, with a smaller proportion addressing sexual risk behaviour, mental illness, and educational attainment.

It is interesting to note that despite relatively high prevalence of engagement in other risk behaviours, we found few studies targeting these behaviours as a multiple risk approach alongside the other behaviours included in this review. For instance, data from cohort studies show that in the UK, $26 \%$ of females and $9 \%$ of males have ever self-harmed by age 16 to 17 years (Kidger 2012), and self-harm is a leading cause of mortality among young people globally (leading to $8 \%$ to $9 \%$ of deaths) (Mokdad 2013). Recent estimates of the prevalence of gambling among young people aged 10 to 24 years have ranged from $0.2 \%$ to $12.3 \%$ (Calado 2016), and road injuries remain a leading cause of disability-adjusted life years (DALYs) and death among young people globally (accounting for $5.4 \%$ of total DALYs for young people aged 10 to 24 years and $14 \%$ to $15 \%$ of deaths among young people aged 15 to 24 respectively) (Gore 2011; Mokdad 2013). Nevertheless, we found no studies that targeted self-harm or gambling alongside other behaviours, and only four studies targeted vehicle-related risk behaviour in multiple risk behaviour interventions. This was also the case for physical inactivity and poor nutrition, which have high prevalence (MacArthur 2012) but were not frequently targeted alongside risk behaviours that may represent greater association with experimentation or 'thrill-seeking'. This may reflect the view that addressing antecedent risks of low levels of activity and poor diet may require distinct approaches owing to clustering patterns of different risk behaviours (Faeh 2006; Meader 2016; Mistry 2009; van Nieuwenhuijzen 2009; Wiefferink 2006), or because of the need to focus on training in specific skills related to addressing a particular risk behaviour, such as self-harm.

We identified a larger number of studies that compared universal school-based interventions versus those targeted at the individual or family level; thus we note that some caution is warranted in interpreting findings regarding the latter types of interventions. School-based programmes have tended to predominate among interventions targeted to adolescents. This may reflect the target age range of participants, thus almost universal coverage of young people, access to large numbers of adolescents, and ease of delivery (Bonnell 2016; Stockings 2016), making schools a highly efficient setting for behaviour change-focused interventions. Most of these 34 school-level interventions $(n=28 ; 82 \%)$ were universal in nature and did not target particular groups, as might be expected in this setting. Although several studies targeted individuals in early adolescence and thus focused on primary prevention, relatively few were initiated among children younger than 10 years $(n=10$ of 28 studies; $36 \%$ ), and so it was not possible to assess whether early intervention led to differential impact for the different outcomes assessed.

As outlined above, data suggest that interventions with beneficial effects were often characterised by multiple components including school-wide changes or family engagement. Recent evidence highlights that combined student and parent programmes can be effective in relation to substance use outcomes (Newton 2017), and whole-school interventions that combine multiple components such as policy changes and parental involvement can prevent smoking and sexual risk behaviour (Langford 2014; Shackleton
2016). We will be conducting further research to explore the impact of distinct components of interventions, combinations of components, or the intensity of intervention components because such additional analyses were not possible here, given the scale and complexity of this review. Similarly, we were not able to examine the specific impact of interventions that targeted particular combinations of behaviours; further analyses are required in this regard.

In contrast to school-based interventions, 17 of 20 (85\%) familylevel studies were targeted to particular populations on the basis of factors such as ethnic group, socioeconomic status, or family status (e.g. being in foster care, having recently divorced parents). Most of these interventions targeted illicit drug use (95\%), antisocial behaviour (85\%), and alcohol use (80\%). Over half (55\%) targeted tobacco use, sexual risk, and mental illness. The particular groups targeted in such interventions showed variability, and heterogeneity was evident among the interventions themselves, making conclusions about these types of interventions difficult.

Although this review provides the first quantified effect estimate for the effects of multiple risk behaviour interventions, we must note additional limitations of the review. Overall, all studies lacked a focus on equity. For instance, data were insufficient to show the impact of interventions in relation to gender, ethnicity, and/or socioeconomic group. In addition, most studies were conducted in the USA (79\%), only two studies in low- or middle-income countries (Matthews 2016; Saraf 2015), and one jointly in an upper-middleincome country and a high-income country (Lana 2014). A scoping review of multiple risk behaviour interventions in adult populations also found a scarcity of studies conducted among minority ethnic groups and lack of studies conducted in the Middle East, Africa, and South America (Meader 2016). Thus, the generalisability of these findings to other geographical settings and educational or cultural contexts is unclear. In 2012 it was reported that $90 \%$ of the world's population of young people aged 10 to 24 years live in low- and middle-income countries (LMICS), and some of the highest rates of tobacco use and overweight and lowest levels of physical activity were evident in these areas (Sawyer 2012), alongside a substantial health burden from injuries, unsafe sex, and alcohol use (Gore 2011; Mokdad 2013). These findings together highlight the need to examine the effectiveness of context-dependent and culturally relevant interventions that may target multiple risk factors or behaviours in LMICs. As we have discussed, we were unable to explore the impact of distinct components of interventions, combinations of components, or the impact of targeting different combinations of behaviours using available data; thus further research is needed to allow a more detailed understanding of the components needed for successful prevention of engagement in multiple behaviours. Last, few studies reported long-term followup data, so the extent to which effects are sustained over the longer term remains unclear.

\section{Quality of the evidence}

We rated the overall quality of evidence for each outcome using the GRADE approach and found the quality to be low or moderate, with the exception of antisocial behaviour, for which we judged the quality of the evidence to be very low overall (see Summary of findings for the main comparison). This was due to several factors. First, a large proportion of studies were at unclear or high risk of bias owing to lack of blinding and lack of clarity around allocation concealment or reporting. As noted above, it is not 
always possible to blind complex public health interventions such as those included in this review, and many studies were published before reporting guidelines were available. Nevertheless, we took such factors into account when considering the quality of included evidence. Second, we downgraded the quality of the evidence on the basis of inconsistency when we found evidence of substantial heterogeneity $\left(\mathrm{I}^{2}\right)$, large between-study variance, and poor overlap between $95 \%$ confidence intervals. It is likely that there are plausible explanations for the heterogeneity observed, but we were unable to explore possible explanations further via subgroup or meta-regression analyses (see Assessment of heterogeneity). Lastly, for certain outcomes, funnel plots suggested possible smallstudy or publication bias.

It should be noted, however, that the use of GRADE to judge quality of evidence from complex public health interventions may have limitations (Movsisyan 2016; Movsisyan 2016b; Rehfeuss 2013); it is unusual for complex interventions to be rated as 'high' quality, and ratings of very low quality compared with that of simple interventions are likely (Movsisyan 2016b). For instance, as stated above, it is generally not feasible or possible to blind study participants, and heterogeneity among these complex interventions is inevitable, owing to factors such as variability in numbers and types of intervention components, modes of intervention delivery, and intervention contexts (Movsisyan 2016b; Rehfeuss 2013).

\section{Potential biases in the review process}

We assessed the possibility of publication bias for several outcomes when we identified a sufficient number of studies (see Risk of bias in included studies). These analyses highlighted the possibility of small-study bias or reporting bias due to an underrepresentation of smaller studies reporting negative findings. However, we conducted searches in all languages, with no geographical restrictions, and we searched a large number of databases, alongside searches of grey literature, so our search for studies was extremely thorough in identifying available studies. We contacted study authors for additional data when data were missing, and we incorporated such data into our analyses.

We note that owing to the poor quality of data reported in many studies, it was necessary to manipulate the data to conduct analyses, including re-analysis of data to account for clustered data (e.g. for school-based studies). We were not able to address issues around randomisation of matched clusters in such randomised controlled trials. We conducted sensitivity analyses to assess the impact of using different intracluster correlation coefficients (ICCS), and findings were unchanged in most cases. In three instances in which summary effect estimates changed, they reached statistical significance, demonstrating the conservative nature of our main analyses.

\section{Agreements and disagreements with other studies or reviews}

\section{Primary outcomes}

\section{Tobacco, alcohol, and/or drug use}

Our findings support a systematic review focused on interventions that target multiple risk behaviours (tobacco use, alcohol use, drug use, and sexual risk behaviour), which reported mixed but broadly beneficial findings (Jackson 2011). Similarly, a systematic review of interventions targeting substance use, sexual risk, and antisocial behaviour reported that such interventions were broadly effective (Hale 2014), although no meta-analysis was conducted and only trials reporting positive findings were included. A Cochrane review that examined effects of interventions on tobacco use reported that social competence curricula (odds ratio (OR) $0.52,95 \%$ confidence interval $(\mathrm{Cl}) 0.30$ to 0.88 ) and combined social competence and social influences curricula were effective in preventing the onset of smoking (OR $0.50,95 \% \mathrm{Cl} 0.28$ to 0.87 ) (Thomas 2013); Langford 2014 also found that tobacco and multiple risk behaviour interventions within the Health-Promoting Schools (HPS) Framework were effective in reducing tobacco use (OR 0.77, $95 \% \mathrm{Cl} 0.64$ to 0.93 ; and OR $0.84,95 \% \mathrm{Cl} 0.76$ to 0.93 , respectively). In addition, a recent systematic review identified weak evidence of benefit of social competence, social influence, and combined interventions in relation to cannabis use among young people (risk ratio (RR) $0.90,95 \% \mathrm{Cl} 0.81$ to 1.01 ; RR $0.88,95 \% \mathrm{Cl} 0.72$ to 1.07 ; and RR $0.79,95 \% \mathrm{Cl} 0.59$ to 1.05 , respectively) (Faggiano 2014), although results showed no clear benefit in relation to hard drugs. Thus, our findings, together with a wider body of evidence, suggest that school-based programmes targeting single or multiple behaviours may be effective in preventing smoking in adolescent populations.

Our finding regarding the impact of universal school-based programmes in relation to alcohol use contrasts with those reported in other reviews. For instance, Foxcroft 2011 reported that psychosocial and developmental prevention programmes can be effective, but that findings were mixed overall, with some studies reporting statistically significant effects and others reporting no effect. Additional systematic reviews have similarly highlighted the mixed findings of reviews of school-based or education- or skills-based prevention interventions, with no clear pattern distinguishing effective from ineffective interventions (Martineau 2013; Stockings 2016), although Strom 2014 found a small but favourable effect among school-based interventions reporting continuous outcomes and no effect among those reporting categorical outcomes. Langford 2014 found no effect of interventions testing the HPS framework to address alcohol use or multiple risk behaviours (OR $0.72,95 \% \mathrm{Cl} 0.34$ to 1.52 ; OR 0.75 , 95\% $\mathrm{Cl} 0.55$ to 1.02 , respectively).

In addition, although we identified few studies overall, we found an absence of evidence to support the effectiveness of family-based interventions in preventing tobacco or alcohol use. Systematic reviews of alcohol-focused parenting interventions have reported small but consistently beneficial effects (Allen 2016; Foxcroft 2011b; Kuntsche 2016; Stockings 2016), and a systematic review of family-based programmes reported a positive effect on prevention of smoking among children and adolescents (Thomas 2015). The contrast in these findings may reflect the combinations of behaviours addressed in this review; our companion review will further investigate the impact of family-based interventions targeted to substance use (Hickman 2014).

\section{Antisocial behaviour and offending}

Evidence indicates that universal school-based programmes may show benefit in reducing antisocial behaviour or offending compared to usual practice. Evidence was less certain in relation to violent offences or school delinquency only, but the summary effect estimate was in the direction of benefit. Our findings support others who have reported that school-based programmes are effective in reducing aggressive behaviour (standardised mean difference 
(SMD) $-0.41,95 \% \mathrm{Cl}-0.56$ to -0.26$)$, with effects maintained at 12 months' follow-up (Mytton 2006). Langford 2014 reported that multi-component school-based interventions reduce bullying victimisation and show promise in reducing perpetration of bullying. Another review has reported beneficial outcomes of parenting programmes aimed at reducing early conduct problems in children three to 12 years of age (SMD $-0.44,95 \% \mathrm{Cl}-0.77$ to -0.11 ) (Furlong 2012), and family and parenting programmes have been reported to reduce the time spent in institutions (weighted mean difference (WMD) 51.34 days, $95 \% \mathrm{Cl} 72.52$ to 30.16 ) and the risk of re-arrest (RR $0.66,95 \% \mathrm{Cl} 0.44$ to 0.98 ) (Woolfenden 2009).

\section{Sexual risk behaviour}

Low-quality evidence suggests that multiple risk behaviour interventions may have little or no effect in reducing sexual risk behaviour, although the average effect was in the direction of benefit and the size of the summary point estimates would be consistent with potential public health benefit at the population level. Among universal school-based interventions, Positive Action showed a strong effect (Beets 2009), and one of two active intervention arms in the Aban Aya trial showed a beneficial effect (Flay 2004a); these study authors reported effects in boys but not girls. The Gatehouse Study, All Stars, and PATHS reported null findings (Bond 2004; McNeal 2004; Shek 2011). Our findings support those of a previous systematic review of multiple risk behaviour interventions (targeting substance use and sexual risk behaviour) (Jackson 2011), which highlighted mixed findings for sexual risk behaviour, including condom use, sexual partners, having had sexual intercourse, and teenage pregnancy.

\section{Physical activity and unhealthy diet}

We found moderate-quality evidence showing that universal school-based multiple risk behaviour interventions are likely to be effective in increasing vigorous activity or fitness among young people (four studies; 6441 participants), with findings suggesting a possible $32 \%$ increase in this outcome associated with such interventions compared to control or usual practice. A Cochrane review of interventions using the HPS Framework also found that physical activity and nutrition interventions are effective in increasing physical activity in students (Langford 2014); Dobbins 2013 reported that school-based programmes could have small but positive effects on physical activity, with possible increases of just under five to 45 minutes more moderate to vigorous physical activity per week, although most studies used selfreported measures. We did not find that interventions had a positive effect on nutrition or body mass index (BMI), but few studies addressed these outcomes and could be included in the meta-analysis (e.g. three universal school-based interventions).

\section{Secondary outcome}

\section{Mental illness and educational attainment}

We noted an absence of evidence to support the effectiveness of school-based interventions in reducing depressive symptoms, although, on average, targeted family programmes appeared to have a beneficial effect over the longer term (four studies; 2386 participants). We found few studies that addressed educational attainment; therefore, although we found no benefit, this finding should be interpreted with caution.

\section{AUTHORS' CONCLUSIONS}

\section{Implications for practice}

For this review, we have conducted quantitative syntheses to obtain the best available estimate of the effectiveness of multiple risk behaviour interventions among young people. We report that school-based programmes provided universally without consideration of individual risk are likely to be effective in preventing tobacco use, alcohol use, and physical inactivity (moderate-quality evidence) and may also be beneficial in relation to illicit drug use (low-quality evidence). We identified that such interventions may also be effective in relation to antisocial behaviour, although the evidence was of very low quality, but low-quality evidence related to sexual risk behaviour was less certain. For these outcomes, we considered the size of the effect to represent potential public health importance at the population level. Because such interventions show promise, there is scope to consider adaptation of universal school-based models to particular contexts and implementation more widely, although we note that some caution is warranted in their interpretation owing to the low or very low quality ratings for evidence related to certain outcomes and the proximity of upper $95 \%$ confidence interval $(\mathrm{Cl})$ levels to one. In contrast to universal school-based interventions, and in the context of identifying fewer such studies, we identified that individual- and family-level interventions may have little or no benefit in relation to the outcomes considered.

Findings reported in this review provide the foundation for research that will assist with commissioning and decision-making around investment or disinvestment in different types of interventions that aim to prevent engagement in multiple risk behaviours among young people. In this way, the review may contribute to shaping future service delivery and the nature of family- or school-based preventive programmes and curricula. Our findings may also play a role in informing national and international guidance around public health interventions and approaches to behaviour change, such as guidelines for preventing smoking, improving physical activity, and preventing drug misuse (such as those of the UK National Institute for Health and Care Excellence (NICE); https:// www.nice.org.uk).

\section{Implications for research}

Conduct of this review has highlighted a number of implications for future research and evaluation. First, interventions were heterogeneous in relation to the age of participants, intervention domains, duration, and outcomes assessed. Although such heterogeneity is to be expected with multiple risk behaviour interventions implemented throughout childhood and adolescence (up to age 18), replication studies of promising models would help to strengthen the evidence base around particular components or intervention characteristics that are effective. We also found a lack of consistency among outcomes assessed and note that greater consistency, or use of a core outcome dataset reflecting outcomes that pose the greatest harm to public health, would assist with quantitative analyses of the effects of interventions.

Furthermore, rigorously conducted and adequately powered randomised controlled trials and replication studies are clearly needed in this field to strengthen available evidence. Interventions must be characterised to a greater extent by adequate sample 
sizes, published protocols, and clear reporting and robust methods, including adjustments for clustered data and imputation for missing data, when necessary. The conduct of comprehensive process evaluations would also be useful to enable detailed exploration and analysis of whether interventions were conducted as planned, any changes that were introduced, mechanisms of action, and the impact of contextual factors on outcomes. In this way, it would be possible to examine how implementation affected outcomes and the potential causal pathways of different interventions (Moore 2015).

Last, given that most interventions were conducted in highincome countries, notably the USA, further research is needed to adapt interventions to wider geographical contexts, enabling the development of tailored and culturally appropriate interventions that are effective in a range of sociodemographic, educational, and geographical environments.

\section{ACKNOWLEDGEMENTS}

We are grateful to Val Hamilton for assistance in devising the search strategies for this review, and to members of the review advisory group (Alison Bell, Professor Selena Gray, Professor Angela Harden, Dr Caroline Jackson, Professor Mike Kelly, and Hazel Miller) and the public involvement advisory group, Advice Leading to Public Health Advancement (ALPHA) Group of the Centre for the Development and Evaluation of Complex Interventions for Public Health Improvement (DECIPHer) for the helpful advice they provided. We are also grateful to Dr Margaret Astin for her assistance and input into the review. 


\section{R E F E R E N C E S}

\section{References to studies included in this review}

\section{Averdijk 2016 \{published data only\}}

Averdijk M, Zirk-Sadowski J, Ribeaud D, Eisner M. Longterm effects of two childhood psychosocial interventions on adolescent delinquency, substance use, and antisocial behavior: a cluster randomized controlled trial. Journal of Experimental Criminology 2016;12:21-47.

\section{Averdijk 2016b \{published data only\}}

Averdijk M, Zirk-Sadowski J, Ribeaud D, Eisner M. Longterm effects of two childhood psychosocial interventions on adolescent delinquency, substance use, and antisocial behavior: a cluster randomized controlled trial. Journal of Experimental Criminology 2016;12:21-47.

\section{Beach 2016 \{published data only\}}

Beach SRH, Barton AW, Lei MK, Mandara J, Wells AC, Kogan SM, et al. Decreasing substance use risk among African American youth: parent-based mechanisms of change. Prevention Science 2016;17:572-83.

\section{Beets 2009 \{published data only\}}

Beets MW. Factors Associated With the Implementation Fidelity of a School-Based Social and Character Development Program: Findings From the Positive Action Program, Hawaii. [PhD thesis]. Oregon State University, Oregon, USA 2007.

Beets MW, Flay BR, Vuchinich S, Acock AC, Li KK, Allred C. School climate and teachers' beliefs and attitudes associated with implementation of the positive action program: a diffusion of innovations model. Prevention Science 2008;9(4):264-75.

Beets MW, Flay BR, Vuchinich S, Snyder FJ, Acock A, Li K-K, et al. Use of a social and character development program to prevent substance use, violent behaviors, and sexual activity among elementary-school students in Hawaii. American Journal of Public Health 2009;99:1438-45.

Snyder F, Flay B, Vuchinich S, et al. Impact of a social-emotional and character development program on school-level indicators of academic achievement, absenteeism, and disciplinary outcomes: a matched-pair, cluster-randomized, controlled trial. Journal of Research on Educational Effectiveness 2010;3:26-55.

Snyder FJ, Acock AC, Vuchinich S, Beets MW, Washburn IJ, Flay BR. Preventing negative behaviors among elementaryschool students through enhancing students' social-emotional and character development. American Journal of Health Promotion 2013;28:50-8.

Snyder FJ, Vuchinich S, Acock A, Washburn IJ, Flay BR. Improving elementary school quality through the use of a social-emotional and character development program: a matched-pair, cluster-randomized, controlled trial in Hawai'i. Journal of School Health 2012;81(1):11-20.

Washburn I, Acock A, Vuchinich S, Snyder F, Li K, Ji P, et al. Effects of a social-emotional and character development program on the trajectory of behaviors associated with social- emotional and character development: findings from three randomized trials. Prevention Science 2011;12(3):314-23.

\section{Bernstein 2010a \{published data only\}}

Bernstein J, Heeren T, Edward E, Dorfman D, Bliss C, Winter M, et al. A brief motivational interview in a pediatric emergency department, plus 10-day telephone follow-up, increases attempts to quit drinking among youth and young adults who screen positive for problematic drinking. Academic Emergency Medicine 2010;17:890-902.

\section{Berry 2009 \{published data only\}}

Berry V, Little M, Axford N, Cusick GR. An evaluation of youth at risk's coaching for communities programme. Howard Journal of Criminal Justice 2009;48:60-75.

\section{Bodin 2011 \{published data only\}}

Bodin $\mathrm{M}$, Leifman $\mathrm{H}$. A randomized effectiveness trial of an adult-to-youth mentoring program in Sweden. Addiction Research and Theory 2011;19(5):438-47.

Bond 2004 \{published data only\}

Bond L, Glover S, Godfrey C, Butler H, Patton GC. Building capacity for system-level change in schools: lessons from the Gatehouse Project. Health Education \& Behavior 2001;28(3):368-83.

Bond L, Patton GC, Glover S, Carlin JB, Butler H, Thomas L. The Gatehouse Project: can a multilevel school intervention affect emotional wellbeing and health risk behaviors. Journal of Epidemiology and Community Health 2004;58:997-1003.

Bond L, Thomas L, Coffey C, Glover S, Butler H, Carlin JB. Longterm impact of the Gatehouse Project on cannabis use of 16year-olds in Australia. Journal of School Health 2004;74(1):23-9.

Patton G, Bond L, Butler H, Glover S. Changing schools, changing health? Design and implementation of the Gatehouse Project. Journal of Adolescent Health 2003;33(4):231-9.

Patton GC, Bond L, Carlin JB, Thomas L, Butler H, Glover S, et al. Promoting social inclusion in schools: a group-randomized trial of effects on student health risk behavior and well-being. American Journal of Public Health 2006;96(9):1582-7.

Patton GC, Glover S, Bond L, Butler H, Godfrey C, Di Pietro G, et al. The Gatehouse Project: a systematic approach to mental health promotion in secondary schools. Australian and New Zealand Journal of Psychiatry 2000;34(4):586-93.

\section{Bonds 2010 \{published data only\}}

Bonds McClain D, Wolchik SA, Winslow E, Tein J-Y, Sandler IN, Millsap RE. Developmental cascade effects of the New Beginnings Program on adolescent adaptation outcomes. Development and Psychopathology 2010;22(4):771-84.

Wolchik SA, Sandler IN, Millsap RE, Plummer BA, Greene SM, Anderson ER, et al. Six-year follow-up of preventive interventions for children of divorce. JAMA 2002;288(15):1874-81. 
Wolchik SA, Sandler IN, Tein JY, Mahrer NE, Millsap RE, Winslow $E$, et al. Fifteen-year follow-up of a randomized trial of a preventive intervention for divorced families: effects on mental health and substance use outcomes in young adulthood. Journal of Consulting \& Clinical Psychology 2013;81:660-73.

\section{Brody 2012 \{published data only\}}

Brody GH, Chen Y-F, Kogan SM, Yu T, Molgaard VK, DiClemente RJ, et al. Family-centered program deters substance use, conduct problems, and depressive symptoms in black adolescents. Pediatrics 2012;129:108-15.

Kogan SM, Brody GH, Molgaard VK, Grange CM, Oliver DAH, Anderson TN, et al. The Strong African American Families teen trial: rationale, design, engagement processes, and familyspecific effects. Prevention Science 2012;13:206-17.

Bush 1989 \{published data only\}

Bush PJ, Zuckerman AE, Theiss PK, Taggart VS, Horowitz C, Sheridan MJ, et al. Cardiovascular risk factor prevention in black schoolchildren: two-year results of the "Know Your Body" program. American Journal of Epidemiology 1989;129:466-82.

Catalano 1999 \{published data only\}

Catalano RF, Gainey RR, Fleming CB, Haggerty KP, Johnson NO. An experimental intervention with families of substance abusers: one-year follow-up of the focus on families project. Addiction 1999;94:241-54.

Conduct Problems Prevention Research Group 2010 \{published and unpublished data\}

Bierman KL, Coie JD, Dodge KA, Greenberg M, Lochman JE, McMahon RJ, et al. Trajectories of risk for early sexual activity and early substance use in the Fast Track Prevention Program. Prevention Science 2014; Vol. 15:33-46.

Conduct Problems Prevention Research Group. A developmental and clinical model for the prevention of conduct disorder: the FAST Track Program. Development and Psychopathology 1992;4:509-27.

Conduct Problems Prevention Research Group. Impact of early intervention on psychopathology, crime, and well-being at age 25. American Journal of Psychiatry 2015;172:59-70.

Conduct Problems Prevention Research Group. Initial impact of the Fast Track Prevention Trial for conduct problems: II. Classroom effects. Journal of Consulting and Clinical Psychology 1999;67(5):648-57.

Conduct Problems Prevention Research Group. Trajectories of risk for early sexual activity and early substance use in the Fast Track Prevention Program. Prevention Science 2014;15(Suppl 1):S33-S46.

\section{Connell 2007 \{published data only\}}

Caruthers AS, Van Ryzin MJ, Dishion TJ. Preventing high-risk sexual behavior in early adulthood with family interventions in adolescence: outcomes and developmental processes. Prevention Science 2014;15:59-69.
Connell AM, Dishion TJ, Yasui M, Kavanagh K. An adaptive approach to family intervention: linking engagement in family-centered intervention to reductions in adolescent problem behavior. Journal of Consulting \& Clinical Psychology 2007;75:568-79.

Fosco GM, Frank JL, Stormshak EA, Dishion TJ. Opening the "Black Box": family check-up intervention effects on selfregulation that prevents growth in problem behavior and substance use. Journal of School Psychology 2013; Vol. 51:455-68.

Stormshak EA, Connell AM, Veronneau M-H, Myers MW, Dishion TJ, Kavavagh K, et al. An ecological approach to promoting early adolescent mental health and social adaptation: family-centred intervention in public middle schools. Child Development 2011;82:209-25.

Van Ryzin MJ, Nowicka P. Direct and indirect effects of a familybased intervention in early adolescence on parent-youth relationship quality, late adolescent health, and early adult obesity. Journal of Family Psychology 2013;27:106-16.

Van Ryzin MJ, Nowicka P. Family-based intervention prevents early adulthood obesity: 10 -years follow up of the family checkup, a randomized controlled trial. Obesity Facts 2012;5:33.

Van Ryzin MJ, Stormshak EA, Dishion TJ. Engaging parents in the family check-up in middle school: longitudinal effects on family conflict and problem behavior through the high school transition. Journal of Adolescent Health 2012;50:627-33.

\section{Cunningham 2012 \{published data only\}}

Cunningham RM, Chermack ST, Shope JT, Bingham CR, Zimmerman MA, Blow FC, et al. Twelve-month outcomes from the "saferteen" study: an alcohol and violence brief intervention for adolescents in the emergency department. Alcoholism Clinical and Experimental Research 2011;35:213A.

Cunningham RM, Chermack ST, Zimmerman MA, Shope JT, Bingham CR, Blow FC, et al. Brief motivational interviewing intervention for peer violence and alcohol use in teens: oneyear follow-up. Pediatrics 2012;129:1083-90.

Walton MA, Chermack ST, Shope JT, Bingham CR, Zimmerman MA, Blow FC, et al. Effects of a brief intervention for reducing violence and alcohol misuse among adolescents. JAMA 2010;304(5):527-35.

Whiteside LK, Cunningham R, Chermack ST, Zimmerman MA, Shope JT, Bingham CR, et al. Dating violence: outcomes following a brief motivational interviewing intervention among at-risk adolescents in an urban ED. Academic Emergency Medicine 2012;19:S140.

D'Amico 2002a \{published data only\}

D'Amico EJ, Fromme K. Brief prevention for adolescent risktaking behavior. Addiction 2002;97:563-74.

DeGarmo 2009 \{published data only\}

DeGarmo DS, Eddy JM, Reid JB, Fetrow RA. Evaluating mediators of the impact of the Linking the Interests of Families and Teachers (LIFT) multimodal preventive intervention on 
substance use initiation and growth across adolescence. Prevention Science 2009;10(3):208-20.

Eddy JM, Reid JB, Fetrow RA. An elementary school-based prevention program targeting modifiable antecedents of youth delinquency and violence: Linking the Interests of Families and Teachers (LIFT). Journal of Emotional and Behavioral Disorders 2000;8(3):165-76.

Eddy JM, Reid JB, Stoolmiller M, Fetrow RA. Outcomes during middle school for an elementary school-based preventive intervention for conduct problems: follow-up results from a randomized trial. Behavior Therapy 2003;34:535-52.

Reid JB, Eddy JM, Fetrow RA, Stoolmiller M. Description and immediate impacts of a preventive intervention for conduct problems. American Journal of Community Psychology 1999;27(4):483-517.

\section{Dolan 2010 \{published and unpublished data\}}

Dolan P, Brady B, O'Regan C, Brumovska T, Canavan J, Forkan C. Big Brothers Big Sisters (BBBS) of Ireland: evaluation study: report 2: qualitative evidence. Child and Family Research Centre, National University of Ireland, Galway, 2011.

Dolan P, Brady B, O'Regan C, Canavan J, Russell D, Forkan C. Big Brothers Big Sisters (BBBS) of Ireland: evaluation study: report 3: summary report. Child and Family Research Centre, National University of Ireland, Galway, 2011.

Dolan P, Brady B, O'Regan C, Russell D, Canavan J, Forkan C. Big Brothers Big Sisters (BBBS) of Ireland: evaluation study: report 1: randomised control trial and implementation report. Child and Family Research Centre, National University of Ireland, Galway, 2010.

\section{Estrada 2015 \{published data only\}}

Estrada Y, Rosen A, Huang S, Tapia M, Sutton M, Willis L, et al. Efficacy of a brief intervention to reduce substance use and human immunodeficiency virus infection risk among Latino youth. Journal of Adolescent Health 2015;57:651-7.

Fearnow-Kenney 2003 \{published data only (unpublished sought but not used)

Fearnow-Kenney MD, Wyrick DL, Jackson-Newsom J, Wyrick CH, Hansen WB. Initial indicators of effectiveness for a high school drug prevention program. American Journal of Health Education 2003;34(2):66-71.

\section{Flay 2004a \{published data only\}}

Benner TA. Aban Aya Youth Project: preventing high-risk behaviors among African-American youths in grades 5-8. In: Card JJ, Benner TA editor(s). Model Programs for Adolescent Sexual Health: Evidence-Based HIV, STI and Pregnancy Prevention Interventions. New York; London: Springer, 2008.

Fagen MC, Flay BR. Sustaining a school-based prevention program: results from the Aban Aya Sustainability Project. Health Education \& Behavior 2009;36(1):9-23.

Flay BR, Graumlich S, Segawa E, Burns JL, Holliday MY, for the Aban Aya Investigators. Effects of 2 prevention programs on high-risk behaviors among African American youth: a randomized trial. Archives of Pediatrics \& Adolescent Medicine 2004;158:377-84.

Ngwe JE, Liu LC, Flay BR, Segawa E, Aban Aya Co-Investigators. Violence prevention among African American adolescent males. American Journal of Health Behavior 2004;Suppl 28:S24-S37.

\section{Freidman 2002 \{published data only\}}

Friedman AS, Terras A, Glassman K. Multimodel substance use intervention program for male delinquents. Journal of Child \& Adolescent Substance Abuse 2002;11:43-65.

\section{Freudenberg 2010 \{published data only\}}

Daniels J, Crum M, Ramaswamy M, Freudenberg N. Creating REAL MEN: description of an intervention to reduce drug use, HIV risk, and rearrest among young men returning to urban communities from jail. Health Promotion Practice 2011;12(1):44-54.

Freudenberg N, Ramaswamy M, Daniels J, Crum M, Ompad DC, Vlahov D. Reducing drug use, human immunodeficiency virus risk, and recidivism among young men leaving jail: evaluation of the REAL MEN re-entry program. Journal of Adolescent Health 2010;47:448-55.

Gonzales 2012 \{published data only (unpublished sought but not used)\}

Carpentier FRD, Mauricio AM, Gonzales NA, Millsap RE, Meza CM, Dumka LE, et al. Engaging Mexican origin families in a schoolbased preventive intervention. Journal of Primary Prevention 2007;28(6):521-46.

Gonzales NA, Dumka LE, Millsap RE, Gottschall A, McClain DB, Wong JJ, et al. Randomized trial of a broad preventive intervention for Mexican American adolescents. Journal of Consulting and Clinical Psychology 2012;80(1):1-16.

Gonzales NA, Wong JJ, Toomey RB, Millsap R, Dumka LW, Maricio AM. School engagement mediates long-term prevention effects for Mexican American adolescents. Prevention Science 2014;15:929-39.

\section{Gottfredson 2010 \{published data only\}}

Gottfredson DC, Cross A, Wilson D, Rorie M, Connell N. An experimental evaluation of the All Stars Prevention Curriculum in a community after school setting. Prevention Science 2010;11:142-54.

Gottfredson DC, Cross AB, Wilson DM, Connell N, Rorie M. A randomised trial of the effects of an enhanced after school program. Unpublished report available from authors. College Park, Maryland, 2009.

\section{Griffin 2006 \{published data only\}}

Griffin KW, Botvin GJ, Nichols TR. Effects of a school-based drug abuse prevention program for adolescents on HIV risk behavior in young adulthood. Prevention Science 2006;7(1):103-12.

\section{Griffin 2009 \{published data only\}}

Griffin JP Jr, Holliday RC, Frazier E, Braithwaite RL. The BRAVE (Building Resiliency and Vocational Excellence) Program: evaluation findings for a career-oriented substance abuse and 
violence preventive intervention. Journal of Health Care for the Poor \& Underserved 2009;20(3):798-816.

\section{Haggerty 2007a \{published data only\}}

Haggerty KP, Skinner ML, MacKenzie EP, Catalano RF. A randomized trial of parents who care: effects on key outcomes at 24-month follow-up.. Prevention Science 2007;8(4):249-60.

\section{Ialongo 1999 \{published data only (unpublished sought but not} used)\}

Furr-Holden CDM, lalongo NS, Anthony JC, Petras H, Kellam SG. Developmentally inspired drug prevention: middle school outcomes in a school-based randomized prevention trial. Drug and Alcohol Dependence 2001;73:149-58.

Ialongo N, Poduska J, Werthamer L, Kellam S. The distal impact of two first-grade preventive interventions on conduct problems and disorders in early adolescence. Journal of Emotional and Behavioral Disorders 2001;9(3):146-60.

lalongo NS, Werthamer L, Kellam SG. Proximal impact of two first-grade preventive interventions on the early risk behaviors for later substance abuse, depression, and antisocial behavior. American Journal of Community Psychology 1999;27(5):599-641.

Storr CL, lalongo NS, Kellam SG, Anthony JC. A randomized controlled trial of two primary school intervention strategies to prevent early onset tobacco smoking. Drug and Alcohol Dependence 2002;66:51-60.

\section{Ialongo 1999b \{published data only\}}

Ialongo NS, Werthamer L, Kellam SG. Proximal impact of two first-grade preventive interventions on the early risk behaviors for later substance abuse, depression, and antisocial behavior. American Journal of Community Psychology 1999;27(5):599-641.

\section{Jalling 2016 \{published data only\}}

Jalling C, Bodin M, Romelsjö A, Källmén H, Durbeej N, Tengström A. Parent programs for reducing adolescent's antisocial behavior and substance use: a randomized controlled trial. Journal of Child and Family Studies 2016;25:811-26.

\section{Jalling 2016b \{published data only\}}

Jalling C, Bodin M, Romelsjö A, Källmén H, Durbeej N, Tengström A. Parent programs for reducing adolescent's antisocial behavior and substance use: a randomized controlled trial. Journal of Child and Family Studies 2016;25:811-26.

\section{Johnson 2015 \{published data only\}}

Johnson SL, Jones V, Cheng TL. Promoting "Healthy Futures" to reduce risk behaviors in urban youth: a randomized controlled trial. American Journal of Community Psychology 2015;56:36-45.

\section{Kellam 2014 \{published data only\}}

Kellam SG, Wang W, Mackenzie ACL, Brown CH, Ompad DC, Or F, et al. The impact of the Good Behavior Game, a universal classroom-based preventive intervention in first and second grades, on high-risk sexual behaviors and drug abuse and dependence disorders into young adulthood. Prevention Science 2014; Vol. 15:6-18.
Kim 2011 \{published data only\}

Kim HK, Leve LD. Substance use and delinquency among middle school girls in foster care: a three-year follow-up of a randomized controlled trial. Journal of Consulting and Clinical Psychology 2011;79(6):740-50.

\section{Kitzman 2010 \{published data only\}}

Kitzman HJ, Olds DL, Cole RE, Hanks CA, Anson EA, Arcoleo KJ, et al. Enduring effects of prenatal and infancy home visiting by nurses on children: follow-up of a randomized trial among children at age 12 years. Archives of Pediatrics \& Adolescent Medicine 2010;164(5):412-8.

\section{Lana 2014 \{published data only\}}

Lana A, Faya-Ornia G, López ML. Impact of a web-based intervention supplemented with text messages to improve cancer prevention behaviors among adolescents: results from a randomized controlled trial. Preventive Medicine 2014;59:54-9.

Lana A, Valle MO, López S, Faya-Ornia G, López ML. Study protocol of a randomized controlled trial to improve cancer prevention behaviors in adolescents and adults using a webbased intervention supplemented with SMS. BMC Public Health 2013; Vol. 13:357.

\section{Li 2002 \{published data only\}}

Li X, Stanton B, Galbraith J, Burns J, Cottrell L, Pack R. Parental monitoring intervention: practice makes perfect. Journal of the National Medical Association 2002;94:364-70.

\section{Li 2011 \{published data only\}}

Bavarian N, Lewis KM, DuBois DL, Acock A, Vuchinich S, Silverthorn N, et al. Using social-emotional and character development to improve academic outcomes: a matchedpair, cluster-randomized controlled trial in low-income, urban schools. Journal of School Health 2013;83:771-9.

Lewis K.M, DuBois D.L, Bavarian N, Acock A, Silverthorn N, Day J, Ji P, Vuchinich S, Flay B.R. Effects of Positive Action on the Emotional Health of Urban Youth:A Cluster-Randomized Trial. Journal of Adolescent Health 2013;53:706-11.

Lewis KM, Bavarian N, Snyder FJ, Acock A, Day J, DuBois DL, et al. Direct and mediated effects of a social-emotional and character development program on adolescent substance use. International Journal of Emotional Education 2012;4(1):56-78.

Lewis KM, Schure MB, Bavarian N, DuBois D, Day J, Ji P, et al. Problem behavior and urban, low-income youth: a randomized controlled trial of Positive Action in Chicago. American Journal of Preventive Medicine 2013;44(6):622-30.

Li K-K, Washburn I, DuBois DL, Vuchinich S, Ji P, Brechling V, et al. Effects of the Positive Action programme on problem behaviours in elementary school students: a matched-pair randomised control trial in Chicago. Psychology \& Health 2011;26(2):187-204.

\section{Lochman 2003a \{published data only\}}

Lochman JE, Wells KC. Effectiveness of the Coping Power Program and of classroom intervention with aggressive 
children: outcomes at a 1-year follow-up. Behavior Therapy 2003;34:493-515.

Lochman JE, Wells KC. The Coping Power Program at the Middle-School transition: universal and indicated prevention effects. Psychology of Addictive Behaviors 2002;16(4S):S40-S54.

Lochman JE, Wells KC, Qu L, Chen L. Three year follow-up of coping power intervention effects: evidence of neighborhood moderation?. Prevention Science 2013;14:364-76.

Lochman 2004a \{published data only\}

Lochman JE, Wells KC. The Coping Power Program for preadolescent aggressive boys and their parents: outcome effects at the 1-year follow-up. Journal of Consulting and Clinical Psychology 2004;72(4):571-8.

\section{LoSciuto 1999 \{published data only\}}

LoSciuto L, Hilbert SM, Fox MM, Porcellini L, Lanphear A. A twoyear evaluation of the Woodrock Youth Development Project. Journal of Early Adolescence 1999;19(4):488-507.

\section{Matthews 2016 \{published data only\}}

Mathews C, Eggers SM, Townsend L, Aarø LE, de Vries PJ, Mason-Jones AJ, et al. Effects of PREPARE, a multi-component, school-based HIV and intimate partner violence (IPV) prevention programme on adolescent sexual risk behaviour and IPV: cluster randomised controlled trial. AIDS Behavior 2016;20(9):1821-40.

\section{McNeal 2004 \{published data only\}}

Harrington NG, Giles SM, Hoyle RH, Feeney GJ, Yungbluth SC. Evaluation of the All Stars character education and problem behavior prevention program: effects on mediator and outcome variables for middle school students. Health Education \& Behavior 2001;28(5):533-46.

McNeal RB Jr, Hansen WB, Harrington NG, Giles SM. How All Stars works: an examination of program effects on mediating variables. Health Education \& Behavior 2004;31(2):165-78.

\section{Melnyk 2013 \{published data only\}}

Melnyk BM, Jacobson D, Kelly S, Belyea M, Shaibi G, Small L, et al. Promoting healthy lifestyles in high school adolescents: a randomized controlled trial. American Journal of Preventive Medicine 2013;45:407-15.

\section{Milburn 2012 \{published data only\}}

Milburn NG, Iribarren FJ, Rice E, Lightfoot M, Solorio R, Rotheram-Borus $\mathrm{MJ}$, et al. A family intervention to reduce sexual risk behavior, substance use, and delinquency among newly homeless youth. Journal of Adolescent Health 2012;50:358-64.

\section{Minnis 2014 \{published data only\}}

Minnis AM, Vandommelen-Gonzalez E, Luecke E, Dow W, Bautista-Arredondo S, Padian NS. Yo Puedo - a conditional cash transfer and life skills intervention to promote adolescent sexual health: results of a randomized feasibility study in San Francisco. Journal of Adolescent Health 2014; Vol. 55:85-92.

\section{Monti 1999 \{published data only\}}

Monti PM, Colby SM, Barnett NP, Spirito A, Rohsenow DJ, Myers $\mathrm{M}$, et al. Brief intervention for harm reduction with alcohol-positive older adolescents in a hospital emergency department. Journal of Consulting \& Clinical Psychology 1999;67(6):989-94.

\section{Morris 2003 \{published data only\}}

Morris P, Michalopoulos C. Findings from the Self-Sufficiency Project: effects on children and adolescents of a program that increased employment and income. Journal of Applied Developmental Psychology 2003;24:201-39.

Morris P, Michalopoulos C. The Self-Sufficiency Project at 36 months. The Self-Sufficiency Project at 36 Months. Social Research and Demonstration Corporation (SRDC). Ottawa: Human Resources Development Canada, 2000:1-146.

\section{Murry 2014 \{published data only\}}

Brody GH, Murry VM, McNair L, Chen Y-F, Gibbons FX, Gerrard M, et al. Linking changes in parenting to parent-child relationship quality and youth self-control: the Strong African American Families Program. Journal of Research on Adolescence 15;1:47-69.

McBride MV, McNair LD, Myers SS, Chen Y-F, Brody GH. Intervention induced changes in perceptions of parenting and risk opportunities among rural African Americans. Journal of Child and Family Studies 2014;23:422-36.

Murry VM, Brody GH. The Strong African American Families - teen trial: rationale, design, engagement processes, and family-specific effects. Journal of Marital and Family Therapy 2004;30(3):271-83.

\section{Nader 1999 \{published data only\}}

Edmundson EW, Luton SC, McGraw SA, Kelder SH, Layman AK, Smyth $\mathrm{MH}$, et al. CATCH - classroom process evaluation in a multicenter trial. Health Education Quarterly 1994;Suppl 2:S27S50.

Elder JP, McGraw SA, Stone EJ, Reed DB, HarshaDW, Greene T, et al. CATCH: process evaluation of environmental factors and programs. Health Education Quarterly 1994;Suppl 2:S107-S127.

Elder JP, Perry CL, Stone EJ, Johnson CC, Yang M, Edmundson EW, et al. Tobacco use measurement, prediction, and intervention in elementary schools in four states: the CATCH study. Preventive Medicine 1996;25:486-94.

Heath EM, Coleman KJ. Adoption and institutionalization of the Child and Adolescent Trial for Cardiovascular Health (CATCH) in El Paso, Texas. Health Promotion Practice 2003;4(2):157-64.

Heath EM, Coleman KJ. Evaluation of the institutionalization of the coordinated approach to child health (CATCH) in a U.S./Mexico border community. Health Education \& Behavior 2002;29(4):444-60.

Hoelscher DM, Feldman HA, Johnson CC, Lytle LA, Osganian SK, Parcel GS, et al. School-based health education programs can be maintained over time: results from the 
CATCH institutionalization study. Preventive Medicine 2004;38(5):594-606.

Johnson CC, Li D, Galati T, Pedersen S, Smyth M, Parcel GS. Maintenance of the classroom health education curricula: results from the CATCH-ON study. Health Education \& Behavior 2003;30(4):476-88.

Kelder SH, Mitchell PD, McKenzie TL, Derby C, Strikmiller PK, Luepker RV, et al. Long-term implementation of the CATCH physical education program. Health Education \& Behavior 2003;30(4):463-75.

Luepker RV, Perry CL, McKinlay SN, Nader PR, Parcel GS, Stone EJ, et al. Outcomes of a field trial to improve children's dietary patterns and physical activity: the Child and Adolescent Trial for Cardiovascular Health (CATCH). JAMA 1996;275(10):768-76.

Lytle LA, Stone EJ, Nichaman MZ, Perry CL, Montgomery DH, Nicklas TA, et al. Changes in nutrient intakes of elementary school children following a school-based intervention: results from the CATCH study. Preventive Medicine 1996;25(4):465-77.

Lytle LA, Ward J, Nader PR, Pedersen S, Williston BJ. Maintenance of a health promotion program in elementary schools: results from the CATCH-ON study key informant interviews. Health Education \& Behavior 2003;30(4):503-18.

McKenzie TL, Li D, Derby CA, Webber LS, Luepker RV, Cribb P. Maintenance of effects of the CATCH physical education program: results from the CATCH-ON study. Health Education \& Behavior 2003;30(4):447-62.

McKenzie TL, Nader PR, Strikmiller PK, Yang M, Stone EJ, Perry CL. School physical education: effect of the Child and Adolescent Trial for Cardiovascular Health. Preventive Medicine 1996;25(4):423-31.

McKenzie TL, Stone EJ, Feldman HA, Epping JN, Yang M, Strikmiller PK, et al. Effects of the CATCH physical education intervention: teacher type and lesson location. American Journal of Preventive Medicine 2001;21(2):101-9.

Nader PR, Sellers DE, Johnson CC, Perry CL, Stone EJ, Cook KC, et al. The effect of adult participation in a school-based family intervention to improve children's diet and physical activity: the Child and Adolescent Trial for Cardiovascular Health. Preventive Medicine 1996;25(4):455-64.

Nader PR, Stone EJ, Lytle LA, Perry CL, Osganian SK, Kelder S, et al. Three-year maintenance of improved diet and physical activity: the CATCH cohort. Archives of Pediatrics \& Adolescent Medicine 1999;153(7):695-704.

Nader PR, Stone EJ, Lytle LA, Perry CL, Osganian SK, Kelder S, et al. Three-year maintenance of improved diet and physical activity: the CATCH cohort. Child and Adolescent Trial for Cardiovascular Health. Archives of Pediatrics \& Adolescent Medicine 1999;153:695-704.

Nader PR, Yang M, Luepker RV, Parcel GS, Pirie P, Feldman HA, et al. Parent and physician response to children's cholesterol values of $200 \mathrm{mg} / \mathrm{dL}$ or greater: the Child and Adolescent Trial for Cardiovascular Health Experiment. Pediatrics 1997;99(5):E5.

Nicklas TA, Dwyer J, Mitchell P, Zive M, Montgomery D, Lytle L, et al. Impact of fat reduction on micronutrient density of children's diets: the CATCH Study. Preventive Medicine 1996;25(4):478-85.

Osganian SK, Ebzery MK, Montgomery DH, Nicklas TA, Evans MA, Mitchell PD, et al. Changes in the nutrient content of school lunches: results from the CATCH Eat Smart Food service intervention. Preventive Medicine 1996;25(4):400-12.

Osganian SK, Hoelscher DM, Zive M, Mitchell PD, Snyder P, Webber LS. Maintenance of effects of the eat smart school food service program: results from the $\mathrm{CATCH}-\mathrm{ON}$ study. Health Education \& Behavior 2003;30(4):418-33.

Osganian SK, Nicklas T, Stone E, Nichaman M, Ebzery MK, Lytle L, et al. Perspectives on the School Nutrition Dietary Assessment Study from the Child and Adolescent Trial for Cardiovascular Health. American Journal of Clinical Nutrition 1995;61(Suppl 1):241S-244S.

Parcel GS, Perry CL, Kelder SH, Elder JP, Mitchell PD, Lytle LA, et al. School climate and the institutionalization of the CATCH program. Health Education \& Behavior 2003;30(4):489-502.

Perry CL, Lytle LA, Feldman H, Nicklas T, Stone E, Zive M, et al. Effects of the Child and Adolescent Trial for Cardiovascular Health (CATCH) on fruit and vegetable intake. Journal of Nutrition Education 1998;30(6):354-6.

Perry CL, Parcel GS, Stone E, Nader P, McKinlay SM, Luepker RV, et al. The Child and AdolescentTrial for Cardiovascular Health (CATCH): overview of the intervention program and evaluation methods. Cardiovascular Risk Factors 1992;2:36-44.

Smith KW, Hoelscher DM, Lytle LA, Dwyer JT, Nicklas TA, Zive MM, et al. Reliability and validity of the Childand Adolescent Trial for Cardiovascular Health (CATCH) food checklist: a self-report instrument to measure fat and sodium intake by middle school students. Journal of the American Dietetic Association 2001;101(6):635-47.

Stone EJ, Osganian SK, McKinlay SM, Wu MC, Webber LS, Luepker RV, et al. Operational design and quality control in the CATCH multicenter trial. Preventive Medicine 1996;25(4):384-99.

Webber LS, Osganian SK, Feldman HA, Wu M, McKenzie TL, Nichaman $\mathrm{M}$, et al. Cardiovascular risk factors among children after a 2 1/2-year intervention - the CATCH Study. Preventive Medicine 1996;25(4):432-41.

Webber LS, Osganian V, Luepker RV, Feldman HA, Stone EJ, Elder JP, et al. Cardiovascular risk factors among third grade children in four regions of the United States. The CATCH Study. Child and Adolescent Trial for Cardiovascular Health. American Journal of Epidemiology 1995;141(5):428-39.

Zucker DM, Lakatos E, Webber LS, Murray DM, McKinlay SM, Feldman HA, et al. Statistical design of the Child and Adolescent Trial for Cardiovascular Health (CATCH): implications of cluster randomization. Controlled Clinical Trials 1995;16(2):96-118. 


\section{Nirenberg 2013 \{published data only\}}

Nirenberg T, Baird J, Longabaugh R, Mello MJ. Motivational counseling reduces future police charges in court referred youth. Accident Analysis and Prevention 2013; Vol. 53:89-99.

\section{O'Neill 2011 \{published data only\}}

O'Neill JM, Clark JK, Jones JA. Promoting mental health and preventing substance abuse and violence in elementary students: a randomized control study of the Michigan Model for Health. Journal of School Health 2011;81(6):320-30.

\section{Olds 1998 \{published data only\}}

Karoly LA, Greenwood PW, Everingham SS, Hoube J, Kilburn MR, Rydell CP, et al. Investing in Our Children: What We Know and Don't Know About the Costs and Benefits of Early Childhood Interventions. Santa Monica, LA: RAND, 1998.

Olds D, Henderson CR Jr, Cole R, Eckenrode J, Kitzman H, Luckey $\mathrm{D}$, et al. Long-term effects of nurse home visitation on children's criminal and antisocial behavior: 15-year follow-up of a randomized controlled trial. JAMA 1998;280(14):1238-44.

Olds DR, Henderson CR Jr, Kitzman HJ, Eckenrode JJ, Cole RE, Tatelbaum RC. Prenatal and home visitation by nurses: recent findings. The Future of Children. Vol. 9, Princeton, New Jersey: Princeton University Press, 1999:44-65.

Pantin 2009 \{published data only\}

Cordova D, Huang S, Lally M, Estrada Y, Prado G. Do parentadolescent discrepancies in family functioning increase the risk of Hispanic adolescent HIV risk behaviors?. Family Process 2014; Vol. 53:348-63.

Pantin H, Prado G, Lopez B, Huang S, Tapia MI, Schwartz SJ, et al. A randomized controlled trial of Familias Unidas for Hispanic adolescents with behavior problems. Psychosomatic Medicine 2009;71:987-95.

\section{Perry 2003a \{published data only\}}

Eischens A, Komro KA, Perry CL, Bosma LM, Farbakhsh K. The association of extracurricular activity participation with substance use among youth in the DARE Plus project. American Journal of Health Education 2004;35(2):68-75.

Komro K, Perry C, Munson K, Stigler M, Farbakhsh K. Reliability and validity of self-report measures to evaluate drug and violence prevention programs. Journal of Child \& Adolescent Substance Abuse 2004;13(3):17-51.

Komro KA, Perry CL, Veblen-Mortenson S, Stigler MH, Bosma LM, Munson KA, et al. Violence-related outcomes of the D.A.R.E. plus project. Health Education \& Behavior 2004;31(3):335-54.

Perry CL, Komro KA, Veblen-Mortenson S, Bosma L, Munson K, Stigler M, et al. The Minnesota DARE PLUS Project: creating community partnerships to prevent drug use and violence. Journal of School Health 2000;70(3):84-8.

Perry CL, Komro KA, Veblen-Mortenson S, Bosma LM, Farbakhsh K, Munson KA, et al. A randomized controlled trial of the middle and junior high school D.A.R.E. and D.A.R.E. Plus programs. Archives of Pediatrics \& Adolescent Medicine 2003;157:178-84.
Piper 2000a \{published data only\}

Piper DL, Moberg DP, King MJ. The healthy for life project: behavioral outcomes. Journal of Primary Prevention 2000;21(1):47-73.

\section{Redding 2015 \{published data only\}}

Redding CA, Prochaska JO, Armstrong K, Rossi JS, Hoeppner BB, Sun X, et al. Randomized trial outcomes of a TTM-tailored condom use and smoking intervention in urban adolescent females. Health Education Research 2015;30:162-78.

\section{Sanchez 2007 \{published data only\}}

Halfors D, Cho H, Sanchez V, Khatapoush S, Kim HM, Bauer D. Efficacy vs effectiveness trial results of an indicated "model" substance abuse program: implications for public health. American Journal of Public Health 2006;96(12):2254-9.

Sanchez V, Steckler A, Nitirat P, Hallfors D, Cho H, Brodish P. Fidelity of implementation in a treatment effectiveness trial of Reconnecting Youth. Health Education Research 2007;22(1):95-107.

\section{Saraf 2015 \{published data only\}}

Saraf DS, Gupta SK, Pandav CS, Nongkinrih B, Kapoor SK, Pradhan SK, et al. Effectiveness of a school based intervention for prevention of non-communicable diseases in middle school children of rural North India: a randomized controlled trial. Indian Journal of Pediatrics 2015;82:354-62.

\section{Schweinhart 1993 \{published data only\}}

Belfield CR, Nores M, Barnett S, Schweinhart L. The High/Scope Perry Preschool Program cost-benefit analysis using data from the age-40 followup. Journal of Human Resources 2006;1:162-90.

Berrueta-Clement JR, Schweinhart LJ, Barnett WS, Epstein AS, Weikart DP. Changed Lives: The Effects of the Perry Preschool Program on Youths through Age 19. Monographs of the High/ Scope Educational Research Foundation, Number Eight. Ypsilanti, Michigan: High/Scope Press, 1984.

Heckman JJ, Moon SH, Pinto R, Savelyet PA, Yavitz A. The rate of return to the HighScope Perry Preschool Program. Journal of Public Economics 2010;94:114-28.

Luster T, McAdoo H. Family and child influences on educational attainment: a secondary analysis of the High/Scope Perry Preschool data. Developmental Psychology 1996;32(1):26-39.

Muennig P, Schweinhart L, Montie J, Neidell M. Effects of a prekindergarten educational intervention on adult health: 37-year follow-up results of a randomized controlled trial. American Journal of Public Health 2009;99(8):1431-7.

Nores M, Belfield CR, Barnett WS, Schweinhart L. Updating the economic impacts of the High/Scope Perry Preschool Program. Educational Evaluation and Policy Analysis 2005;27(3):245-61.

Schweinhart LJ. The High/Scope Perry Preschool study: a case study in random assignment. Evaluation \& Research in Education 2000;14(3-4):137-47. 
Schweinhart LJ, Barnes HV, Weikart DP. Monographs of the High/Scope Educational Research Foundation. Vol. 10, Ypsilanti, Michigan: High/Scope Press, 1993.

Schweinhart LJ, Barnes HV, Weikart DP. Significant Benefits. The High/Scope Perry Preschool Study Through Age 27. Monographs of the High/Scope Educational Research Foundation. Vol. 10, Ypsilanti, Michigan: High/Scope Press, 1993.

Schweinhart LJ, Weikart DP. Young children grow up : the effects of the Perry preschool program on youths through age 15. Series: Monographs of the High/Scope Educational Research Foundation; No. 7. Ypsilanti, Michigan: High/Scope Press, 1980.

Weikart DP, Bond JT, McNeil JT. The Ypsilanti Perry Preschool Project: Preschool Years and Longitudinal Results Through Fourth Grade. Monograph Number 3. Monographs of the High/ Scope Educational Research Foundation. No. 3. Ypsilanti, Michigan: High/Scope Press, 1978.

Weikart DP, Epstein AS, Schweinhart L, Bond JT. The Ypsilanti Preschool Curriculum Demonstration Project. Monographs of the High/Scope Educational Research Foundation. No. 4. Ypsilanti, Michigan: High/Scope Press, 1978.

\section{Schwinn 2014 \{published data only\}}

Schwinn TM, Schinke S, Fang L, Kandasamy S. A web-based, health promotion program for adolescent girls and their mothers who reside in public housing. Addictive Behaviours 2014; Vol. 39:757-60

\section{Shek 2011 \{published data only\}}

Shek DTL. Objective outcome evaluation of the Project P.A.T.H.S. in Hong Kong: findings based on individual growth curve models. TheScientificWorld Journal: Child Health \& Human Development 2010;10:182-91.

Shek DTL, Sun RCF. Effectiveness of the Tier 1 program of Project P.A.T.H.S.: findings based on three years of program implementation. TheScientificWorldJournal: Child Health \& Human Development 2010;10:1509-19.

Shek DTL, Yu L. Prevention of adolescent problem behavior: longitudinal impact of the Project P.A.T.H.S. in Hong Kong. TheScientificWorldJournal: Child Health \& Human Development 2011;11:546-67.

\section{Shetgiri 2011 \{published data only\}}

Shetgiri R, Kataoka S, Lin H, Flores G. A randomized, controlled trial of a school-based intervention to reduce violence and substance use in predominantly Latino high school students. Journal of the National Medical Association 2011;103(9-10):932-40.

\section{Simons-Morton 2005 \{published data only\}}

Simons-Morton B, Chen R, Abroms L, Haynie DL. Latent growth curve analyses of peer and parent influences on smoking progression among early adolescents. Health Psychology 2004;23(6):612-21.

Simons-Morton B, Haynie D, Saylor K, Crump AD, Chen R. Impact analysis and mediation of outcomes: the Going Places program. Health Education \& Behavior 2005;32(2):227-41.
Simons-Morton B, Haynie D, Saylor K, Crump AD, Chen R. The effects of the Going Places program on early adolescent substance use and antisocial behavior. Prevention Science 2005;6(3):187-97.

\section{Tierney 1995 \{published data only\}}

Tierney JP, Baldwin Grossman J, Resch NL. Making a Difference. An Impact Study of Big Brothers Big Sisters. Making a Difference. An Impact Study of Big Brothers Big Sisters. Philadelphia: Public/Private Ventures, 1995:71.

\section{Wagner 2014 \{published data only\}}

Wagner EF, Hospital MM, Graziano JN, Morris SL, Gil AG. A randomized controlled trial of guided self-change with minority adolescents. Journal of Consulting and Clinical Psychology 2014;82:1128-39.

\section{Walker 2002 \{published data only\}}

Walker Z, Townsend J, Oakley L, Donovan C, Smith H, Hurst Z, et al. Health promotion for adolescents in primary care: randomised controlled trial. BMJ 2002;325:524-7.

Walter 1989 \{published data only\}

Walter HJ. Primary prevention of chronic disease among children: the school-based "Know Your Body" intervention trials. Health Education Quarterly 1989;16(2):201-14.

Walter HJ, Hofman A, Connelly PA, Barrett LT, Kost KL. Primary prevention of chronic disease in childhood: changes in risk factors after one year of intervention. American Journal of Epidemiology 1985;122(5):772-81.

\section{Wolfe 2012 \{published data only\}}

Wolfe DA, Crooks C, Jaffe P, Chiodo D, Hughes R, Ellis W, et al. A school-based program to prevent adolescent dating violence: a cluster randomized trial. Archives of Pediatrics and Adolescent Medicine 2009;163(8):692-9.

Wolfe DA, Crooks CV, Chiodo D, Hughes R, Ellis W. Observations of adolescent peer resistance skills following a classroom-based healthy relationship program: a post-intervention comparison. Prevention Science 2012;13:196-205.

\section{References to studies excluded from this review}

\section{Aboutanos 2011 \{published data only\}}

Aboutanos MB, Jordan A, Cohen R, Foster RL, Goodman K, Halfond RW, et al. Brief violence interventions with community case management services are effective for high-risk trauma patients. Journal of Trauma 2011;11:228-36.

\section{Allahverdipour 2009 \{published data only\}}

Allahverdipour H, Bazargan M, Farhadinasab A, Hidarnia A, Bashirian S. Effectiveness of skill-based substance abuse intervention among male adolescents in an Islamic country: case of the Islamic Republic of Iran. Journal of Drug Education 2009;39:211-22.

\section{Arbuthnot 1986 \{published data only\}}

Arbuthnot J, Gordon DA. Behavioral and cognitive effects of a moral reasoning development intervention for high-risk 
behavior-disordered adolescents. Journal of Consulting \& Clinical Psychology 1986;54:208-16.

\section{Baker 2004 \{published data only\}}

Baker V. Preventing Alcohol and Drug Abuse Through Primary Education: An Evaluation of a School-Based Substance Abuse Prevention Program. Dissertation abstracts international: section B: the sciences and engineering 2004; Vol. 64, issue 7B:3513.

\section{Balaji 2011 \{published data only\}}

Balaji M, Andrews T, Andrew G, Patel V. The acceptability, feasibility, and effectiveness of a population-based intervention to promote youth health: an exploratory study in Goa, India. Journal of Adolescent Health 2011;48(5):453-60.

Balvig 2011 \{published data only\} Balvig F, Holmberg L. The ripple effect: a randomized trial of a social norms intervention in a Danish middle school setting. Journal of Scandinavian Studies in Criminology and Crime Prevention 2011;12:3-19.

\section{Bannink 2014 \{published data only\}}

Bannink R, Broeren S, Joosten-van Zwanenburg E, van As E, van de Looij-Jansen P, Raat H. Effectiveness of a Web-based tailored intervention (E-health4Uth) and consultation to promote adolescents' health: randomized controlled trial. Journal of Medical Internet Research 2014;16:e143.

\section{Barlow 2013a \{published data only\}}

Barlow J, Sembi S, Gardner F, Macdonald G, Petrou S, Parsons $\mathrm{H}$, et al. An evaluation of the Parents Under Pressure programme: a study protocol for an RCT into its clinical and cost effectiveness. Trials [Electronic Resource] 2013;14:210.

\section{Barlow 2013b \{published data only\}}

Barlow A, Mullany B, Neault N, Compton S, Carter A, Hastings R, et al. Effect of a paraprofessional home-visiting intervention on American Indian teen mothers' and infants' behavioral risks: a randomized controlled trial. American Journal of Psychiatry 2013;170:83-93.

\section{Barrington 2008 \{published data only\}}

Barrington KD. The Effect of Voluntary, Randomized Drug Testing on Secondary School Students' Self-Reported Rates of Illegal Drug Use: An Analysis of Secondary School Students Living in Rural, Low-Income, South-Central Texas. Dissertation Abstracts International Section A: Humanities and Social Sciences. 2008; Vol. 68(7-A):2734.

\section{Battistich 1996 \{published data only\}}

Battistich V, Schaps E, Watson M, Solomon D. Prevention effects of the Child Development Project: early findings from an ongoing multisite demonstration trial. Journal of Adolescent Research 1996;11:12-35.

\section{Bell 2005 \{published data only\}}

Bell ML, Baker TK, Falb T. Protecting you/protecting me: evaluation of a student-led alcohol prevention and traffic safety program for elementary students. Journal of Alcohol \& Drug Education 2005;49:33-53.

\section{Bennett 1995 \{published data only\}}

Bennett GT. An exploration of patterns of drug use and of the effectiveness of a substance abuse prevention program according to adolescents' level of academic achievement. Dissertation Abstracts International A: Humanities and Social Sciences 1995; Vol. 55:1-3091.

Biggam 2002 \{published data only\}

Biggam FH, Power KG. A controlled, problem-solving, groupbased intervention with vulnerable incarcerated young offenders. International Journal of Offender Therapy \& Comparative Criminology 2002;46(6):678-98.

Bock 2016 \{published data only\}

Bock BC, Barnett NP, Thind H, Rosen R, Walaska K, Traficante R, et al. A text message intervention for alcohol risk reduction among community college students: TMAP. Addictive Behaviors 2016;63:107-13.

\section{Bonell 2010 \{published data only\}}

Bonell CP, Sorhaindo AM, Allen EE, Strange VJ, Wiggins M, Fletcher A, et al. Pilot multimethod trial of a school-ethos intervention to reduce substance use: building hypotheses about upstream pathways to prevention. Journal of Adolescent Health 2010;47:555-63.

Botvin 1994 \{published data only\}

Botvin GJ, Schinke SP, Epstein JA, Diaz T. Effectiveness of culturally focused and generic skills training approaches to alcohol and drug abuse prevention among minority youths. Psychology of Addictive Behaviors 1994;8:116-27.

\section{Botvin 1997 \{published data only\}}

Botvin GJ, Epstein JA, Baker E, Diaz T, Ifill-Williams M. Schoolbased drug abuse prevention with inner-city minority youth. Journal of Child and Adolescent Substance Abuse 1997;6:5-19.

\section{Botvin 2006 \{published data only\}}

Botvin GJ, Griffin KW, Nichols TD. Preventing youth violence and delinquency through a universal school-based prevention approach. Prevention Science 2006;7(4):403-8.

Bradley 2009 \{published data only\}

Bradley EG. The effects of a school-based motivational intervention on adolescent substance abuse. Dissertation Abstracts International B: The Sciences and Engineering 2009;70(6-A):1917.

\section{Brody 2004 \{published data only\}}

Brody GH, Murry VM, Gerrard M, Gibbons FX, Molgaard V, McNair L, et al. The Strong African American Families Program: translating research into prevention programming. Child Development 2004;75(3):900-17.

Bryan 2009 \{published data only\}

Bryan AD, Schmiege SJ, Broaddus MR. HIV risk reduction among detained adolescents: a randomized, controlled trial. Pediatrics 2009;124(6):e1180-8. 


\section{Bryan 2010 \{published data only\}}

Bryan AD, Magnan RE, Ewing SFE, Sabbatieni A, Filbey FM, Hutchison KE. Individual differences in response to psychosocial intervention among high risk adolescents: neurocognitive and genetic factors. Alcoholism: Clinical and Experimental Research. 2010; Vol. 34:130A.

\section{Burke 2010 \{published data only\}}

Burke K, Brennan L, Roney S. A randomised controlled trial of the efficacy of the ABCD Parenting Young Adolescents Program: rationale and methodology. Child and Adolescent Psychiatry and Mental Health 2010;4:22.

\section{Cameron 2015 \{published data only\}}

Cameron D, Epton T, Norman P, Sheeran P, Harris PR, Webb TL, et al. A theory-based online health behaviour intervention for new university students (U@Uni:LifeGuide): results from a repeat randomized controlled trial. Trials 2015;7(16):555.

\section{Campbell-Heider 2009 \{published data only\}}

Campbell-Heider N, Tuttle J, Knapp TR. The effect of positive adolescent life skills training on long term outcomes for highrisk teens. Journal of Addictions Nursing 2009;20:6-15.

\section{Castro 2013 \{published data only\}}

Castro Y, Basen-Engquist K, Fernandez ME, Strong LL, Eakin EG, Resnicow K, et al. Design of a randomized controlled trial for multiple cancer risk behaviors among Spanish-speaking Mexican-origin smokers. BMC Public Health 2013; Vol. 13:237.

\section{Clair-Michaud 2016 \{published data only\}}

Clair-Michaud M, Martin RA, Stein LA, Bassett S, Lebeau R, Golembeske $C$. The impact of motivational interviewing on delinquent behaviors in incarcerated adolescents. Journal of Substance Abuse Treatment 2016;65:13-9.

\section{Clinton-Sherrod 2011 \{published data only\}}

Clinton-Sherrod M, Morgan-Lopez A, Brown J, McMillen B, Cowell A. Incapacitated sexual violence involving alcohol among college women: the impact of a brief drinking intervention. Violence Against Women 2011;17(1):135-54.

\section{Colnes 2001 \{published data only\}}

Colnes RM. The effectiveness of a school-based substance use prevention program. Dissertation Abstracts International B: The Sciences and Engineering 2001; Vol. 61(8-B):4381.

\section{Copeland 2001 \{published data only\}}

Copeland J, Swift W, Howard J, Roffman RA, Stephens RS, Berghuis J. A randomized controlled trial of brief interventions for cannabis problems among young offenders [abstract]. Drug and Alcohol Dependence. 2001; Vol. 63:S3-S178.

\section{Copeland 2010 \{published data only\}}

Copeland AL, Williamson DA, Kendzor DE, Businelle MS, Rash CJ, Kulesza M, et al. A school-based alcohol, tobacco, and drug prevention program for children: the Wise Mind study. Cognitive Therapy and Research 2010;34:522-32.

\section{Crooks 2015 \{published data only\}}

Crooks CV, Scott KL, Broll R, Zwarych S, Hughes R, Wolfe DA. Does an evidence-based healthy relationships program for 9th graders show similar effects for 7 th and 8 th graders? Results from 57 schools randomized to intervention. Health Education Research 2015;30(3):513-9.

\section{Croom 2015 \{published data only\}}

Croom K, Staiano-Coico L, Lesser ML, Lewis DK, Reyna VF, Marchell TC, et al. The glass is half full: evidence for efficacy of alcohol - wise at one university but not the other. Journal of Health Communication 2015;20(6):627-38.

\section{Cuijpers 2002 \{published data only\}}

Cuijpers P, Jonkers R, de Weerdt I, de Jong A. The effects of drug abuse prevention at school: the 'Healthy School and Drugs' project. Addiction 2002;97:67-73.

\section{Cunningham 2015 \{published data only\}}

Cunningham RM, Chermack ST, Ehrlich PF, Carter PM, Booth BM, Blow FC, et al. Alcohol interventions among underage drinkers in the ED: a randomized controlled trial. Pediatrics 2015;136(4):e783-93.

\section{Cupp 2013 \{published data only\}}

Cupp PK, Atwood KA, Byrnes HF, Miller BA, Fongkaew W, Chamratrithirong $A$, et al. The impact of Thai family matters on parent-adolescent sexual risk communication attitudes and behaviors. Journal of Health Communcication 2013; Vol. 18:1384-96

\section{D'Amico 2008 \{published data only\}}

D'Amico EJ, Miles JNV, Stern SA, Meredith LS. Brief motivational interviewing for teens at risk of substance use consequences: a randomized pilot study in a primary care clinic. Journal of Substance Abuse Treatment 2008;35(1):53-61.

Dakof 2015 \{published data only\}

Dakof GA, Henderson CE, Rowe CL, Boustani M, Greenbaum PE, Wang W, et al. A randomized clinical trial of family therapy in juvenile drug court. Journal of Family Psychology 2015;29:232-41.

\section{Danielson 2012 \{published data only\}}

Danielson CK, McCart MR, Walsh K, Arellano MA, White D, Resnick HS. Reducing substance use risk and mental health problems among sexually assaulted adolescents: a pilot randomized controlled trial. Journal of Family Psychology 2012; Vol. 26:628-35.

\section{Dembo 1996 \{published data only\}}

Dembo R, Brown CH, Hunter B, Cervenka K, Wang W. Engaging high risk families in community-based intervention services. American Sociological Association: Conference Papers and Proceedings. 1996:N/A.

\section{Dembo 1998 \{published data only\}}

Dembo R, Shemwell M, Guida J, Schmeidler J, Pacheco K, Seeberger W. A longitudinal study of the impact of a family empowerment intervention on juvenile offender psychosocial 
functioning: a first assessment. Journal of Child and Adolescent Substance Abuse 1998;8:15-54.

Dembo 1999 \{published data only\}

Dembo R, Cervenka KA, Hunter B, Wang W. Engaging high risk families in community based intervention services. Aggression and Violent Behavior 1999;4(1):41-58.

\section{Dembo 2000 \{published data only\}}

Dembo R, Shemwell M, Pacheco K, Seeberger W, Rollie M, Schmeidler J. A longitudinal study of the impact of a family empowerment intervention on juvenile offender psychosocial functioning: an expanded assessment. Journal of Child and Adolescent Substance Abuse 2000;10(2):1-7.

\section{Dembo 2000a \{published data only\}}

Dembo R, Seeberger W, Shemwell M, Klein L, Rollie M, Pacheco $\mathrm{K}$, et al. Psychosocial functioning among juvenile offenders 12 months after family empowerment intervention. Journal of Offender Rehabilitation 2000;32:1-56.

\section{Dermen 2011 \{published data only\}}

Dermen $\mathrm{KH}$, Thomas SN. Randomized controlled trial of brief interventions to reduce college students' drinking and risky sex. Psychology of Addictive Behaviors 2011;25(4):583-94.

\section{Devine 1995 \{published data only\}}

Devine JA, Wright JD, Brody CJ. An evaluation of an alcohol and drug treatment program for homeless substance abusers. Evaluation Review 1995;19(6):620-45.

\section{Dewhirst 2013 \{published data only\}}

Dewhirst S, Leydon G, Tucker L, Roderick P, Harindra V, Dimitrov $B$, et al. Patient views from a feasibility study of a brief intervention to reduce alcohol consumption in a high-risk group in a sexual health clinic. BASHH Meeting, United Kingdom. 15 17 May 2013. 2013; Vol. 24:56.

\section{DeWit 2000 \{published data only\}}

DeWit DJ, Steep B, Silverman G, Stevens-Lavigne A, Ellis K, Smythe $C$, et al. Evaluating an in-school drug prevention program for at-risk youth. Alberta Journal of Educational Research 2000;XLVI(2):117-33.

\section{Dickinson 2013 \{published data only\}}

Dickinson WP, Glasgow RE, Fisher L, Dickinson LM, Christensen SM, Estabrooks PA, et al. Use of a website to accomplish health behavior change: if you build it, will they come? And will it work if they do?. Journal of the American Board of Family Medicine 2013; Vol. 26:168-76.

\section{Dishion 1995 \{published data only\}}

Dishion TJ, Andrews DW. Preventing escalation in problem behaviors with high-risk young adolescents: immediate and 1-year outcomes. Journal of Consulting \& Clinical Psychology 1995;63(4):538-48.

\section{Dishion 2000 \{published data only\}}

Dishion TJ, Kavanagh K. A multilevel approach to familycentered prevention in schools: process and outcome. Addictive Behaviors 2000;25(6):899-911.

\section{Donovan 2015 \{published data only\}}

Donovan E, Das Mahapatra P, Green TC, Chiauzzi E, McHugh K, Hemm A. Efficacy of an online intervention to reduce alcoholrelated risks among community college students. Addiction Research \& Theory 2015;23(5):437-47.

\section{Doolittle 1975 \{published data only\}}

Doolittle JC. Immunizing children against the possible antisocial effects of viewing television violence: a curricular intervention. Perceptual and Motor Skills. University of Wisconsin, Madison, 1980; Vol. 51, issue 2:498.

Dumas 2001 \{published data only\}

Dumas JE, Lynch AM, Laughlin JE, Smith EP, Prinz RJ. Promoting intervention fidelity: conceptual issues, methods, and preliminary results from the EARLY ALLIANCE prevention trial. American Journal of Preventive Medicine 2001;20(Suppl 1):38-47.

Dymnicki 2014 \{published data only\}

Dymnicki AB. Moderating effects of school climate on outcomes for the Multisite Violence Prevention Project Universal Program. Journal of Research on Adolescence 2014;24:383-98.

\section{Eggert 1994a \{published data only\}}

Eggert LL, Thompson EA, Herting JR, Nicholas LJ. Prevention research program: reconnecting at-risk youth. Issues in Mental Health Nursing 1994;15:107-35.

\section{Eggert 1994b \{published data only\}}

Eggert LL, Thompson EA, Herting JR, Nicholas LJ, Dicker BG. Preventing adolescent drug abuse and high school dropout through an intensive school-based social network development program. American Journal of Health Promotion 1994;8(3):202-15

\section{Eisner 2012 \{published data only\}}

Eisner M, Nagin D, Ribeaud D, Malti T. Effects of a universal parenting program for highly adherent parents: a propensity score matching approach. Prevention Science 2012;13:252-66.

\section{Elder 1994 \{published data only\}}

Elder JP, McGraw SA, Stone EJ, Reed DB, Harsha DW, Greene T, et al. CATCH: process evaluation of environmental factors and programs. Health Education Quarterly 1994;Suppl 2:S107-27.

Ellickson 2004 \{published data only\}

Ellickson PL, McCaffrey DF, Ghosh-Dastidar B. Project ALERT reduces initiation of cigarette and marijuana use in 12-14 year olds. Evidence Based Mental Health 2004;7:53.

\section{Elliot 2004 \{published data only\}}

Elliot DL, Goldberg L, Moe EL, Defrancesco CA, Durham MB, HixSmall H. Preventing substance use and disordered eating: initial outcomes of the ATHENA (athletes targeting healthy exercise and nutrition alternatives) program. Archives of Pediatric and Adolescent Medicine 2004;158:1043-9.

Elliot 2008 \{published data only\}

Elliot DL, Goldberg L, Moe EL, Defrancesco CA, Durham MB, McGinnis W, et al. Long-term outcomes of the ATHENA (Athletes Targeting Healthy Exercise \& Nutrition Alternatives) program for 
female high school athletes. Journal of Alcohol \& Drug Education 2008;52(2):73-92.

\section{Epton 2014 \{published data only\}}

Epton T, Norman P, Dadzie AS, Harris PR, Webb TL, Sheeran P, et al. A theory-based online health behaviour intervention for new university students (U@Uni): results from a randomised controlled trial. BMC Public Health 2014;14:563.

\section{Espelage 2013 \{published data only\}}

Espelage DL, Low S, Polanin JR, Brown EC. The impact of a middle school program to reduce aggression, victimization, and sexual violence. Journal of Adolescent Health 2013; Vol. 53:180-6.

\section{Esposito-Smythers 2014 \{published data only\}}

Esposito-Smythers C, Hadley W, Brown LK, McKnight P, Short J, Weismoore J, et al. Alcohol, suicide, HIV prevention for teens in mental health treatment: preliminary results of a clinical trial. Symposium conducted at the annual meeting of the Research Society on Alcoholism, Seattle, WA. 2014; Vol. 38:307a.

\section{Fagen 2009 \{published data only\}}

Fagen MC, Flay BR. Sustaining a school-based prevention program: results from the Aban Aya Sustainability Project. Health Education \& Behavior 2009;36(1):9-23.

\section{Fekkes 2016 \{published data only\}}

Fekkes M, van de Sande MCE, Gravesteijn JC, Pannebakker FD, Buijs GJ, Diekstra RFW, et al. Effects of the Dutch Skills for Life program on the health behavior, bullying, and suicidal ideation of secondary school students. Health Education 2016;116(1):2-15.

\section{Fergusson 2013 \{published data only\}}

Fergusson DM, Boden JM, Horwood LJ. Nine-year follow-up of a home-visitation program: a randomized trial. Pediatrics 2013;131:297-303.

\section{Fishbein 2016 \{published data only\}}

Fishbein D, Miller S, Herman-Stahl M, Williams J, Lavery B, Markovitz L, et al. Behavioral and psychophysiological effects of a yoga intervention on high-risk adolescents: a randomized control trial. Journal of Child and Family Studies 2016;25(2):518-29.

\section{Fleming 2010 \{published data only\}}

Fleming MF, Balousek SL, Grossberg PM, Mundt MP, Brown D, Wiegel JR, et al. Brief physician advice for heavy drinking college students: a randomized controlled trial in college health clinics. Journal of Studies on Alcohol and Drugs 2010;71(1):23-31.

\section{Fraguela 2003 \{published data only\}}

Fraguela JA, Martin AL, Trinanes ER. Drug abuse prevention in the school: four-year follow-up of a programme. Psychology in Spain 2003;7(1):29-38.

\section{Friedmann 2012 \{published data only\}}

Friedmann PD, Green TC, Taxman FS, Harrington M, Rhodes AG, Katz E, et al. Collaborative behavioral management among parolees: drug use, crime and re-arrest in the Step'n Out randomized trial. Addiction 2012;107:1099-108.

Furr-Holden 2003 \{published data only\}

Furr-Holden CDM, Ialongo NS, Anthony JC, Petras H, Kellam SG. The distal impact of two 1st grade randomized preventive interventions on drug sampling by youths: recent evidence from the Baltimore prevention program. Journal of Emotional and Behavioural Disorders. 2003:51.

\section{Gil 2004 \{published data only\}}

Gil AG, Wagner EF, Tubman JG. RESEARCH REPORT: Culturally sensitive substance abuse intervention for Hispanic and African American adolescents: empirical examples from the Alcohol Treatment Targeting Adolescents in Need (ATTAIN) Project. Addiction 2004;99(Suppl 2):140-50.

\section{Giles 2010 \{published data only\}}

Giles SM, Pankratz MM, Ringwalt C, Hansen WB, Dusenbury L, Jackson-Newsom J. Teachers' delivery skills and substance use prevention program outcomes: the moderating role of students' need for cognition and impulse decision making. Journal of Drug Education 2010;40(4):395-410.

\section{Gislason 1995 \{published data only\}}

Gislason T, Yngvadottir A, Benediktsdottir B. Alcohol consumption, smoking and drug abuse among Icelandic teenagers: a study into the effectiveness of the 'Skills for Adolescence' programme. Drugs: Education, Prevention and Policy 1995;2(3):243-58.

\section{Gittman 1994 \{published data only\}}

Gittman E, Cassata M. A prevention program for middle-school high risk youth. Paper presented at the Annual Meeting of the Northeastern Educational Research Association. Ellenville, NY, 1994:1-11.

\section{Gorman 2002 \{published data only\}}

Gorman DM. The 'science' of drug and alcohol prevention: the case of the randomized trial of the life skills training program. International Journal of Drug Policy 2002;13:21-6.

\section{Harmon 1993 \{published data only\}}

Harmon MA. Reducing the risk of drug involvement among early adolescents - an evaluation of drug abuse resistance education (dare). Evaluation Review 1993;17(2):221-39.

\section{Henggeler 2012 \{published data only\}}

Henggeler SW, McCart MR, Cunningham PB, Chapman JE. Enhancing the effectiveness of juvenile drug courts by integrating evidence-based practices. Journal of Consulting and Clinical Psychology 2012; Vol. 80:264-75.

\section{Hidalgo 2015 \{published data only\}}

Hidalgo MA, Kuhns LM, Hotton AL, Johnson AK, Mustanski B, Garofalo R. The MyPEEPS randomized controlled trial: a pilot of preliminary efficacy, feasibility, and acceptability of a grouplevel, HIV risk reduction intervention for young men who have sex with men. Archives of Sexual Behavior 2015;44:475-85. 
Hogue 2015 \{published data only\}

Hogue A, Dauber S, Henderson CE, Bobek M, Johnson C, Lichvar $E$, et al. Randomized trial of family therapy versus non family treatment for adolescent behavior problems in usual care. Journal of Clinical Child \& Adolescent Psychology 2015;44(6):954-69.

\section{Horigian 2015 \{published data only\}}

Horigian VE, Feaster DJ, Robbins MS, Brincks AM, Ucha J, Rohrbaugh MJ, et al. A cross-sectional assessment of the long term effects of brief strategic family therapy for adolescent substance use. American Journal on Addictions 2015;24:637-45.

\section{Horn 2013 \{published data only\}}

Horn K, Branstetter S, Zhang J, Jarrett T, Tompkins NO, AnesettiRothermel A, et al. Understanding physical activity outcomes as a function of teen smoking cessation. Journal of Adolescent Health 2013;53:125-31.

\section{Hunter 1990 \{published data only\}}

Hunter SM, Johnson CC, Little-Christian S, Nicklas TA, Harsha D, Arbeit ML, et al. Heart Smart: a multifaceted cardiovascular risk reduction program for grade school students. American Journal of Health Promotion 1990;4(5):352-60.

\section{Jacobs 2011 \{published data only\}}

Jacobs N, Clays E, De Bacquer D, De Backer G, Dendale P, Thijs $\mathrm{H}$, et al. Effect of a tailored behavior change program on a composite lifestyle change score: a randomized controlled trial. Health Education Research 2011;26(5):886-95.

\section{Jacobs 2016 \{published data only\}}

Jacobs F, Easterbrooks MA, Goldberg J, Mistry J, Bumgarner E, Raskin M, et al. Improving adolescent parenting: results from a randomized controlled trial of a home visiting program for young families. American Journal of Public Health 2016;106(2):342-9.

\section{Jegannathan 2014 \{published data only\}}

* Jegannathan B, Dahlblom K, Kullgren G. Outcome of a schoolbased intervention to promote life-skills among young people in Cambodia. Asian Journal of Psychiatry 2014;9:78-84.

\section{Jekielek 2002 \{published data only\}}

Jekielek SM, Moore KA, Hair EC, Scarupa HJ. Mentoring: a promising strategy for youth development. Child trends research brief. Child Trends. Report ED462660. Washington, DC: Child Trends, 2002:1-10.

\section{Johnson 1990 \{published data only\}}

Johnson CA, Pentz MA, Weber MD, Dwyer JH, Baer N, Mackinnon DP, et al. Relative effectiveness of comprehensive community programming for drug abuse prevention with highrisk and low-risk adolescents. Journal of Consulting \& Clinical Psychology 1990;58(4):447-56.

\section{Johnson 2009 \{published data only\} \\ Johnson KW, Shamblen SR, Ogilvie KA, Collins D, Saylor B. Preventing youths' use of inhalants and other harmful legal products in frontier Alaskan communities: a randomized trial. Prevention Science 2009;10(4):298-312.}

\section{Kellam 1994a \{published data only\}}

Kellam SG, Rebok GW, lalongo N, Mayer LS. The course and malleability of aggressive behavior from early first grade into middle school: results of a developmental epidemiology-based preventive trial. Journal of Child Psychology and Psychiatry 1994;35(2):259-81.

\section{Kellam 1994b \{published data only\}}

Kellam SG, Rebok GW, Ialongo NS, Mayer LS. "The course and malleability of aggressive behavior from early first grade into middle school: Results of a developmental epidemiology-based preventive trial": Erratum. Journal of Child Psychology and Psychiatry 1994;35:983.

\section{Kelleher 1999 \{published data only\}}

Kelleher CC, Fallon UB, McCarthy E, Dineen BD, O'Donnell M, Killian M, et al. Feasibility of a lifestyle cardiovascular health promotion programme for 8-15-year-olds in Irish general practice: results of the Galway Health Project. Health Promotion International 1999;14(3):221-9.

\section{Kennedy 2009 \{published data only\}}

Kennedy C, Chen JL. Changes in childhood risk taking and safety behavior after a peer group media intervention. Nursing Research 2009;58(4):264-73.

\section{Killen 1988 \{published data only\}}

Killen JD, Telch MJ, Robinson TN, Maccoby N, Taylor CB, Farquhar JW. Cardiovascular disease risk reduction for tenth graders. A multiple-factor school-based approach. JAMA 1988;260(12):1728-33.

\section{Killen 1989 \{published data only\}}

Killen JD, Robinson TN, Telch MJ, Saylor KE, Maron DJ, Rich T, et al. The Stanford Adolescent Heart Health Program. Health Education Quarterly 1989;16(2):263-83.

\section{Kilmer 2008 \{published data only\}}

Kilmer B. Does parolee drug testing influence employment and education outcomes? Evidence from a randomized experiment with noncompliance. Journal of Quantitative Criminology 2008;24(1):93-123.

\section{Kosterman 2001 \{published data only\}}

Kosterman R, Hawkins JD, Haggerty KP, Spoth R, Redmond C. Preparing for the Drug Free Years: session-specific effects of a universal parent-training intervention with rural families. Journal of Drug Education 2001;31(1):47-68.

\section{Kruger 2014 \{published data only\}}

Kruger J, Brennan A, Strong M, Thomas C, Norman P, Epton T. The cost-effectiveness of a theory-based online health behaviour intervention for new university students: an economic evaluation. BMC Public Health 2014;14:1011.

\section{Kypri 2005 \{published data only\}}

Kypri K, McAnally HM. Randomized controlled trial of a webbased primary care intervention for multiple health risk behaviors. Preventive Medicine 2005;41(3-4):761-6. 


\section{Lauver 2002 \{published data only\}}

Lauver SC. Assessing the Benefits of an After-School Program for Urban Youth: An Impact and Process Evaluation. Dissertation available from ProQuest. AA13043903. UMI, Ann Arbor, MI, 2002:1-553.

\section{Lees 2014 \{published data only\}}

Lees DG, Fergusson DM, Frampton CM, Merry SN. A pilot study to evaluate the efficacy of adding a structured home visiting intervention to improve outcomes for high-risk families attending the Incredible Years Parent Programme: study protocol for a randomised controlled trial. Trials [Electronic Resource] 2014;15:66.

\section{Letourneau 2013 \{published data only\}}

Letourneau EJ, Henggeler SW, McCart MR, Borduin CM, Schewe PA, Armstrong KS. Two-year follow-up of a randomized effectiveness trial evaluating MST for juveniles who sexually offend. Journal of Family Psychology 2013;27:978-85.

Lewis 2014 \{published data only\}

Lewis MA, Patrick ME, Litt DM, Atkins DC, Kim T, Blayney JA, et al. Randomized controlled trial of a web-delivered personalized normative feedback intervention to reduce alcohol-related risky sexual behavior among college students. Journal of Consulting and Clinical Psychology 2014;82:429-40.

Lewis 2015 \{published data only\}

Lewis MA, Neighbors C. An examination of college student activities and attentiveness during a web-delivered personalized normative feedback intervention. Psychology of Addictive Behaviors 2015;29:162-7.

\section{MacDonald 2007 \{published data only\}}

MacDonald JM, Morral AR, Raymond B, Eibner C. The efficacy of the Rio Hondo Dui Court: a 2-year field experiment. Evaluation Review 2007;31(1):4-23.

\section{Martinez 2005 \{published data only\}}

Martinez C Jr, Eddy JM. Effects of culturally adapted parent management training on Latino youth behavioral health outcomes. Journal of Consulting \& Clinical Psychology 2005;73(4):841-51.

\section{Mathews 2007 \{published data only\}}

Mathews AE, Werch C, Michniewicz M, Bian H. An impact evaluation of two versions of a brief intervention targeting alcohol use and physical activity among adolescents. Journal of Drug Education 2007;37(4):401-16.

Mays 2012 \{published data only\}

Mays D, Peshkin BN, Sharff ME, Walker LR, Abraham AA, Hawkins KB, et al. Correlates of adherence to a telephonebased multiple health behavior change cancer preventive intervention for teens: the Healthy for Life Program (HELP). Health Education \& Behavior 2012;39:18-26.

\section{Muratori 2015 \{published data only\}}

Muratori P, Bertacchi I, Giuli C, Lombardi L, Bonetti S, Nocentini A, et al. First adaptation of coping power program as a classroom-based prevention intervention on aggressive behaviors among elementary school children. Prevention Science 2015;16:432-9.

\section{Murry 2011 \{published data only\}}

Murry VM, Berkel C, Chen YF, Brody GH, Gibbons FX, Gerrard M. Intervention Induced changes on parenting practices, youth self-pride and sexual norms to reduce HIV-related behaviors among rural African American youths. Journal of Youth and Adolescence 2011;40:1147-63.

O'Donnell 1995 \{published data only\}

O'Donnell J, Hawkins JD, Catalano RF, Abbott RD, Day LE. Preventing school failure, drug use, and delinquency among low-income children: long-term intervention in elementary schools. American Journal of Orthopsychiatry 1995;65(1):87-100.

O'Donnell 2010 \{published data only\}

O'Donnell L, Myint UA, Duran R, Stueve A. Especially for daughters: parent education to address alcohol and sexrelated risk taking among urban young adolescent girls. Health Promotion Practice 2010;11 (3 Suppl):70S-8S.

\section{O'Leary-Barrett 2009 \{published data only\}}

O'Leary-Barrett M, Al Khudhairy N, Conrod P. The efficacy of personality-targeted interventions for substance misuse as delivered by educational professionals. Conference Proceedings. 2009; Vol. 33:42A.

\section{O'Leary-Barrett 2010 \{published data only\}}

O'Leary-Barrett M, Topper L, Mackie CJ, Castellanos-Ryan N, Al Khudhairy N, Conrod PJ. The adventure RCT: effective delivery of personality-targeted interventions for substance misuse by educational professionals. Journal of the American Academy of Child \& Adolescent Psychiatry 2010;49(9):954-63.

\section{Osgood 2013 \{published data only\}}

Osgood DW, Feinberg ME, Gest SD, Moody J, Ragan DT, Spoth R, et al. Effects of PROSPER on the influence potential of prosocial versus antisocial youth in adolescent friendship networks. Journal of Adolescent Health 2013:53:174-9.

\section{Patrick 2014 \{published data only\}}

Patrick ME, Lee CM, Neighbors C. Web-based intervention to change perceived norms of college student alcohol use and sexual behavior on spring break. Addictive Behaviors 2014; Vol. 39:600-6.

\section{Peden 2012 \{published data only\}}

Peden NE. The efficacy of individual Community Reinforcement and Family Training (CRAFT) with concerned significant others of problem gamblers. Dissertation Abstracts International, Section B: The Sciences and Engineering 2012;73:2514.

Peeler 2001 \{published data only\}

Peeler CM. An analysis of the effects of a course designed to reduce the frequency of high-risk sexual behavior and heavy drinking (immune deficiency). Dissertation Abstracts International, Section B: The Sciences and Engineering 2001;61(10-B):5546. 
Pierce 2008 \{published data only\}

Pierce JP, James LE, Messer K, Myers MG, Williams RE, Trinidad DR. Telephone counseling to implement best parenting practices to prevent adolescent problem behaviors. Contemporary Clinical Trials 2008;29:324-34.

Poduska 2008 \{published data only\} Poduska JM, Kellam SG, Wang W, Brown C, lalongo NS, Toyinbo P. Impact of the Good Behavior Game, a universal classroom-based behavior intervention, on young adult service use for problems with emotions, behavior, or drugs or alcohol. Drug \& Alcohol Dependence 2008;95 Suppl 1:S29-44.

\section{Prado 2005 \{published data only\}}

Prado $\mathrm{G}$. The efficacy of three interventions to prevent substance use and sex initiation in subgroups of Hispanic adolescents. Dissertation Abstracts International: Section B: The Sciences and Engineering 2005; Vol. 66:1705.

\section{Prado 2007 \{published data only\}}

Prado G, Pantin H, Briones E, Schwartz SJ, Feaster D, Huang S, et al. A randomized controlled trial of a parent-centered intervention in preventing substance use and HIV risk behaviors in Hispanic adolescents. Journal of Consulting \& Clinical Psychology 2007;75(6):914-26.

\section{Prevention Program \{published data only\}}

Vitaro F, Dobkin PL. Prevention of substance use /abuse in early adolescents with behavior problems. ? 1996;41:11-36.

Vitaro F, Dobkin PL, Tremblay RE. A school-based program for the prevention of substance abuse [Programme de prevention des toxicomanies en milieu scolaire]. Journal International de Psychologie 1994;29(4):431-52.

\section{Rhoades 2013 \{published data only\}}

Rhoades KA, Chamberlain P, Roberts R, Leve LD. MTFC for highrisk adolescent girls: a comparison of outcomes in England and the United States. Journal of Child \& Adolescent Substance Abuse 2013;22:435-49.

\section{Rhoades 2014 \{published data only\}}

Rhoades KA, Leve LD, Harold GT, Kim HK, Chamberlain P. Drug use trajectories after a randomized controlled trial of MTFC: associations with partner drug use. Journal of Research on Adolescence 2014;24:40-54.

\section{Ringwalt 1991 \{published data only\}}

Ringwalt C, Ennett ST, Holt KD. An outcome evaluation of project DARE (Drug Abuse Resistance Education). Health Education Research 1991;6(3):327-37.

\section{Robbins 2009 \{published data only\}}

Robbins MS, Szapocznik J, Horigian VE, Feaster DJ, Puccinelli M, Jacobs $P$, et al. Brief strategic family therapy for adolescent drug abusers: a multi-site effectiveness study. Contemporary Clinical Trials 2009;30(3):269-78.

\section{Rohrbach 2010 \{published data only\}}

Rohrbach LA, Gunning M, Sun P, Sussman S. The project

Towards No Drug Abuse (TND) dissemination trial: implementation fidelity and immediate outcomes. Prevention Science 2010;11:77-88.

Ross 1998 \{published data only\}

Ross C, Richard L, Potvin L. One year outcome evaluation of an alcohol and drug abuse prevention program in a Quebec high school. Revue Canadienne Santa Publique 1998;89(3):166-70.

\section{Rotheram-Borus 2012 \{published data only\}}

Rotheram-Borus MJ, Lightfoot M, Kasirye R, Desmond K. Vocational training with HIV prevention for Ugandan youth. AIDS \& Behavior 2012;16:1133-7.

Rowland 2008 \{published data only\}

Rowland MD, Chapman JE, Henggeler SW. Sibling outcomes from a randomized trial of evidence-based treatments with substance abusing juvenile offenders. Journal of Child and Adolescent Substance Abuse 2008;17(3):11-26.

Sakofs 1991 \{published data only\}

Sakofs M, Schuurman D. Assessing the impact of the Wilderness Alternative for Youth Program: an outward bound program for adjudicated youth. Report 1991:21.

\section{Salminen 2005 \{published data only\}}

Salminen M, Vahlberg T, Ojanlatva A, Kivela SL. Effects of a controlled family-based health education/counseling intervention. American Journal of Health Behavior 2005;29(5):395-406.

\section{Samet 2015 \{published data only\}}

Samet JH, Raj A, Cheng DM, Blokhina E, Bridden C, Chaisson CE, et al. HERMITAGE - a randomized controlled trial to reduce sexually transmitted infections and HIV risk behaviors among HIV-infected Russian drinkers. Addiction 2015;110:80-90.

\section{Sanci 2015 \{published data only\}}

Sanci L, Chondros P, Sawyer S, Pirkis J, Ozer E, Hegarty K, et al. Responding to young people's health risks in primary care: a cluster randomised trial of training clinicians in screening and motivational interviewing. PLoS One 2015;10(9):e0137581.

\section{Santisteban 2003 \{published data only\}}

Santisteban DA, Coatsworth J, Perez-Vidal A, Kurtines WM, Schwartz SJ, LaPerriere A, et al. Efficacy of brief strategic family therapy in modifying Hispanic adolescent behavior problems and substance use. Journal of Family Psychology 2003;17(1):121-33.

\section{Schaeffer 2014 \{published data only\}}

Schaeffer CM, Henggeler SW, Ford JD, Mann M, Chang R, Chapman JE. RCT of a promising vocational/employment program for high-risk juvenile offenders. Journal of Substance Abuse Treatment 2014;46:134-43.

\section{Schinke 1986 \{published data only\}}

Schinke SP, Schilling RF, Gilchrist LD. Prevention of drug and alcohol abuse in American Indian youths. Social Work Research Abstracts 1986;N/A:18-9. 
Schinke 1988 \{published data only\}

Schinke SP, Bebel MY, Orlandi MA, Botvin GJ. Prevention strategies for vulnerable pupils: school social work practices to prevent substance abuse. Urban Education. 22nd Edition. Beverly Hills, California: Sage, 1988:510-9.

Schinke 2005 \{published data only\}

Schinke S, Schwinn T. Gender-specific computer-based intervention for preventing drug abuse among girls. American Journal of Drug \& Alcohol Abuse 2005;31(4):609-16.

\section{Scott 1988 \{published data only\}}

Scott KA, Myers AM. Impact of fitness training on native adolescents' self-evaluations and substance use. Canadian Journal of Public Health 1988;79:424-9.

\section{Sheidow 2012 \{published data only\}}

Sheidow AJ, Jayawardhana J, Bradford W, Henggeler SW, Shapiro SB. Money matters: cost-effectiveness of juvenile drug court with and without evidence-based treatments. Journal of Child \& Adolescent Substance Abuse 2012;21:69-90.

\section{Shope 1996 \{published data only\}}

Shope JT, Copeland LA, Marcoux BC, Kamp ME. Effectiveness of a school-based substance abuse prevention program. Journal of Drug Education 1996;26(4):323-37.

\section{Sieving 2014 \{published data only\}}

Sieving RE, McMorris BJ, Secor-Turner M, Garwick AW, Shlafer R, Beckman KJ, et al. Prime time: 18-month violence outcomes of a clinic-linked intervention. Prevention Science 2014;15:460-72.

\section{Sine 1976 \{published data only\}}

Sine R. The comparative effect of a values approach with a factual approach on the drug abuse and smoking behavior of college students. Journal of the American College Health Association 1976;25:113-6.

\section{Skybo 2002 \{published data only\}}

Skybo TA, Ryan-Wenger N. A school-based intervention to teach third grade children about the prevention of heart disease. Pediatric Nursing 2002;28(3):223-35.

\section{Slater 2006 \{published data only\}}

Slater MD, Kelly KJ, Edwards RW, Thurman PJ, Plested BA, Keefe TJ, et al. Combining in-school and community-based media efforts: reducing marijuana and alcohol uptake among younger adolescents. Health Education Research 2006;21(1):157-67.

\section{Slesnick 2005 \{published data only\}}

Slesnick N, Prestopnik JL. Ecologically based family therapy outcome with substance abusing runaway adolescents. Journal of Adolescence 2005;28(2):277-98.

\section{Slesnick 2006 \{published data only\}}

Slesnick N, Bartle-Haring S, Gangamma R. Predictors of substance use and family therapy outcome among physically and sexually abused runaway adolescents. Journal of Marital \& Family Therapy 2006;32(3):261-81.

\section{Slesnick 2007 \{published data only\}}

Slesnick N, Prestopnik JL, Meyers RJ, Glassman M. Treatment outcome for street-living, homeless youth. Addictive Behaviors 2007;32(6):1237-51.

\section{Slesnick 2013 \{published data only\}}

Slesnick N, Guo X, Brakenhoff B, Feng X. Two-year predictors of runaway and homeless episodes following shelter services among substance abusing adolescents. Journal of Adolescence 2013; Vol. 36:787-95.

\section{Snow 1992 \{published data only\}}

Snow DL, Tebes JK, Arthur MW, Tapasak RC. Two-year followup of a social-cognitive intervention to prevent substance use. Journal of Drug Education 1992;22(2):101-14.

\section{Sommers 2013 \{published data only\}}

Sommers MS, Lyons MS, Fargo JD, Sommers BD, McDonald CC, Shope JT, et al. Emergency department-based brief intervention to reduce risky driving and hazardous/harmful drinking in young adults: a randomized controlled trial. Alcoholism, Clinical and Experimental Research 2013; Vol. 37:1753-62.

\section{Spoth 2011 \{published data only\}}

Spoth R, Redmond C, Clair S, Shin C, Greenberg M, Feinberg M. Preventing substance misuse through community-university partnerships: randomized controlled trial outcomes $41 / 2$ years past baseline. American Journal of Preventive Medicine 2011;40(4):440-7.

\section{Stanton 2000 \{published data only\}}

Stanton BF, Li X, Galbraith J, Cornick G, Feigelman S, Kaljee L, et al. Parental underestimates of adolescent risk behavior: a randomized, controlled trial of a parental monitoring intervention. Journal of Adolescent Health 2000;26:18-26.

\section{Stanton 2004 \{published data only\}}

Stanton B, Cole M, Galbraith J, Li X, Pendleton S, Cottrel L, et al. Randomized trial of a parent intervention: parents can make a difference in long-term adolescent risk behaviors, perceptions, and knowledge. Archives of Pediatric and Adolescent Medicine 2004;158:947-55

\section{Stein 2011 \{published data only\}}

Stein L, Lebeau R, Colby SM, Barnett NP, Golembeske C, Monti PM. Motivational interviewing for incarcerated adolescents: effects of depressive symptoms on reducing alcohol and marijuana use after release. Journal of Studies on Alcohol and Drugs 2011;72:497-506.

\section{Stevens 2002 \{published data only\}}

Stevens MM, Olson AL, Gaffney CA, Tosteson TD, Mott LA, Starr P. A pediatric, practice-based, randomized trial of drinking and smoking prevention and bicycle helmet, gun, and seatbelt safety promotion. Pediatrics 2002;109(3):490-7.

\section{St Lawrence 1999 \{published data only\}}

St Lawrence JS, Crosby RA, Belcher L, Yazdani N, Brasfield TL. Sexual risk reduction and anger management interventions for incarcerated male adolescents: a randomized controlled 
trial of two interventions. Journal of Sex Education and Therapy 1999;24:9-17.

\section{St Pierre 1992 \{published data only\}}

St Pierre TL, Kaltreider DL, Mark MM, Aikin KJ. Drug prevention in a community setting: a longitudinal study of the relative effectiveness of a 3-year primary prevention program in boys and girls clubs across the nation. American Journal of Community Psychology 1992;20(6):673-706.

\section{Stuart 1976 \{published data only\}}

Stuart RB, Jayaratne S, Tripodi T. Changing adolescent deviant behaviour through reprogramming the behaviour of parents and teachers: an experimental evaluation. Canadian Journal of Behavioural Science 1976;8(2):132-44.

\section{Taussig 2007 \{published data only\}}

Taussig HN, Culhane SE, Hettleman D. Fostering healthy futures: an innovative preventive intervention for preadolescent youth in out-of-home care. Child Welfare 2007;86(5):113-31.

\section{Taylor 2000 \{published data only\}}

Taylor BJ, Graham JW, Cumsille P, Hansen WB. Modeling prevention program effects on growth in substance use: analysis of five years of data from the Adolescent Alcohol Prevention Trial. Prevention Science 2000;1(4):183-97.

\section{Teesson 2009 \{published data only\}}

Teesson M, Newton NC, Vogl LE, Andrews G. Climate schools: the development and evaluation of an alcohol and cannabis computer-based prevention program for junior high school students. Alcoholism: Clinical and Experimental Research 2009;33:43A.

\section{Tucker 2016 \{published data only\}}

Tucker JS, Edelen MO, Huang W. Effectiveness of parentchild mediation in improving family functioning and reducing adolescent problem behavior: results from a pilot randomized controlled trial. Journal of Youth and Adolescence 2017;46(3):505-15.

\section{Tupe 2014 \{published data only\}}

Tupe R, Narayana G, Kapadia-Kundu N, Storey D, Safi B, Trivedi G. Seeds of prevention: the impact on health behaviors of young adolescent girls in Uttar Pradesh, India, a cluster randomized control trial. Social Science and Medicine 2014;120:169-79.

\section{Valdez 2013 \{published data only\}}

Valdez A, Cepeda A, Parrish D, Horowitz R, Kaplan C. An adapted brief strategic family therapy for gang-affiliated Mexican American adolescents. Research on Social Work Practice 23;4:383-96.

\section{van Rosmalen-Nooijens 2013 \{published data only\}}

van Rosmalen-Nooijens KA, Prins JB, Vergeer M, Wong SH, Lagro-Janssen AL. "Young people, adult worries": RCT of an internet-based self-support method "Feel the ViBe" for children, adolescents and young adults exposed to family violence, a study protocol. BMC Public Health 2013;13:226.

\section{Vartiainen 1986 \{published data only\}}

Vartiainen E, Puska P, Tossavainen K, Viri L, Niskanen E, Moisio S. Prevention of non-communicable diseases: risk factors in youth. The North Karelia Youth Project (1984-88). Health Promotion 1986;1(3):269-83.

Villalbi 1993 \{published data only\} Villalbi JR, Auba J, Garcia Gonzalez A. Results of a school addictive substances abuse prevention program: the Barcelona PASE pilot project. Gaceta Sanitaria 1993;35:70-7.

\section{Vitaro 1994 \{published data only\}}

Vitaro F, Dobkin PL, Tremblay RE. A school-based program for the prevention of substance abuse. Journal International de Psychologie 1994;29(4):431-52.

Weikart 1988 \{published data only\}

Weikart DP. A perspective in High/Scope's early education research. Early Child Development and Care. Great Britain: Gordon \& Breach Science Publishers, Inc, 1988; Vol. 33:29-40.

\section{Weikart 1991 \{published data only\}}

Weikart DP, Schweinhart LJ. Disadvantaged children and curriculum effects. New Directions for Child Development 1991;53:57-64.

\section{Werch 2005 \{published data only\}}

Werch C, Moore MJ, DiClemente CC, Bledsoe R, Jobli E. A multihealth behavior intervention integrating physical activity and substance use prevention for adolescents. Prevention Science 2005;6(3):213-26.

\section{Werch 2005a \{published data only\}}

Werch CEC, Moore MM, DiClemente CC, Owen DM, Carlson JM, Jobli E. Single vs. multiple drug prevention: is more always better? A pilot study. Substance Use \& Misuse 2005;40(8):1085-101.

\section{Werch 2008 \{published data only\}}

Werch CE, Moore MJ, DiClemente CC. Brief image-based health behavior messages for adolescents and their parents. Journal of Child and Adolescent Substance Abuse 2008;17(4):19-40.

\section{Wilson 2012a \{published data only\}}

Wilson DB, Jones RM, McClish D, Westerberg AL, Danish S. Fruit and vegetable intake among rural youth following a school-based randomized controlled trial. Preventive Medicine 2012;54:150-6.

\section{Wilson 2012b \{published data only\}}

Wilson H, Scult M, Wilcher M, Chudnofsky R, Malloy L, Fricchione $\mathrm{G}$, et al. Implementing a mind-body medicine relaxation training program in an urban high school: changes in health behaviors, perceived stress, and anxiety. $B M C$ Complementary and Alternative Medicine 2012;12(Suppl 1):P298.

Winters 2012 \{published data only\}

Winters KC, Fahnhorst T, Botzet A, Lee S, Lalone B. Brief intervention for drug-abusing adolescents in a school setting: 
outcomes and mediating factors. Journal of Substance Abuse Treatment 2012;42(3):279-88.

\section{Wodarski 1987 \{published data only\}}

Wodarski JS. Teaching adolescents about alcohol and driving: a two year follow-up. Journal of Drug Education 1987;17(4):327-44.

\section{Wu 2003 \{published data only\}}

Wu Y, Stanton BF, Galbraith J, Kaljee L, Cottrell L, Li X, et al. Sustaining and broadening intervention impact: a longitudinal randomized trial of 3 adolescent risk reduction approaches. Pediatrics 2003;111(1):e32-8.

Yabiku 2007 \{published data only\}

Yabiku S, Kulis S, Marsiglia FF, Lewin B, Nieri T, Hussaini S. Neighborhood effects on the efficacy of a program to prevent youth alcohol use. Substance Use \& Misuse 2007;42(1):65-87.

Yilmaz 2015 \{published data only\}

Yilmaz G, Demirli CN, Karacan CD. An intervention to preschool children for reducing screen time: a randomized controlled trial. Child: Care, Health \& Development 2015;41:443-9.

\section{Zatzick 2014 \{published data only\}}

Zatzick D, Russo J, Lord SP, Varley C, Wang J, Berliner L, et al. Collaborative care intervention targeting violence risk behaviors, substance use, and posttraumatic stress and depressive symptoms in injured adolescents: a randomized clinical trial. JAMA Pediatrics 2014;168:532-9.

\section{References to ongoing studies}

\section{Allara 2015 \{published data only\}}

Allara E, Angelini P, Gorini G, Bosi S, Carreras G, Gozzi C, et al. A prevention program for multiple health-compromising behaviors in adolescence: baseline results from a cluster randomized controlled trial. Preventive Medicine 2015;71:20-6.

\section{Barbosa Filho 2015 \{published data only\}}

Barbosa FVC, Lopes AS, Lima AB, de Souza EA, Gubert Fdo A, Silva KS, et al. Rationale and methods of a cluster-randomized controlled trial to promote active and healthy lifestyles among Brazilian students: the "Fortaleca sua Saude" program. BMC Public Health 2015;15:1212.

\section{Letona 2013 \{published data only\}}

Letona P, Gittelsohn J, Chacon V, Ramirez-Zea M, Caballero B. Process evaluation of a community-based pilot intervention for chronic disease prevention in Guatemalan school-aged children. FASEB Journal 2013;27(1):Suppl 1055.22.

\section{Pendergrass 2014 \{published data only\}}

Pendergrass T, Fiellin L, Hieftje K, Duncan L. A videogame intervention for risk reduction and prevention in young minority teens. Journal of Adolescent Health 2014;(1):S72.

\section{Piotrowski 2014 \{published data only\}}

Piotrowski $\mathrm{H}$. Evidence of reduction of aggression and violent behaviors after the positive youth potential curriculum intervention in the sixth grade: a 12-month follow-up in a rural Indiana middle school cluster randomized controlled trial. Journal of Adolescent Health 2014;(1):S26.

\section{Sanci 2012 \{published data only\}}

Sanci L, Grabsch B, Chondros P, Shiell A, Pirkis J, Sawyer S, et al. The prevention access and risk taking in young people (PARTY) project protocol: a cluster randomised controlled trial of health risk screening and motivational interviewing for young people presenting to general practice. BMC Public Health 2012; Vol. 12:400.

Sanci LA, Chondros P, Ambresin AE, Grabsch B, Sawyer SM, Pirkis J, et al. Psychosocial risk screening and counselling for young people attending primary care: a cluster randomised trial. Turk Pediatri Arsivi 2013;48:91.

\section{Standage 2013 \{published data only\}}

Standage M, Cumming SP, Gillison FB. A cluster randomized controlled trial of the be the best you can be intervention: effects on the psychological and physical well-being of school children. BMC Public Health 2013; Vol. 13:666.

\section{Tzelepis 2015 \{published data only\}}

Tzelepis FP, Wiggers CL, Kypri J, Bonevski B, McElduff P, Hill MA, et al. Targeting multiple health risk behaviours among vocational education students using electronic feedback and online and telephone support: protocol for a cluster randomised trial. BMC Public Health 2015;15:550.

\section{Additional references}

\section{Akobeng 2005}

Akobeng AK. Principles of mevidence based Medicine. Archive of Disease in Childhood 2005;90:840-4.

\section{Allen 2016}

Allen ML, Garcia-Huidobro D, Porta C, Curran D, Patel R, Miller J, et al. Effective parenting interventions to reduce youth substance use: a systematic review. Pediatrics 2016;138(2):pii: e20154425.

\section{Basen-Engquist 1996}

Basen-Engquist K, Edmundson EW, Parcel GS. Structure of health risk behavior among high school students. Journal of Consulting and Clinical Psychology 1996;64(4):764-75.

\section{Beyers 2004}

Beyers JM, Toumbourou JW, Catalano RF, Arthur MW, Hawkins JD. A cross-national comparison of risk and protective factors for adolescent substance use: the United States and Australia. Journal of Adolescent Health 2004;35:3-16.

\section{Biglan 2004}

Biglan A, Brennan PA, Foster SL, Holder H. Helping Adolescents At Risk - Prevention of Multiple Problem Behaviors. London: The Guildofrd Press, 2004.

\section{Bonnell 2016}

Bonnell C, Fletcher A, Jamal F, Aveyard P, Markham W. Where next with theory and research on how the school environment 
influences young people's substance use?. Health and Place 2016;40:91-7.

\section{Borenstein 2009}

Borenstein M, Hedges LV, Higgins JPT, Rothstein HR. Introduction to Meta-Analysis. West Sussex, UK: John Wiley and Sons Ltd, 2009.

\section{Brody 2005}

Brody GH, Murry VM, McNair L, Chen Y-F, Gibbons FX, Gerrard M, et al. Linking changes in parenting to parent-child relationship quality and youth self-control: the Strong African American Families Program. Journal of Research on Adolescence 2005;15(1):47-69.

\section{Burke 1997}

Burke V, Milligan RAK, Beilin LJ, Dunbar D, Spencer M, Balde E, et al. Clustering of health-related behaviors among 18-year-old Australians. Preventive Medicine 1997;26:724-33.

\section{Calado 2016}

Calado F, Alexandre J, Griffiths MD. Prevalence of adolescent problem gambling: a systematic review of recent research. Journal of Gambling Studies 2016;33(2):1-28.

\section{Campbell 2012}

Campbell R. Population-level intervention for preventing multiple risk behaviours in individuals aged 8-25 years (ongoing).. Cochrane Database of Systematic Reviews 2012.

\section{Carney 2016}

Carney T, Myers BJ, Lous J, Okwundu CI. Brief school-based interventions and behavioural outcomes for substanceusing adolescents. Cochrane Database of Systematic Reviews 2016;Issue 1:CD008969.

\section{Catalano 2012}

Catalano RF, Fagan AA, Loretta EG, Greenberg MT, Irwin Jr, CE, Ross DA, Shek DTL. Worldwide application of prevention science in adolescent health. Lancet 2012;379:1653-64.

\section{Chen 1995}

Chen K, Kandel DB. The natural history of drug use from adolescence to the mid-thirties in a general population sample. American Journal of Public Health 1995;85(1):41-7.

\section{Connell 2009}

Connell CM, Gilreath TD, Hansen NB. A multiprocess latent class analysis of HTE co-occurrence of substance use and sexual risk behaviour among adolescents. Journal of Studies on Alcohol and Drugs 2009;70:943-51.

\section{de Looze 2014}

de Looze M. Cross-national evidence for the clustering and psychosocial correlates of adolescent risk behaviours in 27 countries. European Journal of Public Health 2014;25(1):50-6.

\section{de Winter 2016}

de Winter AF, Visser L, Verhulst FC, Vollebergh WAM, Reijneveld SA. Longitudinal patterns and predictors of multiple health risk behaviors among adolescents: the TRAILS study. Preventive Medicine 2016;84:76-82.

\section{DerSimonian 1986}

DerSimonian R, Laird N. Meta-analysis in clinical trials. Controlled Clinical Trials 1986;7:177-88.

\section{Djousse 2009}

Djousse L, Driver JA, Gaziano JM. Relation between modifiable lifestyle factors and lifetime risk of heart failure. JAMA 2009;302(4):394-400.

\section{Dobbins 2013}

Dobbins M, Husson H, DeCorby K, LaRocca RL. School-based physical activity programs for promoting physical activity and fitness in children and adolescents aged 6 to 18. Cochrane Database of Systematic Reviews 2013;Issue 2:Art No.: CD007651.

\section{Eckenrode 2010}

Eckenrode J, Campa M, Luckey DW, Henderson CR Jr, Cole R, Kitzman $\mathrm{H}$, et al. Long-term effects of prenatal and infancy nurse home visitation on the life course of youths: 19-year follow-up of a randomized trial. Archives of Pediatrics Adolescent Medicine 2010;164(1):9-15.

\section{Faeh 2006}

Faeh D, Viswanathan B, Chiolero A, Warren W, Bovet P. Clustering of smoking, alcohol drinking and cannabis use in adolescents in a rapidly developing country. BMC Public Health 2006;6:169-76.

\section{Faggiano 2014}

Faggiano F, Minozzi S, Versino E, Buscemi D. Universal schoolbased prevention for illicit drug use. Cochrane Database of Systematic Reviews 2014;Issue 12:Art. No: CD003020.

\section{Fellmeth 2011}

Fellmeth GLT, Nurse J, Heffernan C, Habibula S, Sethi D. Educational and skills-based interventions for preventing relationship and dating violence in adolescents and young adults. Cochrane Database of Systematic Reviews 2011, Issue 7. [DOI: 10.1002/14651858.CD004534.pub2]

\section{Foxcroft 2011}

Foxcroft DR, Tsertsvadze A. Universal school-based prevention programs for alcohol misuse in young people. Cochrane Database of Systematic Reviews 2011;5:CD009113.

\section{Foxcroft 2011b}

Foxcroft DR, Tsertsvadze A. Universal family-based prevention programs for alcohol misuse in young people. Cochrane Database of Systematic Reviews 2011;Issue 9.:Art. No.: CD009308.

\section{Fuller 2015}

Fuller $\mathrm{E}$ (editor). Smoking, drinking and drug use among young people in England in 2014. Health and Social Care Information Centre. A survey carried out for the Health and Social Care Information Centre by NatCen Social Research and the National Foundation for Educational Research. 2015. 


\section{Furlong 2012}

Furlong M, McGilloway S, Bywater T, Hutchings J, Smith SM, Donnelly M. Behavioural and cognitive-behavioural groupbased parenting programmes for early-onset conduct problems in children aged 3 to 12 years. Cochrane Database of Systematic Reviews 2012;15(2):CD008225.

\section{Gibbons 2007}

Gibbons FX, Gerrard M, Ouellette JA, Burzette R. Cognitive antecedents to adolescent health risk: discriminating between behavioral intention and behavioral willingness. Psychology and Health 2007;13(2):319-39.

\section{Gore 2011}

Gore FM, Bloem PJ, Patton GC, Ferguson J, Joseph V, Coffey C, et al. Global burden of disease in young people aged $10-24$ years: a systematic analysis. Lancet 2011;377:2093-102.

\section{Guyatt 2008}

Guyatt G, Oxman AD, Gunn EV, Kunz R, Falck-Ytter Y, AlonsoCoello P, et al. GRADE: an emerging consensus on rating quality of evidence and strength of recommendations. $B M J$ 2008;336:924.

\section{Hale 2014}

Hale DR, Fltzgerald-Yau N, Viner RM. A systematic review of effective interventions for reducing multiple health risk behaviors in adolescence. American Journal of Public Health 2014;104:19-41.

\section{Hale and Viner 2016}

Hale DR, Viner RM. The correlates and course of multiple health risk behaviour in adolescence. BMC Public Health 2016;16:458-70.

\section{Hales 2010}

Hales J, Nevill C, Pudney S, Tipping S. Longitudinal Analysis of the Offending, Crime and Justice Survey 2003-06. Research Report 19. Home Office, London 2010.

\section{Hall 2016}

Hall WD, Patton G, Stockings E, Weier M, Lynskey M, Morley KI, et al. Why young people's substance use matters for global health. Lancet Psychiatry 2016;3:265-79.

\section{Hawkins 2005}

Hawkins JD, Kosterman R, Catalano RF, Hill KG, Abbott RD. Promoting positive adult functioning through social development intervention in childhood: long-term effects from the Seattle Social Development Project. Archives of Pediatrics Adolescent Medicine 2005;159(1):25-31.

\section{Hawkins and Weis 1985}

Hawkins JD, Weis JG. The social development model: an integrated approach to delinquency prevention. Journal of Primary Prevention 1985;6(2):73-97.

\section{Hickman 2014}

Hickman M, Caldwell DM, Busse H, MacArthur G, Faggiano F, Foxcroft DR, et al. Individual-, family-, and school-level interventions for preventing multiple risk behaviours relating to alcohol, tobacco and drug use in individuals aged 8 to 25 years. Cochrane Database of Systematic Reviews 2014, Issue Issue 11. Art. No.: CD011374.. [DOI: 10.1002/14651858.CD011374]

\section{Higgins 2008}

Higgins JPT, Green S (editors). Cochrane Handbook for Systematic Reviews of Interventions. Chichester: John Wiley and Sons, 2008.

\section{Higgins 2018}

Higgins J, Lasserson T, Chandler J, Tovey D, Churchill R. Methodological Expectations of Cochrane Intervention Reviews (MECIR). Standards for the conduct and reporting of new Cochrane Intervention Reviews, reporting of protocols and the planning, conduct and reporting of updates. http:// community.cochrane.org/mecir-manual 2018;Version 1.05.

\section{Jackson 2010}

Jackson C, Haw S, Frank J. Adolescent and young adulthood health in Scotland: interventions that address multiple risk behaviours or take a generic approach to risk in youth. Adolescent and Young Adulthood Health in Scotland: Interventions That Address Multiple Risk Behaviours or Take a Generic Approach to Risk in Youth. Edinburgh: SCHPRP, 2010.

\section{Jackson 2011}

Jackson C, Geddes R, Haw S, Frank J. Interventions to prevent substance use and risky sexual behaviour in young people: a systematic review. Addiction 2011;107:733-47.

\section{Jackson 2012}

Jackson C, Sweeting H, Haw S. Clustering of substance use and sexual risk behaviour in adolescence: analysis of two cohort studies. BMJ Open 2012;2:e000661.

\section{Jessor 1991}

Jessor R. Risk behavior in adolescence: a psychosocial framework for understanding and action. Journal of Adolescent Health 1991;12(8):597-605.

\section{Jessor 1992}

Jessor R. Risk behavior in adolescence: a psychosocial framework for understanding and action. Developmental Review 1992;12:374-90.

\section{Junger 2001}

Junger M, Stroebe W, van der Laan AM. Delinquency, health behaviour and health. British Journal of Health Psychology 2001;6:103-20.

\section{Kidger 2012}

Kidger J, Heron J, Lewis G, Evans J, Gunnell D. Adolescent selfharm and suicidal thoughts in the ALSPAC cohort: a self-report survey in England. BMC Psychiatry 2012;12(1):69.

\section{King 2015}

King K, Meader N, Wright K, Graham H, Power C, Petticrew M, et al. Characteristics of interventions targeting multiple lifestyle risk behaviours in adult populations: a systematic scoping review. PLOS ONE 2015;10(1):e0117015. 


\section{Kipping 2014}

Kipping RR, Smith M, Heron J, Hickman M, Campbell R. Multiple risk behaviour in adolescence and socio-economic status: findings from a UK birth cohort. European Journal of Public Health 2014;25(1):44-9.

\section{Kuh 2003}

Kuh D, Ben-Shlomo Y, Lynch J, Hallqvist J, Power C. Life course epidemiology. Journal of Epidemiology and Community Health 2003;57:778-83.

\section{Kuntsche 2016}

Kuntsche S, Kuntsche E. Parent-based interventions for preventing or reducing adolescent substance use: a systematic literature review. Clinical Psychology Review 2016;45:89-101.

\section{Lancet 2012}

The Lancet Series on Adolescent Health. Adolescent Health 2012. Lancet 2012; Vol. 379:https://www.thelancet.com/series/ adolescent-health-2012 (accessed April 2017).

\section{Lancet Commission on Adolescent Health \& Wellbeing}

Lancet Commission on Adolescent Health \& Wellbeing. Our future: a Lancet commission on adolescent health and wellbeing. Lancet 2016; Vol. http://www.thelancet.com/ commissions/adolescent-health-and-wellbeing (accessed May 2017).

\section{Langford 2014}

Langford R, Bonnell CP, Jones HE, Pouliou T, Murphy SM, Waters E, et al. The WHO Health Promoting School framework for improving the health and well-being of students and their academic achievement. Cochrane Database of Systematic Reviews 2014, Issue Issue 4. Art. No.: CD008958. [DOI: 10.1002/14651858.CD008958.pub2]

\section{Leatherdale 2010}

Leatherdale ST, Ahmed R. Alcohol, marijuana and tobacco use among Canadian youth: do we need more multi-substance prevention programming?. Journal of Primary Prevention 2010;31:99-108.

\section{Lewis 2012}

Lewis KM, Bavarian N, Snyder FJ, Acock A, Day J, DuBois DL, et al. Direct and mediated effects of a social-emotional and character development program on adolescent substance use. International Journal of Emotional Education 2012;4(1):56-78.

\section{Lewis 2013}

Lewis KM, Schure MB, Bavarian N, DuBois DL, Day J, Ji P, et al. Problem behavior and urban, low-income youth: a randomized controlled trial of Positive Action in Chicago. Americal Journal of Preventive Medicine 2013;44(6):622-30.

\section{Livingstone 2010}

Livingstone N, Macdonald G, Carr N. Restorative justice conferencing for reducing recidivism in young offenders. Cochrane Database of Systematic Reviews 2010, Issue 12. [DOI: 10.1002/14651858.CD008898]

\section{MacArthur 2012}

MacArthur G, Kipping R, White J, Chittleborough C, Lingam R, Pasch K, et al. Individual-, family-, and school-level interventions targeting multiple risk behaviours in young people (Protocol). Cochrane Database of Systematic Reviews 2012;Issue 6:Art. No. CD009927.

\section{Mahalik 2013}

Mahalik JR, Levine CR, McPherran LC, Doyle LA, Markowitz AJ, Jaffee SR. Changes in health risk behaviors for males and females from early adolescence through early adulthood. Health Psychology 2013;32(6):685-94.

\section{Martineau 2013}

Martineau F, Tyner E, Lorenc T, Petticrew M, Lock K. Populationlevel interventions to reduce alcohol-related harm: an overview of systematic reviews. Preventive Medicine 2013;57:278-96.

\section{Mason 2010}

Mason WA, Hitch JE, Kosterman R, McCarty CA, Herrenkohl TI, Hawkins JD. Growth in adolescent delinquency and alcohol use in relation to young adult crime, alcohol use disorders, and risky sex: a comparison of youth from low-versus middleincome backgrounds. Journal of Child Psychology and Psychiatry 2010;51(12):1377-85.

\section{McCambridge 2011}

McCambridge J, McAlaney RR. Adult consequences of late adolescent alcohol consumption: a systematic review of cohort studies. PLoS One 2011;8(2):e1000413.

\section{McVie 2005}

McVie S, Bradshaw P. Adolescent smoking, drinking and drug use. The Edinburgh Study of Youth Transitions and Crime. Edinburgh: Centre for Law and Society, 2005.

\section{Meader 2016}

Meader N, King K, Moe-Byrne T, Wright K, Graham H, Petticrew $\mathrm{M}$, et al. A systematic review on the clustering and co-occurrence of multiple risk behaviours. BMC Public Health 2016;16:657-66.

\section{Mendenhall 2017}

Mendenhall E, Kohrt BA, Norris SA, Ndetei D, Prabhakaran D. Non-communicable disease syndemics: poverty, depression, and diabetes among low-income populations. Lancet 2017;389(10072):951-63.

\section{Mistry 2009}

Mistry R, McCarthy WJ, Yancey AK, Lu Y, Patel M. Resilience and patterns of health risk behaviors in California adolescents. Preventive Medicine 2009;48:291-7.

\section{Moffitt 1993}

Moffit TE. Adolescence-limited and life-course-persistent antisocial behavior: a developmental taxonomy. Psychological Review 1993;100(4):674-701.

\section{Mokdad 2013}

Mokdad AH, Forouzanfar MH, Daoud F, Mokdad AA, El Bcheraoui C, Moradi-Lakeh M, et al. Global burden of 
diseases, injuries, and risk factors for young people's health during 1990-2013: a systematic analysis for the Global Burden of Disease Study 2013. Lancet 2016;387:2383-401.

\section{Moore 2015}

Moore GF, Audrey S, Barker M, Bond L, Bonnell C, Hardeman W, et al. Process evaluation of complex interventions: Medical Research Council guidance. BMJ 2015;350:h1258.

\section{Movsisyan 2016}

Movsisyan A, Melendez-Torres GJ, Montgomery P. Users identified challenges in applying GRADE to complex interventions and suggested an extension to GRADE. Journal of Clinical Epidemiology 2016;70:191-9.

\section{Movsisyan 2016b}

Movsisyan A, Melendez-Torres GJ, Montgomery P. Outcomes in systematic reviews of complex interventions never reached "high" GRADE ratings when compared with those of simple interventions. Journal of Clinical Epidemiology 2016;78:22-33.

\section{Murry 2009}

Murry VM, Berkel C, Brody G, Miller S, Chen Y. Linking parental socialization to interpersonal protective processes, academic self-presentation, and expectations among rural African American youth. Cultural Diversity and Ethnic Minority Psychology 2009;15(1):1-10.

\section{Mytton 2006}

Mytton J, DiGuiseppi C, Gough D, Taylor RS, Logan S. Schoolbased secondary prevention programmes for preventing violence. Cochrane Database of Systematic Reviews 2006, Issue 3: CD004606. [DOI: 10.1002/14651858.CD004606.pub2]

\section{Newton 2017}

Newton NC, Champion KE, Slade T, Chapman C, Stapinski L, Koning I, et al. A systematic review of combined student- and parent-based programs to prevent alcohol and other drug use among adolescents. Drug and Alcohol Review 2017;36(3):337-51.

\section{NHS 2008}

NHS Scotland. Scottish Schools Adolescent Lifestyle and Substance Use Survey (SALSUS). Smoking, drinking and drug use among 13 and 15 year olds in Scotland in 2008.. NHS Scotland, Edinburgh 2008.

\section{Ortega 2013}

Ortega FB, Konstabel K, Pasquali E, Ruiz JR, Hurtig-Wennlof A, Maestu J, et al. Objectively measured physical activity and sedentary time during childhood, adolescence and young adulthood: a cohort study. PLoS One 2013;8(4):e60871.

\section{Pahl 2010}

Pahl K, Brook DW, Morojele NK, Brook JS. Nicotine dependence and problem behaviours among urban South African adolescents. Journal of Behavioural Medicine 2010;33(2):101-9.

\section{Patton 2012}

Patton GC, Coffey C, Cappa C, Currie D, Riley L, Gore F, et al. Health of the world's adolescents: a synthesis of internationally comparable data. Lancet 2012;379:1665-75.

\section{Patton 2014}

Patton GC, Ross DA, Santelli JS, Sawyer SM, Viner RM, Kleinert S. Next steps for adolescent health: a Lancet Commission. Lancet 2014;383:385-86.

\section{Rehfeuss 2013}

Rehfeuss EA, Akl EA. Current experience with applying the GRADE approach to public health interventions: an empirical study. BMC Public Health 2013;13:9.

\section{Reid 1999}

Reid JB, Eddy JM, Fetrow RA, Stoolmiller M. Description and immediate impacts of a preventive intervention for conduct problems. American Journal of Community Psychology 1999;27(4):483-517.

\section{Resnick 2012}

Resnick MD, Catalano RF, Sawyer SM, Viner R, Patton GC. Seizing the opportunities of adolescent health. Lancet 2012;379:1564-6.

\section{Sawyer 2012}

Sawyer SM, Afifi RA, Bearinger LH, Blakemore SJ, Dick B, Ezeh AC, Patton GC. Adolescence: a foundation for future health. Lancet 2012;379:1630-40.

\section{Shackleton 2016}

Shackleton N, Jamal F, Viner RM, Dickson K, Patton G, Bonnell C. School-based interventions going beyond health education to promote adolescent health: systematic review of reviews. Journal of Adolescent Health 2016;58:382-96.

\section{Sheehan 2017}

Sheehan P, Sweeny K, Rasmussen B, Wils A, Friedman HS, Mahon J, et al. Building the foundations for sustainable development: a case for global investment in the capabilities of adolescents. Lancet 2017;S0140-6736(17):http:// dx.doi.org/10.1016/S0140-6736(17)30872-3.

\section{Shepard and Dickstein 2009}

Shepard SA, Dickstein S. Preventive intervention for early childhood behavioral problems: an ecological perspective. Child \& Adolescent Psychiatric Clinics of North America 2009;18(3):687-706.

\section{Silins 2015}

Silins E, Fergusson DM, Patton GC, Horwood LJ, Olsson CA, Hutchinson DM, et al. Adolescent substance use and educational attainment: an integrative data analysis comparing cannabis and alcohol from three Australasian cohorts. Drug and Alcohol Dependence 2015;156:90-6.

\section{Sterne 2011}

Sterne JAC, Sutton AJ, loannidis JPA, Terrin N, Jones DR, Lau J, et al. Recommendations for examining and interpreting funnel plot asymmetry in meta-analyses of randomised controlled trials. BMJ 2011;342:d4002.

\section{Stockings 2016}

Stockings E, Hall WD, Lynskey M, Morley KI, Reavley N, Strang J, et al. Prevention, early intervention, harm reduction, and 
treatment of substance use in young people. Lancet Psychiatry 2016;3:280-96.

\section{Stone 1996}

Stone EJ, Osganian SK, McKinlay SM, Wu MC, Webber LS, Luepker RV, et al. Operational design and quality control in the CATCH multicenter trial. Preventive Medicine 1996;25:384-99.

\section{Strom 2014}

Strom HK, Adolfsen F, Fossum S, Kaiser S, Martinussen M. Effectiveness of school-based preventive interventions on adolescent alcohol use: a meta-analysis of randomized controlled trials. Substance Abuse Treatment Prevention and Policy 2014;9(1):1-11.

\section{Thomas 2013}

Thomas RE, McLellan J, Perera R. School-based programmes for preventing smoking. Cochrane Database of Systematic Reviews 2013;4:Art No.: CD001293..

\section{Thomas 2015}

Thomas RE, Baker PRA, Thomas BC, Lorenzetti DL. Familybased programmes for preventing smoking by children and adolescents. Cochrane Database of Systematic Reviews 2015; Issue 2:Art. No.: CD004493..

\section{Tinner 2018}

Tinner L, Caldwell D, Hickman M, MacArthur GJ, Gottfredson D, Lana Perez A, et al. Examining subgroup effects by socioeconomic status of public health interventions targeting 1 multiple risk behaviour in adolescence.. BMC Public Health. Accepted for publication..

\section{Tremblay 1995}

Tremblay RE, Pagani-Kurtz L, Mâsse LC, Vitaro F, Pihl RO. A bimodal preventive intervention for disruptive kindergarten boys: its impact through mid-adolescence. Journal of Consulting and Clinical Psychology 1995;63(4):560-8.

\section{van Nieuwenhuijzen 2009}

van Nieuwenhuijzen M, Junger M, Velderman MK, Wiefferink KH, Paulussen TWGM, Hox J, et al. Clustering of healthcompromising behavior and delinquency in adolescents and adults in the Dutch population. Preventive Medicine 2009;48:572-8.

\section{CHARACTERISTICS OF STUDIES}

Characteristics of included studies [ordered by study ID]

\section{Viner 2006}

Viner RM, Haines MM, Head JA, Bhui K, Taylor S, Stansfeld SA, et al. Variations in associations of health risk behaviors among ethnic minority early adolescents. Journal of Adolescent Health 2006;38(1):55.

\section{Wanner 2006}

Wanner B, Vitaro F, Ladouceur R, Brendgen M, Tremblay RE. Joint trajectories of gambling, alcohol and marijuana use during adolescence: a person- and variable-centered developmental approach. Addictive Behaviors 2006;31:566-80.

\section{Waters 2012}

Waters E, de Silva-Sanigorski A, Hall BJ, Brown T, Campbell KJ, Gao Y, et al. Interventions for preventing obesity in children. Cochrane Database of Systematic Reviews 2011, Issue 12. [DOI: 10.1002/14651858.CD001871.pub3]

\section{Webster-Stratton and Taylor 2001}

Webster-Stratton C, Taylor T. Nipping early risk factors in the bud: preventing substance abuse, delinquency, and violence in adolescence through interventions targeted at young children (0-8 years). Prevention Science 2001;2(3):165-92.

\section{Wiefferink 2006}

Wiefferink C, Peters L, Hoekstra F, Dam G, Buijs G, Paulussen T. Clustering of health-related behaviors and their determinants: possible consequences for school health interventions. Prevention Science 2006;7(2):127-49.

\section{Woolfenden 2009}

Woolfenden S, Williams KJ, Peat J. Family and parenting interventions in children and adolescents with conduct disorder and delinquency aged 10-17. Cochrane Database of Systematic Reviews 2001, Issue 2. [DOI: 10.1002/14651858.CD003015]

\section{World Health Organization 2014}

World Health Organization. Health for the World's Adolescents: A Second Chance in the Second Decade. Report 2014:http:// www.who.int/maternal_child_adolescent/documents/seconddecade/en/ (accessed September 2018).

* Indicates the major publication for the study

Averdijk 2016

$\begin{array}{ll}\text { Methods } & \text { Study design: cluster RCT } \\ & \text { Intervention } \operatorname{arm}(\mathbf{s}): \text { PATHS } \\ \text { Comparator } \operatorname{arm}(\mathbf{s}): \text { Triple P, PATHS + Triple P, Control } \\ \text { Sample size calculation performed: no } \\ \text { Subgroups prespecified: N/A; no subgroups }\end{array}$


Averdijk 2016 (Continued)

\author{
Subgroup analyses: no \\ Start date: 2004-2005 academic year \\ Duration of follow-up: 8 years \\ Number of follow-ups: 2 \\ Follow-up time points: waves 5 (2011) and 6 (2013) \\ ICC (if reported): at age 13 years, the median ICC at the school level was 0.02 (range 0.01 to 0.03 across \\ outcomes). Across youth-reported measures at this age, the median ICC was 0.01 . At age 15 years, the \\ median ICC at the school level was also low, at 0.01 (range 0.01 to 0.05 across outcomes). Across youth- \\ reported measures at this age, the median ICC was 0.01 .
}

Participants

Number of schools randomised: 56 (PATHS: 14, Triple P: 14, PATHS + Triple P: 14, control: 14)

Number of participants randomised (total and by arm): 1675 (PATHS: 360, Triple P: 339, PATHS + Triple P: 306, control: 356)

Age (range or mean (SD)) or grade at the start: 7.45 years

Gender: $52 \%$ male, $48 \%$ female

Ethnicity: not reported

SES: SES was based on coding the caregiver's current profession and was transformed into an International Socio-Economic Index of occupational status (ISEI) score. We took the highest ISEI score of the 2 caregivers across waves 1 and 5/6 (mean 45.83, SD 18.72).

Inclusion criteria: all year 1 primary school children in Zurich, Switzerland, in the academic year 2004-2005

Exclusion criteria: not reported

Interventions

Randomisation before or after baseline survey: not reported

Duration of the intervention (excluding follow-up): 12 months

Description of the intervention arm(s): (1) PATHS: a 1-year programme that includes 46 lessons. Lessons addressed problem-solving skills, social relationships, self-regulation, rule understanding, emotion understanding, and positive self-esteem. Teachers received 2 days of training. Five coaches were trained and supervised. Coaches visited each class 4 to 6 times throughout the implementation period. Additional measures to support implementation included a refresher seminar held at midterm, a PATHS newsletter, and coach-led group meetings, where issues related to the programme could be discussed. (2) Triple P: Level 4 Triple P was implemented. Its core element was a group-based course with 4 units of 2 to 2.5 hours. Units addressed themes such as positive parenting, techniques to support desired behaviours, and routines to help avoid escalation of conflicts. Additionally, the programme included up to 4 follow-up telephone contacts with each participant. Courses were delivered by licenced facilitators selected in collaboration with Triple P Switzerland.

Brief description of the theoretical model: PATHS is a research-based programme that places particular importance on emotional learning processes. Triple P is based on cognitive-behavioural therapy.

\begin{tabular}{ll}
\hline Outcomes & Primary: delinquency, substance use, antisocial behaviour \\
\hline Setting & Country: Switzerland; Place: Zurich \\
& Setting: school (PATHS); community (Triple P) \\
& Focus: universal (PATHS); parent/family (Triple P) \\
\hline
\end{tabular}




\section{Method (qualitative or quantitative): both}

\section{Descriptions:}

\section{PATHS}

Adherence to the intervention: data collected to monitor implementation included teacher and child questionnaires, as well as classroom observations. Indicators suggest high overall implementation quality. Coaches assessed a total of 308 PATHS lessons. Depending on the assessed aspect (e.g. child motivation, quality of classroom management, cooperation with coaches), between $74 \%$ and $81 \%$ of lessons were rated as high quality.

Intensity of the intervention: teachers taught PATHS an average of 67 minutes per week, with an average of 2.4 sessions per week.

\section{Triple P}

Acceptability of the intervention: participant overall satisfaction with the programme was 4.33 (standard deviation $(S D)=0.89$ ) and provider competency was rated at 4.65 (SD 0.73) on a 5-point scale.

Reach of the intervention: parents of $27.0 \%$ of the children attended at least 1 session; parents of $2.2 \%$ of the children attended only 1 session; parents of $1.7 \%$ of the children attended only 2 sessions; parents of $4.3 \%$ of the children attended 3 sessions; and parents of $18.8 \%$ of the children completed all 4 sessions.

Adherence to the intervention: the implementation team organised 41 Triple P courses, of which 33 were held in German, 3 in Turkish, 2 each in Portuguese and Albanian, and 1 in English. Course providers estimated that $93 \%$ of the full course material was delivered during the sessions. About $60 \%$ of those who had attended the training reported that they used a significant part of the learned techniques 3 months after the intervention. Parents who completed the programme were more likely to come from breadwinner families, to be Swiss, to have high socioeconomic status (SES), and to be highly integrated in neighbourhood social networks. However, compliers did not differ from non-compliers with regards to parenting problems or child problem behaviours.

\section{Statistics}

Sample size: eligible N = 90 schools; enrolled N = 56 schools $(62 \%)$; PATHS = 14, Triple P = 14, control = 14, PATHS + Triple $P=14$

$\mathrm{N}=1675$ children: $\mathrm{PATHS}=360$, Triple $\mathrm{P}=339$, control $=356, \mathrm{PATHS}+$ Triple $\mathrm{P}=306$

Unit of analysis: individuals and schools

Method to promote equivalence between groups: blocking

Statistical models: multi-level model. Models incorporated 2 levels: youths (Level 1 ) and school at treatment (Level 2).

\section{Baseline differences adjustment: no}

Repeated measures methods in analysis: not reported

Funding: Swiss National Science Foundation, Jacobs Foundation, Swiss Federal Office of Public Health, Canton of Zurich Ministry of Education, Julius Baer Foundation

Randomisation method, e.g. block, stratification, computer: schools were first blocked by school size and socioeconomic background of the catchment area. Schools in disadvantaged school districts were slightly over-represented in the sample.

Clustering accounted for in sample size calculation (if relevant): not reported

Cluster randomisation methods to account for clustering in analysis: yes

\section{Risk of bias}


Averdijk 2016 (Continued)

\begin{tabular}{|c|c|c|}
\hline Bias & Authors' judgement & Support for judgement \\
\hline $\begin{array}{l}\text { Random sequence genera- } \\
\text { tion (selection bias) }\end{array}$ & Unclear risk & Not reported \\
\hline $\begin{array}{l}\text { Allocation concealment } \\
\text { (selection bias) }\end{array}$ & Unclear risk & Not reported \\
\hline $\begin{array}{l}\text { Blinding of participants } \\
\text { and personnel (perfor- } \\
\text { mance bias) } \\
\text { All outcomes }\end{array}$ & High risk & Participants not blinded \\
\hline $\begin{array}{l}\text { Blinding of outcome as- } \\
\text { sessment (detection bias) } \\
\text { All outcomes }\end{array}$ & High risk & Self-reported outcome measures \\
\hline $\begin{array}{l}\text { Incomplete outcome data } \\
\text { (attrition bias) } \\
\text { All outcomes }\end{array}$ & Low risk & $\begin{array}{l}\text { Low attrition; missing data were handled with robust full-information maxi- } \\
\text { mum-likelihood estimation (FIML) }\end{array}$ \\
\hline $\begin{array}{l}\text { Selective reporting (re- } \\
\text { porting bias) }\end{array}$ & Unclear risk & $\begin{array}{l}\text { Teacher- and student-reported outcomes; also restricted dataset with strict in- } \\
\text { clusion criteria; no protocol }\end{array}$ \\
\hline Other bias & Low risk & \\
\hline
\end{tabular}

Averdijk 2016b

Methods

Study design: cluster RCT

Intervention arm(s): PATHS

Comparator arm (s): Triple P, PATHS + Triple P, control

Sample size calculation performed: no

Subgroups prespecified: N/A; no subgroups

Subgroup analyses: no

Start date: $2004-2005$ academic year

Duration of follow-up: 8 years

Number of follow-ups: 2

Follow-up time points: waves 5 (2011) and 6 (2013)

ICC (if reported): at age 13 years, the median ICC at the school level was 0.02 (range 0.01 to 0.03 across outcomes). Across youth-reported measures at this age, the median ICC was 0.01 . At age 15 years, the median ICC at the school level was also low, at 0.01 (range 0.01 to 0.05 across outcomes). Across youthreported measures at this age, the median ICC was 0.01 .

Participants

Number of schools randomised: 56 (PATHS: 14, Triple P: 14, PATHS + Triple P: 14, control: 14)

Number of participants randomised (total and by arm): 1675 (PATHS: 360, Triple P: 339, PATHS + Triple P: 306, control: 356)

Age (range or mean (SD)) or grade at the start: 7.45 years 
Gender: $52 \%$ male, $48 \%$ female

Ethnicity: not reported

SES: SES was based on coding the caregiver's current profession and was transformed into an International Socio-Economic Index of occupational status (ISEI) score. We took the highest ISEI score of the 2 caregivers across waves 1 and 5/6 (mean 45.83, SD 18.72)

Inclusion criteria: all year 1 primary school children in Zurich, Switzerland, in the academic year 2004-2005

Exclusion criteria: not reported

Interventions

Randomisation before or after baseline survey: not reported

Duration of the intervention (excluding follow-up): 12 months

Description of the intervention arm(s): (1) PATHS: 1-year programme that includes 46 lessons. Lessons addressed problem-solving skills, social relationships, self-regulation, rule understanding, emotion understanding, and positive self-esteem. Teachers received 2 days of training. Five coaches were trained and supervised. Coaches visited each class 4 to 6 times throughout the implementation period. Additional measures to support implementation included a refresher seminar held at midterm, a PATHS newsletter, and coach-led group meetings, where issues related to the programme could be discussed. (2) Triple P: Level 4 Triple P was implemented. Its core element was a group-based course with 4 units of 2 to 2.5 hours. Units addressed themes such as positive parenting, techniques to support desired behaviours, and routines to help avoid escalation of conflicts. Additionally, the programme included up to 4 follow-up telephone contacts with each participant. Courses were delivered by licenced facilitators selected in collaboration with Triple P Switzerland.

Brief description of the theoretical model: PATHS is a research-based programme that places particular importance on emotional learning processes. Triple $\mathrm{P}$ is based on cognitive-behavioural therapy.

Outcomes Primary: delinquency, substance use, antisocial behaviour

\begin{tabular}{ll}
\hline Setting & Country: Switzerland; Place: Zurich \\
& Setting: school (PATHS); community (Triple P) \\
& Focus: universal (PATHS); parent/family (Triple P)
\end{tabular}

Process measures Process data reported: yes

Method (qualitative or quantitative): both

Description

PATHS

Adherence to the intervention: data collected to monitor implementation included teacher and child questionnaires, as well as classroom observations. Indicators suggest high overall implementation quality. Coaches assessed a total of 308 PATHS lessons. Depending on the assessed aspect (e.g. child motivation, quality of classroom management, co-operation with coaches), between $74 \%$ and $81 \%$ of the lessons were rated as high quality.

Intensity of the intervention: teachers taught PATHS an average of 67 minutes per week, for an average of 2.4 sessions per week.

\section{Triple $\mathbf{P}$}

Acceptability of the intervention: participants' overall satisfaction with the programme was 4.33 (standard deviation $(S D)=0.89)$, and provider competency was rated at 4.65 (SD 0.73) on a 5-point scale. 
Reach of the intervention: parents of $27.0 \%$ of the children attended at least 1 session; parents of $2.2 \%$ of the children attended only 1 session; parents of $1.7 \%$ of the children attended only 2; parents of $4.3 \%$ of the children attended 3 ; and parents of $18.8 \%$ of the children completed all 4 sessions.

Adherence to the intervention: the implementation team organised 41 Triple P courses, of which 33 were held in German, 3 in Turkish, 2 each in Portuguese and Albanian, and 1 in English. Course providers estimated that $93 \%$ of the full course material was delivered during the sessions. About $60 \%$ of those who had attended the training reported using a significant part of the learned techniques 3 months after the intervention. Parents who completed the programme were more likely to come from breadwinner families, to be Swiss, to have high socioeconomic status (SES), and to be highly integrated into neighbourhood social networks. However, compliers did not differ from non-compliers with regards to parenting problems or child problem behaviours.

Statistics

Sample size: eligible $N=90$ schools; enrolled $N=56$ schools $(62 \%) ;$ PATHS = 14, Triple $P=14$, control = 14, PATHS + Triple $\mathrm{P}=14$

$\mathrm{N}=1675$ children: $\mathrm{PATHS}=360$, Triple $\mathrm{P}=339$, control $=356$, PATHS + Triple $\mathrm{P}=306$

Unit of analysis: individuals and schools

Method to promote equivalence between groups: blocking

Statistical models: multi-level model. Models incorporated 2 levels: youths (Level 1 ) and school at treatment (Level 2).

\section{Baseline differences adjustment: no}

Repeated measures methods in analysis: not reported

Funding: Swiss National Science Foundation, Jacobs Foundation, Swiss Federal Office of Public Health, Canton of Zurich Ministry of Education, Julius Baer Foundation

Randomisation method, e.g. block, stratification, computer: schools were first blocked by school size and socioeconomic background of the catchment area. Schools in disadvantaged school districts were slightly over-represented in the sample.

Clustering accounted for in sample size calculation (if relevant): not reported

Cluster randomisation methods to account for clustering in analysis: yes

\section{Risk of bias}

\begin{tabular}{lll}
\hline Bias & Authors' judgement & Support for judgement \\
\hline $\begin{array}{l}\text { Random sequence genera- } \\
\text { tion (selection bias) }\end{array}$ & Unclear risk & Not reported \\
\hline $\begin{array}{l}\text { Allocation concealment } \\
\text { (selection bias) }\end{array}$ & Unclear risk & Not reported \\
\hline $\begin{array}{l}\text { Blinding of participants } \\
\text { and personnel (perfor- } \\
\text { mance bias) } \\
\text { All outcomes }\end{array}$ & High risk & Participants not blinded \\
\hline $\begin{array}{l}\text { Blinding of outcome as- } \\
\text { sessment (detection bias) } \\
\text { All outcomes }\end{array}$ & High risk & \\
\hline
\end{tabular}


Averdijk 2016b (Continued)

Incomplete outcome data Low risk Low attrition; missing data were handled with robust full-information maxi(attrition bias) mum-likelihood estimation (FIML)

All outcomes

\begin{tabular}{|c|c|c|}
\hline $\begin{array}{l}\text { Selective reporting (re- } \\
\text { porting bias) }\end{array}$ & Unclear risk & $\begin{array}{l}\text { Teacher- and student-reported outcomes; also restricted dataset with strict in- } \\
\text { clusion criteria; no protocol }\end{array}$ \\
\hline
\end{tabular}

Other bias Low risk

Beach 2016

Methods

Study design: RCT at the level of the family

Intervention arm(s): ProSAAF

Comparator arm (s): control

Sample size calculation performed: no

Subgroups prespecified: N/A; no subgroups

Subgroup analyses: no

Start date: not reported

Duration of follow-up: 9 months

Number of follow-ups: 1

Follow-up time points: 6 months post intervention

ICC (if reported):

Participants

Number of schools randomised: N/A

Number of participants randomised (total and by arm): 139 (intervention: 70, control: 69)

Age (range or mean (SD)) or grade at the start: intervention: 10.89 years (0.87), control: 11.14 years (0.85)

Gender: intervention: 50\% male, 50\% female; control: $61 \%$ male, 39\% female

Ethnicity: 100\% African American

SES: men's median education level was high school or GED (ranging from less than grade 9 to a doctorate or professional degree); women's median education level was some college or trade school (ranging from less than grade 9 to a master's degree). Mean monthly income was USD1894 (range USD200 to USD5000) for men and USD1195 (range USD0 to USD6000) for women. Most men (79.6\%) and women $(61.0 \%)$ reported full- or part-time employment.

Inclusion criteria: families were required to self-identify as an African American couple with a child between the ages of 10 and 13. Couples had to be living together, partnered for 2 years or longer, and coparenting the target child together for at least 1 year. Both parents and the youth had to be willing to answer questions about their experiences inside and outside the family. Couples had to be willing to spend 6 weeks engaged in an in-home educational programme if they were randomly assigned to the intervention condition and to not be planning to move out of the study area during the intervention period. The target child also had to express willingness to participate in the individual and family portions of the session. 
Exclusion criteria: single parent household, family enrolled in another programme, child not within age limits, child not African American

Randomisation before or after baseline survey: after
Interventions
Duration of the intervention (excluding follow-up): 6 weeks
Description of the intervention: (1) ProSAAF: a trained African American facilitator visited each cou-
ple's home for 6 consecutive weeks and facilitated a 2-hour session with co-parenting adults and chil-
dren. The facilitator guided couples through video instruction and modelling, structured activities, and
specific topics for discussion. The first 60 minutes of each session focused on the couple's relationship.
The next 30 minutes of each session focused on parenting topics (e.g. school, peers, children's devel-
opment, discipline). The facilitator then met with the target child for a youth activity (e.g. self-esteem,
peer pressure, understanding parents) while the couple took a break in a different room. After the 15-
minute youth activity, the entire family came back together to meet with the facilitator for a 15-minute
family activity (e.g. discussions, games).

Brief description of the theoretical model: based on effective interventions and stress-spillover theory; psychoeducational framework

Description of the comparator: control: after baseline, couples were mailed the book, 12 Hours to a Great Marriage (Markman et al, 2004) and an accompanying workbook

Outcomes

Primary: parental monitoring, racial socialisation, positive self-concept, conduct problems, substance use initiation
Country: USA; State: Georgia

Setting: families (home)

Focus: African Ameican parent/family

Process measures Process data reported: yes

Method (qualitative or quantitative): both

\section{Description}

Reach of the intervention: families participated in 6 primary sessions (with 97\% participating in all 6). $91.5 \%$ of intervention families participated in a booster session.

Fidelity of the intervention: all sessions were audiotaped to allow monitoring of treatment implementation. A subsample of sessions was coded for adherence to intervention guidelines, with $20 \%$ coded by more than 1 rater on a scale of 0 to $100 \%$ adherence. All facilitators contributed to the sample of tapes to be rated. The intraclass correlation between raters was 0.94 . Mean fidelity adherence score across facilitators was $92.1 \%$ (SD 7.10).

Intensity of the intervention: a trained African American facilitator visited each couple's home for 6 consecutive weeks and facilitated a 2-hour session with co-parenting adults and children. One booster session was scheduled approximately 2 months after programme completion and approximately 2 months before post-test assessment was used to reinforce material covered during the main course of instruction.

Statistics

Sample size: eligible $N=483$ families; enrolled N = 206 families (43\%); I = 105 families; C = 101 families

Unit of analysis: individuals

Method to promote equivalence between groups: blocking

Statistical models: structural equation modelling

Baseline differences adjustment: ProSAAF couples were, on average, around 3 years older; analyses of intervention effects therefore controlled for couple's average age. 
Repeated measures methods in analysis: N/A; 1 follow-up only

Equity: data for parents on education, income, employment status

Funding: Grant R01 HD069439 awarded to Steven R. H. Beach and grant P30 DA027827 awarded to Gene H. Brody

Randomisation method, e.g. block, stratification, computer: block randomisation was performed by county of residence and marital status to ensure group equivalence.

Clustering accounted for in sample size calculation (if relevant): N/A

Cluster randomisation methods to account for clustering in analysis: N/A

\section{Risk of bias}

\begin{tabular}{|c|c|c|}
\hline Bias & Authors' judgement & Support for judgement \\
\hline $\begin{array}{l}\text { Random sequence genera- } \\
\text { tion (selection bias) }\end{array}$ & Unclear risk & Not reported \\
\hline $\begin{array}{l}\text { Allocation concealment } \\
\text { (selection bias) }\end{array}$ & Unclear risk & Not reported \\
\hline $\begin{array}{l}\text { Blinding of participants } \\
\text { and personnel (perfor- } \\
\text { mance bias) } \\
\text { All outcomes }\end{array}$ & High risk & Not reported but participants and personnel unlikely to be blinded. \\
\hline $\begin{array}{l}\text { Blinding of outcome as- } \\
\text { sessment (detection bias) } \\
\text { All outcomes }\end{array}$ & High risk & Not reported; self-reported outcomes only \\
\hline $\begin{array}{l}\text { Incomplete outcome data } \\
\text { (attrition bias) } \\
\text { All outcomes }\end{array}$ & High risk & $\begin{array}{l}\text { Attrition }>30 \% \text {; higher proportion of participants in intervention arm (9\% vs } \\
0 \%) \text { refused post-test assessment }\end{array}$ \\
\hline $\begin{array}{l}\text { Selective reporting (re- } \\
\text { porting bias) }\end{array}$ & Low risk & No protocol but all specified outcomes presented \\
\hline Other bias & Low risk & \\
\hline
\end{tabular}

\section{Beets 2009}

\section{Methods}

Study name: Positive Action (Hawaii)

Study design (e.g. RCT, cluster RCT): cluster RCT

Intervention $\operatorname{arm}(\mathbf{s})$ : Positive Action intervention

Comparator arm (s): standard education (as usual)

Sample size calculation performed: not reported

Subgroups prespecified: yes

Subgroup analyses: yes, by gender (boys vs girls)

Start date: $2001-2002$ 
Beets 2009 (Continued)

Duration of follow-up: immediately post intervention only

Number of follow-ups: 1

Follow-up time points: immediately post intervention, at the end of grade 5

ICC (if reported): student violent behaviours (0.06), substance use (0.05), and sexual activity (0.28)

Participants

Number of schools randomised: 20 overall (intervention: 10, control: 10)

Number of participants randomised (total and by arm): N/S

Age (range or mean (SD)) or grade at the start: N/S; range from 6 to 8 years at baseline

Gender: 50:50 among responders

Ethnicity: Hawaiian or part Hawaiian (26.1\%), multiple ethnic backgrounds (22.6\%), non-Hispanic White (8.6\%), African American (1.6\%), American Indian (1.7\%), other Pacific Islander (4.7\%), Japanese (4.6\%), other Asian (20.6\%), other (7.8\%), unknown (1.6\%)

SES: not reported

Inclusion criteria: schools meeting criteria (1) $25 \%$ of students received free or reduced-price lunch; (2) Hawaiian schools were in the lower 3 quartiles of SAT scores and (3) were located on Oahu, Maui, or Molokai; (4) public schools from kindergarten to fifth or sixth grade (i.e. not specialised schools); (5) annual school stability rate $>80 \%$

Exclusion criteria: students who left the study schools during the study period

Description of the intervention: programme is a school curriculum with school-wide, family, and community components designed to improve behaviour, character, and academic performance. It involves children in kindergarten to 12 th grade. The programme has a detailed curriculum with almost daily lessons and a school-wide climate programme undertaken by the principal and a Positive Action co-ordinator or committee, with family and community involvement components. Sequenced elementary school curriculum of 140 lessons per grade per academic year is delivered in 15- to 20-minute periods. During the academic year of 35 weeks, total programme time is approximately 35 hours. Teachers delivered the intervention; teachers, administrators, and support staff attended training at the start of the academic year. Booster sessions of 30 to 50 minutes were provided for each school at least once over the academic year. Content of lessons was grouped over 6 units: self-concept; mind and body positive action, social and emotional actions; getting along with others; being honest; and self-improvement. Involved structured discussions and activities with teachers and structured or semi-structured small group activities including games, role-play, and skill practice between students

Brief description of the theoretical model: theory of self-concept, consistent with theories of triadic influence (loosely stated)

Description of the comparator: students in the control group received their usual school curriculum

Outcomes

Primary outcomes: substance use for alcohol, drugs, tobacco; violent behaviours (carrying a knife or razor to cause harm, threatening to cut or stab someone, cut or stabbed someone, carried a gun, shot someone); sexual activity (voluntary heterosexual sex)

Secondary outcomes: teacher reports of substance use and violent behaviours

Setting

Country: USA; State: Hawaii (schools on 3 islands: Oahu, Maui, Molokai)

Setting: school

Focus: universal 
Beets 2009 (Continued)

Process measures
Process data reported: not reported

Method (qualitative or quantitative): N/A

Description: N/A

Statistics

Sample size: $N=1993 ; 86 \%(n=1714)$ of children responded to the questionnaire at fifth grade; teachers reported on 1225 children. Intervention: 10 schools ( $=976$ in analysis); control: 10 schools ( $n=738$ in analysis)

Unit of randomisation: school

Unit of analysis: individual

Method to promote equivalence between groups:

Stratification: eligible schools were stratified based on year 2000 school report card data by an index including percentage of students receiving free or reduced-price school lunch, school size, \% student stability, and student ethnic distribution. Additional characteristics of the school (i.e. student-teacher ratios and expenditure/student) and characteristics of student populations (i.e. proportion of gifted, special education, and English as a second language students) were also used, as were indicators of student behavioural and school performance outcomes (i.e. disciplinary referrals, suspension rates, and standardised achievement scores)

Statistical models: 2-level over-dispersion random-effects Poisson models; generalised linear latent and mixed models

Baseline differences adjustment: no significant differences on the index tests or teacher reports of negative student behaviour between intervention and control groups

Repeated measures methods in analysis: N/A; only baseline and 1 follow-up reported

Notes

Equity: descriptive data at follow-up: ethnicity primarily Hawaiian or part Hawaiian (26.1\%), multiple ethnic backgrounds (22.6\%), non-Hispanic White (8.6\%), African American (1.6\%), American Indian (1.7\%), other Pacific Islander (4.7\%), Japanese (4.6\%), other Asian (20.6\%), other (7.8\%), and unknown $(1.6 \%)$

Funding National Institute on Drug Abuse (grants DA13474 and DA018760)

Randomisation method, e.g. block, stratification, computer: stratification of the 111 schools was based on an index, resulting in 19 strata with at least 3 similar schools in each; 1 intervention and 1 control school were then randomly assigned in each stratum

Clustering accounted for in sample size calculation (if relevant): not reported

Cluster randomisation methods to account for clustering in analysis: 2-level random-effects models (binary and Poisson) were implemented to account for the heirarchical nature of the study design and the clustering

\section{Risk of bias}

\begin{tabular}{lll}
\hline Bias & Authors' judgement & Support for judgement \\
\hline $\begin{array}{l}\text { Random sequence genera- } \\
\text { tion (selection bias) }\end{array}$ & Unclear risk & Not reported \\
\hline $\begin{array}{l}\text { Allocation concealment } \\
\text { (selection bias) }\end{array}$ & Unclear risk & Not reported \\
\hline $\begin{array}{l}\text { Blinding of participants } \\
\text { and personnel (perfor- } \\
\text { mance bias) }\end{array}$ & High risk & $\begin{array}{l}\text { Participants, children, teachers, and project staff not blinded to intervention } \\
\text { allocation and receipt }\end{array}$ \\
\hline
\end{tabular}


Beets 2009 (Continued)

All outcomes

Blinding of outcome assessment (detection bias)

All outcomes
High risk

Self-reported outcome measures

Insufficient information provided

Incomplete outcome data Unclear risk $\quad$ Insufficient information provided
(attrition bias)

All outcomes

\begin{tabular}{lll}
\hline $\begin{array}{l}\text { Selective reporting (re- } \\
\text { porting bias) }\end{array}$ & Unclear risk & No published protocol \\
\hline Other bias & Unclear risk & Impact of addition of new students and loss of students from schools unclear \\
\hline
\end{tabular}

\title{
Bernstein 2010a
}

Methods

\author{
Study name: Reaching Adolescents for Prevention \\ Study design: RCT
}

Intervention arm(s): Brief Motivational interview (I)

Comparator arm (s): 2 control arms: standard assessed control (AC), minimally assessed control (MAC)

Sample size calculation performed: yes

Subgroups prespecified: yes, with the exception of post-traumatic stress disorder checklist civilian version $(P C L-C)$ score

Subgroup analyses: age groups: 14 to 17 and 18 to 21 years; high-risk and low-risk AUDIT score, PCL-C negative or positive

Start date: April 2004

Duration of follow-up: 12 months

Number of follow-ups: 2

Follow-up time points: 3 months for I and AC groups, 12 months post baseline for I, AC, and MAC groups

ICC (if reported): N/A

\section{Participants}

Number of schools randomised: N/A

Number of participants randomised (total and by arm): $\mathrm{N}=1202$ eligible; $\mathrm{N}=853$ enrolled $(71 \%)$; intervention $(\mathrm{I})=283 / 853$, assessment control $(A C)=284 / 853$, minimal assessment control $(M A C)=$ $286 / 853$

Age (range or mean (SD)) or grade at the start: 14 to 21 years; $13 \% \leq 17$ years; $87 \% \geq 18$ years. $21 \%$ were in high school; $22 \%$ had dropped out of high school; $57 \%$ had graduated

Gender: $55 \%$ female, $45 \%$ male

Ethnicity: hospital population: American Indian/Alaskan Native $=2 \%$; Asian $=1.4 \%$; Black $/$ African American $=51.3 \%$; Hispanic $/$ Latino $=19.2 \%$; Native Hawaiian $/$ Pacific Islander $=0.5 \%$; White $=25.6 \%$

SES: not reported 
Inclusion criteria: patients were invited during screening if reported binge drinking (i.e. 5 or more drinks in 2 hours for males, 4 or more drinks in 2 hours for females) and/or high-risk behaviours in conjunction with alcohol use (i.e. unplanned or unprotected sex, driving or riding with a drunk driver, injury, fighting, car crash, or arrest); or an AUDIT score of $\geq 4$ for those aged 14 to 17 , or $\geq 8$ for those aged 18 to 21. Patients had to be able to communicate in English, Spanish, Haitian, Creole, or Cape Verdan Creole and were alert and oriented.

Exclusion criteria: could not be interviewed in privacy from accompanying family members, planned to leave the area in the next 3 months, could not provide reliable contact information for follow-up procedures, were currently in a residential substance use treatment facility, were in custody or were institutionalised, presented for a rape exam or a psychiatric evaluation for suicide precautions, parents opted out for patients $<18$ years of age

Interventions

Randomisation before or after baseline survey: after screening for eligibility

Duration of the intervention (excluding follow-up): $<3$ months

Description of the intervention: Reaching Adolescents for Prevention (RAP): the intervention group received a peer-conducted motivational intervention of 20 to 30 minutes of structured conversation, referral to community resources and drug treatment services, and a booster telephone call at 10 days post enrolment, in addition to assessment. Standard assessment involved a battery of instruments described in Data Collection. Components of the intervention included (1) engagement and permission to raise the subject, (2) establishing context, (3) brief feedback, information, and norms; exploration of the pros and cons of consuming mind-altering substances; reinforcing movement towards behaviour change, (4) generating a menu of options, (5) calling up assets and instilling hope, (6) discussing challenges of change, and (7) generating a prescription for change

\section{Brief description of the theoretical model: N/S}

Description of the comparator: AC group received the battery of standard assessment instruments, a brief alcohol use handout, and appointments for reassessment at 3 and 12 months; MAC group received a brief handout of advice about the risks associated with alcohol use, a list of community resources and adolescent treatment facilities, and a follow-up appointment at 1 year.

Outcomes

Primary outcomes: abstinence at 12 months (abstinence defined as zero alcohol consumption in the last 30 days), change in alcohol use (use defined by number of drinking days, number of drinks/typical day, number of binge episodes/month; binge defined as $>5$ drinks/ occasion for males and $>4$ drinks/ occasion for females), intention to quit using, cutting back on use or changing circumstances of use, alcohol-related injury, driving under the influence, fighting, unprotected or unplanned sex. accepting a ride from a drinking driver

Secondary outcomes: none

\begin{tabular}{ll}
\hline Setting & Country: USA;State: Boston, Massachusetts \\
Setting: accident and emergency (secondary care) & Focus: targeted \\
\hline Process measures & Process data reported: yes \\
Method (qualitative or quantitative): quantitative & $\begin{array}{l}\text { Description: adherence to intervention: adherence to the intervention algorithm was assessed weekly } \\
\text { by investigators and the project co-ordinator; interventions were taped when permission was granted } \\
\text { in a separate consent process. Randomly selected intervention tapes were discussed weekly by multi- } \\
\text { ple raters in a consensus process, using a checklist of key elements of the intervention }\end{array}$
\end{tabular}

Statistics

Sample size: $\mathrm{N}=1202$; eligible: $\mathrm{N}=853$ enrolled $(71 \%)$, intervention $(\mathrm{I})=283 / 853$, assessment control $(A C)=284 / 853$, minimal assessment control $(M A C)=286 / 853$

Unit of randomisation: individual 
Unit of analysis: individual

Method to promote equivalence between groups: stratification by age groups 14 to 17 and 18 to 21 years, blocking to balance after every 9 participants

Statistical models: categorical data: generalised estimating equation (GEE) logistical regression for adjusted ORs; continuous data: mixed effect linear regression models for adjusted least squares means at baseline, 3 months, and 12 months

Baseline differences adjustment: all baseline measures were adjusted for

Repeated measures methods in analysis: used regression models: interaction $\mathrm{P}$ value tests whether the difference in adjusted means between intervention groups changed from 3 to 12 months; main effects $P$ value tests whether there was a difference between groups across the 2 time points convictions; no significant differences at baseline between the 3 randomised groups for age, sex, race, or primary language

Funding: NIAAA Youth Alcohol Prevention Center, Boston University, 2006-2009

Randomisation method, e.g. block, stratification, computer: computer-generated lists, blocked to balance assignment after every 9 participants and stratified by age group (14 to 17 years and 18 to 21 years). Randomisation was performed in 2 stages: the first envelope was opened after enrolment was assigned to the participant to either MAC or assessed status (I or C). If the card stated "assessment", the participant completed the standard battery of questionnaires. A second sealed envelope, inside the original envelope, was opened after assessment to allocate the participant to AC or I

Clustering accounted for in sample size calculation (if relevant): N/A

Cluster randomisation methods to account for clustering in analysis: N/A

\begin{tabular}{|c|c|c|}
\hline \multicolumn{3}{|l|}{ Risk of bias } \\
\hline Bias & Authors' judgement & Support for judgement \\
\hline $\begin{array}{l}\text { Random sequence genera- } \\
\text { tion (selection bias) }\end{array}$ & Low risk & Computer-generated lists used \\
\hline $\begin{array}{l}\text { Allocation concealment } \\
\text { (selection bias) }\end{array}$ & Low risk & Two-stage assignment using a double opaque envelope system \\
\hline $\begin{array}{l}\text { Blinding of participants } \\
\text { and personnel (perfor- } \\
\text { mance bias) } \\
\text { All outcomes }\end{array}$ & High risk & $\begin{array}{l}\text { Personnel delivering intervention components and participants were not } \\
\text { blinded. }\end{array}$ \\
\hline $\begin{array}{l}\text { Blinding of outcome as- } \\
\text { sessment (detection bias) } \\
\text { All outcomes }\end{array}$ & High risk & $\begin{array}{l}\text { Research assistants conducting baseline and follow-up assessments were } \\
\text { blinded; self-reported outcome measures were used. }\end{array}$ \\
\hline $\begin{array}{l}\text { Incomplete outcome data } \\
\text { (attrition bias) } \\
\text { All outcomes }\end{array}$ & High risk & $\begin{array}{l}\text { High attrition: } 28 \% \text { lost to follow-up at end of intervention; may differ between } \\
\text { arms }\end{array}$ \\
\hline $\begin{array}{l}\text { Selective reporting (re- } \\
\text { porting bias) }\end{array}$ & Unclear risk & No published protocol \\
\hline Other bias & Low risk & None identified \\
\hline
\end{tabular}




\section{Study design: RCT}

Intervention $\operatorname{arm}(\mathbf{s})$ : residential programme + mentoring

Comparator arm (s): no CfC

Sample size calculation performed: not reported

Subgroups prespecified: none

Subgroup analyses: none

Start date: not reported

Duration of follow-up: immediately post intervention

Number of follow-ups: 3

Follow-up time points: 2 throughout intervention period and 1 immediately post intervention

ICC (if reported): N/A

\section{Participants}

\section{Number of schools randomised: N/A}

Number of participants randomised (total and by arm): 63 overall (intervention: 32, control: 31)

Age (range or mean (SD)) or grade at the start: 15 to 18 years

Gender: not reported

Ethnicity: not reported

SES: not reported

Inclusion criteria: age mid-late teens, antisocial behaviour in more than 1 area (e.g. bullying), exclusion from school, behaviour problems at home, crime in the local community, $\geq 1$ of 5 key risk factors from the logic model

Exclusion criteria: not reported

Randomisation before or after baseline survey: not clear; possibly after baseline survey, as only 63 of the 331 eligible were randomised

Duration of the intervention (excluding follow-up): 1-week residential programme + 9 months (mentor and young person)

Description of the intervention: Coaching for Community ( $\mathrm{CfC})$. The programme has 2 components: the first component is a 5-day residential retreat of course-room exercises, physical activity, and goal setting; the second component involves 9 months of support from an adult mentor (once/month meeting with a 'committed partner' or mentor) with the aim of helping the person achieve goals set during the residential component. At this meeting, programme staff and volunteers work with young people on a themed goal. Themes are chosen and developed by local CfC organisers and include relationship building; personal aspirations; drug awareness; sexual health; community awareness and team working; car crime; driving education; vocational skills; and self-expression.

$\mathrm{CfC}$ has a strong focus on community involvement; both local programme staff and volunteers are from an area near to where the target young people live.

The 'committed partners' attend a shorter version of the same residential programme; each partner is required to make contact with an assigned young person directly or indirectly at least 3 times/week. 
Brief description of the theoretical model: residential coursework is based on 'distinction-based learning'. A structured series of topics are explored by the course leader and participants: relationship to rules; the meaning of giving and keeping one's word; the role of the coach; learning from what one already knows; the hold of the past over the present; distinguishing 'fact' from 'interpretation'; creating a breakthrough; handling breakdowns; and the meaning of responsibility. The objective is to help participants see what is possible for them.

Description of the comparator: receipt of usual services delivered by youth services team within the local authority (but not Coaching for Communities intervention)

List relevant outcomes
Primary outcomes: from logic model: reduced offending; reduced drug alcohol, substance misuse; in-
creased or renewed involvement in education, training, or employment
Secondary outcomes: residential and mentoring would improve self-esteem; reduce impulsivity; in-
crease aspirations; improve positive outlook; reduce need for antisocial peer networks; reduce negativ-
ity

Country: England; State: N/S

Setting: individual (community)

Focus: targeted: young people with antisocial behaviour referred from children's services (e.g. social services, education, youth welfare, justice services) tion

Method (qualitative or quantitative): quantitative

Description: an index assessing the quality of volunteers was developed to examine whether differences in experience, qualifications, or personal characteristics (e.g. persistence) impacted young people's responses to the programme. Analysis showed that the 'quality' of the volunteer did not have a significant impact on the response. Registers and records of all meetings and contacts were kept to measure a dose-response with CfC. Analysis showed that the number of times a young person had weekly contact with his/her volunteer (mentor) had no significant impact on outcomes measured. However, the number of monthly meetings attended by a young person was significantly related to increased improvements $(P<0.05)$; this led study authors to suggest a dose-response for the trainer-led component (programme staff and mentor) of the programme.

Acceptability of the intervention: the large number of dropouts after enrolment could suggest that this type of intervention was not popular among youths.

Adherence to the intervention: few enrolled participants completed the full programme, suggesting low adherence.

Intensity of the intervention: continuous over 9 months

Unit of randomisation: individual

Unit of analysis: individual

Method to promote equivalence between groups: not reported

Statistical models: generalised linear model using baseline (T1) as a covariate or fixed factor

Baseline differences adjustment: data were analysed using GLM, taking the baseline (T1) into account as a covariate or fixed factor. No further details given 
Berry 2009 (Continued)

Repeated measures methods in analysis: not reported

Notes

Equity: not reported

Funding: youth at risk voluntary organisation. Seems to be a pilot study; most resources were spent on developing the programme, and only 1 trial was conducted out of 5 that were proposed. Study authors acknowledge attrition and suggest several more trials of the intervention need to be conducted.

Randomisation method, e.g. block, stratification, computer: programme in Microsoft Excel

Clustering accounted for in sample size calculation (if relevant): N/A

Cluster randomisation methods to account for clustering in analysis: N/A

\section{Risk of bias}

\begin{tabular}{|c|c|c|}
\hline Bias & Authors' judgement & Support for judgement \\
\hline $\begin{array}{l}\text { Random sequence genera- } \\
\text { tion (selection bias) }\end{array}$ & Unclear risk & Excel programme used; no description provided \\
\hline $\begin{array}{l}\text { Allocation concealment } \\
\text { (selection bias) }\end{array}$ & Unclear risk & Not described \\
\hline $\begin{array}{l}\text { Blinding of participants } \\
\text { and personnel (perfor- } \\
\text { mance bias) } \\
\text { All outcomes }\end{array}$ & High risk & $\begin{array}{l}\text { Participants were informed that they were candidates for the programme; ran- } \\
\text { dom allocation was performed in the presence of programme providers. }\end{array}$ \\
\hline $\begin{array}{l}\text { Blinding of outcome as- } \\
\text { sessment (detection bias) } \\
\text { All outcomes }\end{array}$ & High risk & $\begin{array}{l}\text { Participants completed self-report questionnaires; some data were collected } \\
\text { from parents/guardians by self-report and interview. }\end{array}$ \\
\hline $\begin{array}{l}\text { Incomplete outcome data } \\
\text { (attrition bias) } \\
\text { All outcomes }\end{array}$ & High risk & $\begin{array}{l}\text { Attrition } 37 \% \text { in intervention group and } 29 \% \text { in control group; large quantity of } \\
\text { missing data and imbalance between the } 2 \text { groups }\end{array}$ \\
\hline $\begin{array}{l}\text { Selective reporting (re- } \\
\text { porting bias) }\end{array}$ & Low risk & $\begin{array}{l}\text { No published protocol; all specified outcomes in logic model reported at the } \\
\text { start (T1) and end (T5) of the programme }\end{array}$ \\
\hline Other bias & High risk & $\begin{array}{l}\text { Significant baseline differences between intervention and control groups for } \\
\text { key outcomes at Time 1: antisocial peers and volume of offending; data for } \\
\text { time points } 2 \text { to } 4 \text { not reported }\end{array}$ \\
\hline
\end{tabular}

Bodin 2011

Methods

Study name: no study name given

Study design: RCT at the level of the individual

Intervention $\operatorname{arm}(\mathbf{s})$ : mentoring

Comparator arm (s): no intervention. Every second month during the follow-up period, participants were contacted by research staff for a short telephone call and were asked about contacts with nonparental adults (frequency and perceived quality). For this, they received an honorarium every 4 months.

\section{Sample size calculation performed: yes}


Subgroups prespecified: N/A; no subgroups

Subgroup analyses: no

Start date: October 2007 to April 2008

Duration of follow-up: 12 months

Number of follow-ups: 1

Follow-up time points: 12 months

ICC (if reported): N/A

\title{
Participants
}

\section{Number of schools randomised: N/A}

Number of participants randomised (total and by arm): 128 recruited: 65 to intervention, 63 to control

Age (range or mean (SD)) or grade at the start: 14 years

Gender: $61.5 \%$ girls in intervention group; $57.1 \%$ girls in control group

Ethnicity: $24.6 \%$ parents foreign-born in intervention group; $33.3 \%$ parents foreign-born in control group

SES: not reported

Inclusion criteria: age 14 years, attending a programme school; students with self-reported need for additional adult contacts

Exclusion criteria: current or past regular use of illicit drugs, repeated delinquent behaviour or single acts of violence against persons, ongoing contacts with psychiatric or social services

\begin{abstract}
Interventions
Randomisation before or after baseline survey: after

Duration of the intervention (excluding follow-up): 1 year

Description of the intervention: mentoring, in which mentor and mentee meet at least every second week for 2 to 4 hours on every occasion over the course of 1 year (minimum of 2 school semesters). Every pair is given 2000 SEK (180GBP/280USD) to spend during meetings, which take place outside of school and work hours; pairs choose activities of their own preferences. The programme aims to establish a safe and supportive relationship that benefits the young person in terms of social, emotional, and academic development, making the young person less likely to use alcohol and drugs.
\end{abstract}

Brief description of the theoretical model: no theory given, but study authors state that in line with a model proposed by Rhodes (2005), the intervention works on the assumption that healthy relationships with adult role models promote positive development and prevent problematic behaviours among young people

Description of the comparator: no intervention

Outcomes

\section{List relevant outcomes}

Primary outcome: substance use

Secondary outcomes: delinquency, mental health, quality of life, behavioural problems, social competence, grade point average

Setting

Country: Sweden;State: N/A

Setting: community

Focus: targeted 
Bodin 2011 (Continued)

Process measures

Process data reported: yes

Method (qualitative or quantitative): quantitative

\section{Description}

Acceptability of the intervention: half of the assigned youth (50.8\%) had met with their mentor during the stipulated period, with 11.7 meetings on average (SD 4.1). Among those not completing the intervention, the average number of meetings was 4.5 (SD 4.0). Completers were more likely to have positive views around the intervention, e.g. feeling good when with their mentors, trusting their mentors.

Adherence to the intervention: 2 of 65 in the intervention group dropped out; 27 are recorded as having discontinued the intervention

Intensity of the intervention: mentor and mentee met at least every second week for 2 to 4 hours over a period of at least 2 school semesters

\section{Statistics}

Sample size: 128 recruited: 65 to intervention, 63 to control

Unit of randomisation: individual

Unit of analysis: individual

Method to promote equivalence between groups: stratification by school

Statistical models: logistical regression model

Baseline differences adjustment: yes

Repeated measures methods in analysis: N/A

Notes Equity: outlined above

Funding: Office of the National Drug Policy Coordinator, Institute for Evidence-based Social Work Practice at the National Board of Health and Welfare, Centre for Dependency Disorders, Stockholm County Council

Randomisation method, e.g. block, stratification, computer: blocks of 2; coin toss

Clustering accounted for in sample size calculation (if relevant): N/A

Cluster randomisation methods to account for clustering in analysis: N/A

\section{Risk of bias}

\begin{tabular}{lll}
\hline Bias & Authors' judgement & Support for judgement \\
\hline $\begin{array}{l}\text { Random sequence genera- } \\
\text { tion (selection bias) }\end{array}$ & Low risk & Used coin toss \\
\hline $\begin{array}{l}\text { Allocation concealment } \\
\text { (selection bias) }\end{array}$ & Low risk & Used sealed envelopes \\
\hline $\begin{array}{l}\text { Blinding of participants } \\
\begin{array}{l}\text { and personnel (perfor- } \\
\text { mance bias) } \\
\text { All outcomes }\end{array}\end{array}$ & High risk & Not possible to blind participants with an intervention of this nature \\
\hline
\end{tabular}

$\begin{array}{lll}\text { Blinding of outcome as- } & \text { Unclear risk } & \text { No information provided } \\ \text { sessment (detection bias) } & \end{array}$

All outcomes 
Bodin 2011 (Continued)

$\begin{array}{ll}\begin{array}{l}\text { Incomplete outcome data } \\ \text { (attrition bias) }\end{array} & \text { Low risk } \\ \text { Allowing data managed by carrying forward pre-test score, but overall attrition } \\ \text { very low (3.1\%) }\end{array}$
(attrition bias) very low (3.1\%)

All outcomes

Selective reporting (re- Unclear risk No protocol published; primary and secondary outcomes not clear porting bias)

Other bias Low risk No other sources of bias identified

Bond 2004

Methods

Study name: The Gatehouse Project

Study design: cluster RCT

Intervention $\operatorname{arm}(\mathbf{s})$ : whole-school intervention

Comparator arm (s): N/S

Sample size calculation performed: yes

Subgroups prespecified: none

Subgroup analyses: NA

Start date: 1997

Duration of follow-up: 4 years

Number of follow-ups: 2

Follow-up time points: end of school years 1997, 1998, 1999, 2001 (end of school year, 1 year, 2 years, 4 years)

ICC (if reported): 0.01 to 0.06

Participants

Number of schools randomised: 26

Number of participants randomised (total and by arm): 12 (intervention), 14 (control)

Age (range or mean (SD)) or grade at the start: 13 to 14 years

Gender: $52 \%$ female (intervention), 54\% female (control)

Ethnicity: non-Australian born: 16\% intervention, 9\% control. Student with first language other than English: 24\% intervention, 22\% control

SES: N/S

Inclusion criteria: N/S

Exclusion criteria: N/S

Interventions

Randomisation before or after baseline survey: before

Duration of the intervention (excluding follow-up): 24 months

Description of the intervention: The Gatehouse Project was a school-based primary prevention programme, which included both institutional and individual focused components to promote the emotional and behavioural well-being of young people in secondary schools. Components of the intervention included establishment and support of an adolescent health team, identification of risk and pro- 
Bond 2004 (Continued)

tective factors in the social and learning environment from student surveys, and use of these data for identification of effective strategies to address these issues. A curriculum component was also implemented.

Brief description of the theoretical model: health promoting schools framework, ecological approach

Description of the comparator: N/S

Outcomes Primary outcomes: depression, alcohol use, tobacco use, marijuana use, bullying

Secondary outcomes: poor availability of attachments, arguments with 3 or more people

\begin{tabular}{ll}
\hline Setting & Country: Australia; State: Victoria \\
& Setting: secondary schools \\
& Focus: universal \\
\hline Process measures & Process data reported: yes \\
Method (qualitative or quantitative): both & Description: field notes, informant interviews, school audit \\
\hline Sample size: 1335 intervention, 1342 control \\
Unit of randomisation: school \\
Unit of analysis: school \\
Method to promote equivalence between groups: stratification \\
Statistical models: ordinal logit models \\
Baseline differences adjustment: N/S \\
Repeated measures methods in analysis: no \\
\hline
\end{tabular}

Notes Equity: baseline information on gender, ethnicity, parental separation, non-Australian born

Funding: grants from the Queen's Trust for Young Australians, Victorian Health Promotion Foundation, National Health and Medical Research Council and Department of Human Services, Victoria, Murdoch Children's Research Institute, Sydney Myer Fund, Catholic Education Office

Randomisation method, e.g. block, stratification, computer: stratification by school administration

Clustering accounted for in sample size calculation (if relevant): yes

Cluster randomisation methods to account for clustering in analysis: yes

\section{Risk of bias}

\begin{tabular}{lll}
\hline Bias & Authors' judgement & Support for judgement \\
\hline $\begin{array}{l}\text { Random sequence genera- } \\
\text { tion (selection bias) }\end{array}$ & Unclear risk & No details provided \\
\hline $\begin{array}{l}\text { Allocation concealment } \\
\text { (selection bias) }\end{array}$ & Unclear risk & No details provided \\
\hline
\end{tabular}


Bond 2004 (Continued)

Blinding of participants Unclear risk Not blinded but intervention was integrated with usual curriculum and adminand personnel (perforistrative processes mance bias)

All outcomes

\begin{tabular}{lll}
\hline Blinding of outcome as- & Unclear risk & No details provided \\
sessment (detection bias) &
\end{tabular}

All outcomes

Incomplete outcome data Low risk (attrition bias)

Low levels of attrition and non-differential rates between study arms

All outcomes

\begin{tabular}{lll}
\hline $\begin{array}{l}\text { Selective reporting (re- } \\
\text { porting bias) }\end{array}$ & Unclear risk & No protocol available \\
\hline Other bias & Unclear risk & $\begin{array}{l}\text { Proportion responding to baseline survey differed between study arms (81\% } \\
\text { from intervention schools, } 68 \% \text { from comparison schools). }\end{array}$
\end{tabular}

Bonds 2010

Methods

Study name: New Beginnings

\section{Study design: RCT}

Intervention $\operatorname{arm}(\mathbf{s})$ : 2: mother only programme, mother plus child programme

Comparator arm (s): literature control condition

Sample size calculation performed: yes

Subgroups prespecified: N/A; no subgroup analyses carried out

Subgroup analyses: none

Start date: 1 March 1992

Duration of follow-up: 15 years post intervention

Number of follow-ups: 4

Follow-up time points: 3 months, 6 months, 6 years, and 15 years post intervention

ICC (if reported): N/A

\section{Participants}

\section{Number of schools randomised: N/A}

\section{Number of participants randomised (total and by arm): N/A}

Age (range or mean (SD)) or grade at the start: child mean age 10.4 years (SD 1.1) at baseline

Gender: parents were all mothers (female); $49 \%$ of children were female

Ethnicity: mother: Caucasian (88\%), non-Hispanic (8\%), Hispanic (2\%), African American (2\%), Asian/ Pacific Islander (1\%), and other (1\%)

SES: $98 \%$ of mothers had a minimum of high school education.

Inclusion criteria: parents must have divorced in last 24 months, must have 1 child aged 9 to 12 years, mother is the primary resident parent, mother is not remarried or living with another partner (both at 
Exclusion criteria: children who scored $>17$ on the Children's Depression Inventory, endorsed an item about suicidal ideation, or were at $>97$ th percentile on the externalising subscale of the Child Behaviour Checklist

Description of the intervention: the mother only programme targeted mother-child relationship quality ( 6 sessions), effective discipline ( 3 sessions), interparental conflict, and father's access to the child. A total of 11 sessions were delivered, each lasting 1.75 hours. Two individual sessions were also delivered, each of which lasted for 1 hour. In the mother plus child programme, the aforementioned mother intervention was delivered, along with a separate child programme. The child programme also consisted of 11 sessions ( 1.75 hours' duration) and focused on increasing effective coping, reducing negative thoughts about divorce-related stressors, and improving mother-child relationship quality. Recognition of feelings, deep breathing techniques, problem identification and resolution, positive cognitive reframing, and challenging common negative appraisals were all reinforced. Information was delivered via presentations, videos, role-plays, and modelling of behaviours. Communication skills were reinforced via child-mother communicative role-play sessions. In the literature control condition, both mother and child were sent 3 books on divorce adjustment and a syllabus to guide reading over a 6-week period.

Brief description of the theoretical model: a combination of elements from the person-environment transactional framework and a risk and protective factor model. A model by Cummings, Davies, and Campbell (2000) integrates these 2 models; it is called the cascading pathway model

Description of the comparator: standard literature control; participants given reading materials

Outcomes Primary outcomes: symptoms of externalising and internalising disorders, substance use, risky sexual behaviours, diagnosed mental disorders

Secondary outcomes: N/S

Setting

Country: USA; State: Phoenix

Setting: family

Focus: targeted; recently divorced parents (within last 2 years) and their child (aged 9 to 12 years)

\section{Process measures Process data reported: yes}

Method (qualitative or quantitative): quantitative

\section{Description}

Adherence to the intervention: audiotapes of interviews were reviewed; an experienced interviewer intermittently conducted live observations of interviews and provided real-time feedback. Detailed manuals of required intervention delivery were distributed to programme facilitators, and extensive training and supervision were provided throughout. Both mother and child group leaders were required to score at or above $89 \%$ on quizzes about the intervention. Independent raters scored each delivered session between 1 and 3, with 1 meaning not complete and 3 meaning complete. lies 
Statistical models: full information maximum likelihood methods

Baseline differences adjustment: N/S

Repeated measures methods in analysis: no

$\begin{array}{ll}\text { Notes } & \text { Equity: N/S } \\ & \text { Funding: National Institute of Mental Health grants (1R01MH071707-01A2, 5P30MH068685, }\end{array}$

5T32MH018387) supported the study.

Randomisation method: computer software programme

Clustering accounted for in sample size calculation (if relevant): no

Cluster randomisation methods to account for clustering in analysis: N/A

\begin{tabular}{lll}
\hline Risk of bias & & \\
\hline Bias & Authors' judgement & Support for judgement \\
\hline $\begin{array}{l}\text { Random sequence genera- } \\
\text { tion (selection bias) }\end{array}$ & Low risk & Randomisation software used \\
\hline $\begin{array}{l}\text { Allocation concealment } \\
\text { (selection bias) }\end{array}$ & Unclear risk & Insufficient information provided \\
\hline $\begin{array}{l}\text { Blinding of participants } \\
\text { and personnel (perfor- } \\
\text { mance bias) }\end{array}$ & High risk & Blinding unlikely to have been successful, given the nature of the intervention \\
All outcomes & & \\
\hline
\end{tabular}

\begin{tabular}{|c|c|c|}
\hline $\begin{array}{l}\text { Blinding of outcome as- } \\
\text { sessment (detection bias) } \\
\text { All outcomes }\end{array}$ & High risk & Self-reported outcome measures \\
\hline
\end{tabular}

\begin{tabular}{|c|c|c|}
\hline $\begin{array}{l}\text { Incomplete outcome data } \\
\text { (attrition bias) } \\
\text { All outcomes }\end{array}$ & Low risk & $\begin{array}{l}\text { Relatively low attrition rates at all follow-up time points; full maximum likeli- } \\
\text { hood statistical methods accounted for missing data }\end{array}$ \\
\hline
\end{tabular}

\begin{tabular}{lll}
\hline $\begin{array}{l}\text { Selective reporting (re- } \\
\text { porting bias) }\end{array}$ & Unclear risk & No published protocol \\
\hline Other bias & Low risk & No other forms of bias evident \\
\hline
\end{tabular}

Brody 2012

\section{Study design: RCT}

Intervention arm: school-, classroom-, and family-based nutrition, physical activity, and smoking prevention intervention

Comparator arm: control (standard curriculum)

Sample size calculation performed: yes

Subgroups prespecified: none reported 
Brody 2012 (Continued)

\title{
Subgroup analyses: no
}

Start date: not given

Duration of follow-up: 22 months

Number of follow-ups: 2

Follow-up time points: 5 months; 22 months

Intracluster correlation coefficient: N/A

\begin{abstract}
Participants
Number of schools randomised: N/A

Number of participants randomised: 502 total: 252 intervention, 250 control

Age (range or mean (SD)) or grade at the start: 16 years

Gender: $51 \%$ female

Ethnicity: the study involved African American families only

Socioeconomic status: mean household monthly gross income was $\$ 1482.50 .63 .8 \%$ of families lived below federal poverty standards, and $18 \%$ lived within $150 \%$ of the poverty threshold

Inclusion criteria: youth aged 15 or 16 years at pre-test; self-identification as African American

Exclusion criteria: not given

Interventions

Randomisation before or after baseline survey: after
Duration of the intervention (excluding follow-up): 3 to 6 months

Description of the intervention: 5 consecutive meetings held in the community with separate caregiver and adolescent skill-building curricula and a family curriculum. Each meeting included separate 1hour concurrent training sessions for caregivers and adolescents, followed by a 1-hour session in which families practised the skills they had learnt in the separate sessions.
\end{abstract}

Brief description of the theoretical model: no details given

Description of the comparator: attention control named 'Fuel for Families', which included 5 sessions on nutrition, exercise, and informed consumer behaviour

Outcomes Primary outcomes: preventing increases in conduct problems, substance use, substance use problems, depressive symptoms among rural African American adolescents

Secondary outcomes: healthful behaviours: how often during the past 7 days participants exercised and consumed fruit, vegetables, $100 \%$ fruit juices, milk, and carbonated beverages

Setting

Country: USA; Place: Georgia

Setting: community facilities

Focus: targeted to African American families in rural Georgia

Process measures Process data reported: yes

Method (qualitative or quantitative): quantitative

Description: prevention supervisors reviewed videos of each week's session and provided feedback to group leaders. For each group, 2 parents, 2 youths, and 2 family sessions were selected randomly and scored for adherence to and coverage of the prevention curriculum. Coverage of curriculum components exceeded $80 \%$ for both SAAF-T and 'Fuel for Families' sessions. 
Brody 2012 (Continued)

Sample size: 502 families

Unit of randomisation: family

Unit of analyses: individual

Methods to promote equivalence between groups: not given

Statistical models: a zero-inflated Poisson regression model was used to test the study regression hypothesis for conduct problems, substance use, and substance use problems. Ordinary least squares regression was used to examine the hypothesis for depressive symptoms.

Baseline differences adjustment: sociodemographic risk, adolescent gender, and pre-test levels of outcomes were controlled; this allowed each analysis to test the possibility that SAAF-T participation caused differences in rates of change in outcomes across time.

Repeated measures methods in analysis: N/A

Notes

Equity: data regarding socioeconomic status are reported: mean household monthly gross income was $\$ 1482.50 .63 .8 \%$ families lived below federal poverty standards and $18 \%$ lived within $150 \%$ of the poverty threshold. $51 \%$ of the sample were female.

Funding: Award numbers R01DA021736 and P30DA027827 from the National Institute on Drug Abuse

Randomisation method: not given

Clustering accounted for in sample size calculation: N/A

Cluster randomisation methods to account for clustering in analysis: N/A

\section{Risk of bias}

\begin{tabular}{|c|c|c|}
\hline Bias & Authors' judgement & Support for judgement \\
\hline $\begin{array}{l}\text { Random sequence genera- } \\
\text { tion (selection bias) }\end{array}$ & Unclear risk & No information given \\
\hline $\begin{array}{l}\text { Allocation concealment } \\
\text { (selection bias) }\end{array}$ & Unclear risk & No information given \\
\hline $\begin{array}{l}\text { Blinding of participants } \\
\text { and personnel (perfor- } \\
\text { mance bias) } \\
\text { All outcomes }\end{array}$ & Unclear risk & Control group was an attention control. \\
\hline $\begin{array}{l}\text { Blinding of outcome as- } \\
\text { sessment (detection bias) } \\
\text { All outcomes }\end{array}$ & Low risk & Researchers were blind to allocation. \\
\hline $\begin{array}{l}\text { Incomplete outcome data } \\
\text { (attrition bias) } \\
\text { All outcomes }\end{array}$ & Low risk & $\begin{array}{l}\text { Low attrition; at } 5 \text { months, no differences were evident among study variables } \\
\text { or demographic characteristics based on attrition status }\end{array}$ \\
\hline $\begin{array}{l}\text { Selective reporting (re- } \\
\text { porting bias) }\end{array}$ & Unclear risk & No protocol available \\
\hline Other bias & Low risk & No other sources of bias identified \\
\hline
\end{tabular}


Bush 1989

\section{Methods}

Study name: Know Your Body 2

Study design (e.g. RCT, cluster RCT): 3-arm cluster randomised trial

Intervention arm(s): (1) 3 schools: full KYB curriculum and screening in the fall each year; full screening results in a Health Passport for students and parents. (2) 3 schools: KYB curriculum and screening in the fall each year; only parents received results of cholesterol tests; other screening results were available in Health Passport to students and parents

Comparator arm (s): 3 schools: no KYB curriculum; health screening in the fall of each school year; screening results not provided to students; parents received screening results of control participants

\section{Sample size calculation performed: N/S}

Subgroups prespecified: none reported

\section{Subgroup analyses: N/A}

Start date: 1983 (fall) was the year that the intervention commenced.

Duration of follow-up: this study was evaluated after 2 years of intervention.

Number of follow-ups: 2 in this paper

Follow-up time points: wave 1: at the start of grade 5 (12 months); wave 2: at the start of grade 6 (24 months)

ICC (if reported): not reported

Participants

Number of schools randomised: 9 schools crossed by treatment condition and socioeconomic level

Number of participants randomised (total and by arm): not clear

Age (range or mean (SD)) or grade at the start: age: mean 10.5 years. Start at grade 4 to 6 in elementary school

Gender: $54 \%$ female

Ethnicity: all African American

SES: low $36.4 \%$; middle $28.6 \%$; high $35 \%$

Inclusion criteria: students attending grades 4 to 6 at 9 public elementary schools in 1983

Exclusion criteria: students not returning a parental signed consent form

Interventions

Randomisation before or after baseline survey: not reported

Duration of the intervention (excluding follow-up): 5 years

Description of the intervention: Know Your Body (KYB)

School-based education composed of a taught curriculum, screening and feedback, parental involvement, and newsletter. The core curriculum was delivered weekly; other components included a quarterly newsletter entitled 'The Pacesetter', which contained health news for parents; parent-teacher meetings; and a parent-community advisory board. Family physicians were informed about the intervention and were sent the cholesterol results of participants. See previous Know Your Body study for detailed information.

Brief description of the theoretical model: social learning theory was used to develop the "Know Your Body" curriculum, which aims to change risk-related behaviour leading to heart disease through values clarification, goal setting, modelling, rehearsal, feedback on screening results, and reinforcement. The PRECEDE model was also used. 
Bush 1989 (Continued)

Description of the comparator: control participants did not receive the "Know Your Body" curriculum and were not provided with any of their screening results. Only the parents of control participants received screening results. The second intervention group also received the "Know Your Body" curriculum and the health screening. However, only their parents received the results of their cholesterol tests - students were not provided with the results to add to their 'health passport'.

Outcomes

List relevant outcomes

Primary outcomes: smoking cessation (serum thiocyanate); physical activity: fitness score, ponderosity index; cardiovascular risk factors: triceps skinfold thickness

Secondary outcomes: health knowledge, psychosocial variables, attitudes

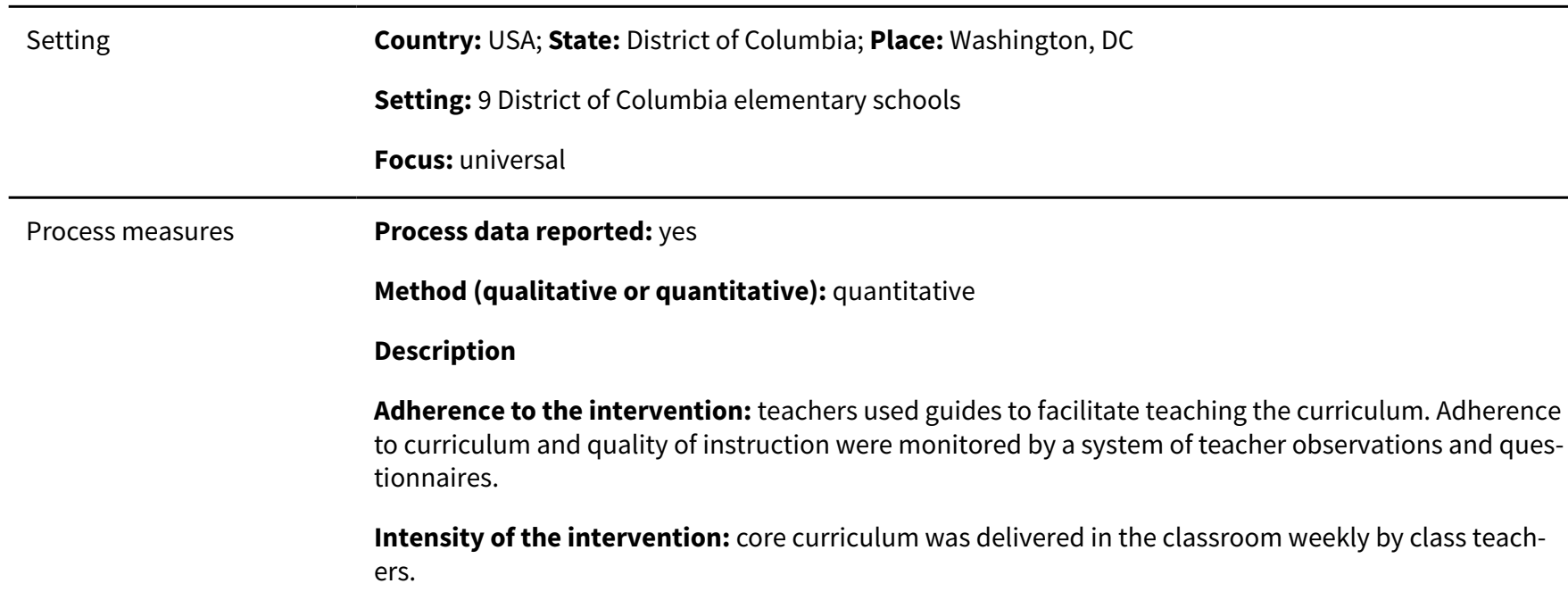

Statistics

Sample size: baseline $\mathrm{N}=1234$

Unit of randomisation: schools

Unit of analysis: schools

Method to promote equivalence between groups: stratification of schools on socioeconomic status by ranking to the \% of students eligible for Title I (federal school lunches); rank order was divided into tertiles; however, students were unevenly distributed between control and intervention groups by grade, gender, and SES. Blocking: a school was selected from each of the 3 SES strata and was randomly assigned to control or intervention conditions.

Statistical models: multiple regression

Baseline differences adjustment: multiple regression analysis was used to adjust the observed difference in scores between control and intervention groups for age, sex, socioeconomic status, and baseline risk factor value.

Repeated measures methods in analysis: not reported; results provided for 2-year assessment only; year 1 results not reported

Notes Equity: baseline only

Funding: National Heart, Lung and Blood Institute, National Institutes of Health

The study may have been stopped after 3 years because of high attrition rates at the time of analysis.

Randomisation method, e.g. block, stratification, computer: randomised blocks; schools ranked by $\%$ students eligible for Title 1, and rank order divided into 3 tertiles; 3 schools were randomly selected from each of the socioeconomic levels

Clustering accounted for in sample size calculation (if relevant): not reported 
Bush 1989 (Continued)

Cluster randomisation methods to account for clustering in analysis: not reported

\section{Risk of bias}

\begin{tabular}{|c|c|c|}
\hline Bias & Authors' judgement & Support for judgement \\
\hline $\begin{array}{l}\text { Random sequence genera- } \\
\text { tion (selection bias) }\end{array}$ & Unclear risk & 3 schools randomly selected from random sequence generation not provided \\
\hline $\begin{array}{l}\text { Allocation concealment } \\
\text { (selection bias) }\end{array}$ & Unclear risk & No information provided \\
\hline $\begin{array}{l}\text { Blinding of participants } \\
\text { and personnel (perfor- } \\
\text { mance bias) } \\
\text { All outcomes }\end{array}$ & High risk & Participants not blinded \\
\hline $\begin{array}{l}\text { Blinding of outcome as- } \\
\text { sessment (detection bias) } \\
\text { All outcomes }\end{array}$ & High risk & Self-reported outcomes and participants unblinded \\
\hline $\begin{array}{l}\text { Incomplete outcome data } \\
\text { (attrition bias) } \\
\text { All outcomes }\end{array}$ & High risk & $\begin{array}{l}\text { Of baseline participants, } 431(41.4 \%) \text { were available for rescreening after } 2 \\
\text { years of intervention. }\end{array}$ \\
\hline $\begin{array}{l}\text { Selective reporting (re- } \\
\text { porting bias) }\end{array}$ & Unclear risk & No published protocol \\
\hline Other bias & High risk & $\begin{array}{l}\text { Few data were reported about control and intervention groups and compar- } \\
\text { isons between groups; however, baseline differences between groups were ad- } \\
\text { justed in the analysis. }\end{array}$ \\
\hline
\end{tabular}

\section{Catalano 1999}

\section{Methods}

Study name: Focus on Families

Study design (e.g. RCT, cluster RCT): cluster RCT - although not stated

Intervention arm(s): standard methadone and FoF programme

Comparator arm (s): standard methadone treatment, which includes some individual and group counselling

Sample size calculation performed: not reported; however study authors state reduced power due to blocking of children by age before randomisation

Subgroups prespecified: parents and children

Subgroup analyses: none

Start date: not reported

Duration of follow-up: 8 months post intervention

Number of follow-ups: 2

Follow-up time points: wave 1: 6 months after parent training (11 months post recruitment); wave 2: 12 months after parent training (17 months post recruitment) 
Catalano 1999 (Continued)

\section{ICC (if reported): no}

Participants

Number of schools randomised: N/A

Number of participants randomised (total and by arm): 78 experimental, 57 control

Age (range or mean (SD)) or grade at the start: children aged 3 to 14 years at baseline; only children $\geq$ 6 years were interviewed. Mean age 10.4 years among those completing baseline interview and 6 - or 12 month follow-up $(\mathrm{N}=105)$

Gender: female parent $75 \%$

Ethnicity: parents: white 77\%, African American 18\%, other 5\%

SES: unemployed $66 \%$, incarcerated before methadone programme $68 \%$

Inclusion criteria: parents have to (1) be on methadone treatment at 1 of 2 participating clinics for a minimum of 90 days before participation; (2) have 1 or more children aged 3 to 14 years living with them at least $50 \%$ of the time; (3) reside not more than 25 miles from the methadone clinic.

Exclusion criteria: not reported

Duration of the intervention (excluding follow-up): 4 months of parent training and 9 months of case management (which began 1 month before parent training). Intervention was provided over 9 months in total.

\section{Description of the intervention: Focus on Families}

FoF combined parent skills training with home-based case management services.

(1) The skills training component involved 53 hours of training in small groups of 6 to 10 families. This consisted of an initial 5-hour family retreat and 32 90-minute twice-weekly meetings (4 months). Children attended 12 sessions to provide the opportunity for families to practice new skills in a controlled environment.

(2) Case management: case managers provided home-based services to families using a standardised manual for about 9 months; this began 1 month before the start of parent training sessions, continued through the group training period of 4 months, then a further 4 months. Case managers aimed to provide 1 home visit and 2 phone calls per week.

Brief description of the theoretical model: The project is based on a social development model described by Catalano and Hawkins (1996). Empirical information about risk and protective factors is organised to form a developmental theory of antisocial behaviour

Description of the comparator: standard methadone treatment alone, along with some individual and group counselling sessions

\section{List relevant outcomes}

\section{Primary outcomes:}

Child outcomes at 6 and 12 months' follow-up (adjusted mean SD or \%)

Family factors: rules index; parental recognition; attachment scale; involvement index

Peer factors: negative peers

School: school attachment; grades

Problem behaviours: \% use of cigarettes, or alcohol, or marijuana; delinquency scale

Secondary outcomes: unclear which were primary and which were secondary outcomes 
Catalano 1999 (Continued) Setting

Country: USA; State: Washington; Place: Seattle

Setting: 2 methadone clinics

Focus: targeted: parents in methadone treatment and their children

Process measures Process data reported: yes

Method (qualitative or quantitative)

\section{Description}

Adherence to the intervention: 11 (13\%) did not attend any parent training session; $42(51 \%)$ attended at least $50 \%(16)$ of the sessions.

Reach of the intervention: 61 (74\%) were actively engaged; home-based case management lasted an average of 9 months but ranged from 3 to 12 months; case managers met clients an average of 6 times monthly, spending 54 hours on average with the family.

Intensity of the intervention: continuous for 9 months

Statistics

Sample size: eligible $N=185$ parents; enrolled $N=144$ parents ( $78 \%$ ) from 130 families; I = 75 families; $\mathrm{C}=55$ families

$\mathrm{N}=178$ children: $\mathrm{I}=97, \mathrm{C}=81$ (137 adults and 105 children completed a baseline interview)

Unit of randomisation: families

Unit of analysis: individuals

Method to promote equivalence between groups: blocking

Statistical models: ANCOVA at 6- and 12-month follow-up control for baseline; logistical regression (dichotomous); repeated measures analysis

Baseline differences adjustment: adjusted for parents: ANCOVA used to assess experimental and control group differences at 6 - and 12-month follow-up, controlling for baseline measures. Children: several indicators were missing at baseline for children who later became eligible for the extensive interview schedule.

Repeated measures methods in analysis: repeated measures analyses of variance were completed on the more limited sample with complete data at baseline, 6-month follow-up, and 12-month follow-up.

Notes

Equity: data for parents on ethnicity, marital status, education, employment, incarceration

Funding National Institute on Drug Abuse

Incentives were used to encourage recruitment and retention - money; transport; child care; tickets for games, the zoo, etc.

Baseline data were missing for young children (<6 years) who later became eligible for interviewing.

At 6 and 12 months' follow-up, 9\% of children (aged 6 or older) had not lived with their FoF parent at any time over the previous 6 months.

Randomisation method, e.g. block, stratification, computer: blocked on race, parents' age at first drug use, whether parents lived with a spouse or partner, and children's age. A higher proportion of eligible families were assigned to the experimental than to the control group.

Clustering accounted for in sample size calculation (if relevant): not reported

Cluster randomisation methods to account for clustering in analysis: no 
Catalano 1999 (Continued)

Risk of bias

\begin{tabular}{|c|c|c|}
\hline Bias & Authors' judgement & Support for judgement \\
\hline $\begin{array}{l}\text { Random sequence genera- } \\
\text { tion (selection bias) }\end{array}$ & Unclear risk & Not reported \\
\hline $\begin{array}{l}\text { Allocation concealment } \\
\text { (selection bias) }\end{array}$ & Unclear risk & Not reported \\
\hline $\begin{array}{l}\text { Blinding of participants } \\
\text { and personnel (perfor- } \\
\text { mance bias) } \\
\text { All outcomes }\end{array}$ & High risk & No blinding of participants or study personnel \\
\hline $\begin{array}{l}\text { Blinding of outcome as- } \\
\text { sessment (detection bias) } \\
\text { All outcomes }\end{array}$ & High risk & Not reported; child interviews likely to be high risk \\
\hline $\begin{array}{l}\text { Incomplete outcome data } \\
\text { (attrition bias) } \\
\text { All outcomes }\end{array}$ & Low risk & $\begin{array}{l}\text { Attrition did not vary by condition at either follow-up time point. For children: } \\
\text { as with parents, attrition did not differ significantly by group assignment. }\end{array}$ \\
\hline $\begin{array}{l}\text { Selective reporting (re- } \\
\text { porting bias) }\end{array}$ & Unclear risk & No published protocol \\
\hline Other bias & Unclear risk & $\begin{array}{l}\text { Potential biases of missing data from children who were not living with par- } \\
\text { ents during part of the intervention and those aged } 6 \text { years or younger; cluster- } \\
\text { ing not accounted for, relatively small sample size }\end{array}$ \\
\hline
\end{tabular}

Conduct Problems Prevention Research Group 2010

Study name: Fast Track

Study design: cluster RCT at the level of the school

Intervention arm(s): Fast Track (delivered at classroom level)

Comparator arm(s): control group

Sample size calculation performed: N/S

Subgroups prespecified: N/S

Subgroup analyses: high-risk and moderate-risk groups; male and female; African American and European American; by site

Start date: 1991

Duration of follow-up: 19 years (up to age 25)

Number of follow-ups: data collected while intervention ongoing up to 12th grade

Follow-up time points: 12th grade ( 2 years post intervention); age 25 ( 9 years post intervention)

ICC (if reported): N/S

\footnotetext{
Participants

Number of schools randomised: 55 elementary schools
} 
Conduct Problems Prevention Research Group 2010 (Continued)

Number of participants randomised (total and by arm): 445 randomised to intervention, 446 randomised to control

Age (range or mean (SD)) or grade at the start: children aged 0 to 12 years; mean age at baseline 6.58 years (SD 0.48)

Gender: $69 \%$ of sample were male.

Ethnicity: 51\% African American, 47\% European American, 2\% other ethnicity

SES: $35 \%$ of families were in the lowest socioeconomic class.

Inclusion criteria: children were screened in kindergarten for conduct problems. The first gate of the screening procedure employed teacher-reported classroom conduct problems, using the Teacher Observation of Child Adjustment-Revised Authority Acceptance score. Children scoring in the highest 40\% within the cohort and site were solicited for screening of parent-rated home problem behaviours, using a 22-item instrument based on the Child Behavior Checklist.

Exclusion criteria: Low risk score for aggressive and disruptive behaviour.

Interventions

\author{
Randomisation before or after baseline survey: N/S \\ Duration of the intervention (excluding follow-up): 10 years
}

Description of the intervention: Elementary school phase: intervention families were offered group intervention during a 2-hour "enrichment program" that included children's social skill "friendship groups", parent training groups, guided parent-child interaction sessions, and paraprofessional tutoring in reading. Tutors provided 3 additional 30 -minute sessions per week in reading and peer pairing to improve friendships with classmates. Teacher consultation and the teacher-implemented social-cognitive skill development Fast Track PATHS (Promoting Alternative Thinking Strategies) curriculum were implemented universally in grade 1 to 5 classrooms at intervention schools (except in Durham, North Carolina, where it was prohibited) to promote social-emotional competence. After grade 1, criterion-referenced assessments adjusted the prescribed dosage to match need. Middle and early high school phase (grades 6 to 10): During grades 5 and 6 , children received a middle school transition programme, and 4 parent-youth groups addressed topics of adolescent development; alcohol, tobacco, and drugs; and decision-making. In grades 7 and 8, 8 youth forums addressed vocational opportunities, life skills, and summer employment opportunities. In grades 7 to 10, individualised interventions addressed parent monitoring, peer affiliation, academic achievement, and social cognition. All children received Oyserman's School-to-Jobs possible-selves intervention aimed at examining emerging identity.

Brief description of the theoretical model: developmental model of conduct disorders

Description of the comparator: N/S

Outcomes List relevant outcomes

Primary outcomes: conduct problems, school failure, poor social relations, externalising problems, internalising problems, substance abuse problems

Secondary outcomes: crime, risky sexual behaviour, well-being, aggression, education/employment

Setting

Country: USA;State: Durham, North Carolina; Nashville, Tennessee; rural Pennsylvania; Seattle, Washington

Setting: primary school (grades 1 to 10$)$

Focus: targeted school-based intervention 
Conduct Problems Prevention Research Group 2010 (Continued)

Description: high intervention fidelity was ensured by manualisation, regular cross-site training, and weekly clinical supervision. Fidelity was assessed through monthly ratings of quality of implementation made on the basis of direct observation of teacher instruction. For all 3 cohorts, 4 4-point Likert scale ratings were obtained (ranging from low skilled to highly skilled performance).

$96 \%$ of parents and $98 \%$ of children attended at least 1 group session during grade 1 . Of these families, $79 \%$ of parents and $90 \%$ of children attended at least $50 \%$ of the sessions offered.

Statistics

Sample size: 891 were recruited; at baseline, 445 were in the intervention arm and 446 in the control arm

Unit of randomisation: school

Unit of analysis: school

Method to promote equivalence between groups: matching of schools into sets that were equivalent in school size, achievement level, poverty, and ethnic/racial diversity. Sets of schools were then randomly assigned to intervention or control groups.

Statistical models: standard linear regression models for continuous outcomes, logit models for dichotomous outcomes, negative binomial models for count and crime variables

Baseline differences adjustment: analyses controlled for 22 pre-intervention covariates

Repeated measures methods in analysis: N/A

Notes Equity: baseline data reported

Funding: supported by National Institute of Mental Health (NIMH) grants R18 MH48043, R18 MH50951, R18 MH50952, R18 MH50953, K05MH00797, and K05MH01027; and supported in part by Department of Education grant S184U30002; and National Institute on Drug Abuse grants DA16903, DA017589, DA015226, K05DA015226, and P30DA023026. The Center for Substance Abuse Prevention and the National Institute on Drug Abuse also provided support through a memorandum of agreement with NIMH.

Randomisation method, e.g. block, stratification, computer: not clear

Clustering accounted for in sample size calculation (if relevant): not clear

Cluster randomisation methods to account for clustering in analysis: yes; analyses take into account sampling at the school level and clustering at the classroom level

\section{Risk of bias}

\begin{tabular}{lll}
\hline Bias & Authors' judgement & Support for judgement \\
\hline $\begin{array}{l}\text { Random sequence genera- } \\
\text { tion (selection bias) }\end{array}$ & Unclear risk & Information not given \\
\hline $\begin{array}{l}\text { Allocation concealment } \\
\text { (selection bias) }\end{array}$ & Unclear risk & Information not given \\
\hline $\begin{array}{l}\text { Blinding of participants } \\
\text { and personnel (perfor- } \\
\text { mance bias) }\end{array}$ & High risk & No blinding of participants or those who delivered the intervention \\
All outcomes & \\
\hline $\begin{array}{l}\text { Blinding of outcome as- } \\
\text { sessment (detection bias) } \\
\text { All outcomes }\end{array}$ & Unclear risk & $\begin{array}{l}\text { Self-reported outcomes, although those conducting the interviews were blind- } \\
\text { ed }\end{array}$ \\
\hline $\begin{array}{l}\text { Incomplete outcome data } \\
\text { (attrition bias) }\end{array}$ & Unclear risk & $\begin{array}{l}\text { Missing data were imputed, but the information provided is not sufficiently } \\
\text { clear. }\end{array}$ \\
\hline
\end{tabular}


Conduct Problems Prevention Research Group 2010 (Continued)

All outcomes

Selective reporting (re- Unclear risk Insufficient information given
porting bias)

Other bias Low risk No other sources of bias identified

Study design (e.g. RCT, cluster RCT): cluster RCT individuals randomised by classroom from 3 middle schools N =998; also randomised to a Family Resource Centre (FRC) at each of the 3 schools

Intervention $\operatorname{arm}(\mathbf{s}): \mathrm{N}=500$

Universal intervention: $\mathrm{N}=500$

Selected intervention (FCU): $N=115$ (in addition to universal intervention)

Indicated intervention: $\mathrm{N}=88$ of 115 in the FCU received further interventions.

Comparator $\operatorname{arm}(\mathbf{s}): \mathrm{N}=498$

No intervention

Sample size calculation performed: no; CACE analysis was used to accommodate non-compliance; no information about impact of clustering on sample size

Subgroups prespecified: CACE analysis uses covariates, but these were not prespecified.

Subgroup analyses: gender, ethnicity, biological father present, family conflict, deviant peers, teacher report of risk

Start date: not reported

Duration of follow-up: 6 years including intervention period

\section{Number of follow-ups: 5}

Follow-up time points: wave 1: grade 6, age 11 to 12 years; wave 2: grade 7 , age 12 to 13 years; wave 3: grade 8, age 13 to 14 years; wave 4: grade 9, age 14 to 15 years; wave 5: grade 11 , age 16 to 17 years; wave 6: age 19 years (arrests only)

ICC (if reported): not reported

\section{Participants}

\section{Number of schools randomised: not clear}

Number of participants randomised (total and by arm): $N=998(90 \%)$ adolescents and their families from 3 urban middle schools participated; intervention $n=500$; control $n=498$

Age (range or mean (SD)) or grade at the start: 11 to 12 years. Start in spring of grade 6

Gender: $53 \%$ male, $47 \%$ female

Ethnicity: Caucasian 42.3\%, African American 29.1\%, Latino 6.8\%, Asian American 5.2\%, other 16.4\%

SES: across the 3 schools, $35 \%, 89 \%$, and $39 \%$ of families received free/reduced price lunch.

Inclusion criteria: all sixth grade students

Exclusion criteria: not reported 
Connell 2007 (Continued)

Interventions

\section{Randomisation before or after baseline survey: not clear}

Duration of the intervention (excluding follow-up): intervention by FRC was provided only in middle school up to grade 8 ( 2.5 school years).

\section{Description of the intervention}

Family Check-Up (FCU)

Students were randomly assigned to a family-centred intervention in sixth grade and were offered a multi-level intervention that included (1) a universal classroom-based intervention, (2) the Family Check-Up, and (3) family management treatment.

FCU is part of the Adolescent Transitions Programme (ATP) and is modelled on the Life Skills Training Programme; the ATP links universal, selected, and indicated family interventions.

The intervention has 3 parts:

(1) A universal classroom-based intervention

The universal intervention was designed to support positive parenting practices and to engage parents of high-risk youths in the selected interventions (FCU).

The universal intervention involved a family resource centre (FRC) at each of the 3 participating schools, available to those in the intervention group. The FRC provided brief consultations with parents, telephone consultations, feedback to parents on their child's behaviour at school, and access to videotapes and books. The FRC parent consultant also conducted 6 in-class lessons to students, known as the SHAPe curriculum. The 6 sessions focused on school success, health decisions, building positive peer groups, the cycle of respect, coping with stress and anger, and solving problems peacefully.

Brief parent-student activities designed to motivate family management were also included.

FRC was discontinued when students entered high school.

(2) FCU: a selected intervention to improve parenting practices; consists of 3 brief sessions of an initial interview, a family assessment involving videotaping at home whilst engaging in a variety of tasks, and feedback focusing on motivation for parenting. Although all families could receive the FCU, families of high-risk youths, determined by teacher ratings, were specifically offered the FCU in grades 7 and 8 .

(3) Family management treatment - indicated services for families provided through behavioural parent group intervention, individual-based behaviour family therapy, and multi-systemic family therapy

Brief description of the theoretical model: intervention was modelled on the Life Skills Training Programme.

Description of the comparator: school as usual, including regular services offered by the schools but no access to any of the intervention services available to families in the intervention condition

\section{Outcomes List relevant outcomes}

Primary outcomes: adolescent substance use: tobacco, alcohol, drugs; problem behaviours: antisocial behaviour and offending

Secondary outcomes: arrests, family conflict, deviant peer involvement
Country: USA; State: Oregon;Place: Portland

Setting: 3 urban public Title 1 middle schools; 20\% of the school population qualified for special education services

Focus: universal: all 3 schools served an at-risk population of youth and families; all families could receive the FCU; families of high-risk youths, determined by teacher ratings, were specifically offered the FCU in grades 7 and 8 
Connell 2007 (Continued)

Process measures

Process data reported: yes

Method (qualitative or quantitative)

Description

Adherence to the intervention: $25 \%$ of families engaged in selected and indicated levels.

Intensity of the intervention: FRC parent consultant conducted 6 in-class lessons referred to as the SHAPe Curriculum for students. The FCU was a brief 3-session intervention based on motivational interviewing and modelled on Drinker's Check-Up.

Statistics

Sample size: $\mathrm{N}=1110$ eligible in sixth grade: $\mathrm{N}=998(90 \%)$ adolescents and their families from 3 urban middle schools participated; intervention $n=500$, control $n=498$

Unit of randomisation: individual

Unit of analysis: individual

Method to promote equivalence between groups: not reported

Statistical models: Complier Average Causal Effect (CACE) analysis (to infer a causal effect of treatment in the presence of departures from randomised intervention - a better term than 'non-compliance')

Baseline differences adjustment: not clear if there were any noticeable baseline differences

Repeated measures methods in analysis: used CACE model for analysing trajectory over time

Equity: some characteristics given for those invited to participate but not for those at baseline who were randomised nor at follow-up

Funding: National Institute on Drug Abuse, National Institute on Alcohol Abuse and Alcoholism

Associated paper: Stormshak 2011: not clear if this is a subsample of Connell 2007, or a different population

Randomisation method, e.g. block, stratification, computer: no information on random sequence generation was provided.

Clustering accounted for in sample size calculation (if relevant): students from 3 schools were assigned to 3 family resource centres but were individually randomised. No information about impact of clustering on sample size

Cluster randomisation methods to account for clustering in analysis: Complier Average Cause Effect analysis - CACE is based on comparisons of randomised groups and accounts for compliance rather than differences between randomised groups

\section{Risk of bias}

\begin{tabular}{lll}
\hline Bias & Authors' judgement & Support for judgement \\
\hline $\begin{array}{l}\text { Random sequence genera- } \\
\text { tion (selection bias) }\end{array}$ & Unclear risk & No information on random sequence generation was provided. \\
\hline $\begin{array}{l}\text { Allocation concealment } \\
\text { (selection bias) }\end{array}$ & Unclear risk & No information provided \\
\hline $\begin{array}{l}\text { Blinding of participants } \\
\text { and personnel (perfor- } \\
\text { mance bias) } \\
\text { All outcomes }\end{array}$ & High risk & Not possible to be blind to intervention status \\
\hline
\end{tabular}


Connell 2007 (Continued)

\begin{tabular}{|c|c|c|}
\hline $\begin{array}{l}\text { Blinding of outcome as- } \\
\text { sessment (detection bias) }\end{array}$ & High risk & $\begin{array}{l}\text { CIDI was administered blind to intervention status; many other measures were } \\
\text { self-reported. }\end{array}$ \\
\hline
\end{tabular}

\begin{tabular}{lll}
\hline $\begin{array}{l}\text { Incomplete outcome data } \\
\text { (attrition bias) } \\
\text { All outcomes }\end{array}$ & Low risk & $\begin{array}{l}\text { Similar numbers in each arm at follow-up; study authors used FIML estimation } \\
\text { to account for missing data }\end{array}$ \\
\hline $\begin{array}{l}\text { Selective reporting (re- } \\
\text { porting bias) }\end{array}$ & Unclear risk & No published protocol \\
\hline Other bias & Low risk & No other sources of bias identified \\
\hline
\end{tabular}

Study design (e.g. RCT, cluster RCT): RCT at the level of the individual

\section{Intervention $\operatorname{arm}(\mathbf{s})$ :}

(1) Therapist-delivered brief intervention

(2) Computer-delivered brief intervention

Comparator arm(s): no active intervention; participants received a trifold brochure with community resources listed and explained

Sample size calculation performed: yes; based on a 15\% reduction in occurrence of risk behaviour and $\mathrm{P}<0.05$ significance; 107 individuals per arm was required to achieve $80 \%$ power

Subgroups prespecified: N/A; no subgroup analyses conducted

\section{Subgroup analyses: N/A}

Start date: September 2006

Duration of follow-up: 12 months post intervention

\section{Number of follow-ups: 3}

Follow-up time points: 3,6 , and 12 months post baseline

ICC (if reported): N/A (non-cluster RCT)

\section{Number of schools randomised: N/A}

Number of participants randomised (total and by arm): 726 adolescents randomised at baseline

Age (range or mean (SD)) or grade at the start: mean 16.8 years (SD 1.3)

Gender: $43.5 \%$ male

Ethnicity: $55.9 \%$ African American

SES: $57.4 \%$ of participants' families were in receipt of family assistance

Inclusion criteria: adolescents aged 14 to 18 years, presenting to an emergency department with medical illness/injury and a previous history of violent behaviour and alcohol consumption 
Exclusion criteria: adolescents seeking care for acute sexual assault, suicidal ideation, altered mental status, and those with lack of consent and medically unstable (abnormal vital signs) were all excluded from the study.

Interventions

Randomisation before or after baseline survey: after

Duration of the intervention (excluding follow-up): median times for each intervention arm were as follows: computerised brief intervention (29 minutes) and therapist brief intervention ( 37 minutes).

Description of the intervention: study examined 2 delivery modes (therapist (TBI) or computer (CBI)) of essentially the same brief intervention in terms of format and content. In the TBI group, a tablet computer provided feedback from screening and baseline surveys and delivered age- and sex-specific normative information. Adolescents completed computerised checklists to identify reasons to stay away from violence and drinking. Based on participants' risk behaviours, the computer selected various role-play scenarios. The therapist guided participants through these. In the CBI group, an interactive multimedia computer programme was developed and delivered via tablets, and audio headphones were utilised. It had a cartoon theme, with a virtual "buddy" that participants could 'hang out with' throughout the duration of the intervention. Participants could choose the ethnicity, gender, and age of the buddy. The buddy guided participants through each stage of the intervention, providing feedback on choices made and identifying reasons to stay away from alcohol and violence. The buddy also provided participants with feedback from their peer-to-peer interaction, highlighting possible consequences of their choices and informing them of the best possible course of action in each role-play scenario.

Brief description of the theoretical model: An adaptation of traditional motivational interviewing (MI) techniques underpinned the brief interventions. MI promotes motivation to change in a respectful, non-confrontational manner, encompassing choice, responsibility, and self-efficacy, and developing a discrepancy between current behaviour and future goals and inner values. Norm-resetting and skills training were also encouraged via the brief intervention.

Description of the comparator: Participants assigned to the control group receive a trifold brochure with community resources.

Outcomes

Primary outcomes: peer aggression, violence consequences, alcohol misuse (defined by Audit-C score), binge drinking, alcohol consequences

Secondary outcomes: percentage experiencing peer violence

Setting: emergency department

Focus: targeted; hazardous and harmful adolescent drinkers attending emergency department unit

Process data reported: no

Method (qualitative or quantitative): N/A

Description: N/A

Sample size: 726 adolescents randomised at baseline

Unit of randomisation: individual

Unit of analysis: individual

Method to promote equivalence between groups: stratification by gender and age; blocking of 21 ( 7 blocks per arm)

Statistical models: generalised estimating equations

Baseline differences adjustment: yes; school dropout rate baseline imbalance, which was adjusted for in the final analysis 
Repeated measures methods in analysis: yes; generalised estimating equations accounted for correlated nature of data from repeated measures at baseline and at 3, 6, and 12 months post baseline

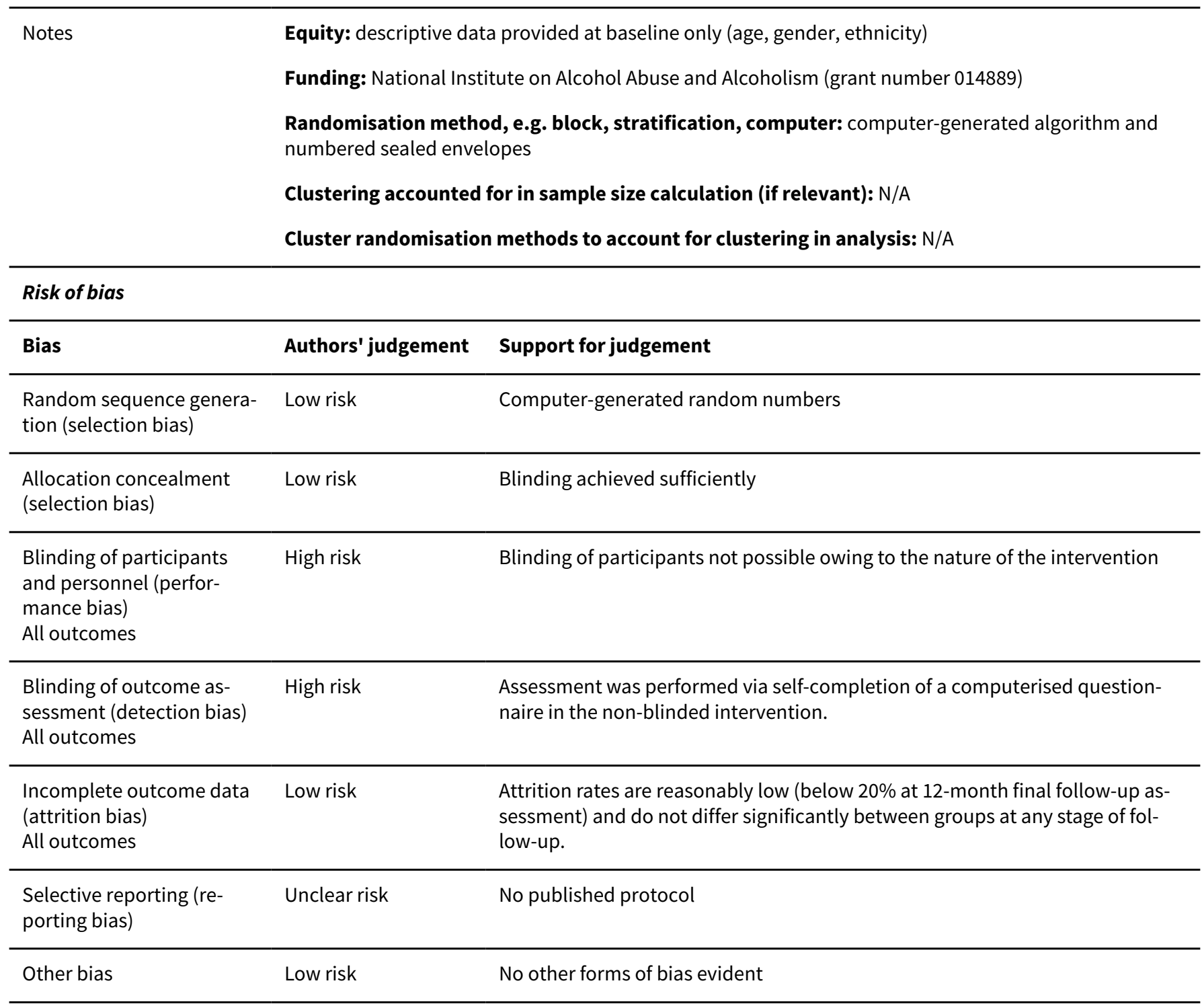

(1) RSTP

(2) DARE-A

Comparator $\operatorname{arm}(\mathbf{s}):$ no intervention

Sample size calculation performed: not reported

Subgroups prespecified: no 
Subgroup analyses: subgroups were examined during the analysis: all analyses were repeated including only participants reporting engagement in that particular behaviour before the programmes were implemented.

Start date: not reported

Duration of follow-up: 6 months (4 months post intervention)

Number of follow-ups: 2

Follow-up time points: wave 1: 2 months post intervention (post-test); wave 2: 6 months' follow-up

ICC (if reported): N/A

Participants

Number of schools randomised: 1 school

Number of participants randomised (total and by arm): $N=300$ at baseline; RSTP $n=75$; DARE-A $n=$ 75 ; control $n=150$

Age (range or mean (SD)) or grade at the start: 14 to 19 years (mean = 16 years)

Gender: $58 \%$ females, $41 \%$ males ( $1 \%$ missing data)

Ethnicity: 63\% Caucasian, 17\% Hispanic, 10\% African American, 2\% Asian, 8\% 'other'

SES: family income: $<\$ 20,000 /$ year $=2 \% ; \$ 20,000$ to $\$ 30,000 /$ year $=15 \% ; \$ 30,000$ to $\$ 40,000 /$ year $=$ $17 \%$; $\$ 40,000$ to $\$ 50,000 /$ year $=21 \% ; \$ 50,000$ to $\$ 60,000 /$ year $=18 \%$; > $\$ 60,000 /$ year $=24 \%$; missing data $=3 \%$

Inclusion criteria: not reported

Exclusion criteria: freshmen because they were younger than the legal driving age

\section{RSTP}

The Risk Skills Training Program (RSTP) consisted of a 50-minute interactive group session. Negotiation and acceptance is the focal point, encouraging youth to change their risk-taking behaviour. Adolescents received confidential, personalised, written, and graphical feedback on their own risk-taking behaviour, peers' risk-taking behaviour, and their perception of their peers' risk-taking behaviour. The graphical feedback was clearly explained and discussed in detail during the session. Graphs showed the number of alcoholic drinks reported in a 1-week period and the number of times drug use was reported in the past 3 months (all self-report).

Individuals then discussed personalised feedback in a group setting among peers. A group discussion of peer influence followed and focused on evidence that youth tend to overestimate their peers' risktaking behaviour, which can influence their personal risk-taking behaviour.

Adolescents were provided with personal, confidential feedback on positive outcome expectancies that they endorsed related to alcohol use. The following discussion focused on differences between effects of the drug vs effects of expecting to receive a drug. Positive and negative consequences of risktaking were discussed, emphasising how to avoid negative consequences, such as a drinking driving accident or losing the respect of parents/peers because of excessive substance use. A skills training component then focused on how to anticipate risky situations and how to implement safer decisions in the future about substance use, driving after drinking, and riding with a drinking driver.

\section{DARE-A}

Drug Abuse and Resistance Education-A (DARE-A) was a 50-minute programme led by a certified DARE instructor that focused on increasing knowledge and understanding of the deleterious effects of sub- 
stance use. Topics were based on relative importance and significance to the theme of DARE and to the current sample (i.e. a suburban sample).

Four topics were selected: (1) drug use and abuse - how mind-altering substances change the way the mind and body function; (2) drugs and the law - the need for laws and school behaviour codes, and how breaking codes can interfere with the rights and safety of all people concerned; (3) consequences - examination of all costs and consequences of drug use to individuals and communities; and (4) assertive resistance - by recognising pressures that may influence a young person to use substances with demonstration of assertive resistance to such pressures.

Brief description of the theoretical model: DARE-A based on DARE programme. The Risk Skills Training Programme is based in part on the Alcohol Skills Training Programme (Fromme 1994) and the Brief Alcohol Screening and Intervention in College Students (BASICS) (Dimeff 1998).

Description of the comparator: no details given

Primary outcomes: weekly drinking; risky drinking; drug use: smoked marijuana, drugs other than marijuana, mixed drugs with alcohol; driving after drinking (DUI), riding with a drinking driver (RDD)

Secondary outcomes: alcohol outcomes and expectancies; perception of peer risky drinking, peer drug use, and peer DUI and RDD

\section{Setting}

Country: USA;State: not reported

Setting: a single mid-sized suburban high school

Focus: universal

Process data reported: An independent rater, considered an expert in the prevention and intervention field, rated 6 randomly selected audiotapes of the sessions (3 DARE-A and 3 RSTP) for adherence to the protocol $(0=$ no adherence to $6=$ substantial adherence $)$ on the following:

Student participation and amount of lecture during the session ( $1=$ none to $5=$ a lot)

Overall quality of the presentation $(0=$ poor to $6=$ excellent $)$

Method (qualitative or quantitative): both

\section{Description}

Acceptability of the intervention: the RSTP was characterised by more student participation and interaction (mean 4.3; SD 0.58) and less lecture (mean 3.0; SD 0) than the DARE-A session (mean 2.0; SD 0 for student participation and mean 5.0; SD 0 for lecture). Overall quality of the programmes was rated as follows: RSTP: mean 5.3; SD 0.58; DARE-A: mean 2.0; SD 0.

Adherence to the intervention: Results showed that both leaders followed their respective protocols (M adherence RSTP 5.3, SD 0.25: M adherence DARE-A 4.7, SD 0.29). The DARE-A leader discussed costs and consequences of drug use and did not discuss expectancies; the RSTP leader discussed personalised feedback and expectancies but did not discuss legal implications of substance use.

Intensity of the intervention: one 50-minute session. Positive anecdotal feedback from students

Unit of randomisation: individual

Unit of analysis: individual

Method to promote equivalence between groups: data were examined for non-normalcy, and outliers from each of the 3 arms were removed from the analysis - outlier rates did not differ between arms. 


\title{
Statistical models: MANOVA
}

Baseline differences adjustment: data were examined for non-normalcy, and outliers from each of the 3 arms were removed from the analysis - outlier rates did not differ between arms.

Repeated measures methods in analysis: MANOVA used for analyses

\begin{tabular}{ll}
\hline Notes & Equity: baseline values reported \\
Funding: National Institute on Alcohol Abuse and Alcoholism (NIAAA) FIRST award number R29- \\
AA09135. NIAAA training grant 5T32-AA07471 and a Hogg Foundation Grant, Austin, Texas
\end{tabular}

Randomisation method, e.g. block, stratification, computer: not reported

Clustering accounted for in sample size calculation (if relevant): N/A

Cluster randomisation methods to account for clustering in analysis: $\mathrm{N} / \mathrm{A}$

\section{Risk of bias}

\begin{tabular}{|c|c|c|}
\hline Bias & Authors' judgement & Support for judgement \\
\hline $\begin{array}{l}\text { Random sequence genera- } \\
\text { tion (selection bias) }\end{array}$ & Unclear risk & Not reported \\
\hline $\begin{array}{l}\text { Allocation concealment } \\
\text { (selection bias) }\end{array}$ & Unclear risk & Not reported \\
\hline $\begin{array}{l}\text { Blinding of participants } \\
\text { and personnel (perfor- } \\
\text { mance bias) } \\
\text { All outcomes }\end{array}$ & High risk & $\begin{array}{l}\text { Blinding of participants and personnel not possible; all participants attended } \\
\text { the same school }\end{array}$ \\
\hline $\begin{array}{l}\text { Blinding of outcome as- } \\
\text { sessment (detection bias) } \\
\text { All outcomes }\end{array}$ & High risk & Self-reported outcomes in unblinded intervention \\
\hline $\begin{array}{l}\text { Incomplete outcome data } \\
\text { (attrition bias) } \\
\text { All outcomes }\end{array}$ & High risk & $\begin{array}{l}\text { Significantly more control participants had dropped out by the 2-month post- } \\
\text { test in comparison to both intervention arms; attrition at } 6 \text { months' follow-up } \\
\text { was 39\%, but no significant differences were found between groups }\end{array}$ \\
\hline $\begin{array}{l}\text { Selective reporting (re- } \\
\text { porting bias) }\end{array}$ & Unclear risk & No published protocol \\
\hline Other bias & Unclear risk & Possibility of contamination, as all participants attend the same school \\
\hline
\end{tabular}

DeGarmo 2009

Methods

\author{
Study name: LIFT \\ Study design (e.g. RCT, cluster RCT): cluster RCT at the level of the school \\ Intervention $\operatorname{arm}(\mathbf{s})$ : Linking the Interests of Families and Teachers intervention \\ Comparator $\operatorname{arm}(\mathbf{s})$ : standard education and support services provided by respective schools \\ Sample size calculation performed: N/S \\ Subgroups prespecified: N/A
}


DeGarmo 2009 (Continued)

\author{
Subgroup analyses: no \\ Start date: identification and recruitment of schools in 1991-1993 \\ Duration of follow-up: 7 years \\ Number of follow-ups: 2 \\ Follow-up time points: 1 year and 7 years post baseline \\ ICC (if reported): yes; .00 to .01 for classrooms $(n=17)$
}

\begin{abstract}
Participants
Number of schools randomised: 12

Number of participants randomised (total and by arm): 671 students in total

Age (range or mean (SD)) or grade at the start: age of youth not stated; grades 5 to 12 (baseline and last follow-up)

Gender: female (51\%), male (49\%)

Ethnicity: European American: intervention (87.4\%), control (81.3\%); African American: intervention (1.3\%), control (2.4\%); American Indian: intervention (2.4\%), control (3.8\%); Asian/Pacific Islander: intervention (1.3\%), control (4.5\%); Hispanic: intervention (5.1\%), control (4.8\%); other: intervention (2.4\%), control (3.1\%)
\end{abstract}

SES: annual income: less than $\$ 15,000$ : intervention (21\%), control (27\%); $\$ 15,000$ to $\$ 30,000$ : intervention (33\%), control (30\%); $\$ 30,000$ to $\$ 50,000$ : intervention (57\%), control $(31 \%)$; more than $\$ 50,000$ : intervention (10\%), control (12\%)

Inclusion criteria: schools in catchment areas with rates above the area median of $8.9 \%$ households with at least 1 juvenile arrest

Exclusion criteria: $\mathrm{N} / \mathrm{S}$

\begin{abstract}
Interventions
Randomisation before or after baseline survey: before

Duration of the intervention (excluding follow-up): 10 weeks

Description of the intervention: multi-component intervention consisting of (1) parent management training, (2) child social and problem-solving skills training, and (3) school recess component

Brief description of the theoretical model: not explicitly stated; the intervention is based on a developmental model centred on moment-to-moment social interaction processes

Description of the comparator: control schools and participants received no psychosocial prevention services from the research team, but schools were paid $\$ 2000$ in unrestricted funds. Students in both control and intervention schools had access to all regular individual and group psychosocial services provided by their school, such as psychological testing, counselling, prevention programmes (e.g. DARE), and special education services.
\end{abstract}

\begin{tabular}{ll}
\hline Outcomes & List relevant outcomes \\
& Primary outcomes: antisocial behaviour, substance use \\
& Secondary outcomes: N/S \\
\hline Setting & Country: USA; State: Pacific NorthWest \\
& Setting: school \\
& Focus: universal \\
\hline Process measures & Process data reported: yes
\end{tabular}




\section{Description}

Acceptability of the intervention: satisfaction of parents and teachers was assessed; $94 \%$ of parents would recommend the programme to other parents, $79 \%$ reported the programme to be 'quite/very' helpful, and teachers reported the intervention as helpful (first grade teachers more so than fifth grade teachers).

Adherence to the intervention: family participation in the programme was recorded; delivery of classroom component of intervention was recorded using checklists ( $92.5 \%$ of 'critical components' on the checklist were completed, as rated by teachers and independent assessors); in the parent training group, $96.5 \%$ of items on the checklist were endorsed as completed, and interventionists reported covering $94 \%$ of the curriculum overall.

Sample size: 351 youth
Unit of randomisation: school
Unit of analysis: individual
Method to promote equivalence between groups: N/S
Statistical models: heirarchical Cox survival models, latent growth curve analysis, repeated measures
multi-variate analysis of variance, bootstrap sampling
Baseline differences adjustment: $\mathrm{N} / \mathrm{S}$
Repeated measures methods in analysis: yes; latent growth curve modelling, controlling for deviant
peer association as a time-varying covariate, and repeated measures multi-variate analysis of variance

Notes Equity: N/S

Funding: Prevention and Behavioural Medicine Research Branch (National Institute of Mental Health, USA; Grant R01 MH054248), P30 MH 46690, and a centre for infrastructure development grant from the McConnell Clark Foundation

Randomisation method, e.g. block, stratification, computer: not clearly stated

Clustering accounted for in sample size calculation (if relevant): yes, random regression applied in the analysis, accounting for clustering by including 'school' as a variable

Cluster randomisation methods to account for clustering in analysis: yes

\section{Risk of bias}

\begin{tabular}{lll}
\hline Bias & Authors' judgement & Support for judgement \\
\hline $\begin{array}{l}\text { Random sequence genera- } \\
\text { tion (selection bias) }\end{array}$ & Low risk & Drawing of lots \\
\hline $\begin{array}{l}\text { Allocation concealment } \\
\text { (selection bias) }\end{array}$ & Unclear risk & Insufficient detail regarding allocation concealment \\
\hline $\begin{array}{l}\text { Blinding of participants } \\
\text { and personnel (perfor- } \\
\text { mance bias) } \\
\text { All outcomes }\end{array}$ & High risk & $\begin{array}{l}\text { Participants unlikely to have been adequately blinded owing to the nature of } \\
\text { the intervention }\end{array}$ \\
\hline
\end{tabular}


DeGarmo 2009 (Continued)

Incomplete outcome data Low risk Attrition relatively low and no more than $18 \%$ at all follow-up time points, av(attrition bias) eraging $13.5 \%$ overall; study authors accounted for missing data using FIML

All outcomes method

Selective reporting (re- Unclear risk No published protocol

porting bias)

Other bias Low risk No other forms of bias evident

Dolan 2010

Methods

Study name: Big Brothers Big Sisters Ireland

Study design (e.g. RCT, cluster RCT): RCT

Intervention arm(s): Big Brothers Big Sisters (Ireland)

Comparator arm (s): treatment as usual/waitlist control

Sample size calculation performed: yes; target of 200 participants for "sufficient statistical power, with an expected effect size of a Cohen's $d$ of just under 0.2"

Subgroups prespecified: yes; age, duration, family and community context, interpersonal history

Subgroup analyses: adherence to recommended match lengths and frequency of meeting; perceived quality or closeness of the match; family context; age; gender

Start date: summer 2007

Duration of follow-up: 24 months post baseline

Number of follow-ups: 4

Follow-up time points: scheduled to be 12,18 , and 24 months after baseline. In reality, this was (on average) $10.52,15.71$, and 21.18 months after baseline

ICC (if reported): N/A

\section{Number of schools randomised: N/A}

Number of participants randomised (total and by arm): 164 overall (intervention: 84, control: 80)

Age (range or mean (SD)) or grade at the start: age at completion of baseline survey: $5 \% 10$ years, $23 \% 11$ years, $26 \% 12$ years, $23 \% 13$ years, $16 \% 14$ years, $5 \% 16$ years, $2 \% 16$ years

Gender: $49 \%$ male, $51 \%$ female

Ethnicity: $87 \%$ Irish, $7 \%$ Irish Traveller, $2 \%$ any other white background, $1 \%$ African, $1 \%$ any other Asian background, $1 \%$ other

SES: not reported

Inclusion criteria: aged 10 to 18 years. Meets 1 or more criteria for referral: culturally or economically disadvantaged, exhibits poor social skills, has few friends, lacks adequate support and attention of a stable adult, is an underachiever in school, is overly dependent, is insecure and does not trust adults, has other siblings with significant problems with social or community adjustment, has a poor self-concept, is introverted, shy, or withdrawn, shows early signs of antisocial behaviour, has needs that are appropriate for volunteer intervention

Exclusion criteria: none stated 
Dolan 2010 (Continued)

Interventions

\section{Randomisation before or after baseline survey: N/S}

Duration of the intervention (excluding follow-up): average 12 months ( $7 \%=0$ to 6 months, $36 \%=7$ to 12 months, $57 \%$ = over 12 months)

Description of the intervention: adapted from the Big Brothers Big Sisters (USA) programme, to an Irish context. Young people are matched with an older mentor and meet for 1 to 2 hours per week for at least 1 year. The match is supposed to be primarily about friendship and interests.

Brief description of the theoretical model: Rhodes' model of youth mentoring, 'Pathways of mentoring influence'. A strong friendship between mentor and mentee will result in a number of positive outcomes, including reduced engagement in risk behaviour, through the mentor being a positive role model and providing a safe place to talk about issues such as drugs and alcohol.

Description of the comparator: offered regular project activities by the Foróige Services, also received the intervention after study end (delayed control)

Outcomes Primary outcomes: alcohol use, cannabis use, emotional symptoms (subscale of SDQ), conduct problems (subscale of SDQ), academic performance

Secondary outcomes: N/S

Setting

Country: Ireland; State: Western Ireland

Setting: individual (community)

Focus: targeted: 'at-risk' individuals referred by services

Process measures Process data reported: yes

Method (qualitative or quantitative): both

Description: $\mathrm{N}$ and \% of various process and intervention acceptability, reach, and intervention fidelity outcomes reported, alongside 21 in-depth interviews with youth/mentor matches, which also explored intervention acceptability

Statistics Sample size: 164

Unit of randomisation: individual

Unit of analysis: individual

Method to promote equivalence between groups: N/S

Statistical models: multi-level regression analysis. Analyses were conducted for 5 imputed datasets, then results were pooled across these.

Baseline differences adjustment: none reported

Repeated measures methods in analysis: no; repeated measures analysis would not work for their data because the times between interviews were not equal - they did not happen at the scheduled times

Notes

Equity: see 'Participants' section above. No additional baseline measures assessed regarding 'equity'

Funding: "Funding for this study was provided by Foróige, with support from The Atlantic Philanthropies".

Randomisation method, e.g. block, stratification, computer: stratified randomisation, conducted by the mathematics department at NUI, Galway. Participants blocked by gender and location

Clustering accounted for in sample size calculation (if relevant): N/A 
Dolan 2010 (Continued)

Cluster randomisation methods to account for clustering in analysis: N/A

\section{Risk of bias}

\begin{tabular}{|c|c|c|}
\hline Bias & Authors' judgement & Support for judgement \\
\hline $\begin{array}{l}\text { Random sequence genera- } \\
\text { tion (selection bias) }\end{array}$ & Unclear risk & $\begin{array}{l}\text { Insufficient information provided: "Those who consented to take part in the } \\
\text { study were randomly assigned to either the intervention or control condition } \\
\text { using a stratified random approach" }\end{array}$ \\
\hline $\begin{array}{l}\text { Allocation concealment } \\
\text { (selection bias) }\end{array}$ & Unclear risk & Lack of clarity provided \\
\hline $\begin{array}{l}\text { Blinding of participants } \\
\text { and personnel (perfor- } \\
\text { mance bias) } \\
\text { All outcomes }\end{array}$ & High risk & Blinding was not possible given the nature of this intervention. \\
\hline $\begin{array}{l}\text { Blinding of outcome as- } \\
\text { sessment (detection bias) } \\
\text { All outcomes }\end{array}$ & High risk & Self-reported outcome measures only \\
\hline $\begin{array}{l}\text { Incomplete outcome data } \\
\text { (attrition bias) } \\
\text { All outcomes }\end{array}$ & Low risk & $\begin{array}{l}\text { Moderate attrition rates at final post-intervention follow-up. Study authors ac- } \\
\text { counted for missing data with multiple imputation. }\end{array}$ \\
\hline $\begin{array}{l}\text { Selective reporting (re- } \\
\text { porting bias) }\end{array}$ & Unclear risk & Protocol not published \\
\hline Other bias & High risk & $\begin{array}{l}\text { A lot of variability for when follow-up interviews actually occurred; on average, } \\
\text { working with a mentor began } 6.48 \text { (SD 3.64) months after the baseline inter- } \\
\text { view. This means that follow-up interviews may have been conducted } 6,12 \text {, } \\
\text { or } 18 \text { months after the intervention started. Dosage of the intervention varied } \\
\text { considerably between matches (see pages } 34 \text { to } 35 \text { for brief discussion of this } \\
\text { in Report 2). }\end{array}$ \\
\hline
\end{tabular}

Estrada 2015

Methods

Study design: RCT at the level of the family

Intervention $\operatorname{arm}(\mathbf{s})$ : Familias Unidas

Comparator arm (s): Community Practice Condition (CPC)

Sample size calculation performed: not reported

Subgroups prespecified: no

Subgroup analyses: N/A

Start date: not reported

Duration of follow-up: 24 months post baseline

Number of follow-ups: 3

Follow-up time points: 6,12 , and 24 months after baseline

ICC (if reported): N/A 
Estrada 2015 (Continued)

Participants
Number of schools randomised: N/A

Number of participants randomised (total and by arm): 160 (intervention: 72, control: 88)

Age (range or mean (SD)) or grade at the start: intervention: 15.3 years (0.89), control: 15.3 years (0.85)

Gender: intervention: $48.6 \%$ male, $51.4 \%$ female; control: $53.4 \%$ male, $46.6 \%$ female

Ethnicity: $100 \%$ Latino; $54.4 \%$ of adolescents were born in the USA. Of the foreign-born adolescents, $45.2 \%$ had been living in the USA for $<3$ years, $28.8 \%$ for 3 to 9 years, and $26 \%$ for $>9$ years (these adolescents/parents were primarily born in Cuba, Honduras, and Nicaragua).

SES: family income: intervention; control

$\$ 0$ to $\$ 9,999: 38.0 \% ; 39.8 \%$

$\$ 10,000$ to $\$ 19,999: 32.4 \% ; 34.1 \%$

$\$ 20,000$ to $\$ 29,999: 18.3 \% ; 11.4 \%$

$\geq \$ 30,000: 11.3 \% ; 14.8 \%$

Inclusion criteria: ninth-grade Latino adolescents and their primary caregivers in Miami-Dade County public high schools

Exclusion criteria: not reported

\section{Randomisation before or after baseline survey: not reported}

Duration of the intervention (excluding follow-up): 6 weeks

Description of the intervention: Familias Unidas: consisted of 5 parent group sessions, 3 parent-homework assignments, and 1 (parent-adolescent communication) family visit. Sessions were parent centred, with adolescents' participation in intervention activities limited to the family visit. Sessions took place on a weekly basis and consisted of topics such as enhancing communication and managing adolescent peer pressure. Each parent group session was 2 hours, and the family visit was 1 hour.

Brief description of the theoretical model: not reported

\section{Description of the comparator}

CPC: consisted of school-based HIV risk reduction intervention delivered by Miami-Dade County Public Schools health science teachers in classroom format and using portions of the evidence-based curriculum

\section{Outcomes}

Primary: substance use, sexual risk behaviour

Secondary: positive parenting, parental involvement, parent-adolescent communication

\section{Setting}

Country: USA; State: Miami

Setting: families (home)

Focus: Latino adolescents and their primary caregivers

Process measures

Process data reported: yes

Method (qualitative or quantitative): quantitative

Description: participants attended a mean number of 3.62 sessions (standard deviation $=2.09$ ), and $50 \%$ of families completed 5 or more of 6 sessions.

Statistics

ale size: eligible $N=239$ families; enrolled $N=160$ families (67\%); I = 72 families; $C=88$ families

Unit of randomisation: families

Unit of analysis: individual 
Method to promote equivalence between groups: not reported

Statistical models: growth curve analyses were used to estimate individual trajectories of change and to test for differences between conditions over time (b-intercept).

Baseline differences adjustment: baseline measures were comparable between groups.

Repeated measures methods in analysis: growth curve analyses

Notes

Equity: baseline data reported

Funding: Centers for Disease Control and Prevention, grant number U01PS000671

Randomisation method, e.g. block, stratification, computer: not reported

Clustering accounted for in sample size calculation (if relevant): N/A

Cluster randomisation methods to account for clustering in analysis: N/A

\begin{tabular}{|c|c|c|}
\hline \multicolumn{3}{|l|}{ Risk of bias } \\
\hline Bias & Authors' judgement & Support for judgement \\
\hline $\begin{array}{l}\text { Random sequence genera- } \\
\text { tion (selection bias) }\end{array}$ & Unclear risk & Not reported \\
\hline $\begin{array}{l}\text { Allocation concealment } \\
\text { (selection bias) }\end{array}$ & Unclear risk & Not reported \\
\hline $\begin{array}{l}\text { Blinding of participants } \\
\text { and personnel (perfor- } \\
\text { mance bias) } \\
\text { All outcomes }\end{array}$ & High risk & Blinding was not possible given the nature of this intervention. \\
\hline $\begin{array}{l}\text { Blinding of outcome as- } \\
\text { sessment (detection bias) } \\
\text { All outcomes }\end{array}$ & High risk & Not reported; self-reported outcomes only \\
\hline $\begin{array}{l}\text { Incomplete outcome data } \\
\text { (attrition bias) } \\
\text { All outcomes }\end{array}$ & Low risk & Low attrition and attrition comparable between groups \\
\hline $\begin{array}{l}\text { Selective reporting (re- } \\
\text { porting bias) }\end{array}$ & Low risk & No published protocol but all specified outcomes reported \\
\hline Other bias & Unclear risk & Control condition is described as an HIV prevention intervention. \\
\hline
\end{tabular}

Fearnow-Kenney 2003

Methods Study name: All Stars Senior

Study design: RCT at the level of the school

Intervention $\operatorname{arm}(\mathbf{s})$ : All Stars Senior

Comparator arm(s): no intervention/usual practice

Sample size calculation performed: N/S 
Fearnow-Kenney 2003 (Continued)

Subgroups prespecified: N/A; no subgroups

Subgroup analyses: no subgroups

Start date: 1999

Duration of follow-up: 1 school year

Number of follow-ups: 1

Follow-up time points: post-test

ICC (if reported): not reported

\section{Participants}

Number of schools randomised: 3 to intervention, 3 to control

Number of participants randomised (total and by arm): 406 randomised to intervention, 247 to control

Age (range or mean (SD)) or grade at the start: 13 to 19 years

Gender: male-to-female ratio 40:60\% (0.6 M:1 F in intervention group; 0.8 M:1 F in control group)

Ethnicity: intervention: white 46.2\%, African American 44.2\%, Asian 1.9\%, Hispanic 1.4\%, Native American 1.2\%, Pacific Islanders or others 5.1\%; control: white 53.6\%, African American 27.8\%, Asian 7.9\%, Hispanic 2.0\%, Native American 2.4\%, Pacific Islander or other $6.3 \%$

SES: not reported

Inclusion criteria: $\mathrm{N} / \mathrm{S}$

Exclusion criteria: $\mathrm{N} / \mathrm{S}$

Duration of the intervention (excluding follow-up): 1 school year

Description of the intervention: All Stars Senior targeted (1) adoption of positive normative beliefs, (2) commitment to avoid high-risk behaviour, (3) lifestyle incongruence (e.g. perceiving substance use to not fit with one's desired lifestyle), (4) beliefs about social and psychological consequences, and (5) resistance skills. Teachers were asked to implement a minimum of 2 All Stars Senior activities per week of health instruction. Activities and implementation were led by the teacher.

Brief description of the theoretical model: not reported

Description of the comparator: no intervention/usual practice

Outcomes

Primary outcomes: lifestyle incongruence for drug use, nutrition and stress, normative beliefs regarding drug use, drunkenness, smoking, marijuana use, teacher perceptions of the programme

Secondary outcomes: exercise, bonding to school

\begin{tabular}{ll}
\hline Setting & Country: USA;State: North Carolina \\
& Setting: school \\
& Focus: young people aged 13 to 19 years \\
\hline Process measures & Process data reported: yes \\
Method (qualitative or quantitative): quantitative \\
Description: 4 of the 5 treatment teachers participated in a focus group. Teachers reported that All \\
Stars Senior had been successfully incorporated into their teaching, and that the interactive lessons \\
were effective in involving all students. They also intended to use the programme in the future. They
\end{tabular}


Fearnow-Kenney 2003 (Continued)

liked the system that linked activities with major high school health textbooks. They also preferred shorter activities because of the competing time demands of teaching and fulfilling administrative duties. The greatest concern expressed by teachers involved the degree of training and experience needed to implement the activities, as the approach was new and complex; it was suggested that training be incorporated.

Six focus groups were conducted with groups of 8 to 10 students each. Students enjoyed activities that required active student involvement (e.g. debates, role-plays) and allowed them to learn about the attitudes and behaviours of their classmates. Students especially enjoyed discussing sensitive topics. Overall, students reported that the programme improved their health class and made it more interesting. Suggestions were made to improve the Wellness Journal and several class activities.

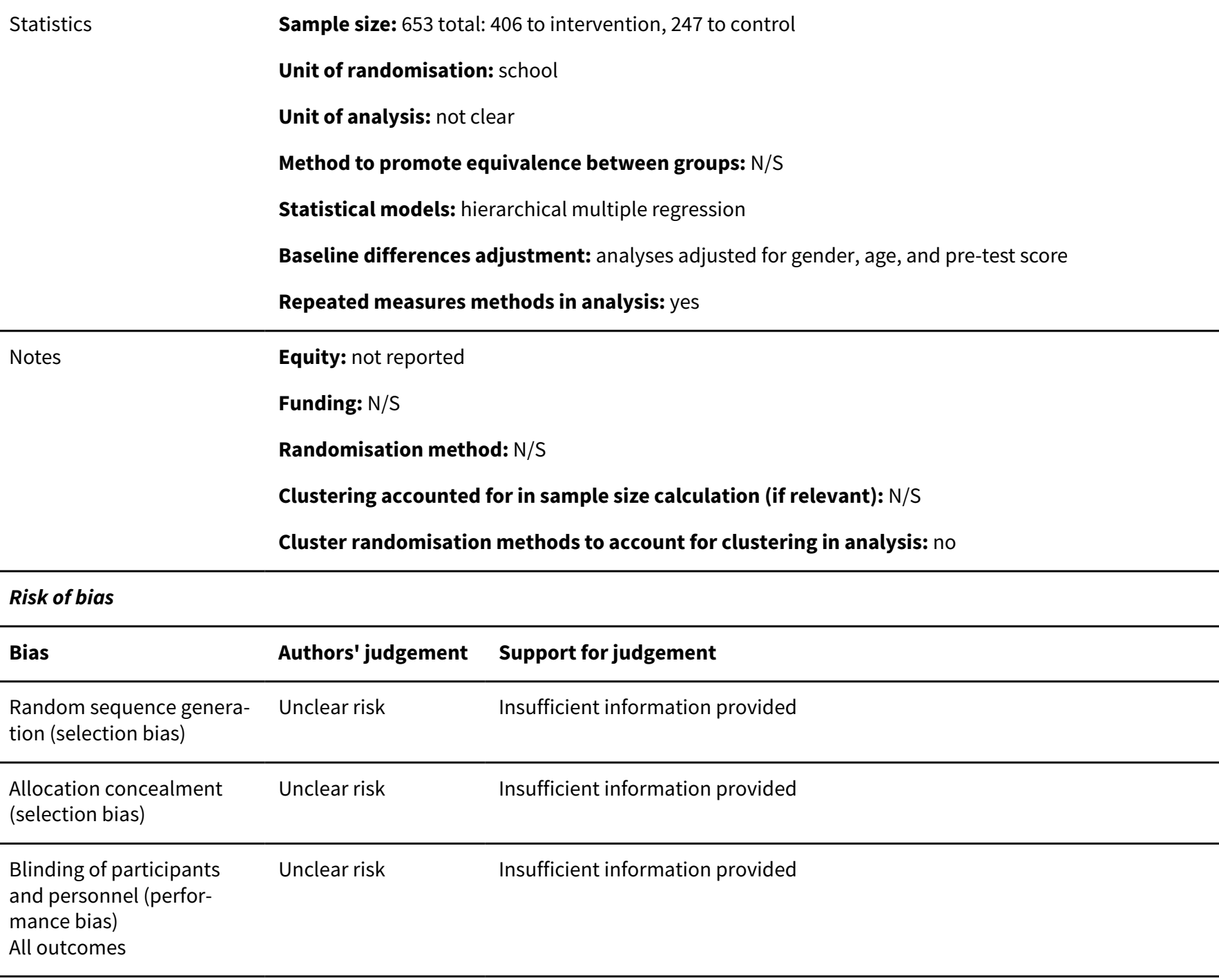

Blinding of outcome as- Unclear risk Insufficient information provided
sessment (detection bias) All outcomes

Incomplete outcome data Unclear risk Insufficient information provided
(attrition bias)
All outcomes

\begin{tabular}{ll}
\hline $\begin{array}{l}\text { Selective reporting (re- } \\
\text { porting bias) }\end{array}$ & Unclear risk \\
\hline
\end{tabular}


Fearnow-Kenney 2003 (Continued)

Other bias Unclear risk Insufficient information provided

Flay 2004a

Methods

Study name: Aban Aya

Study design (e.g. RCT, cluster RCT): cluster RCT at the level of the school $(n=12)$

Intervention $\operatorname{arm}(\mathbf{s}): 2$

(1) Social Development Curriculum (SDC)

(2) Social/Community Intervention (SCI)

Comparator arm(s): Health Enhancement Curriculum (HEC)

Sample size calculation performed: not clear

Subgroups prespecified: no

Subgroup analyses: boys and girls

Start date: school year 1994 to 1995

Duration of follow-up: 3 years

Number of follow-ups: 4

Follow-up time points: wave 1: end of grade 5 (year 1); wave 2: end of grade 6 (year 2); wave 3: end of grade 7 (year 3 ); wave 4 : end of grade 8 (year 4 )

ICC: not reported

Participants

Number of schools randomised: 12

Number of participants randomised (total and by arm): no information about the original numbers in each arm at baseline

Age (range or mean (SD)) or grade at the start: mean age at start 10.8 years (SD 0.6); start fifth grade, followed-up at end of fifth, sixth, seventh, eighth grades

Gender: final sample $49.5 \%$ male

Ethnicity: schools were 91\% African American; all participants were African American.

SES: grade 5 - 77\% received federally subsidised school lunches; $47 \%$ lived with 2 parents

Inclusion criteria: school inclusion criteria: enrolment $>80 \%$ African American and $<10 \%$ Latino or Hispanic students; grades kindergarten to grade 8 (or grade 6 if students were tracked to 1 middle school); more than 500 students enrolled; not on probation or slated for reorganisation; not a specially designated school (e.g. academic centre); moderate mobility $(<50 \%$ annual turnover, e.g. approximately $<25 \%$ transferred in and $<25 \%$ transferred out)

Exclusion criteria: not reported

Interventions

Randomisation before or after baseline survey: not reported; likely to be after randomisation of eligible schools

Duration of the intervention (excluding follow-up): 4 years (1994 through 1998)

Description of the intervention 
Flay 2004a (Continued)

\begin{abstract}
Aban Aya
The SDC teaches cognitive-behavioural skills to build self-esteem and empathy, manage stress and anxiety, develop interpersonal relationships, resist peer pressure, and develop decision-making, problem-solving, conflict-resolution, and goal-setting skills. Application of these skills enables youths to avoid violence, provocative behaviour, school delinquency, drug use, and unsafe sexual behaviour. SDC is classroom based with 16 to 21 lessons/year from grade 5 to 8 .

The SCl included the SDC with parental support, school climate, and community components to influence all children's social domains. The parent support programme reinforced skills and promoted child-parent communication. Each SCl school formed a local school task force of school personnel, students, parents, community advocates, and project staff to implement programme components, propose changes in school policy, develop other school-community liaisons supportive of school-based efforts, and solicit community organisations to conduct activities to support the $\mathrm{SCl}$.
\end{abstract}

Brief description of the theoretical model: theories of behaviour change drew on the theory of triadic influence and focused on risk and protective factors and skills related to target behaviours. The study also incorporated Nguzo Saba principles to promote African American cultural values.

Description of the comparator: HEC involved the same number of lessons as SDC and taught some of the same skills (e.g. decision-making, problem-solving). The focus was on promoting healthy behaviours related to nutrition, physical activity, and general health care. It integrated the importance of cultural pride and communalism.

Outcomes

Primary outcomes: violence, school delinquency, substance use from grade 5 to 8 ; provoking behaviour, recent sexual intercourse, condom use from grade 6 to 8

Secondary outcomes: N/A

Setting

Country: USA; State: Chicago, Illinois

Setting: 12 schools in a metropolitan area (9 inner city, 3 near-suburban)

Focus: targeted: predominantly African American schools, high-risk samples

Process measures Process data reported: yes, in relation to sustaining the programme

Method (quantitative or qualitative): both

Description: interviews were conducted with key actors on school and research teams; surveys were completed indicating the percentage of the lesson that the parent educator (PE) taught and the classroom management techniques used (i.e. to provide ratings of programme implementation); and PEs gave their perspectives on lesson delivery. Visits were made to study schools for observation.

Statistics

Sample size: eligible schools $n=141$ inner city; $n=14$ near-suburban. $N=1153$ fifth grade students eligible for analysis.

Unit of randomisation: schools

Unit of analysis: individuals

Method to promote equivalence between groups: stratification and blocking

Statistical models: hierarchical models to accommodate nesting of data for time within participants, participants within schools, and missing data. Continuous outcomes - mixed models; ordinal outcomes - GEE

Baseline differences adjustment: not reported

Repeated measures methods in analysis: accounted for in GEE model

Notes

Equity: final sample was $49.5 \%$ male; approximately $77 \%$ received federally subsidised school lunches, and $47 \%$ lived in 2-parent households 
Flay 2004a (Continued)

Funding: National Institute on Drug Abuse, National Institute for Child Health and Human Development

Randomisation method: eligible schools were stratified into 4 quartiles of risk; a randomised block design was used to assign 2 inner city schools from the middle of the highest risk quartile, 1 inner city school from the middle of the second risk quartile, and 1 near-suburban school (from the second quartile) to each condition.

Clustering accounted for in sample size calculation (if relevant): not reported

Cluster randomisation methods to account for clustering in analysis: hierarchical statistical models were used to accommodate nested observations (time within participants, participants within schools). Mixed models were used for continuous outcomes, generalised estimating equations for ordinal outcomes.

\section{Risk of bias}

\begin{tabular}{lll}
\hline Bias & Authors' judgement & Support for judgement \\
\hline $\begin{array}{l}\text { Random sequence genera- } \\
\text { tion (selection bias) }\end{array}$ & Unclear risk & Random sequence generation information not provided \\
\hline $\begin{array}{l}\text { Allocation concealment } \\
\text { (selection bias) }\end{array}$ & Unclear risk & No information provided \\
\hline $\begin{array}{l}\text { Blinding of participants } \\
\text { and personnel (perfor- } \\
\text { mance bias) }\end{array}$ & Unclear risk & $\begin{array}{l}\text { Attention control. "The control condition was the health enhancement cur- } \\
\text { riculum (HEC). It consisted of the same number of lessons as the SDC and } \\
\text { taught some of the same skills (eg, decision making and problem solving), but } \\
\text { with a focus on promoting healthy behaviours related to nutrition, physical ac- } \\
\text { tivity, and general health care" }\end{array}$ \\
\hline
\end{tabular}

Blinding of outcome as- High risk

Students were assessed by self-reports, not validated.

sessment (detection bias)

All outcomes

Incomplete outcome data High risk $\quad 38 \%$ attrition from baseline to grade $8 ; 20 \%$ turnover per year
(attrition bias)

All outcomes

Selective reporting (re- Unclear risk No published protocol porting bias)

Other bias Unclear risk Lack of detail about participants at baseline

Freidman 2002

Methods Study name: Botvin LIfe Skills Training, Prothrow-Stith Anti-Violence Program and Values Clarification

Study design (e.g. RCT, cluster RCT): RCT

Intervention $\operatorname{arm}(\mathbf{s})$ : Group A - basic residential programme plus LST, AV, and VC

Comparator arm(s): Group C - basic residential programme

Sample size calculation performed: none, but data were adjusted in the analysis to ensure adequate power

Subgroups prespecified: none 
Freidman 2002 (Continued)

\section{Subgroup analyses: none}

Start date: not reported

Duration of follow-up: 6 months post discharge

Number of follow-ups: 2

Follow-up time points: wave 1: group A - post assessment at time of discharge from SGH, at 9 months after admission; wave 2: group A - follow-up assessment at 6 months post discharge ( 15 months after admission to SGH)

ICC (if reported): N/A

Participants

Number of schools randomised: N/A

Number of participants randomised (total and by arm): $N=201 ;$ group $A=110$; group $C=91$

Age (range or mean (SD)) or grade at the start: age range 13 to 18 years; mean age 15.5 years; mean years of education 8.5 years

Gender: male

Ethnicity: group A, group C

Black $76 \%, 69 \%$

White $14 \%, 17 \%$

Puerto Rican 7\%, 9\%

Asian 2\%, 3\%

SES: low or lower middle class (Hollingshead rating of head of household 6.3)

Business manager $3 \%, 1 \%$

Administrative $9 \%, 10 \%$

Clerical/sales $11 \%, 6 \%$

Inclusion criteria: adolescent males aged 13 to 18 years residing at SGH who were convicted of at least 1 illegal offence serious enough to be brought before a court of law

Exclusion criteria: youths who were AWOL from SGH; or were considered unmanageable in the usual classroom setting and were transferred to "cluster D"; or who were removed from SGH and were committed at another facility; or whose parents did not consent to study participation

Interventions

Randomisation before or after baseline survey: before randomisation - "all new admissions received the project's pre-intervention assessment"

Duration of the intervention (excluding follow-up): unclear duration: 55 sessions for classroom programme; 20 sessions of 55 minutes each for anti-violence programme

\section{Description of the intervention}

Botvin Life Skills Training (LST) model; Prothrow-Stith Anti-Violence Program (AV); and Values Clarification Procedure (VC)

(1) Group A (intervention group) used a cognitive-behavioural social learning model to understand effects of substances and to learn to deal with influence or coercion, to control behaviour, and to enhance personal and social skills; and a social learning model to control violent tendencies and to develop a set of socially acceptable and desirable values. The programme involved instruction, demonstration, feedback reinforcement, and behavioural rehearsal (practice during class) for 20 sessions, over a 4 week period. 
(2) Prothrow-Stith Anti-Violence Programme (AV) involved 20 sessions of 55 minutes to increase awareness of the causes and effects of violence, factors leading to violence, prevention, and alternatives to violence.

(3) Values Clarification is a social-cognitive procedure that directs young people to develop and adopt their own, individualised value system. The VC procedure consists of 7 operations labelled as "process valuing" to assist participants to become more aware of the values implied by their behaviour. Typical areas explored are politics, religion, work, love, sex, family, friends, and drugs.

Brief description of the theoretical model: The Botvin Life Skills Training (LST) model asserts that substance use behaviour is the result of interplay of social and intra-personal factors (e.g. cognition, attitudes, expectations, personality).

The Prothrow-Stith Anti-Violence Programme (AV) and VC are social-cognitive procedures.

Description of the comparator: basic residential programme

$\begin{array}{ll}\text { Outcomes } & \text { List relevant outcomes } \\ & \text { Primary outcomes: drug use; alcohol use; illegal offences; illegal violent behaviour; drug selling; } \\ \text { school problems }\end{array}$

Secondary outcomes: not clear

Setting Country: USA; State: Pennsylvania; Place: Philadelphia

Setting: residential facility for court-adjudicated adolescent males (St Gabriel's Hall - SGH)

Focus: targeted: inner city, low SES, court-adjudicated males convicted of at least 1 offence

Process measures

Process data reported: acceptability of the intervention, adherence to the intervention, intensity of the intervention

Method (qualitative or quantitative): quantitative

\section{Description}

Acceptability of the intervention: In the "Information about cigarettes, alcohol and drugs" section of the Botvin LST modality, $84 \%$ of participants reported that it "was interesting to me"; $82 \%$ reported, "I learned something that I didn't know before"; and $82 \%$ reported that it "was useful or helpful to me". A high percentage of responses were positive about Botvin LST "Information about Social Skills" section.

Responses to 10 different questions regarding their degree of interest in, and the helpfulness to them, of the following:

For the P-S Anti-Violence modality, the mean response of participants to the first 9 questions was midway between "A fair amount" and "A lot". The tenth question was not as acceptable ("Did you like this course better than most other school courses?"), with a mean response of approximately "A little".

Adherence to the intervention: group A participants attended an average of 34.2 classroom sessions of 55 scheduled sessions of the triple-modality classroom programme.

Intensity of the intervention: LST Programme: 12 curriculum units conducted in 20 sessions, over a 4week period. Anti-Violence Programme: conducted in 20 sessions of 55 minutes each; Values Clarification: no information on intensity

Statistics Sample size: $N=201$

Unit of randomisation: individual

Unit of analysis: individual

Method to promote equivalence between groups 
Statistical models: multiple regression

Baseline differences adjustment: dependent variable was regressed individually on each covariate to observe the significant zero-order correlations; only significant covariates were used in the multiple regression analysis.

\section{Repeated measures methods in analysis: N/A}

Equity: baseline data by allocation group
Funding: Center for Substance Abuse Prevention
Within the participant group, those who attended more of the P-S Anti-Violence sessions and were rat-
ed as showing more critical thinking about problems were found at follow-up to report significantly
less violent behaviour. Conversely, those rated as "offering more comments" during these sessions, re-
ported significantly more violent behaviour at follow-up.

Randomisation method, e.g. block, stratification, computer: randomisation method was planned but was not described.

Clustering accounted for in sample size calculation (if relevant): N/A

Cluster randomisation methods to account for clustering in analysis: N/A

\section{Risk of bias}

\begin{tabular}{|c|c|c|}
\hline Bias & Authors' judgement & Support for judgement \\
\hline $\begin{array}{l}\text { Random sequence genera- } \\
\text { tion (selection bias) }\end{array}$ & Unclear risk & A planned randomisation procedure was not described. \\
\hline $\begin{array}{l}\text { Allocation concealment } \\
\text { (selection bias) }\end{array}$ & Unclear risk & Not reported \\
\hline $\begin{array}{l}\text { Blinding of participants } \\
\text { and personnel (perfor- } \\
\text { mance bias) } \\
\text { All outcomes }\end{array}$ & High risk & Participants and personnel cannot be blinded to allocation status. \\
\hline $\begin{array}{l}\text { Blinding of outcome as- } \\
\text { sessment (detection bias) } \\
\text { All outcomes }\end{array}$ & High risk & Self-reported outcomes \\
\hline $\begin{array}{l}\text { Incomplete outcome data } \\
\text { (attrition bias) } \\
\text { All outcomes }\end{array}$ & High risk & $\begin{array}{l}\text { Although attrition was relatively low (16\%) at follow-up, results show signifi- } \\
\text { cant differences between allocation groups of those who were not retrieved. }\end{array}$ \\
\hline $\begin{array}{l}\text { Selective reporting (re- } \\
\text { porting bias) }\end{array}$ & Unclear risk & No published protocol \\
\hline Other bias & Unclear risk & $\begin{array}{l}\text { Participants in intervention and control arms were from the same residential } \\
\text { home. No information about the original numbers in each arm at baseline. } \\
\text { Baseline differences in original sample not reported - only those included in } \\
\text { analyses reported }\end{array}$ \\
\hline
\end{tabular}

Methods Study name: REAL MEN


Intervention arm(s): 3 experimental arms: (1) REAL MEN delivered only in educational sessions, (2) REAL MEN delivered only in community settings, and (3) REAL MEN delivered in both settings

Comparator arm(s): no REAL MEN intervention, just a jail-based discharge planning session, as per usual practice

\section{Sample size calculation performed: N/S}

Subgroups prespecified: unclear

Subgroup analyses: by extent of engagement with the programme

Start date: 2003 (recruitment commenced)

Duration of follow-up: 12 months post discharge

Number of follow-ups: 1 (12 months' follow-up)

Follow-up time points: 12 months post intervention

ICC (if reported): N/A

\section{Participants}

\section{Number of schools randomised: N/A}

Number of participants randomised (total and by arm): 552 participants at baseline: 277 randomised to intervention, 275 randomised to control

Age (range or mean (SD)) or grade at the start: mean age 18 years at baseline

Gender: $100 \%$ male

Ethnicity: African American (55.8\%), Latino (38.1\%), missing data (6.1\%)

SES: N/S

Inclusion criteria: males aged 16 to 18 years, individuals determined to be eligible for release within 12 months of enrolment and who plan to return to the Bronx, Brooklyn, or Manhattan once discharged

Exclusion criteria: individuals with psychiatric conditions that would preclude participation in a group intervention

\section{Randomisation before or after baseline survey: after}

Duration of the intervention (excluding follow-up): 18 hours (jail-based intervention), 12 hours (intervention within community setting)

Description of the intervention: consisted of 2 core components: jail and community. The jail component consisted of 5 educational sessions (getting ready for going home, staying healthy to stay free - what about HIV, being a REAL MAN in today's world, sex in the risk zone, and my people, my pride). These 5 sessions collectively took 18 hours to deliver. An additional 3 educational sessions were offered in the community after participant discharge (drugs in your life, getting the information you need to stay free, and staying free and healthy for life). Collectively, these took 12 hours to be delivered. The community component of the REAL MEN intervention was optional and consisted of family meetings, open groups for discussion, special excursions, drug treatment, healthcare services, and referrals to other community services, as deemed necessary.

Brief description of the theoretical model: no

Description of the comparator: control group receive jail-based discharge planning session.

Outcomes Primary outcomes: drug use, risky sexual behaviour, criminal justice involvement, education/work post release 
Freudenberg 2010 (Continued)

\section{Secondary outcomes: N/S}

Country: USA, New York City
Setting $\quad$ Focus: targeted; male participants recruited in prisons

\begin{tabular}{ll}
\hline Process measures & Process data reported: no \\
& Method (qualitative or quantitative): N/A \\
& Description: N/A \\
\hline Statistics & Sample size: 552 participants at baseline \\
Unit of randomisation: individual \\
Unit of analysis: individual \\
Method to promote equivalence between groups: none stated \\
Statistical models: logistical regression and odds ratios, ordinary least squares regression \\
Baseline differences adjustment: all were adjusted for. \\
Repeated measures methods in analysis: N/A; only 1 follow-up period reported (at 12 months post \\
baseline)
\end{tabular}

Notes

Equity: descriptive data (age, ethnicity, prior arrests) provided at both baseline and follow-up

Funding: National Institute of Drug Abuse grant number R01 DA014725

Randomisation method, e.g. block, stratification, computer: N/S

Clustering accounted for in sample size calculation (if relevant): N/A; non-CRCT

Cluster randomisation methods to account for clustering in analysis: N/A

\begin{tabular}{|c|c|c|}
\hline \multicolumn{3}{|l|}{ Risk of bias } \\
\hline Bias & Authors' judgement & Support for judgement \\
\hline $\begin{array}{l}\text { Random sequence genera- } \\
\text { tion (selection bias) }\end{array}$ & Unclear risk & Method of random sequence generation not reported \\
\hline $\begin{array}{l}\text { Allocation concealment } \\
\text { (selection bias) }\end{array}$ & Unclear risk & Not reported \\
\hline $\begin{array}{l}\text { Blinding of participants } \\
\text { and personnel (perfor- } \\
\text { mance bias) } \\
\text { All outcomes }\end{array}$ & High risk & Not possible to blind participants owing to the nature of this intervention \\
\hline $\begin{array}{l}\text { Blinding of outcome as- } \\
\text { sessment (detection bias) } \\
\text { All outcomes }\end{array}$ & High risk & Self-reported outcomes in unblinded intervention \\
\hline $\begin{array}{l}\text { Incomplete outcome data } \\
\text { (attrition bias) } \\
\text { All outcomes }\end{array}$ & Unclear risk & Insufficient information provided \\
\hline
\end{tabular}


Freudenberg 2010 (Continued)
Selective reporting (re-
Unclear risk
No published protocol porting bias)

Other bias

Low risk

No other forms of bias evident

Gonzales 2012

Study name: Bridges to High School (Bridges/Puentes)
Study design (e.g. RCT, cluster RCT): RCT at the level of the family
Intervention $\operatorname{arm}(\mathbf{s})$ : Bridges to High School
Comparator arm(s): control
Sample size calculation performed: N/S
Subgroups prespecified: N/A
Subgroup analyses: N/A
Start date: not given
Duration of follow-up: 5 years post intervention
Number of follow-ups: 3
Follow-up time points: 1,2 , and 5 years post intervention
ICC (if reported): N/A

Participants

Number of schools randomised: N/A

Number of participants randomised (total and by arm): 338 to intervention, 178 to control

Age (range or mean (SD)) or grade at the start: 12.3 years (SD 0.54)

Gender: $49.2 \%$ males, $50.8 \%$ females; $98.6 \%$ participated with mothers; $55.8 \%$ participated with fathers

Ethnicity: all participants were Mexican American adolescents.

SES: not given

Inclusion criteria: adolescent of Mexican descent, at least 1 caregiver of Mexican descent who was interested in participating, willingness of the family to be randomly assigned to the 9-week intervention or a brief workshop

Exclusion criteria: none given

Description of the intervention: Bridges to High School Program (Bridges) was a combined parentand youth-focused intervention that aimed to bring parents and students together to increase school engagement and decrease mental health symptoms and risky behaviours following the middle school transition. The programme involved 9 weekly evening group sessions ( 2 hours total) at the adolescents' schools and 2 home visits (pre-intervention and mid-programme). The 9 sessions included separate simultaneous 1.25 -hour groups for adolescents and parents followed by a 0.75 -hour conjoint family session. The parenting group aimed to increase effective parenting practices, family cohesion, and promo- 
tion of school engagement. The adolescent groups aimed to increase adolescents' coping efficacy, academic engagement, and family cohesion.

Brief description of the theoretical model: social development model

Description of the comparator: brief 1.5-hour evening workshop for parents and adolescents in which participants received handouts on school resources, discussed barriers to school success, and discussed barriers to middle school success. No teaching of specific skills to promote school success was included.

Outcomes

Primary outcomes: mental health, substance use, school dropout, externalising symptoms

Secondary outcomes: mediators were measured including effective parenting, family cohesion, adolescent coping efficacy, and school engagement.

\section{Setting}

Country: USA (southwestern metropolitan area)

Setting: home and community

Focus: family programme targeted to Mexican Americans

Process measures Process data reported: yes

Method (qualitative or quantitative): quantitative and qualitative

Description: of families randomised to Bridges/Puentes, $64 \%$ attended at least 5 and $33 \%$ attended all 9 sessions. Spanish families attended significantly more sessions; the mean number of sessions was $5.81,4.50$, and 6.15 , respectively, for mothers, fathers, and adolescents in the Spanish sample, and $4.69,3.57$, and 4.91, respectively, for mothers, fathers, and adolescents in the English sample. These attendance statistics include families that did not attend any sessions (11.29\% Spanish, 22.80\% English).

Video recordings of the intervention sessions were coded for adherence by independent raters who determined the extent to which the programme curriculum, both content and processes, was delivered as specified in the programme manual. Across cohorts, group leaders received a median score of $90 \%$ correct on tests of session content before each session. Videos for all intervention sessions were coded for adherence by independent raters, with an average inter-rater agreement of $90 \%$. Results indicated that $91 \%$ of adolescent and $88 \%$ of parent programme components were delivered.

Control groups were not videotaped. However, the control workshop was rated positively by parents (mean 4.44, SD 0.52; 1 = awful, 5 = wonderful) and teens (mean 4.34, SD 0.78) at the post-test interview, and ratings did not differ significantly from parent (mean 4.51, SD 0.55) and teen ratings (mean 4.39, SD 0.79) of the intervention.

Unit of analysis: individual (parent and adolescent)

Method to promote equivalence between groups: random number generator was used programmed with the appropriate probabilities to randomise all families that completed wave 1 data collection. A greater proportion of families were randomised to the intervention condition to ensure adequate intervention group size at each school. A greater proportion of English families were also randomised to the intervention group vs the control group (70:30) compared to Spanish families (60:40) because pilot testing showed higher retention for Spanish families.

Statistical models: ANCOVA and path models to test mediated effects. Missing data were handled using full information maximum likelihood

Baseline differences adjustment: yes

Repeated measures methods in analysis: not clear 
Gonzales 2012 (Continued)

Notes

Equity: baseline data reported

Funding: National Institute of Mental Health grant R01 MH64707 and grant T32 MH018387

Randomisation method, e.g. block, stratification, computer: random number generator

Clustering accounted for in sample size calculation (if relevant): N/A

Cluster randomisation methods to account for clustering in analysis: N/A

\section{Risk of bias}

\begin{tabular}{|c|c|c|}
\hline Bias & Authors' judgement & Support for judgement \\
\hline $\begin{array}{l}\text { Random sequence genera- } \\
\text { tion (selection bias) }\end{array}$ & Low risk & Random number generator used \\
\hline $\begin{array}{l}\text { Allocation concealment } \\
\text { (selection bias) }\end{array}$ & Unclear risk & Lack of clarity provided \\
\hline $\begin{array}{l}\text { Blinding of participants } \\
\text { and personnel (perfor- } \\
\text { mance bias) } \\
\text { All outcomes }\end{array}$ & High risk & $\begin{array}{l}\text { Not clear how alternative intervention was communicated to participants, and } \\
\text { not clear whether those delivering the intervention were blind }\end{array}$ \\
\hline $\begin{array}{l}\text { Blinding of outcome as- } \\
\text { sessment (detection bias) } \\
\text { All outcomes }\end{array}$ & Low risk & $\begin{array}{l}\text { Teachers were blind to random assignment, but students self-reported out- } \\
\text { comes. }\end{array}$ \\
\hline $\begin{array}{l}\text { Incomplete outcome data } \\
\text { (attrition bias) } \\
\text { All outcomes }\end{array}$ & Low risk & $\begin{array}{l}\text { Language was the only baseline variable found to be associated with missing } \\
\text { data; this was included in all analyses for missing data adjustments. }\end{array}$ \\
\hline $\begin{array}{l}\text { Selective reporting (re- } \\
\text { porting bias) }\end{array}$ & Unclear risk & Insufficient information provided \\
\hline Other bias & Low risk & No other sources of bias identified \\
\hline
\end{tabular}

\section{Gottfredson 2010}

\section{Methods}

Study name: All Stars

Study design (e.g. RCT, cluster RCT): cluster RCT

Intervention arm(s): All Stars prevention curriculum implemented in a year-long school-based after-school programme

Comparator $\operatorname{arm}(\mathbf{s})$ : treatment as usual with 1 after-school activity per month. A total of 8 occurred.

Sample size calculation performed: power calculated for that sample size

Subgroups prespecified: no

Subgroup analyses: none

Start date: start of school year in 2006

Duration of follow-up: at end of school year 
Gottfredson 2010 (Continued)

\section{Number of follow-ups: 1}

Follow-up time points: wave 1: post-test at end of school year

ICC (if reported): 0.000 to 0.0025 at Time 1

Participants Number of schools randomised: 5

Number of participants randomised (total and by arm): $\mathrm{N}=447 ; 5$ middle schools randomised to All Stars or control within each school; All Stars N $=224$, control $N=223$

Age (range or mean (SD)) or grade at the start: 11 to 14 years (mean 12.2 years), grades 6 to 8 (grade 6 $=42.3 \%$, grade $7=33.2 \%$, grade $8=24.5 \%$ )

Gender: 54\% male

Ethnicity: 70\% African American, 17\% Caucasian, 2\% Latino, 8\% Mixed race, 2\% a mixture of other races

SES: of 416 included cases, 59\% were eligible for subsidised school meals.

Inclusion criteria: All Stars programme was part of a larger study to test the effectiveness of an enhanced ASP model described in a report (Gottfredson 2009). Five urban schools volunteered to participate, and all students were invited.

Exclusion criteria: outcome analysis excluded those who did not provide outcome data (blank/refusal) and those who left Maryland schools.

Randomisation before or after baseline survey: not reported

Duration of the intervention (excluding follow-up): 1 school year (32 weeks)

\section{Description of the intervention}

\section{All Stars after-school programme (ASP)}

The ASP ran alongside the All Stars intervention and was operated for 3 hours/day and 3 days/week for 96 days (or 32 weeks) over the school year. All 5 sites followed the same daily schedule. The programme is divided into "core" (14 lessons, intention to prevent substance use and reduce bullying/violence/other conduct problems) and "plus" (13 lessons designed to re-enforce changes in attitudes and behaviours). In "core", lifestyle incongruence awareness is built, youths clarify their beliefs regarding prevalence and acceptability of risk behaviour by peers (normative behaviour), challenges are set (i.e. youths make public commitments to abstain from drugs), bonding is promoted with schools/community groups and other positive social organisations, and positive parental attention is provided through parent-child communication and parental modelling of student activities. In "plus", additional developmental skills are taught, including goal setting, persistence, a 5-step process for decision-making, and resistance skills.

An All Stars lesson is designed to be delivered in 1 session; ASP sites delivered each lesson in blocks of 45 minutes over 2 days.

Brief description of the theoretical model: social learning theory

Description of the comparator: control condition was "treatment as usual", except that members of the control group were invited to attend 1 after-school activity per month. Sites usually planned a special event or party for the days that control students were invited to attend. A total of 8 occurred.

Outcomes

\section{List relevant outcomes}

Primary outcomes: 13 scales were used to assess last month's drug use, disruptive classroom behaviour, aggression, delinquency, and 9 mediators targeted by All Stars Core and Plus curricula.

Secondary outcomes: tobacco, alcohol, marijuana, inhalant, other drug initiation 
Gottfredson 2010 (Continued)

Setting

Country: USA; State: Maryland; Place: Baltimore

Setting: students from 5 urban middle schools previously part of an All Stars school-based programme were invited to take part in the after-school programme.

Focus: universal

Process measures

Process data reported: yes

Method (qualitative or quantitative): quantitative

Description

Adherence to the intervention: Graduate student observers (attended 3 days of training) and programme staff assessed implementation of the programme. 80 site visits were made (14 to 18 visits/site) covering 66 All Stars sessions. Observers and staff members completed fidelity checklists. These checklists were used to rate the overall quality of the lesson, the level of student engagement, and the extent to which each activity in the lesson met its objective.

Reach of the intervention: the study design required enrolment of 50 treatment students/site; the number of youths registered varied across sites ( 36 to 60 ). On average, students attended 36 of 96 possible days (38\%). A low level of activity modification was reported by group leaders - 0.34 (range 0 to 2; $0=$ no modification; 2 = high levels of modification). Site A reported a significantly higher level of modification at the 0.05 level (0.67) when compared with other sites. The sites delivered an average of 26 of 27 possible All Stars lessons over the mean 102 lessons. Staff delivered lessons 'most of the time' for the planned 45 minutes.

Intensity of the intervention: All Stars programme delivered for 3 hours/day and 3 days/week for 96 days (or 32 weeks) approximately 8 months of the school year

Statistics

\section{Sample size: $N=447$}

Unit of randomisation: individuals

Unit of analysis: individuals

Method to promote equivalence between groups: not reported

Statistical models: programme effects were measured by a series of regression models controlling for pre-test levels of age, gender, race, and school site; OLS regression was used for continuous variables; delinquent behaviour was measured with binomial regression, and bonding with Poisson regression

Baseline differences adjustment: post-test means were adjusted for pre-test level of each variable, age, gender, ethnicity, and school site.

Repeated measures methods in analysis: N/A; only baseline and 1 follow-up presented

Notes

Equity: treatment and control students did not differ in demographic characteristics: age, grade, family income, gender, race, single-parent household, receipt of subsidised meals, or maternal education.

Funding: US Department for Education, Institute of Educational Sciences, University of Maryland Grant number R305F050069

Randomisation method, e.g. block, stratification, computer: students were randomised into treatment and control conditions by the principal investigator, using a random number generator in SPSS by a 50:50 ratio; randomisation appears to be blocked by school.

Clustering accounted for in sample size calculation (if relevant): clustering was accounted for in the analyses by a dummy variable.

Cluster randomisation methods to account for clustering in analysis: the number of clusters was < 10 , so dummy variables for schools were entered in all outcome analyses to correct for intercept differences between schools. 
Gottfredson 2010 (Continued)

Risk of bias

\begin{tabular}{|c|c|c|}
\hline Bias & Authors' judgement & Support for judgement \\
\hline $\begin{array}{l}\text { Random sequence genera- } \\
\text { tion (selection bias) }\end{array}$ & Low risk & Random number generator used \\
\hline $\begin{array}{l}\text { Allocation concealment } \\
\text { (selection bias) }\end{array}$ & Unclear risk & No information reported on whether or not allocation concealment occurred \\
\hline $\begin{array}{l}\text { Blinding of participants } \\
\text { and personnel (perfor- } \\
\text { mance bias) } \\
\text { All outcomes }\end{array}$ & High risk & Not possible to blind schools/participants \\
\hline $\begin{array}{l}\text { Blinding of outcome as- } \\
\text { sessment (detection bias) } \\
\text { All outcomes }\end{array}$ & High risk & Student self-reports and teacher reports likely to be high risk \\
\hline $\begin{array}{l}\text { Incomplete outcome data } \\
\text { (attrition bias) } \\
\text { All outcomes }\end{array}$ & Low risk & $\begin{array}{l}\text { Missing data were minimal for most measures used in the analyses. Missing } \\
\text { data were imputed for decision-making when losses exceeded } 5 \% \text {. }\end{array}$ \\
\hline $\begin{array}{l}\text { Selective reporting (re- } \\
\text { porting bias) }\end{array}$ & Unclear risk & No published protocol \\
\hline Other bias & Low risk & None \\
\hline
\end{tabular}

\section{Griffin 2006}

\section{Methods}

Study name: Life Skills Training

Study design (e.g. RCT, cluster RCT): cluster randomised N = 56 schools; 2 intervention arms and 1 control arm

Intervention $\operatorname{arm}(\mathbf{s})$ : LST with 1-day training workshop for providers; LST with videotaped training

Comparator $\operatorname{arm}(\mathbf{s})$ : treatment as usual

Sample size calculation performed: not reported

Subgroups prespecified: no

Subgroup analyses: none

Start date: 1985 (month not specified)

Duration of follow-up: 10-year follow-up post intervention; followed up to age 24 in 1998

Number of follow-ups: 2

Follow-up time points: wave 1: grade 9 (post intervention); wave 2: high school seniors (approximately 3 years post intervention) and in young adulthood at approximately 11 years post intervention

ICC (if reported): not reported 
Number of participants randomised (total and by arm): baseline survey $N=5569$ before intervention started (grade 7)

Age (range or mean (SD)) or grade at the start: median age at final follow-up 24.6 years; grade 7 at start; grades 7, 8, 9 given intervention; grades 10 and 12 at follow-up

Gender: not reported

Ethnicity: majority white (91\%) at final follow-up

SES: participants primarily from middle class areas

Inclusion criteria: not reported

Exclusion criteria: not reported

\section{Life Skills Training (LST)}

Students are taught a range of cognitive-behavioural skills for building self-esteem, resisting peer pressure and media influences, managing anxiety, communicating effectively, developing personal relationships, and asserting one's rights, along with specific skills such as ways to be assertive in situations where there is interpersonal pressure from peers to engage in substance use. Material is provided to reinforce norms against substance use. Teaching is interactive and includes group discussion and skills training techniques such as demonstration, modelling, behavioural rehearsal, feedback and reinforcement, and behavioural "homework" assignments for out-of-class practice.

The 2 intervention groups received a drug abuse prevention programme composed of a primary year of 15 classes in the seventh grade, followed by 10 booster classes in eighth grade, and 5 in the ninth grade, totalling 30 class sessions

Brief description of the theoretical model: Life Skills Training teaches alcohol and drug resistance skills and is designed to facilitate the development of important personal and social skills. The prevention programme aims to reduce substance use by increasing general personal and social competence and to provide adolescents with knowledge and skills to resist social influences to engage in substance use.

Description of the comparator: treatment as usual control group

Outcomes List relevant outcomes

Primary outcomes: $\%$ at high HIV risk at 10-year follow-up post intervention

Secondary outcomes: high-risk substance use, alcohol and marijuana intoxication

Setting

Country: USA; Place: New York

Setting: 56 schools in middle-class suburban and rural areas of New York State

Focus: universal

Process measures

Process data reported: yes

Method (qualitative or quantitative): quantitative

Description

Adherence to the intervention: trained observers monitored randomly selected classes, taught by the teachers providing the prevention programme, over the 3 years of intervention. A quantitative as- 
Griffin 2006 (Continued)

sessment of the completeness of programme implementation was calculated from the proportions of curriculum points and objectives covered during each class session observed, and a cumulative implementation score was calculated for each student receiving the intervention.

Intensity of the intervention: 30 class sessions over 3 years from seventh to ninth grades

Statistics Sample size: $N=56$ schools; $N=5569$ students surveyed; 3815 received intervention; $n=1754$ controls

Unit of randomisation: school

Unit of analysis: individual

Method to promote equivalence between groups: blocking before randomisation

Statistical models: logistical regression of HIV risk index

Baseline differences adjustment: no notable differences

Repeated measures methods in analysis: not reported

Equity: participants were primarily from middle-class suburban and rural areas of New York State; $77.6 \%$ lived in 2-parent families during junior high school. At final follow-up, most were white (91.2\%); almost half (49.6\%) were college graduates; $39.6 \%$ were married or cohabitating; and the median age was 24.6 years (range 23.8 to 27.5 ).

Funding: National Institute on Drug Abuse, National Institutes of Health

Dr. Botvin has a financial interest in the Life Skills Training (LST) programme. His consulting company, National Health Promotion Associates (NHPA), provides teacher training and technical assistance for LST. Dr. Griffin is a consultant to NHPA.

Has the longer-term RCT been reported?

Contact author at kgriffin@med.cornell.edu for data for ninth grade (post intervention) and high school seniors? Time post intervention

Randomisation method, e.g. block, stratification, computer: randomised block design; schools were divided into high, medium, and low smoking prevalence. Schools then were randomised to 1 of 3 conditions within the blocks.

Clustering accounted for in sample size calculation (if relevant): analysis run using PROC GENMOD command to adjust for school clustering effects

Cluster randomisation methods to account for clustering in analysis: yes; PROC GENMOD used in SAS

\section{Risk of bias}

\begin{tabular}{lll}
\hline Bias & Authors' judgement & Support for judgement \\
\hline $\begin{array}{l}\text { Random sequence genera- } \\
\text { tion (selection bias) }\end{array}$ & Unclear risk & Not reported \\
\hline $\begin{array}{l}\text { Allocation concealment } \\
\text { (selection bias) }\end{array}$ & Unclear risk & Not reported \\
\hline
\end{tabular}

Blinding of participants High risk Participants and personnel cannot be blinded to allocation status.
and personnel (perfor-
mance bias)
All outcomes

Blinding of outcome as-

High risk

Self-reported outcomes from participants in unblinded intervention sessment (detection bias) 
Griffin 2006 (Continued)

All outcomes

\begin{tabular}{lll}
\hline $\begin{array}{l}\text { Incomplete outcome data } \\
\text { (attrition bias) } \\
\text { All outcomes }\end{array}$ & High risk & $\begin{array}{l}\text { Low retention rate of the baseline sample completing follow-up: attrition } \\
\text { rates: grade } 9=25 \% ; \text { high school seniors }=40 \% ; 10 \text { years post intervention, } \\
\text { young adults }=63 \%\end{array}$ \\
\hline $\begin{array}{l}\text { Selective reporting (re- } \\
\text { porting bias) }\end{array}$ & High risk & $\begin{array}{l}\text { No published protocol; only the final 10-year post-intervention follow-up data } \\
\text { were reported }\end{array}$ \\
\hline Other bias & Unclear risk & Little information about the conduct of the trial; no sample size calculation
\end{tabular}

Study design (e.g. RCT, cluster RCT): cluster RCT: 4 classrooms were selected 2 years before the start of the intervention, which ran over 3 years (i.e. 3 separate cohorts); 2 intervention classes and 2 control classes/year. A random method was used to assign students to a homeroom classroom at the beginning of the eighth grade. Over the 3 years, a total of 6 intervention and 6 control classes were provided.

Intervention arm(s): Life Skills Curriculum; Violence Prevention Curriculum; Violence Prevention training videos; manhood development training for African Americans

Comparator arm(s): standard curriculum involved school teachers, who were certified instructors, conducting the public school health curriculum including HIV prevention and personal hygiene.

\title{
Sample size calculation performed: no
}

\section{Subgroups prespecified: no}

Subgroup analyses: males and females (descriptive data)

Start date: not reported

Duration of follow-up: 12 months (following October at grade 9)

Number of follow-ups: 2

Follow-up time points: post-test and 1-year follow-up (1 year after baseline)

Follow-up period (post-intervention): 6 months

ICC (if reported): N/A

\section{Participants}

\author{
Number of schools randomised: N/A (12 classrooms) \\ Number of participants randomised (total and by arm): N = 199 \\ Age (range or mean (SD)) or grade at the start: start grade 8; follow-up grade 9 \\ Gender: female I $=42.4 \%, C=31.4 \%$; male I $=57.6 \%, C=68.6 \%$
}

Ethnicity: 99\% African American

SES: $78 \%$ single-parent household

Inclusion criteria: not reported

Exclusion criteria: students who were a threat to themselves or others 
The systematic random probability sampling in sixth grade assigned all students a number assigning them to 1 of the 12 classes 2 years before the intervention commenced. (These must have been stratified by student year.)

Duration of the intervention (excluding follow-up): intervention took place over 3 school years with 3 separate, sequential cohorts. Training was provided over a 9-week period during the eighth grade. Baseline survey at the start of the school year in October. Two classes of intervention students attended the 9-week training sequentially in the first or second semester, so the intervention took place over an 8-month period within the school year.

\section{Description of the intervention}

\section{Building Resiliency and Vocational Excellence (BRAVE)}

School-based ATOD and violence prevention programme. BRAVE programme staff ran the programme during health education class sessions. Classroom sessions ran over 7 to 8 months of the eighth grade year; the 2 intervention classes were run sequentially over the first and second semesters. The training intervention took place in 90-minute sessions 2 to 3 times/week for 9 weeks. Students developed career plans with short- and long-term goals. They were paired as buddies to monitor progress towards their own goals. Participants were also mentored for a minimum of 1 hour/week over the school year.

Students could opt out of the intervention programme and then were rescheduled into alternative health and physical education classes that were not part of the programme.

Brief description of the theoretical model: social learning theory and resiliency

Description of the comparator: standard curriculum

Outcomes List relevant outcomes

Primary outcomes: smokeless tobacco use, cigarette smoking, alcohol drinking, drunk from alcohol, marijuana use, victimhood, perpetration

Secondary outcomes: N/A

Setting Country: USA; State: Atlanta; Place: Georgia

Setting: a single public middle school in an inner city working-poor to middle-class neighbourhood

Focus: targeted: inner city African American majority

Process measures

Process data reported: adherence to the intervention, intensity of the intervention

Method (qualitative or quantitative)

Description

Adherence to the Intervention: The principal investigator used weekly lesson-planning sessions for trainers to review and practise using the training material. Trainers were required to prepare a service delivery schedule documenting the delivery of lesson plan objectives, to encourage adherence to lesson plan content, and to maintain the fidelity of the training.

Intensity of the intervention: programme 2 to 3 times/week for 9 weeks; mentoring 1 hour/week over the school year

Statistics

Sample size: $\mathrm{N}=199$ ( 3 cohorts of grade 8 students: cohort year $1=62$, cohort year $2=93$, cohort year $3=44) ; 21$ excluded owing to missing data or because families had moved, leaving 178 students with baseline, post-test, and follow-up data

Unit of randomisation: classroom first, then individuals assigned to each of the 4 classes for 3 cohorts

Unit of analysis: individuals: data from the 3 cohorts were combined 
Method to promote equivalence between groups: adjustments made during analysis for effects of groups, gender, and covariates

Statistical models: $\mathrm{Chi}^{2}$, t-tests for mean differences and \% change; MANCOVA: adjusted means for group, gender, covariates

\section{Baseline differences adjustment: not reported}

Repeated measures methods in analysis: MANCOVA used for differences between intervention and control groups over time. Baseline ATOD measurements as covariates and post-test and follow-up measurements used to conduct the analysis

Equity: baseline data only
Funding: not reported
Randomisation method, e.g. block, stratification, computer: N/A
Clustering accounted for in sample size calculation (if relevant): N/A
Cluster randomisation methods to account for clustering in analysis: significance of interaction for
group differences

\section{Risk of bias}

\begin{tabular}{|c|c|c|}
\hline Bias & Authors' judgement & Support for judgement \\
\hline $\begin{array}{l}\text { Random sequence genera- } \\
\text { tion (selection bias) }\end{array}$ & High risk & $\begin{array}{l}\text { Systematic random probability sampling in sixth grade: all students were as- } \\
\text { signed a number assigning them to } 1 \text { of the } 12 \text { classes } 2 \text { years before the in- } \\
\text { tervention commenced; however students could opt out of the intervention, } \\
\text { breaking randomisation }\end{array}$ \\
\hline $\begin{array}{l}\text { Allocation concealment } \\
\text { (selection bias) }\end{array}$ & High risk & $\begin{array}{l}\text { School administrators selected } 4 \text { homeroom classrooms ( } 2 \text { intervention and } 2 \\
\text { comparison) each year out of the } 12 \text { available. }\end{array}$ \\
\hline $\begin{array}{l}\text { Blinding of participants } \\
\text { and personnel (perfor- } \\
\text { mance bias) } \\
\text { All outcomes }\end{array}$ & High risk & Participants and personnel cannot be blinded to allocation status. \\
\hline $\begin{array}{l}\text { Blinding of outcome as- } \\
\text { sessment (detection bias) } \\
\text { All outcomes }\end{array}$ & High risk & All self-reports \\
\hline $\begin{array}{l}\text { Incomplete outcome data } \\
\text { (attrition bias) } \\
\text { All outcomes }\end{array}$ & Low risk & High (89\%) response to all surveys; authors state no differential attrition \\
\hline $\begin{array}{l}\text { Selective reporting (re- } \\
\text { porting bias) }\end{array}$ & Unclear risk & $\begin{array}{l}\text { All outcomes reported but data missing for the immediate post-intervention } \\
\text { period: Time } 1 \text { to Time } 2\end{array}$ \\
\hline Other bias & Unclear risk & $\begin{array}{l}\text { This study was conducted in } 1 \text { school only, and intervention and control class- } \\
\text { es were run in the same school year. Potential for contamination bias between } \\
\text { intervention and control classes }\end{array}$ \\
\hline
\end{tabular}

Methods Study name: Parents Who Care




\section{Study design (e.g. RCT, cluster RCT): RCT $(2 \times 3 \times 4)$ design}

Intervention arm(s): PWC parent and adolescent group administered programme (PA); PWC self-administered with weekly telephone support (SA)

Comparator $\operatorname{arm}(\mathbf{s})$ : no treatment

Sample size calculation performed: not reported

Subgroups prespecified: African American (AA), European American (EA)

Subgroup analyses: $E A$ and $A A$

Start date: not reported

Duration of follow-up: 2 years post intervention +10 weeks

\section{Number of follow-ups: 3}

Follow-up time points: wave 1: post-test 7 or 10 weeks; wave 2: 1-year follow-up post intervention; wave 3: 2-year follow-up post intervention

ICC (if reported): no

\section{Participants}

\section{Number of schools randomised: N/A}

Number of participants randomised (total and by arm): $N=331$ youths and parents; $S A n=107$ families; PA $n=118$ families; control $n=106$

Age (range or mean (SD)) or grade at the start: mean age 13.7 years; eighth grade

Gender: not reported

Ethnicity: 163/331 African American (49.2\%); 168/331 European American (50.8\%)

SES: not reported

Inclusion criteria: families with an African American or European American eighth grader at home, English as the primary language, living in the area for the following 6 months

Exclusion criteria: not reported

\section{Parents Who Care (PWC)}

PWC is a 7- or 10-session universal substance abuse and problem behaviour preventive intervention for families with at least 1 early adolescent teenager. Components involve parenting, youth, and family. The SA group completed a video and workbook within 10 weeks, plus family consultant contact by phone weekly to record activities, motivate families to use the materials, and enable implementation. The PA group met for 7 consecutive weekly sessions (between 2 and 2.5 hours in duration) involving a review of the video components of the curriculum, practice of skills, family interaction skills, and completion of sections of the workbook. The workbook contained 7 chapters addressing (1) roles: relating to your teen; (2) risks: identifying and reducing them; (3) protection: bonding with your teen to strengthen resilience; (4) tools: working with your family to solve problems; (5) involvement: allowing everybody to contribute; (6) policies: setting family policies on health and safety issues; and (7) supervision: supervising without invading.

Brief description of the theoretical model: programme is based on a social development model that specifies the mechanisms and causal pathways of risk and protective factors that contribute to both healthy and maladaptive lifestyles. The PWC programme is designed to enhance protection in families 
by teaching parents ways to provide children with opportunities to contribute towards the family, to take advantage of opportunities, and to use reward and recognition strategies to promote bonding.

Description of the comparator: no treatment control

\begin{tabular}{ll}
\hline Outcomes & List relevant outcomes \\
& Primary outcomes: non-violent delinquency; violent behaviour; initiation of drug use: cigarettes, alco- \\
hol, marijuana, illegal drugs; initiation of sex \\
Secondary outcomes: N/A
\end{tabular}

Setting

Country: USA; State: Washington;Place: Seattle

Setting: family - 1 parent and teenager were paired

Focus: universal

Process measures

Process data reported: yes

Method (qualitative or quantitative): quantitative

Description

\section{Acceptability of the intervention}

On a 1 to 4 scale ( 1 = very satisfied, $4=$ not at all satisfied):

SA parents had a mean rating of 1.2 for satisfaction, and PA parents had a mean rating of 1.4 for satisfaction.

PA group: $82 \%$ of students and $93.7 \%$ of parents reported that they would recommend the programme to a friend.

Parents reported overall satisfaction with the PWC video (1.6 for PA, 1.7 for SA) and workbook (1.5 for $\mathrm{PA}, 1.6$ for SA).

Students reported a lower satisfaction rating with the video (2.1 for PA video, 1.9 for SA) and workbook (2.2 for PA, 2.0 for SA).

\section{Adherence to the intervention}

PA: each family session was independently observed and rated noting coverage of up to 123 individual programme content items per session. After 7 weeks, the overall content covered per group ranged from $75.5 \%$ to $88.3 \%$, with an average of $82.3 \%$.

SA: quality of programme delivery was measured by asking assigned families how supported they felt by the family consultants (mean $=5.53$ on a scale from $1=$ not at all to $6=$ very) and how well the consultants followed through with phone calls at the appointment times (mean $=5.24$ ).

\section{Reach of the intervention}

SA: family consultants achieved $57 \%$ telephone contact with families.

PA: families were exposed to nearly all of the video segments and workbook activities (does not report how many families attended).

\section{Intensity of the intervention}

7-week intervention period for both PA and SA groups 
Unit of analysis: individuals

Method to promote equivalence between groups: at recruitment, families were stratified on race (168 EA/163 AA) and gender (170 male/161 female), then were randomly assigned to the 3 experimental conditions.

Statistical models: multi-level mixed models account for changes over time and use all available data (incomplete records are included).

Baseline differences adjustment: baseline levels were entered as covariates in all analyses to reduce the potential for baseline race differences among intervention outcomes.

Repeated measures methods in analysis: repeated measures mixed-model regressions for delinquent and violent behaviour over time from post-test to 24 months' follow-up

Funding: Grant \# R01-DA121645-05 from the National Institute on Drug Abuse

Randomisation method, e.g. block, stratification, computer: at recruitment, families were stratified by race and gender, then were randomly assigned to 1 of 3 conditions.

Clustering accounted for in sample size calculation (if relevant): no

Cluster randomisation methods to account for clustering in analysis: none

\section{Risk of bias}

\begin{tabular}{lll}
\hline Bias & Authors' judgement & Support for judgement \\
\hline $\begin{array}{l}\text { Random sequence genera- } \\
\text { tion (selection bias) }\end{array}$ & Unclear risk & No information on how the random sequence was generated \\
\hline $\begin{array}{l}\text { Allocation concealment } \\
\text { (selection bias) }\end{array}$ & Unclear risk & No information on how allocation was concealed \\
\hline $\begin{array}{l}\text { Blinding of participants } \\
\text { and personnel (perfor- } \\
\text { mance bias) } \\
\text { All outcomes }\end{array}$ & High risk & Blinding not possible \\
\hline
\end{tabular}

Blinding of outcome as- High risk Outcomes measured by teen self-report surveys

sessment (detection bias)

All outcomes

\begin{tabular}{|c|c|c|}
\hline $\begin{array}{l}\text { Incomplete outcome data } \\
\text { (attrition bias) }\end{array}$ & Low risk & $\begin{array}{l}\text { Attrition }<10 \% \text {. Study authors accounted for missing data using multiple im- } \\
\text { putation. }\end{array}$ \\
\hline
\end{tabular}

All outcomes putation.

Selective reporting (re- Unclear risk No published protocol
porting bias)

Other bias Low risk No other sources of bias


Study design (e.g. RCT, cluster RCT): cluster RCT (school as blocking factor and classrooms as unit of randomisation)

Intervention $\operatorname{arm}(\mathbf{s}):$ FSP and CC

Comparator arm(s): control

Sample size calculation performed: N/S

Subgroups prespecified: N/S

Subgroup analyses: gender

Start date: 1993

Duration of follow-up: up to 7 years

Number of follow-ups: 5

Follow-up time points: end of intervention; 1 year, 4,5 , and 6 years

ICC (if reported): not reported

Participants

Number of schools randomised: N/A

Number of participants randomised (total and by arm): 230 to CC, 229 to FSP, 219 to control

Age (range or mean (SD)) or grade at the start: mean age 6.2, SD 0.34

Gender: $53.2 \%$ male

Ethnicity: 86.8\% African American; 13.2\% European American

SES: $62.3 \%$ had free or reduced-price lunch; 2-parent household: $32.7 \%$ in CC arm; $41.3 \%$ in FSP arm; $44.9 \%$ in control arm

Inclusion criteria: children in elementary schools in Baltimore

Exclusion criteria: N/A

\section{Randomisation before or after baseline survey: before}

Duration of the intervention (excluding follow-up): 1 school year

Description of the intervention: CC intervention involved curriculum enhancement, behaviour management (a weekly meeting to promote group problem-solving skills), and an additional strategy for unresponsive children. FSP intervention involved training for teachers in parent-teacher communication and partnership building; weekly home-school learning and communication activities; and a series of 9 workshops for parents led by the first grade teacher and the school psychologist or social worker.

Brief description of the theoretical model: life course/social field theory: mal/adapting to earlier social task demands leads to later mal/adaption across related fields

Description of the comparator: control classrooms

Outcomes

Primary outcomes: aggressive and shy behaviour

Secondary outcomes: substance use, affective disorder, conduct disorder

Setting

Country: USA, Baltimore

Setting: primary school

Focus: universal 
lalongo 1999 (Continued)

Process measures

\section{Process data reported: yes}

Method (qualitative or quantitative): qualitative

Description: in all but 2 of the 9 CC classrooms, teachers implemented over $50 \%$ of the intervention protocol. The median $\%$ of implementation as designed was $64.4 \%$, mean $59.9 \%$, SD $17 \%$. In the FSP arm, parents/caregivers attended 4.02 sessions on average (SD 2.38), with a median of 5 (range 0 to 7 ) of the 7 core sessions offered. $12.7 \%$ of parents/caregivers did not attend any workshops, but $35.3 \%$ of parents attended at least 6 of 7 workshops. Among take-home activities, parents completed 39.15 (SD 16.54 ) of the 64 activities, or $60.93 \% ; 35.7 \%$ completed $75 \%$ or more of the activities, whereas $2.3 \%$ did not complete any activities.

\section{Statistics}

Sample size: 678 children invited, 597 children recruited

Unit of randomisation: classroom

Unit of analysis: individual

Method to promote equivalence between groups: N/A

Statistical models: mixed model using SAS Proc Mixed

Baseline differences adjustment: yes

Repeated measures methods in analysis: not clear
Equity: baseline data provided

Funding: NIH/National Institute of Mental Health: National Institute of Mental Health (R01MH57005); R01 DA11796 from the National Institute on Drug Abuse, and grants R01 MH40859, T32 MH14592, and T32 MH18834 from the National Institute of Mental Health. National Institute on Drug Abuse Institutional Training Grant (DA07292); National Institute of Mental Health (Epidemiologic Prevention Center for Early Risk Behaviors), NIMH 5 PO MH38725

Randomisation method, e.g. block, stratification, computer: blocking on school

Clustering accounted for in sample size calculation (if relevant): not clear

Cluster randomisation methods to account for clustering in analysis: yes

\section{Risk of bias}

\begin{tabular}{lll}
\hline Bias & Authors' judgement & Support for judgement \\
\hline $\begin{array}{l}\text { Random sequence genera- } \\
\text { tion (selection bias) }\end{array}$ & Low risk & Computer-generated algorithm used for random sequence generation \\
\hline $\begin{array}{l}\text { Allocation concealment } \\
\text { (selection bias) }\end{array}$ & Unclear risk & Insufficient information provided \\
\hline $\begin{array}{l}\text { Blinding of participants } \\
\text { and personnel (perfor- } \\
\text { mance bias) } \\
\text { All outcomes }\end{array}$ & High risk & Not blinded \\
\hline
\end{tabular}

\begin{tabular}{lll}
$\begin{array}{l}\text { Blinding of outcome as- } \\
\text { sessment (detection bias) } \\
\text { All outcomes }\end{array}$ & High risk & Self-reported outcomes by individuals not blinded to intervention condition \\
\hline $\begin{array}{l}\text { Incomplete outcome data } \\
\text { (attrition bias) }\end{array}$ & Low risk & $\begin{array}{l}\text { Study authors state that baseline characteristics were balanced between } \\
\text { groups and attrition was low. }\end{array}$
\end{tabular}


lalongo 1999 (Continued)

All outcomes

Selective reporting (re- Unclear risk $\quad$ Lack of protocol and outcomes reported over multiple years of follow-up
porting bias)

Other bias Unclear risk Possibility of contamination due to randomisation of classrooms within
schools

Study design (e.g. RCT, cluster RCT): cluster RCT (school as blocking factor and classrooms as unit of randomisation)

Intervention $\operatorname{arm}(\mathbf{s})$ : FSP and CC

Comparator arm s): control

Sample size calculation performed: N/S

Subgroups prespecified: N/S

Subgroup analyses: gender

Start date: 1993

Duration of follow-up: up to 7 years

Number of follow-ups: 5

Follow-up time points: end of intervention; 1 year, 4,5 , and 6 years

ICC (if reported): not reported

Number of participants randomised (total and by arm): 230 to CC, 229 to FSP, 219 to control

Age (range or mean (SD)) or grade at the start: mean age 6.2, SD 0.34

Gender: $53.2 \%$ male

Ethnicity: 86.8\% African American; 13.2\% European American

SES: $62.3 \%$ had free or reduced-price lunch; 2-parent household: $32.7 \%$ in CC arm; $41.3 \%$ in FSP arm; $44.9 \%$ in control arm

Inclusion criteria: children in elementary schools in Baltimore

Exclusion criteria: N/A

\section{Randomisation before or after baseline survey: before}

Duration of the intervention (excluding follow-up): 1 school year

Description of the intervention: CC intervention involved curriculum enhancement, behaviour management (a weekly meeting to promote group problem-solving skills), and an additional strategy for unresponsive children. FSP intervention involved training for teachers in parent-teacher communication and partnership building; weekly home-school learning and communication activities; and a series of 9 workshops for parents led by the first grade teacher and the school psychologist or social worker. 
Brief description of the theoretical model: life course/social field theory: mal/adapting to earlier social task demands leads to later mal/adaption across related fields

Description of the comparator: control classrooms

\begin{tabular}{|c|c|}
\hline Outcomes & $\begin{array}{l}\text { Primary outcomes: aggressive and shy behaviour } \\
\text { Secondary outcomes: substance use, affective disorder, conduct disorder }\end{array}$ \\
\hline Setting & $\begin{array}{l}\text { Country: USA, Baltimore } \\
\text { Setting: primary school } \\
\text { Focus: universal }\end{array}$ \\
\hline Process measures & $\begin{array}{l}\text { Process data reported: yes } \\
\text { Method (qualitative or quantitative): qualitative } \\
\text { Description: in all but } 2 \text { of the } 9 \text { CC classrooms, teachers implemented over } 50 \% \text { of the intervention } \\
\text { protocol. The median } \% \text { of implementation as designed was } 64.4 \% \text {, mean } 59.9 \% \text {, SD } 17 \% \text {. In the FSP } \\
\text { arm, parents/caregivers attended } 4.02 \text { sessions on average (SD } 2.38 \text { ), median } 5 \text { (range } 0 \text { to } 7 \text { ) of the } 7 \\
\text { core sessions offered. } 12.7 \% \text { of parents/caregivers did not attend any workshops, but } 35.3 \% \text { of parents } \\
\text { attended at least } 6 \text { of } 7 \text { workshops. Among take-home activities, parents completed } 39.15 \text { (SD 16.54) of } \\
\text { the } 64 \text { activities, or } 60.93 \% .35 .7 \% \text { completed } 75 \% \text { or more of the activities, whereas } 2.3 \% \text { did not com- } \\
\text { plete any activities. }\end{array}$ \\
\hline
\end{tabular}

Statistics

Sample size: 678 children invited, 597 children recruited

Unit of randomisation: classroom

Unit of analysis: individual

Method to promote equivalence between groups: N/A

Statistical models: mixed model using SAS Proc Mixed

Baseline differences adjustment: yes

Repeated measures methods in analysis: not clear

\begin{tabular}{|c|c|c|}
\hline \multirow[t]{5}{*}{ Notes } & \multicolumn{2}{|c|}{ Equity: baseline data provided } \\
\hline & \multicolumn{2}{|c|}{$\begin{array}{l}\text { Funding: NIH/National Institute of Mental Health: National Institute of Mental Health (R01MH57005); } \\
\text { R01 DA11796 from the National Institute on Drug Abuse, and grants R01 MH40859, T32 MH14592, and } \\
\text { T32 MH18834 from the National Institute of Mental Health. National Institute on Drug Abuse Institution- } \\
\text { al Training Grant (DA07292); National Institute of Mental Health (Epidemiologic Prevention Center for } \\
\text { Early Risk Behaviors), NIMH } 5 \text { PO MH38725 }\end{array}$} \\
\hline & \multicolumn{2}{|c|}{ Randomisation method, e.g. block, stratification, computer: blocking on school } \\
\hline & \multicolumn{2}{|c|}{ Clustering accounted for in sample size calculation (if relevant): not clear } \\
\hline & \multicolumn{2}{|c|}{ Cluster randomisation methods to account for clustering in analysis: yes } \\
\hline \multicolumn{3}{|l|}{ Risk of bias } \\
\hline Bias & Authors' judgement & Support for judgement \\
\hline $\begin{array}{l}\text { Random sequence genera- } \\
\text { tion (selection bias) }\end{array}$ & Low risk & Computer-generated algorithm used for random sequence generation \\
\hline
\end{tabular}




\begin{tabular}{|c|c|c|}
\hline $\begin{array}{l}\text { Allocation concealment } \\
\text { (selection bias) }\end{array}$ & Unclear risk & Insufficient information provided \\
\hline $\begin{array}{l}\text { Blinding of participants } \\
\text { and personnel (perfor- } \\
\text { mance bias) } \\
\text { All outcomes }\end{array}$ & High risk & Not blinded \\
\hline $\begin{array}{l}\text { Blinding of outcome as- } \\
\text { sessment (detection bias) } \\
\text { All outcomes }\end{array}$ & High risk & Self-reported outcomes by individuals not blinded to intervention condition \\
\hline $\begin{array}{l}\text { Incomplete outcome data } \\
\text { (attrition bias) } \\
\text { All outcomes }\end{array}$ & Low risk & $\begin{array}{l}\text { Study authors state that baseline characteristics were balanced between } \\
\text { groups and attrition was low. }\end{array}$ \\
\hline $\begin{array}{l}\text { Selective reporting (re- } \\
\text { porting bias) }\end{array}$ & Unclear risk & Lack of protocol and outcomes reported over multiple years of follow-up \\
\hline Other bias & Unclear risk & $\begin{array}{l}\text { Possibility of contamination due to randomisation of classrooms within } \\
\text { schools }\end{array}$ \\
\hline
\end{tabular}

\begin{tabular}{l} 
Jalling 2016 \\
\hline Methods \\
Intervention arm(s): Comet \\
Comparator arm(s): ParentSteps, Control \\
Sample size calculation performed: yes \\
Subgroups prespecified: unclear if this was prespecified although mentioned in Methods \\
Subgroup analyses: adolescents with poor functioning at baseline, adolescents 12 to 17 years old only \\
for comparability with other studies \\
Start date: waves of recruitment/cohort: between 23 September 2008 and 19 October 2010 \\
Duration of follow-up: 6 months \\
Number of follow-ups: 1 \\
Follow-up time points: 6 months \\
ICC (if reported): N/A
\end{tabular}

Participants

Number of schools randomised: N/A

Number of participants randomised (total and by arm): 271 families (Comet: 99, ParentSteps: 86 , control: 86)

Age (range or mean (SD)) or grade at the start: Comet: 14.6 years (1.67), ParentSteps: 14.5 years (1.63), control: 14.7 years (1.89)

Gender: Comet: $38.4 \%$ female, ParentSteps: $45.7 \%$ female, control: $58.0 \%$ female

Ethnicity: foreign-born mother: Comet 19.8\%; ParentSteps 20.0\%; control 20.9\% 
Jalling 2016 (Continued)

SES: parent is employed: Comet $86.4 \%$, ParentSteps $84.5 \%$, control $84.1 \%$. Parent has university degree: Comet $28.4 \%$, ParentSteps $17.1 \%$, control $28.0 \%$

Inclusion criteria: eligible participants were parents/caregivers and their adolescent children 12 to 18 years old who were at risk of consolidating antisocial behaviour. When screening parents for participation, antisocial risk behaviour in adolescence was indicated by single-item descriptions: delinquent behaviour; bullying; repeated conflicts regarding family rules; any use of alcohol, tobacco, and/or drugs; or excessive computer use. Participation also necessitated living in 1 of the 5 participating municipalities, and the adolescent had to live at least part-time with the participating parent or caregiver.

Exclusion criteria: adolescent's ongoing psychotherapy, treatment for alcohol or drug use, out-ofhome placement, parents' participation in another parent programme

Interventions

Randomisation before or after baseline survey: before

Duration of the intervention (excluding follow-up): Comet: 9 weeks, ParentSteps: 6 weeks

Description of the intervention

Comet

Consisted of 9 parent sessions and an optional booster session, each lasting 2 to 2.5 hours. It was delivered by certified group leaders (all are social workers who had 6 days of training and 2 booster training sessions). Principal programme components are rehearsals of the use of reinforcement principles (e.g. encouragement and praise, ignoring minor problems) through role-play and home assignments whereby parents practise and develop these principles in their daily lives. Parents are instructed to keep a diary to document their interactions with their adolescent, and home assignments are followed up in subsequent sessions. Video vignettes are used in each session to enhance learning. Examples of themes covered include taking initiatives for spending time together with the adolescent, dealing with rejection, performing basic interactional (behavioural) analysis, providing positive communication and encouragement, solving problems, and defining rules and consequences.

\section{ParentSteps}

Consisted of 6 parent sessions, each lasting 1.5 to 2 hours. ParentSteps is delivered by certified group leaders (all are social workers who had 1 day of training) using video vignettes, group discussions, and home assignments. Themes for the 6 sessions and home assignments are Love and limits; Encouragement and consequences; Risks and protection; Stress, fights, and different points of view; Youth, parents, and alcohol; and Youth, parents, and drugs.

Brief description of the theoretical model: Comet is based on operant learning and social learning principles. ParentSteps is based on the resilience model.

Description of the comparator: control (wait-list): after 6-month follow-up, parents in the control group were offered Comet 12-18 or ParentSteps in accordance with their preferences.

Outcomes

Primary: adolescent externalising behaviour, including antisocial behaviour, delinquency, alcohol use, and drug use

Secondary: adolescent psychosocial dysfunction

Setting

Country: Sweden; State: Huddinge, Solna, Sundbyberg, Nacka, and Stockholm

Setting: families (home)

Focus: parents/caregivers and their adolescent children who were at risk of consolidating antisocial behaviour

Process measures

Process data reported: yes

Method (qualitative or quantitative): quantitative

\section{Description}


Reach of the intervention: of the 6 total sessions, parents in ParentSteps attended a mean of 4.7 sessions (SD 1.44). Of the 9 total Comet 12-18 sessions, only every second session was assessed and mean attendance for 4.5 sessions was 3.6 (SD 1.07). With dropout defined as absence during the 2 last sessions, the dropout rate in ParentSteps was $14.1 \%$. It was impossible to estimate the dropout rate in Comet 12-18 owing to lack of data from the 2 last sessions.

Fidelity/Adherence to the intervention: only social workers who were certified as programme deliverers and were active as group leaders were involved in the trial. Comet 12-18 leaders reported that $78 \%$ of the 73 sections in the manual were fulfilled "to a full extent", $17 \%$ "to a greater extent", $3 \%$ "to at least half", $1 \%$ "to a lesser extent", and $1 \%$ "not at all". ParentSteps leaders' self-assessments showed that $83 \%$ had fulfilled the manual sections "to a full extent", $13 \%$ "to a greater extent", $2 \%$ "to at least half", $1 \%$ "to a lesser extent", and 1\% "not at all". At the 6-month follow-up, 32 parents reported that they themselves or their adolescent child had participated in another intervention targeting the adolescent such as seeing a school counsellor or a therapist at a child and adolescent psychiatry unit. These were equally distributed across groups (Comet 12-18 14.1\%, ParentSteps 14.3\%, and control $12.2 \%)$, and they were kept for analysis.

Statistics

Sample size: eligible N = 605 families; enrolled N = 271 families (45\%); Comet: 99, ParentSteps: 86, control: 86

Unit of randomisation: families

Unit of analysis: individual

Method to promote equivalence between groups: not reported

Statistical models: analyses of normally distributed outcome data were performed with the general linear model (GLM) repeated measures ANOVA, and skewed data on the SRD were first log-transformed.

Baseline differences adjustment: gender and age were added to the ANOVA models as secondary explanatory factors.

Repeated measures methods in analysis: N/A. Only 1 follow-up

Equity: number of adolescents who completed baseline measures (total and by arm): 237 (Comet: 86 , ParentSteps: 70, control: 81)

Funding: not reported

Randomisation method, e.g. block, stratification, computer: the randomisation sequence was generated by a research assistant who drew 1 of 3 folded opaque pieces of paper from a bowl. The paper was then put back in the bowl for the next family to be randomised.

Clustering accounted for in sample size calculation (if relevant): N/A

Cluster randomisation methods to account for clustering in analysis: N/A

\section{Risk of bias}

\begin{tabular}{lll}
\hline Bias & Authors' judgement & Support for judgement \\
\hline $\begin{array}{l}\text { Random sequence genera- } \\
\text { tion (selection bias) }\end{array}$ & High risk & $\begin{array}{l}\text { The randomisation sequence was generated by a research assistant who drew } \\
1 \text { of } 3 \text { folded opaque pieces of paper from a bowl. Only } 3 \text { lots }\end{array}$ \\
\hline $\begin{array}{l}\text { Allocation concealment } \\
\text { (selection bias) }\end{array}$ & High risk & Only 3 lots - highly predictable \\
\hline $\begin{array}{l}\text { Blinding of participants } \\
\begin{array}{l}\text { and personnel (perfor- } \\
\text { mance bias) }\end{array}\end{array}$ & High risk & Blinding was not possible given the nature of this intervention. \\
\hline \begin{tabular}{l} 
All outcomes \\
\hline
\end{tabular}
\end{tabular}


Jalling 2016 (Continued)

Blinding of outcome as- High risk Not reported; self-reported outcomes only sessment (detection bias)

All outcomes

\begin{tabular}{lll}
\hline $\begin{array}{l}\text { Incomplete outcome data } \\
\text { (attrition bias) } \\
\text { All outcomes }\end{array}$ & Low risk \\
\hline $\begin{array}{l}\text { Selective reporting (re- } \\
\text { porting bias) }\end{array}$ & Low risk & No published protocol, but all specified outcomes were reported \\
\hline Other bias & Unclear risk & $\begin{array}{l}\text { The number of conducted parent groups differed between the } 2 \text { programmes } \\
\text { because of the limited capacity of social services to include trial parents in } \\
\text { their regular Comet } 12-18 \text { group. Some groups included only } 2 \text { trial parents; in } \\
\text { 2 groups, the whole group comprised parents participating in the trial. In Par- } \\
\text { entSteps, almost all parents in each group participated in the trial. }\end{array}$
\end{tabular}

Study design: RCT at the level of the family
Intervention $\operatorname{arm}(\mathbf{s})$ : Comet
Comparator $\operatorname{arm}(\mathbf{s}):$ ParentSteps, Control
Sample size calculation performed: yes
Subgroups prespecified: unclear if it was prespecified although mentioned in Methods
Subgroup analyses: adolescents with poor functioning at baseline; adolescents 12 to 17 years old only
for comparability with other studies

Start date: waves of recruitment/cohort: between 23 September 2008 and 19 October 2010

Duration of follow-up: 6 months

Number of follow-ups: 1

Follow-up time points: 6 months

ICC (if reported): N/A

Number of participants randomised (total and by arm): 271 families (Comet: 99, ParentSteps: 86, control: 86)

Age (range or mean (SD)) or grade at the start: Comet: 14.6 years (1.67), ParentSteps: 14.5 years (1.63), control: 14.7 years (1.89)

Gender: Comet: $38.4 \%$ female, ParentSteps: $45.7 \%$ female, control: $58.0 \%$ female

Ethnicity: foreign-born mother: Comet 19.8\%; ParentSteps 20.0\%; control 20.9\%

SES: parent is employed: Comet $86.4 \%$; ParentSteps $84.5 \%$; control $84.1 \%$. Parent has university degree: Comet 28.4\%; ParentSteps 17.1\%; control 28.0\%

Inclusion criteria: eligible participants were parents/caregivers and their adolescent children 12 to 18 years old who were at risk of consolidating antisocial behaviour. When screening parents for participation, antisocial risk behaviour in adolescence was indicated by single-item descriptions: delinquent behaviour; bullying; repeated conflicts regarding family rules; any use of alcohol, tobacco, and/or drugs; 
Jalling 2016b (Continued)

or excessive computer use. Participation also necessitated living in 1 of the 5 participating municipalities, and the adolescent had to live at least part-time with the participating parent or caregiver.

Exclusion criteria: adolescent's ongoing psychotherapy, treatment for alcohol or drug use, out-ofhome placement, parents' participation in another parent programme

Interventions

Randomisation before or after baseline survey: before

Duration of the intervention (excluding follow-up): Comet: 9 weeks, ParentSteps: 6 weeks

Description of the intervention

Comet

Consisted of 9 parent sessions and an optional booster session, each lasting 2 to 2.5 hours. It was delivered by certified group leaders (all are social workers who had 6 days of training and 2 booster training sessions). Principal programme components are rehearsals of the use of reinforcement principles (e.g. encouragement and praise, ignoring minor problems) through role-play and home assignments whereby parents practise and develop these principles in their daily lives. Parents are instructed to keep a diary to document their interactions with their adolescent, and home assignments are followed up in subsequent sessions. Video vignettes are used in each session to enhance learning. Examples of themes covered include taking initiative for spending time together with the adolescent, dealing with rejection, performing basic interactional (behavioural) analysis, providing positive communication and encouragement, solving problems, and defining rules and consequences.

\section{ParentSteps}

Consisted of 6 parent sessions, each lasting 1.5 to 2 hours. ParentSteps is delivered by certified group leaders (all are social workers who had 1 day of training) using video vignettes, group discussions, and home assignments. Themes for the 6 sessions and home assignments are Love and limits; Encouragement and consequences; Risks and protection; Stress, fights, and different points of view; Youth, parents, and alcohol; and Youth, parents, and drugs.

Brief description of the theoretical model: Comet is based on operant learning and social learning principles. ParentSteps is based on the resilience model.

Description of the comparator: control (wait-list): after the 6-month follow-up, parents in the control group were offered Comet 12-18 or ParentSteps in accordance with their preferences.

Outcomes

Primary: adolescent externalising behaviour, including antisocial behaviour, delinquency, alcohol use, and drug use

Secondary: adolescent psychosocial dysfunction

Setting

Country: Sweden; State: Huddinge, Solna, Sundbyberg, Nacka, and Stockholm

Setting: families (home)

Focus: parents/caregivers and their adolescent children who were at risk of consolidating antisocial behaviour

Process measures Process data reported: yes

Method (qualitative or quantitative): quantitative

Description

Reach of the intervention: of the 6 total sessions, parents in ParentSteps attended a mean of 4.7 sessions (SD 1.44). Of the 9 total Comet 12-18 sessions, only every second session was assessed and mean attendance for 4.5 sessions was 3.6 (SD 1.07). With dropout defined as absence during the 2 last sessions, the dropout rate in ParentSteps was $14.1 \%$. It was impossible to estimate the dropout rate in Comet 12-18 owing to lack of data from the 2 last sessions. 
Fidelity/Adherence to the intervention: Only social workers who were certified as programme deliverers and were active as group leaders were involved in the trial. Comet 12-18 leaders reported that $78 \%$ of the 73 sections in the manual were fulfilled "to a full extent", $17 \%$ "to a greater extent", $3 \%$ "to at least half", $1 \%$ "to a lesser extent", and $1 \%$ "not at all". ParentSteps leaders' self-assessments showed that $83 \%$ had fulfilled the manual sections "to a full extent", $13 \%$ "to a greater extent", $2 \%$ "to at least half", $1 \%$ "to a lesser extent", and 1\% "not at all". At the 6-month follow-up, 32 parents reported that they themselves or their adolescent child had participated in another intervention targeting the adolescent such as seeing a school counsellor or a therapist at a child and adolescent psychiatry unit. These were equally distributed across groups (Comet 12-18 14.1\%, ParentSteps 14.3\%, control 12.2\%), and they were kept for analysis.

Statistics

Sample size: eligible N = 605 families; enrolled N = 271 families (45\%); Comet: 99, ParentSteps: 86, control: 86

Unit of randomisation: families

Unit of analysis: individual

Method to promote equivalence between groups: not reported

Statistical models: analyses of normally distributed outcome data were performed with the general linear model (GLM) repeated measures ANOVA, and skewed data on the SRD were first log-transformed.

Baseline differences adjustment: gender and age were added to the ANOVA models as secondary explanatory factors.

Repeated measures methods in analysis: N/A. Only 1 follow-up

Equity: number of adolescents who completed baseline measures (total and by arm): 237 (Comet: 86, ParentSteps: 70, control: 81)

Funding: not reported

Randomisation method, e.g. block, stratification, computer: the randomisation sequence was generated by a research assistant who drew 1 of 3 folded opaque pieces of paper from a bowl. The paper was then put back in the bowl for the next family to be randomised.

Clustering accounted for in sample size calculation (if relevant): N/A

Cluster randomisation methods to account for clustering in analysis: N/A

\section{Risk of bias}

\begin{tabular}{lll}
\hline Bias & Authors' judgement & Support for judgement \\
\hline $\begin{array}{l}\text { Random sequence genera- } \\
\text { tion (selection bias) }\end{array}$ & High risk & $\begin{array}{l}\text { The randomisation sequence was generated by a research assistant who drew } \\
1 \text { of } 3 \text { folded opaque pieces of paper from a bowl. Only } 3 \text { lots }\end{array}$ \\
\hline $\begin{array}{l}\text { Allocation concealment } \\
\text { (selection bias) }\end{array}$ & High risk & Only 3 lots - highly predictable \\
\hline
\end{tabular}

Blinding of participants

High risk Blinding was not possible given the nature of this intervention. and personnel (performance bias)

All outcomes

\begin{tabular}{|c|c|c|}
\hline $\begin{array}{l}\text { Blinding of outcome as- } \\
\text { sessment (detection bias) } \\
\text { All outcomes }\end{array}$ & High risk & Not reported; self-reported outcomes only \\
\hline
\end{tabular}


Jalling 2016b (Continued)

All outcomes

Selective reporting (re- Low risk $\quad$ No published protocol, but all specified outcomes were reported
porting bias)

\begin{tabular}{|c|c|c|}
\hline Other bias & Unclear risk & $\begin{array}{l}\text { The number of conducted parent groups differed between the } 2 \text { programmes } \\
\text { because of the limited capacity of social services to include trial parents in } \\
\text { their regular Comet } 12-18 \text { group. Some groups included only } 2 \text { trial parents; in } \\
2 \text { groups, the whole group comprised parents participating in the trial. In Par- } \\
\text { entSteps, almost all parents in each group participated in the trial. }\end{array}$ \\
\hline
\end{tabular}

\begin{tabular}{ll}
\hline Study design: RCT \\
Intervention $\operatorname{arm}(\mathbf{s}):$ Healthy Futures \\
Comparator $\operatorname{arm}(\mathbf{s}):$ control \\
Sample size calculation performed: not reported \\
Subgroups prespecified: no \\
Subgroup analyses: career planning subgroup \\
Start date: 2008 to 2011 \\
Duration of follow-up: 6 months \\
Number of follow-ups: 1 \\
Follow-up time points: 6 months \\
ICC (if reported): N/A \\
\hline
\end{tabular}

Participants

Number of schools randomised: N/A

Number of participants randomised (total and by arm): 200 (Healthy Futures: 101, control: 99)

Age (range or mean (SD)) or grade at the start: Healthy Futures: 16.59 years (2.08), control: 16.77 years (1.98)

Gender: Healthy Futures: $42.6 \%$ male, control: 38.4\% male

Ethnicity: black; 97\% Healthy Futures, 94.9\% control

SES: education level: Healthy Futures: high school student $66.3 \%$, high school grad/GED 28.7\%, dropout no GED 5.0\%. Control: 68.7\%, 25.3\%, 6.1\%

Maternal education: Healthy Futures: some college 36.6\%, high school diploma/GED 41.6\%, dropout no GED 14.9\%. Control: 36.4\%, 34.3\%, 25.3\%

Inclusion criteria: between the ages of 14 and 21 , had attended or were currently attending high school in the local public school system, not currently enrolled in a self-contained classroom (indicating receipt of intense special education services)

Exclusion criteria: not reported

Interventions

\section{Randomisation before or after baseline survey: after}

Duration of the intervention (excluding follow-up): 3 sessions over a period of 5 months 
Description of the intervention: all participants were invited to participate in bi-annual job and college fairs held at the clinic and received a monthly newsletter containing information about local opportunities to build their resume. Participants in the intervention arm also received 3 in-person Motivational Interviewing (MI) sessions (approximately 1 every other month), which took place at the clinic, with follow-up contact provided via phone or email after each session (i.e. in the in-between month). $\mathrm{MI}$ sessions were facilitated by master's level educators trained in MI. Activities in each session provided opportunities for youth to discuss their goals for the future, identify barriers to accomplishing these goals (including involvement in risk behaviours), practise the skills necessary to accomplish these goals (e.g. research careers, explore jobs and educational programmes, develop their resume, complete applications), and link them to community resources.

Brief description of the theoretical model: based on social cognitive theory and positive youth development

Description of the comparator: participants in the comparison group were invited to participate in biannual job and college fairs held at the clinic and received a monthly newsletter containing information about local opportunities to build their resume

Country: USA; State: N/S
Setting: individuals (clinic)
Focus: universal but recruited through paediatric primary care clinic in area with high proportion of
African American and low-income population

Process measures Process data reported: yes

Method (qualitative or quantitative): qualitative

Description: fidelity to the principles of MI was assessed through supervisor oversight and case management meetings but was not validated.

Unit of randomisation: individual

Unit of analysis: individual

Method to promote equivalence between groups: based on educational level and future aspirations, youth were divided into 3 groups: career planning (i.e. freshman or sophomore youth; $n=72$ ), job (i.e. junior or senior students who did not desire to go to college; out of school youth; $n=43$ ), or college (i.e. junior or senior students who desired to go to college; $n=55)$.

Statistical models: linear or binomial regression model

Baseline differences adjustment: linear or binomial regression model controlling for participant's age, gender, and baseline outcome finding

Repeated measures methods in analysis: N/A. Only 1 follow-up

Notes Equity: baseline data reported

Funding: Zanvyl and Isabelle Krieger Foundation, Health Research and Services Administration Grant Number T32 HP1004, the DC-Baltimore Research Center on Child Health Disparities P20 MD000198 from the National Institute on Minority Health and Health Disparities, and the Eunice Kennedy Shriver National Institute of Child Health and Human Development (NICHD) Grant Number 1K24HD052559

Randomisation method, e.g. block, stratification, computer: youth were randomised to intervention and comparison arms via numbered sealed envelopes. Based on a random number generator, youth within groups were then randomly assigned to a condition. 


\section{Clustering accounted for in sample size calculation (if relevant): N/A}

Cluster randomisation methods to account for clustering in analysis: N/A

\section{Risk of bias}

\begin{tabular}{lll}
\hline Bias & Authors' judgement & Support for judgement \\
\hline $\begin{array}{l}\text { Random sequence genera- } \\
\text { tion (selection bias) }\end{array}$ & Low risk & Random number generator \\
\hline
\end{tabular}

\begin{tabular}{lll}
\hline $\begin{array}{l}\text { Allocation concealment } \\
\text { (selection bias) }\end{array}$ & High risk & Numbered sealed envelopes but not opaque \\
\hline $\begin{array}{l}\text { Blinding of participants } \\
\text { and personnel (perfor- } \\
\text { mance bias) }\end{array}$ & High risk & Blinding was not possible given the nature of this intervention. \\
All outcomes & \\
\hline
\end{tabular}

\section{Blinding of outcome as- High risk} sessment (detection bias)

All outcomes Outcome assessor blinded, but outcomes self-reported by participants who
were not blind to intervention allocation

Incomplete outcome data Low risk Low attrition and attrition comparable between groups
(attrition bias)
All outcomes

\begin{tabular}{lll}
\hline $\begin{array}{l}\text { Selective reporting (re- } \\
\text { porting bias) }\end{array}$ & Low risk & $\begin{array}{l}\text { No published protocol, but all specified outcomes were reported and sub- } \\
\text { groups were built into the randomisation schedule }\end{array}$ \\
\hline Other bias & Unclear risk & $\begin{array}{l}\text { Control group received some career advice and had opportunities to attend } \\
\text { career workshops; insufficient detail about reporting of subgroup data }\end{array}$ \\
\hline
\end{tabular}

Kellam 2014

\section{Methods}

Study name: Good Behaviour Game (GBG)

Study design: cluster RCT (schools were randomised and classrooms within schools were randomised to receive intervention or control)

Intervention arm: GBG

Comparator arm: internal control group; external control group

Sample size calculation performed: yes

Subgroups prespecified: not clear

Subgroup analyses: by gender, risk group

Start date: 1985

Duration of follow-up: up to 12 years

Number of follow-ups: at least 8

Follow-up time points: end of third grade through end of sixth grade (up to 4 years following end of intervention); from 8 to 9 years to age 14 years; from 19 to 21 years (equivalent to approximately 12 years following the end of the intervention) 
Kellam 2014 (Continued)

Intracluster correlation coefficient: not given

Participants

Number of schools randomised: 19 schools in total, 41 classrooms

Number of participants randomised: $\mathrm{N}=1196$ children recruited (407 in GBG and internal control); 238 children in intervention group at baseline; 169 in control group at baseline

Age (range or mean (SD)) or grade at the start: first grade ( 6 years)

Gender: $49.1 \%$ male

Ethnicity: $75 \%$ African American, $22.6 \%$ Caucasian, 2.4\% other

Socioeconomic status: $51.8 \%$ had free or reduced-price lunch status

Inclusion criteria: children in Baltimore elementary schools

Exclusion criteria: children in special education classrooms

Duration of the intervention: 2 school years

Description of the intervention arm(s): classroom team-based behaviour management strategy that promotes good behaviour by rewarding teams that do not exceed maladaptive behaviour standards. Teachers assign students to heterogeneous, gender-balanced teams. Teachers highlight rules of classroom behaviour, and teams are rewarded if members exhibited 4 or fewer behaviours during game sessions. Initially, prizes are delivered immediately after the game, but as the year progresses, the game becomes longer and less predictable and rewards are deferred.

Brief description of the theoretical model: life course/social field theory

Description of the comparator arm(s): receive customary school programmes

$\begin{array}{ll}\text { Outcomes } & \text { Primary outcomes: aggression and disruptive behaviour } \\ & \text { Secondary outcomes: tobacco smoking, depression, anxiety, drug use, alcohol use, high-risk sexual } \\ \text { behaviours, suicidality }\end{array}$

Setting Country: USA; Place: Baltimore

Setting: primary school

Focus: universal

Process measures Process data reported: yes

Method: qualitative

Description: GBG was played 3 times per week for 10 minutes. Duration increased 10 minutes per game period every 3 weeks, up to maximum 3 hours.

Unit of randomisation: classroom

Unit of analyses: individual-, classroom- and school-level data for different analyses

Methods to promote equivalence between groups: 3 or 4 schools matched in each of 5 urban areas by socioeconomic status, size of school, and ethnicity

Statistical models: general growth mixture modelling 
Baseline differences adjustment: controlled for larger proportion of missing data from 1 school - postulated as due to large classroom size. Differences in depressive symptoms mitigated by analysis

Repeated measures methods in analysis: yes

Notes

Equity: results presented by gender and risk class

Funding: National Institute of Mental Health and National Institute on Drug Abuse; NIMH Grants R01 MH 42968, P50 MH 38725, R01 MH 40859, and T32 MH018834, with supplements from NIDA for each of the cited research grants

Randomisation method: multi-level design in which researchers selected 5 large urban areas within Baltimore City, matched sets of schools in each area, and randomly assigned which type of intervention would be tested in which elementary schools from these urban areas. All children entering first grade in classrooms within these schools were assigned in a balanced manner; classrooms were then randomly assigned to the classroom intervention condition.

Clustering accounted for in sample size calculation: N/S

Cluster randomisation methods to account for clustering in analysis: yes, in later papers

\begin{tabular}{|c|c|c|}
\hline \multicolumn{3}{|l|}{ Risk of bias } \\
\hline Bias & Authors' judgement & Support for judgement \\
\hline $\begin{array}{l}\text { Random sequence genera- } \\
\text { tion (selection bias) }\end{array}$ & Unclear risk & Insufficient information \\
\hline $\begin{array}{l}\text { Allocation concealment } \\
\text { (selection bias) }\end{array}$ & Unclear risk & Insufficient information \\
\hline $\begin{array}{l}\text { Blinding of participants } \\
\text { and personnel (perfor- } \\
\text { mance bias) } \\
\text { All outcomes }\end{array}$ & High risk & $\begin{array}{l}\text { Within schools, it was likely that students knew which intervention they were } \\
\text { receiving and teachers knew which intervention they were delivering. }\end{array}$ \\
\hline $\begin{array}{l}\text { Blinding of outcome as- } \\
\text { sessment (detection bias) } \\
\text { All outcomes }\end{array}$ & High risk & Results are based on peer and teacher reports. \\
\hline $\begin{array}{l}\text { Incomplete outcome data } \\
\text { (attrition bias) } \\
\text { All outcomes }\end{array}$ & Unclear risk & $\begin{array}{l}\text { Imputation conducted for certain variables, but insufficient information over- } \\
\text { all }\end{array}$ \\
\hline $\begin{array}{l}\text { Selective reporting (re- } \\
\text { porting bias) }\end{array}$ & Unclear risk & Data not always presented in full for each subgroup \\
\hline Other bias & Low risk & No other sources of bias identified \\
\hline
\end{tabular}

$\operatorname{Kim} 2011$

Methods

Study name: Middle School Success

Study design (e.g. RCT, cluster RCT): RCT at the level of the family

Intervention arm(s): Middle School Success intervention

Comparator arm(s): usual services provided by child welfare system 
Kim 2011 (Continued)

\author{
Sample size calculation performed: N/S \\ Subgroups prespecified: N/A \\ Subgroup analyses: no \\ Start date: N/S \\ Duration of follow-up: 36 months post baseline \\ Number of follow-ups: 4 \\ Follow-up time points: $6,12,24$, and 36 months post baseline \\ ICC (if reported): N/A
}

Participants

Number of schools randomised: N/A

Number of participants randomised (total and by arm): 48 allocated to intervention, 52 to control

Age (range or mean (SD)) or grade at the start: mean 11.54 years at baseline

Gender: $100 \%$ female

Ethnicity: European American (63\%), Latino (10\%), African American (9\%), Native American (4\%), multi-racial (14\%)

SES: caregiver annual family income used as proxy (intervention/control): $\$ 24,999$ or below (22.7\%/17.8\%), $\$ 25,000$ to $\$ 59,999$ (47.8\%/51.1\%), $\$ 60,000+(29.5 \% / 31.1 \%)$

Inclusion criteria: in relative or non-relative foster care within the 2 study catchment areas (Pacific NorthWest USA)

Exclusion criteria: not reported

\title{
Randomisation before or after baseline survey: before
}

Duration of the intervention (excluding follow-up): 3 months throughout school summer holiday

Description of the intervention: the intervention had 2 main components: 6 sessions of group-based care management training for foster parents, and 6 sessions of group-based skills building sessions for girls

\section{Brief description of the theoretical model: none}

Description of the comparator: usual services provided by the child welfare system, including services such as referrals to individual or family therapy, parenting classes for biological parents, and case monitoring

\begin{tabular}{ll}
\hline Outcomes & $\begin{array}{l}\text { Primary outcomes: not specifically stated; hypothesised to be delinquency, substance use, internalis- } \\
\text { ing and externalising behaviours, and prosocial behaviour } \\
\text { Secondary outcomes: N/S }\end{array}$ \\
\hline Setting & Country: USA; State: Pacific NorthWest \\
& Setting: family \\
& $\begin{array}{l}\text { Focus: targeted; girls in foster care, who are about to enter middle school (in final year of elementary } \\
\text { school) }\end{array}$ \\
\hline Process measures & Process data reported: yes \\
Method (qualitative or quantitative): quantitative
\end{tabular}


Kim 2011 (Continued)

\section{Description}

Adherence to the intervention: the interventionists were supervised weekly and video recordings of sessions took place, which were reviewed and feedback relayed on a regular basis

Intensity of the intervention: 2 weekly meetings for a 3-week duration (for both caregiver and youth group sessions), plus $1 \times 2$-hour support session every week for 1 year for both caregivers and youth

Sample size: 100 (48 intervention, 52 control)
Unit of randomisation: individual (family)
Unit of analysis: individual
Method to promote equivalence between groups: N/S (none)
Statistical models: full information maximum likelihood estimation
Baseline differences adjustment: yes, for severity of neglect
Repeated measures methods in analysis: N/S

Notes Equity: N/A

Funding: National Institute of Mental Health (MH054257) and the National Institute on Drug Abuse (DA023920, DA024672, DA027091), both from the US Public Health Service

Randomisation method, e.g. block, stratification, computer: coin-flip

Clustering accounted for in sample size calculation (if relevant): N/A

Cluster randomisation methods to account for clustering in analysis: N/A

\section{Risk of bias}

\begin{tabular}{|c|c|c|}
\hline Bias & Authors' judgement & Support for judgement \\
\hline $\begin{array}{l}\text { Random sequence genera- } \\
\text { tion (selection bias) }\end{array}$ & Low risk & Coin-flip used as randomisation method \\
\hline $\begin{array}{l}\text { Allocation concealment } \\
\text { (selection bias) }\end{array}$ & Unclear risk & Insufficient information provided \\
\hline $\begin{array}{l}\text { Blinding of participants } \\
\text { and personnel (perfor- } \\
\text { mance bias) } \\
\text { All outcomes }\end{array}$ & High risk & Self-reported outcome measures used \\
\hline $\begin{array}{l}\text { Blinding of outcome as- } \\
\text { sessment (detection bias) } \\
\text { All outcomes }\end{array}$ & High risk & Self-reported outcome measures used \\
\hline $\begin{array}{l}\text { Incomplete outcome data } \\
\text { (attrition bias) } \\
\text { All outcomes }\end{array}$ & Low risk & $\begin{array}{l}\text { Relatively low rates of attrition and no differential attrition between study } \\
\text { arms/subgroups. Accounted for missing data using FIML estimation }\end{array}$ \\
\hline $\begin{array}{l}\text { Selective reporting (re- } \\
\text { porting bias) }\end{array}$ & Unclear risk & No published protocol \\
\hline Other bias & Low risk & No other forms of bias evident \\
\hline
\end{tabular}


Comparator arm(s): standard care

Sample size calculation performed: yes, partially stated

Subgroups prespecified: yes

Subgroup analyses: intention to treat: available case analysis

Start date: June 1990

Duration of follow-up: 12 years

Number of follow-ups: 2

Follow-up time points: 3 and 12 years post baseline

ICC (if reported): N/A

Number of participants randomised (total and by arm): 743 women (515 intervention, 228 control)

Age (range or mean (SD)) or grade at the start: from birth

Gender: all female

Ethnicity: 92.1\% African American

SES: $85.1 \%$ were from households with annual incomes below UW federal poverty guidelines

Inclusion criteria: females, $<29$ weeks' gestation, first pregnancy, possessing certain sociodemographic risk characteristics ( 2 or more of unemployed, less than 12 years of education in total, unmarried)

\section{Exclusion criteria: $\mathrm{N} / \mathrm{S}$}

\section{Randomisation before or after baseline survey: after \\ Duration of the intervention (excluding follow-up): 2.5 years}

Description of the intervention: women received home visitation services, delivered by nurses, throughout pregnancy and up to 2 years after the birth of the child. During home visits, nurses (1) promoted improvements in both women and other family members, pregnancy-outcomes related behaviour, and behaviour affecting the health and development of the child; (2) helped women build supportive relationships with family members and friends; and (3) sign-posted women to other services and support avenues. Nurses followed a protocol and used behavioural change mechanisms to attempt to achieve a reduction in female self-reported alcohol, tobacco, and drug use; they advised women on the signs and symptoms of common pregnancy-related complications. After birth, nurses helped mothers improve physical and emotional care of the child and helped them with problem-solving and goal-setting regarding education, work, and planning of future pregnancies.

Brief description of the theoretical model: epidemiology and theories of child development and behaviour change, namely, human ecology, self-efficacy, and human attachment

Description of the comparator: free transportation for scheduled prenatal care plus developmental screening and referral for the child at 6,12 , and 24 months of age 
Kitzman 2010 (Continued)

Outcomes

Primary outcomes: use of alcohol, cigarettes, and marijuana; externalising and internalising problems; arrests; academic achievement

Secondary outcomes: special education; grade retention; conduct grades; sustained attention

\begin{tabular}{ll}
\hline Setting & Country: USA; State: Memphis \\
& Setting: family home \\
& Focus: targeted; females at less than 29 weeks' gestation with sociodemographic risk characteristics \\
\hline Process measures & Process data reported: no \\
Method (qualitative or quantitative): N/A & Description: N/A
\end{tabular}

Sample size: 743 women (515 intervention, 228 control)

Unit of randomisation: individual (females recruited)

Unit of analysis: individual (mother and also child)

Method to promote equivalence between groups: paper described only results of the 3-year follow-up: stratification based on maternal age, employment, gestational age, geographic residence, annual household income

Statistical models: generalised linear models (and same, with negative binomial error assumption added into the model), logistical regression modelling

Baseline differences adjustment: yes; all significant baseline differences were adjusted for

Repeated measures methods in analysis: yes; mixed models accounted for repeated measures

Equity: N/S
Funding: National Institutes of Health Research (1R01MH68790-01) and National Institute of Mental
Health and the Office of Juvenile Justice and Delinquency Prevention (2004-52854-CO-JS0). Earlier
phases of this study were supported by the National Institute of Mental Health (grant R01-MH61428-01),
the National Institute of Child Health and Human Development (grant R01-HD-043492), the National In-
stitute of Nursing Research (grant NR01-01691-05), the Bureau of Maternal and Child Health (grant MCJ
360579), the Administration for Children and Families (grants 90PJ0003 and 90PD0215/01), the Office of
the Assistant Secretary for Planning and Evaluation (Department of Health and Human Services), and
the National Center for Child Abuse and Neglect through a transfer of funds to the National Institute of
Nursing Research (grant R01NR01691)

Randomisation method, e.g. block, stratification, computer: N/S

Clustering accounted for in sample size calculation (if relevant): N/A

Cluster randomisation methods to account for clustering in analysis: N/A

\section{Risk of bias}

\begin{tabular}{lll}
\hline Bias & Authors' judgement & Support for judgement \\
\hline $\begin{array}{l}\text { Random sequence genera- } \\
\text { tion (selection bias) }\end{array}$ & Unclear risk & Computer-generated randomisation \\
\hline $\begin{array}{l}\text { Allocation concealment } \\
\text { (selection bias) }\end{array}$ & Unclear risk & No information provided \\
\hline
\end{tabular}




\section{Kitzman 2010 (Continued)}

Blinding of participants and personnel (perfor-

High risk Blinding not possible owing to the nature of the intervention mance bias)

All outcomes

\begin{tabular}{lll}
\hline Blinding of outcome as- & High risk & Self-reported outcome measures \\
sessment (detection bias) & &
\end{tabular}

All outcomes

Incomplete outcome data Low risk

(attrition bias)

Attrition not overly high $(<30 \%)$ and never $>10 \%$ throughout all waves of fol-

All outcomes low-up

\begin{tabular}{lll}
\hline $\begin{array}{l}\text { Selective reporting (re- } \\
\text { porting bias) }\end{array}$ & Unclear risk & No published protocol
\end{tabular}

Other bias Low risk No other forms of bias evident

Lana 2014

\begin{tabular}{ll}
\hline Study name: Prevencanadol & Study design: RCT \\
Intervention arm(s): Prevencanadol intervention \\
Comparator arm(s): control \\
Sample size calculation performed: yes \\
Subgroups prespecified: N/A \\
Subgroup analyses: no \\
Start date: 2009 \\
Duration of follow-up: 9 months \\
Number of follow-ups: 1 \\
Follow-up time points: post-test \\
ICC (if reported): N/A
\end{tabular}

Participants

Number of schools randomised: N/A

Number of participants randomised (total and by arm): 2001 total; 1014 intervention, 987 control

Age (range or mean (SD)) or grade at the start: percentage of each age by intervention group: 12 years $23.6 ; 13$ years $40.6 ; 14$ years $26.5 ; 15$ years or more 9.2 . Control group: 12 years $20.5 ; 13$ years 42.7 ; 14 years $27.4 ; 15$ years or more 9.4. Experimental group: 12 years $26.6 ; 13$ years $38.5 ; 14$ years $25.7 ; 15$ years or more 9.2

Gender: for both study arms, female 54.8\%. Control group: female 54.2\%. Experimental group: female $55.4 \%$

Ethnicity: participants from Mexico 78.0\%; the remainder were from Spain. Control group: $80.6 \%$ from Mexico. Experimental group: $75.6 \%$ from Mexico

SES 
Lana 2014 (Continued)

Father studies: primary $6.9 \%$; secondary $43.5 \%$; university $49.6 \%$

Mother studies: primary $6.4 \%$; secondary $39.7 \%$; university $53.9 \%$

Control

Father studies: primary $7.7 \%$; secondary $42 \%$; university $50.3 \%$

Mother studies: primary $7.6 \%$; secondary $37.6 \%$; university $54.8 \%$

Experimental group

Father studies: primary 6\%; secondary $45.1 \%$; university $78.9 \%$

Mother studies: primary 5.2\%; secondary $41.8 \%$; university $53.0 \%$

Inclusion criteria: Spanish and Mexican students in secondary education 12 to 16 years of age who voluntarily accepted to participate. Access to the Internet at home or at school was required.

Exclusion criteria: N/A

Interventions

Randomisation before or after baseline survey: after

Duration of the intervention (excluding follow-up): 9 months (1 academic year)

Description of the intervention: The website included several sections to show how to prevent and treat main cancer risk behaviours using the theoretical framework of the ASE model, that is, (1) emphasising advantages of following the recommendations and disadvantages of risk behaviours, (2) creating a healthy online social environment, and (3) strengthening skills to avoid risk behaviours. The section with the highest educational capacity contained problems or challenges that students had to solve. They were related both to subjects of their curriculum (e.g. Math, Literature, Science) and to risk behaviour prevention. The website provided other services such as expert dietetic advice after analysis of common homemade recipes and 24-hour food recalls, peer-starred educational videos, forums and chat lines to discuss cancer-related topics, documents and web links with selected information, and online educational games. Moreover, adolescents who had provided a cell phone number received weekly text messages to encourage compliance with healthy behaviours.

Brief description of the theoretical model: the Transtheoretical Model of Behavior Change and the ASE Model (or I-Change Model), which affirms that the purpose of carrying out a behaviour mainly depends on attitude, social influence, and self-efficacy

Description of the comparator: not clear

Outcomes Primary outcomes: smoking, unhealthy diet, alcohol consumption, obesity, sedentary lifestyle, sun exposure

Secondary outcomes: N/A

Setting Country: Spain and Mexico; State: N/A

Setting: secondary school (web-based programme)

Focus: universal

Process measures Process data reported: no

Method (qualitative or quantitative): N/A

Description: N/A

Statistics

Sample size: 2001 randomised

Unit of randomisation: individual 
Lana 2014 (Continued)

Unit of analysis: individual

Method to promote equivalence between groups: N/A

Statistical models: generalised linear models; binary logistical regression; multi-nomial logistical regression

Baseline differences adjustment: yes

Repeated measures methods in analysis: N/A

Notes

Equity: student academic level: experimental arm: very good $21.5 \%$; good $47.3 \%$; fair $28.2 \%$; bad/very bad $2.9 \%$; control group: very good $21.4 \%$; good $47.0 \%$; fair $27.8 \%$; bad/very bad $3.8 \%$. Health status: experimental arm: very good $41.4 \%$; good $36.5 \%$; fair $19.2 \%$; bad/very bad $2.9 \%$; control group: very good 39.6\%; good 39.5\%; fair 17.9\%; bad/very bad 3.1\%

Funding: Spanish Ministry of Health (Reference: FISS 08PI080544).

Randomisation method: computerised process; no further details given

Clustering accounted for in sample size calculation (if relevant): N/A

Cluster randomisation methods to account for clustering in analysis: N/A

\section{Risk of bias}

\begin{tabular}{lll}
\hline Bias & Authors' judgement & Support for judgement \\
\hline $\begin{array}{l}\text { Random sequence genera- } \\
\text { tion (selection bias) }\end{array}$ & Low risk & Computerised process \\
\hline $\begin{array}{l}\text { Allocation concealment } \\
\text { (selection bias) }\end{array}$ & Unclear risk & Insufficient information \\
\hline $\begin{array}{l}\text { Blinding of participants } \\
\text { and personnel (perfor- } \\
\text { mance bias) }\end{array}$ & High risk & Intervention not blinded \\
All outcomes & & \\
\hline
\end{tabular}

Blinding of outcome as- High risk Self-reported outcome data

sessment (detection bias)

All outcomes

Incomplete outcome data High risk Missing outcome data not balanced across groups; high attrition
(attrition bias)

All outcomes

\begin{tabular}{l}
$\begin{array}{l}\text { Selective reporting (re- Low risk } \\
\text { porting bias) }\end{array}$ \\
\hline
\end{tabular}

Other bias Low risk No other sources of bias identified

Study design (e.g. RCT, cluster RCT): RCT at the level of the family (parent/child dyad)

Intervention arm(s): Informed Parents and Children Together (imPACT) intervention 
Comparator arm(s): "Goal for It" intervention; 22-minute video on education and career training and handout of workbook for achieving long-term goals. No health-specific information was delivered to control group participants.

Sample size calculation performed: not reported

Subgroups prespecified: N/A

Subgroup analyses: not carried out

Start date: summer 1997

Duration of follow-up: 12 months post intervention

Number of follow-ups: 3

Follow-up time points: 2,6 , and 12 months post intervention

ICC (if reported): N/A

\title{
Participants
}

\section{Number of schools randomised: N/A}

Number of participants randomised (total and by arm): 237 dyads

Age (range or mean (SD)) or grade at the start: parent respondents: baseline: median age 13.6 years; youth respondents: 12 -month follow-up. Intervention: mean age 14.52 years (1.41 SD). Control; 14.28 (1.41 SD)

Gender: parent respondents: baseline (only reported): $51 \%$ male, $49 \%$ female; youth respondents: 12 month follow-up: intervention ( $53 \%$ male, $47 \%$ female), control ( $47 \%$ male, $53 \%$ female), overall ( $49 \%$ male, $51 \%$ female)

\section{Ethnicity: N/S}

SES: N/S

Inclusion criteria: for the adolescent/parent dyad to be eligible, the adolescent had to spend at least $50 \%$ of his/her time with the parent (parent is defined as biological, legal, or other guardian). The child had to be 12 to 16 years of age. Only 1 parent and 1 child were eligible for inclusion, per family.

Exclusion criteria: N/S

Interventions

\author{
Randomisation before or after baseline survey: after \\ Duration of the intervention (excluding follow-up): $<90$ minutes (brief family intervention) \\ Description of the intervention \\ imPACT
}

A culturally tailored video, developed via qualitative work with families and children, lasting $22 \mathrm{~min}$ utes. The video includes excerpts from qualitative interviews, including parental concepts of risk involvement, risk prevention, precise meanings of 'parenting' and 'monitoring', youth-youth discussions on importance of parental monitoring, and adolescent risk and protective factors. A condom demonstration follows, along with 6 key messages directed towards parents: monitor children, talk about sex with children before they engage in it, know about AIDS, learn how to use a condom, emphasise selfprotection, including abstinence (condom use/contraception), and emphasise drug/alcohol use posing risks to sexual behaviour. Parents and youth were then asked to play a gender-specific vignette with a pre-assigned role before swapping over. Parents were given their own copy of the 22-minute video and were provided additional literature.

Brief description of the theoretical model: $\mathrm{N} / \mathrm{S}$ 
Li 2002 (Continued)

Description of the comparator: the 'Goal for It' programme, which consists of a 22-minute video entitled "Goal for it: a guide for adolescents and parents on education and career training", and a workbook for recording the process of working through the 4-step process in achieving a long-term goal

Outcomes Primary outcomes: not reported; hypothesised to be the following: tobacco, alcohol, and marijuana use; selling drugs; carrying a weapon; having sex; staying out all night; fighting

Secondary outcomes: not reported

$\begin{array}{ll}\text { Setting } & \text { Country: USA; State: N/S } \\ \text { Setting: family (home) } \\ \text { Focus: targeted: African American parent-adolescent dyads }\end{array}$

Process measures Process data reported: no

Method (qualitative or quantitative): N/A

Description: intensity of the intervention: 1 session, 60 to 90 minutes in duration

Sample size: total of 179 families
Unit of randomisation: family dyad
Unit of analysis: individual (parent/adolescent)
Method to promote equivalence between groups: not reported
Statistical models: ANCOVA, t-statistic
Baseline differences adjustment: age and gender controlled by ANCOVA model
Repeated measures methods in analysis: no
Equity: N/S
Funding: supported by the National Institute of Mental Health (4R01-MH54983)
Randomisation method, e.g. block, stratification, computer: random number table
Clustering accounted for in sample size calculation (if relevant): not reported
Cluster randomisation methods to account for clustering in analysis: not applicable

Risk of bias

\begin{tabular}{lll}
\hline Bias & Authors' judgement & Support for judgement \\
\hline $\begin{array}{l}\text { Random sequence genera- } \\
\text { tion (selection bias) }\end{array}$ & Low risk & Random number tables used to generate random sequence \\
\hline $\begin{array}{l}\text { Allocation concealment } \\
\text { (selection bias) }\end{array}$ & Unclear risk & Lack of clarity around method and roles of different personnel involved \\
\hline $\begin{array}{l}\text { Blinding of participants } \\
\text { and personnel (perfor- } \\
\text { mance bias) } \\
\text { All outcomes }\end{array}$ & High risk & $\begin{array}{l}\text { Highly unlikely to be possible, given the nature of this intervention; self-report- } \\
\text { ed outcomes }\end{array}$ \\
\hline
\end{tabular}

$\begin{aligned} & \text { Blinding of outcome as- } \\ & \text { sessment (detection bias) }\end{aligned} \quad$ High risk $\quad$ Self-reported outcome measures used


Li 2002 (Continued)

All outcomes

Incomplete outcome data Unclear risk $\quad 24 \%$ attrition could be related to 12 -month outcomes.
(attrition bias)

(attrition bias)

All outcomes

\begin{tabular}{lll}
\hline $\begin{array}{l}\text { Selective reporting (re- } \\
\text { porting bias) }\end{array}$ & Unclear risk & No published protocol; primary outcomes not stated \\
\hline Other bias & Low risk & No other forms of bias evident \\
\hline
\end{tabular}

\section{Li 2011}

\section{Methods}

Study name: Positive Action Chicago

Study design (e.g. RCT, cluster RCT): cluster RCT at the level of the school; a matched-pair, randomised-control design (i.e. schools with similar characteristics were matched into pairs, then schools within a pair were allocated randomly to either programme or control conditions)

Intervention arm(s): Positive Action

Comparator arm(s): schools in the control condition received PA programme and materials after the end of the trial period.

\section{Sample size calculation performed: no}

Subgroups prespecified: covariates of age, gender, ethnicity; newcomers and stayers specified before the results section

Subgroup analyses: assessment by exposure - newcomers/stayers

Start date: fall 2004-2005 school year (baseline fall 2004)

Duration of follow-up: over 6 years of programme implementation (grades 3 to 8 )

\section{Number of follow-ups: 4}

Follow-up time points:

Wave 1: spring 2005 (6 months)

Wave 2: fall 2005 (12 months)

Wave 3: spring 2006 (18 months)

Wave 4: spring 2007 (end of grade 5; 2.5 years)

Wave 5: fall 2008

Wave 6: spring 2009

Wave 7: spring 2010 (end of grade 8; 5.5 years)

ICC (if reported): not reported but interaction was reported as significant or not in some analyses

Participants

Number of schools randomised: 68 elementary schools eligible, 18 schools agreed to participate; 7 well-matched schools were created ( 7 intervention and 7 control)

Number of participants randomised (total and by arm): at baseline, intervention $\mathrm{n}$ 310; control $\mathrm{n}$ 310 (numbers were rounded to the nearest 10); up to 1170 total in later analyses 
Age (range or mean (SD)) or grade at the start: grade 3 students aged 8 to 9 years, mean 8.6 to 8.7 years

Gender: boys: control $44.9 \%$, intervention $48.2 \%$

\section{Ethnicity:}

White: intervention $7.54 \%$, control $6.09 \%$

African American: intervention 48.2\%, control 40.7\%

Hispanic American: intervention 23.61\%, control 26.6\%

Asian American: intervention 3.28\%, control 1.28\%

Other or mixed ethnicity: intervention $17.38 \%$, control $25.32 \%$

SES: percentage students receiving a free lunch: control: mean 81.46, SD 3.81; intervention: 85.51, SD 4.56

Inclusion criteria: schools in the Chicago Public Schools system

Exclusion criteria: schools using PA curricula or a similar programme; non-community schools; enrolment $<50$ or $>140$ children/grade; student mobility $>40 \% ; 50 \%$ pass rate in Illinois State Assessment Test (ISAT); $<50 \%$ of students receiving free lunches

Description of the intervention: Positive Action is an educational programme consisting of a series of scripted lessons for each grade level Pre-K through 12th. The programme is in kit form and includes all materials required for implementation. Lessons are scoped and sequenced, allowing schools to create a unified environment. No training is required, and the lessons are easy to use. This programme teaches positive actions for the physical, intellectual, social, and emotional areas of the self. In this trial, programme schools received the $\mathrm{K}$ to 8 portion of the PA classroom curriculum and school/staff training from the programme developer; also included were kits for school preparation, school-wide climate development, counsellors, and family classes. The K to 8 PA classroom curriculum is scoped and sequenced, consisting of over 140 15-minute age-appropriate lessons per grade designed to be taught 4 days per week. Website: www.positiveaction.net

Brief description of the theoretical model: multiple health behaviour theories (i.e. Ajzen 1991). Positive Action works through the Thoughts-Actions-Feelings Circle, where positive thoughts lead to positive actions, positive actions lead to positive feelings about self, and positive feelings lead to more positive thoughts.

Description of the comparator: treatment as usual

Secondary outcomes: social and emotional character development, academic outcomes, depression, anxiety, positive affect, life satisfaction

\begin{tabular}{ll}
\hline Setting & Country: USA; State: Chicago \\
& Setting: 14 public urban elementary schools \\
& Focus: universal \\
\hline Process measures & Process data reported: yes \\
& Method (qualitative or quantitative): quantitative
\end{tabular}


Li 2011 (Continued)

\section{Description}

Adherence to the intervention: programme providers regularly contacted school principals and administrators to ensure adherence to the PA programme. Workshops for teachers were held during the second and third years of the study. Teachers in intervention schools completed a Unit Implementation Report at the end of each of the 6 units of the programme - approximately every 6 weeks. Reports show some variability in adherence between schools.

Intensity of the intervention: variability between schools in all implementation indices (i.e. programme benchmarks), especially in the first year, with improvements over time. By the end of the third year, 1 school was still implementing at a low level ( $<50 \%$, on average, across all benchmarks), 4 at a moderate level (between $50 \%$ and $60 \%$ ), and 2 at moderate to high levels (between $60 \%$ and $70 \%$ ).

Statistics

Sample size: 68 elementary schools eligible; 18 schools agreed to participate; 7 well-matched schools were created ( 7 intervention and 7 control); Intervention $n$ 310; control $n$ 310 (numbers were rounded to the nearest 10)

Unit of randomisation: schools

Unit of analysis: school

Method to promote equivalence between groups: matched pairs were generated. Equivalence between PA and control schools was tested at school and student levels. With controls for demographics and clustering, PA and control groups were not significantly different in terms of selected variables.

Statistical models: missing values were imputed via switching regression; MID (Multiple Imputation then Deletion) methods were used in the analyses; 3-level Poisson models

Baseline differences adjustment: self-reported variables of age, gender, ethnicity, and baseline problem behaviours were included in analyses as covariates

Repeated measures methods in analysis: not reported

Equity: baseline and follow-up in Table S1 plus baseline measures (given in online table for paper) with some discussion in text: Gender composition and age at baseline were not significantly different between programme and control conditions, whereas differences in ethnicity composition were significant. There were more African American students and fewer students in the other/mixed ethnicity group for the control condition compared to the PA condition. With controls for demographic variables and clustering of students, PA students were not significantly different from control students in reported rates of problem behaviours.

Funding: IES research grant. Dr Flay's spouse holds a significant financial interest in Positive Action, Inc.

Randomisation method, e.g. block, stratification, computer: random number generation function in Microsoft Excel was used to randomise schools to treatment or control conditions.

Clustering accounted for in sample size calculation (if relevant): not reported

Cluster randomisation methods to account for clustering in analysis: yes

\section{Risk of bias}

\begin{tabular}{lll}
\hline Bias & Authors' judgement & Support for judgement \\
\hline $\begin{array}{l}\text { Random sequence genera- } \\
\text { tion (selection bias) }\end{array}$ & Low risk & Microsoft Excel random number generation used \\
\hline $\begin{array}{l}\text { Allocation concealment } \\
\text { (selection bias) }\end{array}$ & Unclear risk & $\begin{array}{l}\text { Study authors stated that allocation to condition was blinded until partici- } \\
\text { pants were assigned but provided no further details. }\end{array}$ \\
\hline
\end{tabular}




\section{Li 2011 (Continued)}

Blinding of participants High risk Not able to blind as school-based intervention and personnel (performance bias)

All outcomes

\begin{tabular}{lll}
\hline $\begin{array}{l}\text { Blinding of outcome as- } \\
\text { sessment (detection bias) } \\
\text { All outcomes }\end{array}$ & High risk & Self-reported outcomes \\
\hline $\begin{array}{l}\text { Incomplete outcome data } \\
\text { (attrition bias) }\end{array}$ & Unclear risk & $\begin{array}{l}\text { Study authors reported conducting multiple imputation, but attrition of en- } \\
\text { rolled participants is high, and approximately half the final sample were new- } \\
\text { comers. }\end{array}$ \\
\hline
\end{tabular}

\begin{tabular}{lll}
\hline $\begin{array}{l}\text { Selective reporting (re- } \\
\text { porting bias) }\end{array}$ & Unclear risk & No published protocol \\
\hline Other bias & Low risk & No other sources of bias identified \\
\hline
\end{tabular}

Lochman 2003a

$\begin{array}{ll}\text { Methods } & \text { Study name: Coping Power } \\ \text { Study design (e.g. RCT, cluster RCT): cluster RCT: } 17 \text { schools, } 60 \text { classrooms }\end{array}$

Intervention arm(s): universal intervention classroom + indicated intervention (IU); universal intervention classroom + indicated control (U); universal comparison classroom + indicated intervention (I)

Comparator arm(s): universal comparison classroom + indicated control

Sample size calculation performed: not reported

Subgroups prespecified: none

Subgroup analyses: none reported

Start date: September 1997

Duration of follow-up: 12 months post intervention

Number of follow-ups: 3

Follow-up time points: wave 1 summer 1998; wave 2 post intervention 1999; wave 3 one year post intervention at the end of grade 6 or 7

ICC (if reported): not reported

\section{Participants}

Number of schools randomised: 17

Number of participants randomised (total and by arm): $N=245$ children randomised ( 61 to indicated + universal; 59 to indicated; 62 to universal; 63 to control)

Age (range or mean (SD)) or grade at the start: age not reported. Sampled at end of grade 4; start at grade 5 , finish end of grade 7

Gender: 2:1 ratio boys:girls; across the 4 cells, the number of boys ranged from $63 \%$ to $68 \%$

Ethnicity: across the 4 cells, African American children ranged from $75 \%$ to $81 \%$.

SES: the 4 cells were equivalent for demographic factors, teacher-estimated cognitive functioning, and aggressive behaviour at screening. 
Lochman 2003a (Continued)

Inclusion criteria: fourth grade teachers from 17 elementary schools were asked to rate the aggressiveness of children in their classes. The most aggressive children (31\%) were eligible for the trial depending upon parental consent.

Exclusion criteria: children not selected by the screening process

Interventions

Randomisation before or after baseline survey: after summer baseline assessment (Time 1)

Duration of the intervention (excluding follow-up): 16 months

Description of the intervention

Coping Power (at middle school transition) (CP)

Classroom intervention (known as Coping With the Middle School Transition: CMST) children and parents were offered 4 parent meetings ( 3 during grade 5 year and 1 at grade 6); teachers had five 2-hour in-service meetings to promote home-school involvement and to address concerns about the transition to middle school in the fifth grade year.

The Coping Power programme has a child and parent focus. The child component is 16 months with 22 group sessions during the fifth grade year and 12 group sessions for the sixth grade, usually held at school. Group sessions were led by a school guidance counsellor and a school-family programme specialist; sessions were 40 to 50 minutes with 5 to 8 children/group.

Individual child sessions were held at school for 30 minutes approximately 1 to 2 months.

CP parent component ran for the same 16 months: 11 parent group sessions were held at grade 5 , and 5 sessions at grade 6 . Meetings included groups of 12 parents or parent dyads with 2 leaders.

Brief description of the theoretical model: the Coping Power parent component was derived from social learning theory-based parent training programmes.

Description of the comparator: comparator condition children received services as usual.

Outcomes Primary outcomes: delinquency, substance use, school aggression, school bonding

Secondary outcomes: N/A

Setting Country: USA; State: not reported

Setting: children from 17 elementary schools, parents, and teachers; high proportion of inner city and high-poverty schools

Focus: targeted at children at risk of aggressive/disruptive behaviour

Process data reported: adherence to the intervention, reach of the intervention, intensity of the intervention

Method (qualitative or quantitative): quantitative

\section{Description}

Adherence to the intervention: intervention manuals were provided for parent and child components, along with structured guidelines for the classroom intervention. All grant-funded staff and school counsellors received 10 hours of training before and during the intervention.

Reach of the intervention: classroom intervention: teacher attendance at meetings was 63\%; parent meetings were attended by $21 \% ; 84 \%$ of children attended CP sessions and received a mean of 6 of a possible 8 individual sessions over the 16 months

CP parent group attendance was $26 \%$ with a mean of 6.5 individual contacts.

Intensity of the intervention: CP programme ran over 16 months with 34 child group sessions and 16 parent group sessions. In addition, children had individual bimonthly sessions. 
Lochman 2003a (Continued)

Statistics
Sample size: grade 4 students were screened for aggressive behaviour by class teachers using a screening instrument; eligible children from 17 elementary schools $N=473$; number from consenting parents $n=245.60$ classrooms: 31 classroom Intervention, 29 control

Unit of randomisation: classrooms

Unit of analysis: individuals

Method to promote equivalence between groups: initial randomisation may have been blocked by school. Each school had at least 1 class receiving the classroom intervention and 1 class as the control. Within these classes, the target children (approximately 4/class) were randomised further to CP intervention or non-CP cells.

Statistical models: general linear model ANOVA, MANCOVA, factorial analyses for main effects and interactions

Baseline differences adjustment: equivalence analyses using GLM ANOVA

Repeated measures methods in analysis: repeat measures ANOVA and MANCOVA
Equity not reported

Funding: grant from the Center for Substance Abuse Prevention (KD1 SP08633; UR6 5907956). Additional support provided by grants from the National Institute for Drug Abuse (R01 DA08453; R01 DA16135), the US Department of Justice (2000CKWX0091), and the Centers for Disease Control and Prevention (R49/CCR418569)

Randomisation method, e.g. block, stratification, computer: not reported but may have been blocked by school

Clustering accounted for in sample size calculation (if relevant): no

Cluster randomisation methods to account for clustering in analysis: not reported

\section{Risk of bias}

\begin{tabular}{|c|c|c|}
\hline Bias & Authors' judgement & Support for judgement \\
\hline $\begin{array}{l}\text { Random sequence genera- } \\
\text { tion (selection bias) }\end{array}$ & Unclear risk & Not reported \\
\hline $\begin{array}{l}\text { Allocation concealment } \\
\text { (selection bias) }\end{array}$ & Unclear risk & Not reported \\
\hline $\begin{array}{l}\text { Blinding of participants } \\
\text { and personnel (perfor- } \\
\text { mance bias) } \\
\text { All outcomes }\end{array}$ & High risk & Teachers, parents, and children not blinded \\
\hline $\begin{array}{l}\text { Blinding of outcome as- } \\
\text { sessment (detection bias) } \\
\text { All outcomes }\end{array}$ & High risk & $\begin{array}{l}\text { Teacher and parent assessors not blinded in first year of intervention. Teachers } \\
\text { were not informed of intervention status in the 1-year follow-up study. }\end{array}$ \\
\hline $\begin{array}{l}\text { Incomplete outcome data } \\
\text { (attrition bias) } \\
\text { All outcomes }\end{array}$ & High risk & $\begin{array}{l}\text { Attrition varied between } 83 \% \text { at the start for children (Time } 1 \text { ) and } 51 \% \text { for } \\
\text { teachers (Times } 1 \text { to } 3 \text { ); these values are based on the } \% \text { of completed out- } \\
\text { come assessments. }\end{array}$ \\
\hline $\begin{array}{l}\text { Selective reporting (re- } \\
\text { porting bias) }\end{array}$ & Unclear risk & No published protocol \\
\hline
\end{tabular}


Lochman 2003a (Continued)

Other bias Unclear risk Risk of contamination related to randomisation of classrooms within schools

Lochman 2004a

Methods Study name: Coping Power

Study design (e.g. RCT, cluster RCT): cluster RCT: 11 elementary schools; all male students in grades 4 and 5 were screened by teachers $(N=1578)$. Eligible $N=546$. Parental consent provided for $N=183$.

Intervention $\operatorname{arm}(\mathbf{s}): 2$

Child Intervention $(\mathrm{Cl}) \mathrm{N}=60$

Child plus Parent Intervention (CPI) N = 63

Control arm(s): services as usual

Control (C) $N=60$

Normative sample $(\mathrm{N}=63)$ to represent boys not at risk

Sample size calculation performed: target sample size of 180 boys; no method reported

Subgroups prespecified: none

Subgroup analyses: none reported

Start date: winter 1997

Duration of follow-up: 12 months post intervention

Number of follow-ups: 1

Follow-up time points: 1-year follow-up at the end of sixth or seventh grade

ICC (if reported): interaction values reported but not ICC

Participants

Number of schools randomised: 11

Number of participants randomised (total and by arm): indicated and universal

Age (range or mean (SD)) or grade at the start: age not reported; 2 annual cohorts of fourth and fifth grade boys followed up to grade 6 or 7

Gender: male $100 \%$

Ethnicity: not reported

SES: mean family income levels for the 3 at-risk conditions were 5.4 for $\mathrm{Cl}, 5.6$ for $\mathrm{CPI}$ (child plus parent), and 5.8 for control.

Inclusion criteria: boys in fourth or fifth grade elementary school were screened by teachers for inclusion via multiple-gating; boys with a raw score of 7 or more were eligible for inclusion at Gate 1 ; the Teacher Report Form at Gate 2 required parental consent; the Gate 3 final screen was the Child Behaviour Checklist.

Exclusion criteria: children not meeting the screening score cutoff

Interventions

Randomisation before or after baseline survey: baseline survey conducted at Time 1 along with screening, so appears to be before randomisation

Duration of the intervention (excluding follow-up): 15 months 
Lochman 2004a (Continued)

\section{Description of the intervention}

\section{Coping Power}

Children: Coping Power child sessions included components involving behavioural and personal goalsetting, awareness of feelings and associated physiological arousal, use of coping self-statements, distraction techniques and relaxation methods when provoked and made angry, organisational and study skills, perspective taking and attribution retraining, social problem-solving skills, and use of refusal skills for peer pressure and neighbourhood-based problems.

Eight intervention sessions were scheduled in year 1 , and 25 in year 2; group sessions for 4 to 6 boys lasted 40 to 60 minutes and were led by a staff family-school program specialist and a school guidance counsellor.

Parents: parents learned skills on identifying prosocial and disruptive behavioural targets, rewarding appropriate child behaviours, giving effective instructions and establishing age-appropriate rules and expectations, applying effective consequences to negative child behaviour, and establishing ongoing family communication through weekly family meetings. Parents also learned to support the social cognitive skills that children learn in the Coping Power child component and to use stress management skills to remain calm and in control during stressful or irritating disciplinary interactions with their children.

The Coping Power parent intervention consisted of 16 parent group sessions over the same 15-month period and was delivered in groups of 4 to 6 single parents or couples, with groups usually meeting at the boys' schools.

Brief description of the theoretical model: social learning theory

Description of the comparator: services as usual within schools

Outcomes

\section{List relevant outcomes}

Primary outcomes:

Times 1 and 3: child-rated substance use; child-reported overt and covert delinquency

Time 3: teacher-rated child improvement over the follow-up year; parent-reported substance use by child

Secondary outcomes: none reported

Setting

Country: USA; State: not reported

Setting: 11 schools (start of the trial was in an elementary school setting; end of the trial was in a middle school setting)

Focus: targeted at boys at risk of aggressive or disruptive behaviour

Process measures

Process data reported: adherence to the intervention, reach of the intervention, intensity of the intervention

Method (qualitative or quantitative): quantitative

\section{Description}

Adherence to the intervention: intervention staff scheduled 1.4 individual meetings/month with target children. Intervention staff rated the level of accomplishment for each objective at the end of each intervention session, and checklists were reviewed by supervisors in weekly supervision sessions. Additionally, some intervention sessions were audiotaped or videotaped. Supervisors reviewed taped sessions on a random basis, and supervisors directly observed the delivery of some sessions. All grantfunded staff and school counsellors received 10 hours of training before and during the intervention and weekly supervision of their intervention work. 
Reach of the intervention: attendance at child group sessions was $83 \%$, and attendance at parent sessions was $49 \%$.

Intensity of the intervention: intervention involved 33 group meetings with boys over 15 months ( 2 sessions/month).

Statistics

Sample size: screened $N=1578$ across 2 cohorts in fourth and fifth grades. Eligible $N=546$. Parental consent provided for $\mathrm{N}=183$; a normative sample of 63 boys was also selected from the screened sample

Unit of randomisation: not clear whether individuals, classes, or schools

Unit of analysis: individuals

Method to promote equivalence between groups: not reported

Statistical models: general linear model ANOVA to assess attrition differences. Intervention effects: ANOVA, MANCOVA, or ANCOVA using general linear models. Factorial analyses of main effects and interaction effects when significant. Regression coefficients reported in later paper (2013)

Baseline differences adjustment: no significant differences between attrited and non-attrited participants on the aggression screening score, nor in race or teacher ratings of boys' cognitive abilities. Thus, the available sample for analyses at Time 3 is representative of the original sample at baseline.

Repeated measures methods in analysis: ANCOVA used in analyses

Notes Equity: limited; mean family income levels for each condition

Funding: National Institute for Drug Abuse Grant R01 DA08453. Additional support to complete the article provided to John E. Lochman by Center for Substance Abuse Prevention Grants KD1 SP08633 and UR6 5907956, US Department of Justice Grant 2000CKWX0091, Centers for Disease Control and Prevention Grant R49/CCR418569, and National Institute of Drug Abuse Grant R01 DA16135

Randomisation method, e.g. block, stratification, computer: not reported

Clustering accounted for in sample size calculation (if relevant): not reported

Cluster randomisation methods to account for clustering in analysis: not reported

\section{Risk of bias}

\begin{tabular}{lll}
\hline Bias & Authors' judgement & Support for judgement \\
\hline $\begin{array}{l}\text { Random sequence genera- } \\
\text { tion (selection bias) }\end{array}$ & Unclear risk & Not reported \\
\hline $\begin{array}{l}\text { Allocation concealment } \\
\text { (selection bias) }\end{array}$ & Unclear risk & Not reported \\
\hline
\end{tabular}

Blinding of participants High risk Children, parents, teachers, and programme staff not blinded

and personnel (perfor-

mance bias)

All outcomes

\begin{tabular}{lll}
$\begin{array}{l}\text { Blinding of outcome as- } \\
\text { sessment (detection bias) } \\
\text { All outcomes }\end{array}$ & High risk & $\begin{array}{l}\text { Teachers completing child behaviour assessment at end of follow-up were } \\
\text { blind to intervention status; parents reported substance use by their children }\end{array}$ \\
\hline $\begin{array}{l}\text { Incomplete outcome data } \\
\text { (attrition bias) } \\
\text { All outcomes }\end{array}$ & High risk & $\begin{array}{l}\text { Large proportion not followed up ( 30\%); comparison of other variables be- } \\
\text { tween remaining participants and those included at baseline not given }\end{array}$
\end{tabular}


Lochman 2004a (Continued)
Selective reporting (re-
Unclear risk
No published protocol porting bias)

Other bias

Low risk

No other issues identified

LoSciuto 1999

Methods

Study name: Woodrock Youth Development Project

Study design (e.g. RCT, cluster RCT): cluster RCT at the level of the group (classrooms in schools)

Intervention arm(s): multi-component Woodrock Youth Development Project

Comparator arm(s): standard education classes in schools

Sample size calculation performed: study authors stated that "statistical power was felt adequate to detect any significant effects of a moderate magnitude"; however, no formal sample size calculation was presented, and it is unclear what this premise is based upon.

Subgroups prespecified: N/A; no subgroup analysis undertaken

Subgroup analyses: none

Start date: 1995 (cohort 1), 1996 (cohort 2)

Duration of follow-up: N/S; post-test conducted soon after intervention delivery finished

Number of follow-ups: 1

Follow-up time points: immediately post test (after 2 years of intervention delivery)

ICC (if reported): not reported

Participants

Number of schools randomised: classrooms were randomised - number not stated

Number of participants randomised (total and by arm): 718 were included at baseline (pre-test) assessment.

Age (range or mean (SD)) or grade at the start: mean age of post-test participants at pre-test completion was 10.18 years (1.73 SD); overall age of cohort at baseline was not described.

Gender: described only for students who completed post-test (50\% female)

Ethnicity: described only for students who completed post-test (45\% Latino, 18.9\% white, $12.4 \%$ African American, 10.2\% Asian, 2.2\% American Indian, 11.3\% other)

SES: N/S

Inclusion criteria: N/S

Exclusion criteria: $\mathrm{N} / \mathrm{S}$

Interventions

Randomisation before or after baseline survey: before programme implementation

Duration of the intervention (excluding follow-up): 2 academic school years

Description of the intervention: the Woodrock Youth Development Project (YDP) is a multi-component alcohol, tobacco, and drug (ATD) prevention programme. Components included (1) education, including human relations and life skills seminars in which role-playing and other simulations relevant to drug-use situations are included; (2) psychosocial support, including peer mentoring, tutoring, and a programme of structured, alternative, extracurricular activities; and (3) family and community supports, including counselling and outreach. Classroom activities focus on raising awareness about the 
dangers of ATD use, promoting healthful attitudes about ATD use, and fostering self-esteem by providing positive images of students' ethnic and racial heritages and encouraging appreciation of other cultural traditions. Peer mentoring, extracurricular activities, parenting classes, and home visits were included.

Brief description of the theoretical model: not described

Description of the comparator: completed pre-test and post-test measures only

\begin{tabular}{ll}
\hline Outcomes & Primary outcomes: alcohol, tobacco, and other drug use; aggressive behaviour \\
& Secondary outcomes: N/A \\
\hline Setting & Country: USA; Place: Philadelphia \\
& Setting: 4 public schools (with additional family and community components) \\
& $\begin{array}{l}\text { Focus: targeted; deprived community, with large } \% \text { of children from families receiving financial assis- } \\
\text { tance from the state }\end{array}$
\end{tabular}

\begin{tabular}{ll}
\hline Process measures & Process data reported: no \\
& Method (qualitative or quantitative): N/A \\
& Description: N/A
\end{tabular}

Sample size: 822 students invited to participate in the study, of which 718 were included at baseline (pre-test) assessment

Unit of randomisation: classroom

Unit of analysis: individual

Method to promote equivalence between groups: $\mathrm{N} / \mathrm{S}$

Statistical models: ANCOVA

Baseline differences adjustment: no

Repeated measures methods in analysis: N/A; only post-test assessment carried out

\begin{tabular}{|c|c|c|}
\hline \multirow[t]{5}{*}{ Notes } & \multicolumn{2}{|l|}{ Equity: not described } \\
\hline & \multicolumn{2}{|c|}{$\begin{array}{l}\text { Funding: funded by the Centre for Substance Abuse Prevention (US DHHS), grant number 5H86- } \\
\text { SP03094 }\end{array}$} \\
\hline & \multicolumn{2}{|c|}{ Randomisation method, e.g. block, stratification, computer: N/S } \\
\hline & \multicolumn{2}{|c|}{ Clustering accounted for in sample size calculation (if relevant): no } \\
\hline & \multicolumn{2}{|c|}{ Cluster randomisation methods to account for clustering in analysis: none } \\
\hline \multicolumn{3}{|l|}{ Risk of bias } \\
\hline Bias & Authors' judgement & Support for judgement \\
\hline $\begin{array}{l}\text { Random sequence genera- } \\
\text { tion (selection bias) }\end{array}$ & Unclear risk & Not stated \\
\hline $\begin{array}{l}\text { Allocation concealment } \\
\text { (selection bias) }\end{array}$ & Unclear risk & Not stated \\
\hline
\end{tabular}


LoSciuto 1999 (Continued)

Blinding of participants High risk Blinding unlikely to have been possible owing to the nature of the trial and personnel (performance bias)

All outcomes

\begin{tabular}{lll}
\hline $\begin{array}{l}\text { Blinding of outcome as- } \\
\text { sessment (detection bias) } \\
\text { All outcomes }\end{array}$ & High risk & Self-reported outcome measures only \\
\hline $\begin{array}{l}\text { Incomplete outcome data } \\
\text { (attrition bias) } \\
\text { All outcomes }\end{array}$ & Low risk & $\begin{array}{l}\text { Attrition rates relatively low in both intervention and control groups and over- } \\
\text { all }(<15 \%)\end{array}$ \\
\hline $\begin{array}{l}\text { Selective reporting (re- } \\
\text { porting bias) }\end{array}$ & Unclear risk & No published protocol \\
\hline $\begin{array}{l}\text { Other bias } \\
\text { High risk }\end{array}$ & $\begin{array}{l}\text { Randomisation occurred at the level of the classroom; contamination is an is- } \\
\text { sue. }\end{array}$
\end{tabular}

Matthews 2016

Methods

Study design: cluster RCT

Intervention arm(s): PREPARE

Comparator arm(s): control

Sample size calculation performed: yes

Subgroups prespecified: not clear but described in Methods

Subgroup analyses: attendance at educational programme

Start date: February 2013

Duration of follow-up: 12 months

Number of follow-ups: 2

Follow-up time points: 6 months, 12 months

ICC (if reported): sexual debut: 0.016; condom use at last sex: insufficient statistical power to calculate; number of sexual partners: 0.049; IPV victimisation: 0.022; IPV perpetration: 0.024

Participants

Number of schools randomised: 42 (PREPARE: 20, control: 22)

Number of participants randomised (total and by arm): 6244 (PREPARE: 3302, control: 2942)

Age (range or mean (SD)) or grade at the start: PREPARE: 13.71 years (0.99), control: 13.7 years (1.07)

Gender: PREPARE: $37.9 \%$ male, control: $41.5 \%$ male

Ethnicity: not reported

SES: PREPARE: 5.98 (1.68), control 5.99 (1.65)

Inclusion criteria: adolescents in grade 8 (average age 13) in public high schools in the Western Cape

Exclusion criteria: schools with grade 12 pass rates below $40 \%$ (indicating their inability to deliver on the core educational mandate); schools with pass rates above $97 \%$ (indicating well-resourced schools 


\section{Randomisation before or after baseline survey: before}

Duration of the intervention (excluding follow-up): 21 weeks

Description of the intervention

PREPARE

Consisted of an educational programme, a school health service, and a school safety programme. The educational programme consisted of 21 sessions delivered once a week, immediately when school ended, on the school premises. The session duration ranged from 1 to 1.5 hours. Sessions included up to 25 participants, and educational methods were interactive and skills-based. Staff employed by the PREPARE project, who had been screened for positive gender norms and comfort with sexuality education and condom demonstrations, facilitated the programme in groups of up to 25 participants. Facilitators received a 2-week training course and subsequent weekly training, supervision, and session preparation support.

The school health service (SHS) was delivered by a nurse from the public clinic nearest to the school premises, once a week immediately after school ended. The service was modelled on the new South African Integrated School Health Policy, was free, and involved sexual and reproductive health (SRH) education, identification of need for SRH services or commodities, and referral for such services or commodities to the nearest community clinic, where they were provided free of charge. Some clinics were also able to send a health promoter to assist with health education.

The school safety programme comprised 2 initiatives. School safety teams were invited to a 2-day training at a central venue, conducted by the PREPARE team with the Centre for Justice and Crime Prevention (CJCP) (a non-government organisation). We implemented "Photovoice", a carefully piloted programme for 20 randomly selected students at each school, facilitated by 2 PREPARE researchers.

Brief description of the theoretical model: Jewkes conceptual framework, social cognition models including the Reasoned Action Framework and the I-Change theoretical model

Description of the comparator: participants in the control schools received school as usual, which excluded the after-school programme, the school health service, and the safety programme.

Outcomes Primary: sexual risk behaviours (sexual debut, number of sexual partners, condom use, contraception use)

Secondary: intimate partner violence (IPV), incidence of conceptions

$\begin{array}{ll}\text { Setting } & \text { Country: South Africa; State: Western Cape } \\ \text { Setting: school } \\ \text { Focus: universal }\end{array}$

Process measures Process data reported: yes

Method (qualitative or quantitative): both

\section{Description}

Acceptability of the intervention: the intervention was highly acceptable to participants in the intervention arm, with three-quarters (1003; 75.1\%) rating the PREPARE after-school sessions as "excellent" or "very good"; 262 (19.5\%) rating them as "good" or "fair"; and 32 (2.3\%) rating them as "bad" to "extremely bad". The remaining 39 (2.9\%) selected "I did not attend".

Reach of the intervention: in intervention schools, the mean $(M)$ number of PREPARE educational sessions attended was 8.02 (standard deviation (SD): 7.44; range 0 to 21). The PREPARE school nurse 
was visited by $17.3 \%$ of trial participants in intervention schools ( $14.9 \%$ of boys and $18.7 \%$ of girls). The number among the 20 randomly selected participants who attended Photovoice varied between 7 and 20. An overview of exposure at the school level shows that 7 schools were exposed to all 4 components of the intervention (educational sessions, school health service, school safety training, and Photovoice); 7 schools had all components except Photovoice; 2 schools had all components except the health service; 2 schools had all components except the safety training; and 2 schools were exposed only to the educational sessions and the school safety training.

Fidelity/Adherence to the intervention: average facilitator performance scores ranged from 32.3 to 58.7 out of a maximum score of 65 . Eleven of the 15 facilitators scored above 50/65, indicating that sessions were conducted with a moderately high degree of fidelity by most facilitators. Three facilitators scored below 50. On closer inspection, 2 of these 3 facilitators scored low on interactions with learners, yet had acceptable scores on session fidelity and facilitation skills. The other facilitator had low scores in all 3 dimensions, but none of the scores was below 25 . We implemented the after-school educational programme in all 20 intervention schools, but in 2 schools we were unable to complete it. In 1 of the 2 , the programme was interrupted by a religious fast. In the other, the school could no longer find a free afternoon for the sessions. We trained nurses and health promoters for 17 of the 20 intervention schools, and the school health service operated in 17 schools. Public health services did not have the capacity to provide school health nurses for 2 of the remaining schools, and in 1, the school was not able to allocate an afternoon session for the school health service. School safety teams for 18 of the 20 intervention schools participated in the school safety training, and we trained 53 school safety team delegates in total. None of the schools implemented participatory safety audits or developed safety plans during the 6 months following safety training. We implemented the Photovoice programme in 10 of the 20 intervention schools. In 2 of these schools, we did not complete implementation of Photovoice because students did not feel safe taking photographs on the school premises (1 school) and because attendance had dwindled ( 1 school).

Unit of randomisation: schools

Unit of analysis: individual

Method to promote equivalence between groups: we stratified schools into 2 strata based on the grade 12 pass rate, which we assumed was an indication of how well the school functioned and its potential ability to benefit from the PREPARE programme. We found that the pass rate was correlated with the amount of school fees charged, indicating that it is also a reflection of socioeconomic status.

Statistical models: regression was undertaken to provide outcomes at 6 and 12 months with odds ratios for dichotomous variables and coefficients for continuous variables.

Baseline differences adjustment: analyses adjusted for baseline demographics (age, gender, socioeconomic status), the baseline measure in question

Repeated measures methods in analysis: not reported

Funding: EC Health research programme (under the 7th Framework Programme). Grant Agreement number: 241945

Randomisation method, e.g. block, stratification, computer: random number generator

Clustering accounted for in sample size calculation (if relevant): yes

Cluster randomisation methods to account for clustering in analysis: clustering was accounted for by the complex samples approach.

\section{Risk of bias}

Bias Authors' judgement Support for judgement


Matthews 2016 (Continued)

\begin{tabular}{l}
$\begin{array}{l}\text { Random sequence genera- Low risk } \\
\text { tion (selection bias) }\end{array}$ \\
\hline
\end{tabular}

Allocation concealment Low risk
(selection bias)

To ensure allocation sequence concealment, a statistician at the South African (selection bias)

Medical Research Council who did not have any knowledge of the schools allocated them within each stratum to intervention and control arms of the study.

Blinding of participants High risk Blinding unlikely to have been possible owing to the nature of the trial and personnel (perfor-

mance bias)

All outcomes

\begin{tabular}{lll}
\hline $\begin{array}{l}\text { Blinding of outcome as- } \\
\text { sessment (detection bias) } \\
\text { All outcomes }\end{array}$ & High risk & Not reported; self-reported outcomes \\
\hline $\begin{array}{l}\text { Incomplete outcome data } \\
\text { (attrition bias) } \\
\text { All outcomes }\end{array}$ & Low risk & Low attrition and attrition comparable between groups \\
\hline $\begin{array}{l}\text { Selective reporting (re- } \\
\text { porting bias) }\end{array}$ & Unclear risk & No published protocol; subgroups not prespecified. \\
\hline Other bias & Unclear risk & $\begin{array}{l}\text { Multiple outcomes have been reported, including additional secondary out- } \\
\text { comes. }\end{array}$
\end{tabular}

McNeal 2004

\section{Methods}

\section{Study name: All Stars}

Study design (e.g. RCT, cluster RCT): cluster RCT, 3 arms (8 schools in intervention: 5 specialist schools and 3 teacher schools; 6 control schools)

Intervention arm(s): (1) specialist (All Stars instructors) - 5 schools, $n=629$ students; (2) teacher (class teachers) -3 schools, $n=287$

Comparator arm(s): usual treatment (attendance at Health Education classes taught by teachers) - 6 schools, $n=739$

\section{Sample size calculation performed: not reported}

Subgroups prespecified: no

Subgroup analyses: N/A

Start date: not reported

Duration of follow-up: 1 year post intervention

Number of follow-ups: 2

Follow-up time points: wave 1: post-test at end of academic year; wave 2: 1-year follow-up post-test ICC (if reported): not reported

\section{Participants}

\section{Number of schools randomised: 14}

Number of participants randomised (total and by arm): at analysis: $N=1655$; schools $N=14$; specialist $n=629$ students; teacher $n=287$ students; control $n=739$ students 
Age (range or mean (SD)) or grade at the start: mode at pre-test 12 years (range 11 to 13 years); grades 6 and 7

Gender: $55 \%$ female (at time of analysis)

Ethnicity: white 69\%; African American 25\%; Hispanic 6\% (at the time of analysis)

SES: not reported

Inclusion criteria: not reported

Exclusion criteria: not reported

Interventions

Randomisation before or after baseline survey: before

Duration of the intervention (excluding follow-up): 1 academic year

Description of the intervention

All Stars

All Stars is a character education and problem behaviour prevention programme delivered through curriculum, homework, group, and 1-on-1 sessions. All Stars target mediators include students identifying an ideal lifestyle and how they perceive that drug use, sex, and violence may influence that lifestyle; students' beliefs about peer norms in relation to abstinence from drugs, sex, and violence; personal commitments to avoid drugs, sex, and violent behaviour and to develop stronger feelings of attachment and acceptance at school.

The curriculum consists of 22 sessions: 14 whole-classroom sessions in class time; 4 small-group sessions outside of class with small groups of students selected to be assistants; and 4 one-on-one sessions between All Stars instructors and students. Each classroom session is designed to address 1 of the mediating variables. The programme is interactive, with debates, games, and discussion. Homework is designed to promote interaction with parents and children, involving parents in the programme.

Brief description of the theoretical model: social learning theory

Description of the comparator: treatment as usual

Outcomes List relevant outcomes

Primary outcomes: use of cigarettes, alcohol, marijuana, and inhalants; antisocial behaviour and offending; adolescent sexual activity

Secondary outcomes: mediator variables

Setting Country: USA; State: Kentucky;Place: Lexington and Louisville

Setting: 14 middle schools in 2 large cities

Focus: universal

Process measures

Process data reported: acceptability of the intervention, adherence to the intervention

Method (qualitative or quantitative): quantitative

\section{Description}

Acceptability of the intervention: $85 \%$ of sessions were rated as very good or excellent across $90 \%$ of observed sessions; teaching was rated as effective, and most or all objectives were achieved; most or all students were involved.

Adherence to the intervention: ratings across sessions were high. In $90 \%$ of observed sessions, most or all of the teaching methods were rated as effective, most or all of the objectives were achieved, and 
McNeal 2004 (Continued)

most or all of the students were involved. Eighty-five per cent of sessions were rated as very good or excellent overall. The subjective quality of delivery by the specialists was judged to be superior to that of the teachers.

Statistics

Sample size: (Harrington 2001) eligible $N=2289$; at analysis, $N=1655$; schools $N=14$; specialist $n=629$ students; teacher $n=287$ students; control $n=739$ students

Unit of randomisation: schools

Unit of analysis: individuals

Methods to promote equivalence between groups: stratification and hierarchical cluster analysis

Statistical models: analysis in a $3 \times 3$ factorial design (Time: pre-test, post-test, follow-up) $\times($ Condition: control, specialist, teacher), with repeat measures on the first factor

Baseline differences adjustment: all analyses include student demographic attributes and baseline values for mediators as covariates.

Repeated measures methods in analysis: repeat measures on the first factor, factorial ANOVA

Funding: subcontract to N. G. Harrington, University of Kentucky, by Tanglewood Research, Inc., Clemmons, North Carolina

The McNeal 2004 paper extends the analysis of Harrington 2001 by separating substance use scales into alcohol, cigarettes, marijuana, and inhalants.

Randomisation method, e.g. block, stratification, computer: specialist and control schools were stratified by school size, gender, ethnicity, and \% receiving free or reduced-price school meals. The teacher condition was added after this assignment. Hierachical cluster analysis was used to give matched pairs of schools by city. One school in each pair was randomly assigned to the treatment condition. The paired schools then were randomly ordered for approach. Treatment schools were approached first.

Clustering accounted for in sample size calculation (if relevant): not reported

Cluster randomisation methods to account for clustering in analysis: hierarchical linear modelling to account for clustering

\section{Risk of bias}

\begin{tabular}{lll}
\hline Bias & Authors' judgement & Support for judgement \\
\hline $\begin{array}{l}\text { Random sequence genera- } \\
\text { tion (selection bias) }\end{array}$ & Unclear risk & Not reported \\
\hline $\begin{array}{l}\text { Allocation concealment } \\
\text { (selection bias) }\end{array}$ & Unclear risk & Not reported but unlikely given the method \\
\hline $\begin{array}{l}\text { Blinding of participants } \\
\text { and personnel (perfor- } \\
\text { mance bias) }\end{array}$ & High risk & Not possible to blind staff and students to intervention status \\
$\begin{array}{l}\text { All outcomes } \\
\begin{array}{l}\text { Blinding of outcome as- } \\
\text { sessment (detection bias) } \\
\text { All outcomes }\end{array}\end{array}$ & High risk & $\begin{array}{l}\text { Students were assessed by self-report questionnaires administered in the } \\
\text { classroom by project staff. }\end{array}$ \\
\hline $\begin{array}{l}\text { Incomplete outcome data } \\
\text { (attrition bias) }\end{array}$ & Low risk & $\begin{array}{l}\text { Invalid or missing values were imputed and did not exceed 1.2\% for any vari- } \\
\text { able (post-test) and 5.7\% at follow-up. }\end{array}$ \\
\hline
\end{tabular}


McNeal 2004 (Continued)

All outcomes

Selective reporting (re- $\quad$ Unclear risk $\quad$ No published protocol
porting bias)

Other bias Low risk No other issues identified

Melnyk 2013

Methods

Study name: COPE (Creating Opportunities for Personal Empowerment)

Study design: cluster RCT at the level of the school

Intervention arm: COPE

Comparator arm: Healthy Teens attention control programme

Sample size calculation performed: yes

Subgroups prespecified: N/A

Subgroup analyses: none

Start date: January 2010

Duration of follow-up: 6 months

Number of follow-ups: 2

Follow-up time points: post-test and 6 months following end of intervention

Intracluster correlation coefficient: not reported

\section{Participants}

\section{Number of schools randomised: 11}

Number of participants randomised: 807 total, 374 intervention, 433 control

Age (range or mean (SD)) or grade at the start: mean age 14.75 years intervention; 14.74 years control

Gender: $54.5 \%$ female in intervention, $49.20 \%$ female in control

Ethnicity: American Native: $2.8 \%$ intervention, 4\% control; Asian: 2\% intervention, $5.7 \%$ control; black: $8.4 \%$ intervention, $11.2 \%$ control; white: $8.7 \%$ intervention, $18.8 \%$ control; Hispanic: $76.8 \%$ intervention, $59.6 \%$ control; other: $1.4 \%$ intervention, $0.7 \%$ control

\section{Socioeconomic status: N/S}

Inclusion criteria: adolescents aged 14 to 16 years who were enrolled in a health education course. Teens of any gender, ethnicity, or SES and those who could read and speak English

Exclusion criteria: medical condition that would prevent participation in the physical exercise component

Description of the intervention arm(s): a 15-session educational and cognitive-behavioural skills building programme that incorporated 15 to 20 minutes of physical activity in each session. Areas covered included healthy lifestyles, self-esteem, goal-setting, and problem-solving; stress and coping; emotional regulation; effective communication; overcoming barriers to goal progression; food and nutrition information (e.g. portion sizes, nutrients, food groups, snacks); and physical activity. Homework 
Melnyk 2013 (Continued)

activities were conducted to reinforce the content of the programme, and 4 parent newsletters were sent home for review.

Brief description of the theoretical model: cognitive-behavioural theory

Description of the comparator arm(s): attention control "Healthy Teens", which provided the same number and length of sessions
Primary outcomes: healthy lifestyle behaviours, BMI

Secondary outcomes: mental health, alcohol and drug use, social skills, academic performance

\begin{tabular}{|c|c|c|}
\hline \multirow[t]{7}{*}{ Statistics } & \multirow{2}{*}{\multicolumn{2}{|c|}{$\begin{array}{l}\text { Sample size: } 807 \text { participants } \\
\text { Unit of randomisation: school }\end{array}$}} \\
\hline & & \\
\hline & \multicolumn{2}{|c|}{ Unit of analyses: individual accounting for clustering } \\
\hline & \multicolumn{2}{|c|}{ Methods to promote equivalence between groups: N/S } \\
\hline & \multicolumn{2}{|c|}{$\begin{array}{l}\text { Statistical models: repeated logistical regression models using generalised estimating equations were } \\
\text { used to analyse binary outcomes. }\end{array}$} \\
\hline & \multicolumn{2}{|c|}{ Baseline differences adjustment: yes } \\
\hline & \multicolumn{2}{|c|}{ Repeated measures methods in analysis: yes } \\
\hline \multirow[t]{5}{*}{ Notes } & \multicolumn{2}{|l|}{ Equity: N/A } \\
\hline & \multicolumn{2}{|c|}{ Funding: NIH/National Institute of Nursing Research (1R01NR012171) } \\
\hline & \multicolumn{2}{|c|}{ Randomisation method: unclear } \\
\hline & \multicolumn{2}{|c|}{ Clustering accounted for in sample size calculation: yes } \\
\hline & \multicolumn{2}{|c|}{$\begin{array}{l}\text { Cluster randomisation methods to account for clustering in analysis: generalised estimating equa- } \\
\text { tions }\end{array}$} \\
\hline \multicolumn{3}{|l|}{ Risk of bias } \\
\hline Bias & Authors' judgement & Support for judgement \\
\hline $\begin{array}{l}\text { Random sequence genera- } \\
\text { tion (selection bias) }\end{array}$ & Unclear risk & Lack of information \\
\hline
\end{tabular}




\title{
Melnyk 2013 (Continued)
}

\begin{tabular}{l}
$\begin{array}{l}\text { Allocation concealment } \\
\text { (selection bias) }\end{array}$ \\
\hline
\end{tabular}

\begin{tabular}{|c|c|c|}
\hline $\begin{array}{l}\text { Blinding of participants } \\
\text { and personnel (perfor- } \\
\text { mance bias) } \\
\text { All outcomes }\end{array}$ & Unclear risk & $\begin{array}{l}\text { Study authors stated that this was a blinded cluster RCT that compared the in- } \\
\text { tervention vs an attention control. }\end{array}$ \\
\hline $\begin{array}{l}\text { Blinding of outcome as- } \\
\text { sessment (detection bias) } \\
\text { All outcomes }\end{array}$ & High risk & Self-report measures \\
\hline $\begin{array}{l}\text { Incomplete outcome data } \\
\text { (attrition bias) } \\
\text { All outcomes }\end{array}$ & Unclear risk & Insufficient information \\
\hline $\begin{array}{l}\text { Selective reporting (re- } \\
\text { porting bias) }\end{array}$ & High risk & Reporting of certain outcomes not consistent with protocol \\
\hline Other bias & Low risk & No other sources of bias \\
\hline
\end{tabular}

\section{Milburn 2012}

Study name: STRIVE
Study design (e.g. RCT, cluster RCT): cluster RCT at the level of the family
Intervention $\operatorname{arm}(\mathbf{s})$ : received the Support To Re-Unite, Involve, and Value Each other (STRIVE) inter-
vention
Comparator arm(s): standard care, based on referring agency. If family was not referred, a referral was
made based on each respective family's needs.

\section{Sample size calculation performed: N/S}

Subgroups prespecified: N/A; no subgroup analysis conducted

Subgroup analyses: no

Start date: March 2006

Duration of follow-up: 12 months post intervention

\section{Number of follow-ups: 3}

Follow-up time points: 3,6 , and 12 months post intervention

ICC (if reported): not reported

\author{
Number of schools randomised: N/A \\ Number of participants randomised (total and by arm): 68 to intervention, 83 to control \\ Age (range or mean (SD)) or grade at the start: intervention (14.7 years, 1.3 SD), control (14.9 years, \\ $1.5 \mathrm{SD})$, overall (14.8 years, $1.4 \mathrm{SD}$ ) \\ Gender: baseline: intervention (male: $22.1 \%$, female: $77.9 \%$ ), control (male: $43.4 \%$, female: $56.6 \%$ ), \\ overall (male: $33.8 \%$, female: $66.2 \%$ )
}


Ethnicity: baseline: intervention (Hispanic: $61.8 \%$, white: $11.8 \%$, African American: $17.6 \%$, other: $8.8 \%$ ); control (Hispanic: $61.4 \%$, white: $10.8 \%$, African American: $22.9 \%$, other: $4.8 \%$ ); overall (Hispanic: $61.6 \%$, white: $11.3 \%$, African American: $20.5 \%$, other: $6.6 \%$ )

\section{SES: N/S}

Inclusion criteria: away from home for 2 or more nights in the past 6 months (but not more than 6 months), having the potential to return home

Exclusion criteria: no current abuse/neglect, no active psychosis, no current substance intoxication

Randomisation before or after baseline survey: after
Interventions
months' duration in total)
Description of the intervention
STRIVE
Consisted of 5 sessions, each lasting 1.5 to 2 hours. All sessions re-inforced the importance of establish-
ing a positive family climate, resolving family issues via conflict resolution (to improve family function-
ing), recognising and managing feelings, increasing positive affirmations, and learning and practising
problem-solving skills. Learning is cumulative, and all sessions complement each other, with each pro-
gressing from the last and building upon previous content delivered.

Brief description of the theoretical model: session content based upon cognitive-behavioural theories, designed to improve families' problem-solving and conflict resolution skills

Description of the comparator: standard care by the agencies that referred them. For families not receiving any services, appropriate referrals were given based on their needs.

\begin{tabular}{ll} 
Outcomes & $\begin{array}{l}\text { Primary outcomes: self-reported alcohol use, substance use, risky sexual behaviour, and delinquent } \\
\text { behaviour } \\
\text { Secondary outcomes: N/S }\end{array}$ \\
\hline Setting & Country: USA; State: California \\
& $\begin{array}{l}\text { Setting: family (intervention took place 'usually' within a family's home, but this was decided upon by } \\
\text { family members themselves) } \\
\text { Focus: targeted; newly homeless youth and their families }\end{array}$
\end{tabular}

Process measures Process data reported: yes; all quantitative estimates

Method (qualitative or quantitative): quantitative

\section{Description}

Acceptability of the intervention: $76 \%$ of families completed all 5 sessions; $16 \%$ attended 1 to 2 sessions, and only 1 family attended no sessions. The Working Alliance Inventory (range 12 to 84) measured satisfaction in both adolescents and parents/guardians. The mean adolescent score was 72.0 (12.5 SD), and the mean parent score was 76.4 (10.0 SD), indicating high satisfaction.

Adherence to the intervention: An intervention manual was created to increase intervention fidelity. $98 \%$ of tasks were implemented with fidelity (according to a 13-item bespoke scale assessing fidelity of session delivery.)

Intensity of the intervention: intervention sessions typically lasted 1.5 to 2 hours and occurred once weekly. 
Unit of randomisation: adolescent (and subsequent attached parent)

Unit of analysis: individual

Method to promote equivalence between groups: not reported

Statistical models: random-intercept regression models

Baseline differences adjustment: analyses adjusted for gender, as this was not balanced between study groups

Repeated measures methods in analysis: random intercept model accounted for repeated measures taken in each individual.

Funding: study funded by the National Institute of Mental Health (NIMH R01-MH070322)

Randomisation method, e.g. block, stratification, computer: computerised coin toss

Clustering accounted for in sample size calculation (if relevant): N/A

Cluster randomisation methods to account for clustering in analysis: N/A

\section{Risk of bias}

\begin{tabular}{|c|c|c|}
\hline Bias & Authors' judgement & Support for judgement \\
\hline $\begin{array}{l}\text { Random sequence genera- } \\
\text { tion (selection bias) }\end{array}$ & Low risk & Computerised coin toss used to randomise participants \\
\hline $\begin{array}{l}\text { Allocation concealment } \\
\text { (selection bias) }\end{array}$ & Unclear risk & Not stated \\
\hline $\begin{array}{l}\text { Blinding of participants } \\
\text { and personnel (perfor- } \\
\text { mance bias) } \\
\text { All outcomes }\end{array}$ & High risk & $\begin{array}{l}\text { Participants and intervention team not blinded to study allocation; self-report- } \\
\text { ed outcome measures }\end{array}$ \\
\hline $\begin{array}{l}\text { Blinding of outcome as- } \\
\text { sessment (detection bias) } \\
\text { All outcomes }\end{array}$ & High risk & Self-reported outcomes \\
\hline $\begin{array}{l}\text { Incomplete outcome data } \\
\text { (attrition bias) } \\
\text { All outcomes }\end{array}$ & High risk & $\begin{array}{l}\text { Although baseline differences were non-significant between those who did } \\
\text { and did not complete follow-up, overall rates of attrition were very high (up to } \\
54 \% \text { at } 12 \text {-month follow-up). }\end{array}$ \\
\hline $\begin{array}{l}\text { Selective reporting (re- } \\
\text { porting bias) }\end{array}$ & Unclear risk & No published protocol \\
\hline Other bias & Low risk & No other forms of bias evident \\
\hline
\end{tabular}

Minnis 2014

$\begin{array}{ll}\text { Methods } & \text { Study name: Yo Puedo } \\ & \text { Study design: cluster RCT; participant social networks randomised }\end{array}$


Intervention arm: Yo Puedo intervention

Comparator arm: control

Sample size calculation performed: N/S

Subgroups prespecified: none performed

Subgroup analyses: no

Start date: June 2011 to January 2012

Duration of follow-up: 6 months from baseline (end of intervention)

Number of follow-ups: 1

Follow-up time points: 6 months (end of intervention)

Intracluster correlation coefficient: not reported

Participants

Number of schools randomised: N/A

Number of participants randomised: 79 intervention, 83 control

Age (range or mean (SD)) or grade at the start: intervention: 16.8 years, control: 16.9 years

Gender: intervention: $49.4 \%$ female, control: $53.0 \%$ female

Ethnicity: intervention: Latino (88.6\%), control: Latino (83.1\%)

Socioeconomic status: Maternal education less than high school: Int 46.8\%, Cont 38.6\%. Crowded housing conditions: Int $48.1 \%$, Cont $47.0 \%$. Household social service use in past 6 months: Int $59.5 \%$, Cont $54.2 \%$.

Inclusion criteria: Latino participants 16 to 21 years of age, residing in San Francisco

Exclusion criteria: current pregnancies/parents, non-English or non-Spanish speaker

Duration of the intervention: 6 months

\section{Yo Puedo}

\section{Description of the intervention $\operatorname{arm}(\mathrm{s})$}

Conditional cash transfer payments were dependent on 24 pre-specified participation and performance goals and attendance at 8 weekly sessions of life skills training classes on sexual health promotion and dating violence throughout the first 2 months of the intervention period.

Brief description of the theoretical model: social learning theory briefly mentioned; unclear how it informed intervention components. Behavioural economics also referenced

Description of the comparator $\operatorname{arm}(\mathbf{s})$ : standard community services

\begin{tabular}{ll} 
Outcomes & Primary outcomes: reduction in sex \\
& $\begin{array}{l}\text { Secondary outcomes: reduction in o } \\
\text { lence) }\end{array}$ \\
\hline Setting & Country: USA;Place: San Francisco \\
& Setting: school and community \\
& Focus: targeted
\end{tabular}


Minnis 2014 (Continued)

Process measures

\section{Process data reported: yes}

Method: quantitative and qualitative methods

Description: qualitative feasibility data in the manuscript under review; adherence and acceptability utilised quantitative methods, with $\mathrm{N}$ and \%. Median number of life skills sessions attended (out of 8 ) (n $=2$, range 0 to 8 ), attended at least 1 session (52\%), median number of goals completed (out of 24$)(n=$ 2 , range 0 to 14). median conditional cash transfer amount earned ( $\$ 30$, range $\$ 0$ to $\$ 200)$, completed at least 1 goal and attended at least 1 group session (46\%), completed at least 1 goal and attended 3 or more group sessions (39\%)

\section{Sample size: $\mathrm{N} / \mathrm{S}$}

Unit of randomisation: social network group

Unit of analyses: individual

Methods to promote equivalence between groups: N/S

Statistical models: linear and logistical regression models

Baseline differences adjustment: no; no baseline differences between intervention and control arms

Repeated measures methods in analysis: not required; only 1 follow-up period immediately post intervention

\section{Notes}

Equity: various baseline measures collected (e.g. educational, relationship and sexual risk behaviour, risk profile measures). Results not analysed by subgroup

Funding: Eunice Kennedy Shriver National Institute of Child Health and Human Development (NICHD) at the National Institutes of Health (R21 HD066192; PI, Minnis) and supported by an NICHD career development award to A.M. Minnis (K01 HD47434)

Randomisation method: N/S

Clustering accounted for in sample size calculation: $\mathrm{N} / \mathrm{S}$

Cluster randomisation methods to account for clustering in analysis: yes; adjusted at social network level for all analyses

\section{Risk of bias}

\begin{tabular}{lll}
\hline Bias & Authors' judgement & Support for judgement \\
\hline $\begin{array}{l}\text { Random sequence genera- } \\
\text { tion (selection bias) }\end{array}$ & Unclear risk & Insufficient information provided \\
\hline $\begin{array}{l}\text { Allocation concealment } \\
\text { (selection bias) }\end{array}$ & Unclear risk & Insufficient information provided \\
\hline $\begin{array}{l}\text { Blinding of participants } \\
\text { and personnel (perfor- } \\
\text { mance bias) }\end{array}$ & High risk & $\begin{array}{l}\text { Even though at baseline both participants and interviewer were blinded to al- } \\
\text { location, this might not be true for follow-up (6-month) assessment. Because } \\
\text { participants were recruited from the same community/location, unmasking of } \\
\text { allocation might have occurred; control might have realised that intervention } \\
\text { participants were receiving payments. }\end{array}$ \\
\hline $\begin{array}{l}\text { Blinding of outcome as- } \\
\text { sessment (detection bias) } \\
\text { All outcomes }\end{array}$ & High risk & $\begin{array}{l}\text { Even though at baseline both participants and interviewer were blinded to al- } \\
\text { location, this might not be true for follow-up (6-month) assessment. Because } \\
\text { participants were recruited from the same community/location, unmasking of } \\
\text { allocation might have occurred; control might have realised that intervention } \\
\text { participants were receiving payments. }\end{array}$ \\
\hline
\end{tabular}


Minnis 2014 (Continued)
Incomplete outcome data
Unclear risk
High retention rate but not clear how this differed between groups (attrition bias)

All outcomes

Selective reporting (re- Low risk Expected outcomes reported
porting bias)

Other bias Low risk No other sources of bias

Monti 1999

Methods

Study name: study not named (brief motivational interview)

Study design (e.g. RCT, cluster RCT): RCT at the level of the individual

Intervention arm(s): theoretical underpinning in motivational interviewing, with a focus on empathy, developing discrepancy, self-efficacy, and personal choice regarding alcohol and alcohol-related behaviours. The motivational interview consisted of 5 stages: (1) introducing and reviewing circumstances of the event, (2) exploring motivation (including pros and cons), (3) providing personalised and computerised assessment feedback, (4) imagining the future, and (5) establishing goals. Participants in the intervention arm also received handouts given to control participants (see below).

Comparator $\operatorname{arm}(\mathbf{s})$ : standard care, in line with normal procedure at emergency department settings, and handout of a leaflet stating the importance of avoiding drinking driving behaviour, with a list of alcohol-related agencies listed on the back

Sample size calculation performed: not stated

Subgroups prespecified: none

Subgroup analyses: none

Start date: N/S

Duration of follow-up: 6 months post intervention

Number of follow-ups: 2

Follow-up time points: 3-month and 6-month follow-up

ICC (if reported): N/A

Participants

Number of schools randomised: N/A

Number of participants randomised (total and by arm): 94 (52 intervention, 42 control)

Age (range or mean (SD)) andor grade at the start: baseline mean age: intervention (18.4 years, 0.5 SD), control (18.3 years, $0.5 \mathrm{SD}$ ), overall (18.4 years, $0.5 \mathrm{SD}$ )

Gender: baseline: intervention (65\% male, 35\% female), control (62\% male, 38\% female), overall (64\% male, $36 \%$ female)

Ethnicity: baseline: intervention (79\% white, 10\% African American, 8\% Asian/East Indian, 4\% Hispanic), control (81\% white, $17 \%$ African American, $2 \%$ Asian/East Indian), overall (80\% white, $13 \%$ African American, 5\% Asian/East Indian, 2\% Latino)

SES: N/S

Inclusion criteria: emergency department attendees 18 to 19 years of age who had a positive blood alcohol concentration or had reported drinking alcohol before the injury/event precipitating treatment were eligible for inclusion into the study. 
Monti 1999 (Continued)

Exclusion criteria: patients who were suicidal, in police custody, or non-English-speaking; had failed a mental status exam; or had suffered serious traumatic injury

Interventions

\section{Randomisation before or after baseline survey: after}

Duration of the intervention (excluding follow-up): 30 minutes (brief intervention)

Description of the intervention: a brief motivational interview that included reviewing the event precipitating arrival to the emergency room, exploring the motivation (including pros and cons) for alcohol use, obtaining a computerised personal assessment and feedback on an individual's drinking behaviour and associated harms, looking to the future regarding alcohol and alcohol-related behaviour, and setting goals

Brief description of the theoretical model: motivational interviewing

Description of the comparator: standard care, designed to be consistent with general practice for treating alcohol-involved teens in an urgent care setting. A handout was provided that included information on avoiding drinking and driving, along with a list of local treatment agencies.

\section{Outcomes}

\section{List relevant outcomes}

Primary outcomes: reduced alcohol-related outcomes, motor vehicle offences (drinking driving, other moving violations), and alcohol-related injuries

Secondary outcomes: N/A

\begin{tabular}{|c|c|}
\hline Setting & $\begin{array}{l}\text { Country: USA } \\
\text { Setting (e.g. school, prison, family): emergency department } \\
\text { Focus: targeted; individuals } 18 \text { to } 19 \text { years of age with drink-related emergency department atten- } \\
\text { dances }\end{array}$ \\
\hline Process measures & $\begin{array}{l}\text { Process data reported: adherence to the intervention } \\
\text { Method (qualitative or quantitative): quantitative } \\
\text { Description: both interventionists and patients completed a 14-item rating scale, which assessed the } \\
\text { degree to which motivational interviewing principles in general and the session protocol in particular } \\
\text { had been adhered to. Additionally, every } 3 \text { months, interventionists were videotaped as they delivered } \\
\text { a motivational interview to naive research staff and were rated in terms of intervention fidelity. }\end{array}$ \\
\hline
\end{tabular}

Sample size: 94 individuals randomised
Unit of randomisation: individual
Unit of analysis: individual
Method to promote equivalence between groups: N/S
Statistical models: ANOVA and logistical regression
Baseline differences adjustment: N/A
Repeated measures methods in analysis: no

Notes Equity: N/S

Funding: partial support from the National Institute on Alcohol Abuse and Alcoholism (grant number 5R01-AA09892), a Department of VA Research Career Scientist Award, and a VA Merit Review grant by the Medical Research Office of Research and Development

Randomisation method, e.g. block, stratification, computer: N/S 
Monti 1999 (Continued)

\section{Clustering accounted for in sample size calculation (if relevant): N/A}

Cluster randomisation methods to account for clustering in analysis: N/A

\section{Risk of bias}

\begin{tabular}{lll}
\hline Bias & Authors' judgement & Support for judgement \\
\hline $\begin{array}{l}\text { Random sequence genera- } \\
\text { tion (selection bias) }\end{array}$ & Unclear risk & Insufficient information provided \\
\hline $\begin{array}{l}\text { Allocation concealment } \\
\text { (selection bias) }\end{array}$ & Unclear risk & Insufficient information provided \\
\hline $\begin{array}{l}\text { Blinding of participants } \\
\text { and personnel (perfor- } \\
\text { mance bias) } \\
\text { All outcomes }\end{array}$ & High risk & No blinding \\
\hline
\end{tabular}

\begin{tabular}{lll}
\hline Blinding of outcome as- & High risk & Self-reported outcome measures \\
sessment (detection bias) &
\end{tabular}

All outcomes

\begin{tabular}{|c|c|c|}
\hline $\begin{array}{l}\text { Incomplete outcome data } \\
\text { (attrition bias) } \\
\text { All outcomes }\end{array}$ & Low risk & $\begin{array}{l}\text { Low overall attrition rates and no differential attrition between study arms or } \\
\text { by subgroups (gender) }\end{array}$ \\
\hline
\end{tabular}

\begin{tabular}{lll}
\hline $\begin{array}{l}\text { Selective reporting (re- } \\
\text { porting bias) }\end{array}$ & Unclear risk & No published protocol \\
\hline Other bias & Low risk & No other forms of bias identified \\
\hline
\end{tabular}

Morris 2003

Study name: Self-Sufficiency Project
Study design (e.g. RCT, cluster RCT): cluster RCT
Intervention arm(s): self-sufficiency project
Comparator arm(s): control group
Sample size calculation performed: not reported
Subgroups prespecified: yes
Subgroup analyses: preschool, middle childhood, and adolescents. Risky behaviours reported only for
adolescents
Start date: parents selected from welfare rolls between November 1992 and March 1995
Duration of follow-up: 3 years (36 months) post baseline
Number of follow-ups: 2
Follow-up time points:
Wave 1: 18 months post baseline
Wave 2: 36 months post baseline


Morris 2003 (Continued)

\section{ICC (if reported): no}

Participants Number of schools randomised: N/A

Number of participants randomised (total and by arm): intervention arm: 1654 families and 2582 children; control arm: 1605 families and 2496 children

\section{Age (range or mean (SD)) or grade at start: 3 age groups}

Preschoolers ( $n=1043$ children): aged 0 to 2 years at randomisation/baseline

Middle childhood ( $n=2158$ children): aged 3 to 8 years at randomisation/baseline

Adolescents ( $n=1417$ children): aged 9 to 15 years at randomisation/baseline

Gender: $97 \%$ female parents

Ethnicity: SSP: First Nations 8.78\%; Asian 4.79\%; French-speaking 13.91\%; control group: First Nations 8.32\%; Asian 5.07\%; French-speaking 13.40\%

SES: number of months on income assistance in prior 3 years - parents: 10 to 23 months $=22.22 \%$; 24 to 35 months $=34.95 \%$; all 36 months $=42.84 \%$

Inclusion criteria: single parents in British Columbia and New Brunswick on welfare for at least 1 year. To be eligible for the study, an income assistance recipient had to be a single parent at least 19 years of age receiving welfare in the current month and in at least 11 of the previous 12 months.

Exclusion criteria: those not meeting eligibility criteria

Interventions

Randomisation before or after baseline survey: states that baseline survey was done at the time of random assignment (from the 2000 Report, the baseline sample who completed an interview was larger than the sample analysed because of withdrawals due to ineligibility and withdrawals soon after intake)

Duration of the intervention (excluding follow-up): parents could receive the financial supplement for up to 3 years.

Description of the intervention: Self-Sufficiency Project (SSP). The programme offered temporary earning supplements to single parents who moved to full-time employment.

Brief description of the theoretical model: not a specific named theory, but study authors draw on theories from economics and psychology

Description of the comparator: control group not offered SSP financial supplement. Both programme and control group members receive all regular benefits associated with income assistance if they continue to qualify for income assistance. Both groups also have access to existing community services and resources not funded by SSP.

\section{Outcomes List relevant outcomes}

Primary outcomes: academic achievement (from scores in reading, writing, math in younger children; writing, math, science in 15- to 18-year-olds; English, math, science in 10- to 18-year-olds); school behaviour problems; frequency of delinquent acts; substance use; risk of depression; cognitive functioning; positive social behaviour; average achievement; delinquent activity; smoking; drinking weekly or more often; drug use

\section{Secondary outcomes: N/A}

Setting

Country: Canada; Provinces: British Columbia, New Brunswick;Places: Vancouver, St John, Moncton, Fredericton

Setting: school, home, government administration offices 
Morris 2003 (Continued)

Focus: targeted at low-income single-parent families from 2 provinces in Canada

Process measures $\quad \begin{aligned} & \text { Process data reported: no } \\ & \text { Method (qualitative or quantitative): N/A } \\ & \text { Description: context: economic situation: although many US and Canadian policies are similar, there } \\ & \text { are at least } 2 \text { important differences. Canadian safety net programmes are more likely to be universal } \\ & \text { rather than means-tested, and Canada has national health insurance. }\end{aligned}$

Statistics

Sample size: families 3259; children 5078 aged 3 to 18 years (those with 36-month follow-up data)

Unit of randomisation: families

Unit of analysis: children

Method to promote equivalence between groups: not reported

Statistical models: factor analysis

Baseline differences adjustment: sample attrition did not make a difference between baseline characteristics of programme and control participants.

Repeated measures methods in analysis: analysis did not appear to take account of repeated measures when this was appropriate, but for risky behaviour outcomes, only baseline and 36-month follow-up data were presented.

\section{Notes}

Equity: baseline characteristics of parents were provided.

Funding: Human Resources Development Canada (HDRC)

Randomisation method, e.g. block, stratification, computer: approximately 6000 single parents in $\mathrm{BC}$ and NB who had been on welfare for at least 1 year were randomly selected from the welfare rolls between November 1992 and March 1995.

Clustering accounted for in sample size calculation (if relevant): not reported

Cluster randomisation methods to account for clustering in analysis: standard errors on measures analysed at the level of the child (rather than the family) were adjusted for shared variance between siblings in the same family, using standard errors developed by White (1980). This adjustment allows for non-independence among sample members that may arise for any reason, including because children belong to the same family, neighbourhood, or province.

\section{Risk of bias}

\begin{tabular}{lll}
\hline Bias & Authors' judgement & Support for judgement \\
\hline $\begin{array}{l}\text { Random sequence genera- } \\
\text { tion (selection bias) }\end{array}$ & Unclear risk & Lack of detail provided \\
\hline $\begin{array}{l}\text { Allocation concealment } \\
\text { (selection bias) }\end{array}$ & Unclear risk & Lack of detail provided \\
\hline $\begin{array}{l}\text { Blinding of participants } \\
\begin{array}{l}\text { and personnel (perfor- } \\
\text { mance bias) } \\
\text { All outcomes }\end{array}\end{array}$ & High risk & Families knew about the intervention. \\
\hline $\begin{array}{l}\text { Blinding of outcome as- } \\
\text { sessment (detection bias) } \\
\begin{array}{l}\text { All outcomes } \\
\hline\end{array}\end{array}$ & High risk & Self-reports for behaviour outcomes; some administrative data \\
\hline
\end{tabular}


Morris 2003 (Continued)

Incomplete outcome data Unclear risk $\quad 81 \%$ response rate across all child outcomes, but direct assessments with old(attrition bias) er children yielded $64 \%$ response rate, so outcomes of older children may be

All outcomes biased, although non-respondents were similar at baseline in programme and control groups

\begin{tabular}{lll}
\hline $\begin{array}{l}\text { Selective reporting (re- } \\
\text { porting bias) }\end{array}$ & Unclear risk & No published protocol \\
\hline Other bias & Low risk & No other issues identified \\
\hline
\end{tabular}

Murry 2014

Methods

Study name: Stronger African American Families (SAAF)

Study design: cluster RCT at the level of the group (counties)

Intervention arm: SAAF

Comparator arm: control group

Sample size calculation performed: not explicitly stated

Subgroups prespecified: N/A

Subgroup analyses: no

Start date: not reported

Duration of follow-up: 65 months

Number of follow-ups: 3

Follow-up time points: 3 months following end of intervention, 29 months, and 65 months

Intracluster correlation coefficient: ICC for intervention-targeted parenting 0.01 , responsive-supportive parent-child relationships 0.00 , children's self-control 0.00 . All ICCs $<0.05$

Participants

Number of schools randomised: N/A

Number of participants randomised: 371 to intervention, 299 to control

Age (range or mean (SD)) or grade at the start: 11 years (mean 11.2 years)

Gender: $54 \%$ female

Ethnicity: African American

Socioeconomic status: $46.3 \%$ of participants lived below federal poverty standards, and another $50.4 \%$ lived within $150 \%$ of the poverty threshold. Families' median household income was $\$ 1655.00$ per month.

Inclusion criteria: African American mothers and 11-year-old children living in rural counties in Georgia, USA

Exclusion criteria: none

Interventions

Timing of randomisation: before baseline survey

Duration of the intervention: 7 weeks 
Description of the intervention $\operatorname{arm}(\mathbf{s})$ : the aim of the intervention was to strengthen family processes and use parental expectations and norms around sexual activity and alcohol/drug use to protect adolescents from engaging in these behaviours. Workshops and group sessions were included, with the aim of providing training in communication along with skills practice after sessions. Mothers were taught skills related to monitoring and providing non-punitive discipline, expectations around alcohol use, communication strategies around sex, and techniques for adaptive racial socialisation. Young people learned about biding by rules, strategies to resist alcohol or drug use, and behavioural strategies when encountering racism. Jointly, mothers and young people engaged in activities designed to increase family cohesion.

Brief description of the theoretical model: social learning theory, problem behaviour theory, Gibbons and Gerrard's cognitive model of adolescent risk behaviour

Description of the comparator arm(s): control families received 3 leaflets by post: 1 described adolescent development, another described stress management, and the third focused on encouraging exercise.

\begin{tabular}{ll} 
Outcomes & Primary outcomes: risky sexual behaviour, a \\
& $\begin{array}{l}\text { Secondary outcomes: parenting behaviour, } \\
\text { parent-child relationships }\end{array}$ \\
\hline Setting & Country: USA; Place: rural Georgia \\
& Setting: community facilities \\
& Focus: targeted to African American families
\end{tabular}

Process measures Process data reported: yes

Method: quantitative

Description: Fidelity/ adherence: approximately $65 \%$ of pre-tested families participated in 5 or more sessions, with $44 \%$ attending all 7 sessions. Each team of group leaders was videotaped while conducting intervention sessions, so that their fidelity in presenting the prevention programme could be assessed. Coverage of curriculum components exceeded .80 for parent, youth, and family sessions. Reliability of fidelity assessments exceeded .80 for all 3 types of sessions. Intensity: the programme involved two 1-hour sessions consecutively, once per week for 7 weeks (14 hours in total).

Unit of randomisation: counties

Unit of analyses: individual level (but clustering considered)

Methods to promote equivalence between groups: not reported

Statistical models: structural equation modelling

Baseline differences adjustment: yes

Repeated measures methods in analysis: yes

Notes Equity: N/A

Funding: National Institutes of Health, National Institute of Mental Health, and National Institute on Alcohol Abuse and Alcoholism

Randomisation method: not clear

Clustering accounted for in sample size calculation: not clear

Cluster randomisation methods to account for clustering in analysis: yes 
Murry 2014 (Continued)

Risk of bias

\begin{tabular}{|c|c|c|}
\hline Bias & Authors' judgement & Support for judgement \\
\hline $\begin{array}{l}\text { Random sequence genera- } \\
\text { tion (selection bias) }\end{array}$ & Unclear risk & Insufficient information \\
\hline $\begin{array}{l}\text { Allocation concealment } \\
\text { (selection bias) }\end{array}$ & Unclear risk & Insufficient information \\
\hline $\begin{array}{l}\text { Blinding of participants } \\
\text { and personnel (perfor- } \\
\text { mance bias) } \\
\text { All outcomes }\end{array}$ & High risk & $\begin{array}{l}\text { Not possible to blind participants and personnel to an intervention of this na- } \\
\text { ture }\end{array}$ \\
\hline $\begin{array}{l}\text { Blinding of outcome as- } \\
\text { sessment (detection bias) } \\
\text { All outcomes }\end{array}$ & High risk & Self-reported outcomes \\
\hline $\begin{array}{l}\text { Incomplete outcome data } \\
\text { (attrition bias) } \\
\text { All outcomes }\end{array}$ & Low risk & $\begin{array}{l}\text { No differential attrition effects detected. Overall low attrition (15\%) at } 65 \\
\text { months post intervention. Study authors accounted for missing data using the } \\
\text { FIML estimation method. }\end{array}$ \\
\hline $\begin{array}{l}\text { Selective reporting (re- } \\
\text { porting bias) }\end{array}$ & High risk & Lack of clarity in presentation of data for all outcomes \\
\hline Other bias & Low risk & No other issues identified \\
\hline
\end{tabular}

Nader 1999

Methods

\author{
Study name: $\mathrm{CATCH}$ \\ Study design: cluster RCT \\ Intervention arm(s): school-based CATCH intervention \\ Comparator $\operatorname{arm}(\mathbf{s})$ : standard education control group \\ Sample size calculation performed: yes
}

Subgroups prespecified: no subgroup analysis conducted

Subgroup analyses: N/A

Start date: January 1992 (middle third grade)

Duration of follow-up: 3 years post intervention (grades 6 to 8 )

Number of follow-ups: 4

Follow-up time points:

Wave 1: grade 5 - approximately 3 years post baseline

Wave 2: grade 6 - approximately 4 years post baseline

Wave 3: grade 7 - approximately 5 years post baseline

Wave 4: grade 8 - approximately 6 years post baseline ( 3 years post intervention) 


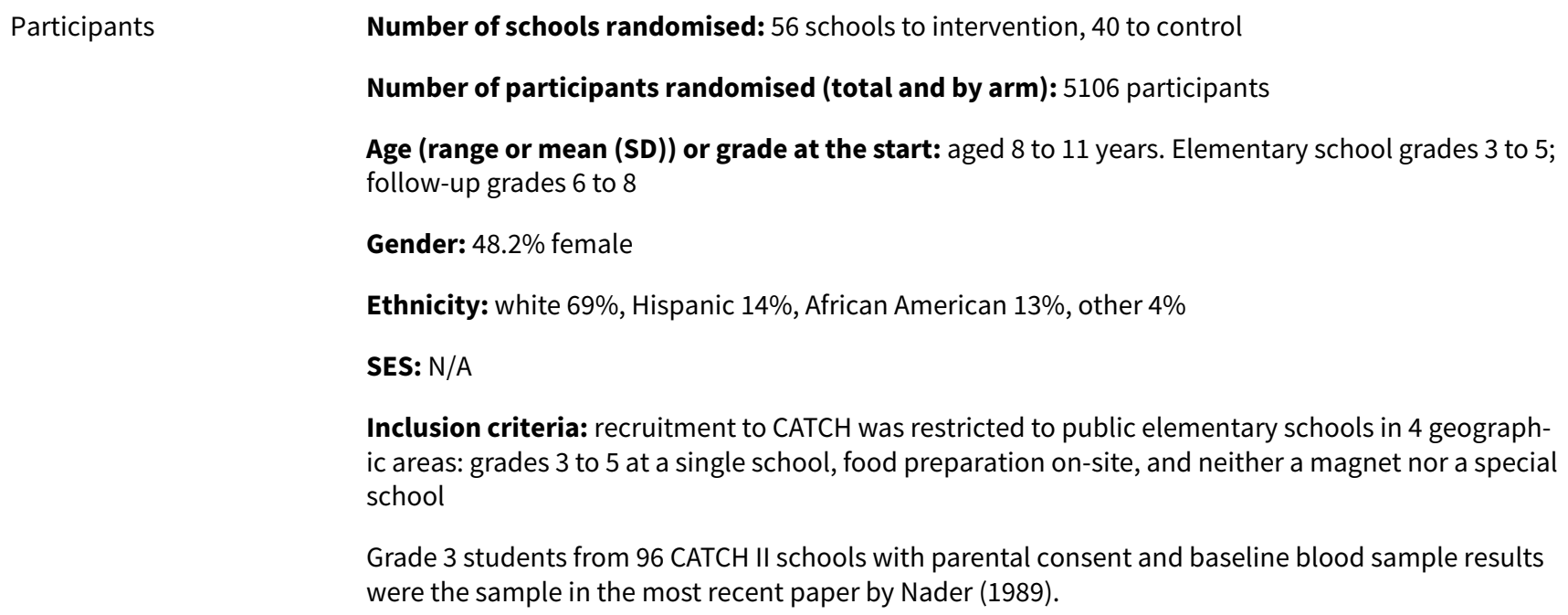

Inclusion criteria: recruitment to CATCH was restricted to public elementary schools in 4 geographic areas: grades 3 to 5 at a single school, food preparation on-site, and neither a magnet nor a special school

Grade 3 students from 96 CATCH II schools with parental consent and baseline blood sample results were the sample in the most recent paper by Nader (1989).

Exclusion criteria: schools serving as "magnet" schools for children with special interests or handicaps

\section{Duration of the intervention (excluding follow-up): 3 years}

Description of the intervention: (1) school component - tobacco policy, school meals (Eat Smart), physical education enhancement, classroom curricula; (2) family component - family fun nights, homebased curricula, school food service changes, and PE enhancement as continuous programmes over the 3 school years

Brief description of the theoretical model: social cognitive theory, social learning theory, organisational change theory

Description of the comparator: usual school health education control group

Outcomes

Primary outcomes: CATCH III outcomes included self-reported daily intakes of dietary fat and saturated fat, levels of moderate to vigorous physical activity, and psychosocial factors.

Secondary outcomes: physiological risk factors: total serum cholesterol, high-density lipoprotein (HDL) cholesterol, and apolipoprotein B levels; body mass index (BMI); skinfold thickness; blood pressure; (self-reported) smoking behaviour
Country: USA; State: California, Louisiana, Minnesota, and Texas;Place: San Diego, New Orleans, Minneapolis, Houston

Setting: grade 3 students recruited from 96 public elementary schools

Focus: universal

Process measures
Process data reported: an extensive process evaluation provided data to describe the quality of how the programme was implemented, how well the activities delivered fitted the original design, to whom services were delivered, the extent to which the target population was reached, and details on external programmes that may have competing effects (Stone 1996).

Method (qualitative or quantitative): quantitative

\section{Description}


Adherence to the intervention: the physical activity programme was assessed by the System for Observing Fitness Instruction Time (SOFIT). Every school was visited twice during each of the 6 semesters of the study by trained observers who used SOFIT when observing the type and intensity of children's activities and the behaviours of PE teachers and specialists. Details are provided in Stone 1996.

Statistics

Sample size: $\mathrm{N}=5106$ enrolled at baseline; 4 centres; 56 intervention schools, 2989 children; 40 control elementary schools, 2117 children

Unit of randomisation: school

Unit of analysis: individuals and schools

Method to promote equivalence between groups: stratification by site

Statistical models: individual-level measures analysed by ANCOVA with baseline values as covariate. School level measures analysed by repeat measures ANCOVA

Baseline differences adjustment: the repeated-measures model included individual-level covariates (sex, race, sex $\times$ race interaction, and, in some cases, age, height, BMI, or total energy intake).

Repeated measures methods in analysis: multi-variate repeated measures analysis for most outcome variables at both school and individual levels

Funding: National Heart, Lung and Blood Institute, National Institutes of Health

Randomisation method, e.g. block, stratification, computer: N/A

Clustering accounted for in sample size calculation (if relevant): study authors state: the CATCH trial (96 schools) includes an adequate number of units to permit a meaningful statistical analysis (Zucker 1995).

Cluster randomisation methods to account for clustering in analysis: yes

\section{Risk of bias}

\begin{tabular}{|c|c|c|}
\hline Bias & Authors' judgement & Support for judgement \\
\hline $\begin{array}{l}\text { Random sequence genera- } \\
\text { tion (selection bias) }\end{array}$ & Unclear risk & No information provided \\
\hline $\begin{array}{l}\text { Allocation concealment } \\
\text { (selection bias) }\end{array}$ & Unclear risk & No information provided \\
\hline $\begin{array}{l}\text { Blinding of participants } \\
\text { and personnel (perfor- } \\
\text { mance bias) } \\
\text { All outcomes }\end{array}$ & High risk & Participants and personnel not blinded to intervention \\
\hline $\begin{array}{l}\text { Blinding of outcome as- } \\
\text { sessment (detection bias) } \\
\text { All outcomes }\end{array}$ & High risk & Self-reported outcomes \\
\hline $\begin{array}{l}\text { Incomplete outcome data } \\
\text { (attrition bias) } \\
\text { All outcomes }\end{array}$ & Low risk & $\begin{array}{l}\text { Attrition less than } 30 \% \text {; minor statistically significant differences in participa- } \\
\text { tion rates according to sex, race, and site on selected measures }\end{array}$ \\
\hline $\begin{array}{l}\text { Selective reporting (re- } \\
\text { porting bias) }\end{array}$ & Low risk & All expected outcomes reported \\
\hline
\end{tabular}


Nader 1999 (Continued)
Other bias
Low risk
No other forms of bias identified

Nirenberg 2013

Study name: ROAD
Study design: RCT at the level of the individual
Intervention arm: motivational intervention with typical community service (MI)
Comparator arm: (MI-H); enhanced prototypical community service (CS)
Sample size calculation performed: yes
Subgroups prespecified: N/A
Subgroup analyses: none
Start date: N/S
Duration of follow-up: 6 months
Number of follow-ups: 2
Follow-up time points: post-test and 6-month follow up
Intracluster correlation coefficient: N/A

Participants

Number of schools randomised: N/A

Number of participants randomised: $\mathrm{N}=990$ participants, 332 to MI, 323 to $\mathrm{MI}-\mathrm{H}$, and 335 to control

Age (range or mean (SD)) or grade at the start: mean age: 18.1 years MI, 18.0 years MI-H, 18 years control

Gender: male: $73.8 \% \mathrm{MI}, 70 \% \mathrm{MI}-\mathrm{H}, 71.9 \%$ control

Ethnicity: white: $86 \% \mathrm{MI}, 91 \% \mathrm{MI}-\mathrm{H}, 89 \%$ control; Hispanic: $5.4 \% \mathrm{MI}, 5 \% \mathrm{MI}-\mathrm{H}, 4.5 \%$ control

Socioeconomic status: N/A

Inclusion criteria: aged 16 to 20 years; referred by the Rhode Island Judicial System to the 20-hour ROAD programme as the result of high-risk driving (e.g. speeding, reckless driving) and/or an alcohol/drug charge to attend the programme; a driver

\section{Exclusion criteria: N/A}

Description of the intervention arm(s): 4 group motivational sessions of 3 hours each were conducted, plus 1 individual session of 1 hour. The first group session was an introduction; the second was an interactive educational session on types of motor vehicle crashes, injuries, safety, and high-risk driving behaviours, with a focus on decision-making. Topics included the pros and cons of high-risk driving and alcohol use, feedback around peer norms, and change plans. Participants were encouraged to implement their change plan over the next week and discuss their success or failure at the next session, along with barriers to or supports for change. Participants completed 6 hours of experimental learning at the trauma centre of a hospital $(2 \times 3$-hour sessions) or through community service. The third session enabled reflections on experiences in the community or hospital and the change plan. The final session focused on preparation for change. Further issues relevant to participants and their change plans were discussed in the individual sessions. 
Nirenberg 2013 (Continued)

\section{Brief description of the theoretical model: N/A}

Description of the comparator arm(s): community service - part of participants' court-mandated sanctions (requirement to complete 20 hours of community service)

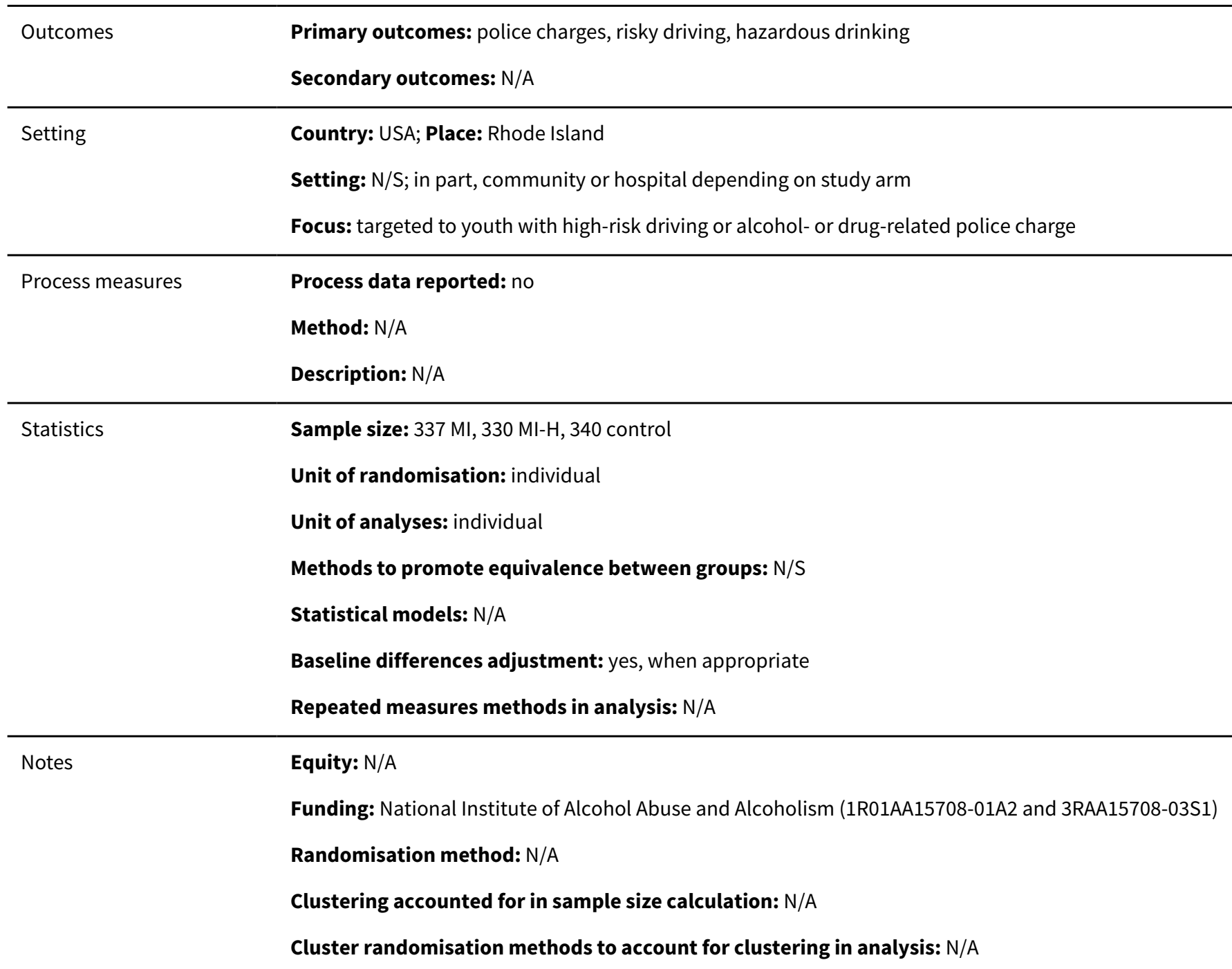

\section{Risk of bias}

\begin{tabular}{lll}
\hline Bias & Authors' judgement & Support for judgement \\
\hline $\begin{array}{l}\text { Random sequence genera- } \\
\text { tion (selection bias) }\end{array}$ & Unclear risk & Insufficient information \\
\hline $\begin{array}{l}\text { Allocation concealment } \\
\text { (selection bias) }\end{array}$ & Unclear risk & Insufficient information \\
\hline $\begin{array}{l}\text { Blinding of participants } \\
\text { and personnel (perfor- } \\
\text { mance bias) } \\
\text { All outcomes }\end{array}$ & High risk & Blinding not discussed by study authors \\
\hline
\end{tabular}

$\begin{array}{lll}\text { Blinding of outcome as- } & \text { High risk } & \text { Self-reported outcomes } \\ \text { sessment (detection bias) }\end{array}$


Nirenberg 2013 (Continued)

All outcomes

Incomplete outcome data Low risk Missing data balanced in numbers across intervention groups
(attrition bias)

All outcomes

Selective reporting (re- $\quad$ Low risk $\quad$ No protocol but expected outcomes reported
porting bias)

porting bias)

Other bias Low risk

No other sources of bias. Note that the risky driving behaviour questionnaire was added to the baseline assessment battery after data collection had commenced.

Intervention arm(s): Michigan Model for Health curriculum

Comparator $\operatorname{arm}(\mathbf{s})$ : standard education

\section{Sample size calculation performed: N/S}

Subgroups prespecified: N/A

\section{Subgroup analyses: none}

Start date: 2005 (recruitment commenced)

Duration of follow-up: 6 weeks after final intervention component delivered, towards end of grade 5 Number of follow-ups: 4

Follow-up time points: 1 week after grade 4 classroom intervention finished, then 5 to 6 weeks after, and again 1 week after grade 5 classroom intervention finished, and finally 5 to 6 weeks after

ICC (if reported): N/S

Participants

Number of schools randomised: 52

Number of participants randomised (total and by arm): 2512 total, 1345 in intervention schools, 1167 in control schools

Age (range or mean (SD)) or grade at the start: aged 9 to 11 years (mean 9.56); grade 4 to 5

Gender: $54 \%$ male, $46 \%$ female

Ethnicity: 54\% Caucasian, 38\% African American, 8\% other

SES: $46 \%$ of students eligible for free or reduced-price school meals (range $11.1 \%$ to $97.9 \%$ )

Inclusion criteria: N/S

Exclusion criteria: N/S

Interventions

Randomisation before or after baseline survey: before

Duration of the intervention (excluding follow-up): over 2 years (grades 4 and 5) 
Description of the intervention: the Michigan Model for Health was a health promotion intervention consisting of 52 lessons (20 to 50 minutes long) delivered over a 2-year period (grade 4 to 5). Lessons consisted of social and emotional health, alcohol, tobacco, other drugs, safety, nutrition, and physical activity. As well as mastery of techniques, skill development and practice are delivered.

Brief description of the theoretical model: based on principles of the health belief model and social learning theory

Description of the comparator: no intervention; no training or curriculum until after completion of the study

Outcomes

Primary outcomes: social and emotional health skills, interpersonal communication, drug refusal skills, intention to use drugs, initiation of and past 30-day drug/alcohol use, aggressive behaviour, prosocial behaviour

Secondary outcomes: N/S
Country: USA; State: Michigan and Indiana

Setting: elementary schools

Focus: universal
Process data reported: yes

Method (qualitative or quantitative): quantitative

\section{Description}

Acceptability of the intervention: \% adhering to the 'same' instructor delivering 3 post-baseline follow-up assessments within the same class ( $89 \%$ in grade $4,93 \%$ in grade 5 )

Adherence to the intervention: implementation fidelity was assessed via adherence to protocol. Delivery of the same order of lessons and no more than 3 lessons delivered in 1 week was assessed. Teachers in the intervention schools completed a survey every 2 weeks, reporting numbers and types of lessons taught. $96 \%$ of grade 4 and $92 \%$ of grade 5 teachers completed surveys. All lessons in grade 4 $(92 \%)$ and grade $5(94 \%)$ were reported by teachers as being delivered. Finally, over $90 \%$ of implementation fidelity assessors reported that teachers delivered the intervention programme with fidelity and provided adequate support.

Intensity of the intervention: grade 4: 24 lessons delivered over 12-week period; grade 5: 28 lessons delivered over 14-week period

Statistics

Sample size: 52 schools/2512 individuals

Unit of randomisation: school

Unit of analysis: individual

Method to promote equivalence between groups: N/S

Statistical models: mixed linear modelling, binary logistical regression

Baseline differences adjustment: N/A - no baseline differences

Repeated measures methods in analysis: N/S

Notes

Equity: N/S

Funding: supported by grants from the Michigan Department of Education and the Michigan Department of Community Health

Randomisation method, e.g. block, stratification, computer: N/S 
O'Neill 2011 (Continued)

Clustering accounted for in sample size calculation (if relevant): yes, via statistical methods deployed

Cluster randomisation methods to account for clustering in analysis: mixed linear modelling

\section{Risk of bias}

\begin{tabular}{lll}
\hline Bias & Authors' judgement & Support for judgement \\
\hline $\begin{array}{l}\text { Random sequence genera- } \\
\text { tion (selection bias) }\end{array}$ & Unclear risk & Insufficient information provided \\
\hline $\begin{array}{l}\text { Allocation concealment } \\
\text { (selection bias) }\end{array}$ & Unclear risk & Insufficient information provided \\
\hline $\begin{array}{l}\text { Blinding of participants } \\
\text { and personnel (perfor- } \\
\text { mance bias) } \\
\text { All outcomes }\end{array}$ & High risk & Intervention not blinded \\
\hline
\end{tabular}

\begin{tabular}{lll}
\hline $\begin{array}{l}\text { Blinding of outcome as- } \\
\text { sessment (detection bias) }\end{array}$ & High risk & $\begin{array}{l}\text { Although study authors state that staff carrying out follow-up measurements } \\
\text { are blinded, it is unclear whether analysis staff are blinded; outcomes mea- } \\
\text { sll outcomes }\end{array}$
\end{tabular}

\begin{tabular}{|c|c|c|}
\hline $\begin{array}{l}\text { Incomplete outcome data } \\
\text { (attrition bias) } \\
\text { All outcomes }\end{array}$ & High risk & $\begin{array}{l}\text { Students who did not complete any questionnaires in fourth grade or fifth } \\
\text { grade were excluded from the evaluation sample. African American partici- } \\
\text { pants and 'other' participants exhibited lower attrition rates than Caucasian } \\
\text { participants ( } 36 \% \text { and } 43 \% \text {, compared with } 49 \% \text {, respectively). Significant dif- } \\
\text { ferences were also evident between those lost to follow-up and those still re- } \\
\text { tained in the study, although details regarding attrition by study arm are lack- } \\
\text { ing. }\end{array}$ \\
\hline
\end{tabular}

\begin{tabular}{lll}
\hline $\begin{array}{l}\text { Selective reporting (re- } \\
\text { porting bias) }\end{array}$ & Unclear risk & No protocol available \\
\hline Other bias & Low risk & No other forms of bias identified \\
\hline
\end{tabular}

Olds 1998

\begin{tabular}{ll}
\hline Study name: Nurse Family Partnership \\
Study design (e.g. RCT, cluster RCT): RCT at the level of the individual \\
Intervention arm(s): nurse family partnership intervention (1), and nurse family partnership + trans- \\
portation costs (2) \\
Comparator arm(s): originally 2 separate control arms; both were provided sensory and developmen- \\
tal screening for the child at 12 and 24 months of age, with referral for further clinical evaluation and \\
treatment as needed. However, in addition, the second control arm received free transportation via \\
a taxi voucher system for prenatal and well-child care through to the child's second birthday. These 2 \\
groups were combined to form a single control group owing to similar rates of attendance. \\
Sample size calculation performed: N/S \\
Subgroups prespecified: yes; low-income, unmarried sample; mothers who smoke during pregnancy \\
Subgroup analyses: as above \\
Start date: April 1978 (recruitment commenced)
\end{tabular}


Olds 1998 (Continued)

Duration of follow-up: 15 years

Number of follow-ups: 1

Follow-up time points: 15 years post baseline

ICC (if reported): N/S

Participants

Number of schools randomised: N/A

Number of participants randomised (total and by arm): $N=116$; screening only control, $n=94$; screening and free transportation control, $n=90$; nurse visitation during pregnancy only, $n=100$; nurse visitation through to child's second birthday, $\mathrm{n}=116$

Age (range or mean (SD)) or grade at the start: $<19$ years at study registration

Gender: mothers: all female adolescents were control (55\% male), FNP (44\% male), FNP + financial assistance (55\%)

Ethnicity: reported only for mothers: control (white $=90 \%)$, FNP (white $=91 \%)$, FNP + financial assistance $($ white $=86 \%)$

SES: low socioeconomic status household: control (64\%), FNP (70\%), FNP + financial assistance (61\%). Percentage of mothers in work: control (39\%), FNP (36\%), FNP + financial assistance (31\%). Mother receiving public financial assistance: control (9\%), FNP (10\%), FNP + financial assistance (13\%). Father working: control (70\%), FNP (70\%), FNP + financial assistance (67\%). Father receiving public assistance: control (4\%), FNP (3\%), FNP + financial assistance (3\%). Maternal education (years of education completed): control (11.2), FNP (11.6), FNP + financial assistance (11.1). Husband/boyfriend years of education completed: control (11.4), FNP (11.7), FNP + financial assistance (11.5)

Inclusion criteria: N/S; see 'focus' for more information

Exclusion criteria: N/S

Interventions

Randomisation before or after baseline survey: before

Duration of the intervention (excluding follow-up): 2 years

Description of the intervention: in the 2 treatment groups that received nurse home visitation, nurses promoted 3 aspects of maternal functioning: maternal personal development (i.e. work, family planning education), positive health-related behaviours, and competent care of their children. Nurses acted as a link between parents and achievement towards each of the above 3 aims. Further, they encouraged friends and family members of the parent they were visiting to play a proactive role in supporting mothers in the programme and their development.

Brief description of the theoretical model: theories of human ecology, self-efficacy, and human attachment. These 3 theories suggest that behaviour change is a function of families' social context, as well as individuals' self-belief, motivations, and emotions.

Description of the comparator: sensory and developmental screening; sensory and developmental screening plus free transportation for prenatal and well-child care through to child's second birthday.

Outcomes

Primary outcomes: reduction in child criminal and antisocial behaviour (including substance use)

Secondary outcomes: not specifically stated. Study authors report on child reports of running away from home, sexual risk behaviours (initiation, number of sexual partners).

Setting

Country: USA; Place: New York

Setting: home/family environment

Focus: targeted; pregnant women recruited. Those with no previous live births, who were less than 25 weeks' pregnant, and who were 19 years of age or younger, unmarried, and/or of low socioeconomic 
Olds 1998 (Continued)

status were actively recruited. Women without these SES characteristics were permitted to enrol provided they had no previous live births.

\begin{tabular}{|c|c|}
\hline \multirow[t]{3}{*}{ Process measures } & Process data reported: yes (regarding intensity of visits only) \\
\hline & Method (qualitative or quantitative): quantitative \\
\hline & $\begin{array}{l}\text { Details: nurses completed an average of } 9 \text { and } 7 \text { visits throughout pregnancy, respectively. } 23 \text { and } 26 \\
\text { visits were made from birth to the child's second birthday. Women who had fewer coping resources (i.e. } \\
\text { limited belief in their control over life experiences) received a larger number of nurse home visits. }\end{array}$ \\
\hline
\end{tabular}

Sample size: 400 individuals randomised at baseline
Unit of randomisation: individual
Unit of analysis: individual
Method to promote equivalence between groups: stratification by the following socioeconomic
characteristics: maternal ethnicity, marital status, geographical region of residence
Statistical models: general linear model
Baseline differences adjustment: yes; all differences adjusted for in analysis
Repeated measures methods in analysis: N/A; only 15-year follow-up point reported
Equity: analysis conducted according to parental receipt of public assistance and various other socioe-
conomic status proxies and gender/smoking status at pregnancy
Funding: work contributing to the study was supported by the Senior Research Scientist Award 1-K05-
MH01382-01 (for Dr Olds) and grants from the Prevention Research and Behavioural Medicine branch of
the National Institute of Mental Health (R01-MH49381); and from the Assistant Secretary for Planning
and Evaluation, Department of Health and Human Services (96ASPE278A); the Bureau of Maternal and
Child Health, Department of Health and Human Services (Washington, DC); the Robert Wood Johnston
Foundation (Princeton, NJ); and the W.T. Grant Foundation, the Ford Foundation, and the Common-
wealth Fund (all New York).

Randomisation method, e.g. block, stratification, computer: N/S

Clustering accounted for in sample size calculation (if relevant): N/S

Cluster randomisation methods to account for clustering in analysis: $\mathrm{N} / \mathrm{S}$

\begin{tabular}{lll}
\hline Risk of bias & & \\
\hline Bias & Authors' judgement & Support for judgement \\
\hline $\begin{array}{l}\text { Random sequence genera- } \\
\text { tion (selection bias) }\end{array}$ & Low risk & Drew cards \\
\hline $\begin{array}{l}\text { Allocation concealment } \\
\text { (selection bias) }\end{array}$ & Unclear risk & Insufficient information provided \\
\hline $\begin{array}{l}\text { Blinding of participants } \\
\text { and personnel (perfor- } \\
\text { mance bias) } \\
\text { All outcomes }\end{array}$ & High risk & Blinding not possible for participants owing to the nature of this study \\
\hline $\begin{array}{l}\text { Blinding of outcome as- } \\
\text { sessment (detection bias) } \\
\text { All outcomes }\end{array}$ & High risk & \begin{tabular}{l} 
\\
\hline
\end{tabular} \\
\hline
\end{tabular}


Olds 1998 (Continued)

\begin{tabular}{|c|c|c|}
\hline $\begin{array}{l}\text { Incomplete outcome data } \\
\text { (attrition bias) }\end{array}$ & Low risk & $\begin{array}{l}\text { Low rates of attrition and similar rates between intervention and control } \\
\text { groups }\end{array}$ \\
\hline
\end{tabular}

All outcomes groups

Selective reporting (re- Unclear risk No published protocol
porting bias)

Other bias Low risk No other forms of bias identified

Study design (e.g. RCT, cluster RCT): RCT at the level of the family (adolescent + primary caregiver)

Intervention arm(s): the Familias Unidas curriculum consisted of sessions on enhancing parental involvement, increasing family functioning via effective child supervision, and increasing family communications about drug/alcohol abuse and risky sexual practices

Comparator arm(s): community control group participants were given 3 referrals to agencies within their catchment area providing services for youth with problem behaviour. No other intervention activities were provided to these participants.

Sample size calculation performed: N/S

Subgroups prespecified: N/A

Subgroup analyses: no

Start date: January 2004

Duration of follow-up: 30 months post intervention

Number of follow-ups: 3

Follow-up time points: 6,18 , and 30 months post baseline

ICC (if reported): N/A

Participants

Number of schools randomised: N/A

Number of participants randomised (total and by arm): 213; 109 to intervention group, 104 to control

Age (range or mean (SD)) or grade at the start: adolescents: mean 13.8 years (0.76 SD), grade 8 at baseline. Primary caregivers: mean 40.0 years (6.5 SD)

Gender: Familias Unidas: male (62.4\%), female (37.6\%); Community Control: male (65.4\%), female $(34.6 \%)$

\section{Ethnicity: N/S}

SES: mean annual family income (intervention/control arm): $\leq \$ 9999(29.4 \% / 30.8 \%), \$ 10,000$ to $\$ 19,999$ (38.5\%/44.2\%), \$20,000 to \$29,999 (18.3\%/12.5\%), $\geq \$ 30,000$ (13.8\%/12.5\%)

Inclusion criteria: adolescents $\geq 1$ standard deviation above average (score of 24) on any 1 of the 3 Revised Behaviour Problem Checklist subscales: conduct disorder, socialised aggression, or attention problems

Exclusion criteria: families who planned to move out of the catchment area of the 3 recruited schools within the study period, families who planned to move out of the Southern Florida area within the 3- 
Pantin 2009 (Continued)

year duration of the study, adolescents who refused assent, families with scheduling conflicts that pre-

vented primary caregivers from attending intervention sessions

Randomisation before or after baseline survey: after
Duration of the intervention (excluding follow-up): N/S
Description of the intervention: intervention involves working with parents to develop effective par-
enting skills and to provide necessary knowledge. It aims to increase parental investment within the
family, foster proactive connections between family and peers/school, and garner additional support
for parents. Hispanic-specific cultural issues are integrated into all aspects of the intervention.
Brief description of the theoretical model: intervention draws on ecodevelopmental theory, which
proposes that risk and protective processes operating at different levels create an overall risk and pro-
tective profile. Promoting protective factors within the family and between the family and other sys-
tems aims to address risk at multiple levels and prevent risks from compounding one another.

Description of the comparator: 3 referrals to agencies in their catchment area that serve young people with behaviour problems

Outcomes

Primary outcomes: lower initiation of/reduction in prevalence of substance use; initiation of sexual intercourse, condom wearing, and externalising behaviours

Secondary outcomes: N/A

Setting

Country: USA;State: Miami, Florida

Setting: family/home environment

Focus: targeted; Hispanic adolescents in grade 8 with behavioural problems and their primary caregivers were recruited

Process measures

Process data reported: yes

Method (qualitative or quantitative): quantitative

Description

Adherence to the intervention: all group sessions were video-recorded. Independent raters assigned scores between 0 (not at all/very poor) and 6 (extensively/excellent) based on both adherence measures and perceived quality of sessions. The average rating for the 3 intervention modules was 4.18 (0.18 SD).

Intensity of the intervention: $9 \times 2$-hour group sessions and $10 \times 1$-hour family visits. Families also received $4 \times 1$-hour booster sessions throughout the follow-up period (at 10, 16, 22, and 28 months).

\author{
Sample size: N/S \\ Unit of randomisation: individual
}

Unit of analysis: individual

Method to promote equivalence between groups: balanced on adolescent gender, years in the United States (i.e. 0 to 3, 3 to 10, or > 10), having initiated substance use (yes, no), and having initiated oral, vaginal, or anal sex (yes, no)

Statistical models: growth curve modelling (assessed initiation and prevalence of substance use), 2part growth curve (initiation of sexual intercourse and frequency of using condoms), confirmatory factor analysis with maximum likelihood (assessed family functioning trajectories), and ANOVA (assessed family functioning between baseline and 6 months, between intervention and control groups, respectively) 
Pantin 2009 (Continued)

Baseline differences adjustment: all were adjusted for (baseline levels of substance use in the substance use growth curve analysis; baseline levels of family functioning in the family functioning analysis; and baseline levels of positive parenting and parental monitoring for the positive parenting and parental monitoring analyses, respectively).

Repeated measures methods in analysis: yes

Notes Equity: baseline data only

Funding: National Institute on Drug Abuse (NIDA) grant DA017462 partially supported the study financially.

Randomisation method, e.g. block, stratification, computer: an urn randomisation programme was used, which balanced on adolescent gender, years in the United States (i.e. 0 to 3, 3 to 10, or > 10), having initiated substance use (yes/no) and having initiated oral, vaginal, or anal sex (yes/no)

Clustering accounted for in sample size calculation (if relevant): N/A

Cluster randomisation methods to account for clustering in analysis: N/A

\section{Risk of bias}

\begin{tabular}{|c|c|c|}
\hline Bias & Authors' judgement & Support for judgement \\
\hline $\begin{array}{l}\text { Random sequence genera- } \\
\text { tion (selection bias) }\end{array}$ & Unclear risk & Insufficient information provided \\
\hline $\begin{array}{l}\text { Allocation concealment } \\
\text { (selection bias) }\end{array}$ & Unclear risk & Insufficient information provided \\
\hline $\begin{array}{l}\text { Blinding of participants } \\
\text { and personnel (perfor- } \\
\text { mance bias) } \\
\text { All outcomes }\end{array}$ & High risk & Blinding not possible \\
\hline $\begin{array}{l}\text { Blinding of outcome as- } \\
\text { sessment (detection bias) } \\
\text { All outcomes }\end{array}$ & High risk & Self-reported outcomes \\
\hline $\begin{array}{l}\text { Incomplete outcome data } \\
\text { (attrition bias) } \\
\text { All outcomes }\end{array}$ & Low risk & $\begin{array}{l}\text { Low level of attrition, similar between groups; statistical models accounted for } \\
\text { missing data (and assumed this was at random) plus ITT analysis conducted }\end{array}$ \\
\hline $\begin{array}{l}\text { Selective reporting (re- } \\
\text { porting bias) }\end{array}$ & Unclear risk & No published protocol; 1 outcome reported at only 1 time point \\
\hline Other bias & Low risk & No other forms of bias identified \\
\hline
\end{tabular}

Perry 2003a

Methods

Study name: DARE

Study design: school-based RCT

Intervention arm(s): DARE

Comparator arm(s): DARE-Plus, control

Sample size calculation performed: N/A 
Subgroups prespecified: yes; analyses conducted separately because substantial interactions with sex were noted

Subgroup analyses: by gender

Start date: 1999

Duration of follow-up: end of intervention

Number of follow-ups: 2

Follow-up time points: spring 2000, spring 2001 (end of intervention)

ICC (if reported): not reported

Participants

Number of schools randomised: 24

Number of participants randomised (total and by arm): 2226 DARE, 2221 DARE-Plus, 1790 control

Age (range or mean (SD)) or grade at the start: seventh grade ( $~ 12$ years)

Gender: $51.6 \%$ male

Ethnicity: $67.3 \%$ white, $7.5 \%$ African American, $12.7 \%$ Asian American, 3.6\% Hispanic, 4\% American Indian, $4.9 \%$ mixed or other ethnic groups

SES: N/A

Inclusion criteria: middle and junior high schools with a seventh grade including $\geq 200$, but specific inclusion criteria not reported

Exclusion criteria: N/A

Interventions

Randomisation before or after baseline survey: N/A

Duration of the intervention (excluding follow-up): 18 months

Description of the intervention: the DARE programme involves a curriculum for skills in resisting drug use and handling violent situations, led by police officers. It also focuses on character building and citizenship skills. DARE-Plus includes a peer-led parental involvement programme entitled "On the Verge", a 4-session programme designed as a teen magazine with classroom activities focused on influences and skills related to peers, social groups, media, and role models. The last part of the magazine included activities to complete with parents at home. Students participated in a theatre production and received 3 postcards that focused on targeting of young people by tobacco companies. Extracurricular activities were included as an additional component of DARE-Plus.

Brief description of the theoretical model: N/S

Description of the comparator: DARE-Plus or control group

Outcomes Primary outcomes: tobacco, alcohol, and marijuana use; violence; victimisation

Secondary outcomes: psychosocial factors associated with multi-drug use and violence (e.g. social skills, expectations, parental rules, perceived access, offers of drugs)

Setting

Country: USA;State: Minnesota

Setting: school

Focus: universal

Process measures Process data reported: no

Method (qualitative or quantitative): N/S 
Perry 2003a (Continued)

\section{Description: N/S}

Statistics

Sample size: 6237 students at baseline (2226 DARE, 2221 DARE-Plus, 1790 control)

Unit of randomisation: school

Unit of analysis: individual (accounting for clustering)

Method to promote equivalence between groups: matching on socioeconomic measures, drug use, and size

Statistical models: growth curve analyses

Baseline differences adjustment: N/A

Repeated measures methods in analysis: yes

Equity: N/S
Funding: National Institute of Drug Abuse
Randomisation method: N/S
Clustering accounted for in sample size calculation (if relevant): N/S
Cluster randomisation methods to account for clustering in analysis: multi-level linear random-co-
efficients model

\section{Risk of bias}

\begin{tabular}{lll}
\hline Bias & Authors' judgement & Support for judgement \\
\hline $\begin{array}{l}\text { Random sequence genera- } \\
\text { tion (selection bias) }\end{array}$ & Unclear risk & Insufficient information \\
\hline $\begin{array}{l}\text { Allocation concealment } \\
\text { (selection bias) }\end{array}$ & Unclear risk & Insufficient information \\
\hline $\begin{array}{l}\text { Blinding of participants } \\
\text { and personnel (perfor- } \\
\text { mance bias) }\end{array}$ & High risk & Lack of blinding \\
All outcomes & & \\
\hline
\end{tabular}

\begin{tabular}{lll}
\hline Blinding of outcome as- & High risk & Self-reported outcomes \\
sessment (detection bias) & \\
All outcomes & \\
\hline
\end{tabular}

\begin{tabular}{|c|c|c|}
\hline $\begin{array}{l}\text { Incomplete outcome data } \\
\text { (attrition bias) }\end{array}$ & Low risk & $\begin{array}{l}\text { Authors state no differential attrition between groups with regard to the main } \\
\text { variables in the analysis }\end{array}$ \\
\hline
\end{tabular}

All outcomes variables in the analysis

Selective reporting (re- Low risk $\quad$ Expected outcomes reported
porting bias)

Other bias Low risk No other forms of bias identified

Piper 2000a

Methods Study name: Healthy for Life


Piper 2000a (Continued)

Study design: school-based RCT

Intervention arm(s): Healthy for Life: Age Appropriate version

Comparator arm(s): Healthy for Life: Intensive version; control

Sample size calculation performed: yes

Subgroups prespecified: N/A

Subgroup analyses: no

Start date: 1987

Duration of follow-up: 12 and 24 months post intervention

Number of follow-ups: 2

Follow-up time points: ninth and tenth grades (12 and 24 months post intervention)

ICC (if reported): not reported

Participants

Number of schools randomised: 21

Number of participants randomised (total and by arm): pre-test total sample 2483; 827 in HFL ageappropriate condition, 758 in intensive condition, 898 in control condition.

Age (range or mean (SD)) or grade at the start: grade 6 ( 11 years) for the age-appropriate version; grade 7 for the intensive version

Gender: $52 \%$ female

Ethnicity: $96 \%$ white

SES: percentage with mothers with college education: $30 \%$ control, $27 \%$ age-appropriate condition, $35 \%$ intensive condition

Inclusion criteria: N/S

Exclusion criteria: $\mathrm{N} / \mathrm{S}$

Interventions

\begin{abstract}
Randomisation before or after baseline survey: after
Duration of the intervention (excluding follow-up): 4-week block over 3 years; or one 12-week block in 1 year

Description of the intervention: a 54-lesson curriculum programme designed to be delivered either in 1 sequential 12-week block (the intensive condition) or in three 4-week segments (the age-appropriate condition) (20 lessons in grade 6, 19 lessons in grade 7, and 19 lessons in grade 8) to an entire cohort of middle schoolers. The programme used 9 teaching strategies considered most effective: social inoculation, use of peer leaders, parent-adult interviews, a parent orientation session at the start of the programme, health advocacy, an emphasis on short-term effects, analysing the influences of advertising and media, encouraging students to make public commitments to healthy behaviour, and an emphasis on peer norms. For the peer component, each classroom elected 3 peer leaders who had a major role in approximately one-third of the curriculum. The 3 peer leaders worked as a team, deciding how to present their activities and learning valuable co-operation, communication, and negotiation skills. The programme included a peer component ( 3 peer leaders were elected who had a role in one-third of the curriculum); a family component (which facilitated communication between teens and family members/other adults); and a community component (which involved sponsoring 1 health event and focusing on 1 policy issue).
\end{abstract}

Brief description of the theoretical model: social influences model 
Piper 2000a (Continued)

Description of the comparator: usual programming. Control schools were provided the opportunity to receive Healthy for Life for a subsequent cohort of students.

\begin{tabular}{|c|c|}
\hline Outcomes & $\begin{array}{l}\text { Primary outcomes: nutrition; alcohol, tobacco, and drug use; sexual behaviour } \\
\text { Secondary outcomes: N/S }\end{array}$ \\
\hline Setting & $\begin{array}{l}\text { Country: USA; State: Wisconsin } \\
\text { Setting: secondary school } \\
\text { Focus: universal }\end{array}$ \\
\hline Process measures & $\begin{array}{l}\text { Process data reported: yes } \\
\text { Method (qualitative or quantitative): both } \\
\text { Description: the process evaluation included maintenance of teacher logs regarding implementation } \\
\text { of each session; observation by a trained observer of a sample of all implementations; interviews with } \\
\text { programme staff, teachers, administrators, and students; and feedback surveys of students and par- } \\
\text { ents. Study authors state that the age-appropriate version experienced some implementation difficul- } \\
\text { ties because it spanned } 3 \text { years in annual, 4-week segments. The programme used approaches that are } \\
\text { not common among most teachers and would be difficult to sustain without significant additional re- } \\
\text { sources and dedicated staffing. In } 3 \text { communities, groups were opposed to any form of sex education, } \\
\text { which hampered implementation. }\end{array}$ \\
\hline
\end{tabular}

\begin{tabular}{|c|c|c|}
\hline \multirow[t]{7}{*}{ Statistics } & \multirow{2}{*}{\multicolumn{2}{|c|}{$\begin{array}{l}\text { Sample size: } 2483 \text { students } \\
\text { Unit of randomisation: school }\end{array}$}} \\
\hline & & \\
\hline & \multicolumn{2}{|c|}{ Unit of analysis: individual - accounting for clustering } \\
\hline & \multicolumn{2}{|c|}{ Method to promote equivalence between groups: stratification } \\
\hline & \multicolumn{2}{|c|}{ Statistical models: hierarchical linear modelling } \\
\hline & \multicolumn{2}{|c|}{ Baseline differences adjustment: yes } \\
\hline & \multicolumn{2}{|c|}{ Repeated measures methods in analysis: not clear } \\
\hline \multirow[t]{5}{*}{ Notes } & \multicolumn{2}{|l|}{ Equity: N/S } \\
\hline & \multicolumn{2}{|c|}{ Funding: National Institute on Drug Abuse and Robert Wood Johnson Foundation } \\
\hline & \multicolumn{2}{|c|}{ Randomisation method: N/S } \\
\hline & \multicolumn{2}{|c|}{ Clustering accounted for in sample size calculation (if relevant): N/S } \\
\hline & \multicolumn{2}{|c|}{ Cluster randomisation methods to account for clustering in analysis: hierarchical linear modelling } \\
\hline \multicolumn{3}{|l|}{ Risk of bias } \\
\hline Bias & Authors' judgement & Support for judgement \\
\hline $\begin{array}{l}\text { Random sequence genera- } \\
\text { tion (selection bias) }\end{array}$ & Unclear risk & Insufficient information given \\
\hline $\begin{array}{l}\text { Allocation concealment } \\
\text { (selection bias) }\end{array}$ & Unclear risk & $\begin{array}{l}\text { Schools actively selected which of the } 2 \text { intervention arms they wanted to re- } \\
\text { ceive, but study authors provided little detail provided about allocation to } \\
\text { treatment or control. }\end{array}$ \\
\hline
\end{tabular}


Piper 2000a (Continued)
Blinding of participants
High risk
Unblinded intervention and personnel (performance bias)

All outcomes

\begin{tabular}{|c|c|c|}
\hline $\begin{array}{l}\text { Blinding of outcome as- } \\
\text { sessment (detection bias) } \\
\text { All outcomes }\end{array}$ & High risk & Self-reported outcomes \\
\hline $\begin{array}{l}\text { Incomplete outcome data } \\
\text { (attrition bias) } \\
\text { All outcomes }\end{array}$ & High risk & $\begin{array}{l}\text { High attrition rate at } 10 \text { th grade of } 32 \% \text {; at this follow-up, participants from the } \\
\text { intensive condition were under-represented ( } 61 \% \text { present vs } 68 \% \text { overall; P } \\
<.001 \text { ) }\end{array}$ \\
\hline $\begin{array}{l}\text { Selective reporting (re- } \\
\text { porting bias) }\end{array}$ & Low risk & Expected outcomes reported \\
\hline Other bias & Low risk & No other sources of bias identified \\
\hline
\end{tabular}

Redding 2015

Study name: Step by Step
Study design: RCT
Intervention arm: Step by Step, a transtheoretical model-tailored intervention to increase condom
use and decrease smoking
Comparator arm: control
Sample size calculation performed: yes
Subgroups prespecified: N/A
Subgroup analyses: no
Start date: N/S
Duration of follow-up: 18 months
Number of follow-ups: 3
Follow-up time points: 6 -month assessment, 12 months, 18 months
Intracluster correlation coefficient: N/A

\section{Participants}

\section{Number of schools randomised: N/A}

Number of participants randomised: 424 intervention, 404 control

Age (range or mean (SD)) or grade at the start: mean 16.4 years

Gender: $100 \%$ female

Ethnicity: $84 \%$ black

Socioeconomic status: student education level intervention vs control: grade 7 or less - $2 \%$ vs $3 \%$; grade 8 - 14\% vs $13 \%$; grade 9 - 29\% vs $26 \%$; grade 10 - $29.0 \%$ vs $31 \%$; grade 11 - $25 \%$ vs $27 \%$

Inclusion criteria: female, 14 to 17 years of age, not pregnant 


\section{Exclusion criteria: N/S}

\begin{tabular}{|c|c|}
\hline \multirow[t]{5}{*}{ Interventions } & Timing of randomisation: before baseline survey \\
\hline & Duration of the intervention: 9 months \\
\hline & $\begin{array}{l}\text { Description of the intervention arm(s): transtheoretical model (TTM)-tailored multimedia interven- } \\
\text { tion package, including computer-delivered feedback and stage-targeted counselling }\end{array}$ \\
\hline & Brief description of the theoretical model: transtheoretical model \\
\hline & $\begin{array}{l}\text { Description of the comparator arm(s): standard care (SC) education and advice - standard care com- } \\
\text { puter feedback (generic, not stage targeted or tailored) and standard contraceptive educational coun- } \\
\text { selling (SC group participants completed identical survey items using the same computer-delivered } \\
\text { programme) }\end{array}$ \\
\hline \multirow[t]{2}{*}{ Outcomes } & Primary outcomes: consistent condom use, smoking cessation and prevention \\
\hline & Secondary outcomes: N/S \\
\hline \multirow[t]{3}{*}{ Setting } & Country: USA; Place: Philadelphia \\
\hline & Setting: clinic \\
\hline & Focus: targeted to females \\
\hline \multirow[t]{3}{*}{ Process measures } & Process data reported: yes \\
\hline & Method: qualitative \\
\hline & $\begin{array}{l}\text { Description: following sessions, counsellors reported what topics they covered and what activities } \\
\text { they used. Subsequent review of these reports for fidelity revealed that counsellors were much more } \\
\text { ready to discuss condom use than smoking-related topics in sessions. Study authors stated that the } \\
\text { teens were enthusiastic and gave positive feedback supporting the programme's acceptability and us- } \\
\text { ability. }\end{array}$ \\
\hline
\end{tabular}

Statistics

Sample size: 424 intervention, 404 control

Unit of randomisation: individual

Unit of analyses: individual

Methods to promote equivalence between groups: stratified by baseline stage of condom use

Statistical models: repeated measures regression analysis using the generalised estimating equation method

Baseline differences adjustment: N/S

Repeated measures methods in analysis: yes

Notes

Equity: data at baseline as above

Funding: National Cancer Institute at the National Institutes of Health (grant numbers RO1 CA63745, PO1 CA50087, to JOP)

Randomisation method: not clear

Clustering accounted for in sample size calculation: N/A

Cluster randomisation methods to account for clustering in analysis: N/A 
Redding 2015 (Continued)

\begin{tabular}{|c|c|c|}
\hline Bias & Authors' judgement & Support for judgement \\
\hline $\begin{array}{l}\text { Random sequence genera- } \\
\text { tion (selection bias) }\end{array}$ & Unclear risk & Study authors stated computer-generated but gave insufficient information \\
\hline $\begin{array}{l}\text { Allocation concealment } \\
\text { (selection bias) }\end{array}$ & Unclear risk & Insufficient information about the role of the computer \\
\hline $\begin{array}{l}\text { Blinding of participants } \\
\text { and personnel (perfor- } \\
\text { mance bias) } \\
\text { All outcomes }\end{array}$ & Unclear risk & Unblinded intervention \\
\hline $\begin{array}{l}\text { Blinding of outcome as- } \\
\text { sessment (detection bias) } \\
\text { All outcomes }\end{array}$ & High risk & $\begin{array}{l}\text { Follow-up surveys at } 12 \text { and } 18 \text { months were blinded, but outcomes were self- } \\
\text { reported. }\end{array}$ \\
\hline $\begin{array}{l}\text { Incomplete outcome data } \\
\text { (attrition bias) } \\
\text { All outcomes }\end{array}$ & Low risk & Multiple imputation for missing data using various approaches \\
\hline $\begin{array}{l}\text { Selective reporting (re- } \\
\text { porting bias) }\end{array}$ & Low risk & Expected outcomes reported \\
\hline Other bias & Low risk & No other sources of bias identified \\
\hline
\end{tabular}

Sanchez 2007

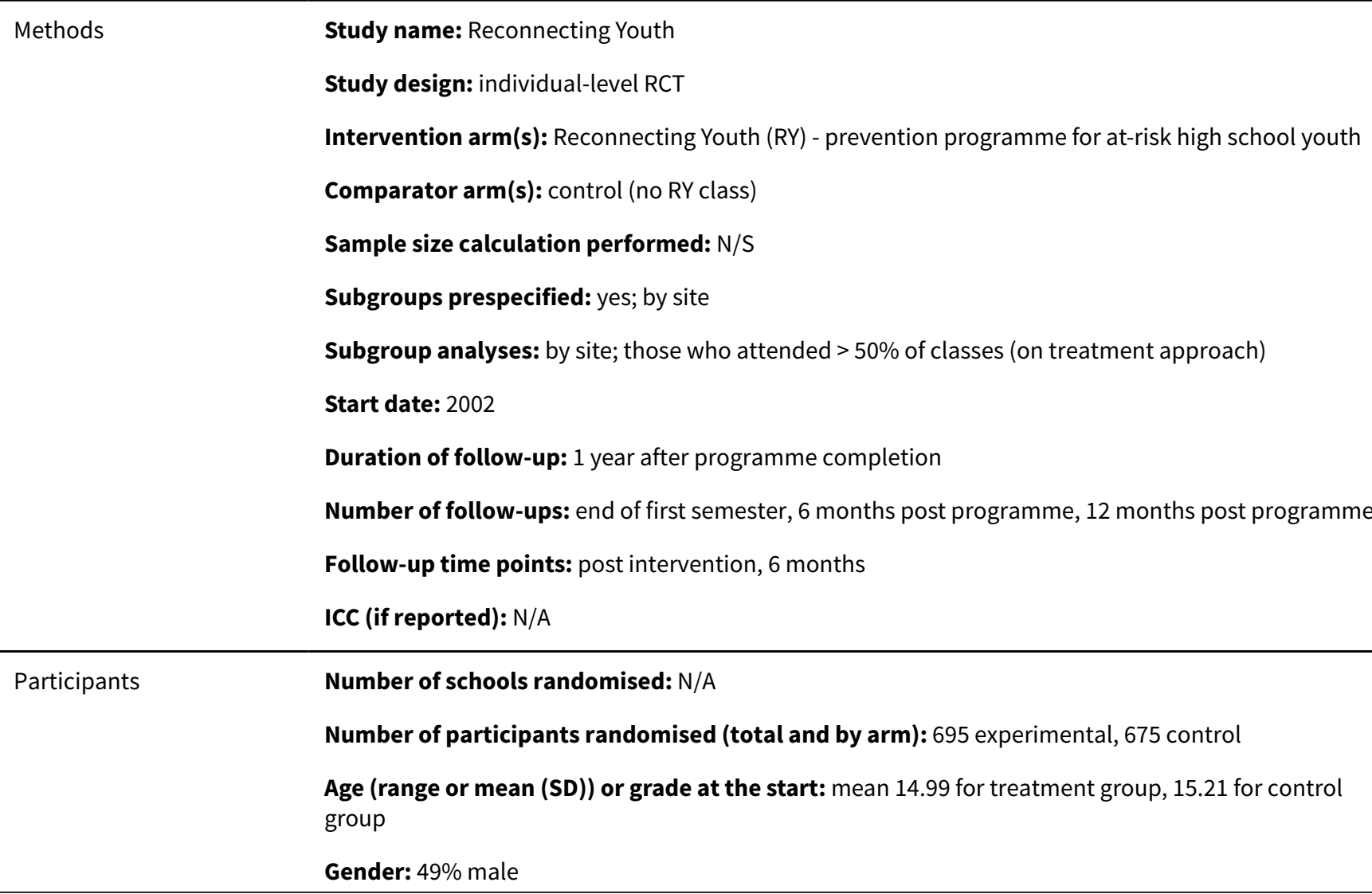


Ethnicity: site A: $87 \%$ Hispanic, 9\% black, 4\% white, 4\% American Indian or other race; site B: $40 \%$ Asian/Pacific Islander, 21\% Hispanic, 15\% black, 10\% white, 12\% American Indian or other race

SES: site A: $90 \%$ qualified for the federal free or reduced-price lunch programme; site B: $61 \%$ of students qualified for free or reduced-price lunch

Inclusion criteria: criteria for high-risk status included being in the top $25 \%$ for truancy and the bottom $50 \%$ for GPA, or being referred for participation by a school teacher or counsellor.

Exclusion criteria: N/S

Duration of the intervention (excluding follow-up): autumn 2002 to autumn 2004

Description of the intervention: Reconnecting Youth (RY) is a 1-semester class that aims to improve academic achievement, reduce or prevent drug use, and improve mood management (depression, anger, anxiety). A 5-unit written curriculum with 55 core and 24 booster lessons is included. The programme is implemented in a small group of 10 to 12 students. The 2 main components are skills training and group development, including social support from teacher and peers. The first unit comprises 10 lessons introducing students to the model, followed by units focused on self-monitoring, self-esteem, decision-making, personal control, and decision-making. Students learn to self-monitor their attendance, moods, and drug use on a daily basis, and to develop achievable goals based on these data. In all, $50 \%$ to $60 \%$ of each class session is allocated to skills building; $20 \%$ to monitoring and reporting back on homework; and $20 \%$ to a structured discussion/feedback process for student issues, problems, and celebrations.

Brief description of the theoretical model: guided by strain, social learning and control theories

Description of the comparator: high-risk students not assigned to take the RY class

Outcomes

Primary outcomes: educational outcomes, substance use, mental health (anger, depression, anxiety), school connectedness, peer bonding

Secondary outcomes: N/S

Setting

Country: USA; State: Pacific Coast (Site B) and South West (Site A)

Setting: secondary school

Focus: targeted to persons experimenting with drugs or other risk-related behaviours

Process measures Process data reported: yes

Method (qualitative or quantitative): quantitative

Description: teacher logs, attendance records, classroom observations, and student questionnaires were completed. Average attendance was $79 \%$ (SD 22.43), with a range of $4 \%$ to $100 \%$. The mean percentage of total lessons taught was $74 \%$ (SD 0.11 , range $56 \%$ to $92 \%$ ). Teachers taught $90 \%$ of core lessons on average, but $38 \%$ of booster lessons (range $4 \%$ to $83 \%$ ). The mean percentage of time spent on life skills building was $56 \%$, just exceeding the $50 \%$ mark denoting adequate adherence to this programme component. Mean scores on group development and life skills building were 2.43 and 2.49, respectively, out of a total of 5 , indicating that implementation quality was between being 'below expectations' and 'meeting the study protocol'. Teacher help and peer help scores were 17.07 (SD 1.03) and 15.70 (SD 1.86), respectively, out of 20 , suggesting relatively high levels of student satisfaction.

Statistics

Sample size: response rate not stated; 1370 randomised

Unit of randomisation: individual

Unit of analysis: individual

Method to promote equivalence between groups: N/S 
Statistical models: ANCOVA models using general linear models, accounting for clustering

Baseline differences adjustment: yes

Repeated measures methods in analysis: N/S

Equity: data provided at baseline, as outlined above
Funding: National Institute on Drug Abuse
Randomisation method: N/S
Clustering accounted for in sample size calculation (if relevant): N/A
Cluster randomisation methods to account for clustering in analysis: yes

\section{Risk of bias}

\begin{tabular}{|c|c|c|}
\hline Bias & Authors' judgement & Support for judgement \\
\hline $\begin{array}{l}\text { Random sequence genera- } \\
\text { tion (selection bias) }\end{array}$ & Unclear risk & Insufficient information \\
\hline $\begin{array}{l}\text { Allocation concealment } \\
\text { (selection bias) }\end{array}$ & Unclear risk & Insufficient information \\
\hline $\begin{array}{l}\text { Blinding of participants } \\
\text { and personnel (perfor- } \\
\text { mance bias) } \\
\text { All outcomes }\end{array}$ & High risk & Programme unblinded \\
\hline $\begin{array}{l}\text { Blinding of outcome as- } \\
\text { sessment (detection bias) } \\
\text { All outcomes }\end{array}$ & High risk & Self-reported outcomes \\
\hline $\begin{array}{l}\text { Incomplete outcome data } \\
\text { (attrition bias) } \\
\text { All outcomes }\end{array}$ & Low risk & $\begin{array}{l}\text { Low attrition rates in each group; study authors state that attrition rates by } \\
\text { group were not significant }(P>.05) \text { at either follow-up. No differences were } \\
\text { found in attrition by site. }\end{array}$ \\
\hline $\begin{array}{l}\text { Selective reporting (re- } \\
\text { porting bias) }\end{array}$ & High risk & $\begin{array}{l}\text { Study authors state that } 25 \text { outcome and mediator variables were examined; } \\
\text { not clear if all are presented. Some inconsistency in presentation of outcome } \\
\text { data at both follow-ups }\end{array}$ \\
\hline Other bias & Unclear risk & Possibility of contamination by students in the same school \\
\hline
\end{tabular}

Saraf 2015

Intervention arm: school-, classroom- and family-based nutrition, physical activity, smoking prevention

Comparator arm: control (standard school curriculum)

Sample size calculation performed: yes

Subgroups prespecified: none reported

Subgroup analyses: no 
Saraf 2015 (Continued)

Start date: April 2009

Duration of follow-up: immediately post intervention

Number of follow-ups: 1

Follow-up time points: immediately post intervention

Intracluster correlation coefficient: not reported

Number of schools randomised: overall 40 (intervention 20, control 20)
Number of participants randomised: overall 2348 (intervention 1026, control 1322)
Age (range or mean (SD)) or grade at the start: overall 12.4 years (mean)
Gender: overall 46.5\% female, 53.5\% male
Ethnicity: N/S
Socioeconomic status: N/S
Inclusion criteria: N/S
Exclusion criteria: N/S
Timing of randomisation: after baseline data collection
Duration of the intervention: 9 months
Description of the intervention arm(s): (1) school component: aims to create an enabling environ-
ment in schools by forming school health committee, a school action plan; adopting policies regarding
physical activity, tobacco, and healthy foods; improving school environment via posters and bulletin
boards; and creating competitions for students concerning non-communicable diseases; (2) classroom
component: involves students engaging in health-promoting activities via lectures, films, peer discus-
sion, and classes; and (3) family/community component: builds connectivity with family/community
via pamphlet distribution, school-holiday assignments, listing healthy foods, and organising a family
orientation regarding non-communicable diseases during parent/teacher association meetings and an-
nual functions

Brief description of the theoretical model: N/S

Description of the comparator $\operatorname{arm}(\mathrm{s}): \mathrm{N} / \mathrm{S}$

Outcomes Primary outcomes: diet, physical activity, tobacco use

Secondary outcomes: N/S

Setting Country: India; Place: Haryana State

Setting: school

Focus: universal

Process measures Process data reported: no

\section{Method: N/A}

Description: describes only how many schools adopted various health-related policies (total $n=19)$, tobacco $(n=16)$, healthy food $(n=14)$, and physical activity $(n=6)$

\begin{tabular}{ll}
\hline Statistics & Unit of randomisation: school \\
& Unit of analyses: individual
\end{tabular}


Methods to promote equivalence between groups: none; study authors created 2 groups based on geographical proximity to the main road (left or right)

Statistical models: mixed-effect regression models

Baseline differences adjustment: N/S

Repeated measures methods in analysis: N/A - only 1 follow-up period

Notes

Equity: no other baseline measures presented

Funding: All India Institute of Medical Sciences, New Delhi

Clustering accounted for in sample size calculation: yes

Cluster randomisation methods to account for clustering in analysis: yes

\section{Risk of bias}

\begin{tabular}{lll}
\hline Bias & Authors' judgement & Support for judgement \\
\hline $\begin{array}{l}\text { Random sequence genera- } \\
\text { tion (selection bias) }\end{array}$ & Low risk & $\begin{array}{l}\text { Villages on 1 side of the main road when passing through villages were consid- } \\
\text { ered 1 group, and those on the other side another group. One group was allo- } \\
\text { cated to intervention, the other to control, based on drawing of lots. }\end{array}$ \\
\hline $\begin{array}{l}\text { Allocation concealment } \\
\text { (selection bias) }\end{array}$ & Unclear risk & Insufficient information provided \\
\hline $\begin{array}{l}\text { Blinding of participants } \\
\begin{array}{l}\text { and personnel (perfor- } \\
\text { mance bias) } \\
\text { All outcomes }\end{array}\end{array}$ & High risk & Participants and personnel not blinded \\
\hline
\end{tabular}

\begin{tabular}{lll}
\hline $\begin{array}{l}\text { Blinding of outcome as- } \\
\text { sessment (detection bias) } \\
\text { All outcomes }\end{array}$ & High risk & Participants not blinded; all outcome measures use self-report \\
\hline $\begin{array}{l}\text { Incomplete outcome data } \\
\text { (attrition bias) } \\
\text { All outcomes }\end{array}$ & High risk & Differential extent of attrition between study arms \\
\hline $\begin{array}{l}\text { Selective reporting (re- } \\
\text { porting bias) }\end{array}$ & Unclear risk & No protocol available \\
\hline $\begin{array}{l}\text { Other bias } \\
\text { Unclear risk }\end{array}$ & $\begin{array}{l}\text { Two groups created pre-randomisation based on geographical proximity to a } \\
\text { road (left vs right). Possibility of cross-contamination from intervention to con- } \\
\text { trol schools }\end{array}$ \\
\hline
\end{tabular}

Schweinhart 1993

$\begin{array}{ll}\text { Methods } & \text { Study name: High/Scope Perry Preschool Program } \\ & \text { Study design (e.g. } \mathbf{R C T} \text {, cluster RCT): RCT } \\ & \text { Intervention } \operatorname{arm}(\mathbf{s}): \text { High/Scope Perry Preschool Program } \\ & \text { Comparator } \operatorname{arm}(\mathbf{s}): \text { no intervention/usual practice } \\ & \text { Sample size calculation performed: not reported }\end{array}$


Subgroups prespecified: no planned subgroups

Subgroup analyses: N/A

Start date: 1962

Duration of follow-up: 36 years post intervention

Number of follow-ups: multiple (see below)

Follow-up time points: annually from ages 3 to 11 , at 14 and 15, at 19, at 27, and currently at ages 39 to 41

ICC (if reported): N/A

Participants

Number of schools randomised: N/A

Number of participants randomised (total and by arm): $N=123$ recruited; randomised to intervention $\mathrm{n}=58$, randomised to control $\mathrm{n}=65$

Age(range or mean (SD)) or grade at the start: age 3 or 4 years

Gender: proportion 0.40 male in both groups (59\% male overall)

Ethnicity: predominantly low-income African American children attended the school.

SES: not reported

Inclusion criteria: children of low socioeconomic status (based on an index score derived from parental income, education, and occupation) and an IQ test score (Stanford-Binet) between 70 and 85

Exclusion criteria: children with any diagnosed physical handicap

Duration of the intervention (excluding follow-up): 2 school years October to May (15 months); wave 0 was 7.5 months since recruited at age 4 years

\section{Description of the intervention}

\section{High/Scope Perry Preschool Program}

(1) a centre-based programme for 2.5 hours each weekday morning (with a child:teacher ratio of approximately 5:1 to $6.25: 1$, and with teachers trained in special education and early childhood)

(2) home visits by teachers to each mother and child for 1.5 hours per week

(3) parent group meetings

The pre-school programme provided organised educational activities to promote the intellectual and social development of young children.

Brief description of the theoretical model: open framework approach to curriculum based on Piaget's constructivist theory of child development.

Description of the comparator: no preschool programme

Outcomes List relevant outcomes

Primary outcomes: educational attainment includes education, employment, criminal activity, social services data, and teenage pregnancy.

Secondary outcomes: not reported 
Schweinhart 1993 (Continued)

Setting: preschool (age 3 to 4 years)

Focus: targeted; children were chosen on the basis of low levels of parental education and socioeconomic status, as well as low Stanford-Binet IQ test scores

\section{Process measures Process data reported: yes}

Method (qualitative or quantitative): qualitative

Description: study authors highlight that teachers were observed and followed the curriculum models closely. Although not part of a planned process evaluation, 12 nationally recognised experts in child development and early childhood education also observed the classrooms; held discussions with project staff and administrators; and recorded their observations in relation to the different curricula involved in the programme. Study authors noted that the consultants highlighted the high degree of staff involvement, enthusiasm, and commitment and involvement of the children in the programme.

Intensity of the intervention: intervention was delivered over 2 preschool years or 15 months and consisted of daily 2.5-hour sessions during the week and a 1.5-hour educational home visit by teachers every 2 weeks

Statistics

Sample size: $N=123$ recruited at ages 3 to 4 years; randomised to intervention $n=58$; randomised to control $n=65$

Unit of randomisation: individuals

Unit of analysis: individuals

Method to promote equivalence between groups: children were matched according to IQ, socioeconomic status, and gender before stratified group randomisation.

Statistical models: multiple and bivariate regression adjusting for family characteristics and parental education

Baseline differences adjustment: coefficients were adjusted for age at midlife interview; gender; IQ at programme entry; mother's education, age, and employment status at programme entry; father's presence in the home at programme entry; and an indicator of father's occupation (skilled or semi-skilled) at programme entry.

Repeated measures methods in analysis: not reported

Equity: programme entry characteristics were provided by group, and overall characteristics at follow-up.

Funding: not reported

Randomisation method, e.g. block, stratification, computer: coin toss

Clustering accounted for in sample size calculation (if relevant): N/A

Cluster randomisation methods to account for clustering in analysis: N/A

Economic outcomes: Cost benefit analysis

Estimates of resources used: the source of resources included operating costs (e.g. teacher salaries, administrative costs) and capital costs (e.g. classrooms, facilities). The initial programme cost is stated as USD17,759 per child (2006 dollars; undiscounted). Unit costs included costs of education USD4325, crime costs USD66,780, welfare costs USD3698, and initial programme costs USD17,759 with 0\% deadweight score. With 50\% deadweight score, these changed to education costs USD6434, crime costs USD75,062, welfare costs USD5547, and initial programme costs USD26,639. Earnings: USD78,010 (note: all in 2006 dollars, with $3 \%$ discount rate)

Point estimate and measure of uncertainty for incremental resource use, costs, and cost-effectiveness: internal rates of return are reported with adjustment for compromised randomisation and $0 \%$ and $50 \%$ deadweight losses and 3\% discounting. Rates are presented for all participants and by 
Schweinhart 1993 (Continued)

gender. The 'all participant' results at 0\% deadweight score include benefit/cost ratio 9.2 (SE 3.5) and IRR to society $8.3 \%$ (SE 2.4). The 'all participant' results at $50 \%$ deadweight score were benefit/cost ratio 6.6 (SE 2.7) and IRR to society 7.7\% (SE 2.6). In contrast, Belfield (2006) presented an unadjusted benefit/cost ratio of 26.6 and IRR to society of 21.0 (net benefits at 3\% discount rate USD229,645 (in 2000 dollars)).

\section{Risk of bias}

\begin{tabular}{|c|c|c|}
\hline Bias & Authors' judgement & Support for judgement \\
\hline $\begin{array}{l}\text { Random sequence genera- } \\
\text { tion (selection bias) }\end{array}$ & High risk & $\begin{array}{l}\text { Random sequence was generated via a reliable method (coin toss); but ran- } \\
\text { domisation was compromised by researchers re-assigning individuals to treat- } \\
\text { ment or control groups after randomisation. }\end{array}$ \\
\hline $\begin{array}{l}\text { Allocation concealment } \\
\text { (selection bias) }\end{array}$ & High risk & $\begin{array}{l}\text { It was not possible to blind researchers or participants to experimental or con- } \\
\text { trol groups during the allocation process. }\end{array}$ \\
\hline $\begin{array}{l}\text { Blinding of participants } \\
\text { and personnel (perfor- } \\
\text { mance bias) } \\
\text { All outcomes }\end{array}$ & High risk & Not blinded during allocation \\
\hline $\begin{array}{l}\text { Blinding of outcome as- } \\
\text { sessment (detection bias) } \\
\text { All outcomes }\end{array}$ & High risk & $\begin{array}{l}\text { Researchers were blinded to the collection of all follow-up data (Muennig } 2009 \\
\text { paper). }\end{array}$ \\
\hline $\begin{array}{l}\text { Incomplete outcome data } \\
\text { (attrition bias) } \\
\text { All outcomes }\end{array}$ & Low risk & Low level of loss to follow-up \\
\hline $\begin{array}{l}\text { Selective reporting (re- } \\
\text { porting bias) }\end{array}$ & Unclear risk & No published protocol \\
\hline Other bias & Unclear risk & $\begin{array}{l}\text { No other forms of bias identified, although multiple outcomes were tested } \\
\text { over multiple years }\end{array}$ \\
\hline
\end{tabular}

Schwinn 2014

Methods

Study name: no study name

Study design: RCT at the level of the family

Intervention arm: a web-based family involvement health promotion programme aimed at drug use, physical activity, and nutrition

Comparator arm: control group - no intervention

Sample size calculation performed: N/S

Subgroups prespecified: no subgroups

Subgroup analyses: N/A

Start date: N/S

Duration of follow-up: 5 months

Number of follow-ups: 2 
Follow-up time points: post-test and 5-month follow-up

Intracluster correlation coefficient: N/A

\begin{abstract}
Participants Number of schools randomised: N/A
Number of participants randomised: 67 recruited; 36 randomised to intervention, 31 to control

Age (range or mean (SD)) or grade at the start: 11.8 years (SD 0.88); intervention $11.8(0.81)$, control $11.9(0.96)$

Gender: females only

Ethnicity: \% white 40.3; black 44.8; Latina 11.9; Asian 3.0. Control: \% white 35.5; black 45.2; Latina 16.1; Asian 3.2. Intervention: \% white 44.4; black 44.4; Latina 8.3; Asian 2.8

Socioeconomic status: Total: \% mothers' education: some high school or less 7.5; completed high school 31.3; vocational school or 2-year college 32.8; 4-year college 25.4; graduate school 3.0. Control: $\%$ mothers' education: some high school or less 3.2; completed high school 32.3; vocational school or 2-year college 41.9; 4-year college 16.1; graduate school 6.5. Intervention: \% mothers' education: some high school or less 11.1; completed high school 30.6; vocational school or 2-year college 25.0; 4-year college 33.3; graduate school 0
\end{abstract}

Total: mothers' employment \%: full-time 44.8; part-time 16.4; homemaker 11.9; student or unemployed 26.9. Control: mothers' employment \%: full-time 41.9; part-time 16.1; homemaker 6.5; student or unemployed 35.5. Intervention: mothers' employment \%: full-time 47.2; part-time 16.7; homemaker 16.7; student or unemployed 19.4 .

$94 \%$ qualify for free/reduced school lunch.

Inclusion criteria: girls aged 10 to 12 years and their mothers, living in subsidised housing

Exclusion criteria: N/S

Interventions

Timing of randomisation: $\mathrm{N} / \mathrm{S}$

Duration of the intervention: 3 weeks

Description of the intervention arm(s): a brief web-based family involvement health promotion programme aimed at drug use, physical activity, and nutrition for adolescent girls, aged 10 to 12 years, who reside in public housing. The programme involved 3 web-based health promotion sessions online completed by mother and daughter together and a 5-step problem-solving process.

\title{
Brief description of the theoretical model: no
}

Description of the comparator arm(s): controls received no intervention materials.

Outcomes

Primary outcomes: daughters: fruit and vegetable intake; physical activity; past-month and past-week use of alcohol, cigarettes, marijuana, heroin, inhalants, methamphetamines, amphetamines, ecstasy, and tranquilisers

Secondary outcomes: mothers: reported past-month and past-week alcohol and cigarette use; mother-daughter closeness; mother-daughter communication; parental monitoring. Daughters: TV and Internet use; perceived stress; drug refusal skills

\begin{tabular}{ll}
\hline Setting & Country: USA; Place: 27 different states \\
& Setting: home \\
& Focus: targeted to girls living in public subsidised housing \\
\hline Process measures & Process data reported: yes \\
& Method: quantitative
\end{tabular}


Description: of the 36 pairs assigned to intervention, 35 completed all 3 sessions and 1 completed 2 sessions. Three sessions of 25 minutes each were included.

\section{Statistics}

Sample size: 86 information packets sent out, 67 participants recruited and randomised (36 intervention, 31 control)

Unit of randomisation: mother-daughter dyads

Unit of analyses: individuals

Methods to promote equivalence between groups: N/S

Statistical models: analysis of covariance; general linear model

Baseline differences adjustment: yes

Repeated measures methods in analysis: yes

Notes

Equity: pre-test baseline characteristics reported

Funding: National Institute on Drug Abuse grant no R21DA24618

Randomisation method: N/S

Clustering accounted for in sample size calculation: N/A

Cluster randomisation methods to account for clustering in analysis: N/A

\section{Risk of bias}

\begin{tabular}{|c|c|c|}
\hline Bias & Authors' judgement & Support for judgement \\
\hline $\begin{array}{l}\text { Random sequence genera- } \\
\text { tion (selection bias) }\end{array}$ & Unclear risk & No information given \\
\hline $\begin{array}{l}\text { Allocation concealment } \\
\text { (selection bias) }\end{array}$ & Unclear risk & No information given \\
\hline $\begin{array}{l}\text { Blinding of participants } \\
\text { and personnel (perfor- } \\
\text { mance bias) } \\
\text { All outcomes }\end{array}$ & Unclear risk & No information given \\
\hline $\begin{array}{l}\text { Blinding of outcome as- } \\
\text { sessment (detection bias) } \\
\text { All outcomes }\end{array}$ & Unclear risk & No information given \\
\hline $\begin{array}{l}\text { Incomplete outcome data } \\
\text { (attrition bias) } \\
\text { All outcomes }\end{array}$ & High risk & $14 \%$ attrition in the intervention arm, but $3.2 \%$ in the control arm \\
\hline $\begin{array}{l}\text { Selective reporting (re- } \\
\text { porting bias) }\end{array}$ & High risk & $\begin{array}{l}\text { Weekly substance use not given for daughters or mothers; composite sub- } \\
\text { stance use items assessed; use of alcohol, cigarettes, and marijuana reported }\end{array}$ \\
\hline Other bias & Unclear risk & $\begin{array}{l}\text { Intervention was targeted at dyads living in public subsidised housing, but it is } \\
\text { not clear whether this eligibility was assessed at recruitment stage, and it was } \\
\text { not presented in the demographics at baseline (only school lunch programme } \\
\text { eligibility is given); therefore it is not known whether the target group was in- } \\
\text { cluded in the study. }\end{array}$ \\
\hline
\end{tabular}




Study name: PATHS (Positive Adolescent Training through Holistic Social Programmes)
Study design: RCT at the level of the school
Intervention arm(s): PATHS
Comparator arm(s): PATHS delayed by 1 year
Sample size calculation performed: not clear
Subgroups prespecified: N/A; no subgroups
Subgroup analyses: no
Start date: not clear
Duration of follow-up: end of intervention
Number of follow-ups: 6 waves of follow-up
Follow-up time points: 2 waves in year 1, 2 waves in year 2, 2 waves in year 3
ICC (if reported): not reported

$\begin{array}{ll}\text { Purticipants } & \text { Number of schools randomised: } 48 \text { (24 to intervention, } 24 \text { to control) } \\ & \text { Number of participants randomised (total and by arm): at baseline: } 7846 \text { total; } 4049 \text { to intervention, } \\ & 3797 \text { to control }\end{array}$

Age (range or mean (SD)) or grade at the start: mean 12 years

Gender: 53\% male in intervention arm, 55\% male in control arm

Ethnicity: N/S

SES: N/S

Inclusion criteria: N/S

Exclusion criteria: $\mathrm{N} / \mathrm{S}$

\section{Randomisation before or after baseline survey: before}

Duration of the intervention (excluding follow-up): 3 school years

Description of the intervention: the programme aims to promote positive development among adolescents in junior secondary school in Hong Kong. The programme includes 2 tiers; both are developed in reference to 15 positive youth development constructs such as social competence, resilience, emotional competence, self-efficacy, and self-determination. The programme provides 20 hours of training each school year. The tier 2 programme is provided for students who display greater psychosocial needs at each grade.

Brief description of the theoretical model: not described

Description of the comparator: the programme was delayed for 1 year.

Outcomes Primary outcomes: delinquency; alcohol, tobacco, and drug use

Secondary outcomes: Internet use; behavioural intentions

Setting

Country: Hong Kong; State: N/A

Setting: junior secondary school 
Shek 2011 (Continued)

Focus: adolescents, with a second tier targeting those with greater psychosocial needs

$\begin{array}{ll}\text { Process measures } & \text { Process data reported: no } \\ & \text { Method (qualitative or quantitative): N/A } \\ & \text { Description: N/A }\end{array}$

Statistics

Sample size: 24 schools in intervention arm, 24 schools in control arm. At baseline, 4049 in intervention arm, 3797 in control arm

Unit of randomisation: school

Unit of analysis: individual

Method to promote equivalence between groups: N/S

Statistical models: individual growth curve modelling

Baseline differences adjustment: no baseline differences identified

Repeated measures methods in analysis: yes

Equity: not reported
Funding: $\mathrm{N} / \mathrm{S}$
Randomisation method: not clear
Clustering accounted for in sample size calculation (if relevant): $\mathrm{N} / \mathrm{S}$
Cluster randomisation methods to account for clustering in analysis: $\mathrm{N} / \mathrm{S}$

\section{Risk of bias}

\begin{tabular}{|c|c|c|}
\hline Bias & Authors' judgement & Support for judgement \\
\hline $\begin{array}{l}\text { Random sequence genera- } \\
\text { tion (selection bias) }\end{array}$ & Unclear risk & Insufficient information reported \\
\hline $\begin{array}{l}\text { Allocation concealment } \\
\text { (selection bias) }\end{array}$ & Unclear risk & Insufficient information reported \\
\hline $\begin{array}{l}\text { Blinding of participants } \\
\text { and personnel (perfor- } \\
\text { mance bias) } \\
\text { All outcomes }\end{array}$ & High risk & Blinding not possible for this type of intervention \\
\hline $\begin{array}{l}\text { Blinding of outcome as- } \\
\text { sessment (detection bias) } \\
\text { All outcomes }\end{array}$ & High risk & Self-reported outcomes \\
\hline $\begin{array}{l}\text { Incomplete outcome data } \\
\text { (attrition bias) } \\
\text { All outcomes }\end{array}$ & High risk & Loss of schools only from intervention arm \\
\hline $\begin{array}{l}\text { Selective reporting (re- } \\
\text { porting bias) }\end{array}$ & High risk & Only statistically significant results reported \\
\hline Other bias & Unclear risk & Insufficient information reported \\
\hline
\end{tabular}


Intervention $\operatorname{arm}(\mathbf{s})$ : school-based curriculum intervention

Comparator $\operatorname{arm}(\mathbf{s})$ : standard education

Sample size calculation performed: yes; a priori power analyses undertaken

Subgroups prespecified: none presented

Subgroup analyses: N/A

Start date: August 2008

Duration of follow-up: immediately post intervention

Number of follow-ups: 1

Follow-up time points: 1 immediately post intervention

ICC (if reported): not reported

\section{Participants}

Number of schools randomised: N/A; students from 1 school only were randomised

Number of participants randomised (total and by arm): 108 overall (intervention 53, control 55)

Age (range or mean (SD)) or grade at the start: intervention 14.4, control 13.9

Gender: intervention: 51\% male, 49\% female; control: 33\% male, $67 \%$ female

Ethnicity: intervention: Latino (81\%), African American (8\%), white (4\%), American Indian/Alaskan Native (2\%), Asian/Pacific Islander (0\%); control: Latino (75\%), African American (9\%), white (4\%), American Indian/Alaskan Native (2\%), Asian/Pacific Islander (4\%), other (6\%)

SES: not reported

Inclusion criteria: enrolment of high-risk students only, with high-risk status based on rate of absence $\geq 80 \%$, 2 or more disciplinary actions in grade 8 , failing 2 or more classes in grade 8 , or high levels of family dysfunction (identified by grade teacher, using proxies such as multiple family moves in grade 8 , perceived lack of parental involvement, or family conflict)

Exclusion criteria: $\mathrm{N} / \mathrm{S}$

Description of the intervention: $28 \times$ weekly peer group sessions, each lasting around 45 minutes, focusing on improving student resilience through activities and counselling to build social skills, communication skills, anger management, conflict resolution, and healthy relationships. Student-led discussions in groups of 6 to 9 students also took place, with violence exposure, alcohol, and smoking the topics of discussion. Students also participated in field trips, community service activities after school, during weekends, and throughout the summer.

Brief description of the theoretical model: N/S

Description of the comparator: control group; not stated what was delivered

\section{Secondary outcomes: N/S}


Shetgiri 2011 (Continued)

$\begin{array}{ll}\text { Setting } & \text { Country: USA; State: California } \\ & \text { Setting: school } \\ \text { Focus: targeted }\end{array}$

\begin{tabular}{ll}
\hline Process measures & Process data reported: no \\
Method (qualitative or quantitative): N/A \\
Description: N/A \\
Acceptability of the intervention: N/S \\
Adherence to the intervention: N/A \\
Intensity of the intervention: N/A \\
Sample size: N/S \\
Unit of randomisation: individual \\
Unit of analysis: individual \\
Method to promote equivalence between groups: N/S \\
Statistical models: ANOVA and GEE \\
Baseline differences adjustment: yes \\
Repeated measures methods in analysis: N/A; 1 follow-up only \\
\hline
\end{tabular}

Notes Equity: study baseline data reported

Funding: study supported in part by Robert Wood Johnson Foundation Clinical Scholars Program and the Clinical Research Scholars Program at the University of Texas Southwestern Medical Center

Randomisation method: rolled die and assigned odd numbers to intervention and even numbers to control

Clustering accounted for in sample size calculation (if relevant): N/A

Cluster randomisation methods to account for clustering in analysis: N/A

\section{Risk of bias}

\begin{tabular}{lll}
\hline Bias & Authors' judgement & Support for judgement \\
\hline $\begin{array}{l}\text { Random sequence genera- } \\
\text { tion (selection bias) }\end{array}$ & Low risk & $\begin{array}{l}\text { "Randomly assigned...by rolling a die and assigning odd numbers to the inter- } \\
\text { vention group and even numbers to the control group" }\end{array}$ \\
\hline $\begin{array}{l}\text { Allocation concealment } \\
\text { (selection bias) }\end{array}$ & Low risk & $\begin{array}{l}\text { "Staff members who enrolled participants were unaware of the allocation of } \\
\text { subjects to intervention or control groups". }\end{array}$ \\
\hline $\begin{array}{l}\text { Blinding of participants } \\
\begin{array}{l}\text { and personnel (perfor- } \\
\text { mance bias) } \\
\text { All outcomes }\end{array}\end{array}$ & High risk & $\begin{array}{l}\text { "After allocation, neither subjects nor staff members conducting the interven- } \\
\text { tion were blinded to group assignment". }\end{array}$ \\
\hline $\begin{array}{l}\text { Blinding of outcome as- } \\
\text { sessment (detection bias) } \\
\text { All outcomes }\end{array}$ & High risk & Self-reported outcome measures used \\
\hline
\end{tabular}


Shetgiri 2011 (Continued)

Incomplete outcome data Low risk Attrition did not differ between study arms (and attrition was $<10 \%$ between (attrition bias) arms).

All outcomes

Selective reporting (re- Unclear risk No protocol published
porting bias)

Other bias Unclear risk

Only 1 school took part in the intervention, and randomisation was carried out at the individual (student) level. Risk of contamination was high between students in intervention and control groups at the same school.

Simons-Morton 2005

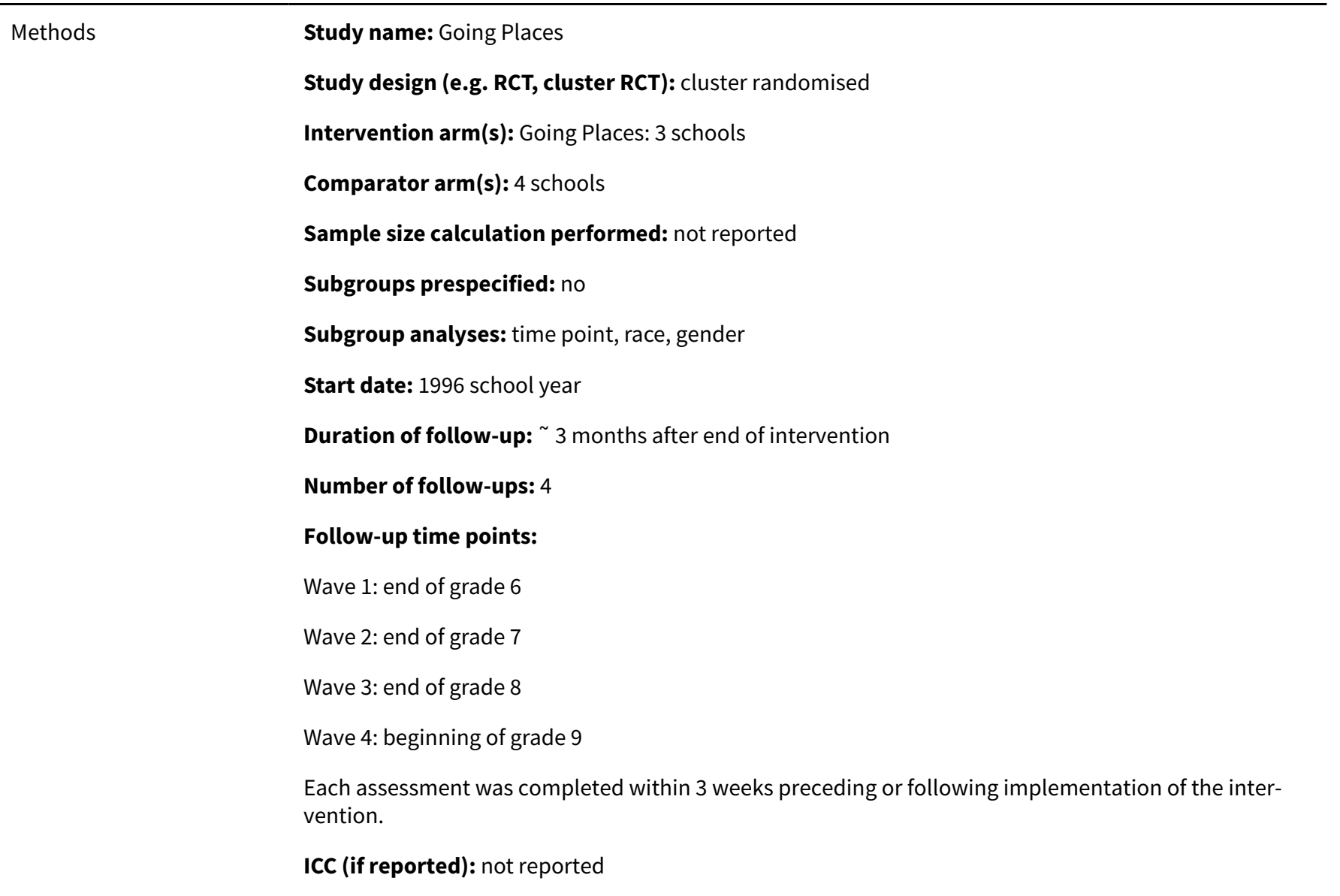

Participants

Number of schools randomised: 7 middle schools

Number of participants randomised (total and by arm): total 2651

Age (range or mean (SD)) or grade at the start: age not reported. Start in grade 6 (middle school), followed to grade 9 (high school)

Gender: male 57\%, female $43 \%$

Ethnicity: subgroups of numbers of black and white participants were reported for smoking stage, drinking stage, and antisocial behaviour at each time point (Table 1, Simons-Morton 2005).

SES: not reported 
Exclusion criteria: students attending special education classes

Duration of the intervention (excluding follow-up): sessions were provided over 3 consecutive school years.

\section{Description of the intervention}

\section{Going Places}

The Going Places programme includes a social skills curriculum, parent education, and school environment enhancement designed to increase academic engagement and commitment to school; alter perceptions, attitudes, and expectations about substance use and antisocial behaviour; and reduce multiple problem behaviours. The social skills curriculum sessions focus on problem-solving, self-control, communication, and conflict resolution skills. Eighteen sessions were delivered in grade 6, 12 in grade 7 , and 6 in grade 8 . Content of sessions typically included a trigger videotape of common problems and problem-solving approaches modelled by actors. Teachers led a brief of the relevant skills, interactive group activity, role-play, and skills practice with constructive feedback.

The school environment enhancement component included social marketing to improve school climate; establish prosocial norms; establish a positive school image; reinforce student achievement; and extend exposure to the concepts of the Going Places curriculum to the wider school environment through the use of information roll-outs, posters, short video segments in social areas, and rewards of "travellers cheques" to students for applying Going Places skills. Rewards also included special activities (e.g. assemblies, year-end field trips).

The parent education component included increasing parental monitoring, involvement, and expectations for academic engagement and problem behaviour. Parents received a 20-minute instructional video on authoritative parenting and a booklet on attentive parenting. Homework was assigned that required parents to be involved.

Brief description of the theoretical model: social development and social cognitive (problem behaviour) theories

Description of the comparator: N/S

Primary outcomes: smoking stage, drinking stage, antisocial behaviour

Secondary outcomes: intermediate outcomes: problem behaving friends; outcome expectancies; social competence; parental expectations

Setting: 7 middle schools in 1 district; 2 successive cohorts of grade 6 students

Focus: universal

The programme addressed sixth graders entering middle school to shape the attitudes and skills thought to motivate them to try hard in school and refrain from problem behaviours.

\section{Process measures Process data reported: yes}

Method (qualitative or quantitative): both

\section{Description}

Adherence to the intervention: Teachers completed $95 \%$ of lessons in grade 6 and $84 \%$ in grade 7 . Teachers completed all core lessons of scheduled observations. Teacher ratings of students fully participating in lessons, including staying on task, were $90 \%$ in grade 6 and $88 \%$ in grade 7 . An average 
Simons-Morton 2005 (Continued)

of $77 \%$ of grade 6 and $65 \%$ of grade 7 students indicated usually or always paying attention to Going Places classes; $70 \%$ considered the programme helpful; $54 \%$ used skills from the programme at school; and $41 \%$ used programme skills outside school. On knowledge tests, $90 \%$ scored $80 \%$ or better. Of 45 parents interviewed, 40 indicated that they or their spouse viewed the video and parenting booklet, of which $80 \%$ reported liking these materials.

Intensity of the intervention: 18 sessions in grade 6,12 in grade 7, and 6 in grade 8

Statistics

Sample size: eligible $\mathrm{N}=2969$; consent obtained for $\mathrm{N}=2651$ in grade 6

Unit of randomisation: schools

Unit of analysis: individuals

Method to promote equivalence between groups: N/S

Statistical models: generic latent growth curve modelling for treatment group effects; the added growth factor represents the effects of treatment

Baseline differences adjustment: ANCOVA to control baseline covariates in outcomes

Repeated measures methods in analysis: growth mixture modelling to identify different trajectories or patterns of response across time that represent groups of individuals with similar behaviour over time. The assumption is made that an individual has a certain trajectory class membership that does not change over time, and that the intervention effects are captured in average slopes for each class. Analysis captures the effects of intervention on changes in the slope of trajectories for each class.

\section{Notes}

Equity: limited data reported

Funding: National Institutes of Health

Randomisation method, e.g. block, stratification, computer: N/S

Clustering accounted for in sample size calculation (if relevant): $\mathrm{N} / \mathrm{S}$

Cluster randomisation methods to account for clustering in analysis: not clear

\section{Risk of bias}

\begin{tabular}{|c|c|c|}
\hline Bias & Authors' judgement & Support for judgement \\
\hline $\begin{array}{l}\text { Random sequence genera- } \\
\text { tion (selection bias) }\end{array}$ & Unclear risk & Not reported \\
\hline $\begin{array}{l}\text { Allocation concealment } \\
\text { (selection bias) }\end{array}$ & Unclear risk & Not reported \\
\hline $\begin{array}{l}\text { Blinding of participants } \\
\text { and personnel (perfor- } \\
\text { mance bias) } \\
\text { All outcomes }\end{array}$ & High risk & Intervention students, parents, and teachers not blinded to the intervention \\
\hline $\begin{array}{l}\text { Blinding of outcome as- } \\
\text { sessment (detection bias) } \\
\text { All outcomes }\end{array}$ & High risk & $\begin{array}{l}\text { Surveys were self-reported by students and were anonymised by using a } \\
\text { unique identifier. }\end{array}$ \\
\hline $\begin{array}{l}\text { Incomplete outcome data } \\
\text { (attrition bias) } \\
\text { All outcomes }\end{array}$ & High risk & $\begin{array}{l}\text { Overall } 50 \% \text { attrition due to lack of consent or incomplete outcomes by grade } \\
9\end{array}$ \\
\hline
\end{tabular}


Simons-Morton 2005 (Continued)
Selective reporting (re-
Unclear risk
No published protocol porting bias)

Other bias

Low risk

No other sources of bias identified

Tierney 1995

Study name: Big Brothers Big Sisters of America (BBBSA)
Study design (e.g. RCT, cluster RCT): randomised controlled trial; participants matched with BBBSA
Intervention $\operatorname{arm}(\mathbf{s})$ : Big Brothers Big Sisters of America Intervention
Comparator arm(s): delayed BBBSA intervention (control group received treatment as usual and had
no active intervention delivered to them)

Sample size calculation performed: not carried out. Agencies had goals on how many youth to recruit based on their individual caseloads

Subgroups prespecified: yes

Subgroup analyses: by gender and by ethnicity

Start date: October 1991 (randomisation first occurred)

Duration of follow-up: 18 months post randomisation

Number of follow-ups: 1

Follow-up time points: 18 months post baseline

ICC (if reported): N/A

Participants

Number of schools randomised: N/A

Number of participants randomised (total and by arm): 1138 youth randomised ( 571 to treatment, 567 to control)

Age (range or mean (SD)) or grade at start: baseline; 10 to 16 years, mean 14.8 years

Gender: boys $=62.4 \%$, girls $=37.6 \%$

Ethnicity: Caucasian $=43.2 \%$, minority ethnic groups $=56.8 \%$

SES: percentage of youth with household income per annum of: $<\$ 10,000(39.7 \%), \$ 10,000$ to $\$ 24,999$ (43\%), $\$ 25,000$ to $\$ 39,999(13.1 \%), \$ 40,000$ to $\$ 54,999(3.3 \%)$, and $\geq \$ 55,000(1 \%)$

Inclusion criteria: youth between 5 and 18 years of age who reside within the agency catchment area, who achieve a minimum level of competency skills, and who agree (along with their parent/guardian) to follow agency rules

Exclusion criteria: inability to complete a telephone interview (usually because of severe physical or learning disabilities), not part of the core BBBSA programme, served under a contractual obligation (i.e. other youth agencies or child protection service agreements)

\section{Interventions \\ Randomisation before or after baseline survey: before \\ Duration of the intervention (excluding follow-up): varies - most mentoring occurred over a period of 12 months or longer \\ Description of the intervention: the BBBSA intervention consists of an agency matching a mentor (termed a Big Brother or a Big Sister, depending on gender) with an adolescent 10 to 16 years of age}


who comes from a single-parent household. The mentor and the adolescent meet around 4 times per month in the first 12 months, engaging in a wide range of activities.

Brief description of the theoretical model: not described

Description of the comparator: no intervention, but on waiting list for Big Brothers Big Sisters

List relevant outcomes
Primary outcomes: N/S, but the following presumed: antisocial activities (including alcohol and drug
use), academic performance, attitudes and behaviours, relationships with family, relationships with
friends, self-concept, and social and cultural enrichment

Secondary outcomes: N/S and N/A

Setting Country: USA;Place: the 8 BBBSA agencies were in the Northeast (Philadelphia and Rochester), the Midwest (Minneapolis, Columbus, and Wichita), the South (Houston and San Antonio), and the Southwest (Phoenix).

Setting: varies; mentoring meetings can take place at various locations within a community. Not explicitly stated

Focus: targeted; youth from single-parent households recruited for mentoring

Process data reported: yes - partially. Parents were asked to evaluate the performance of the volunteer, their satisfaction with the BBBSA agency, and whether they thought the programme had made a difference in their child's life. Interviewers asked treatment youth about their relationship with their Big Brother or Big Sister.

\section{Method (qualitative or quantitative): not clear}

\section{Description: not clear}

Adherence to the intervention: The frequency of youth/mentor meetings was recorded as follows: 2 per week (4.5\%), 1 per week (41.7\%), 3 per month (24.4\%), 2 per month (24.2\%), 1 per month (5.3\%)

Sample size: 1138 youth underwent random assignment.

Unit of randomisation: individual

Unit of analysis: individual

\section{Method to promote equivalence between groups: $\mathrm{N} / \mathrm{S}$}

Statistical models: multi-variate analysis

Baseline differences adjustment: N/A, as no notable differences were evident

Repeated measures methods in analysis: N/A; only baseline and 18-month follow-up reported

Equity: parameters reported at baseline

Funding: Lilly Endowment, Inc., The Commonwealth Fund, The Pew Charitable Trusts, and an anonymous donor

Randomisation method, e.g. block, stratification, computer: N/S

Clustering accounted for in sample size calculation (if relevant): not relevant

Cluster randomisation methods to account for clustering in analysis: $\mathrm{N} / \mathrm{A}$

\section{Risk of bias}


Tierney 1995 (Continued)

\begin{tabular}{lll} 
Bias & Authors' judgement & Support for judgement \\
\hline $\begin{array}{l}\text { Random sequence genera- } \\
\text { tion (selection bias) }\end{array}$ & Unclear risk & No information regarding how the random sequence was generated \\
\hline $\begin{array}{l}\text { Allocation concealment } \\
\text { (selection bias) }\end{array}$ & Unclear risk & $\begin{array}{l}\text { 'Survey subcontractor' carried out random assignment; no description of who } \\
\text { these individuals were }\end{array}$ \\
\hline
\end{tabular}

\begin{tabular}{ll}
\hline $\begin{array}{l}\text { Blinding of participants } \\
\text { and personnel (perfor- }\end{array}$ & High risk \\
Impossible to do owing to the nature of the intervention. Those on the wait-list \\
receive intervention 18 months later than those in the treatment group.
\end{tabular}
mance bias)

All outcomes

\begin{tabular}{lll}
\hline $\begin{array}{l}\text { Blinding of outcome as- } \\
\text { sessment (detection bias) } \\
\text { All outcomes }\end{array}$ & High risk & Self-reported outcomes \\
\hline $\begin{array}{l}\text { Incomplete outcome data } \\
\begin{array}{l}\text { (attrition bias) } \\
\text { All outcomes }\end{array}\end{array}$ & Low risk & Attrition rates moderate and acceptable \\
\hline $\begin{array}{l}\text { Selective reporting (re- } \\
\text { porting bias) }\end{array}$ & Unclear risk & No published protocol; primary outcomes not stated \\
\hline Other bias & Low risk & No other forms of bias identified \\
\hline
\end{tabular}

Wagner 2014

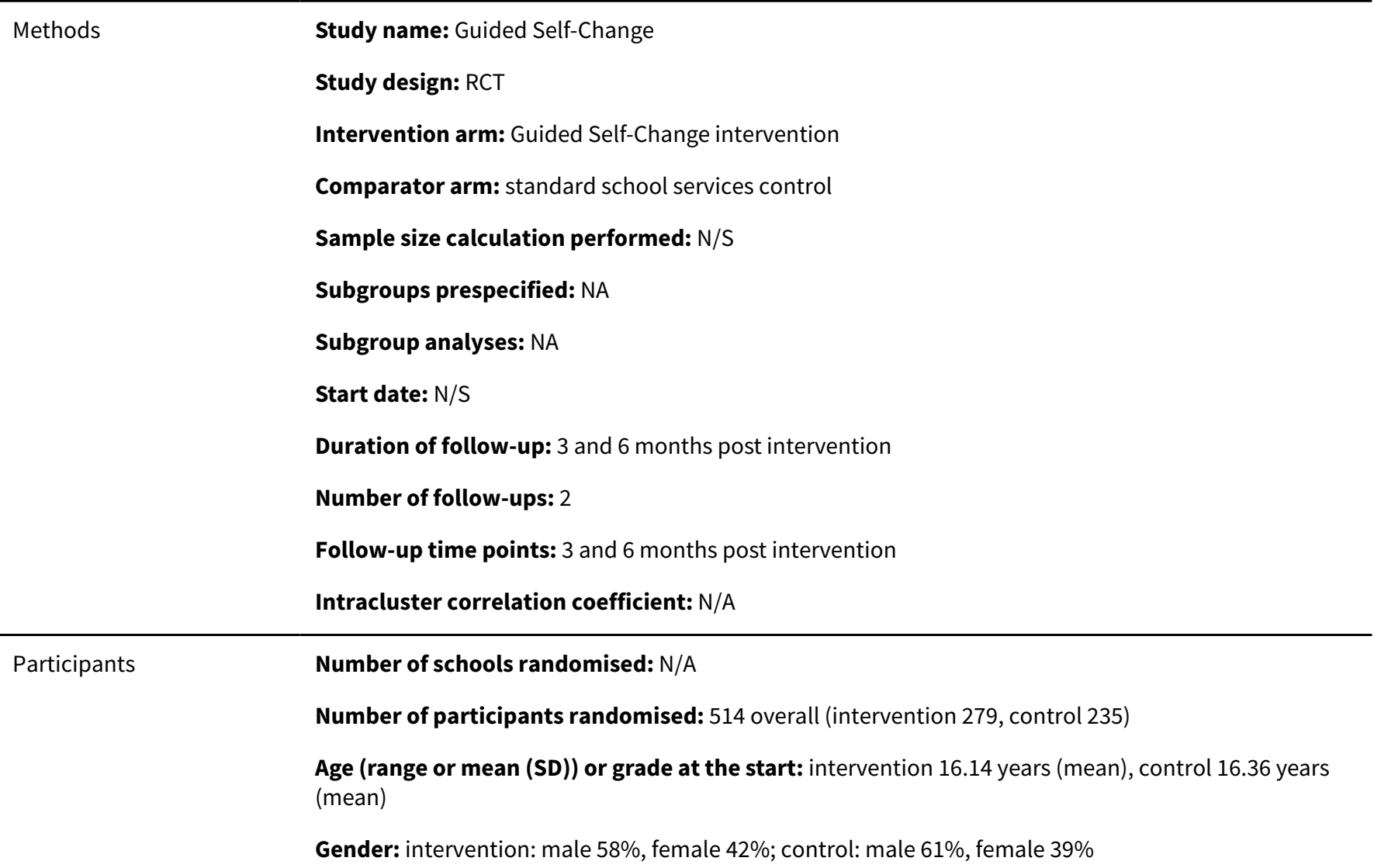


Ethnicity: intervention: Hispanic 56\%, African American 21\%, non-Hispanic white 9\%, other 15\%; control: Hispanic 58\%, African American 25\%, non-Hispanic white 3\%, other $13 \%$

\section{Socioeconomic status: N/S}

Inclusion criteria: 14 to 18 years of age, at least 6 occasions of alcohol or other drug use in past 90 days, at least 1 act of relational or predatory violence in past 90 days

Exclusion criteria: repeated dangerous behaviour such as drinking while driving; current suicidal risk; significant health problems related to drinking (e.g. withdrawal symptoms, significant history of blackouts); pregnancy in females; cognitive impairments or developmental delays, as indicated by school evaluations and educational placement

Duration of the intervention: not clearly stated; intervention lasted for 5 weeks, with an additional 2 weeks for $25 \%$ of participants within the intervention arm

Description of the intervention $\operatorname{arm}(\mathbf{s})$ : one-on-one 5-weekly sessions of "Guided Self-Change" delivered at schools, based on brief motivational interventions and cognitive-behavioural therapies. Major treatment components consisted of weekly self-monitoring of behaviours targeted for change, treatment goal advice, homework assignments exploring high-risk situations and actions, motivational strategies to increase commitment to change, and cognitive relapse prevention procedures.

\section{Brief description of the theoretical model: Guided Self-Change}

Description of the comparator arm(s): school counsellors were available to provide brief alcohol/drug/violence assessments and referral to outside treatment providers. Thus, standard care consisted of education/brief assessment/referral only, which is the standard of care in schools without a formal substance abuse or violence early intervention programme

\begin{tabular}{ll}
\hline Outcomes & Primary outcomes: alcohol use, drug use, aggressive behaviour \\
& $\begin{array}{l}\text { Secondary outcomes: gender and race/ethnicity as moderators of treatment effect, participants' con- } \\
\text { fidence about and importance of changing }\end{array}$ \\
\hline Setting & Country: USA; Place: Miami \\
& Setting: school \\
& Focus: targeted
\end{tabular}

Process measures Process data reported: yes; not a process evaluation

Method: qualitative and quantitative

Description: all intervention sessions recorded; a random $10 \%$ were reviewed to assess adherence to the manual. Mean adherence 4.25 (SD 0.59) out of 5

Unit of randomisation: individual

Unit of analyses: individual

\section{Methods to promote equivalence between groups: N/S}

Statistical models: structural equation modelling

Baseline differences adjustment: N/S

Repeated measures methods in analysis: N/S 
Funding: supported by Grant R01AA013369 from the National Institute on Alcohol Abuse and Alcoholism

Randomisation method: computer programme

Clustering accounted for in sample size calculation: N/A

Cluster randomisation methods to account for clustering in analysis: N/A

\begin{tabular}{|c|c|c|}
\hline \multicolumn{3}{|l|}{ Risk of bias } \\
\hline Bias & Authors' judgement & Support for judgement \\
\hline $\begin{array}{l}\text { Random sequence genera- } \\
\text { tion (selection bias) }\end{array}$ & Low risk & $\begin{array}{l}\text { "Using a random number generator (http://www.random.org/), qualifying par- } \\
\text { ticipants were assigned to receive intervention (odd number) or standard care } \\
\text { (even number)". }\end{array}$ \\
\hline $\begin{array}{l}\text { Allocation concealment } \\
\text { (selection bias) }\end{array}$ & Unclear risk & Insufficient information provided \\
\hline $\begin{array}{l}\text { Blinding of participants } \\
\text { and personnel (perfor- } \\
\text { mance bias) } \\
\text { All outcomes }\end{array}$ & High risk & Participants not blinded, given the nature of this intervention \\
\hline $\begin{array}{l}\text { Blinding of outcome as- } \\
\text { sessment (detection bias) } \\
\text { All outcomes }\end{array}$ & High risk & Self-reported outcome measures only \\
\hline $\begin{array}{l}\text { Incomplete outcome data } \\
\text { (attrition bias) } \\
\text { All outcomes }\end{array}$ & Low risk & $\begin{array}{l}\text { Missing data imputed via the full information maximum likelihood (FIML) } \\
\text { method }\end{array}$ \\
\hline $\begin{array}{l}\text { Selective reporting (re- } \\
\text { porting bias) }\end{array}$ & Unclear risk & Protocol not available \\
\hline Other bias & Unclear risk & $\begin{array}{l}\text { Possibility of contamination due to inclusion of participants in the same } \\
\text { schools }\end{array}$ \\
\hline
\end{tabular}

Walker 2002

Study design (e.g. RCT, cluster RCT): randomised controlled trial

Intervention arm(s): 20-minute primary care surgery brief consultation with practice nurse

Comparator arm(s): standard care (health promotion leaflets sent to homes via mail)

Sample size calculation performed: N/S

Subgroups prespecified: none

Subgroup analyses: N/A

Start date: 1 January 1999

Duration of follow-up: 12 months post intervention

Number of follow-ups: 2 
Walker 2002 (Continued)

Follow-up time points: 3 and 12 months post intervention

ICC (if reported): no

Participants

Number of schools randomised: N/A; teenagers targeted outside of school setting

Number of participants randomised (total and by arm): 1488 total; 746 to intervention, 742 to control

Age (range or mean (SD)) or grade at the start: 14.8 years mean age of study sample

Gender: 478 of participants (49\%) were male

Ethnicity: 868 (89\%) of participants were white/Caucasian

SES: 466 (48\%) of participants' parents were in a professional, managerial, or technical occupation

Inclusion criteria: teenagers 14 to 15 years of age, living in Hertfordshire, and on a general practice register

Exclusion criteria: N/S

Interventions

Randomisation before or after baseline survey: before

Duration of the intervention (excluding follow-up): 20 minutes (brief advice)

Description of the intervention: the intervention consisted of a 20-minute discussion with a practice nurse (in a total of $8 \mathrm{GP}$ practices throughout the UK county of Hertfordshire), during which participants discussed health concerns and made plans to live a healthier lifestyle.

Brief description of the theoretical model: intervention has theoretical underpinnings from self-efficacy theory. The intervention also adhered to American Medical Association guidelines on developing brief interventions for adolescents.

Description of the comparator: the control group were sent health promotion leaflets to their homes.

Outcomes Primary outcomes: study authors state 'mental and physical health' and 'stages of change' (measured tobacco, alcohol, nutrition, physical activity)

Secondary outcomes: N/S

Setting Country: United Kingdom;Place: Hertfordshire

Setting: general practice surgeries

Focus: universal

Process measures

Process data reported: yes

Method (qualitative or quantitative): quantitative

Description

Acceptability of the intervention: $97 \%$ of consultation attendees said they would recommend the intervention to a friend; most said they could talk about what they wanted to talk about; all but 1 individual were satisfied or fairly satisfied with the consultation.

Adherence to the intervention: nurses were observed to assess adherence to protocol. This was followed in most cases.

Statistics

Sample size: 1516 individuals invited, 1488 randomised to 2 intervention arms (98.2\%)

Unit of randomisation: individual 
Walker 2002 (Continued)

Unit of analysis: individual

Method to promote equivalence between groups: stratification according to gender

Statistical models: none (aside from $\mathrm{Chi}^{2}$ analysis)

Baseline differences adjustment: N/A

Repeated measures methods in analysis: N/S

Notes

Equity: baseline data regarding health behaviours are provided.

Funding: NHS Executive - Eastern Region, and Hertfordshire Primary Care Research Network (HertNet)

Randomisation method, e.g. block, stratification, computer: N/S

Clustering accounted for in sample size calculation (if relevant): $\mathrm{N} / \mathrm{S}$

Cluster randomisation methods to account for clustering in analysis: N/A

\section{Risk of bias}

\begin{tabular}{|c|c|c|}
\hline Bias & Authors' judgement & Support for judgement \\
\hline $\begin{array}{l}\text { Random sequence genera- } \\
\text { tion (selection bias) }\end{array}$ & Unclear risk & The randomisation process was not stated. \\
\hline $\begin{array}{l}\text { Allocation concealment } \\
\text { (selection bias) }\end{array}$ & Unclear risk & Details regarding allocation concealment were not stated. \\
\hline $\begin{array}{l}\text { Blinding of participants } \\
\text { and personnel (perfor- } \\
\text { mance bias) } \\
\text { All outcomes }\end{array}$ & High risk & Participants were not blinded and outcomes were provided via self-report. \\
\hline $\begin{array}{l}\text { Blinding of outcome as- } \\
\text { sessment (detection bias) } \\
\text { All outcomes }\end{array}$ & High risk & $\begin{array}{l}\text { All outcomes were provided via self-report. Salivary cotinine was used to con- } \\
\text { firm self-reported smoking status, although study authors did not state the de- } \\
\text { gree of agreement between cotinine levels and subjective self-reported smok- } \\
\text { ing status. }\end{array}$ \\
\hline $\begin{array}{l}\text { Incomplete outcome data } \\
\text { (attrition bias) } \\
\text { All outcomes }\end{array}$ & High risk & $\begin{array}{l}\text { High rates of attrition from enrolment; over } 30 \% \text { in both intervention and con- } \\
\text { trol arms }\end{array}$ \\
\hline $\begin{array}{l}\text { Selective reporting (re- } \\
\text { porting bias) }\end{array}$ & High risk & $\begin{array}{l}\text { No published protocol; not all primary outcomes reported; outcomes from } \\
\text { both 3- and 12-month follow-up not clearly reported }\end{array}$ \\
\hline Other bias & Low risk & No other forms of bias identified \\
\hline
\end{tabular}

Walter 1989

Methods

Study name: Know Your Body

Study design (e.g. RCT, cluster RCT): cluster RCT at the level of the school; 5-year longitudinal study in 22 elementary schools

Intervention arm(s): Know Your Body curriculum: Bronx: 14 schools, $\mathrm{n}=1590$; Westchester: 8 schools, $\mathrm{n}=485$ 
Sample size calculation performed: not reported

Subgroups prespecified: none, but participants were from different school regions

Subgroup analyses: Westchester vs Bronx (by region)

Start date: 1979 in Weschester County; 1980 in the Bronx

Duration of follow-up: followed annually for 5 or 6 years post baseline; intervention finished at end of final year

\section{Number of follow-ups: 2}

Follow-up time points: following 5 and 6 years of intervention

ICC (if reported): not reported

\section{Participants}

Number of schools randomised: 22

Number of participants randomised (total and by arm): intervention: Bronx: 14 schools, $n=1590$; Westchester: 8 schools, $n=485$. Control: Bronx: 8 schools, $n=693$; Westchester: 7 schools, $n=620$

Age (range or mean (SD)) or grade at the start: Bronx: mean age 9.1 years; Westchester: mean age 8.9 years. Start at fourth grade

Gender: Bronx: 51.4\% male; Westchester: 51.5\% male

Ethnicity: Bronx: $48.9 \%$ black, $24.5 \%$ white, $23.2 \%$ Hispanic, 3.3\% other; Westchester: $13.8 \%$ black, $39.3 \%$ white, $2.2 \%$ Hispanic, $4.7 \%$ other

\section{SES: N/S}

Inclusion criteria: grade 4 students in elementary schools in the Bronx district of NYC and Westchester County; other specific inclusion criteria not provided

Exclusion criteria: not reported

Duration of the intervention (excluding follow-up): delivered from grades 4 to 8: 5 years for Bronx schools and 6 years for Westchester schools

\section{Description of the intervention}

\section{Know Your Body (KYB)}

From grades 4 to 9 (Weschester) and in grade 8 (Bronx), intervention groups were taught the KYB curriculum. This programme focuses on nutrition, physical fitness, and cigarette smoking prevention. The curriculum is taught by the classroom teacher for approximately 2 hours/week over the school year. Parents of intervention group children received KYB parent education. Consenting students from intervention and control groups took part in written and risk factor evaluations. Children in the intervention groups received risk factor results in the classroom setting. Those in the control group and parents of both groups received risk factor results by post along with explanatory information and referral to the medical system if considered to be high risk.

Brief description of the theoretical model: the PRECEDE health education planning model contains elements of social learning theory and the health belief model.

Description of the comparator: standard curriculum. Results of risk factor tests are sent home, along with a referral for an individual if he or she was deemed to be at high risk. 
Walter 1989 (Continued)

Primary outcomes: smoking cessation (serum thiocyanate); physical activity: ponderosity index; responsivity index (from Harvard Step Test); cardiovascular risk factors: triceps skinfold thickness, blood pressure, plasma cholesterol

Secondary outcomes: attitudes towards diet, physical activity, and smoking; knowledge related to prevention of coronary heart disease and cancer

Country: USA; State New York City;Place The Bronx and Westchester County
Setting: 2 cohorts of elementary school children; 22 schools in the Bronx, and 15 in Westchester Coun-
ty
$\begin{aligned} & \text { Focus: universal: teacher delivered classroom curriculum, parent education, and periodic examination } \\ & \text { of student risk factor levels }\end{aligned}$

Process measures

Process data reported: yes

Method (qualitative or quantitative): quantitative

\section{Description}

Adherence to the intervention: adherence to teaching protocols was measured by teacher monitoring, including attendance at training workshops and number of lessons taught. Research staff periodically visited classrooms. The ability of teachers to convey the message varied widely. Teacher training time was deemed insufficient.

Intensity of the intervention: KYB curriculum was taught for 2 hours/week over the school year, for 5 or 6 years.

Statistics

Sample size: intervention: Bronx: 14 schools, $n=1590$; Westchester: 8 schools, $n=485$; control: Bronx: 8 schools, $n=693$; Westchester: 7 schools, $n=620$

Unit of randomisation: school

Unit of analysis: school

Method to promote equivalence between groups: assigned by district

Statistical models: average changes in outcome variables were compared between schools in intervention and control groups over the course of the study, in Westchester and the Bronx separately.

Baseline differences adjustment: not reported

Repeated measures methods in analysis: no

\begin{tabular}{ll}
\hline Notes & Equity: baseline only \\
& $\begin{array}{l}\text { Funding: National Heart, Lung and Blood and National Cancer Institute } \\
\text { Randomisation method, e.g. block, stratification, computer: not reported } \\
\text { Clustering accounted for in sample size calculation (if relevant): not reported, but results were } \\
\text { analysed at the school level } \\
\text { Cluster randomisation methods to account for clustering in analysis: not clear }\end{array}$ \\
\hline Risk of bias & Authors' judgement Support for judgement \\
\hline Bias & Unclear risk Not reported \\
\hline $\begin{array}{l}\text { Random sequence genera- } \\
\text { tion (selection bias) }\end{array}$ & \\
\hline
\end{tabular}


Walter 1989 (Continued)

Allocation concealment $\quad$ Unclear risk $\quad$ Not reported
(selection bias)

Blinding of participants

High risk

School randomisation, so individual students were unblinded to the intervenand personnel (perfortion arm mance bias)

All outcomes

\begin{tabular}{ll}
\hline Blinding of outcome as- & High risk \\
sessment (detection bias) &
\end{tabular}

Alloutcomes

All outcomes

Both subjective and objective reports were used, including self-reported measures. Trained professionals took physiological measurements, but it is unclear if they were blinded.

Incomplete outcome data High risk

(attrition bias)

Mean values for risk factors and knowledge scores did not differ at baseline

All outcomes between cohorts and those lost to follow-up. However, no details were given and report does not specify sociodemographic variables or values between groups/districts. There was also a higher rate of unexplained attrition in the Bronx schools.

\begin{tabular}{lll}
\hline $\begin{array}{l}\text { Selective reporting (re- } \\
\text { porting bias) }\end{array}$ & Unclear risk & No published protocol \\
\hline Other bias & Low risk & No other sources of bias identified \\
\hline
\end{tabular}

Wolfe 2012

Methods

Study name: Fourth R: Skills for Youth Relationships

Study design: cluster RCT at the level of the school

Intervention arm: Fouth $\mathrm{R}$, a school-based programme to prevent adolescent dating violence

Comparator arm: control group: schools targeted similar objectives without training or materials

Sample size calculation performed: N/S

Subgroups prespecified: described in methods section

Subgroup analyses: physical dating violence data presented by gender and for all students and for those who have dated in the past 12 months

Start date: September 2004

Duration of follow-up: 2 years

Number of follow-ups: 1

Follow-up time points: 2 years following intervention (2.5 years from baseline)

Intracluster correlation coefficient: physical dating violence 0.02 , physical peer violence 0.01 , problem substance use 0.03 , condom use if sexually active 0.01

\section{Participants}

\section{Number of schools randomised: 20}

Number of participants randomised: 10 schools per study arm; 968 in intervention, 754 in control

Age (range or mean (SD)) or grade at the start: 14 to 15 years

Gender: $52.8 \%$ girls; intervention $51 \%$, control $55 \%$

Ethnicity: participants were predominantly white. 
Wolfe 2012 (Continued)

Socioeconomic status: 1 or both parents employed: intervention $86 \%$, control $85 \%$. Parental education: college diploma or university degree: intervention $55 \%$, control $58 \%$

Inclusion criteria: eligible schools had general student populations. All students within these schools enrolled in the required grade 9 health and physical education curriculum were eligible.

Exclusion criteria: schools participating in the development phase of the programme (2001-2003)

Interventions
Duration of the intervention: 8 months
Description of the intervention arm(s)
The programme was taught in place of the existing health curriculum. It addressed dating violence in
the context of overlapping areas of risk - sexual activity, substance use, and peer violence. It was de-
signed to integrate evidence-based strategies to address these issues. A 21-lesson manualised cur-
riculum was delivered by teachers with specialisation in health and physical education. It consists of
3units, each with seven 75-minute classes on (1) personal safety and injury prevention, (2) healthy
growth and sexuality, and (3) substance use and abuse. These units contained an underlying theme
of healthy, non-violent relationship skills. Detailed lesson plans, video resources, role-play exercises,
rubrics, and handouts were provided for all lessons. Extensive skill development was based on gradu-
ated practice with peers aimed at the development of positive strategies for dealing with pressures and
resolution of conflict without abuse or violence. The curriculum included examples of conflicts faced by
teens, with peer and dating examples used concurrently to increase relevance for youth who were not
dating.

\section{Brief description of the theoretical model: N/S}

Description of the comparator arm(s): usual grade 9 health and physical education curriculum - 21 lessons delivered by teachers covering the same 3 units as the intervention schools, but with no training or background on these topics and no access to a structured curriculum emphasising relationship skills for preventing violence and risk behaviours

Outcomes Primary outcomes: physical dating violence

Secondary outcomes: reductions in peer violence, substance use, unsafe sex

Setting Country: Canada; Place: Ontario

Setting: secondary school

Focus: universal

\section{Process measures Process data reported: yes}

Method: quantitative

Description: $89 \%$ of the intervention lessons were completed according to teacher checklists (89\%, $88 \%$, and $90 \%$ for units 1,2 , and 3 , respectively). The programme included 21 lessons, each of which lasted 75 minutes.

Statistics

Sample size: 2243 invited, 1722 recruited (76.8\%). 1166 potentially eligible students, of which 968 consented to be in the intervention group; 1077 potentially eligible students, of which 754 were included in the control group

Unit of randomisation: school

Unit of analyses: individual, accounting for clustering

Methods to promote equivalence between groups: stratified by size ( $\geq$ or $<500$ students) and location (rural vs urban) 
Wolfe 2012 (Continued)

Statistical models: 2-level hierarchical models for categorical data; random-effects Bernoulli models with school as a random effect

Baseline differences adjustment: yes

Repeated measures methods in analysis: N/A

Notes

Equity: baseline characteristics reported, including risk behaviour data and school characteristics

Funding: this work was supported solely by grant MCT-66913 from the Canadian Institutes of Health Research. The RBC Financial Group is recognised for its support of the Chair in Children's Mental Health (Dr Wolfe), and the Royal Lepage Shelter Foundation is recognised for its support in developing the programme.

Randomisation method: coin toss

Clustering accounted for in sample size calculation: N/A

Cluster randomisation methods to account for clustering in analysis: yes; hierarchical models, random-effects Bernoulli models

\begin{tabular}{|c|c|c|}
\hline \multicolumn{3}{|l|}{ Risk of bias } \\
\hline Bias & Authors' judgement & Support for judgement \\
\hline $\begin{array}{l}\text { Random sequence genera- } \\
\text { tion (selection bias) }\end{array}$ & Low risk & Randomisation by coin toss \\
\hline $\begin{array}{l}\text { Allocation concealment } \\
\text { (selection bias) }\end{array}$ & Unclear risk & No information given \\
\hline $\begin{array}{l}\text { Blinding of participants } \\
\text { and personnel (perfor- } \\
\text { mance bias) } \\
\text { All outcomes }\end{array}$ & Unclear risk & $\begin{array}{l}\text { Students were blinded, but teachers were not; impact of lack of blinding of } \\
\text { teachers is unclear. }\end{array}$ \\
\hline $\begin{array}{l}\text { Blinding of outcome as- } \\
\text { sessment (detection bias) } \\
\text { All outcomes }\end{array}$ & Unclear risk & $\begin{array}{l}\text { Self-reported measures; although students were blinded, study personnel } \\
\text { were not, so blinding could have been broken, which might have affected out- } \\
\text { come assessment }\end{array}$ \\
\hline $\begin{array}{l}\text { Incomplete outcome data } \\
\text { (attrition bias) } \\
\text { All outcomes }\end{array}$ & Low risk & $\begin{array}{l}\text { Low attrition overall, at } 12 \% \text {. Sensitivity analyses were conducted to deter- } \\
\text { mine the robustness of findings relative to missing data. }\end{array}$ \\
\hline $\begin{array}{l}\text { Selective reporting (re- } \\
\text { porting bias) }\end{array}$ & Low risk & No protocol, but all expected outcomes reported \\
\hline Other bias & Low risk & No other sources of bias identified \\
\hline
\end{tabular}

AA: African American.

AC: assessment control.

ANCOVA: analysis of covariance.

ANOVA: analysis of variance.

ASE: Attitude - Social influence - Self-efficacy model.

ASP: after-school programme.

ATD: alcohol, tobacco, and drug.

ATOD: alcohol, tobacco and other drugs.

$\mathrm{AV}$ : anti-violence.

BBBSA: Big Brothers Big Sisters of America.

$B M I$ : body mass index. 
BRAVE: Building Resiliency And Vocational Excellence.

CACE: Complier Average Causal Effect.

$\mathrm{CBI}$ : computer-based intervention.

CC: classroom-centred.

CfC: Coaching for Community.

$\mathrm{Cl}$ : child intervention.

CIDI: Composite International Diagnostic Interview.

CJCP: Centre for Justice and Crime Prevention.

CMST: Coping With the Middle School Transition.

COPE: Creating Opportunities for Personal Empowerment.

$\mathrm{CP}$ : coping power.

CPC: Community Practice Condition.

CPI: child plus parent intervention.

CRCT: cluster randomised controlled trial.

CS: community service.

DARE-A: Drug Abuse and Resistance Education-A.

DUI: driving after drinking.

EA: European American.

FCU: Family Check-Up.

FIML: full-information maximum-likelihood.

FNP: Family Nurse Partnership.

FoF: Focus on Families.

FRC: family resource centre.

FSP: Family Schools Partnership.

GBG: Good Behaviour Game.

GED: graduation equivalency diploma.

GEE: generalised estimating equation.

GLM: general linear model.

GP: general practitioner.

GPA: grade point average.

HDL: high-density lipoprotein.

HRDC: Human Resources Department of Canada.

HEC: Health Enhancement Curriculum.

HFL: Healthy for Life.

ICC: intracluster correlation coefficient.

imPACT: Informed Parents and Children Together.

IPV: intimate partner violence.

IRR: incidence rate ratio.

ISAT: Illinois State Assessment Test.

ISEI: International Socio-Economic Index.

KYB: Know Your Body.

LIFT: Linking the Interests of Families and Teachers.

LST: Life Skills Training.

MAC: minimal assessment control.

MANCOVA: multi-variate analysis of covariance.

MANOVA: multi-variate analysis of variance.

MI: motivational intervention.

MID: multiple imputation then deletion.

MI-H: motivational intervention with exposure to hospital trauma centre.

N/A: not applicable.

NHPA: National Health Promotion Associates.

N/S: not stated.

OR: odds ratio.

PA: parent and adolescent group administered programme.

PATHS: Promoting Alternative Thinking Strategies.

PCL-C: Post-traumatic stress disorder checklist civilian version.

PE: parent educator.

ProSAAF: Protecting Stronger African American Families program.

PWC: Parents Who Care.

RAP: Reaching Adolescents for Prevention.

RCT: randomised controlled trial.

$\mathrm{RDD}$ : riding with a drunk driver.

Individual-, family-, and school-level interventions targeting multiple risk behaviours in young people (Review) 
RSTP: Risk Skills Training Program.

RY: Reconnecting Youth.

SA: self-administered with weekly telephone support.

SAAF: Stronger African American Families.

SAAF-T: Stronger African American Families-Teen.

SafERteens: brief intervention aimed at reducing and preventing violence and alcohol use.

SC: standard care.

$\mathrm{SCl}$ : social/community intervention.

SD: standard deviation.

SDC: Social Development Curriculum.

SDQ: Strengths and Difficulties Questionnaire.

SE: standard error.

SES: socioeconomic status.

SGH: St Gabriel's Hall.

SHS: school health service.

SOFIT: System for Observing Fitness Instruction Time.

$\mathrm{SRH}$ : Sexual and reproductive health.

SSP: Self-Sufficiency Project.

TBI: therapist-based intervention.

TTM: transtheoretical model.

VC: values clarification.

YDP: Youth Development Project.

Characteristics of excluded studies [ordered by study ID]

\begin{tabular}{|c|c|}
\hline Study & Reason for exclusion \\
\hline Aboutanos 2011 & Not randomised \\
\hline Allahverdipour 2009 & Not randomised \\
\hline Arbuthnot 1986 & $\begin{array}{l}\text { Fewer than } 2 \text { outcomes regarding engagement in risk behaviours (only antisocial behaviour and of- } \\
\text { fending measured) }\end{array}$ \\
\hline Baker 2004 & Dissertation; no response from study authors re: associated published journals \\
\hline Balaji 2011 & Community intervention \\
\hline Balvig 2011 & Tobacco, alcohol, and drug outcomes only \\
\hline Bannink 2014 & Less than 6 months combined intervention and follow-up period \\
\hline Barlow 2013a & $\begin{array}{l}\text { Fewer than } 2 \text { outcomes regarding engagement in risk behaviours (only socioemotional adjustment } \\
\text { measured) }\end{array}$ \\
\hline Barlow 2013b & $\begin{array}{l}\text { Fewer than } 2 \text { outcomes regarding engagement in risk behaviours (only externalising and internalis- } \\
\text { ing disorders measured) }\end{array}$ \\
\hline Barrington 2008 & Dissertation; no response from study authors re: associated published journals \\
\hline Battistich 1996 & Not randomised \\
\hline Bell 2005 & Not randomised \\
\hline Bennett 1995 & Dissertation; no response from study authors re: associated published journals \\
\hline Biggam 2002 & Outside of required age range (aged 16 to 21 years, mean $>18.5$ years) \\
\hline
\end{tabular}




\begin{tabular}{|c|c|}
\hline Study & Reason for exclusion \\
\hline Bock 2016 & $\begin{array}{l}\text { Outside of required age range (aged } 16 \text { to } 21 \text { years, mean }>18.5 \text { years); less than } 6 \text { months com- } \\
\text { bined intervention and follow-up period }\end{array}$ \\
\hline Bonell 2010 & Not randomised \\
\hline Botvin 1994 & Not randomised \\
\hline Botvin 1997 & Not randomised \\
\hline Botvin 2006 & Less than 6 months combined intervention and follow-up period \\
\hline Bradley 2009 & Less than 6 months combined intervention and follow-up period \\
\hline Brody 2004 & Measures only participant attitudes and intentions - not actual engagement in risk behaviours \\
\hline Bryan 2009 & $\begin{array}{l}\text { Fewer than } 2 \text { outcomes regarding engagement in risk behaviours (only risky sexual behaviour out- } \\
\text { comes measured) }\end{array}$ \\
\hline Bryan 2010 & Dissertation; study author contacted for associated papers - Bryan 2009 retrieved \\
\hline Burke 2010 & Fewer than 2 outcomes regarding engagement in risk behaviours (none of interest measured) \\
\hline Cameron 2015 & Outside of required age range (aged 16 to 21 years, mean $>18.5$ years) \\
\hline Campbell-Heider 2009 & Not randomised \\
\hline Castro 2013 & Outside of required age range (recruited participants aged 18 years and older) \\
\hline Clair-Michaud 2016 & Fewer than 2 outcomes regarding engagement in risk behaviours (only delinquent behaviours) \\
\hline Clinton-Sherrod 2011 & Less than 6 months combined intervention and follow-up period \\
\hline Colnes 2001 & Tobacco, alcohol, and drug outcomes only \\
\hline Copeland 2001 & Dissertation; no response from study authors re: associated published journals \\
\hline Copeland 2010 & Not randomised \\
\hline Crooks 2015 & Measures only participant attitudes and intentions - not actual engagement in risk behaviours \\
\hline Croom 2015 & Fewer than 2 outcomes regarding engagement in risk behaviours (only alcohol consumption) \\
\hline Cuijpers 2002 & Not randomised \\
\hline Cunningham 2015 & Outside of required age range (aged 16 to 21 years, mean $>18.5$ years) \\
\hline Cupp 2013 & $\begin{array}{l}\text { Fewer than } 2 \text { outcomes regarding engagement in risk behaviours (only sexual risk communication } \\
\text { measured) }\end{array}$ \\
\hline D'Amico 2008 & Less than 6 months combined intervention and follow-up period \\
\hline Dakof 2015 & No control group \\
\hline Danielson 2012 & Clinical intervention (all participants were previously victims of sexual abuse) \\
\hline
\end{tabular}




\begin{tabular}{|c|c|}
\hline Study & Reason for exclusion \\
\hline Dembo 1996 & Less than 6 months combined intervention and follow-up period \\
\hline Dembo 1998 & Not randomised \\
\hline Dembo 1999 & Not randomised \\
\hline Dembo 2000 & Not randomised \\
\hline Dembo 2000a & Not randomised \\
\hline Dermen 2011 & Outside of required age range (mean age 20.7 years) \\
\hline Devine 1995 & Clinical intervention (alcohol and drug treatment programme) \\
\hline Dewhirst 2013 & $\begin{array}{l}\text { Fewer than } 2 \text { outcomes regarding engagement in risk behaviours (sexual risk behaviour outcomes } \\
\text { measured) }\end{array}$ \\
\hline DeWit 2000 & Not randomised \\
\hline Dickinson 2013 & Outside of required age range (recruited participants aged 18 years and older) \\
\hline Dishion 1995 & Not randomised \\
\hline Dishion 2000 & Not randomised \\
\hline Donovan 2015 & Outside of required age range (aged 16 to 21 years, mean $>18.5$ years) \\
\hline Doolittle 1975 & Dissertation; no response from study authors re: associated published journals \\
\hline Dumas 2001 & Less than 6 months combined intervention and follow-up period \\
\hline Dymnicki 2014 & $\begin{array}{l}\text { Fewer than } 2 \text { outcomes regarding engagement in risk behaviours (only violence-related outcomes } \\
\text { measured) }\end{array}$ \\
\hline Eggert 1994a & Not randomised \\
\hline Eggert 1994b & Not randomised \\
\hline Eisner 2012 & $\begin{array}{l}\text { Fewer than } 2 \text { outcomes regarding engagement in risk behaviours (antisocial behaviour and offend- } \\
\text { ing) }\end{array}$ \\
\hline Elder 1994 & $\begin{array}{l}\text { Fewer than } 2 \text { outcomes regarding engagement in risk behaviours (measures only participant atti- } \\
\text { tudes and intentions - not actual engagement in risk behaviours) }\end{array}$ \\
\hline Ellickson 2004 & Tobacco, alcohol, and drug outcomes only \\
\hline Elliot 2004 & Fewer than 2 outcomes regarding engagement in risk behaviours (tobacco use only) \\
\hline Elliot 2008 & Focuses on tobacco, alcohol, and drugs only \\
\hline Epton 2014 & Outside of required age range (aged 16 to 21 years, mean $>18.5$ years) \\
\hline Espelage 2013 & Focus on violence-related outcomes rather than multiple-risk behaviours \\
\hline Esposito-Smythers 2014 & Clinical intervention (participants were in receipt of mental health treatment (secondary care)) \\
\hline
\end{tabular}




\begin{tabular}{|c|c|}
\hline Study & Reason for exclusion \\
\hline Fagen 2009 & $\begin{array}{l}\text { Fewer than } 2 \text { outcomes regarding engagement in risk behaviours (assessed only participant atti- } \\
\text { tudes and intentions) }\end{array}$ \\
\hline Fekkes 2016 & Not randomised \\
\hline Fergusson 2013 & $\begin{array}{l}\text { Fewer than } 2 \text { outcomes regarding engagement in risk behaviours (assesses only socioemotional } \\
\text { problems) }\end{array}$ \\
\hline Fishbein 2016 & Less than 6 months combined intervention and follow-up period \\
\hline Fleming 2010 & Outside of required age range (mean 20.9 years) \\
\hline Fraguela 2003 & Not randomised \\
\hline Friedmann 2012 & Outside of required age range (mean 33.5 years) \\
\hline Furr-Holden 2003 & Dissertation; no response from study authors re: associated published journals \\
\hline Gil 2004 & $\begin{array}{l}\text { Fewer than } 2 \text { outcomes regarding engagement in risk behaviours (assesses only participant atti- } \\
\text { tudes and intentions) }\end{array}$ \\
\hline Giles 2010 & $\begin{array}{l}\text { Fewer than } 2 \text { outcomes regarding engagement in risk behaviours (assesses only participant atti- } \\
\text { tudes and intentions) }\end{array}$ \\
\hline Gislason 1995 & Not randomised \\
\hline Gittman 1994 & Not randomised \\
\hline Gorman 2002 & Not randomised \\
\hline Harmon 1993 & Not randomised \\
\hline Henggeler 2012 & Clinical intervention (most participants were drug addicts recruited from a juvenile drug court) \\
\hline Hidalgo 2015 & Outside of required age range (mean 18.8 years) \\
\hline Hogue 2015 & $\begin{array}{l}\text { Clinical intervention (mental health illness was clinically diagnosed in a large proportion of partici- } \\
\text { pants) }\end{array}$ \\
\hline Horigian 2015 & Clinical intervention \\
\hline Horn 2013 & No control group \\
\hline Hunter 1990 & Not randomised \\
\hline Jacobs 2011 & Outside of required age range (participant age range 25 to 75 years) \\
\hline Jacobs 2016 & Outside of required age range (aged 16 to 21 years, mean $>18.5$ years) \\
\hline Jegannathan 2014 & Study not randomised \\
\hline Jekielek 2002 & Not randomised \\
\hline Johnson 1990 & Not randomised \\
\hline
\end{tabular}




\begin{tabular}{|c|c|}
\hline Study & Reason for exclusion \\
\hline Johnson 2009 & Intervention delivered at community level \\
\hline Kellam 1994a & $\begin{array}{l}\text { Fewer than } 2 \text { outcomes regarding engagement in risk behaviours (antisocial behaviour and offend- } \\
\text { ing) }\end{array}$ \\
\hline Kellam 1994b & $\begin{array}{l}\text { Fewer than } 2 \text { outcomes regarding engagement in risk behaviours (antisocial behaviour and offend- } \\
\text { ing) }\end{array}$ \\
\hline Kelleher 1999 & Not randomised \\
\hline Kennedy 2009 & Fewer than 2 outcomes regarding engagement in risk behaviours (none) \\
\hline Killen 1988 & Less than 6 months combined intervention and follow-up period \\
\hline Killen 1989 & Less than 6 months combined intervention and follow-up period \\
\hline Kilmer 2008 & Outside of required age range (mean age 19.3 years) \\
\hline Kosterman 2001 & Less than 6 months combined intervention and follow-up period \\
\hline Kruger 2014 & Outside of required age range (aged 16 to 21 years, mean > 18.5 years) \\
\hline Kypri 2005 & Less than 6 months combined intervention and follow-up period \\
\hline Lauver 2002 & Dissertation; no response from study authors re: associated published journals \\
\hline Lees 2014 & Fewer than 2 outcomes regarding engagement in risk behaviours (antisocial behaviour only) \\
\hline Letourneau 2013 & Clinical intervention (therapy for sex offenders) \\
\hline Lewis 2014 & Outside of required age range (mean age 20.4 years) \\
\hline Lewis 2015 & Outside of required age range (mean age 20.4 years) \\
\hline MacDonald 2007 & Outside of required age range (mean age 35.4 years) \\
\hline Martinez 2005 & Fewer than 2 outcomes regarding engagement in risk behaviours (none of interest measured) \\
\hline Mathews 2007 & Less than 6 months combined intervention and follow-up period \\
\hline Mays 2012 & Less than 6 months combined intervention and follow-up period \\
\hline Muratori 2015 & $\begin{array}{l}\text { Fewer than } 2 \text { outcomes regarding engagement in risk behaviours (antisocial behaviour and offend- } \\
\text { ing) }\end{array}$ \\
\hline Murry 2011 & Fewer than 2 outcomes regarding engagement in risk behaviours (risky sexual behaviours) \\
\hline O'Donnell 1995 & Not randomised \\
\hline O'Donnell 2010 & Less than 6 months combined intervention and follow-up period \\
\hline O'Leary-Barrett 2009 & Dissertation; study authors contacted and full published paper (O'Leary-Barrett 2010) received \\
\hline O'Leary-Barrett 2010 & Tobacco, alcohol, and drug outcomes only \\
\hline
\end{tabular}




\begin{tabular}{|c|c|}
\hline Study & Reason for exclusion \\
\hline Osgood 2013 & Community-level intervention (randomised at the level of the school district) \\
\hline Patrick 2014 & Less than 6 months combined intervention and follow-up period \\
\hline Peden 2012 & Outside of required age range (recruited participants aged 18 years and older) \\
\hline Peeler 2001 & Outside of required age range (recruited participants aged 18 years and older) \\
\hline Pierce 2008 & $\begin{array}{l}\text { Fewer than } 2 \text { outcomes regarding engagement in risk behaviours (antisocial behaviour and offend- } \\
\text { ing) }\end{array}$ \\
\hline Poduska 2008 & $\begin{array}{l}\text { Fewer than } 2 \text { outcomes regarding engagement in risk behaviours (antisocial behaviour and offend- } \\
\text { ing) }\end{array}$ \\
\hline Prado 2005 & Dissertation; study authors contacted and full published paper (Prado 2007) received \\
\hline Prado 2007 & Not randomised \\
\hline Prevention Program & Tobacco, alcohol, and drug outcomes only \\
\hline Rhoades 2013 & Fewer than 2 outcomes regarding engagement in risk behaviours (antisocial behaviour) \\
\hline Rhoades 2014 & Fewer than 2 outcomes regarding engagement in risk behaviours (antisocial behaviour) \\
\hline Ringwalt 1991 & Less than 6 months combined intervention and follow-up period \\
\hline Robbins 2009 & Clinical intervention (brief strategic family therapy) \\
\hline Rohrbach 2010 & Measures only participant attitudes and intentions - not actual engagement in risk behaviours \\
\hline Ross 1998 & Not randomised \\
\hline Rotheram-Borus 2012 & Outside of required age range (mean age 19 years) \\
\hline Rowland 2008 & Clinical intervention (integration of evidence-based practices into juvenile drug court) \\
\hline Sakofs 1991 & Fewer than 2 outcomes regarding engagement in risk behaviours (none of interest) \\
\hline Salminen 2005 & Not randomised \\
\hline Samet 2015 & Outside of required age range (mean age 30.1 years) \\
\hline Sanci 2015 & Most participants were aged 18 to 24 years. \\
\hline Santisteban 2003 & Less than 6 months combined intervention and follow-up period \\
\hline Schaeffer 2014 & Clinical population (participants had to have DSM classification of substance abuse) \\
\hline Schinke 1986 & Community-level intervention \\
\hline Schinke 1988 & Tobacco, alcohol, and drug outcomes only \\
\hline Schinke 2005 & Less than 6 months combined intervention and follow-up period \\
\hline Scott 1988 & Not randomised \\
\hline
\end{tabular}




\begin{tabular}{|c|c|}
\hline Study & Reason for exclusion \\
\hline Sheidow 2012 & Clinical population (participants had to have DSM classification of substance abuse) \\
\hline Shope 1996 & Not randomised \\
\hline Sieving 2014 & $\begin{array}{l}\text { Fewer than } 2 \text { outcomes regarding engagement in risk behaviours (antisocial behaviour and vio- } \\
\text { lence) }\end{array}$ \\
\hline Sine 1976 & Less than 6 months combined intervention and follow-up period \\
\hline Skybo 2002 & Not randomised \\
\hline Slater 2006 & Community-level intervention \\
\hline Slesnick 2005 & Clinical intervention (ecologically based family therapy) \\
\hline Slesnick 2006 & Clinical intervention (family therapy) \\
\hline Slesnick 2007 & Clinical population (DSM-IV criteria for alcohol or other psychoactive substance use disorders) \\
\hline Slesnick 2013 & Clinical population (DSM-IV criteria for alcohol or other psychoactive substance use disorders) \\
\hline Snow 1992 & Not randomised \\
\hline Sommers 2013 & Outside of required age range (age range 18 to 44 years) \\
\hline Spoth 2011 & Community-level intervention \\
\hline St Lawrence 1999 & Not randomised \\
\hline St Pierre 1992 & Not randomised \\
\hline Stanton 2000 & Measures only participant attitudes and intentions - not actual engagement in risk behaviours \\
\hline Stanton 2004 & Not randomised \\
\hline Stein 2011 & Not randomised \\
\hline Stevens 2002 & Not randomised \\
\hline Stuart 1976 & Not randomised \\
\hline Taussig 2007 & Not randomised \\
\hline Taylor 2000 & Not randomised \\
\hline Teesson 2009 & Dissertation; no response from study authors re: associated published journals \\
\hline Tucker 2016 & Less than 6 months combined intervention and follow-up period \\
\hline Tupe 2014 & $\begin{array}{l}\text { Fewer than } 2 \text { outcomes regarding engagement in risk behaviour (focuses on reproductive health } \\
\text { and hygiene, rather than actual engagement in risk behaviour) }\end{array}$ \\
\hline Valdez 2013 & Therapeutic intervention \\
\hline van Rosmalen-Nooijens 2013 & Fewer than 2 outcomes regarding engagement in risk behaviours (none of interest) \\
\hline
\end{tabular}




\begin{tabular}{|c|c|}
\hline Study & Reason for exclusion \\
\hline Vartiainen 1986 & Not randomised \\
\hline Villalbi 1993 & Measures only participant attitudes and intentions - not actual engagement in risk behaviours \\
\hline Vitaro 1994 & Not randomised \\
\hline Weikart 1988 & Not randomised \\
\hline Weikart 1991 & Not randomised \\
\hline Werch 2005 & Not randomised \\
\hline Werch 2005a & Less than 6 months combined intervention and follow-up period \\
\hline Werch 2008 & Less than 6 months combined intervention and follow-up period \\
\hline Wilson 2012a & Fewer than 2 risk behaviour engagement outcomes (diet-related outcomes only) \\
\hline Wilson 2012b & Not randomised \\
\hline Winters 2012 & $\begin{array}{l}\text { Clinical intervention (brief intervention for individuals meeting DSM-IV criteria for drug and/or mar- } \\
\text { ijuana abuse) }\end{array}$ \\
\hline Wodarski 1987 & Not randomised \\
\hline Wu 2003 & Not randomised \\
\hline Yabiku 2007 & Tobacco, alcohol, and drug outcomes only \\
\hline Yilmaz 2015 & Fewer than 2 multiple-risk behaviour engagement outcomes (sedentary behaviour) \\
\hline Zatzick 2014 & $\begin{array}{l}\text { Clinical population (participants have traumatic injuries, with }>50 \% \text { possessing severe brain in- } \\
\text { juries) }\end{array}$ \\
\hline
\end{tabular}

DSM: Diagnostic and Statistical Manual of Mental Disorders.

Characteristics of ongoing studies [ordered by study ID]

Allara 2015

\begin{tabular}{ll}
\hline Trial name or title & Paesaggi di Prevenzione \\
\hline Methods & Study design: cluster RCT \\
Intervention $\operatorname{arm}(\mathbf{s}):$ Paesaggi di Prevenzione intervention \\
Comparator arm(s): usual school curriculum \\
Sample size calculation performed: yes \\
Country: Italy; State: Emilia-Romagna \\
Setting: school \\
Focus: universal
\end{tabular}


Age (range or mean (SD)) and grade at the start: N/S

Gender: N/S

Ethnicity: N/S

SES: N/S

Inclusion criteria: N/S

Exclusion criteria: $\mathrm{N} / \mathrm{S}$

Duration of the intervention (excluding follow-up): 12 months

Description of the intervention: 2 components: (1) interactive classroom curriculum focusing on reducing initiation of both tobacco and alcohol use, improving the quality of the diet, and increasing the frequency and intensity of physical activity, all tailored to the needs of individual students. Lessons and activities were designed to enhance decision-making, problem-solving, personal and interpersonal skills, stress and emotion management, and communication skills; (2) school policy-level modification comprising a smoking ban inside/outside school premises, prohibition of alcohol use at school events, promotion of healthy foods in school canteens and food outlets, and walking acitvities/team games involving families, students, and school staff

\section{Brief description of the theoretical model: N/S}

Description of the comparator: standard school curriculum

\begin{tabular}{ll}
\hline Outcomes & $\begin{array}{l}\text { Primary: tobacco use, alcohol misuse, nutrition, and physical activity } \\
\text { Secondary: N/S }\end{array}$ \\
\hline Starting date & January 2011 \\
\hline Contact information & Elias Allara; elias.allara@med.unipmn.it \\
\hline Notes & Baseline results available (July 2015) \\
\hline
\end{tabular}

\section{Barbosa Filho 2015}

\begin{tabular}{ll}
\hline Trial name or title & Fortaleça sua Saúde \\
\hline Methods & Study design: cluster RCT \\
& Intervention $\operatorname{arm}(\mathbf{s})$ : Fortaleça sua Saúde \\
& Comparator arm(s): no intervention \\
& Sample size calculation performed: yes \\
& Country: Brazil; State: Fortaleza \\
& Setting: school \\
Focus: universal
\end{tabular}


Number of participants randomised (total and by arm): 639 to intervention, 633 students to control; 548 students in intervention group and 537 in control group at baseline

Age (range or mean (SD)) and grade at the start: grades 7 to 9 (age 12 to 15 years)

Gender: N/S

Ethnicity: N/S

SES: N/S

Inclusion criteria: aged 12 to 15 years enrolled in grades 7 to 9 , attending full-time public schools in Fortaleza, northeastern Brazil

Exclusion criteria: N/S

Randomisation before or after baseline survey: before
Duration of the intervention (excluding follow-up): 4 months
Description of the intervention: training of teachers; class activities focused on discussion of ac-
tive and healthy lifestyles; training tailored to PE teachers to structure active PE classes; active op-
portunities in the school to promote opportunities for physical activity (e.g. supervised 'gym in
school' sessions, availability of space and equipment, new games); and health education (e.g. ban-
ners, classroom materials, pamphlets)

Brief description of the theoretical model: N/S

Description of the comparator: no intervention

\begin{tabular}{ll}
\hline Outcomes & $\begin{array}{l}\text { Primary: physical activity; screen time; intrapersonal, interpersonal, and environmental variables } \\
\text { associated with physical activity }\end{array}$ \\
$\begin{array}{l}\text { Secondary: nutritional status; tobacco use; alcohol use; sexual risk behaviour; academic perfor- } \\
\text { mance; sleep; stress perception }\end{array}$ \\
\hline Starting date & Second semester 2014 \\
\hline Contact information & valtercbf@gmail.com \\
\hline Notes & Protocol \\
\hline
\end{tabular}

Letona 2013

\begin{tabular}{ll}
\hline Trial name or title & Pilas! \\
\hline Methods & Study design: $\mathrm{RCT}$ \\
& Intervention $\operatorname{arm}(\mathbf{s}):$ Pilas! intervention \\
& Comparator arm(s): usual school curriculum \\
& Sample size calculation performed: N/S \\
& Country: Guatemala; State: Guatemala City \\
& Setting: school \\
Focus: universal
\end{tabular}


Letona 2013 (Continued)

Number of participants randomised (total and by arm): N/S

Age (range or mean (SD)) and grade at the start: N/S

Gender: N/S

Ethnicity: N/S

SES: N/S

Inclusion criteria: N/S

Exclusion criteria: N/S

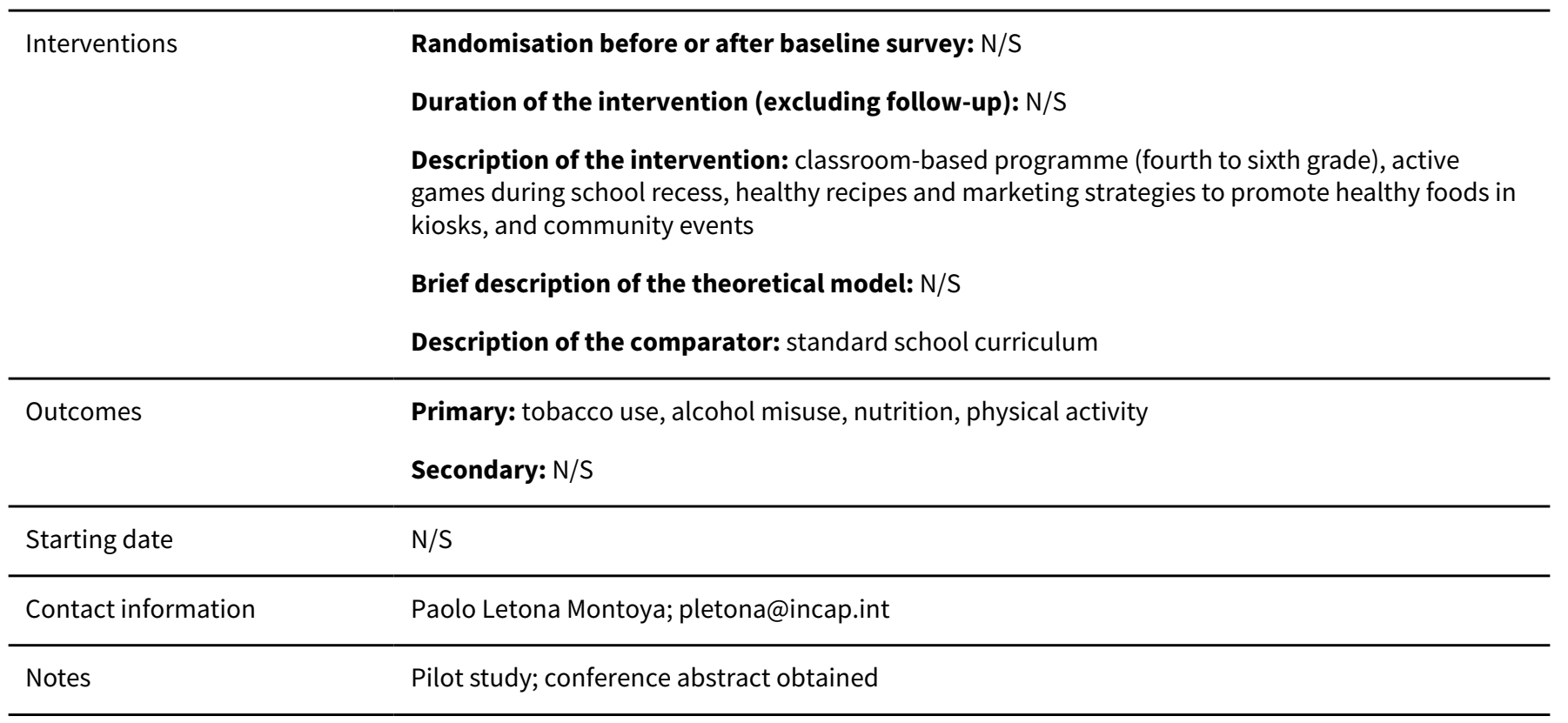

Pendergrass 2014

\begin{tabular}{ll}
\hline Trial name or title & Play-Forward: Elm City Stories \\
\hline Methods & Study design: $\mathrm{RCT}$ \\
& Intervention $\operatorname{arm}(\mathbf{s}):$ video game intervention \\
& Comparator arm(s): N/S \\
& Sample size calculation performed: N/S \\
& Country: USA; State: N/S \\
& Setting: individual \\
& Focus: universal \\
\hline
\end{tabular}

Participants

Number of schools randomised: N/A

Number of participants randomised (total and by arm): N/S

Age (range or mean (SD)) and grade at the start: N/S

Gender: N/S 
Pendergrass 2014 (Continued)

Ethnicity: N/S

SES: N/S

Inclusion criteria: N/S

Exclusion criteria: N/S

Randomisation before or after baseline survey: N/S
Duration of the intervention (excluding follow-up): N/S
Description of the intervention: focus is on preventing HIV and developing skills and knowledge
on preventing HIV-related risk behaviours and other health risk behaviours such as substance use,
via an interactive video game in which the user role-plays a character, making virtual choices and
discovering how such choices go on to affect their lives and how a different set of choices may have
led to different outcomes

Description of the comparator: N/S

\begin{tabular}{ll}
\hline Outcomes & $\begin{array}{l}\text { Primary: increased knowledge about and prevention of HIV-related risk behaviours and other } \\
\text { health risk behaviours } \\
\text { Secondary: N/S }\end{array}$ \\
\hline Starting date & February 2013 \\
\hline Contact information & Ms Lynn Fiellin; lynn.fiellin@yale.edu \\
\hline Notes & Conference presentation abstract only; study authors contacted; full results of RCT in press \\
\hline
\end{tabular}

\section{Piotrowski 2014}

\begin{tabular}{ll}
\hline Trial name or title & Positive Youth Potential \\
\hline Methods & Study design: cluster RCT \\
& Intervention $\operatorname{arm}(\mathbf{s}):$ 3-year Positive Youth Potential classroom-based curriculum \\
& Comparator arm(s): standard health education curriculum \\
& Sample size calculation performed: N/S \\
& Country: USA; State: NorthWest Indiana \\
& Setting: school \\
& Focus: universal \\
\hline
\end{tabular}

Participants

Number of schools randomised: 16

Number of participants randomised (total and by arm): overall 1776; intervention 970, control 806

Age (range or mean (SD)) and grade at the start: N/S

Gender: N/S

Ethnicity: N/S

SES: N/S 
Inclusion criteria: grade 6 students with English comprehension and reading to at least grade 5 level, providing assent and parental consent

\section{Exclusion criteria: N/S}

Interventions

\section{Randomisation before or after baseline survey: N/S}

\section{Duration of the intervention (excluding follow-up): N/S}

Description of the intervention: focuses on positive youth development (with emphasis on a child's possible self and future self), goal orientation, positive school performance, and risk reduction and risk elimination behaviours with sexual activity and other adolescent risk behaviours (such as alcohol, tobacco, drugs, violence, pornography, and bullying). Students are encouraged via risk avoidance and developmental health promotion strategies. Five $\times 45$ to 50 -minute classroom sessions in each of grades 6,7 , and 8 , and a 45 -minute assembly at the end of each grade

\section{Brief description of the theoretical model: N/S}

Description of the comparator: usual health education instruction, after-school activities, or other community activities and instruction about risk behaviours and health. The school general health curriculum usually includes 1 lesson on sexually transmitted disease/HIV prevention. Control group students also attend assemblies at the same times as treatment group students; however, these assemblies focus on topics not related to the Positive Potential instruction, such as general health and exercise. Nationally recognised speakers present to the assembly each year and avoid any content that is presented to treatment groups.

Outcomes Primary: impacting upon sexual intercourse

Secondary: other risk behaviour reduction, including risky sexual behaviours; tobacco, alcohol, and drug use; and violence prevention

\begin{tabular}{ll}
\hline Starting date & 2012 \\
\hline Contact information & Harry Piotrowski; zhp@sprynet.com \\
\hline Notes & Conference presentation abstract only; limited information stated \\
\hline
\end{tabular}

\section{Sanci 2012}

\begin{tabular}{ll}
\hline Trial name or title & Prevention Access and Risk Taking in Young People (PARTY) \\
\hline Methods & Study design: cluster RCT (unit of randomisation = general practice) \\
Intervention arm(s): health professional training, screening, and brief intervention regarding a & range of health risk behaviours following consultation with a general practitioner \\
Comparator arm(s): standard health consultation + assessment completion \\
Sample size calculation performed: yes \\
Country: Australia; State: Victoria, Melbourne \\
Setting: primary care \\
Focus: universal
\end{tabular}

Participants

Number of schools randomised: N/A; 42 general practices randomised to intervention or control

Number of participants randomised (total and by arm): N/S 


\section{Age (range or mean (SD)) and grade at the start: N/S}

Gender: N/S

Ethnicity: N/S

SES: N/S

Inclusion criteria: youth aged 14 to 24 years, attending participating clinicians

Exclusion criteria: physically or mentally unwell patients (e.g. vomiting, febrile, weak, cognitively impaired, psychotic), unable to read or speak English, younger than $18+$ parent refusal of content + judged by clinician to be incompetent to make informed decisions regarding risks and benefits of involvement

Interventions

Randomisation before or after baseline survey: before; baseline sample different from that followed up at 3 and 12 months post intervention

Duration of the intervention (excluding follow-up): 10 to 30 -minute consultation

Description of the intervention: psychosocial health-risk behaviour screening and subsequent intervention to prevent health risk behaviours; also training of general practitioners and nurse practitioners on screening principles, adolescent development, and risky behaviours

\section{Brief description of the theoretical model: N/S}

Description of the comparator: standard consultation with general practitioner and assessment completion at 3 and 12 months post consultation

\begin{tabular}{ll}
\hline Outcomes & $\begin{array}{l}\text { Primary: comparison of intervention and control arms in terms of young people's uptake of health } \\
\text { risk behaviour }\end{array}$ \\
& $\begin{array}{l}\text { Secondary: N/S; general risk behaviour outcomes assessed include smoking, alcohol and other } \\
\text { substance use, risky sexual behaviours, vehicle-related risk behaviour, nutrition, physical activity, } \\
\text { self-harm, antisocial behaviour, and incidences of bullying }\end{array}$ \\
\hline Starting date & 2005 (piloting) \\
\hline Contact information & Lena Sanci; l.sanci@unimelb.edu.au \\
\hline Notes & Trial registration number: ISRCTN16059206 \\
Protocol available; initial results of RCT available as conference presentation abstract only
\end{tabular}

\section{Standage 2013}

Trial name or title Be the Best You Can Be (BtBYCB)

Comparator $\operatorname{arm}(\mathbf{s})$ : standard education control arm

Sample size calculation performed: yes

Country: England; State: Bath and North East Somerset

Setting: school 
Number of participants randomised (total and by arm): 58 classes (1333 pupils overall); intervention 28 classes ( 711 pupils), control 30 classes (622 pupils)

Age (range or mean (SD)) and grade at the start: N/S

Gender: N/S

Ethnicity: N/S

SES: N/S

Inclusion criteria: all year 7 and 8 pupils eligible

Exclusion criteria: none stated, with the exception of refusal of the parent and/or pupil to participate in the intervention

Interventions

\section{Randomisation before or after baseline survey: N/S}

Duration of the intervention (excluding follow-up): intervention duration not clearly stated; 2 talks by Olympians ( 1 at the beginning and 1 at the end of the intervention period); intervention itself consisted of $11 \times 1$-hour classroom-based lessons

Description of the intervention: initial launch event talk by an Olympian; 11 teacher-led classroom sessions (lessons, exercises, and activities) with an Olympian theme, providing students with knowledge and skills needed to realise their goals and ambitions; and a final closing event (talk by another Olympian)

\section{Brief description of the theoretical model: N/S}

Description of the comparator: standard education; control group participants received PSHE tuition as usual

\begin{tabular}{ll}
\hline Outcomes & $\begin{array}{l}\text { Primary: changes in self-perception, well-being, and self-esteem } \\
\text { Secondary: changes in modifiable health risk behaviours (dietary intake, physical activity levels, } \\
\text { tobacco use, and alcohol consumption) }\end{array}$ \\
\hline Starting date & N/S \\
\hline Contact information & Martyn Standage; m.standage@bath.ac.uk \\
\hline Notes & Trial registration number: ISRCTN99443695 \\
\hline
\end{tabular}

Tzelepis 2015

\begin{tabular}{ll}
\hline Trial name or title & No study name \\
\hline Methods & Study design: cluster RCT \\
& Intervention $\operatorname{arm}(\mathbf{s}):$ intervention $\operatorname{arm}$ \\
& Comparator $\operatorname{arm}(\mathbf{s}):$ no intervention \\
& Sample size calculation performed: yes \\
& Country: Australia; State: New South Wales (Hunter region)
\end{tabular}


Focus: universal

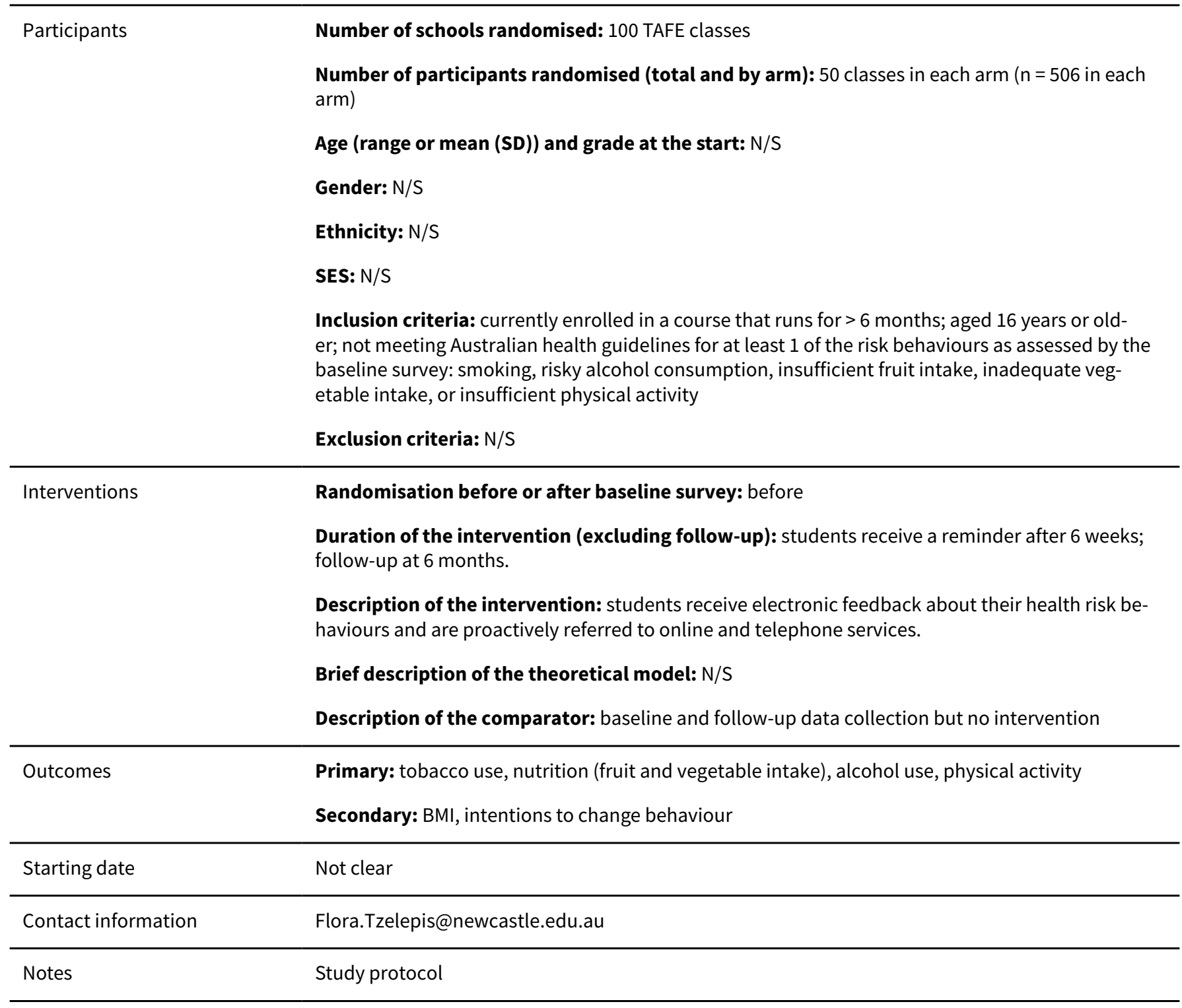

BMI: body mass index.

BtBYCB: Be the Best You Can Be.

N/S: not stated.

PE: physical education.

PSHE: Personal, social, health and economic education.

RCT: randomised controlled trial.

SD: standard deviation.

TAFE: technical and further education.

\section{DATA AND ANALYSES}


Comparison 1. Tobacco

\begin{tabular}{lllll}
\hline Outcome or subgroup title & No. of studies & $\begin{array}{l}\text { No. of partici- } \\
\text { pants }\end{array}$ & Statistical method & Effect size \\
\hline 1 Tobacco Use (short-term) & 15 & & Odds Ratio (Random, 95\% Cl) & Subtotals only \\
\hline 1.1 Individual Targeted & 2 & 521 & Odds Ratio (Random, 95\% Cl) & $0.98[0.35,2.73]$ \\
\hline 1.2 Individual Universal & 2 & 1549 & Odds Ratio (Random, 95\% Cl) & $1.03[0.32,3.27]$ \\
\hline 1.3 Family Targeted & 2 & 313 & Odds Ratio (Random, 95\% Cl) & $0.78[0.40,1.53]$ \\
\hline 1.4 School Universal & 9 & 15354 & Odds Ratio (Random, 95\% Cl) & $0.77[0.60,0.97]$ \\
\hline 2 Tobacco Use (long-term) & 6 & & Odds Ratio (Random, 95\% Cl) & Subtotals only \\
\hline 2.1 Individual Targeted & 1 & 397 & Odds Ratio (Random, 95\% Cl) & $1.08[0.56,2.11]$ \\
\hline 2.2 Family Targeted & 2 & 1177 & Odds Ratio (Random, 95\% Cl) & $0.82[0.32,2.14]$ \\
\hline 2.3 Family Universal & 1 & 237 & Odds Ratio (Random, 95\% Cl) & $0.82[0.38,1.78]$ \\
\hline 2.4 School Universal & 2 & 879 & Odds Ratio (Random, 95\% Cl) & $0.60[0.33,1.09]$ \\
\hline
\end{tabular}

Analysis 1.1. Comparison 1 Tobacco, Outcome 1 Tobacco Use (short-term).

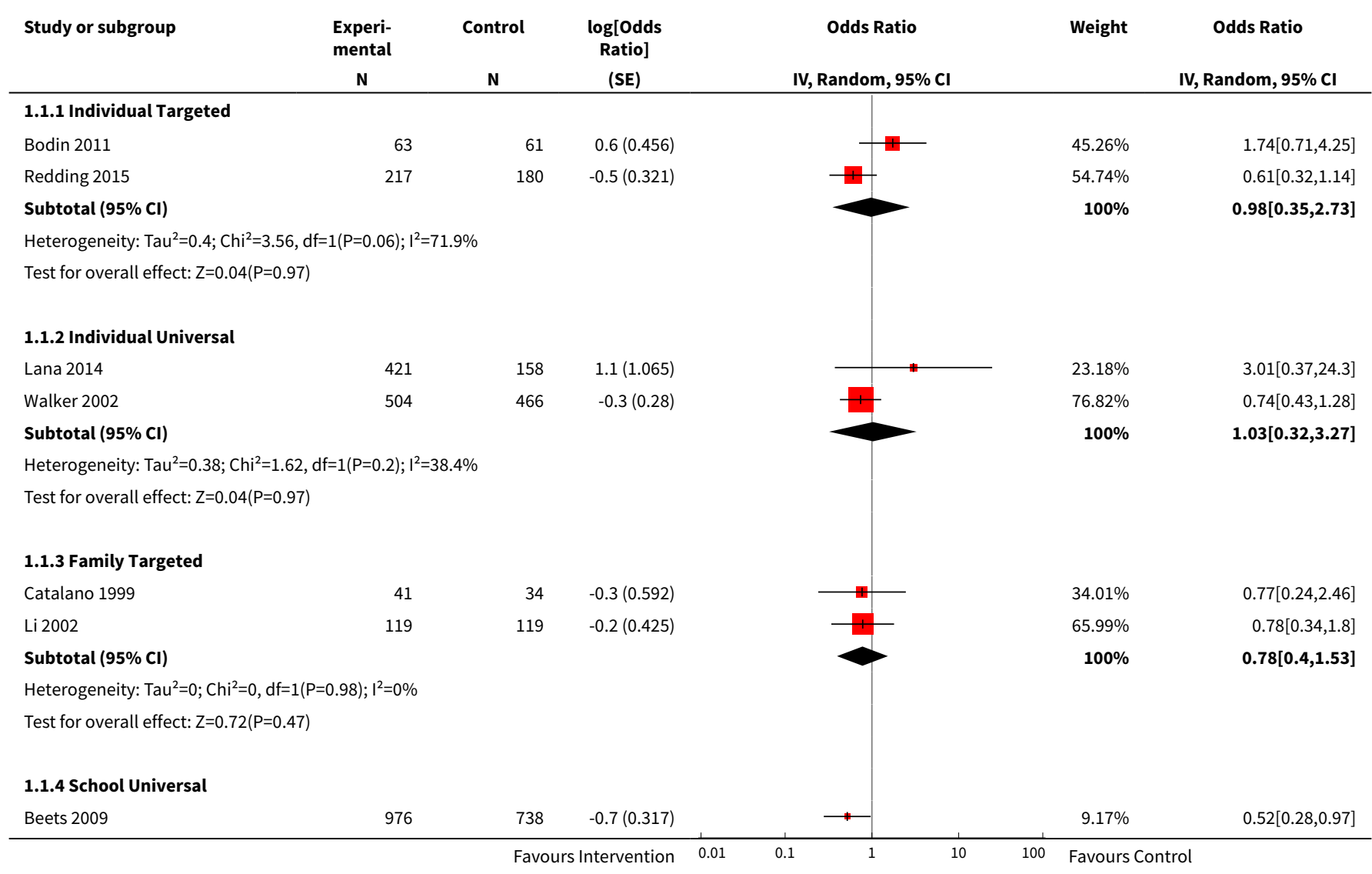




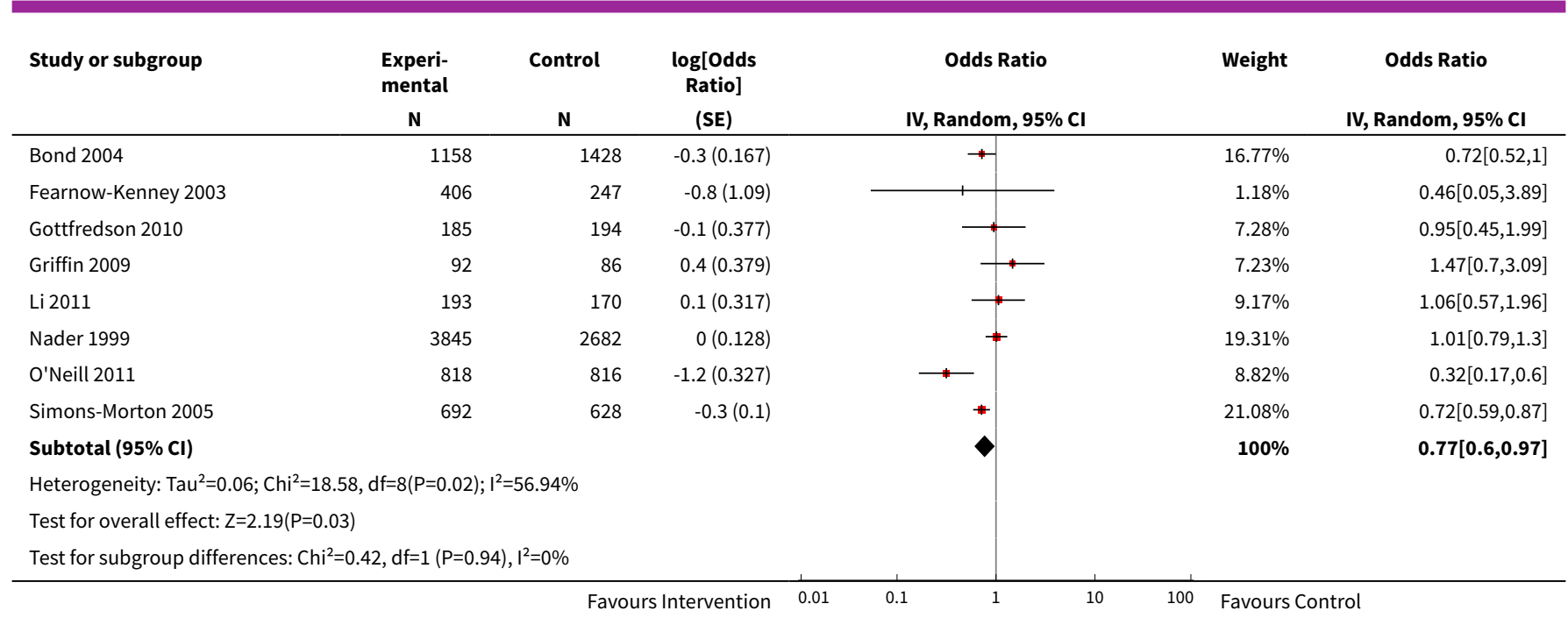

Analysis 1.2. Comparison 1 Tobacco, Outcome 2 Tobacco Use (long-term).

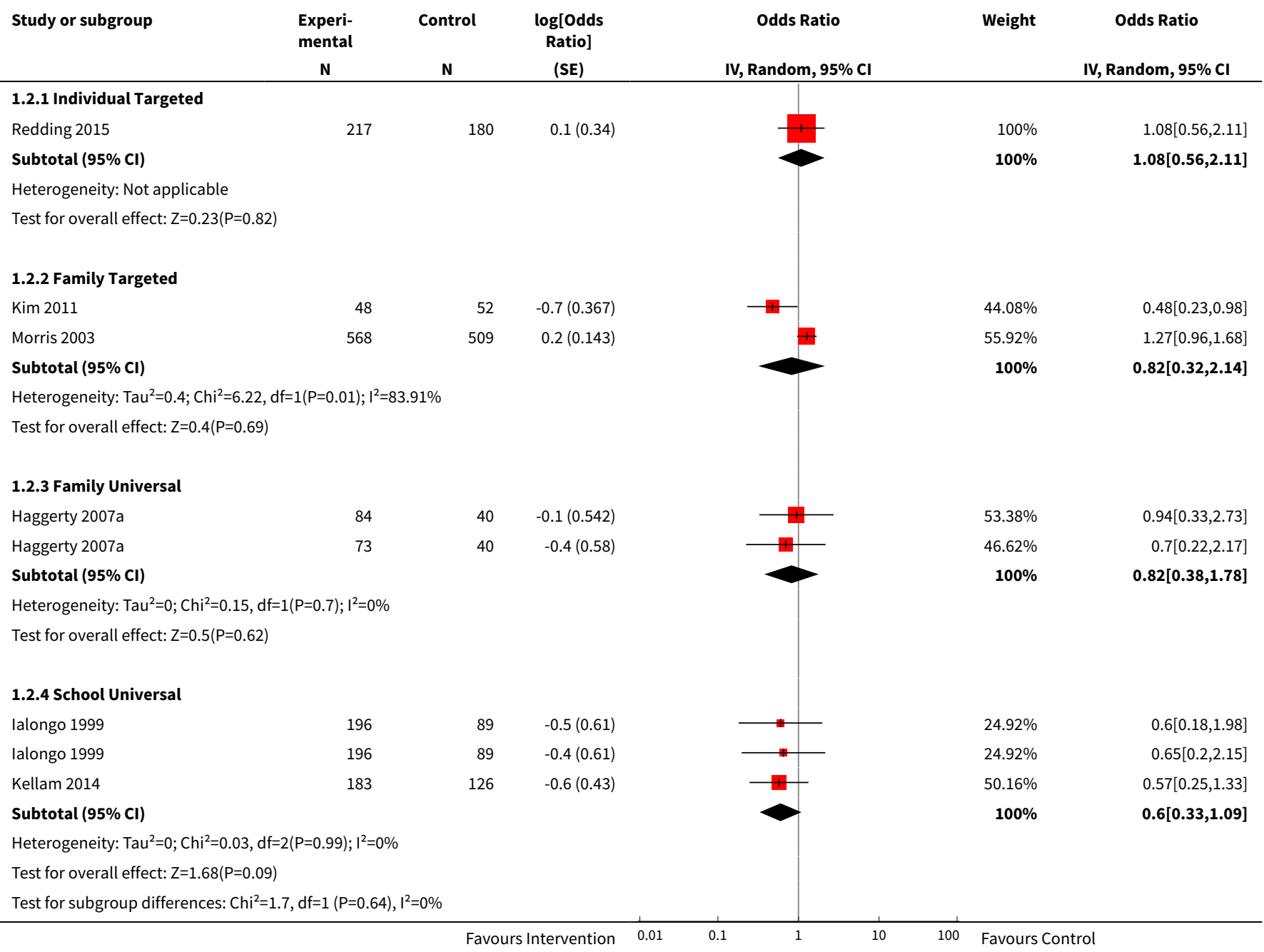


Comparison 2. Alcohol

\begin{tabular}{lllll}
\hline Outcome or subgroup title & No. of studies & $\begin{array}{l}\text { No. of partici- } \\
\text { pants }\end{array}$ & Statistical method & Effect size \\
\hline 1 Alcohol Use (short-term) & 19 & & Odds Ratio (Random, 95\% Cl) & Subtotals only \\
\hline 1.1 Individual Targeted & 4 & 2044 & Odds Ratio (Random, 95\% Cl) & $1.02[0.80,1.31]$ \\
\hline 1.2 Individual Universal & 4 & 1911 & Odds Ratio (Random, 95\% Cl) & $0.80[0.58,1.11]$ \\
\hline 1.3 Family Targeted & 3 & 417 & Odds Ratio (Random, 95\% Cl) & $0.83[0.47,1.46]$ \\
\hline 1.4 School Universal & 8 & 8751 & Odds Ratio (Random, 95\% Cl) & $0.72[0.56,0.92]$ \\
\hline 2 Alcohol Use (long-term) & 7 & & Odds Ratio (Random, 95\% Cl) & Subtotals only \\
\hline 2.1 Family Targeted & 3 & 1417 & Odds Ratio (Random, 95\% Cl) & $1.24[0.69,2.24]$ \\
\hline 2.2 Family Universal & 1 & 237 & Odds Ratio (Random, 95\% Cl) & $0.86[0.47,1.55]$ \\
\hline 2.3 School Targeted & 2 & 762 & Odds Ratio (Random, 95\% Cl) & $0.73[0.52,1.03]$ \\
\hline 2.4 School Universal & 1 & 566 & Odds Ratio (Random, 95\% Cl) & $1.34[0.55,3.27]$ \\
\hline
\end{tabular}

Analysis 2.1. Comparison 2 Alcohol, Outcome 1 Alcohol Use (short-term).

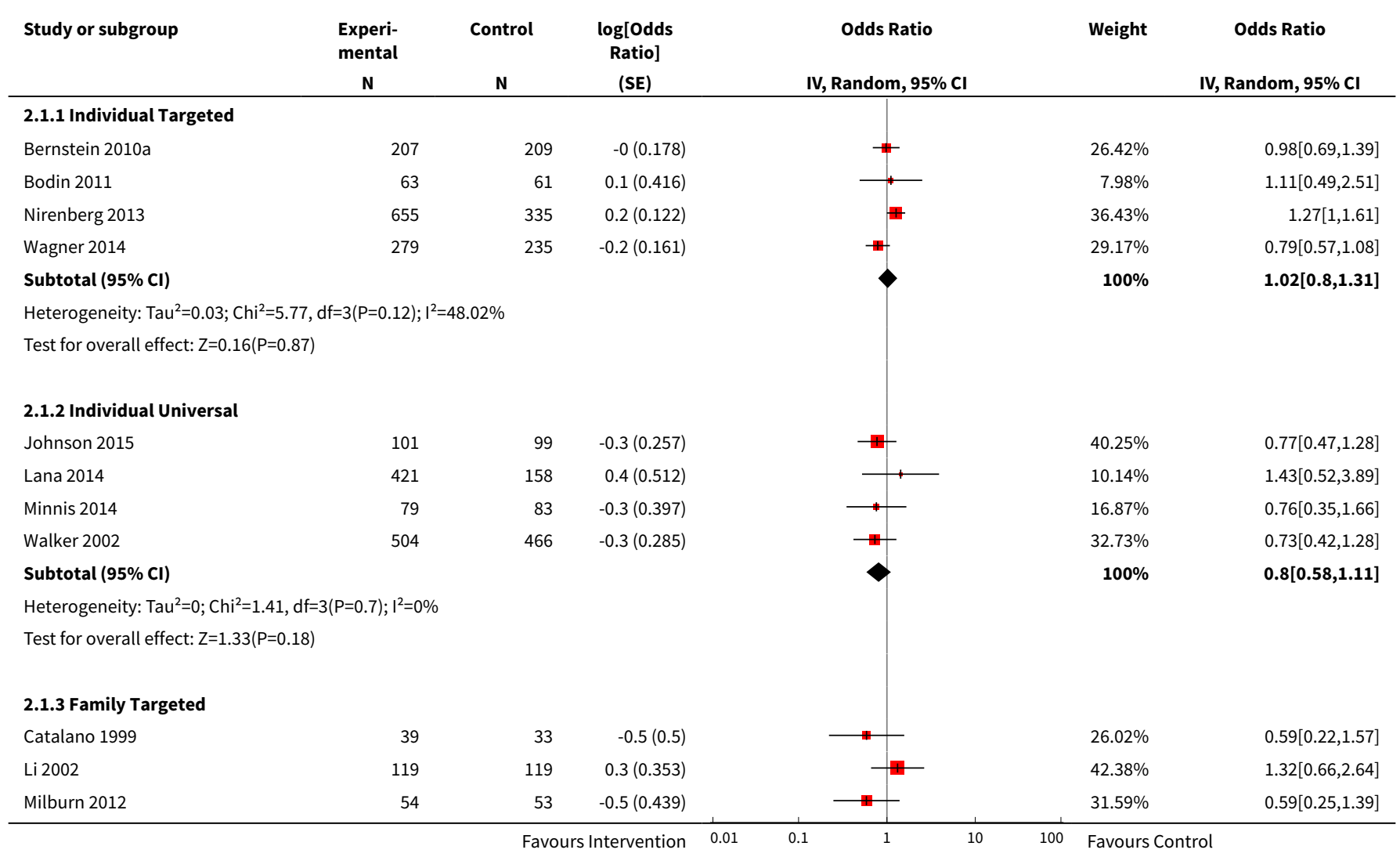




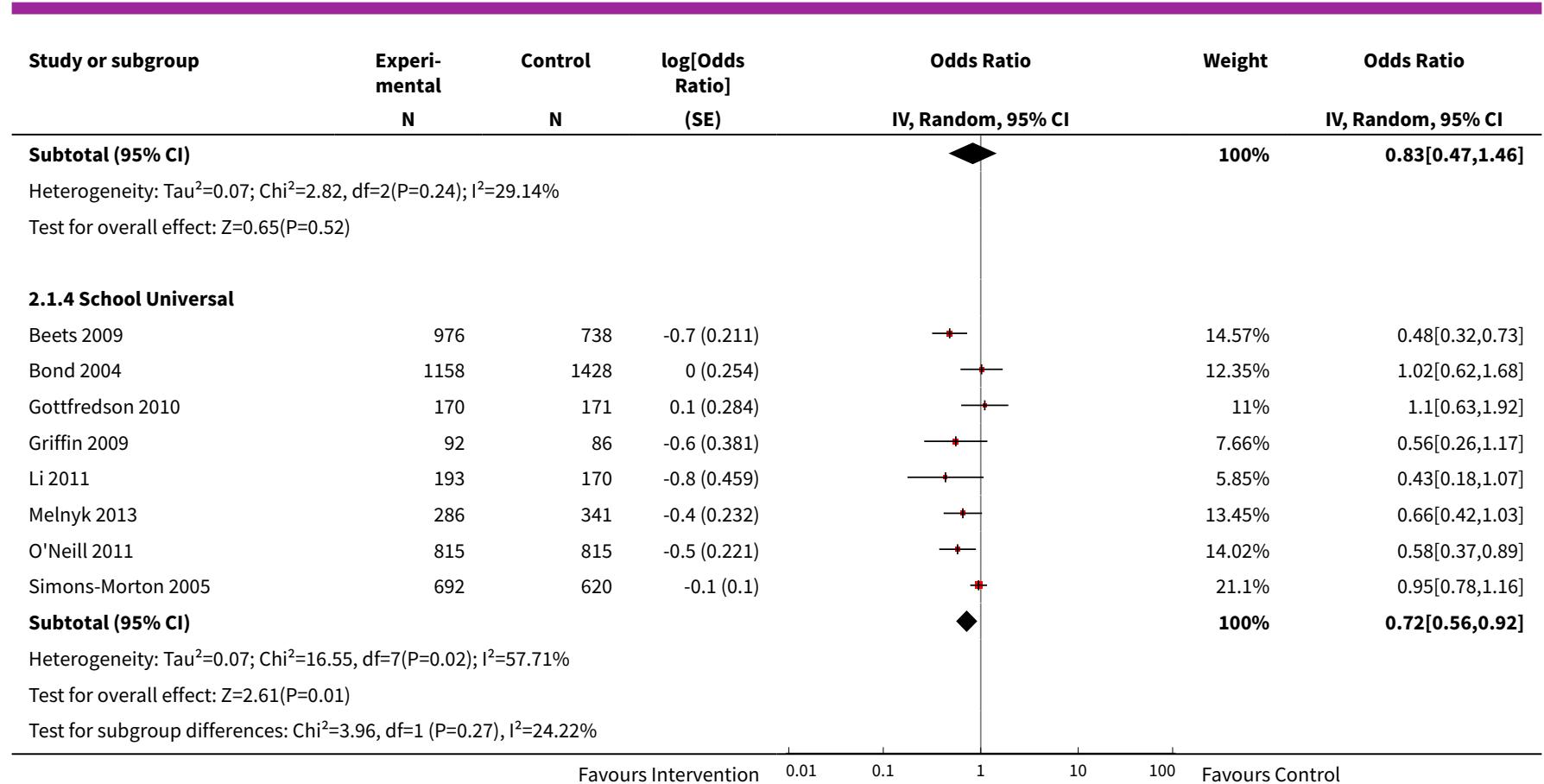

Analysis 2.2. Comparison 2 Alcohol, Outcome 2 Alcohol Use (long-term).

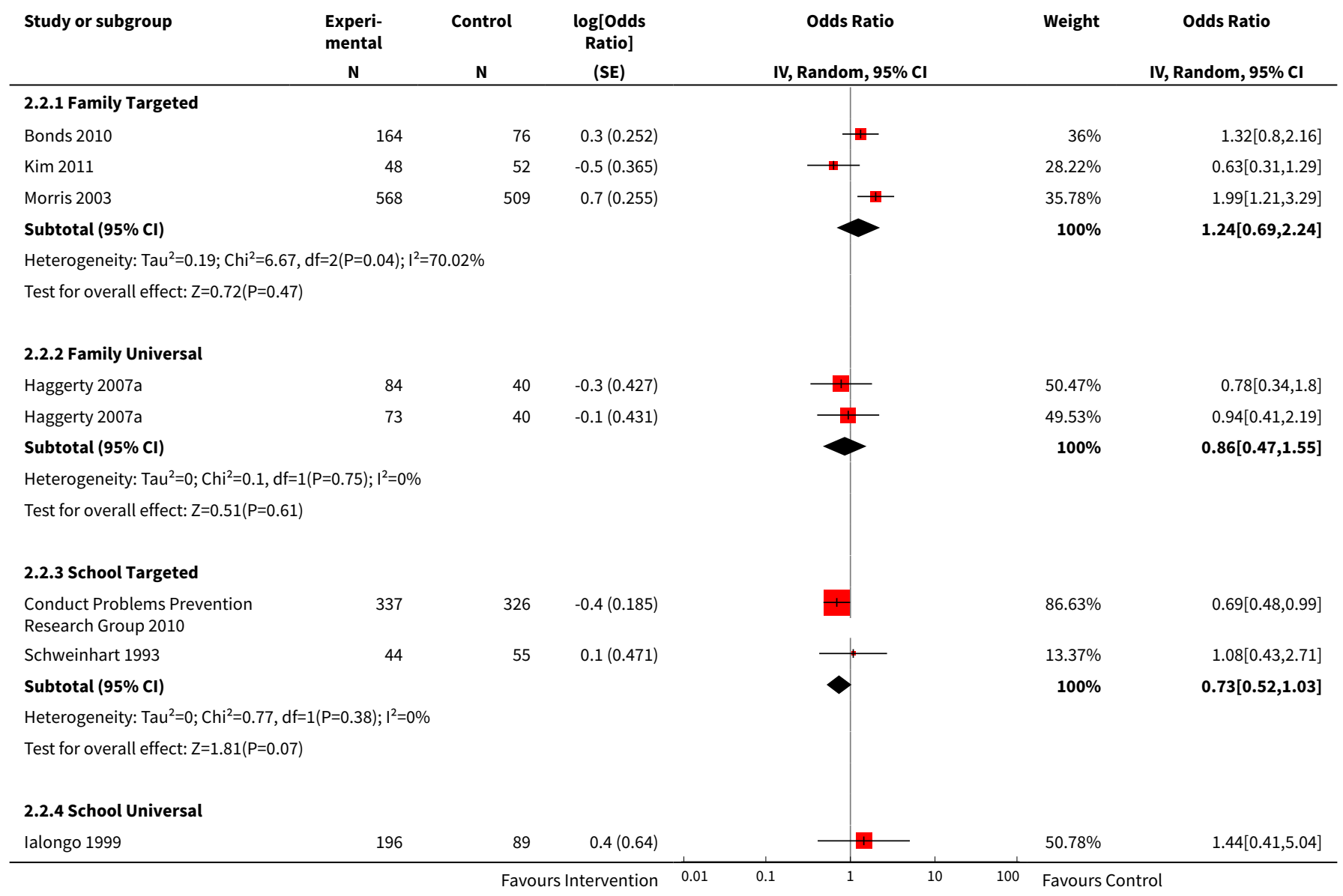




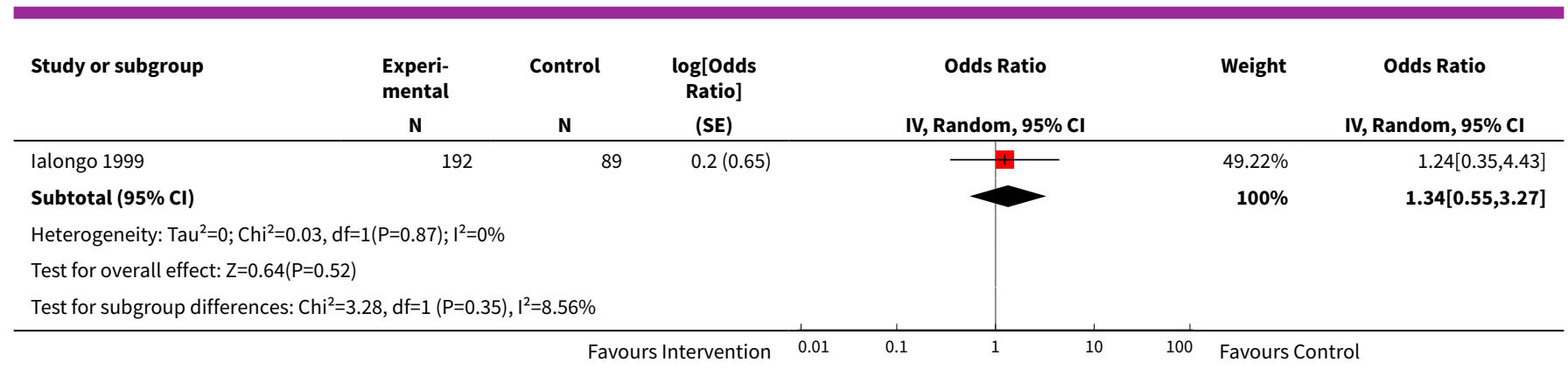

\section{Comparison 3. Binge drinking}

\begin{tabular}{lllll}
\hline Outcome or subgroup title & No. of studies & $\begin{array}{l}\text { No. of partici- } \\
\text { pants }\end{array}$ & Statistical method & Effect size \\
\hline $\begin{array}{l}1 \text { Drunkenness or Excess Drinking } \\
\text { (short-term) }\end{array}$ & 8 & & Odds Ratio (Random, 95\% Cl) & Subtotals only \\
\hline $\begin{array}{lllll}1.1 \text { Individual Targeted } \\
1.2 \text { School Universal }\end{array}$ & 3 & 250 & Odds Ratio (Random, 95\% Cl) & $0.97[0.68,1.37]$ \\
\hline $\begin{array}{l}2 \text { Drunkenness or Excess Drinking } \\
\text { (long-term) }\end{array}$ & 2 & 5494 & Odds Ratio (Random, 95\% Cl) & $0.66[0.41,1.06]$ \\
\hline $\begin{array}{l}2.1 \text { Family Targeted } \\
\text { 2.2 School Targeted }\end{array}$ & 1 & & Odds Ratio (Random, 95\% Cl) & Subtotals only \\
\hline
\end{tabular}

Analysis 3.1. Comparison 3 Binge drinking, Outcome 1 Drunkenness or Excess Drinking (short-term).

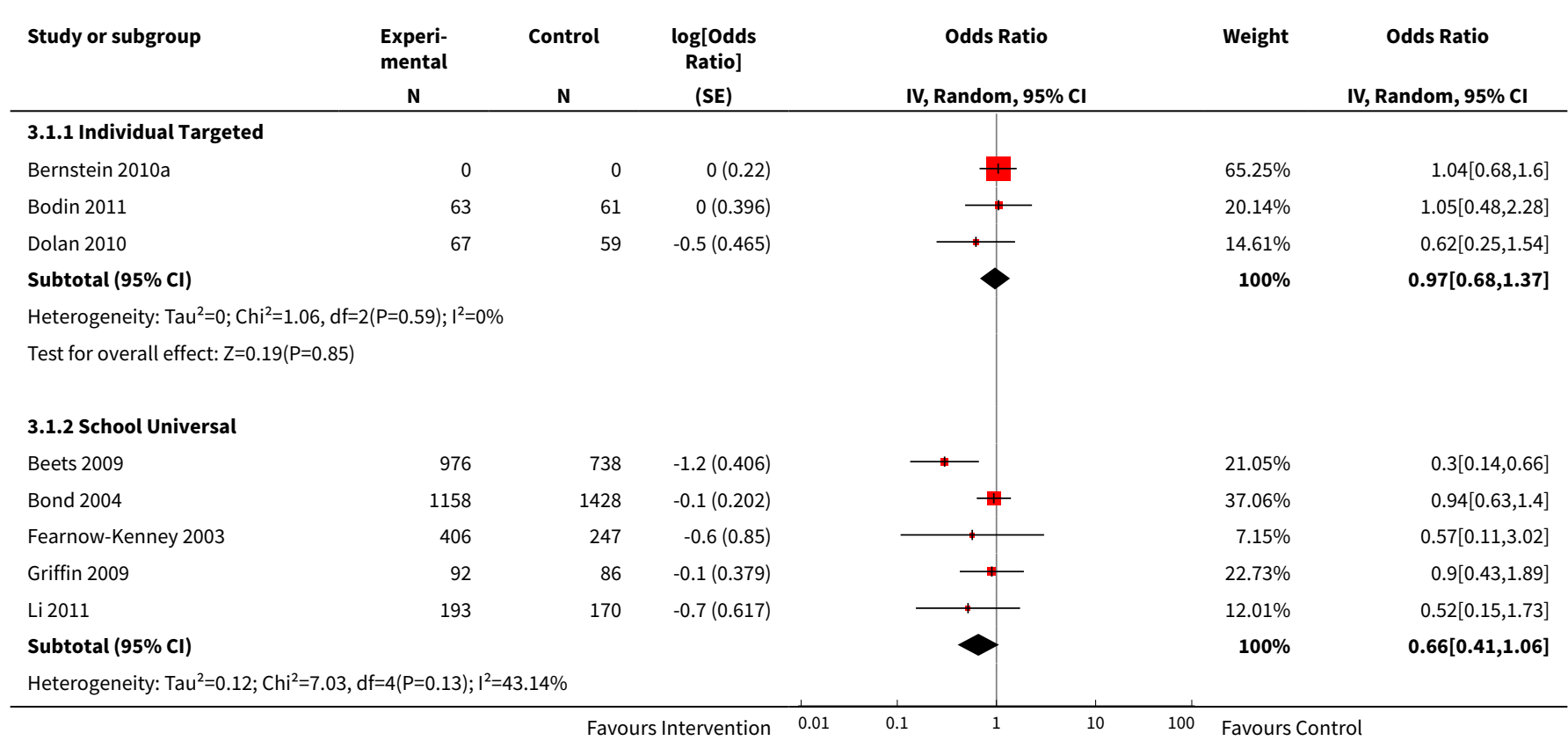




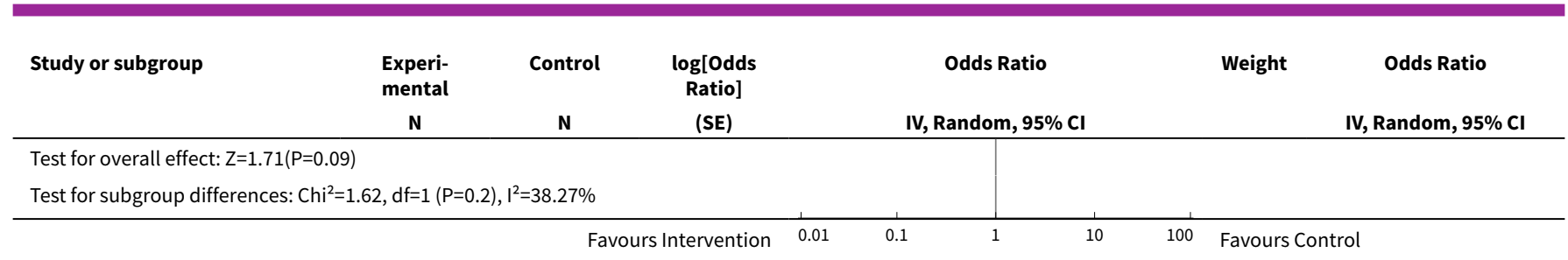

Analysis 3.2. Comparison 3 Binge drinking, Outcome 2 Drunkenness or Excess Drinking (long-term).

\begin{tabular}{|c|c|c|c|c|c|c|}
\hline Study or subgroup & $\begin{array}{c}\text { Inter- } \\
\text { vention } \\
\mathbf{N}\end{array}$ & Control & $\begin{array}{c}\text { log[Odds } \\
\text { Ratio] } \\
\text { (SE) } \\
\end{array}$ & $\begin{array}{c}\text { Odds Ratio } \\
\text { IV, Random, } 95 \% \text { CI }\end{array}$ & Weight & $\begin{array}{c}\text { Odds Ratio } \\
\text { IV, Random, } 95 \% \text { CI }\end{array}$ \\
\hline \multicolumn{7}{|l|}{ 3.2.1 Family Targeted } \\
\hline Bonds 2010 & 164 & 76 & $0.3(0.252)$ & & $100 \%$ & $1.3[0.79,2.13]$ \\
\hline Subtotal $(95 \% \mathrm{Cl})$ & & & & & $100 \%$ & $1.3[0.79,2.13]$ \\
\hline \multicolumn{7}{|l|}{ Heterogeneity: Not applicable } \\
\hline \multicolumn{7}{|l|}{ 3.2.2 School Targeted } \\
\hline $\begin{array}{l}\text { Conduct Problems Prevention } \\
\text { Research Group } 2010\end{array}$ & 354 & 351 & $-0.3(0.155)$ & & $100 \%$ & $0.75[0.55,1.02]$ \\
\hline Subtotal $(95 \% \mathrm{Cl})$ & & & & & $100 \%$ & $0.75[0.55,1.02]$ \\
\hline \multicolumn{7}{|l|}{ Heterogeneity: Not applicable } \\
\hline \multicolumn{7}{|c|}{ Test for subgroup differences: $\mathrm{Chi}^{2}=3.48, \mathrm{df}=1(\mathrm{P}=0.06), \mathrm{I}^{2}=71.27 \%$} \\
\hline
\end{tabular}

\section{Comparison 4. Illicit drug use}

\begin{tabular}{lllll}
\hline Outcome or subgroup title & No. of studies & $\begin{array}{l}\text { No. of partici- } \\
\text { pants }\end{array}$ & Statistical method & Effect size \\
\hline 1 Illicit drug use (short-term) & 11 & & Odds Ratio (Random, 95\% Cl) & Subtotals only \\
\hline 1.1 Individual Targeted & 3 & 638 & Odds Ratio (Random, 95\% Cl) & $0.94[0.71,1.25]$ \\
\hline 1.2 Family Targeted & 1 & 69 & Odds Ratio (Random, 95\% Cl) & $0.74[0.42,1.31]$ \\
\hline 1.3 School Targeted & 2 & 1299 & Odds Ratio (Random, 95\% Cl) & $0.96[0.79,1.18]$ \\
\hline 1.4 School Universal & 5 & 11058 & Odds Ratio (Random, 95\% Cl) & $0.74[0.55,1.00]$ \\
\hline 2 Illicit drug use (long-term) & 9 & & Odds Ratio (Random, 95\% Cl) & Subtotals only \\
\hline 2.1 Family Targeted & 4 & 2032 & Odds Ratio (Random, 95\% Cl) & $0.80[0.52,1.24]$ \\
\hline 2.2 School Targeted & 2 & 819 & Odds Ratio (Random, 95\% Cl) & $1.07[0.19,6.21]$ \\
\hline 2.3 School Universal & 3 & 3338 & Odds Ratio (Random, 95\% Cl) & $0.73[0.56,0.95]$ \\
\hline
\end{tabular}


Analysis 4.1. Comparison 4 Illicit drug use, Outcome 1 Illicit drug use (short-term).

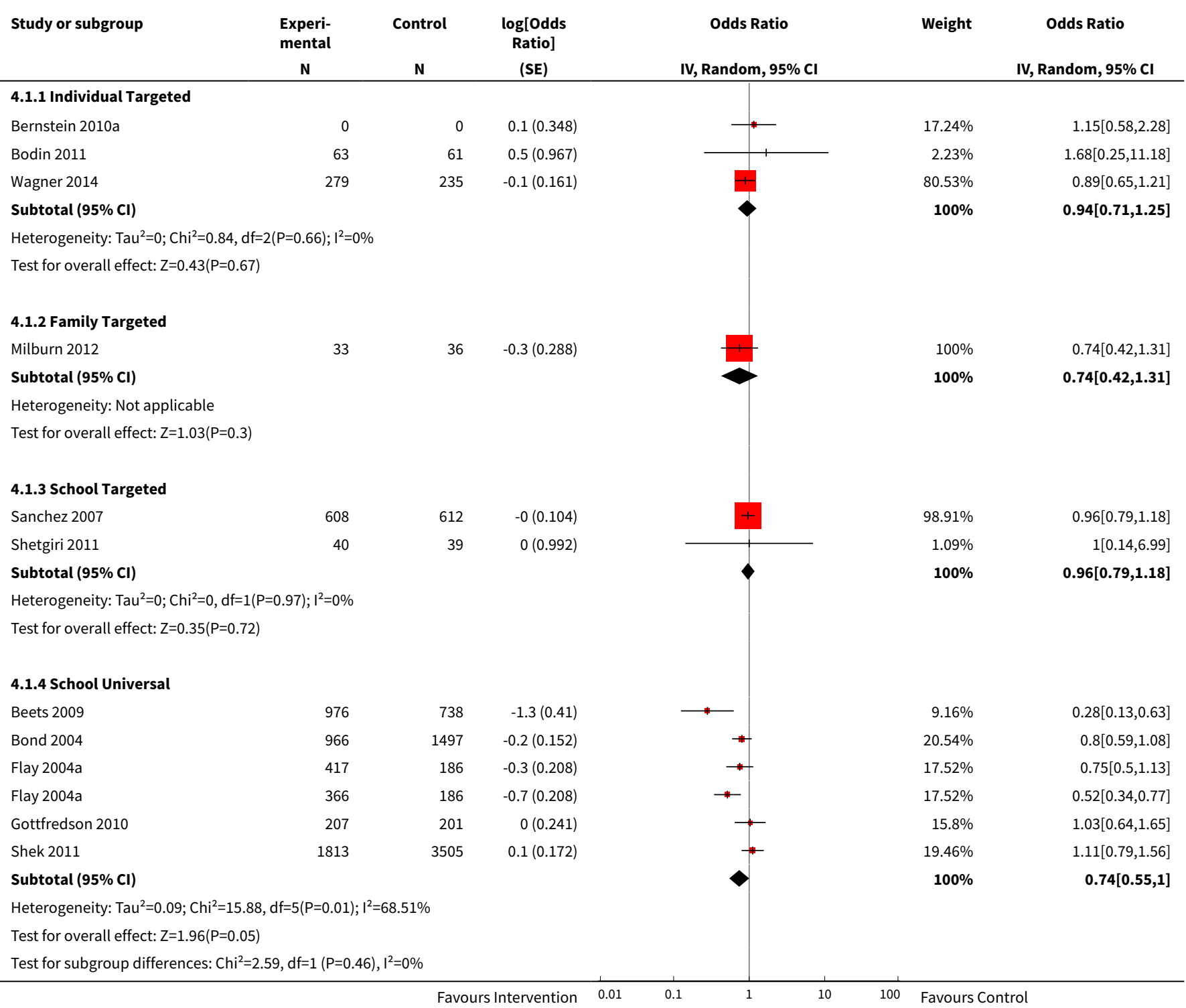

Analysis 4.2. Comparison 4 Illicit drug use, Outcome 2 Illicit drug use (long-term).

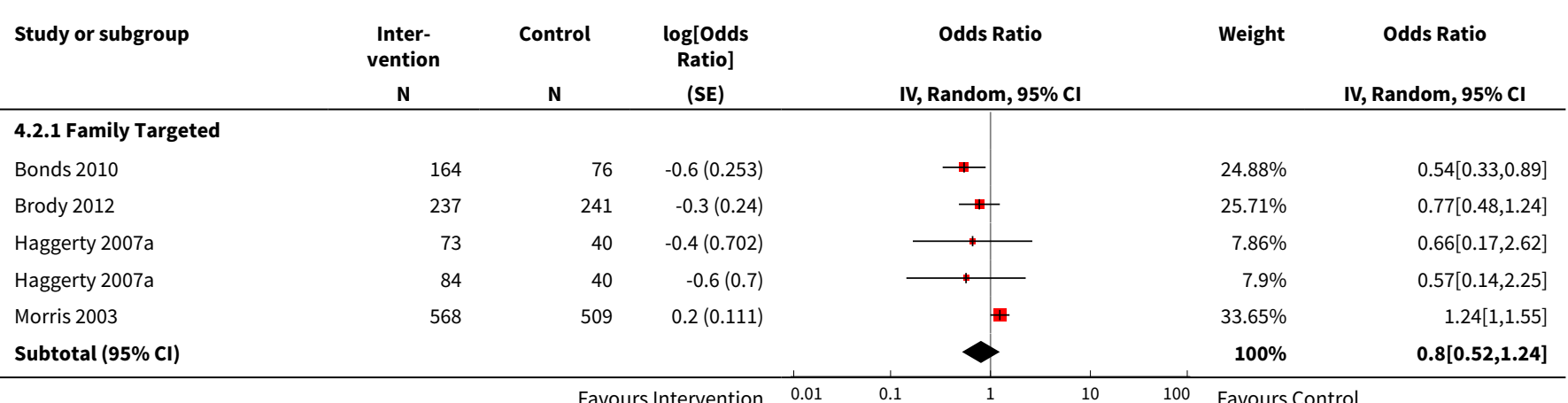




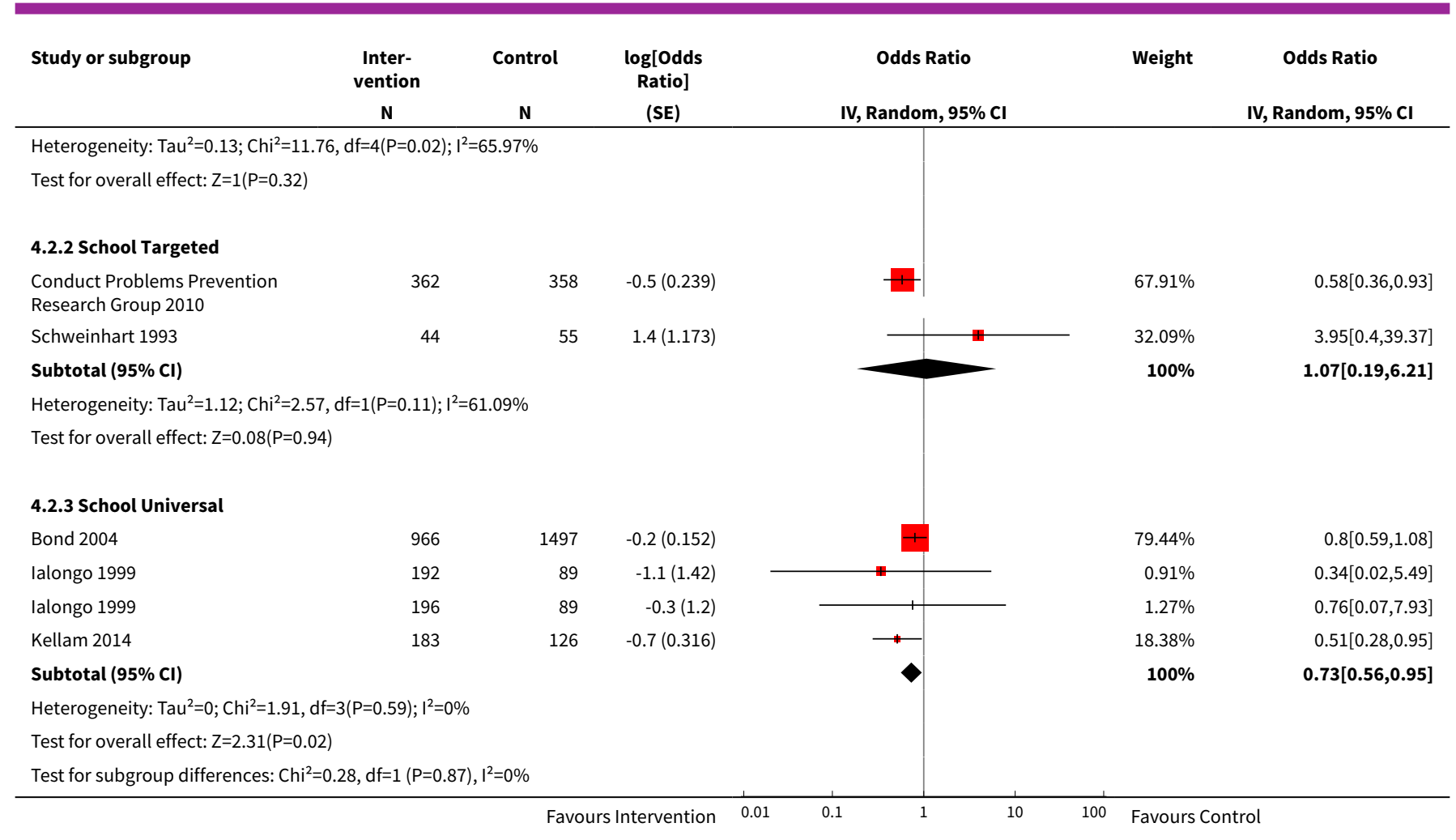

\section{Comparison 5. Cannabis use}

\begin{tabular}{lllll}
\hline Outcome or subgroup title & No. of studies & $\begin{array}{c}\text { No. of partici- } \\
\text { pants }\end{array}$ & Statistical method & Effect size \\
\hline 1 Cannabis Use (short-term) & 12 & & Odds Ratio (Random, 95\% Cl) & Subtotals only \\
\hline 1.1 Individual Targeted & 2 & 126 & Odds Ratio (Random, 95\% Cl) & $1.10[0.69,1.76]$ \\
\hline 1.2 Individual Universal & 2 & 362 & Odds Ratio (Random, 95\% Cl) & $0.69[0.46,1.04]$ \\
\hline 1.3 Family Targeted & 3 & 380 & Odds Ratio (Random, 95\% Cl) & $1.02[0.52,2.02]$ \\
\hline 1.4 School Universal & 5 & 4140 & Odds Ratio (Random, 95\% Cl) & $0.79[0.62,1.01]$ \\
\hline 2 Cannabis Use (long-term) & 6 & & Odds Ratio (Random, 95\% Cl) & Subtotals only \\
\hline 2.1 Family Targeted & 2 & 340 & Odds Ratio (Random, 95\% Cl) & $0.53[0.28,1.02]$ \\
\hline 2.2 Family Universal & 1 & 237 & Odds Ratio (Random, 95\% Cl) & $0.80[0.44,1.45]$ \\
\hline 2.3 School Targeted & 2 & 806 & Odds Ratio (Random, 95\% Cl) & $0.82[0.51,1.32]$ \\
\hline 2.4 School Universal & 1 & 566 & Odds Ratio (Random, 95\% Cl) & $1.13[0.40,3.21]$ \\
\hline
\end{tabular}


Analysis 5.1. Comparison 5 Cannabis use, Outcome 1 Cannabis Use (short-term).

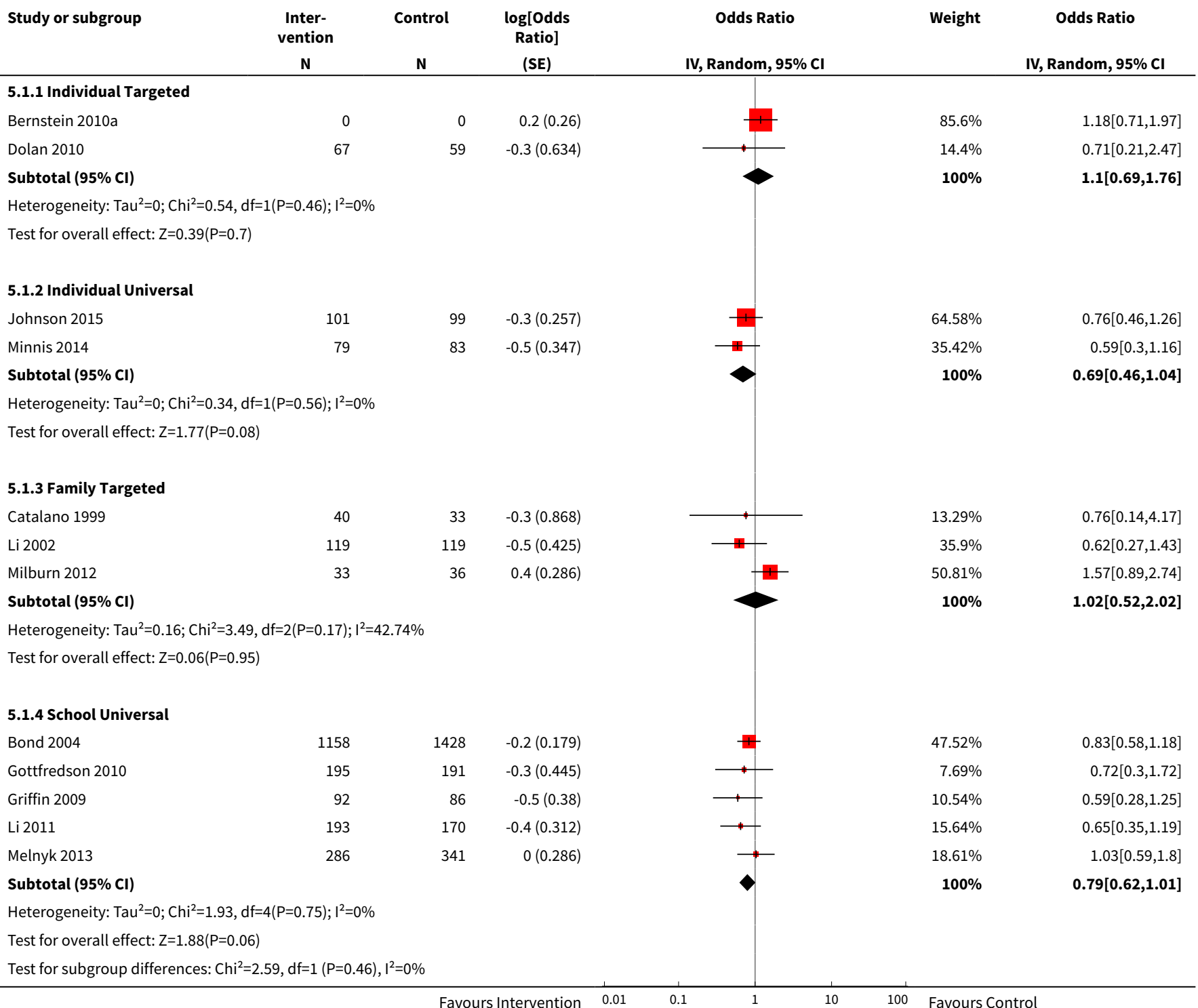

Analysis 5.2. Comparison 5 Cannabis use, Outcome 2 Cannabis Use (long-term).

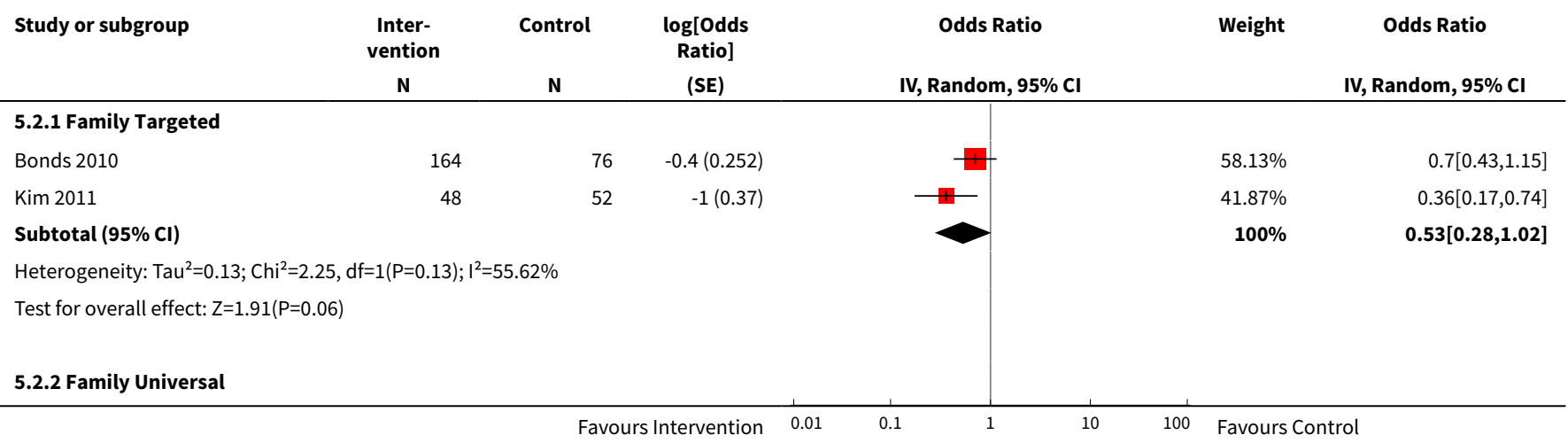




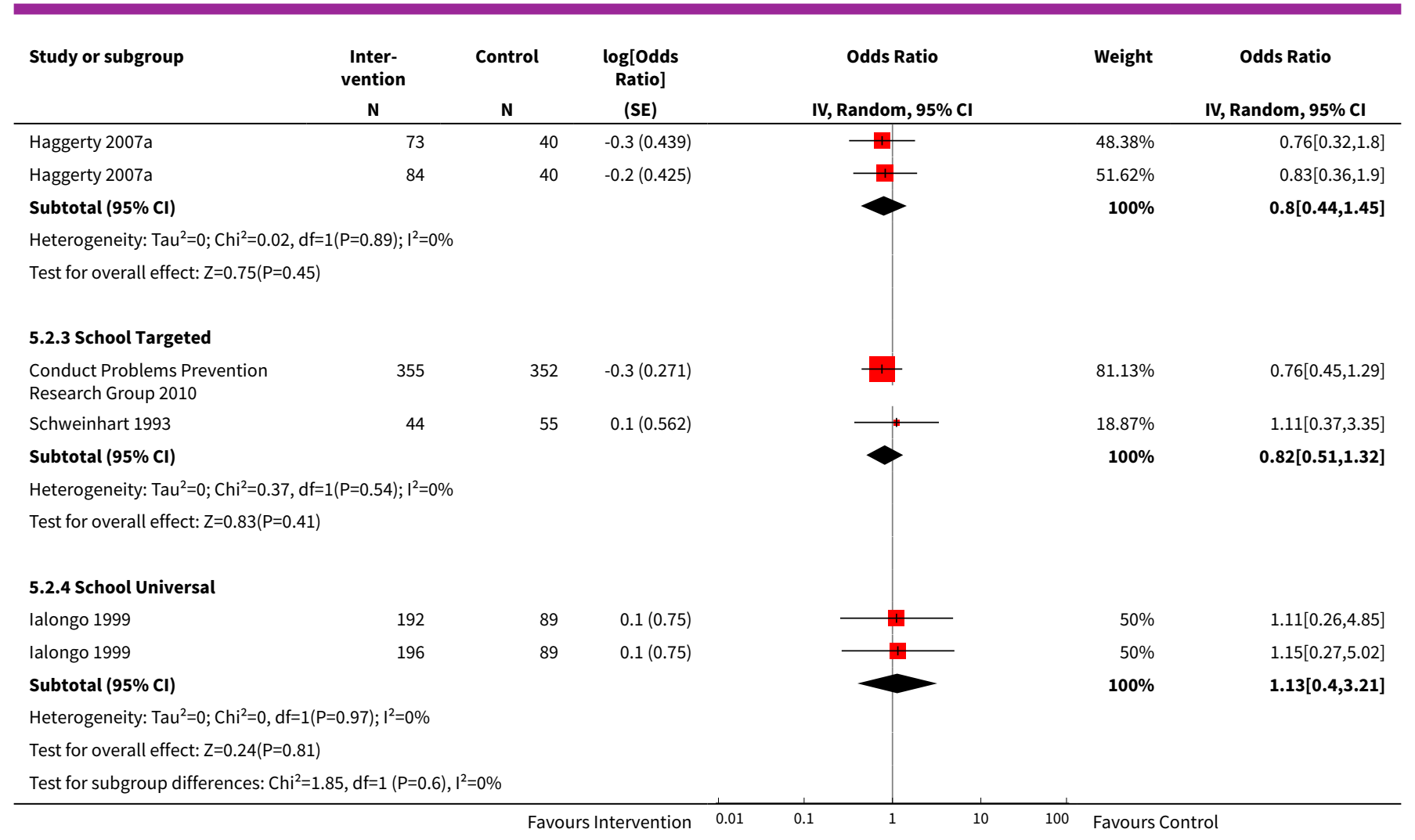

\section{Comparison 6. Alcohol, tobacco, and/or drug use}

\begin{tabular}{|c|c|c|c|c|}
\hline Outcome or subgroup title & No. of studies & $\begin{array}{l}\text { No. of partici- } \\
\text { pants }\end{array}$ & Statistical method & Effect size \\
\hline $\begin{array}{l}1 \text { Composite Substance Use } \\
\text { (short-term) }\end{array}$ & 7 & & Odds Ratio (Random, 95\% Cl) & Subtotals only \\
\hline 1.1 Family Targeted & 2 & 213 & Odds Ratio (Random, 95\% Cl) & $0.81[0.50,1.33]$ \\
\hline 1.2 School Targeted & 2 & 342 & Odds Ratio (Random, 95\% Cl) & $0.55[0.24,1.25]$ \\
\hline 1.3 School Universal & 3 & 7390 & Odds Ratio (Random, 95\% Cl) & $1.03[0.77,1.37]$ \\
\hline $\begin{array}{l}2 \text { Composite Substance Use } \\
\text { (long-term) }\end{array}$ & 6 & & Odds Ratio (Random, 95\% Cl) & Subtotals only \\
\hline 2.1 Family Targeted & 4 & 1622 & Odds Ratio (Random, 95\% Cl) & $0.69[0.47,1.03]$ \\
\hline 2.2 School Universal & 2 & 2145 & Odds Ratio (Random, 95\% Cl) & $1.09[0.94,1.27]$ \\
\hline
\end{tabular}


Analysis 6.1. Comparison 6 Alcohol, tobacco, and/or drug use, Outcome 1 Composite Substance Use (short-term). Study or subgroup

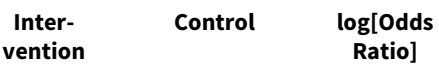
(SE)

Odds Ratio

$\mathbf{N}$

$\mathbf{N}$

IV, Random, $95 \% \mathrm{CI}$

Weight

Odds Ratio

\subsubsection{Family Targeted}

Pantin 2009

84

Schwinn 2014

31

$0.1(0.465)$

Subtotal $(95 \% \mathrm{CI})$

30

$0.1(0.465)$

Heterogeneity: $\mathrm{Tau}^{2}=0 ; \mathrm{Chi}^{2}=0.8, \mathrm{df}=1(\mathrm{P}=0.37) ; \mathrm{I}^{2}=0 \%$

Test for overall effect: $Z=0.84(P=0.4)$

\subsubsection{School Targeted}

Lochman 2003a

Lochman 2003a

Lochman 2003a

Lochman 2004a

Lochman 2004a

$\begin{array}{lll}54 & 18 & -1.3(0.55) \\ 53 & 18 & -0.8(0.544) \\ 53 & 18 & -1.7(0.56) \\ 42 & 22 & 0.2(0.478) \\ 42 & 22 & 0.4(0.479)\end{array}$

Subtotal $(95 \% \mathrm{CI})$

Heterogeneity: $\mathrm{Tau}^{2}=0.6 ; \mathrm{Chi}^{2}=12.87, \mathrm{df}=4(\mathrm{P}=0.01) ; \mathrm{I}^{2}=68.91 \%$

Test for overall effect: $Z=1.43(P=0.15)$

6.1.3 School Universal

$\begin{array}{lrrr}\text { Li } 2011 & 193 & 170 & -0.5(0.353) \\ \text { McNeal 2004 } & 916 & 739 & 0.2(0.188) \\ \text { Shek 2011 } & 1855 & 3517 & 0.1(0.171)\end{array}$

Subtotal $(95 \% \mathrm{Cl})$

Heterogeneity: $\mathrm{Tau}^{2}=0.02 ; \mathrm{Chi}^{2}=2.79, \mathrm{df}=2(\mathrm{P}=0.25) ; \mathrm{I}^{2}=28.22 \%$

Test for overall effect: $Z=0.19(P=0.85)$

Test for subgroup differences: $\mathrm{Chi}^{2}=2.34, \mathrm{df}=1(\mathrm{P}=0.31), \mathrm{I}^{2}=14.53 \%$

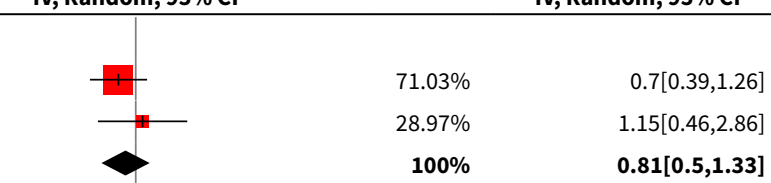

$\begin{array}{lllllll}\text { Favours Intervention } & 0.01 & 0.1 & 1 & 10 & 100 & \text { Favours Control }\end{array}$

Analysis 6.2. Comparison 6 Alcohol, tobacco, and/or drug use, Outcome 2 Composite Substance Use (long-term).

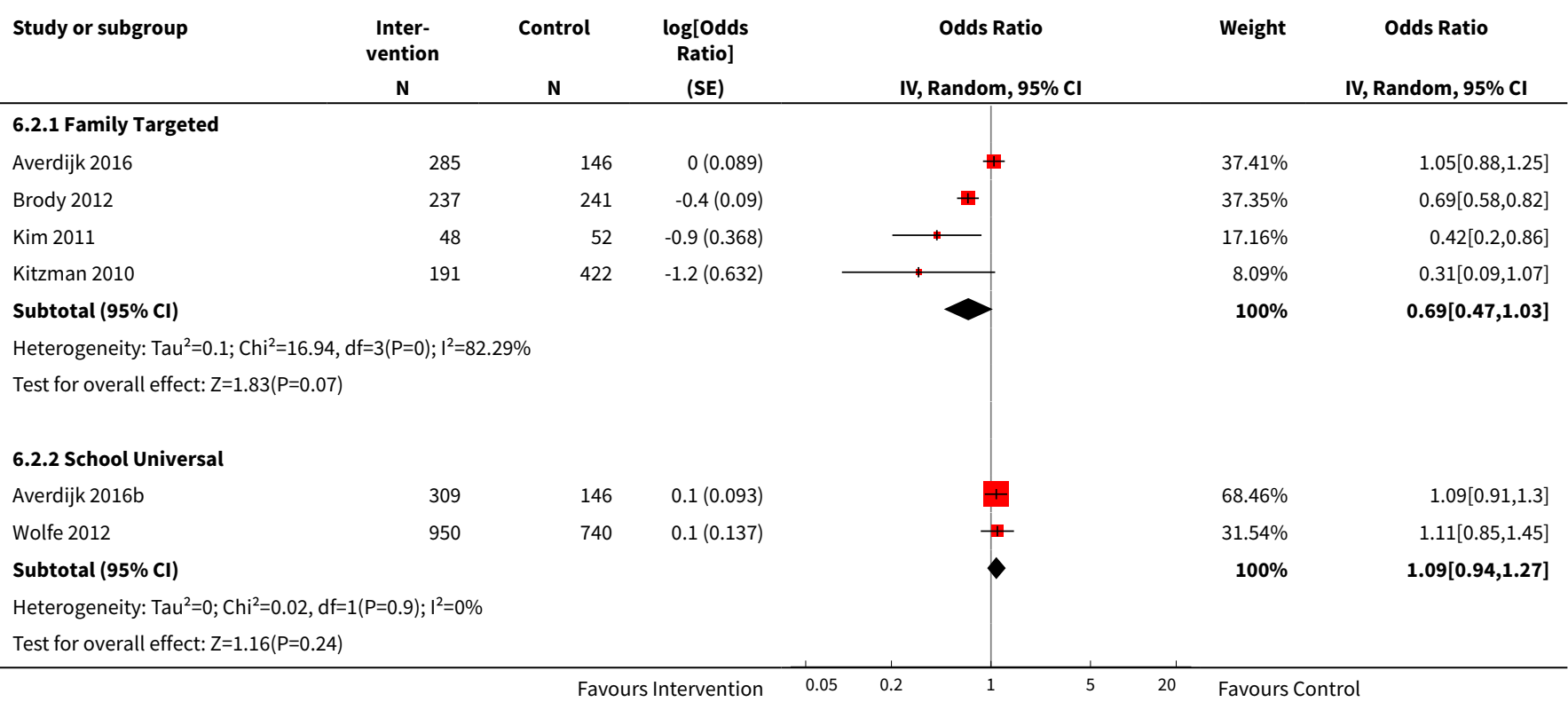




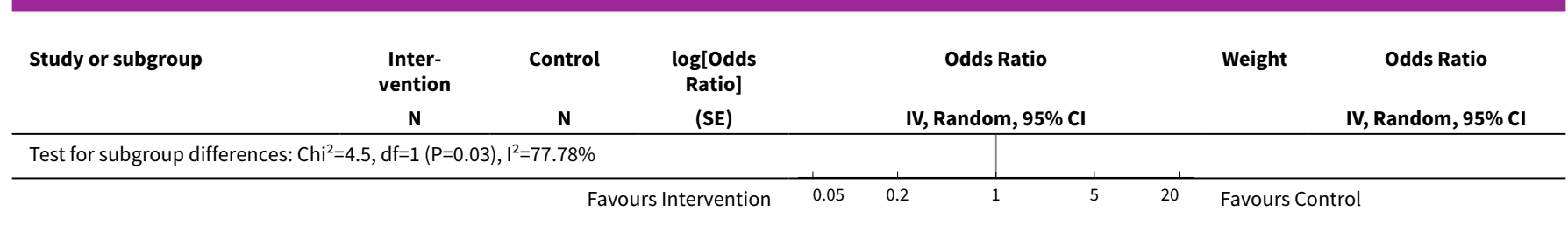

\section{Comparison 7. Antisocial behaviour and offending}

\begin{tabular}{|c|c|c|c|c|}
\hline Outcome or subgroup title & No. of studies & $\begin{array}{l}\text { No. of partici- } \\
\text { pants }\end{array}$ & Statistical method & Effect size \\
\hline $\begin{array}{l}1 \text { Antisocial Behaviour and } \\
\text { Offending - Any (short-term) }\end{array}$ & 27 & & Odds Ratio (Random, 95\% Cl) & Subtotals only \\
\hline 1.1 Individual Targeted & 4 & 764 & Odds Ratio (Random, 95\% Cl) & $1.21[0.92,1.60]$ \\
\hline 1.2 Individual Universal & 1 & 200 & Odds Ratio (Random, 95\% Cl) & $1.02[0.62,1.69]$ \\
\hline 1.3 Family Targeted & 6 & 772 & Odds Ratio (Random, 95\% Cl) & $0.84[0.57,1.24]$ \\
\hline 1.4 Family Universal & 1 & 306 & Odds Ratio (Random, 95\% Cl) & $0.87[0.56,1.35]$ \\
\hline 1.5 School Targeted & 3 & 1531 & Odds Ratio (Random, 95\% Cl) & $0.78[0.59,1.05]$ \\
\hline 1.6 School Universal & 12 & 20756 & Odds Ratio (Random, 95\% Cl) & $0.81[0.66,0.98]$ \\
\hline 2 Violent Offences & 13 & & Odds Ratio (Random, 95\% Cl) & Subtotals only \\
\hline 2.1 Individual Targeted & 2 & 514 & Odds Ratio (Random, 95\% Cl) & $1.11[0.56,2.17]$ \\
\hline 2.2 Family Targeted & 1 & 238 & Odds Ratio (Random, 95\% Cl) & $0.95[0.49,1.84]$ \\
\hline 2.3 Family Universal & 1 & 306 & Odds Ratio (Random, 95\% Cl) & $0.87[0.56,1.35]$ \\
\hline 2.4 School Targeted & 1 & 158 & Odds Ratio (Random, 95\% Cl) & $0.60[0.31,1.16]$ \\
\hline 2.5 School Universal & 8 & 11347 & Odds Ratio (Random, 95\% Cl) & $0.86[0.69,1.07]$ \\
\hline $\begin{array}{l}3 \text { School or General Delin- } \\
\text { quency }\end{array}$ & 14 & & Odds Ratio (Random, 95\% Cl) & Subtotals only \\
\hline 3.1 Individual Targeted & 2 & 250 & Odds Ratio (Random, 95\% Cl) & $1.07[0.61,1.89]$ \\
\hline 3.2 Family Targeted & 4 & 598 & Odds Ratio (Random, 95\% Cl) & $0.80[0.54,1.20]$ \\
\hline 3.3 School Targeted & 3 & 1573 & Odds Ratio (Random, 95\% Cl) & $0.79[0.59,1.06]$ \\
\hline 3.4 School Universal & 5 & 10113 & Odds Ratio (Random, 95\% Cl) & $0.88[0.77,1.00]$ \\
\hline $\begin{array}{l}4 \text { Antisocial Behaviour and } \\
\text { Offending - Any (long term) }\end{array}$ & 11 & & Odds Ratio (Random, 95\% Cl) & Subtotals only \\
\hline 4.1 Family Targeted & 5 & 2486 & Odds Ratio (Random, 95\% Cl) & $0.74[0.54,1.03]$ \\
\hline 4.2 Family Universal & 1 & 304 & Odds Ratio (Random, 95\% Cl) & $0.67[0.43,1.04]$ \\
\hline
\end{tabular}




\begin{tabular}{lllll}
\hline Outcome or subgroup title & No. of studies & $\begin{array}{l}\text { No. of partici- } \\
\text { pants }\end{array}$ & Statistical method & Effect size \\
\hline 4.3 School Targeted & 3 & 1177 & Odds Ratio (Random, 95\% Cl) & $0.71[0.46,1.10]$ \\
\hline 4.4 School Universal & 2 & 4146 & Odds Ratio (Random, 95\% Cl) & $0.91[0.63,1.31]$ \\
\hline
\end{tabular}

Analysis 7.1. Comparison 7 Antisocial behaviour and offending, Outcome 1 Antisocial Behaviour and Offending - Any (short-term).

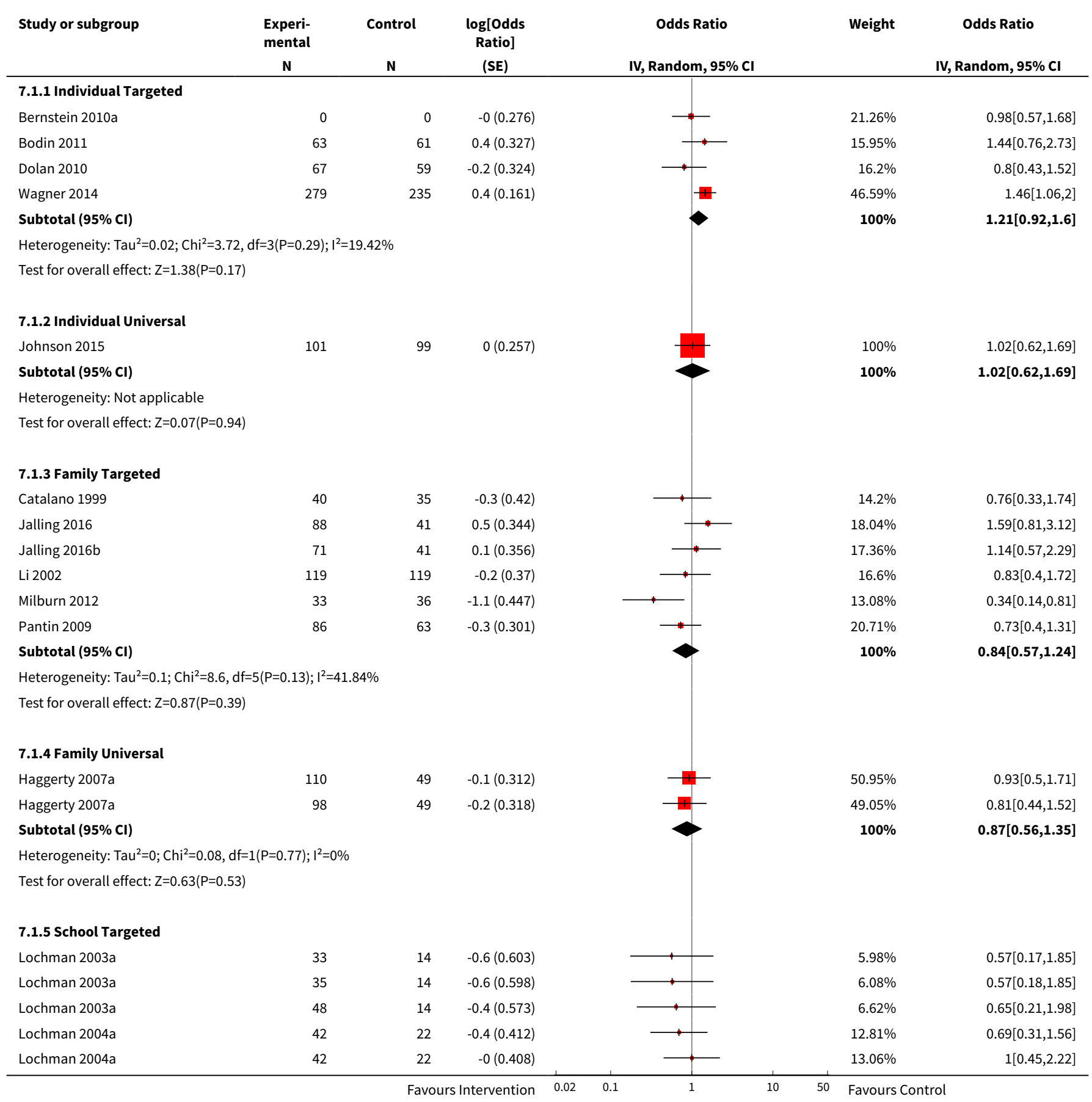




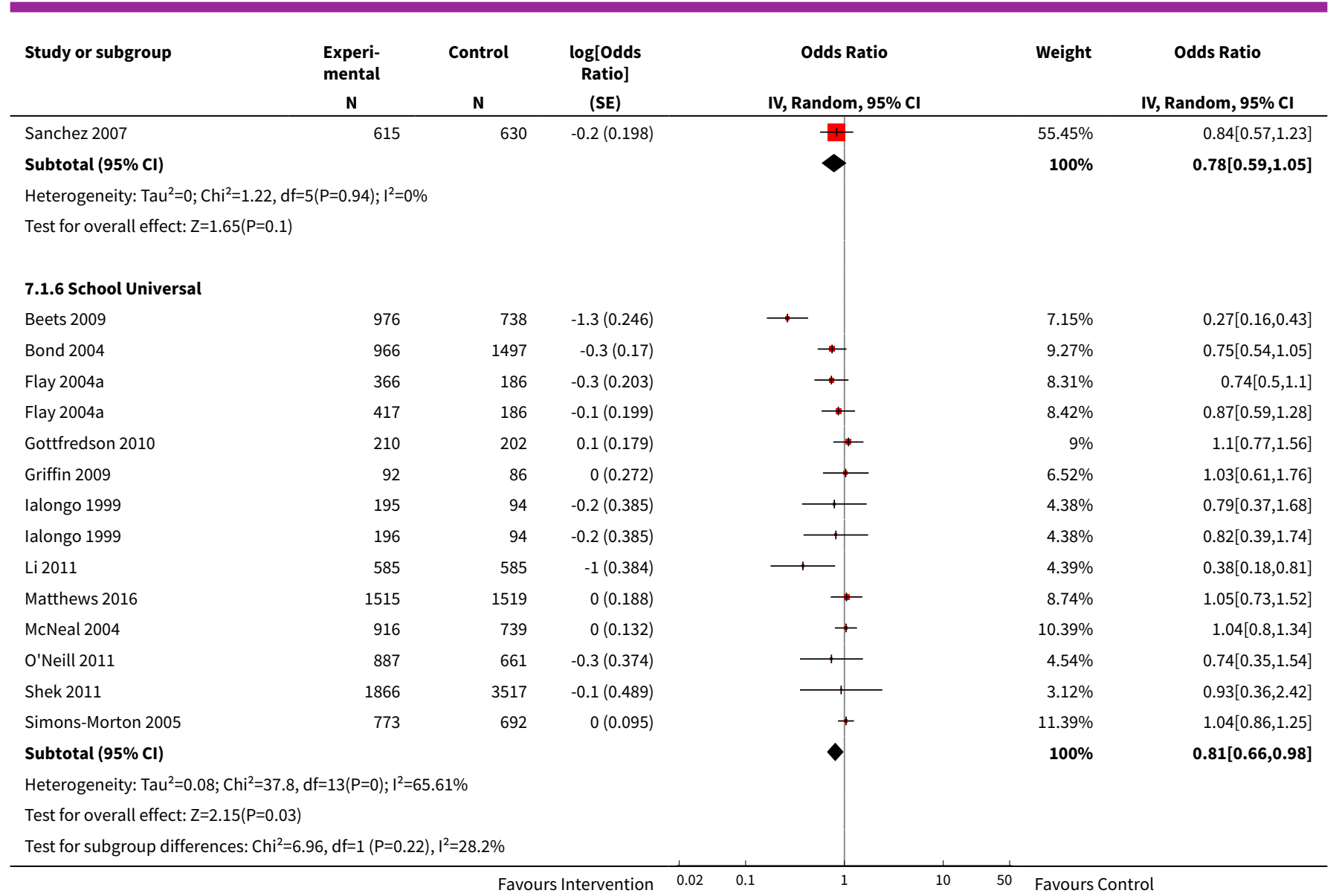

Analysis 7.2. Comparison 7 Antisocial behaviour and offending, Outcome 2 Violent Offences.

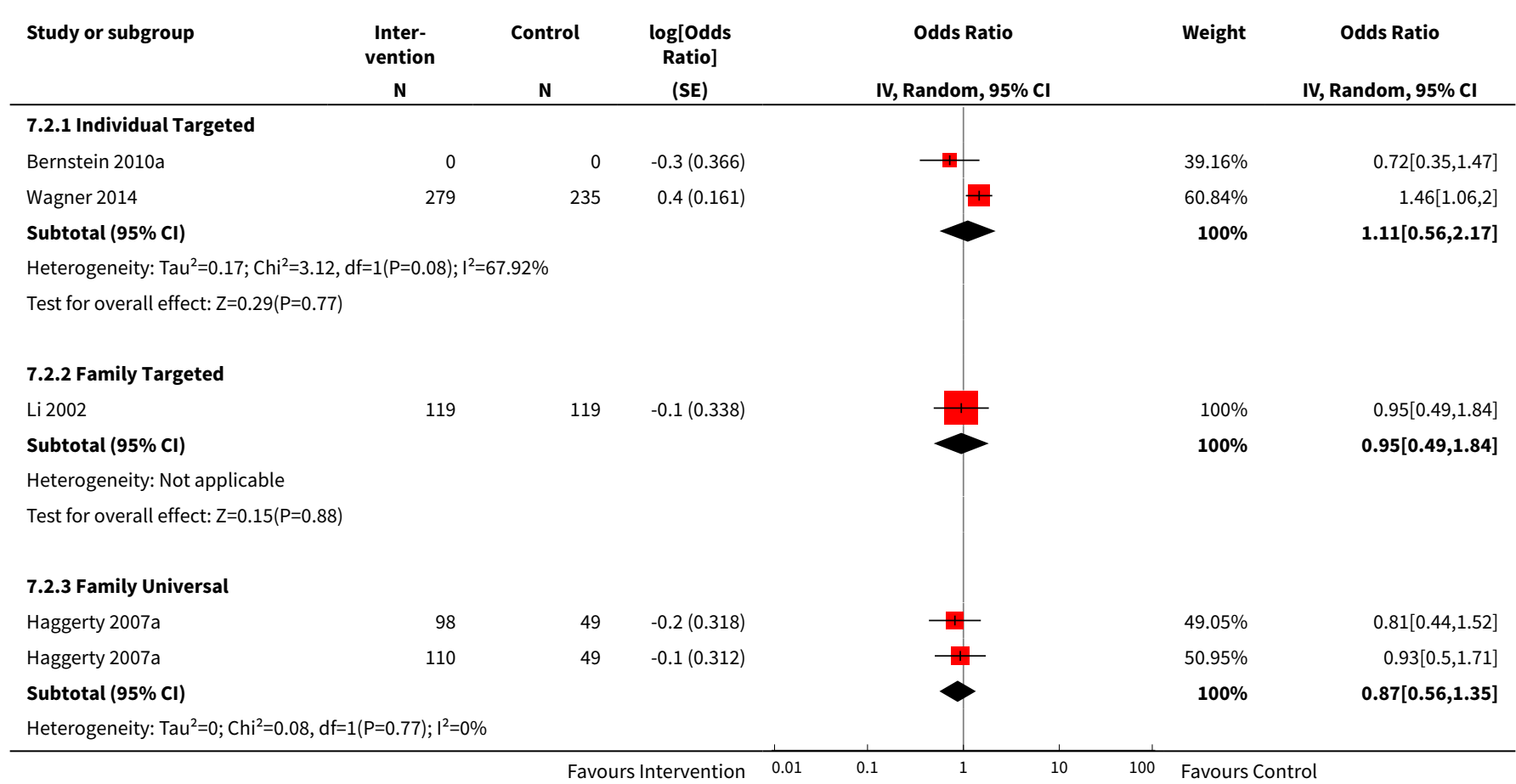




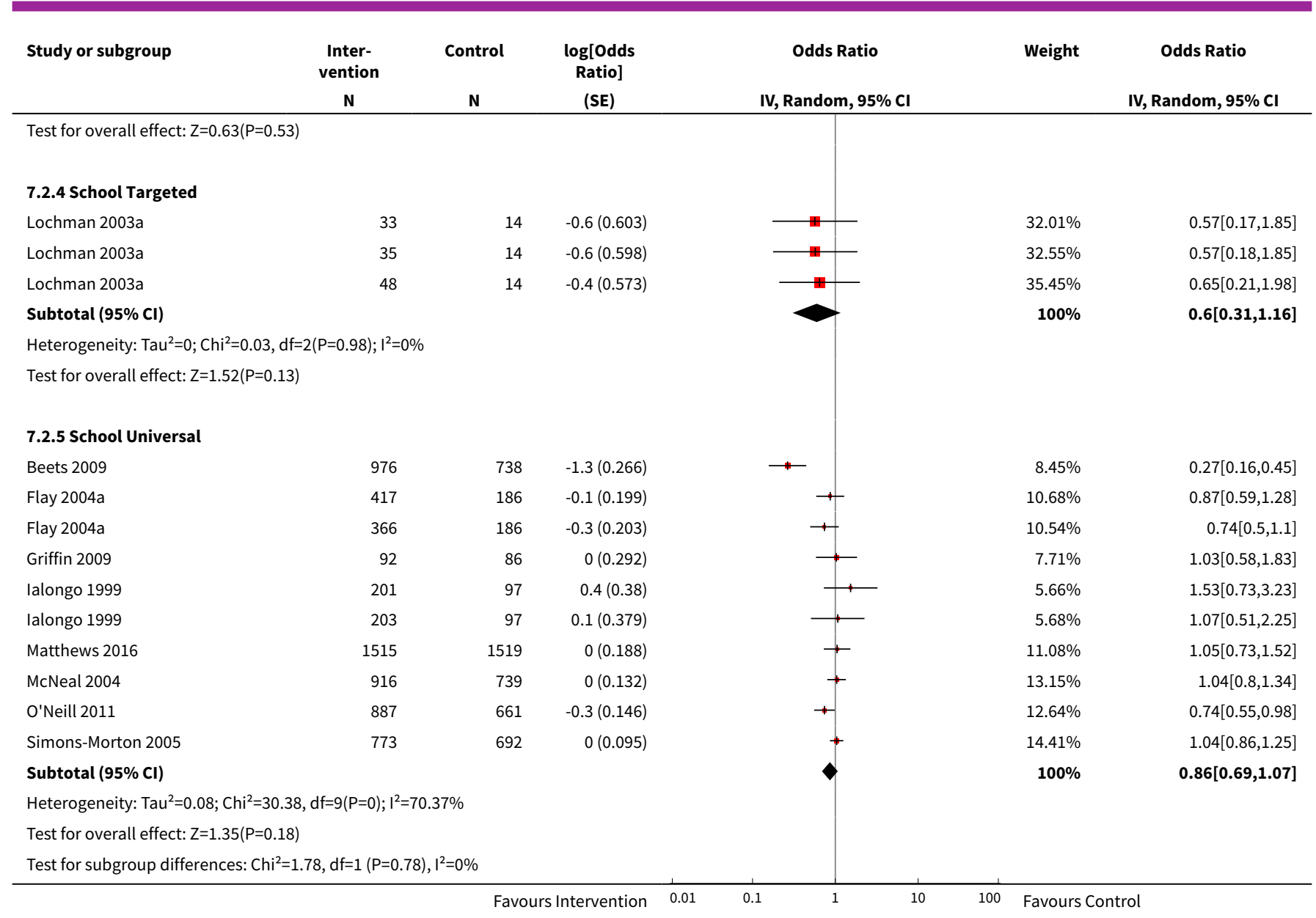

Analysis 7.3. Comparison 7 Antisocial behaviour and offending, Outcome 3 School or General Delinquency.

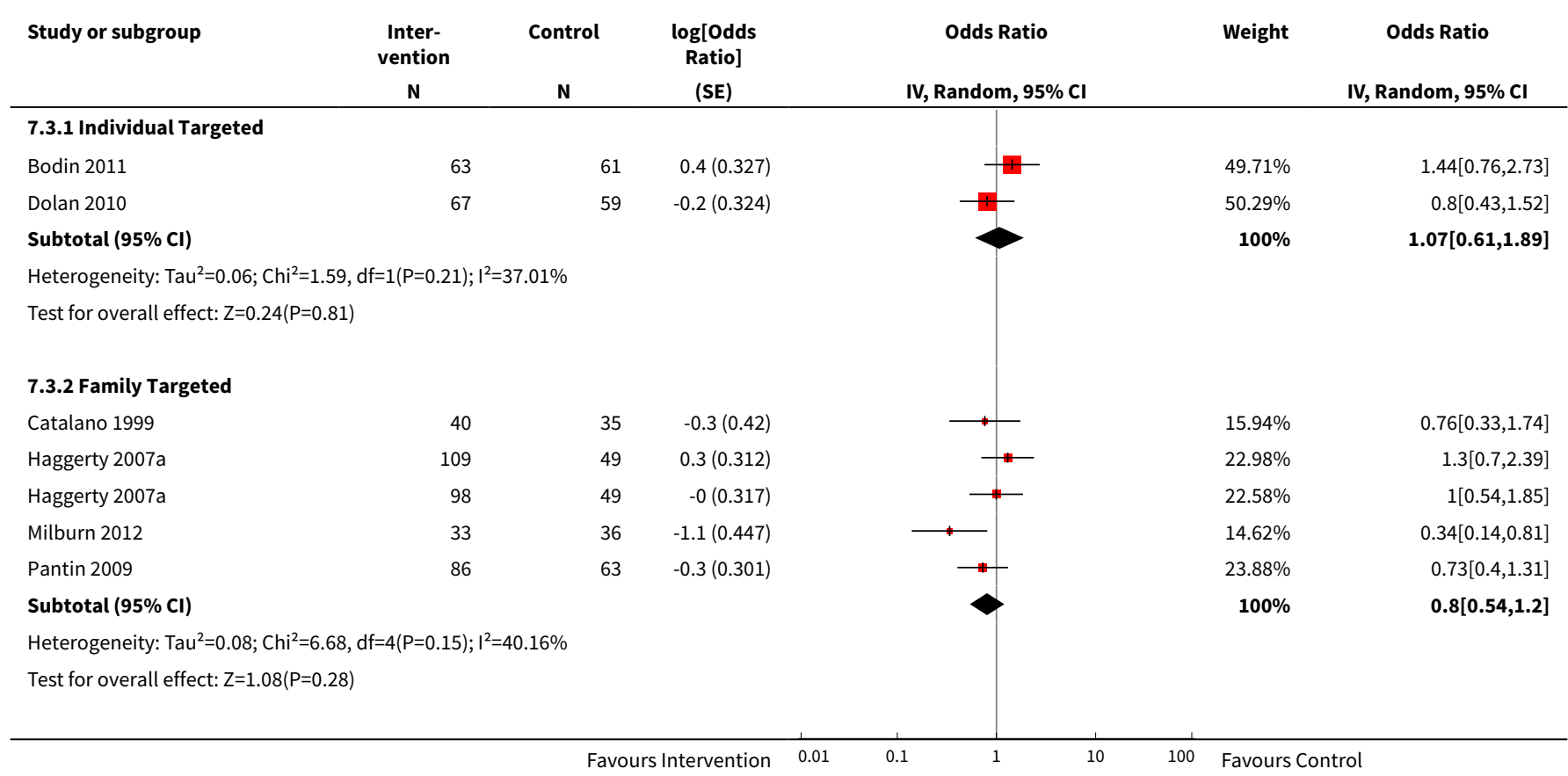




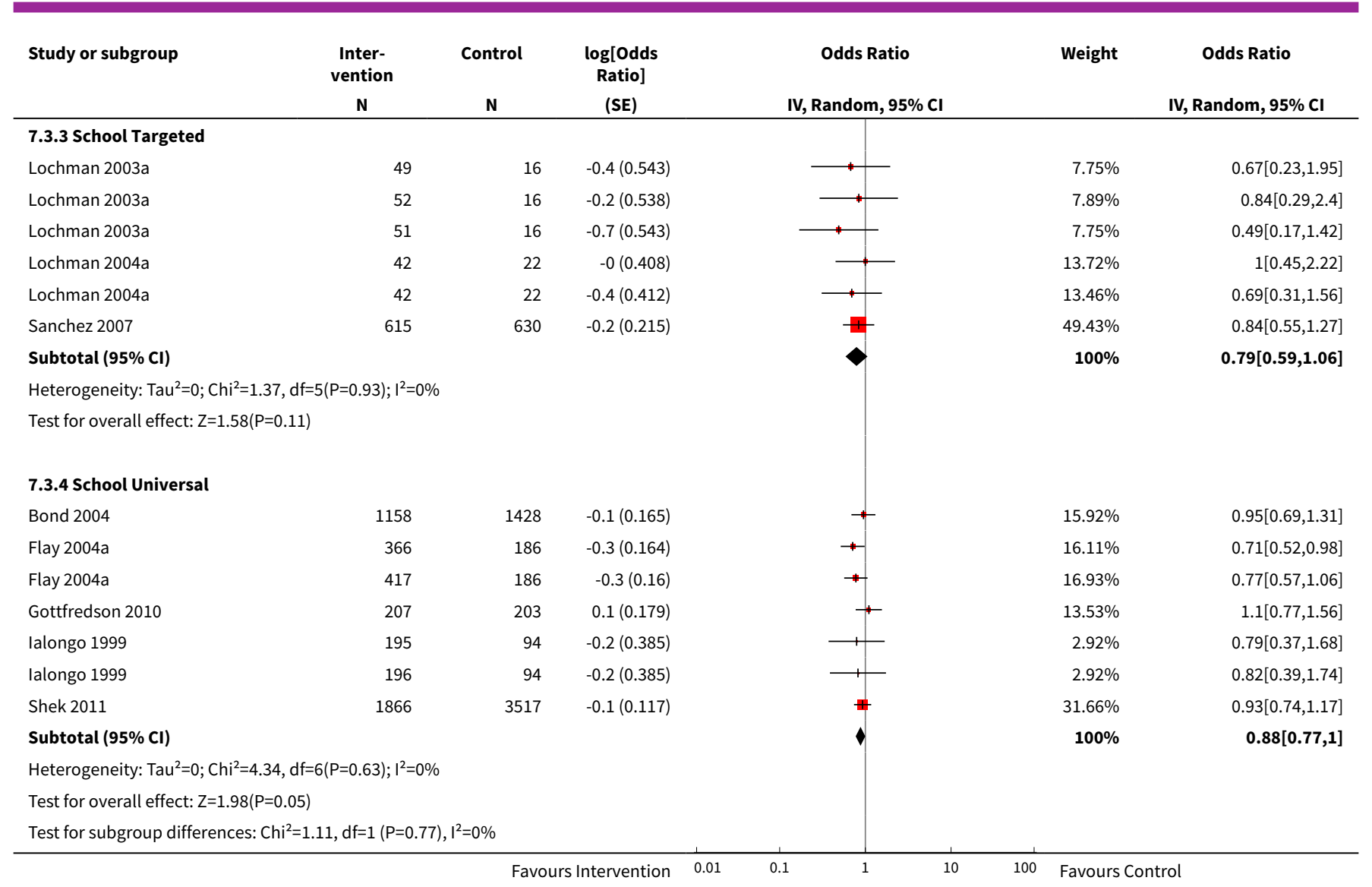

Analysis 7.4. Comparison 7 Antisocial behaviour and offending, Outcome 4 Antisocial Behaviour and Offending - Any (long term).

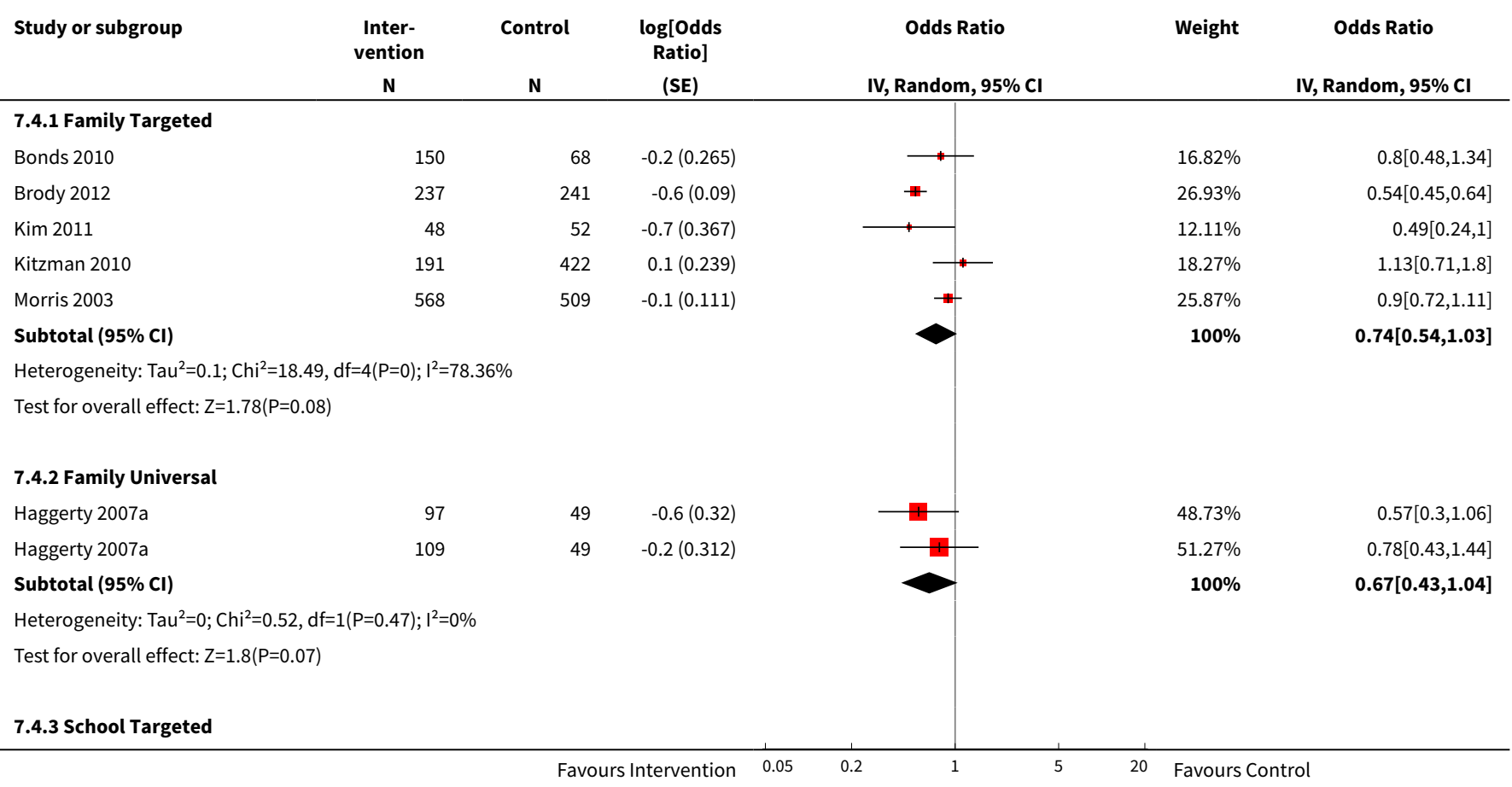




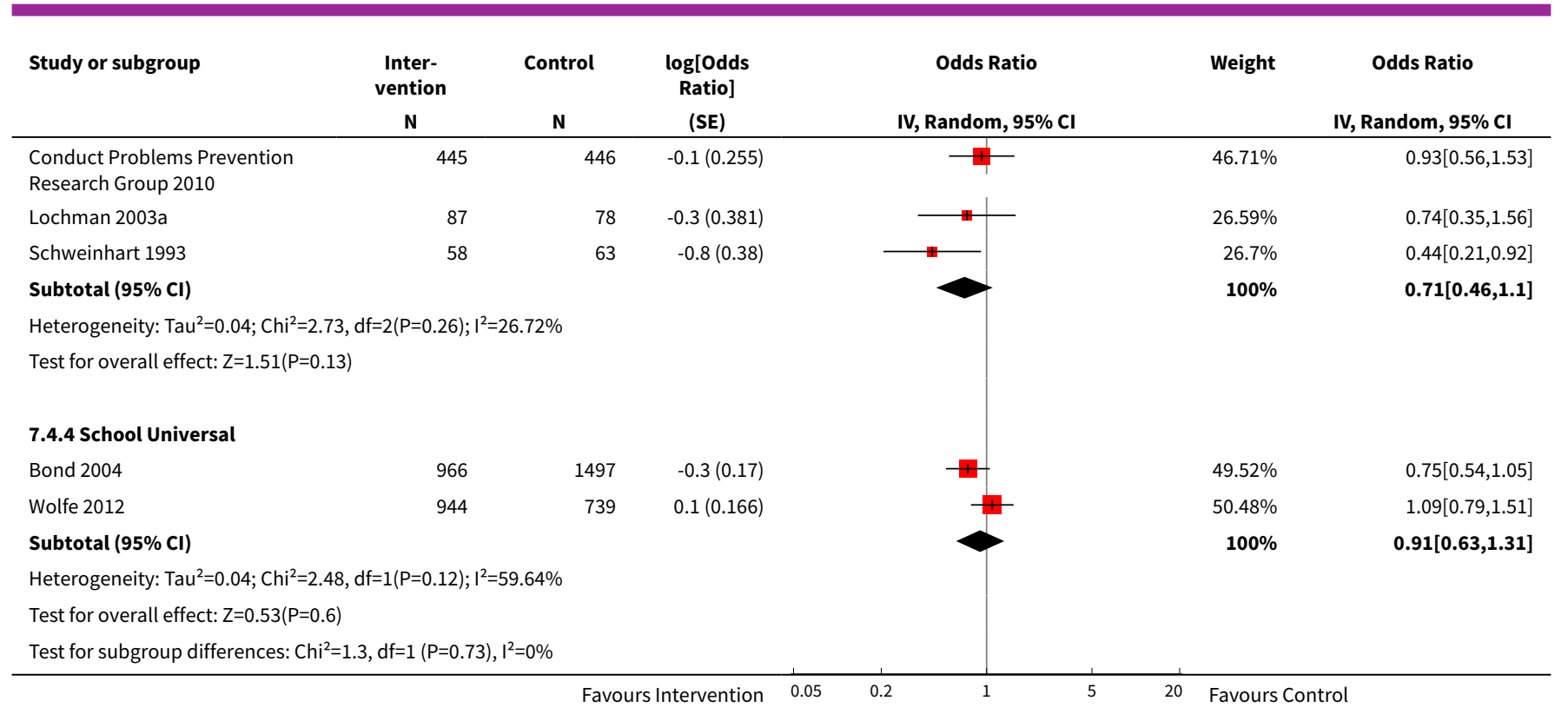

\section{Comparison 8. Vehicle-related risk behaviours}

\begin{tabular}{lllll}
\hline Outcome or subgroup title & No. of studies & $\begin{array}{l}\text { No. of partici- } \\
\text { pants }\end{array}$ & Statistical method & Effect size \\
\hline $\begin{array}{l}1 \text { Driving Under the Influence of Alco- } \\
\text { hol and/or Drugs }\end{array}$ & 2 & & $\begin{array}{l}\text { Odds Ratio (Random, 95\% } \\
\text { Cl) }\end{array}$ & Subtotals only \\
\hline $\begin{array}{l}1.1 \text { Individual Targeted } \\
\text { n }\end{array}$ & 2 & 94 & $\begin{array}{l}\text { Odds Ratio (Random, 95\% } \\
\text { Cl) }\end{array}$ & $0.59[0.14,2.48]$ \\
\hline
\end{tabular}

Analysis 8.1. Comparison 8 Vehicle-related risk behaviours, Outcome 1 Driving Under the Influence of Alcohol and/or Drugs.

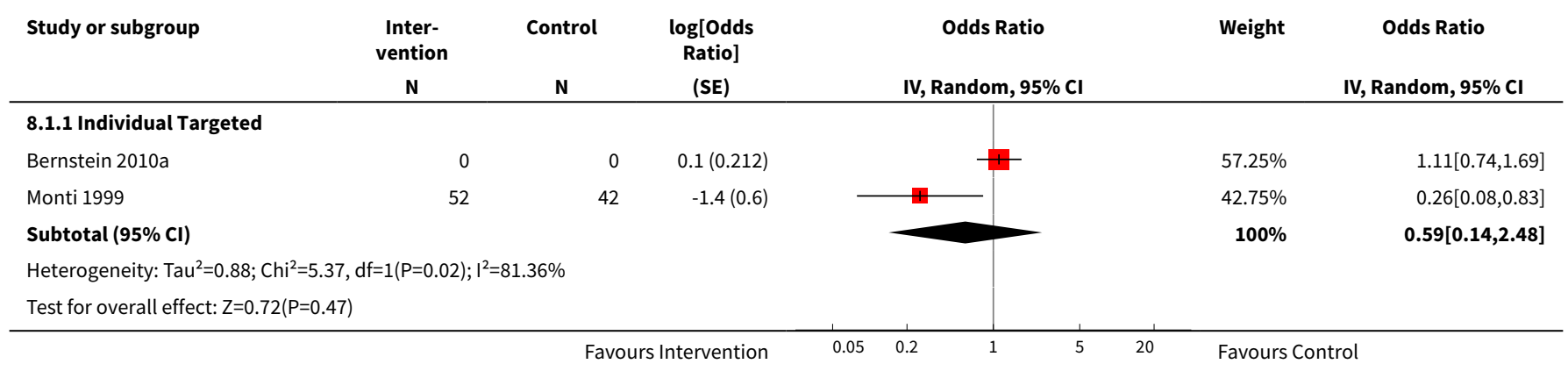


Comparison 9. Sexual risk behaviours

\begin{tabular}{|c|c|c|c|c|}
\hline Outcome or subgroup title & No. of studies & $\begin{array}{l}\text { No. of partici- } \\
\text { pants }\end{array}$ & Statistical method & Effect size \\
\hline $\begin{array}{l}1 \text { Sexual Risk Behaviour } \\
\text { (short-term) }\end{array}$ & 12 & & Odds Ratio (Random, 95\% Cl) & Subtotals only \\
\hline 1.1 Individual Targeted & 2 & 494 & Odds Ratio (Random, 95\% Cl) & $0.73[0.49,1.08]$ \\
\hline 1.2 Individual Universal & 1 & 162 & Odds Ratio (Random, 95\% Cl) & $0.42[0.14,1.25]$ \\
\hline 1.3 Family Targeted & 3 & 371 & Odds Ratio (Random, 95\% Cl) & $0.89[0.55,1.44]$ \\
\hline 1.4 School Universal & 6 & 12633 & Odds Ratio (Random, 95\% Cl) & $0.83[0.61,1.12]$ \\
\hline $\begin{array}{l}2 \text { Sexual Risk Behaviour } \\
\text { (long-term) }\end{array}$ & 8 & & Odds Ratio (Random, 95\% Cl) & Subtotals only \\
\hline 2.1 Individual Targeted & 1 & 461 & Odds Ratio (Random, 95\% Cl) & $0.93[0.64,1.35]$ \\
\hline 2.2 Family Targeted & 2 & 318 & Odds Ratio (Random, 95\% Cl) & $0.47[0.31,0.71]$ \\
\hline 2.3 Family Universal & 1 & 237 & Odds Ratio (Random, 95\% Cl) & $1.12[0.64,1.96]$ \\
\hline 2.4 School Targeted & 1 & 650 & Odds Ratio (Random, 95\% Cl) & $0.62[0.47,0.82]$ \\
\hline 2.5 School Universal & 3 & 3391 & Odds Ratio (Random, 95\% Cl) & $0.74[0.50,1.09]$ \\
\hline
\end{tabular}

\section{Analysis 9.1. Comparison 9 Sexual risk behaviours, Outcome 1 Sexual Risk Behaviour (short-term).}

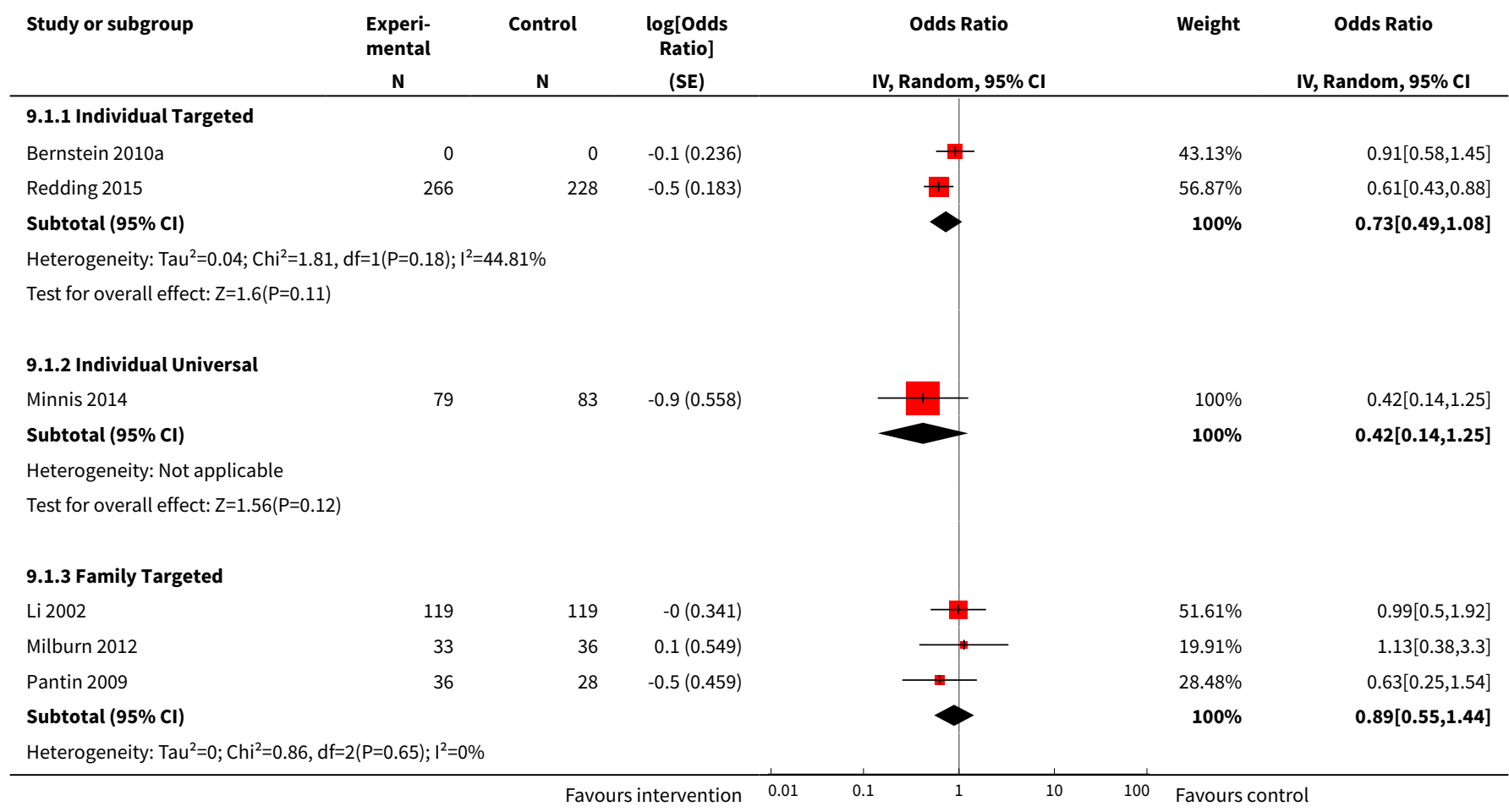




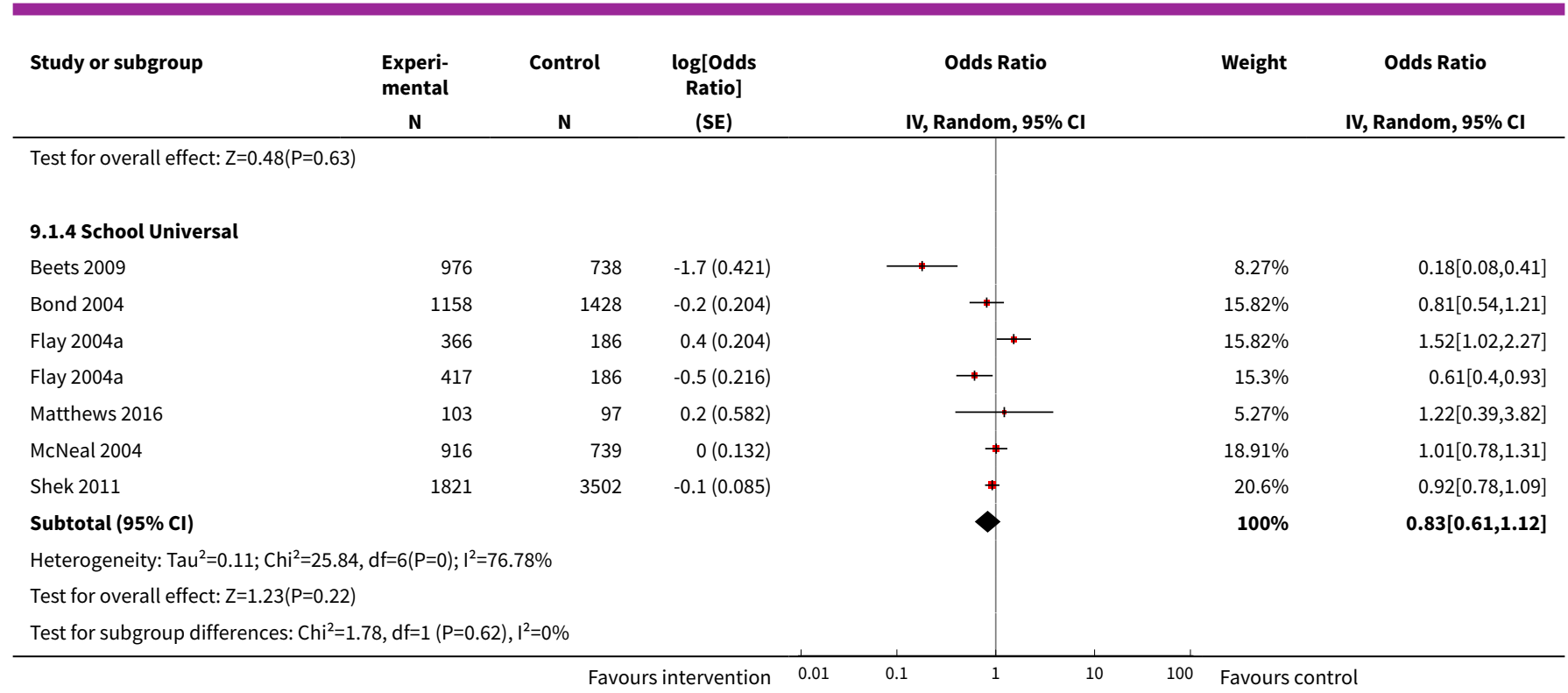

Analysis 9.2. Comparison 9 Sexual risk behaviours, Outcome 2 Sexual Risk Behaviour (long-term).

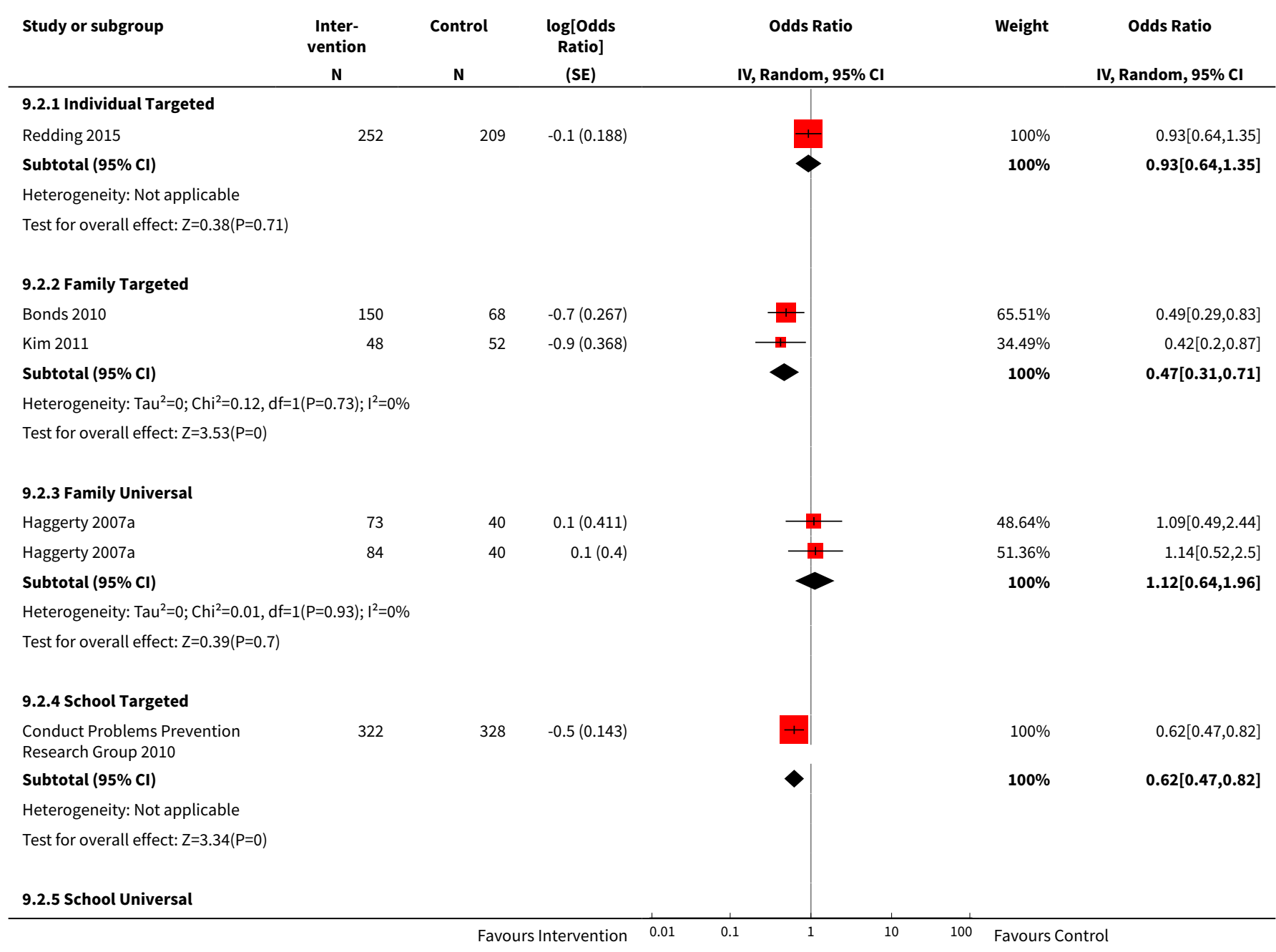




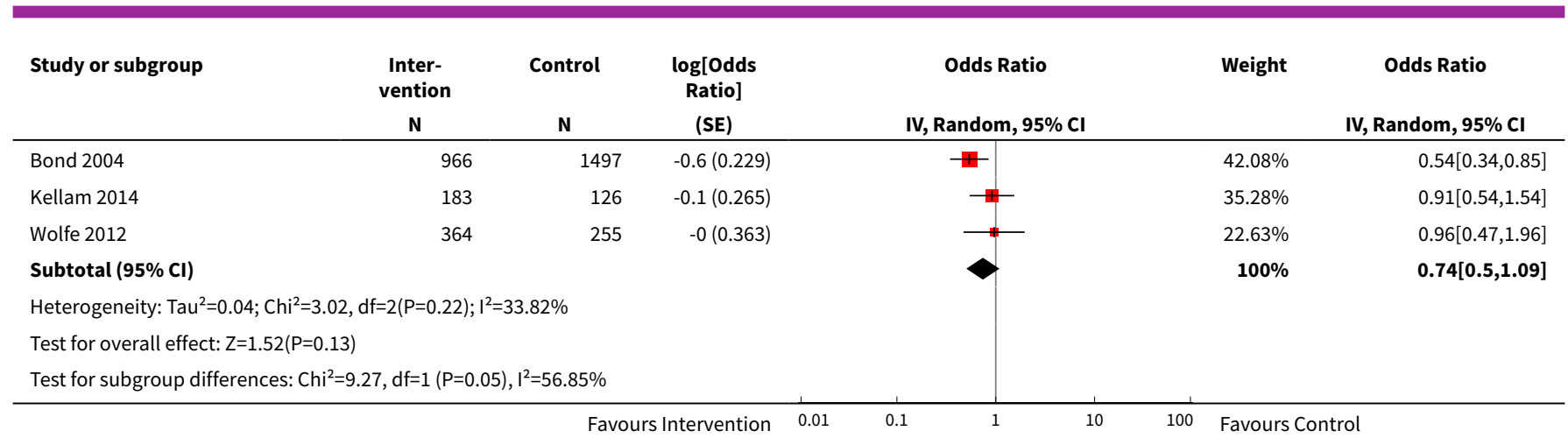

\section{Comparison 10. Physical activity}

\begin{tabular}{lllll}
\hline Outcome or subgroup title & No. of studies & $\begin{array}{l}\text { No. of partici- } \\
\text { pants }\end{array}$ & Statistical method & Effect size \\
\hline 1 Physical Activity & 7 & & Odds Ratio (Random, 95\% Cl) & Subtotals only \\
\hline 1.1 Individual Universal & 2 & 1530 & Odds Ratio (Random, 95\% Cl) & $1.11[0.74,1.67]$ \\
\hline 1.2 Family Targeted & 1 & 61 & Odds Ratio (Random, 95\% Cl) & $0.72[0.29,1.79]$ \\
\hline 1.3 School Universal & 4 & 6441 & Odds Ratio (Random, 95\% Cl) & $1.32[1.16,1.50]$ \\
\hline
\end{tabular}

Analysis 10.1. Comparison 10 Physical activity, Outcome 1 Physical Activity.

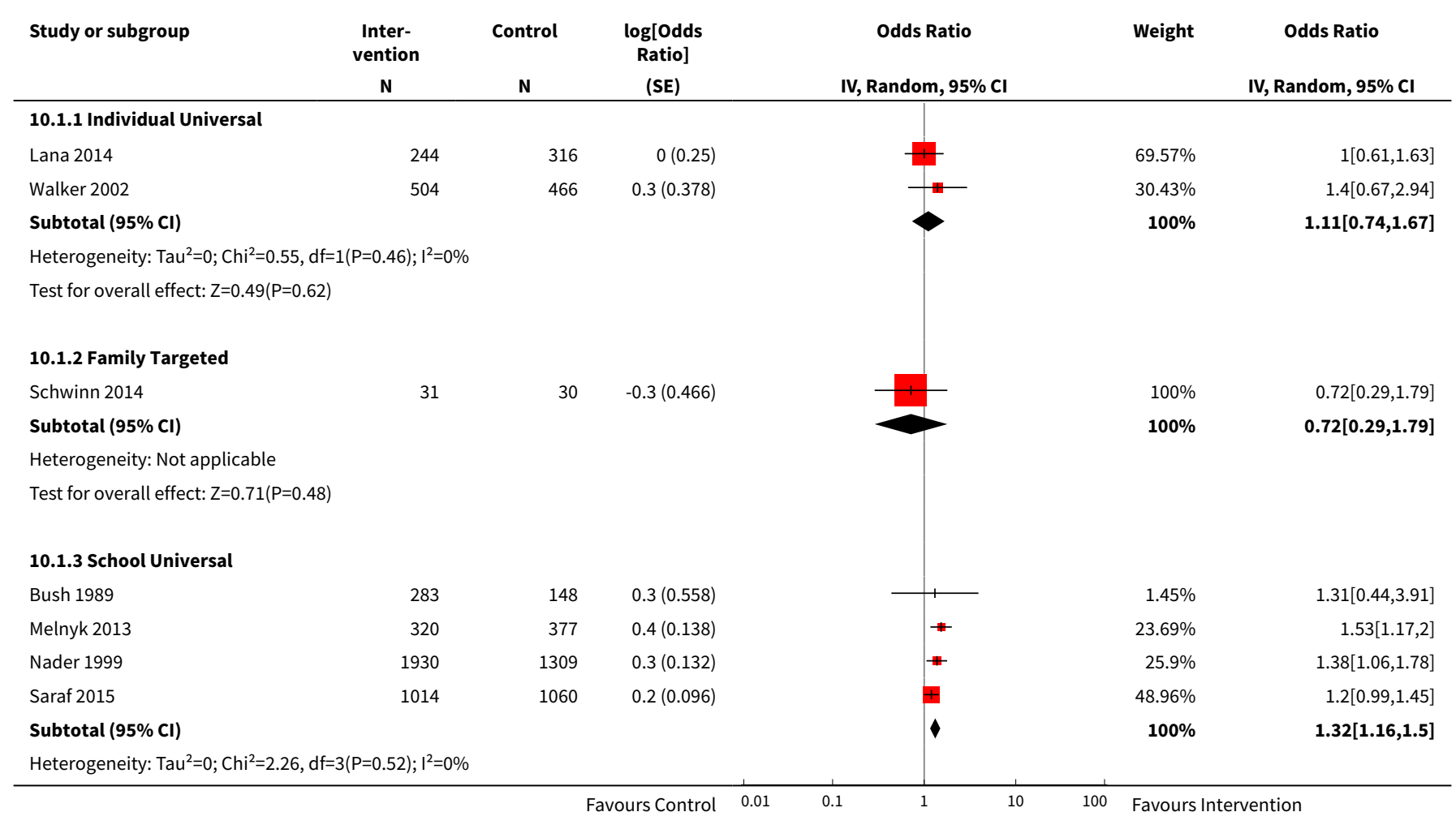




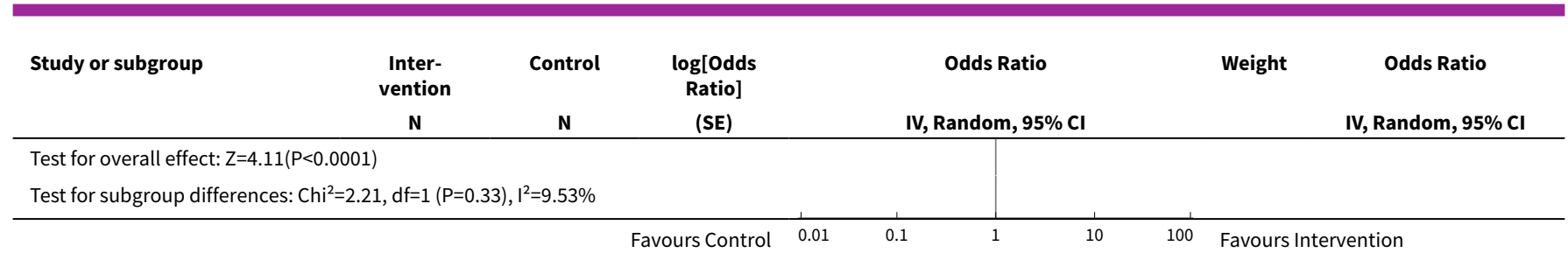

\section{Comparison 11. Mental health}

\begin{tabular}{|c|c|c|c|c|}
\hline Outcome or subgroup title & No. of studies & $\begin{array}{l}\text { No. of partici- } \\
\text { pants }\end{array}$ & Statistical method & Effect size \\
\hline $\begin{array}{l}1 \text { Depressive Symptoms } \\
\text { (short-term) }\end{array}$ & 4 & & Odds Ratio (Random, 95\% Cl) & Subtotals only \\
\hline 1.1 Individual Targeted & 1 & 124 & Odds Ratio (Random, 95\% Cl) & $1.02[0.54,1.93]$ \\
\hline 1.2 School Universal & 3 & 3907 & Odds Ratio (Random, 95\% Cl) & $0.92[0.71,1.20]$ \\
\hline $\begin{array}{l}2 \text { Depressive Symptoms (long- } \\
\text { term) }\end{array}$ & 5 & & Odds Ratio (Random, 95\% Cl) & Subtotals only \\
\hline 2.1 Family Targeted & 4 & 2386 & Odds Ratio (Random, 95\% Cl) & $0.88[0.80,0.98]$ \\
\hline 2.2 School Targeted & 1 & 721 & Odds Ratio (Random, 95\% Cl) & $0.68[0.42,1.09]$ \\
\hline
\end{tabular}

Analysis 11.1. Comparison 11 Mental health, Outcome 1 Depressive Symptoms (short-term).

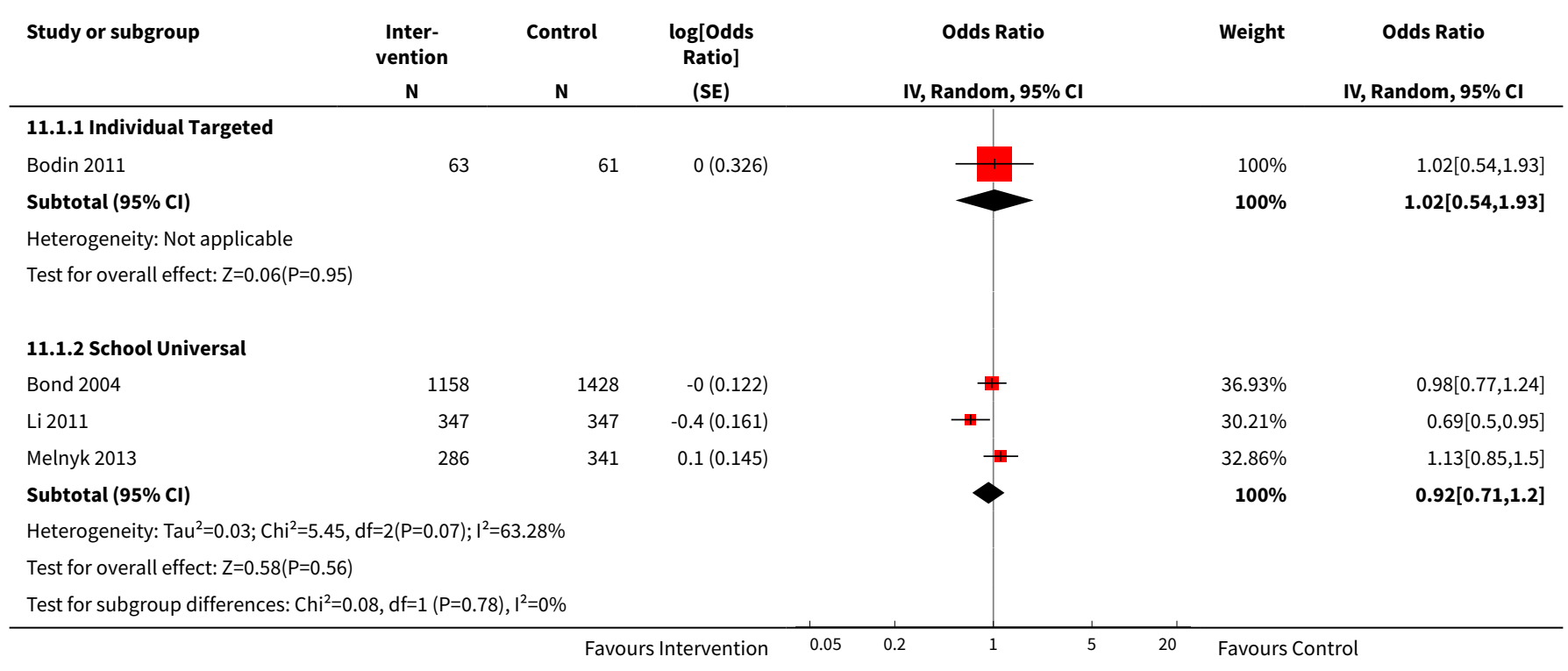




\section{Analysis 11.2. Comparison 11 Mental health, Outcome 2 Depressive Symptoms (long-term).}

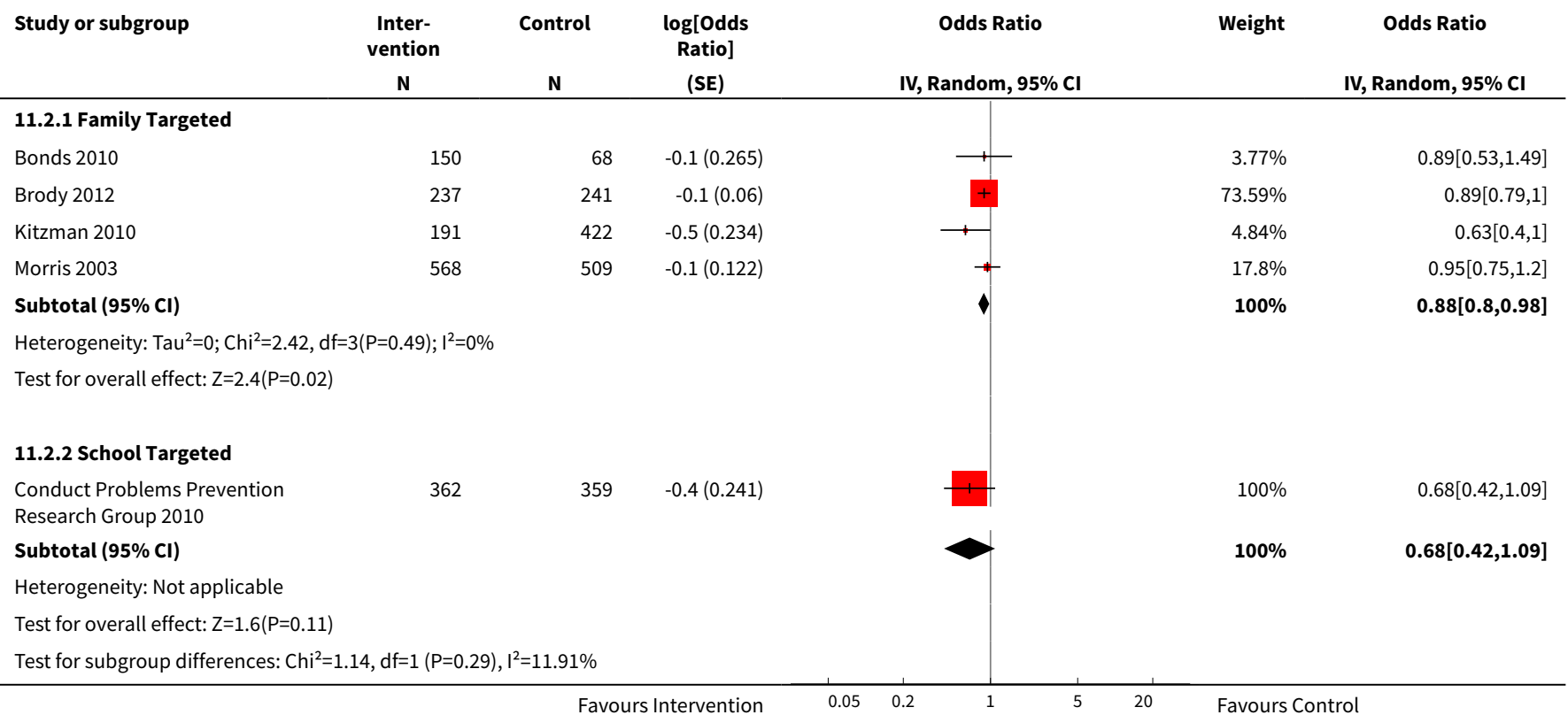

\section{Comparison 12. Unhealthy diet}

\begin{tabular}{lllll}
\hline $\begin{array}{l}\text { Outcome or subgroup ti- } \\
\text { tle }\end{array}$ & No. of studies & $\begin{array}{l}\text { No. of partici- } \\
\text { pants }\end{array}$ & Statistical method & Effect size \\
\hline $1 \mathrm{BMI}$ & 4 & & Odds Ratio (Random, 95\% Cl) & Subtotals only \\
\hline 1.1 Individual Universal & 1 & 579 & Odds Ratio (Random, 95\% Cl) & $0.80[0.48,1.31]$ \\
\hline 1.2 School Universal & 3 & 5017 & Odds Ratio (Random, 95\% Cl) & $0.84[0.60,1.19]$ \\
\hline 2 Unhealthy Diet & 5 & Odds Ratio (Random, 95\% Cl) & Subtotals only \\
\hline 2.1 Individual Universal & 2 & 1549 & Odds Ratio (Random, 95\% Cl) & $0.76[0.42,1.34]$ \\
\hline 2.2 School Universal & 3 & 6441 & Odds Ratio (Random, 95\% Cl) & $0.82[0.64,1.06]$ \\
\hline
\end{tabular}

Analysis 12.1. Comparison 12 Unhealthy diet, Outcome 1 BMI.

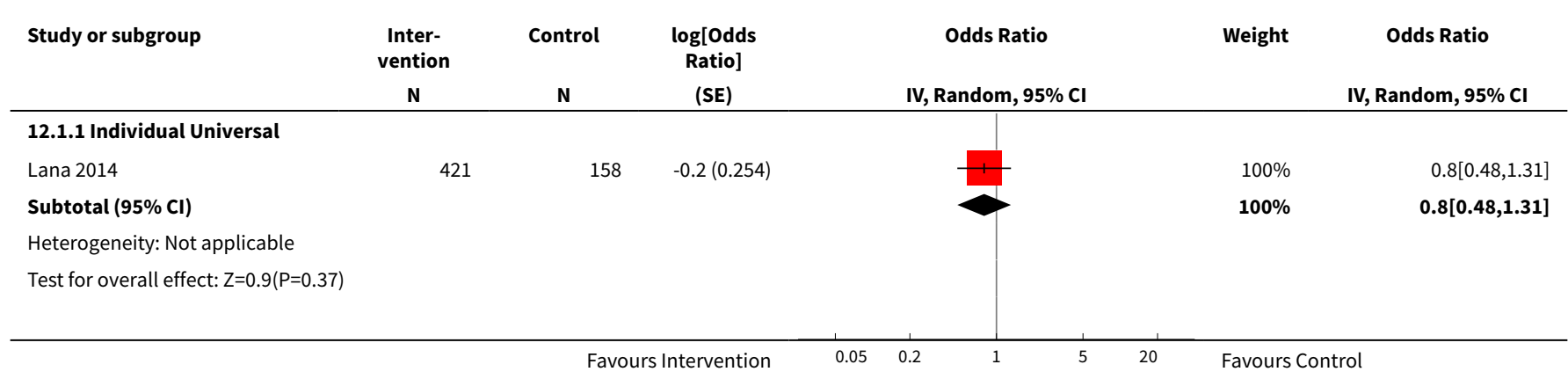




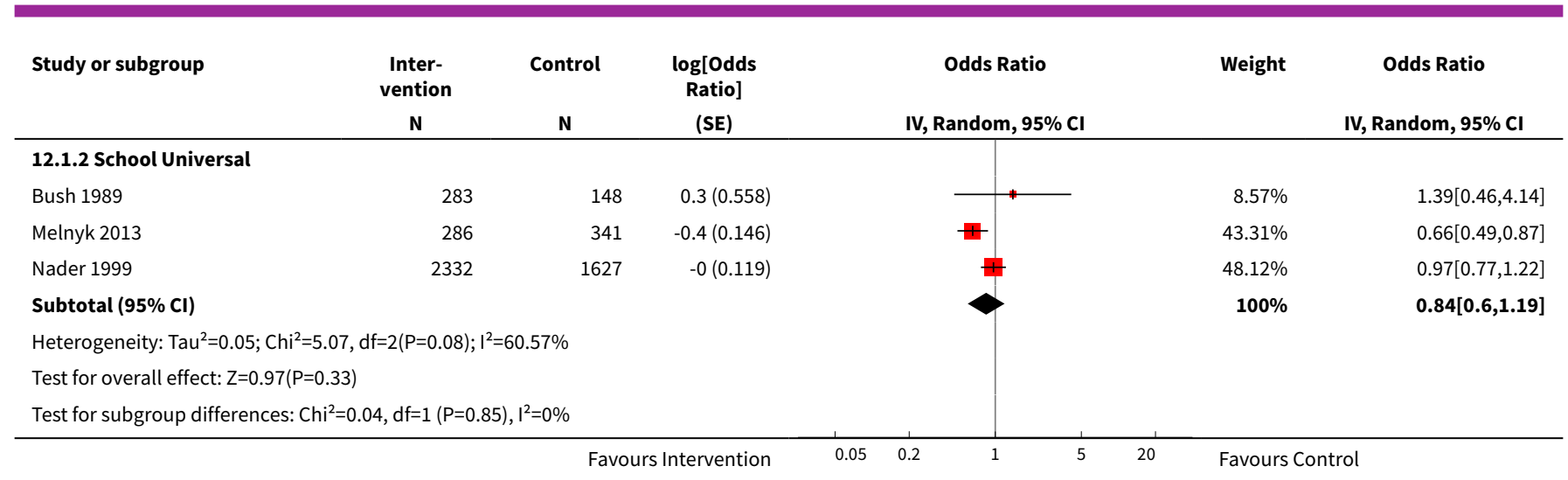

Analysis 12.2. Comparison 12 Unhealthy diet, Outcome 2 Unhealthy Diet.

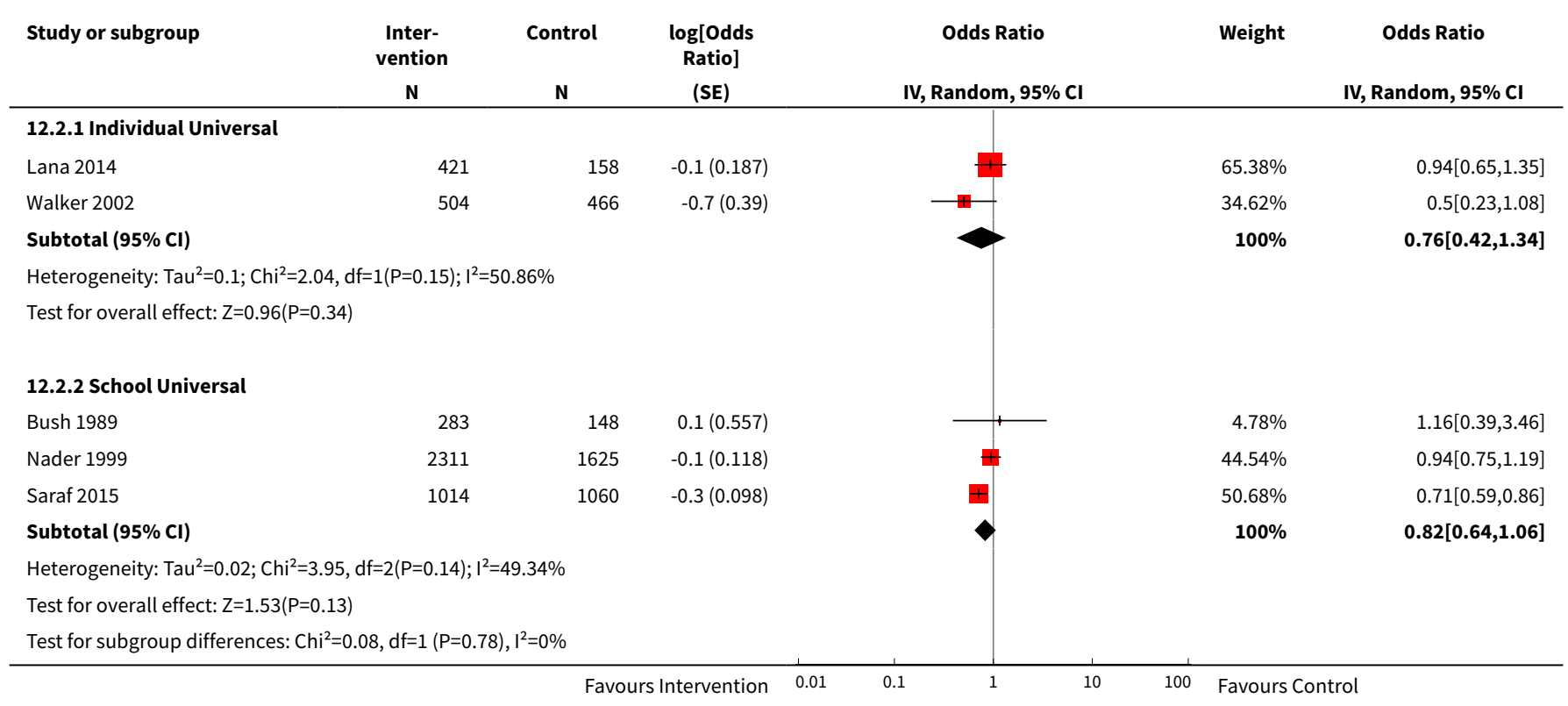

\section{Comparison 13. School-related outcomes}

\begin{tabular}{lllll}
\hline Outcome or subgroup title & No. of studies & $\begin{array}{l}\text { No. of partici- } \\
\text { pants }\end{array}$ & Statistical method & Effect size \\
\hline $\begin{array}{l}1 \text { Academic Performance } \\
\text { (short-term) }\end{array}$ & 5 & & Odds Ratio (Random, 95\% Cl) & Subtotals only \\
\hline 1.1 Individual Targeted & 1 & 126 & Odds Ratio (Random, 95\% Cl) & $1.34[0.71,2.52]$ \\
\hline 1.2 School Targeted & 3 & 1247 & Odds Ratio (Random, 95\% Cl) & $0.91[0.30,2.73]$ \\
\hline 1.3 School Universal & 1 & 579 & Odds Ratio (Random, 95\% Cl) & $0.94[0.62,1.44]$ \\
\hline
\end{tabular}


Analysis 13.1. Comparison 13 School-related outcomes, Outcome 1 Academic Performance (short-term).

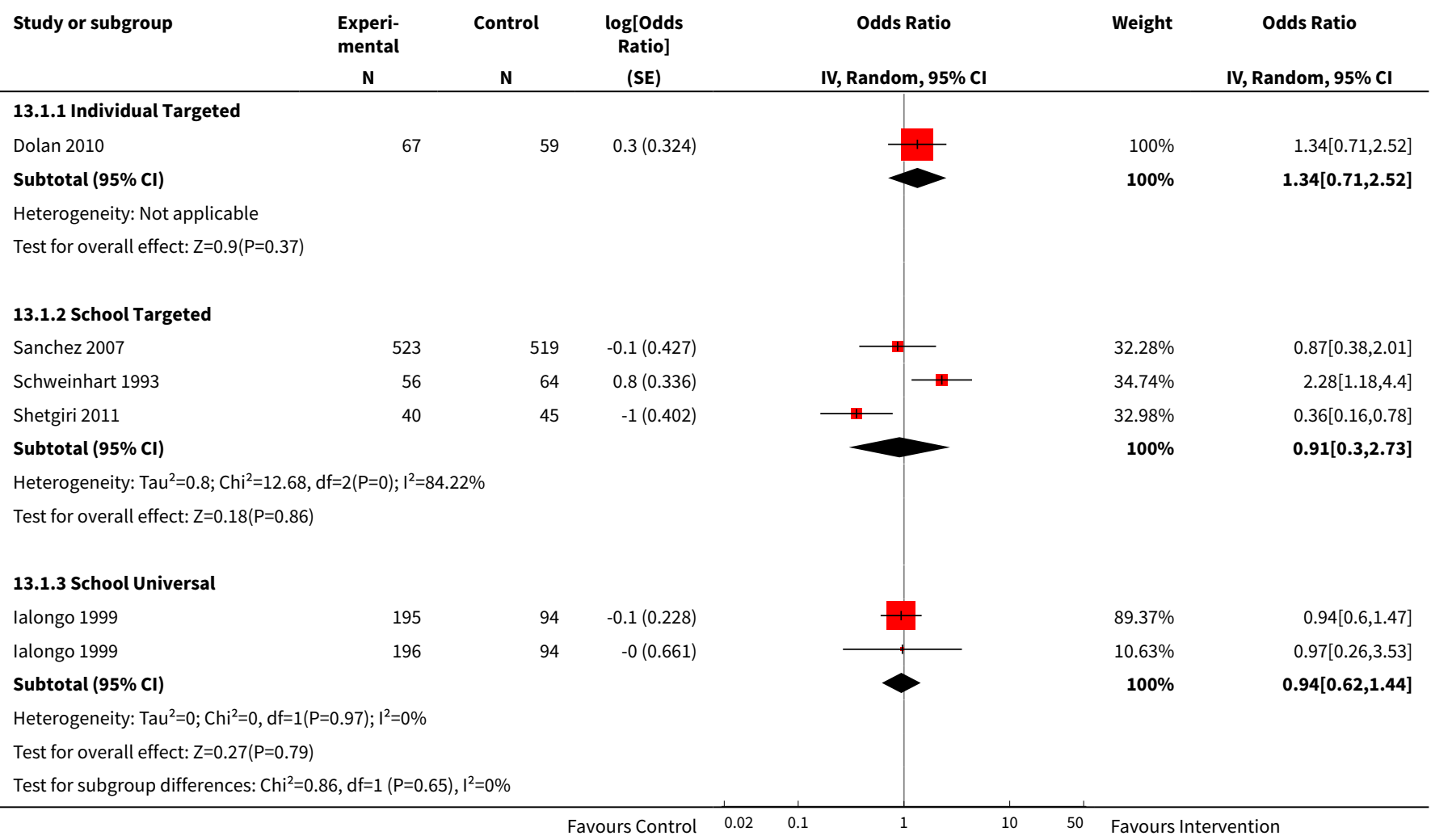

\section{ADDITIONAL TABLES}

Table 1. Intracluster correlation coefficients

\begin{tabular}{|c|c|c|c|c|c|}
\hline Study & Country & Age & $\begin{array}{l}\text { Outcome vari- } \\
\text { able }\end{array}$ & $\begin{array}{l}\text { Reported in- } \\
\text { tracluster } \\
\text { correlation } \\
\text { coefficient }\end{array}$ & $\begin{array}{l}\text { Published or } \\
\text { correspon- } \\
\text { dence (com- } \\
\text { ment) }\end{array}$ \\
\hline
\end{tabular}

\section{ICCs used in primary analyses}

\begin{tabular}{llllll}
\hline Gatehouse Study (Bond 2004) & Australia & 13-14 & Substance use & 0.06 & Published \\
\hline All Stars 2 (Gottfredson 2010) & USA & $11-14$ & Aggression & 0.025 & Published \\
\hline Fourth R (Wolfe 2012) & USA & $14-15$ & Violence & 0.01 & Published \\
\hline All Stars 2 (Gottfredson 2010) & USA & $11-14$ & Delinquency & 0.025 & Published \\
\hline Fourth R (Wolfe 2012) & USA & $14-15$ & Sexual risk behav- & 0.01 & Published \\
\hline Gatehouse Study (Bond 2004) & Australia & $13-14$ & Diet/physical ac- & 0.06 & Published $a$ \\
\hline tivity & & Education & 0.1 & Published \\
\hline
\end{tabular}


Table 1. Intracluster correlation coefficients (Continued)

\begin{tabular}{|c|c|c|c|c|c|}
\hline Gatehouse Study (Bond 2004) & Australia & $13-14$ & Mental illness & 0.01 & Published \\
\hline \multicolumn{6}{|l|}{ ICCs used in sensitivity analyses } \\
\hline $\begin{array}{l}\text { LIFT/All Stars } 2 \text { (DeGarmo 2009; Got- } \\
\text { tfredson 2010) }\end{array}$ & USA & $10 / 11-14$ & Substance use & 0.0 & Published \\
\hline All Stars 2 (Gottfredson 2010) & USA & $11-14$ & Aggression & 0.0 & Published \\
\hline Fourth R (Wolfe 2012) & USA & $14-15$ & Violence & 0.01 & Published \\
\hline All Stars 2 (Gottfredson 2010) & USA & $11-14$ & Delinquency & 0.0 & Published \\
\hline Fourth R (Wolfe 2012) & USA & $14-15$ & $\begin{array}{l}\text { Sexual risk behav- } \\
\text { iour }\end{array}$ & 0.01 & Published \\
\hline $\begin{array}{l}\text { All Stars 2, Gatehouse Study, Fourth R, } \\
\text { LIFT, Positive Action (Chicago) (Bond } \\
\text { 2004; Gottfredson 2010; Wolfe 2012; } \\
\text { DeGarmo 2009; Li 2011) }\end{array}$ & $\begin{array}{l}\text { USA, } \\
\text { Australia }\end{array}$ & $10-15$ & $\begin{array}{l}\text { Diet/physical ac- } \\
\text { tivity }\end{array}$ & 0.0263 & Published $b$ \\
\hline Gatehouse Study (Bond 2004) & Australia & $13-14$ & Education & 0.01 & Published ${ }^{c}$ \\
\hline Gatehouse Study (Bond 2004) & Australia & $13-14$ & Mental illness & 0.01 & Published \\
\hline
\end{tabular}

ICC: intracluster correlation coefficient.

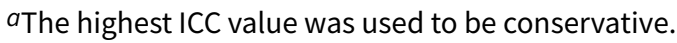

${ }^{b}$ Average ICC value used from across these studies.

CICC related to school engagement.

Table 2. Outcomes not included in meta-analysis

\begin{tabular}{|c|c|c|c|c|}
\hline Author and year & Study name & Categorisation & Outcome & Authors' conclusions \\
\hline \multicolumn{5}{|l|}{ 1. Tobacco use } \\
\hline Bonds 2010 & New Beginnings & Family-Targeted & $\begin{array}{l}\text { Tobacco use dis- } \\
\text { order (including } \\
\text { nicotine with- } \\
\text { drawal and de- } \\
\text { pendence) }\end{array}$ & $\begin{array}{l}\text { No difference between study arms in the propor- } \\
\text { tion of participants meeting criteria for nicotine } \\
\text { use disorder ( } 6.7 \% \text { in each arm) }\end{array}$ \\
\hline Bush 1989 & Know Your Body & School-Universal & $\begin{array}{l}\text { Serum thio- } \\
\text { cyanate (micro- } \\
\text { moles/L) }\end{array}$ & $\begin{array}{l}\text { Mean difference from baseline to } 1 \text { year follow-up } \\
\text { was - } 9.87 \text { (SE } 2.5 \text { ) in the intervention group, and } \\
20.03 \text { (SE 2.68) in the control group }(\mathrm{P}<0.001) \text {. } \\
\text { These data were based on a } 50 \% \text { subsample strat- } \\
\text { ified at baseline, based on measurement after } 1 \\
\text { year of intervention. }\end{array}$ \\
\hline Connell 2007 & Family Check-Up & Family-Universal & $\begin{array}{l}\text { Nicotine abuse/ } \\
\text { dependence }\end{array}$ & $\begin{array}{l}\text { Across treatment and control groups, no significant } \\
\text { differences were found for nicotine abuse/depen- } \\
\text { dence }\left(\mathrm{Chi}^{2}(1,998)=3.09, \mathrm{P}>0.05\right) \text {. No significant } \\
\text { correlation between assignment to experimental } \\
\text { condition(s) and tobacco use over time }\end{array}$ \\
\hline
\end{tabular}


Table 2. Outcomes not included in meta-analysis (Continued)

$\begin{array}{ll}\text { DeGarmo } 2009 \quad \text { LIFT School-Universal } & \begin{array}{l}\text { Initiation of to- } \\ \text { bacco use }\end{array}\end{array}$
With controls for parental drinking and deviant peer association, the intervention was associated with reduced risk of initiation of tobacco use (beta $=-0.10, P<0.01)$. The effect translated to odds ratios of a $10 \%$ reduction in risk for tobacco use.

\begin{tabular}{|c|c|c|c|c|}
\hline Estrada 2015 & $\begin{array}{l}\text { Brief Familias } \\
\text { Unidas }\end{array}$ & Family-Targeted & $\begin{array}{l}\text { Tobacco use in } \\
\text { past } 90 \text { days }\end{array}$ & $\begin{array}{l}\text { Brief Familias Unidas was not significantly effica- } \\
\text { cious in reducing tobacco use (beta }=-0.09, P= \\
0.85 \text { ) in the past } 90 \text { days. }\end{array}$ \\
\hline Gonzales 2012 & $\begin{array}{l}\text { Bridges to High } \\
\text { School }\end{array}$ & Family-Targeted & Substance use & $\begin{array}{l}\text { Study authors report that substance use at fol- } \\
\text { low-up was less in the intervention group than in } \\
\text { the control group for adolescents who engaged in } \\
\text { high levels ( } 85 \text { th percentile) of baseline substance } \\
\text { use ( } d=3.65) \text {. }\end{array}$ \\
\hline
\end{tabular}

\begin{tabular}{|c|c|c|c|c|}
\hline LoSciuto 1999 & $\begin{array}{l}\text { Woodrock Youth } \\
\text { Development } \\
\text { Project }\end{array}$ & School-Universal & $\begin{array}{l}\text { Substance use in } \\
\text { past month (to- } \\
\text { bacco, alcohol, } \\
\text { drugs) }\end{array}$ & $\begin{array}{l}\text { Mean substance use in the past month was } 1.1 \text { for } \\
\text { the intervention group and } 1.15 \text { for the control } \\
\text { group (SMD 0.18) }\end{array}$ \\
\hline McNeal 2004 & All Stars & School-Universal & $\begin{array}{l}\text { Tobacco use in } \\
\text { past } 30 \text { days }\end{array}$ & $\begin{array}{l}\text { The teacher-delivered All Stars programme was as- } \\
\text { sociated with reduced rate of growth in } 30 \text {-day us- } \\
\text { age of cigarettes ( } 7.4 \% \text { to } 7.8 \% \text { ) compared to the } \\
\text { specialist condition ( } 11.0 \% \text { to } 13.8 \% \text { ) and the con- } \\
\text { trol group ( } 15.1 \% \text { to } 17.9 \%) \text {. }\end{array}$ \\
\hline
\end{tabular}

\begin{tabular}{llll}
\hline Olds 1998 & $\begin{array}{l}\text { Nurse Family } \\
\text { Partnership }\end{array}$ & Family-Targeted & $\begin{array}{l}\text { Mean cigarettes } \\
\text { per day }\end{array}$
\end{tabular}

15-year follow-up: incidence of cigarettes smoked per day in past 6 months among those who received nurse visitation through pregnancy (group 3) was 0.91 compared to 1.30 among control participants $(P=0.49)$. Among a subgroup of women from low socioeconomic status (SES) households who were unmarried, the comparison was 1.32 vs 2.50 among control participants $(P=0.07)$. Incidence of cigarettes smoked per day in the past 6 months among those who received nurse visitation until the child's second birthday was $1.28 \mathrm{com}$ pared to 1.30 among control participants $(P=0.76)$. Subgroup analysis of women from low SES households who were unmarried showed that incidence was 1.50 among the intervention group compared to 2.50 among controls $(\mathrm{P}=0.1)$.

$\begin{array}{llll}\text { Perry } 2003 & \begin{array}{l}\text { DARE and DARE- } \\ \text { Plus }\end{array} & \text { School-Universal } & \begin{array}{l}\text { Current smoker } \\ \text { (growth rate) }\end{array}\end{array}$

Growth curve analysis showed that for boys: the growth rate of tobacco use was $0.31(0.05)$ in the control group, $0.28(0.05)$ in the DARE group, and $0.18(0.05)$ in the DARE Plus group (DARE vs control $P=0.28$; DARE Plus vs control $P=0.02$; DARE Plus vs DARE $P=0.08)$. Among girls: the growth rate was $0.28(0.07)$ in the control group, $0.25(0.07)$ in the DARE group, and $0.22(0.07)$ in the DARE Plus group (DARE vs control $P=0.38$; DARE Plus vs control $P=$ 0.25 ; DARE plus vs DARE $P=0.35$ ).

\begin{tabular}{lll}
\hline Piper $2000 \quad$ Healthy for Life $\quad$ School-Universal & $\begin{array}{l}\text { Tobacco use in } \\
\text { past } 30 \text { days }\end{array}$
\end{tabular}

The age-appropriate condition showed no benefit over the control condition at 12-month follow-up (prevalence $24 \%$ in both arms; HLM coefficient 0.18 , SE $0.12, \mathrm{P}>0.1$ ) or at 24-month fol- 
low-up, where prevalence was higher in the intervention group (prevalence $36 \%$ vs $30 \%$ in the control group, coefficient 0.41 , SE $0.2, \mathrm{P}<0.1$ ). Among those receiving the intensive condition, prevalence was similar in both study arms ( 12 months: $22 \%$ vs $24 \%$ in the control group; coefficient - 0.3 , SE 0.17 , $P>0.1 ; 24$ months: $28 \%$ vs $30 \%$ in the control arm; coefficient -0.38 , SE 0.15, $P<0.05$ ).

Saraf $2015 \quad$ (none given) School-Universal Tobacco use

Current smoking (in the past month) changed from $13.1 \%(95 \% \mathrm{Cl} 10.2 \%$ to $15.9 \%)$ to $3.1 \%(95 \% \mathrm{Cl}$ $0.2 \%$ to $5.9 \%)$ in the intervention group; and from $7.7 \%(95 \% \mathrm{Cl} 5.0 \%$ to $10.4 \%)$ to $5.4 \%(95 \% \mathrm{Cl} 2.6 \%$ to $8.2 \%$ ) in the control group (overall difference between groups in pre- to post-change $-7.7(-10.7$ to $-4.7) ; \mathrm{P}<0.01$.

\begin{tabular}{|c|c|c|}
\hline $\begin{array}{l}\text { Schweinhart } \\
1980\end{array}$ & $\begin{array}{l}\text { High/Scope Per- } \\
\text { ry Preschool } \\
\text { Study }\end{array}$ & School-Targeted \\
\hline
\end{tabular}

No impact of the intervention on smoking cigarettes 22 years after the end of the programme: $45 \%$ of those in the intervention group smoked compared to $56 \%$ of those in the control group $(\mathrm{P}=$ $0.231)$. Effect size 0.22

$\begin{array}{llll}\text { Tierney } 1995 & \begin{array}{l}\text { Big Brothers Big } \\ \text { Sisters }\end{array} & \begin{array}{l}\text { Individual-Tar- } \\ \text { geted }\end{array} & \begin{array}{l}\text { Likelihood of } \\ \text { smoking }\end{array}\end{array}$

Those receiving the intervention were reported to be $19.7 \%$ less likely to start smoking compared to controls (males receiving Big Brothers Big Sisters were $24.5 \%$ less likely to start smoking, and females $9.9 \%$ ). Males from an ethnic minority receiving Big Brothers Big Sisters had a 29.9\% increased likelihood of smoking compared to controls, but among females there was a $1.9 \%$ reduction. White males and females receiving the intervention had a $47.9 \%$ and $14.7 \%$ reduced likelihood of smoking, respectively.

Walter $1989 \quad$ Know Your Body School-Universal Smoking

Among the schools in Westchester, results showed a beneficial impact of the intervention: the school mean at the end of the intervention was 3.5\% (SD $4.3 \%$ ) compared to $13.1 \%$ (SD 5.2) among control schools; $P<0.005$. This is equivalent to a $73 \%$ reduction in the rate of initiation of smoking.

\section{Alcohol use}

$\begin{array}{ll}\text { Bonds } 2010 \quad \text { New Beginnings Family-Targeted } & \text { Alcohol use, } \\ & \text { binge drinking, } \\ & \text { age commencing } \\ & \text { drinking }\end{array}$

15-year follow-up: alcohol use in the past month higher in the intervention arm than in the control $\operatorname{arm}(\mathrm{d}=0.23,95 \% \mathrm{Cl}-0.26$ to 0.72$)$. Intervention arm commenced drinking at a mean age 0.47 years younger than the control group $(95 \% \mathrm{Cl}-1.31$ to 0.23 years). Binge drinking in the past year higher in the intervention group than in the control arm $(\mathrm{d}=$ $0.16,95 \% \mathrm{Cl}-0.14$ to 0.46$)$.

\begin{tabular}{lllll}
\hline $\begin{array}{l}\text { Conduct Prob- } \\
\text { lems Prevention } \\
\begin{array}{l}\text { Research Group } \\
2010\end{array}\end{array}$ & Fast Track & School-Targeted & $\begin{array}{l}\text { Binge drinking } \\
\text { problem }\end{array}$ & $\begin{array}{l}\text { The intervention marginally decreased binge drink- } \\
\text { ing at 10-year follow-up (adjusted OR 0.75, 95\% Cl } \\
0.55 \text { to 1.01, P }=0.057) .\end{array}$ \\
\hline Connell 2007 & Family Check-Up & Family-Universal & Alcohol use & $\begin{array}{l}\text { No significant association was noted between as- } \\
\text { signment to experimental condition(s) and alcohol }\end{array}$ \\
\hline
\end{tabular}


abuse/dependence over time $\left(\mathrm{Chi}^{2}(1,998)=0.98, \mathrm{P}\right.$ $>0.05$ ), with the exception of Time 2 , when a correlation between treatment assignment and alcohol use was observed $(r=0.09, P \leq 0.05)$.

\begin{tabular}{|c|c|c|}
\hline $\begin{array}{l}\text { Cunningham } \\
2012\end{array}$ & SafERteens & $\begin{array}{l}\text { Individual-Tar- } \\
\text { geted }\end{array}$ \\
\hline
\end{tabular}

Reduction in the proportion of participants scoring $\geq 3$ on AUDIT-C from $50 \%$ at baseline to $34.4 \%$ at 3 months and $37.3 \%$ at 12 months $(-12.7 \%$ change at 12 months; OR $1.09,95 \% \mathrm{Cl} 0.77$ to 1.56 ) for those in the therapist intervention arm; and a reduction from $45.6 \%$ at baseline to $32.7 \%$ at 3 months and $28.9 \%$ at 12 months $(-16.7 \%$ change at 12 months; OR $0.95,95 \% \mathrm{Cl} 0.66$ to 1.37 ) for those in the computer arm. For controls, a reduction from $47.7 \%$ to $38.1 \%$ at 3 months and $34.7 \%$ at 12 months was evident (-13\% change at 12 months).

\begin{tabular}{|c|c|c|}
\hline $\begin{array}{l}\text { Cunningham } \\
2012\end{array}$ & SafERteens & $\begin{array}{l}\text { Individual-Tar- } \\
\text { geted }\end{array}$ \\
\hline
\end{tabular}

Reduction in the proportion of participants reporting any binge drinking from $52.8 \%$ at baseline to $34.4 \%$ at 3 months and $38.7 \%$ at 12 months $(-14.1 \%$ reduction at 12 months; OR $0.95,95 \% \mathrm{Cl} 0.66$ to 1.36) among those in the therapist group; and a reduction from $48.5 \%$ to $28.8 \%$ at 3 months and $30.3 \%$ at 12 months $(-18.2 \%$ reduction; OR 0.83 , $95 \% \mathrm{Cl} 0.58$ to 1.19 ) among those in the computer group. Similar reductions were seen in the control group: a reduction from $54 \%$ at baseline to $34.6 \%$ at 3 months and $36.1 \%$ at 12 months $(-17.9 \%$ reduction at 12 months).

\begin{tabular}{ll}
\hline Estrada 2015 & $\begin{array}{l}\text { Familias Unidas } \\
\text { - Brief }\end{array}$
\end{tabular}

Brief Familias Unidas was not significantly efficacious in reducing alcohol use (beta $=0.17 ; \mathrm{P}=0.51$ ) in the past 90 days.

\begin{tabular}{llll}
\hline Friedman 2002 & $\begin{array}{l}\text { Botvin Life Skills } \\
\text { Training and An- } \\
\text { ti-violence }\end{array}$ & $\begin{array}{l}\text { Individual-Tar- } \\
\text { geted }\end{array}$ & $\begin{array}{l}\text { Degree of alco- } \\
\text { hol use }\end{array}$ \\
\hline Gonzales 2012 & $\begin{array}{l}\text { Bridges to High } \\
\text { School }\end{array}$ & Family-Targeted & Substance use \\
\hline
\end{tabular}

Alcohol use was decreased among intervention participants compared to controls $(\mathrm{t}=-1.24, \mathrm{P}$ > 0.05).

Study authors report that substance use was less at follow-up in the intervention group compared to the control group for adolescents who engaged in high levels (85th percentile) of baseline substance use $(d=3.65)$.

\begin{tabular}{lll}
\hline Jalling $2016 \quad$ Comet 12-18 Family-Targeted & $\begin{array}{l}\text { Alcohol use (AU- } \\
\text { DIT score) }\end{array}$
\end{tabular}

No significant difference was found between groups: at T2, mean AUDIT score was 7.59 (SD 7.60) in the intervention group vs 6.26 (SD 6.79) in the control group.

\begin{tabular}{lll}
\hline Jalling 2016b $\quad$ ParentSteps & Family-Targeted & $\begin{array}{l}\text { Alcohol use (AU- } \\
\text { DIT score) }\end{array}$
\end{tabular}

No significant difference was found between groups: at T2, mean AUDIT score was 5.10 (SD 6.38) in the intervention group vs 6.26 (SD 6.79) in the control group.

\begin{tabular}{|c|c|c|c|}
\hline Kellam 2008 & $\begin{array}{l}\text { Good Behaviour } \\
\text { Game }\end{array}$ & School-Universal & $\begin{array}{l}\text { Lifetime alcohol } \\
\text { abuse/ depen- } \\
\text { dence }\end{array}$ \\
\hline
\end{tabular}

The Good Behaviour Game (GBG) was associated with a reduction in lifetime alcohol abuse/dependence disorders compared to control: $13 \%$ for GBG vs $20 \%$ for controls $(P=0.08)$. The effect was similar for males and females. 


\section{Table 2. Outcomes not included in meta-analysis (Continued)}

$\begin{array}{lll}\text { Murry } 2014 \quad \text { SAAF } & \text { Family-Targeted } & \begin{array}{l}\text { Escalation of al- } \\ \text { cohol use }\end{array}\end{array}$

Study authors report through structural equation modelling analysis that youth avoidance of risk opportunity situations served a role in delaying initiation and escalation of use of alcohol and other substances as they transitioned from early to late adolescence.

\begin{tabular}{lll}
\hline Monti 1999 & $\begin{array}{l}\text { Alcohol Screen- } \\
\text { ing and Brief In- } \\
\text { tervention }\end{array}$ & $\begin{array}{l}\text { Individual-Tar- } \\
\text { geted }\end{array}$
\end{tabular}

With a $2 \times 2$ (group $\times$ time) repeated measures analysis of variance, time effect showed reductions in alcohol scores $(F(1,79)=24.55, P<0.001)$ with no group differences or interactions.

\begin{tabular}{lll}
\hline Olds 1998 & $\begin{array}{l}\text { Nurse Family } \\
\text { Partnership }\end{array}$ & Family-Targeted
\end{tabular}$\quad$ Alcohol use

15-year follow-up: incidence of days drunk alcohol in past 6 months among those who received nurse visitation through pregnancy (group 3) was 1.81 compared to 1.57 among control participants $(P=0.97)$. Among a subgroup of women from low socioeconomic status (SES) households who were unmarried, the comparison was 1.84 vs 2.49 among control participants $(P=0.41)$. Incidence of days drunk alcohol in past 6 months among those who received nurse visitation until the child's second birthday was 1.87 compared to 1.57 among control participants $(P=0.96)$. Subgroup analysis of women from low SES households who were unmarried show the incidence was 1.09 among the intervention group compared to 2.49 among controls $(P=0.03)$.

\begin{tabular}{|c|c|c|c|}
\hline Perry 2003 & $\begin{array}{l}\text { DARE vs DARE } \\
\text { Plus }\end{array}$ & School-Universal & $\begin{array}{l}\text { Alcohol con- } \\
\text { sumption in past } \\
\text { month }\end{array}$ \\
\hline
\end{tabular}

Growth curve analysis showed that for boys: the growth rate in alcohol use in the past month (mean, SE) was $0.14(0.02)$ for those in the control group, $0.11(0.02)$ for the DARE group $(P=0.12)$, and $0.08(0.02)$ for the DARE Plus group $(P=0.01)$ (DARE Plus vs DARE, $P=0.12)$. Among girls: values were $0.12(0.03)$ for controls, $0.13(0.02)$ for those in the DARE group $(P=0.40)$, and $0.08(0.03)$ for those in the DARE Plus group $(P=0.15)$ (DARE Plus vs DARE, $P=0.10)$.

$\begin{array}{ll}\text { Piper } 2000 \quad \text { Healthy for Life School-Universal } & \begin{array}{l}\text { Alcohol use in } \\ \text { past } 30 \text { days }\end{array}\end{array}$

Results showed a negative treatment effect at 12 months and 24 months of follow-up: in the age-appropriate intervention, prevalence of alcohol use in the past month was 33\% in the intervention group and $28 \%$ in the control group at 12 months (hierarchical linear modelling (HLM) coefficient 0.34 , SE $0.19, \mathrm{P}<0.1$ ). At 24 months, the prevalence of alcohol use in the past month was $48 \%$ in the intervention group and $41 \%$ in the control group at 24 months (HLM coefficient 0.3, SE 0.14, P<0.05). In the intensive version of the intervention, the prevalence of alcohol use at 12 months was $33 \%$ vs $28 \%$ in the control arm (HLM coefficient 0.2, SE 0.09, P $<0.05$ ), and at 24 months, prevalence was $45 \%$ vs $41 \%$ in the control arm (HLM coefficient 0.27 , SE $0.1, \mathrm{P}<0.05)$. 


\section{Table 2. Outcomes not included in meta-analysis (Continued)}

\begin{tabular}{|c|c|c|}
\hline $\begin{array}{l}\text { Schweinhart } \\
1980\end{array}$ & $\begin{array}{l}\text { High/Scope Per- } \\
\text { ry Preschool } \\
\text { Study }\end{array}$ & School-Targeted \\
\hline
\end{tabular}

$\begin{array}{llll}\text { Tierney } 1995 & \begin{array}{l}\text { Big Brothers Big } \\ \text { Sisters }\end{array} & \begin{array}{l}\text { Individual-Tar- } \\ \text { geted }\end{array} & \begin{array}{l}\text { Likelihood of ini- } \\ \text { tiating alcohol } \\ \text { use }\end{array}\end{array}$

No impact of the intervention on drinking alcoholic beverages several or more times a week 22 years after the end of the programme: $16 \%$ of those in the intervention group drank alcohol several or more times a week compared to $26 \%$ of those in the control group. Effect size for drinking alcoholic beverages was $0.27(P=0.141)$.

Those receiving the intervention were $27.4 \%$ less likely to start using alcohol than those in the control group (19.2\% reduction in likelihood among males and $38.8 \%$ among females). The reduction in likelihood was $11.4 \%$ among males from an ethnic minority, $53.7 \%$ among females from an ethnic minority; $34.5 \%$ among white males, and $8.4 \%$ among white females.

\section{Illicit drug use}

\begin{tabular}{ccl}
\hline Connell $2007 \quad$ Family Check-Up $\quad$ Family-Universal & Marijuana use & $\begin{array}{l}\text { Across treatment and control groups, no signifi- } \\
\text { cant differences were found for marijuana abuse/ } \\
\text { dependence }\left(\mathrm{Chi}^{2}(1,998)=0.74, \mathrm{P}>0.05\right) . \mathrm{No} \text { sig- }\end{array}$ \\
& $\begin{array}{l}\text { nificant correlation was noted between assignment } \\
\text { to experimental condition(s) and marijuana use } \\
\text { over time, with the exception of Time } 2(\mathrm{r}=0.10, \mathrm{P} \\
\end{array}$
\end{tabular}

\begin{tabular}{lll}
\hline Bonds $2010 \quad$ New Beginnings $\quad$ Family-Targeted & Marijuana use, \\
& polydrug use, \\
& other drug use
\end{tabular}

6-year follow-up: results showed no significant group effects for drug dependence, drug symptom count, or polydrug use (all $P>0.05$ ).

15-year follow-up: intervention group displayed lower past year polydrug use $(\mathrm{d}=-.44,95 \% \mathrm{Cl}-.88$ to .00$)$ and past year other drug use $(\mathrm{d}=-.06,95 \%$ $\mathrm{Cl}-.11$ to -.00$)$ compared to control group. No difference was observed for marijuana use between intervention and control groups $(\mathrm{d}=.00,95 \% \mathrm{Cl}$ -.47 to .47$)$

\begin{tabular}{|c|c|c|c|c|}
\hline DeGarmo 2009 & LIFT & School-Universal & $\begin{array}{l}\text { Percentage of } \\
\text { participants who } \\
\text { have not used } \\
\text { marijuana }\end{array}$ & $\begin{array}{l}\text { One year post intervention, } 2.2 \% \text { had not used } \\
\text { marijuana in the past year compared to } 2.3 \% \text { in the } \\
\text { control group. }\end{array}$ \\
\hline Estrada 2015 & $\begin{array}{l}\text { Brief Familias } \\
\text { Unidas }\end{array}$ & Family-Targeted & $\begin{array}{l}\text { Illicit drug use } \\
\text { (past } 90 \text { days) }\end{array}$ & $\begin{array}{l}\text { Brief Familias Unidas was not significantly effica- } \\
\text { cious in reducing illicit drug use (beta }=0.03 ; \mathrm{P}= \\
0.93 \text { ) in the past } 90 \text { days. }\end{array}$ \\
\hline Friedman 2002 & $\begin{array}{l}\text { Botvin Life Skills } \\
\text { Training and An- } \\
\text { ti-violence }\end{array}$ & $\begin{array}{l}\text { Individual-Tar- } \\
\text { geted }\end{array}$ & $\begin{array}{l}\text { Degree of drug } \\
\text { use and involve- } \\
\text { ment in selling of } \\
\text { drugs }\end{array}$ & $\begin{array}{l}\text { Among intervention participants compared to con- } \\
\text { trols, data showed a greater reduction in drug use } \\
(\mathrm{t}=-2.58, \mathrm{P}<0.01) \text { and a greater reduction in the } \\
\text { frequency of involvement in the selling of drugs ( } \mathrm{t}= \\
-1.99) \text {. }\end{array}$ \\
\hline $\begin{array}{l}\text { Freudenberg } \\
2010\end{array}$ & REAL MEN & $\begin{array}{l}\text { Individual-Tar- } \\
\text { geted }\end{array}$ & $\begin{array}{l}\text { Daily marijua- } \\
\text { na use in past } 90 \\
\text { days }\end{array}$ & $\begin{array}{l}\text { Intervention associated with reduced odds of daily } \\
\text { marijuana use (OR 0.751). No } 95 \% \text { confidence inter- } \\
\text { val or standard error was provided. }\end{array}$ \\
\hline
\end{tabular}




\section{Table 2. Outcomes not included in meta-analysis (Continued)}

\begin{tabular}{|c|c|c|c|c|}
\hline $\begin{array}{l}\text { Freudenberg } \\
2010\end{array}$ & REAL MEN & $\begin{array}{l}\text { Individual-Tar- } \\
\text { geted }\end{array}$ & $\begin{array}{l}\text { Hard drug use } \\
\text { tried in past } 90 \\
\text { days }\end{array}$ & $\begin{array}{l}\text { Intervention was associated with reduced odds of } \\
\text { trying hard drugs (OR } 0.166, \mathrm{P}<0.05) \text {. No } 95 \% \text { con- } \\
\text { fidence interval or standard error was provided. }\end{array}$ \\
\hline Griffin 2006 & $\begin{array}{l}\text { Life Skills Train- } \\
\text { ing }\end{array}$ & School-Universal & $\begin{array}{l}\text { High-risk sub- } \\
\text { stance use }\end{array}$ & $\begin{array}{l}32.4 \% \text { of participants in the intervention group en- } \\
\text { gaged in high-risk substance use at the young adult } \\
\text { follow-up compared to } 37.1 \% \text { of those in the con- } \\
\text { trol group } 11 \text { years following completion of the in- } \\
\text { tervention. }\end{array}$ \\
\hline
\end{tabular}

$\begin{array}{llll}\text { Jalling 2016 } & \begin{array}{l}\text { Comet } 12-18 \text { and } \\ \text { Parent Steps }\end{array} & \begin{array}{l}\text { Individual-Tar- } \\ \text { geted }\end{array} & \begin{array}{l}\text { Any illicit drug } \\ \text { use }(\%)\end{array}\end{array}$

Higher odds of illicit drug use were evident among those whose parents took part in the study, although 95\% Cls were wide. Comet 12-18: OR 3.52, $95 \% \mathrm{Cl} 1.23$ to 10.10 . ParentSteps OR 3.23, $95 \% \mathrm{Cl}$ 1.06 to 9.08

\begin{tabular}{lll}
\hline McNeal $2004 \quad$ All Stars & School-Universal & $\begin{array}{l}\text { Marijuana use in } \\
\text { past } 30 \text { days }\end{array}$
\end{tabular}

Marijuana use in the past 30 days for those in the specialist arm increased from $3.2 \%$ to $4.1 \%$ in the intervention group and from $5.0 \%$ to $8.7 \%$ in the control group (standardised B coefficient $=0.02, P$ $>0.05$ ). For those in the teacher-delivered arm, the increase was $3.2 \%$ at baseline and follow-up compared to a change from $5.0 \%$ to $8.7 \%$ in the control group (standardised B coefficient -0.01, P > 0.05).

\begin{tabular}{lll}
\hline Piper $2000 \quad$ Healthy for Life & School-Universal & $\begin{array}{l}\text { Marijuana use in } \\
\text { past } 30 \text { days }\end{array}$
\end{tabular}

In the age-appropriate condition, prevalence of marijuana use was similar in the intervention and control groups at 12 -month and 24-month follow-up (prevalence $4 \%$ vs $5 \%$ in the control group; OR $0.77, P>0.1$; and $12 \%$ vs $10 \%$ in the control group; OR $1.28, \mathrm{P}>0.1$, respectively). Among those receiving the intensive version of the programme, findings suggested benefit of the intervention: prevalence $5 \%$ in both arms at 12 months (OR 0.56 , $\mathrm{P}<0.05)$ and prevalence $8 \%$ vs $10 \%$ in the control condition (OR 0.56, $\mathrm{P}<0.05)$.

\begin{tabular}{llll}
\hline Tierney 1995 & $\begin{array}{l}\text { Big Brothers Big } \\
\text { Sisters }\end{array}$ & $\begin{array}{l}\text { Individual-Tar- } \\
\text { geted }\end{array}$ & $\begin{array}{l}\text { Likelihood of } \\
\text { initiating drug } \\
\text { abuse }\end{array}$
\end{tabular}

Overall, receiving the intervention was associated with a $45.8 \%$ reduction in the likelihood of initiating drug abuse (55\% among males and $26.6 \%$ among females). The impact was greatest among males and females from an ethnic minority, among whom results showed a $67.8 \%$ and $72.6 \%$ reduced likelihood of initiating drug use, respectively. White males in the intervention group were $32.7 \%$ less likely to start using drugs compared to white males in the control group, but white females were $49.5 \%$ more likely to start using drugs compared to white females in the control group.

\section{Substance misuse (composite)}

\begin{tabular}{llll}
\hline Beach $2016 \quad$ ProSAAF & Family-Targeted & $\begin{array}{l}\text { Substance use in } \\
\text { lifetime (self-re- }\end{array}$ & $\begin{array}{l}\text { At } 9 \text { months, young people in the intervention } \\
\text { group reported lower levels of substance use initia- }\end{array}$ \\
& $\begin{array}{l}\text { ported use of cig- } \\
\text { arettes, alcohol, }\end{array} \quad$ ciompared to those in the control group (coeffi- \\
&
\end{tabular}

and/or marijua-

na) 
Table 2. Outcomes not included in meta-analysis (Continued)

$\begin{array}{llll}\text { Berry } 2009 & \begin{array}{l}\text { Coaching for } \\ \text { Communities }\end{array} & \begin{array}{l}\text { Individual-Com- } \\ \text { munity }\end{array} & \begin{array}{l}\text { Alcohol and drug } \\ \text { use }\end{array}\end{array} \begin{aligned} & \begin{array}{l}\text { At the end of the intervention, the mean use of al- } \\ \text { cohol and drugs in the past } 30 \text { days was } 0.83 \text { in the } \\ \text { intervention group and } 2.55 \text { in the control group. }\end{array} \\ & \end{aligned}$

\begin{tabular}{|c|c|c|c|c|}
\hline Estrada 2015 & $\begin{array}{l}\text { Brief Familias } \\
\text { Unidas }\end{array}$ & Family-Targeted & $\begin{array}{l}\text { Substance use } \\
\text { (alcohol, tobac- } \\
\text { co, and/or drugs) }\end{array}$ & $\begin{array}{l}\text { Growth curve analyses showed a non-significant } \\
\text { difference in past } 90 \text {-day substance use between } \\
\text { brief Familias Unidas and CPC (beta }=0.24 ; P= \\
0.37 \text { ). }\end{array}$ \\
\hline
\end{tabular}

\begin{tabular}{|c|c|c|c|c|}
\hline $\begin{array}{l}\text { Freudenberg } \\
2010\end{array}$ & REAL MEN & $\begin{array}{l}\text { Individual-Tar- } \\
\text { geted }\end{array}$ & $\begin{array}{l}\text { Alcohol or drug } \\
\text { dependence in } \\
\text { the past year }\end{array}$ & $\begin{array}{l}\text { Reduced odds of alcohol or drug dependence in } \\
\text { the past year following receipt of intervention (OR } \\
0.519, \mathrm{P}<0.05) \text {. No } 95 \% \text { confidence interval or } \\
\text { standard error was provided. }\end{array}$ \\
\hline
\end{tabular}

\begin{tabular}{|c|c|c|c|c|}
\hline Gonzales 2014 & $\begin{array}{l}\text { Bridges to High } \\
\text { School (Bridges/ } \\
\text { Puentes) }\end{array}$ & Family-Targeted & Substance use & $\begin{array}{l}\text { Intervention status was associated with a reduc- } \\
\text { tion in substance use at } 2 \text { years and } 5 \text { years post- } \\
\text { test (unstandardised regression coefficients }-0.3 \\
\text { and }-0.13 \text {, respectively). }\end{array}$ \\
\hline
\end{tabular}

\begin{tabular}{lll}
\hline Griffin 2006 & Life Skills Train- & School-Universal \\
ing & $\begin{array}{l}\text { High-risk sub- } \\
\text { stance use }\end{array}$
\end{tabular}

$32.4 \%$ of participants in the intervention group engaged in high-risk substance use at the young adult follow-up compared to $37.1 \%$ of those in the control group.

\begin{tabular}{lll}
\hline LoSciuto 1999 & $\begin{array}{l}\text { Woodrock Youth } \\
\text { Development }\end{array}$ & School-Universal \\
& Project & $\begin{array}{l}\text { Substance use in } \\
\text { past month }\end{array}$
\end{tabular}

Participation in the programme was associated with higher average scores for lifetime substance use $(F(1,711)=6.10, P=0.01$, Cohen's $d=0.19)$ and past month substance use $(F(1,712)=5.93, P=0.02$, Cohen's $d=0.18)$. The data could not be adjusted for clustering owing to insufficient information reported.

\begin{tabular}{lll}
\hline Olds 1998 & $\begin{array}{l}\text { Nurse Family } \\
\text { Partnership }\end{array}$ & Family-Targeted
\end{tabular} Drug use

At 15-year follow-up, data showed no significant difference in the incidence of days of drug use in the past 6 months between intervention and control groups. Among those who received nurse visitation during pregnancy, incidence was 3.55 vs 2.28 among controls $(P=0.49)$ (low $S E S$, unmarried subgroup: 9.38 vs $4.04, P=0.01$ ). Among those who received nurse visitation until the child's second birthday, incidence was 2.04 vs 2.28 in the control group $(\mathrm{P}=0.54)$ (low $\mathrm{SES}$, unmarried subgroup: 2.5 vs 4.04 among controls, $P=0.24$ ).

\section{Antisocial behaviour and offending}

\begin{tabular}{lllll}
\hline Averdijk 2016 & Triple P & Family-Targeted & Delinquency & $\begin{array}{l}\text { No substantial effect of the intervention was found } \\
\text { at long-term follow-up (age 15 years, beta }=0.004, \\
95 \% \mathrm{Cl}-0.15 \text { to } 0.15 ; \mathrm{ES}=0.002)\end{array}$ \\
\hline Averdijk 2016 & PATHS & School-Universal & Delinquency & $\begin{array}{l}\text { No substantial effect of the intervention was found } \\
\text { at long-term follow-up (age 15 years, beta }=-0.04, \\
95 \% \mathrm{Cl}-0.19 \text { to 0.11; } \mathrm{ES}=-0.022) .\end{array}$ \\
\hline Beach 2016 & ProSAAF & Family-Targeted & $\begin{array}{l}\text { Conduct prob- } \\
\text { lems }\end{array}$ & $\begin{array}{l}\text { Follow-up revealed a beneficial effect of the inter- } \\
\text { vention on conduct problems: coefficient for con- } \\
\text { duct problems -0.54, SE 0.22, } \mathrm{t}=2.42, \mathrm{P}=0.05 .\end{array}$ \\
\hline
\end{tabular}


Table 2. Outcomes not included in meta-analysis (Continued)

\begin{tabular}{|c|c|c|c|c|}
\hline Berry 2009 & $\begin{array}{l}\text { Coaching for } \\
\text { Communities }\end{array}$ & $\begin{array}{l}\text { Individual-Tar- } \\
\text { geted }\end{array}$ & $\begin{array}{l}\text { Variety and vol- } \\
\text { ume of offending }\end{array}$ & $\begin{array}{l}\text { For variety of offending, the mean in the interven- } \\
\text { tion group was } 3.5 \text { vs } 5.95 \text { in the control group at } \\
\text { the end of intervention; and for volume of offend- } \\
\text { ing, the mean in the intervention group was } 18.1 \text { vs } \\
23.9 \text { in the control group. }\end{array}$ \\
\hline
\end{tabular}

\begin{tabular}{|c|c|c|c|c|}
\hline $\begin{array}{l}\text { Conduct Disor- } \\
\text { ders Prevention } \\
\text { Research Group } \\
2010\end{array}$ & Fast Track & School-Targeted & $\begin{array}{l}\text { Antisocial per- } \\
\text { sonality disorder } \\
\text { (ASPD) }\end{array}$ & $\begin{array}{l}10 \text { years post intervention, the prevalence of being } \\
\text { in the DSM-IV clinical range for ASPD was lower in } \\
\text { the intervention group than in the control group } \\
\text { (OR } 0.60,95 \% \mathrm{Cl} 0.39 \text { to } 0.93, \mathrm{P}=0.022 \text { ). }\end{array}$ \\
\hline
\end{tabular}

Connell 2007 Family Check-Up Family-Universal Antisocial Behav- Across treatment and control groups, no signifi-
iour cant differences were found for marijuana abuse/ dependence $\left(\mathrm{Chi}^{2}(1,781)=0.69, \mathrm{P}>0.05\right)$. No significant correlation between assignment to experimental condition(s) and antisocial behaviour over time

\begin{tabular}{|c|c|c|c|}
\hline $\begin{array}{l}\text { Cunningham } \\
2012\end{array}$ & SafERteens & $\begin{array}{l}\text { Individual-Tar- } \\
\text { geted }\end{array}$ & $\begin{array}{l}\text { Any peer aggres- } \\
\text { sion }\end{array}$ \\
\hline
\end{tabular}

A reduction of $34.3 \%$ in the proportion reporting any severe peer aggression at 3 months (from $82.7 \%$ ), increasing to a $43.3 \%$ reduction at 12 months (OR 1.36, 95\% Cl 0.87 to 2.12) for the therapist group. For the computer group, a reduction of $21.3 \%$ was evident at 3 months, and $26.2 \%$ at 12 months (OR $0.88,95 \% \mathrm{Cl} 0.57$ to 1.34 ). For controls, a $16.4 \%$ reduction was evident at 3 months, increasing to $25.9 \%$ at 12 months.

\begin{tabular}{|c|c|c|c|}
\hline $\begin{array}{l}\text { Cunningham } \\
2012\end{array}$ & SafERteens & $\begin{array}{l}\text { Individual-Tar- } \\
\text { geted }\end{array}$ & $\begin{array}{l}\text { Any peer victimi- } \\
\text { sation or peer vi- } \\
\text { olence }\end{array}$ \\
\hline
\end{tabular}

Reduction of $10.4 \%$ at 3 months and $22.7 \%$ at 12 months for those in the therapist group (baseline $47.6 \%$ ) (OR $1.25,95 \% \mathrm{Cl} 0.87$ to 1.79 ); and reduction of $2.5 \%$ at 3 months and $17.4 \%$ at 12 months for the computer group (OR 1.06, 95\% Cl 0.73 to 1.52). Among those in the control group, results showed a $4.7 \%$ increase at 3 months but a $12.3 \%$ reduction in reported experience of peer violence at 12 months.

\begin{tabular}{lll}
\hline DeGarmo $2009 \quad$ LIFT School-Universal & $\begin{array}{l}\text { Percentage ar- } \\
\text { rested or de- } \\
\text { tained }\end{array}$
\end{tabular}

At initial follow-up, 300 days post intervention, $0.6 \%$ of those in the intervention group had been detained or arrested vs $4.1 \%$ in the control group. 2.5 years post intervention ( 900 days), $5.1 \%$ had been arrested/detained in the intervention group vs $10.3 \%$ in the control group.

\begin{tabular}{lllll}
\hline Friedman 2002 & $\begin{array}{l}\text { Botvin Life Skills } \\
\text { Training and An- } \\
\text { ti-violence }\end{array}$ & $\begin{array}{l}\text { Individual-Tar- } \\
\text { geted }\end{array}$ & $\begin{array}{l}\text { Degree of illegal } \\
\text { offences }\end{array}$ & $\begin{array}{l}\text { Among intervention participants vs controls, there } \\
\text { was a slight reduction in the degree of illegal of- } \\
\text { fences (t=-1.53). }\end{array}$ \\
\hline $\begin{array}{l}\text { Freudenberg } \\
2010\end{array}$ & REAL MEN & $\begin{array}{l}\text { Individual-Tar- } \\
\text { geted }\end{array}$ & $\begin{array}{l}\text { Criminal justice } \\
\text { outcomes (re-ar- } \\
\text { rest, re-incarcer- } \\
\text { ation, problem- } \\
\text { atic behaviour) }\end{array}$ & $\begin{array}{l}\text { Intervention associated with reduced odds of re- } \\
\text { arrest (OR 0.871). No 95\% confidence interval or } \\
\text { standard error was provided. Odds of re-incarcer- } \\
\text { ation 1.019; the intervention was associated with } \\
\text { reduced odds of often engaging in problematic be- } \\
\text { haviour (OR 0.789) }\end{array}$ \\
\hline Gonzales 2014 & $\begin{array}{l}\text { Bridges to High } \\
\text { School (Bridges/ } \\
\text { Puentes) }\end{array}$ & Family-Targeted & $\begin{array}{l}\text { Externalising } \\
\text { symptoms }\end{array}$ & $\begin{array}{l}\text { Intervention associated with small reduction in } \\
\text { externalising symptoms at 2 and 5 years post-test }\end{array}$ \\
\hline
\end{tabular}


Table 2. Outcomes not included in meta-analysis

(Continued)

(unstandardised regression coefficients -0.02 and -0.01 , respectively)

\begin{tabular}{|c|c|c|c|}
\hline Kellam 2008 & $\begin{array}{l}\text { Good Behaviour } \\
\text { Game (GBG) }\end{array}$ & School-Universal & $\begin{array}{l}\text { Lifetime antiso- } \\
\text { cial personality } \\
\text { disorder (ASPD) }\end{array}$ \\
\hline
\end{tabular}

At 12 years' follow-up (participants were aged 19 to 21 years), overall rates of ASPD were lower for those in the GBG groups (17\%) vs internal controls $(25 \%)(P=0.07)$.

$\begin{array}{ll}\text { LoSciuto } 1999 & \text { Woodrock Youth School-Universal Aggression } \\ & \text { Development } \\ & \text { Project }\end{array}$

No strong evidence showed a greater reduction in aggression in experimental vs control groups at post-test $(F(1,342)=2.95, P=0.09$, Cohen's $d$ $=0.19$ ). Insufficient data were available to adjust these findings for clustering of participants by classroom.

\begin{tabular}{llll}
\hline Olds 1998 & $\begin{array}{l}\text { Nurse Family } \\
\text { Partnership }\end{array}$ & Family-Targeted & $\begin{array}{l}\text { Major delinquent } \\
\text { acts }\end{array}$
\end{tabular}

At 15-year follow-up, results showed no difference between intervention and control groups in the mean number of major delinquent acts committed: mean 2.79 among those who received nurse visitation through pregnancy vs 3.02 in the control group $(P=0.93)$. Among a subgroup of women from low socioeconomic status (SES) households who were unmarried, the comparison was 3.45 vs $4.09(P=$ 0.60).

Among those receiving nurse visitation through to the child's second birthday, the comparison was 3.57 vs $3.02(P=0.48)$. Among a subgroup of women from low SES households who were unmarried, the comparison was 3.99 vs $4.09(P=0.77)$.

$\begin{array}{llll}\text { Olds } 1998 & \begin{array}{l}\text { Nurse Family } \\ \text { Partnership }\end{array} & \text { Family-Targeted } & \begin{array}{l}\text { Mean number of } \\ \text { arrests }\end{array}\end{array}$

Differences between groups were evident regarding the incidence of arrests at 15-year follow-up. For those visited during pregnancy, the incidence of arrests among children was 0.16 vs $0.36(P=$ $0.005)$; among a subgroup of women from low SES households who were unmarried, the comparison was 0.15 vs $0.45(P=0.02)$.

Among those visited through pregnancy and infancy, their children were arrested a mean of 0.17 times vs 0.36 times among controls $(P=0.005)$; and among a subgroup of women from low SES households who were unmarried, the comparison was 0.20 vs $0.45(P=0.03)$.

\begin{tabular}{llll}
\hline Olds 1998 & $\begin{array}{l}\text { Nurse Family } \\
\text { Partnership }\end{array}$ & Family-Targeted & $\begin{array}{l}\text { Externalising } \\
\text { problems }\end{array}$
\end{tabular}

At 15-year follow-up, results showed no difference between intervention and control groups in the mean number of externalising problems: mean 13.65 among those who received nurse visitation through pregnancy vs 13.73 in the control group $(P=0.95)$. Among a subgroup of women from low socioeconomic status (SES) households who were unmarried, the comparison was 15.63 vs $14.18(\mathrm{P}=$ 0.42).

Among those receiving nurse visitation through to the child's second birthday, the comparison was 13.88 vs $13.73(P=0.89)$ Among a subgroup of women from low SES households who were un- 
Table 2. Outcomes not included in meta-analysis

(Continued)

married, the comparison was 11.85 vs $14.18(\mathrm{P}=$ 0.17).

\begin{tabular}{|c|c|c|c|}
\hline Perry 2003 & $\begin{array}{l}\text { DARE vs DARE } \\
\text { Plus }\end{array}$ & School-Universal & Physical victimi- \\
\hline
\end{tabular}

Among boys, those in DARE-Plus schools were less likely than those in control schools to show increases in victimisation (growth rate $-0.1, \mathrm{SE} 0.04, \mathrm{P}$ $=0.02$ ); there was no difference between DARE and control (growth curve rate, mean $-0.03, \mathrm{SE} 0.04, \mathrm{P}=$ 0.18). No differences were evident between groups among girls.

\begin{tabular}{|c|c|c|c|c|}
\hline $\begin{array}{l}\text { Schweinhart } \\
1980\end{array}$ & $\begin{array}{l}\text { High/Scope Per- } \\
\text { ry Preschool Pro- } \\
\text { gram }\end{array}$ & School-Targeted & $\begin{array}{l}\text { Carried a gun } \\
\text { or knife once or } \\
\text { more often }\end{array}$ & $\begin{array}{l}\text { At } 10 \text {-year follow-up (when participants were age } \\
15), 13 \text { of } 44(29.5 \%) \text { in the intervention group had } \\
\text { carried a gun or knife once or more compared to } 15 \\
\text { of } 55(27 \%) \text { in the control group. }\end{array}$ \\
\hline
\end{tabular}

Shetgiri 2011 [No study name] School-Targeted Been in trouble with the police in the past 12 months
Eighteen per cent of those in the intervention group had been in trouble with the police in the past 12 months at follow-up post intervention ( $21 \%$ at baseline) compared to $26 \%$ of those in the control group at follow-up ( $32 \%$ at baseline) $(P=0.41)$.

\begin{tabular}{llll}
\hline Tierney 1995 & $\begin{array}{l}\text { Big Brothers Big } \\
\text { Sisters }\end{array}$ & $\begin{array}{l}\text { Individual-Tar- } \\
\text { geted }\end{array}$ & $\begin{array}{l}\text { Hitting, stealing, } \\
\text { and damaging } \\
\text { property }\end{array}$
\end{tabular}

Participation in the intervention was associated with a $32 \%$ reduction in the number of times participants hit someone compared to control. The percentage reduction was greater in females than in males ( $43 \%$ vs $25 \%)$. Results showed a greater reduction among females from an ethnic minority than among white females ( $48 \%$ reduction vs $2 \%$ reduction), and a greater reduction was observed in white males (45\%) than in males from an ethnic minority (4\%). Data show a $19 \%$ reduction in the number of times participants in the intervention group vs the control group stole something and little change ( $0.15 \%$ reduction) in the number of times participants damaged property. Findings show a $16 \%$ reduction in the number of times participants in the intervention group took something from a store compared to controls, and a $17 \%$ reduction in the number of times participants did risky things. Little change was evident in relation to behavioural conduct ( $1 \%$ reduction in intervention vs control) and the number of times participants were involved in a fight ( $1 \%$ reduction in intervention vs control).

\section{Vehicle-related risk behaviour}

\begin{tabular}{|c|c|c|c|c|}
\hline $\begin{array}{l}\text { Schweinhart } \\
1980\end{array}$ & $\begin{array}{l}\text { High/Scope Per- } \\
\text { ry Preschool } \\
\text { Study }\end{array}$ & School-Targeted & Wearing seatbelt & $\begin{array}{l}\text { Among those in the intervention group, } 24 \text { of } 56 \\
(43 \%) \text { wore a seatbelt sometimes or never } 22 \text { years } \\
\text { after the end of the programme compared to } 40 / 61 \\
(66 \%) \text { of those in the control group. Effect size for } \\
\text { wearing a seatbelt was } 0.37(P=0.052) \text {. }\end{array}$ \\
\hline D'Amico 2002 & $\begin{array}{l}\text { Risk Skills Train- } \\
\text { ing vs DARE }\end{array}$ & School-Universal & $\begin{array}{l}\text { Driving under the } \\
\text { influence/riding } \\
\text { with a drinking } \\
\text { driver }\end{array}$ & $\begin{array}{l}\text { No differences were observed at 6-month follow-up } \\
\text { in relation to driving after drinking and riding with } \\
\text { a drinking driver (mean values for baseline and 6- } \\
\text { month follow-up: risk skills training programme } \\
\text { group: mean 1.25 (SD 3.30) to } 0.95 \text { (SD 2.20); DARE- }\end{array}$ \\
\hline
\end{tabular}


Table 2. Outcomes not included in meta-analysis

(Continued)

A group: mean 0.75 (SD 1.42) to 0.67 (SD 1.26); control group: mean 1.58 (SD 5.32) to 1.32 (4.42).

\begin{tabular}{|c|c|c|c|}
\hline Nirenberg 2013 & ROAD & $\begin{array}{l}\text { Individual-Tar- } \\
\text { geted }\end{array}$ & $\begin{array}{l}\text { Speeding and } \\
\text { distracted dri- } \\
\text { ving }\end{array}$ \\
\hline
\end{tabular}

Scores for speeding and distracted driving were lower in the control group (community service) than in the combined motivational interviewing study arms 6 months post intervention ( $\mathrm{t}(607)=$ -2.32; $P=0.02$ ) (i.e. the control group reported less of the behaviour) (Log+1 transformed mean values: control 2.49 (SD 1.57); combined MI 2.81 (SD 1.53)). No difference between groups was evident in relation to dangerous driving factor scores ( $\mathrm{t}(607)$ $=-0.21, P=0.84)($ Log +1 transformed means: control 1.39 (SD 1.46); combined MI 1.34 (SD 1.39)) or scores for alcohol, drugs, and driving (Log+1 mean values: control 0.58 (SD 1.14); combined MI 0.60 (SD 1.14)).

\section{Sexual risk behaviour}

\begin{tabular}{|c|c|c|c|c|}
\hline Bonds 2010 & New Beginnings & Family-Targeted & $\begin{array}{l}\text { Number of sexu- } \\
\text { al partners }\end{array}$ & $\begin{array}{l}\text { Significant group effect for number of sexual part- } \\
\text { ners (control mean }=1.65 \text {, intervention mean }= \\
0.68, P=0.01, d=0.49)\end{array}$ \\
\hline Estrada 2015 & $\begin{array}{l}\text { Brief Familias } \\
\text { Unidas }\end{array}$ & Family-Targeted & $\begin{array}{l}\text { Inconsistent con- } \\
\text { dom use in past } \\
90 \text { days }\end{array}$ & $\begin{array}{l}\text { Growth curve analyses showed no significant dif- } \\
\text { ferences in unsafe sexual intercourse, defined as } \\
\text { inconsistent condom use, during the past } 90 \text { days } \\
\text { between brief Familias Unidas and CPC (beta = } \\
0.26, P=0.25 \text { ). }\end{array}$ \\
\hline
\end{tabular}

\begin{tabular}{|c|c|c|c|}
\hline $\begin{array}{l}\text { Freudenberg } \\
2010\end{array}$ & REAL MEN & $\begin{array}{l}\text { Individual-Tar- } \\
\text { geted }\end{array}$ & $\begin{array}{l}\text { Engaged in risky } \\
\text { sexual behaviour }\end{array}$ \\
\hline
\end{tabular}

No difference was observed between the intervention arm and the control arm in relation to the proportion of participants engaging in risky sexual behaviour in the past 90 days (OR 0.856 , no $95 \% \mathrm{Cl}$ given, but $\mathrm{P}>0.05)$.

$\begin{array}{lll}\text { Griffin } 2006 & \text { Life Skills Train- } & \text { School-Universal } \\ \text { ing } & \begin{array}{l}\text { Multiple sexual } \\ \text { partners }\end{array}\end{array}$

$21.3 \%$ of those in the intervention group had multiple sex partners at age 24 years ( 11 years following the end of the intervention) vs $24.5 \%$ of those in the control group.

$\begin{array}{ll}\text { Griffin } 2006 & \text { Life Skills Train- School-Universal Condom use } \\ \text { ing }\end{array}$

Results showed no difference across experimental conditions in relation to condom use at age 24 years ( 11 years following the end of the intervention): $78.7 \%$ of the intervention group reported that they did not always use condoms vs $78.2 \%$ of controls $\left(\mathrm{Chi}^{2}(1)=0.05, \mathrm{P}<0.82\right)$.

\begin{tabular}{llll}
\hline McBride Murry & SAAF (Stronger & Family-Targeted & Sexual behaviour \\
2014 & African American & \\
& Families) &
\end{tabular}

The effect size of the intervention on post-test sexual behaviour was 0.01 , although study authors state that detecting a substantial effect size was unlikely with a sample of $<1000$ and owing to the length of time between the programme and longerterm (65-month) follow-up. Using structural equation modelling, study authors also report that participation in SAAF led to protection in engagement in sexual risk behaviour through an indirect mechanism involving increased intervention-targeted parenting practices (beta $=0.35, \mathrm{P}<0.01$ ), which were associated in turn with increased youth self- 
Table 2. Outcomes not included in meta-analysis

(Continued)

pride (beta $=0.25, \mathrm{P}<0.05$ ), in turn associated with increased protective sexual norms (beta $=0.7, \mathrm{P}<$ 0.01 ), in turn associated with reduced sexual risk behaviour (beta $=-0.22, \mathrm{P}<0.01$ ).

\begin{tabular}{|c|c|c|c|c|}
\hline Olds 1998 & $\begin{array}{l}\text { Nurse Family } \\
\text { Partnership }\end{array}$ & Family-Targeted & $\begin{array}{l}\text { Lifetime inci- } \\
\text { dence of sex } \\
\text { partners in past } 6 \\
\text { months }\end{array}$ & $\begin{array}{l}\text { At } 15 \text {-year follow-up: among those visited during } \\
\text { pregnancy, the mean number of sex partners was } \\
1.10 \text { vs } 1.56(P=0.48) \text {; and among a subgroup of } \\
\text { women from low SES households who were un- } \\
\text { married, the mean number of sex partners in the } \\
\text { past } 6 \text { months was } 2.23 \text { vs } 2.48 \text { ( } P=0.73) \text {. Among } \\
\text { those visited during pregnancy and infancy, mean } \\
\text { incidence of sex partners was } 1.16 \text { vs } 1.56 \text { ( } P= \\
0.90) \text {; and for the subgroup of women from low SES } \\
\text { households who were unmarried, mean was } 0.92 \text { vs } \\
2.48(P=0.003) \text {. }\end{array}$ \\
\hline
\end{tabular}

Piper $2000 \quad$ Healthy for Life School-Universal Sexual intercourse in past 30 days
Students were followed up in the ninth and 10th grades, at 12 and 24 months. Students in the ageappropriate condition reported higher rates of intercourse than those in the control group (13\% vs $11 \%$; HLM coefficient 0.4, SE $0.16, P<0.05$ ) at 12 months; at 24 months, prevalence was $23 \%$ vs $19 \%$ (HLM coefficient 0.32, SE 0.2, P > 0.1). The intensive condition had no effect on rates of intercourse in the 2 groups at 12 months (prevalence $15 \%$ vs $11 \%$ in the control arm, HLM coefficient 0.25, SE 0.21, P $>0.1$ ) nor at 24 months (prevalence $21 \%$ vs $19 \%$ in the control arm; HLM coefficient -0.07, SE 0.15, P > $0.1)$.

\section{Physical inactivity}

\begin{tabular}{|c|c|c|c|c|}
\hline Bush 1989 & Know Your Body & School-Universal & Fitness score & $\begin{array}{l}\text { Study authors highlight that significant changes } \\
\text { were observed in a favourable direction in relation } \\
\text { to fitness. The observed difference between inter- } \\
\text { vention and control group mean change after } 2 \\
\text { years of intervention was - } 0.28 \text { (SE 0.19); and - } 0.38 \\
\text { (SE 0.15) after adjustment for baseline value, age, } \\
\text { sex, and socioeconomic status. }\end{array}$ \\
\hline
\end{tabular}

\begin{tabular}{llll}
\hline O'Neill 2016 & $\begin{array}{l}\text { Michigan Model } \\
\text { for Health }\end{array}$ & $\begin{array}{l}\text { School-Universal } \\
\text { Physical activity }\end{array}$ & $\begin{array}{l}\text { Six weeks following the intervention, results } \\
\text { showed a significant intervention effect for physi- } \\
\text { skills }\end{array}$
\end{tabular}$\quad \begin{aligned} & \text { cal activity skills: } F[53,590.79]=4.42, P=0.001 .\end{aligned}$

$\begin{array}{ll}\text { Saraf } 2015 \quad \text { (none given) } & \begin{array}{l}\text { Total time spent } \\ \text { watching TV } \\ \text { (minutes) }\end{array}\end{array}$

Weak evidence for a reduction in time spent watching television in the intervention group: reported reduction from $70.4 \%(95 \% \mathrm{Cl} 67.0 \%$ to $73.8 \%)$ at pre-test to $56.1 \%(95 \% \mathrm{Cl} 53.9 \%$ to $58.4 \%)$ at posttest $(\mathrm{P}<0.05)$. In comparison, a slight increase in time spent watching TV was observed in the control group: $56.4 \%$ (95\% $\mathrm{Cl} 53.9 \%$ to $58.9 \%$ ) at pretest increasing to $57.9 \%(95 \% \mathrm{Cl} 55.2 \%$ to $60.8 \%)$ at post-test; overall difference $15.8(95 \% \mathrm{Cl} 15.7$ to 16.9) $(\mathrm{P}<0.01)$.

\begin{tabular}{lllll}
\hline Tierney 1995 & $\begin{array}{l}\text { Big Brothers Big } \\
\text { Sisters }\end{array}$ & $\begin{array}{l}\text { Individual-Tar- } \\
\text { geted }\end{array}$ & $\begin{array}{l}\text { Number of times } \\
\text { participated in } \\
\text { an outdoor activ- } \\
\text { ity }\end{array}$ & $\begin{array}{l}\text { Overall, researchers reported a 23\% reduction } \\
\text { in the number of times participants participated } \\
\text { in an outdoor activity. The effect was greater for } \\
\text { males than for females (25\% vs 18\% reduction). }\end{array}$
\end{tabular}


Data show a greater reduction among females from a minority ethnic group (43\%) than among males from an ethnic minority group (14\%); and a greater reduction among white males (29\%) than among males from an ethnic minority (14\%). Data for white females were not available.

$\begin{array}{llll}\text { Walter } 1989 \quad \text { Know Your Body } & \text { School-Universal } & \begin{array}{l}\text { Recovery index } \\ \text { score }\end{array}\end{array}$

In Westchester, the recovery index in the intervention group changed by -0.7 per year vs -1.4 in the control group (overall difference in school means $0.7(95 \% \mathrm{Cl}-0.1$ to 1.5$))$. Among schools in the Bronx, the rate of change per year in the intervention group was -2.5 vs -2.5 in the control group (difference in school means $0.0,95 \% \mathrm{Cl}-1.3$ to 1.3 ).

\section{Nutrition}

\begin{tabular}{|c|c|c|c|c|}
\hline O’Neill 2016 & $\begin{array}{l}\text { Michigan Model } \\
\text { for Health }\end{array}$ & School-Universal & $\begin{array}{l}\text { Nutritional be- } \\
\text { haviours }\end{array}$ & $\begin{array}{l}\text { Six weeks following the intervention, results show } \\
\text { a significant effect on nutritional behaviours: } \\
F[53,213.47]=2.32, P=0.04 \text {. }\end{array}$ \\
\hline
\end{tabular}

Walter 1989 Know Your Body School-Universal BMI

In Westchester, data showed no change per year among intervention schools (mean 0.0 (SD 0.1)) vs a change of 0.1 per year (SD 0.1) among control schools (difference $-0.1,95 \% \mathrm{Cl}-0.3$ to 0.1 ). In the Bronx, the rate of change per year among intervention schools was 0.1 (SD 0.1) vs 0.2 (SD 0.1) among control schools (difference $-0.1,95 \% \mathrm{Cl}-0.3$ to 0.1 ).

Walter 1989 Know Your Body School-Universal Plasma total
cholesterol (mg/
$\mathrm{dL})$

In Westchester, the rate of change in total cholesterol was $-2.1 \mathrm{mg} / \mathrm{dL} / \mathrm{y}$ (SD 1.0) among intervention schools but $-0.4 \mathrm{mg} / \mathrm{dL} / \mathrm{y}$ (SD 0.7) among control schools - equivalent to a net mean change in total cholesterol of $-1.7 \mathrm{mg} / \mathrm{dL} / \mathrm{y}$ ( -2.7 to $-0.7 \mathrm{mg} / \mathrm{dL})$. Among intervention schools in the Bronx, the rate of change was $-2.6 \mathrm{mg} / \mathrm{dL} / \mathrm{y}$ (SD 1.5) vs - 1.6 (SD 1.8) among control schools - equivalent to a difference of $-1.0 \mathrm{mg} / \mathrm{dL} / \mathrm{y}(95 \% \mathrm{Cl}-2.3$ to $0.3 \mathrm{mg} / \mathrm{dL})$.

\begin{tabular}{lll}
\hline Walter $1989 \quad$ Know Your Body & School-Universal & $\begin{array}{l}\text { Total fat (\% of to- } \\
\text { tal kcal) }\end{array}$
\end{tabular}

In Westchester, the net mean reduction in total fat intake between intervention and control schools was $-3.6 \%(95 \% \mathrm{Cl}-7.1$ to $-0.1 \%)$; in the Bronx, the net mean reduction in total fat intake was $-1.9 \%$ ( $95 \% \mathrm{Cl}-7.1$ to $3.3 \%)$. Data are presented from a random subsample of the total study population.

\begin{tabular}{llll}
\hline Walter 1989 Know Your Body & School-Universal & $\begin{array}{l}\text { Systolic blood } \\
\text { pressure } \\
(\mathrm{mmHg})\end{array}$ & $\begin{array}{l}\text { Among schools in Westchester, systolic blood pres- } \\
\text { sure changed by } 0.6 \mathrm{mmHg} \text { (SD 0.8) vs 0.8 mmHg } \\
\text { (SD 0.6) in the control group, for an overall differ- } \\
\text { ence of }-0.2 \mathrm{mmHg} \mathrm{(-1.0} \mathrm{to} \mathrm{0.6} \mathrm{mmHg).}\end{array}$ \\
\hline
\end{tabular}

\section{Mental health}

Bonds 2010
New Beginnings
Family-Targeted
Internalising disorder, externalising disorder 6-year follow-up: the MPCP intervention arm had significantly fewer externalising problems $(-0.11$, SE 0.11) compared to the control group $(0.08, \mathrm{SE}$ $0.14)(P=0.02)$. There was no difference between intervention and control in the number of internal- 
ising problems nor in the mental disorder symptom count $(P \geq 0.05)$.

15-year follow-up: lower proportion of intervention group participants with (1) internalising disorder diagnosed in past 9 years; intervention: $4.55 \%$ (SD 2.69), control: $16.7 \%$ (SD 3.25, OR 0.26), and (2) externalising disorder diagnosed in past 9 years; intervention: 0\% (SD 0), control: 3.64\% (SD 0.04).

$\begin{array}{llll}\text { Gonzales } 2014 & \begin{array}{l}\text { Bridges to High } \\ \text { School (Bridges/ } \\ \text { Puentes) }\end{array} & \text { Family-Targeted } & \begin{array}{l}\text { Internalising } \\ \text { symptoms }\end{array}\end{array}$

Intervention was associated with slight increase Puentes) in internalising symptoms at 2 years post-test (unstandardised regression coefficient 0.42 ) but a small reduction in internalising symptoms at 5 years post-test (unstandardised regression coefficient -0.02).

\begin{tabular}{|c|c|c|c|}
\hline Kellam 2008 & $\begin{array}{l}\text { Good Behavior } \\
\text { Game }\end{array}$ & School-Universal & $\begin{array}{l}\text { Lifetime major } \\
\text { depressive dis- } \\
\text { order and gen- } \\
\text { eralised anxiety } \\
\text { disorder }\end{array}$ \\
\hline
\end{tabular}

At 12 years following intervention, when participants were aged 19 to 21 years, unadjusted rates of lifetime major depressive disorder were lower for the GBG group (10\%) than for the control group $(15 \%)(P=0.27)$. The difference was slightly larger for males than for females (males: $9 \%$ for GBG, $14 \%$ for controls; females: $12 \%$ for GBG, $15 \%$ for controls). Overall rates of generalised anxiety disorder were small and did not differ by intervention condition ( $2 \%$ for GBG, $3 \%$ for control; $P=0.37$ ).

\begin{tabular}{llll}
\hline Olds 1998 & $\begin{array}{l}\text { Nurse Family } \\
\text { Partnership }\end{array}$ & Family-Targeted & $\begin{array}{l}\text { Internalising } \\
\text { problems }\end{array}$
\end{tabular}

Results showed no difference between study arms in the mean number of internalising problems at 15-year follow-up: for those visited during pregnancy, mean 11.19 vs $10.58, P=0.46$; and among a subgroup of women from low SES households who were unmarried, mean 11.15 vs $10.82, \mathrm{P}=0.8$.

For those visited through pregnancy and infancy, no difference between groups was evident: mean 11.66 vs $10.58, P=0.19$; among the subgroup of women from low SES households who were unmarried, mean 9.85 vs $10.82, \mathrm{P}=0.44$.

\begin{tabular}{llll}
\hline Cho 2005 & Reconnecting & School-Targeted & Anger \\
(Sanchez 2007, & Youth & & \\
Hallfors 2006) & &
\end{tabular}

Findings regarding depression and anxiety were not reported. However, study authors report that at 6-month follow-up, a negative outcome was observed for those in the experimental arm compared to those in the control arm: main programme effect: $F=-3.62, P=0.058$ (i.e. those in the intervention arm showed greater frequency of anger compared to those in the control arm).

\begin{tabular}{|c|c|c|c|}
\hline Walker 2002 & (none given) & $\begin{array}{l}\text { Individual-Uni- } \\
\text { versal }\end{array}$ & $\begin{array}{l}\text { Mental health } \\
\text { score }\end{array}$ \\
\hline
\end{tabular}

Data show no difference in change in mental health score between intervention and control participants at 3 or 12 months. However, among young people who scored 16 or more on the depression scale (indicating probable depression), there was a greater reduction in mental health score than among those in the control group (-8.1 intervention, -1.4 control, $95 \%$ confidence interval $(\mathrm{Cl})$ for mean difference -0.3 to $-13.3, P=0.04$ at 3 months; 
Table 2. Outcomes not included in meta-analysis

-1.6 intervention, 4.4 control, $95 \% \mathrm{Cl}-0.5$ to $-11.5, \mathrm{P}$ $=0.03$ ).

\section{Educational attainment}

\begin{tabular}{llll}
\hline Berry 2009 & $\begin{array}{ll}\text { Coaching for } \\
\text { Communities }\end{array}$ & Individual-Tar- & In education/em- \\
& geted & ployment
\end{tabular}

At follow-up (post intervention), $85 \%$ of those in the intervention group were in education or employment vs $59 \%$ of those in the control group $(\mathrm{P}<$ 0.05).

\begin{tabular}{llll}
\hline Bond 2004 & $\begin{array}{l}\text { Gatehouse } \\
\text { Project }\end{array}$ & School-Universal & $\begin{array}{l}\text { Low school at- } \\
\text { tachment }\end{array}$
\end{tabular}$\quad$\begin{tabular}{l}
$\begin{array}{l}\text { Two years following the intervention, the OR for } \\
\text { low school attachment was } 1.21(95 \% \mathrm{Cl} 0.93 \text { to } \\
1.57) .\end{array}$ \\
\hline
\end{tabular}

\begin{tabular}{|c|c|c|c|c|}
\hline $\begin{array}{l}\text { Conduct Prob- } \\
\text { lems Prevention } \\
\text { Research Group } \\
2010\end{array}$ & Fast Track & School-Targeted & $\begin{array}{l}\text { Graduated from } \\
\text { high school or } \\
\text { graduate equiva- } \\
\text { lency diploma }\end{array}$ & $\begin{array}{l}\text { At long-term follow-up, the adjusted OR for gradu- } \\
\text { ation from high school or a graduate equivalency } \\
\text { diploma was } 0.93 \text { ( } 95 \% \mathrm{Cl} 0.68 \text { to } 1.27, \mathrm{P}=0.654) \text {. }\end{array}$ \\
\hline
\end{tabular}

\begin{tabular}{|c|c|c|c|c|}
\hline $\begin{array}{l}\text { Freudenberg } \\
2010\end{array}$ & REAL MEN & $\begin{array}{l}\text { Individual-Tar- } \\
\text { geted }\end{array}$ & $\begin{array}{l}\text { Enrolled in ed- } \\
\text { ucational or vo- } \\
\text { cational pro- } \\
\text { grammes in the } \\
\text { past year }\end{array}$ & $\begin{array}{l}\text { Receipt of the intervention was associated with in - } \\
\text { creased odds of being enrolled in educational/vo- } \\
\text { cational programmes (OR } 1.330 \text { ). No } 95 \% \mathrm{Cl} \text { or } \\
\text { standard error was provided. }\end{array}$ \\
\hline
\end{tabular}

\begin{tabular}{|c|c|c|c|c|}
\hline Friedman 2002 & $\begin{array}{l}\text { Life Skills Train- } \\
\text { ing and Anti-vio- } \\
\text { lence Program }\end{array}$ & $\begin{array}{l}\text { Individual-Tar- } \\
\text { geted }\end{array}$ & School problems & $\begin{array}{l}\text { No difference was evident between groups in rela- } \\
\text { tion to school problems }(t=0.91, P>0.05) \text {. }\end{array}$ \\
\hline
\end{tabular}

\begin{tabular}{|c|c|c|c|c|}
\hline Gonzales 2014 & $\begin{array}{l}\text { Bridges to Hlgh } \\
\text { School (Bridges/ } \\
\text { Puentes) }\end{array}$ & Family-Targeted & $\begin{array}{l}\text { High school } \\
\text { dropout (no high } \\
\text { school degree or } \\
\text { equivalent and } \\
\text { no attendance } \\
\text { at high school } \\
\text { at } 12 \text { th grade as- } \\
\text { sessment) }\end{array}$ & $\begin{array}{l}\text { The path coefficient estimate for high school } \\
\text { dropout at 5-year follow-up was not statistically } \\
\text { significant (unstandardised regression coefficient } \\
-0.16 \text { ), but an indirect effect of the intervention } \\
\text { was identified through school engagement (un- } \\
\text { standardised regression coefficient }-0.062,95 \% \mathrm{Cl} \\
-0.517 \text { to }-0.001 \text { ). }\end{array}$ \\
\hline
\end{tabular}

\begin{tabular}{llll}
\hline Kellam 2014 Good Behavior & School-Universal \\
Game & $\begin{array}{l}\text { High school } \\
\text { graduation }\end{array}$ & $\begin{array}{l}\text { High school graduation rates were slightly higher } \\
\text { for those in the GBG arm (72\%) than for those in } \\
\text { the control arm (64\%), and this effect was larger for }\end{array}$ \\
& & $\begin{array}{l}\text { males than for females (68\% vs } 54 \%, \text { respectively). } \\
\text { However, these data were not adjusted for cluster- } \\
\text { ing. }\end{array}$
\end{tabular}

\begin{tabular}{|c|c|c|c|c|}
\hline Kitzman 2010 & $\begin{array}{l}\text { Nurse Family } \\
\text { Partnership }\end{array}$ & Family-Targeted & $\begin{array}{l}\text { Academic } \\
\text { achievement } \\
\text { (grade point av- } \\
\text { erage - GPA) }\end{array}$ & $\begin{array}{l}\text { The GPA for grades } 1 \text { to } 6 \text { for those in the interven- } \\
\text { tion group was } 2.39(0.04) \text { vs } 2.48(0.05) \text { for those in } \\
\text { the control group ( } P=0.19 \text {, mean difference } 0.09 \\
(-0.04 \text { to } 0.22) \text { ). For PIAT scores (reading and maths) } \\
\text { at } 12 \text { years, the mean difference was } 1.27 \text { ( }-0.44 \text { to } \\
2.98)(P=0.14) \text {. Among families of lower socioeco- } \\
\text { nomic status, those in the intervention group had } \\
\text { higher PIAT scores in reading and math at age } 12 \\
\text { (ES } 0.25, P=0.009) \text {, higher GPAs and group-based } \\
\text { achievement test scores in reading and math in } \\
\text { grades } 1 \text { through } 6 \text { (ES } 0.18, P=0.03 ; E S 0.22, P= \\
0.02 \text {, respectively), and higher GPAs in reading and } \\
\text { math in grades } 4 \text { through } 6 \text { (ES } 0.18, P=0.047) \text {. }\end{array}$ \\
\hline
\end{tabular}


Table 2. Outcomes not included in meta-analysis (Continued)

\begin{tabular}{|c|c|c|c|c|}
\hline Li 2011 & Positive Action & School-Universal & $\begin{array}{l}\text { Suspension from } \\
\text { school }\end{array}$ & $\begin{array}{l}\text { No difference between study arms was observed } \\
\text { at follow-up in relation to suspensions from scho } \\
\text { (IRR } 0.58,95 \% \mathrm{Cl} 0.15 \text { to } 2.26 \text { ). }\end{array}$ \\
\hline LoSciuto 1999 & $\begin{array}{l}\text { Woodrock Youth } \\
\text { Development } \\
\text { Project }\end{array}$ & School-Universal & $\begin{array}{l}\text { School atten- } \\
\text { dance }\end{array}$ & $\begin{array}{l}\text { Participants in the intervention group reported } \\
\text { better average scores for self-reported school at } \\
\text { tendance }(F(1,705)=12.18, P<0.01 \text {, Cohen's } d= \\
0.26) \text {. Insufficient data were available to adjust } \\
\text { these findings for clustering of participants by } \\
\text { classroom. }\end{array}$ \\
\hline
\end{tabular}

\begin{tabular}{llll}
\hline Melnyk 2003 COPE & School-Universal & $\begin{array}{l}\text { Academic com- } \\
\text { petence }\end{array}$ & $\begin{array}{l}\text { Academic competence was slightly higher in the in- } \\
\text { tervention group than in the control group (adjust- } \\
\text { ed mean } 97.97,95 \% \mathrm{Cl} 96.35 \text { to } 99.59 ; \mathrm{vs} 95.69,95 \%\end{array}$ \\
& & $\mathrm{Cl} 94.21$ to 97.18$),$ respectively. $\mathrm{F}=4.03, \mathrm{P}=0.05$.
\end{tabular}

\begin{tabular}{|c|c|c|c|c|}
\hline Morris 2003 & $\begin{array}{l}\text { Self-Sufficiency } \\
\text { Project }\end{array}$ & Family-Targeted & $\begin{array}{l}\text { Dropped out of } \\
\text { school (aged } 15 \\
\text { to } 18 \text { ) }\end{array}$ & $\begin{array}{l}\text { At } 36 \text {-month follow-up, math score at age } 12 \text { to } \\
14 \text { was } 0.45 \text { in the intervention group compared } \\
\text { to } 0.46 \text { in the control group (ES -0.03); and aver- } \\
\text { age achievement was } 3.43 \text { compared to } 3.54 \text { in the } \\
\text { control group (ES }-0.11 \text { ). Child-reported average } \\
\text { achievement was } 3.50 \text { in the intervention group vs } \\
3.57 \text { in the control group (ES -0.09). }\end{array}$ \\
\hline
\end{tabular}

\begin{tabular}{|c|c|c|c|c|}
\hline Morris 2003 & $\begin{array}{l}\text { Self-Sufficiency } \\
\text { Project }\end{array}$ & Family-Targeted & $\begin{array}{l}\text { Average achieve- } \\
\text { ment (self-re- } \\
\text { ported) }\end{array}$ & $\begin{array}{l}\text { Self-reported average achievement was similar } \\
\text { between intervention and control groups at } 36 \\
\text { months of follow-up (effect size -0.09). }\end{array}$ \\
\hline
\end{tabular}

\begin{tabular}{|c|c|c|c|}
\hline Olds 1998 & $\begin{array}{l}\text { Nurse Family } \\
\text { Partnership }\end{array}$ & Family-Targeted & $\begin{array}{l}\text { Mean long-term } \\
\text { school suspen- } \\
\text { sions }\end{array}$ \\
\hline
\end{tabular}

Data show no difference between study arms in the mean number of long-term school suspensions at 15-year follow-up. For those visited during pregnancy: mean 0.0 vs $0.04, P=1.0$; among a subgroup of women from low SES households who were unmarried: mean 0.01 vs $0.15, P=0.97$.

For those visited through pregnancy and infancy, no difference between groups was evident: mean 0.01 vs $0.04, \mathrm{P}=1.0$; among the subgroup of women from low SES households who were unmarried, mean 0.04 vs $0.15, P=0.25$.

\begin{tabular}{|c|c|c|c|}
\hline $\begin{array}{l}\text { Schweinhart } \\
1993\end{array}$ & $\begin{array}{l}\text { High/Scope Per- } \\
\text { ry Preschool Pro- } \\
\text { gram }\end{array}$ & School-Targeted & $\begin{array}{l}\text { Total school } \\
\text { achievement }\end{array}$ \\
\hline
\end{tabular}

Those in the intervention group had higher total achievement (mean 122.2, SD 41.6) than those in the control group (mean 94.5, SD 35.5) at 9-year follow-up.

\begin{tabular}{llll}
\hline Tierney 1995 & $\begin{array}{l}\text { Big Brothers Big } \\
\text { Sisters }\end{array}$ & $\begin{array}{l}\text { Individual-Tar- } \\
\text { geted }\end{array}$ & $\begin{array}{l}\text { Grade point av- } \\
\text { erage }\end{array}$
\end{tabular}
Educational impacts of the intervention were more pronounced among girls than among boys. Overall, those receiving the intervention overall had a high- er grade point average (GPA) than those given con- trol (average 2.71 vs 2.63). The difference was par- ticularly marked among girls from an ethnic minor- ity (average GPA 2.83 vs 2.62 for those in the con- trol group).

\begin{tabular}{llll}
\hline Tierney 1995 & $\begin{array}{l}\text { Big Brothers Big } \\
\text { Sisters }\end{array}$ & $\begin{array}{l}\text { Individual-Tar- } \\
\text { geted }\end{array}$ & $\begin{array}{l}\text { Truancy/skip- } \\
\text { ping school }\end{array}$
\end{tabular}

Participants of Big Brothers Big Sisters showed a $52 \%$ reduction in the number of times they skipped a day of school and a $37 \%$ reduction in the number of times they skipped class. The impact was greater 
among girls than boys, for instance those in the intervention group showed $84 \%$ reduction compared to $4 \%$ reduction for skipping a day of school. The reduction was greater for white females than for females from an ethnic minority (92\% reduction vs $78 \%$ reduction, respectively, for skipping a day of school; $72 \%$ vs $46 \%$ reduction for skipping class). Among white males compared to males from an ethnic minority, the reduction was similar for skipping a day of school, but a greater impact was evident among ethnic minority males than among white males for skipping class ( $22 \%$ vs $12 \%$ reduction).

\section{Teenage pregnancy}

\begin{tabular}{|c|c|c|c|c|}
\hline Olds 1998 & $\begin{array}{l}\text { Nurse Family } \\
\text { Partnership }\end{array}$ & Family-Targeted & $\begin{array}{l}\text { Ever pregnant } \\
\text { or made some- } \\
\text { one pregnant in } \\
\text { the previous } 6 \\
\text { months }\end{array}$ & $\begin{array}{l}\text { At age } 15 \text { ( } 13 \text { years following the intervention), } \\
5 \text { of } 176(2.8 \%) \text { in the intervention group had ever } \\
\text { been pregnant or made someone pregnant com- } \\
\text { pared to } 4 \text { of } 148(2.7 \%) \text { in the control group (OR } \\
1.04 \text {, SE } 0.65) .\end{array}$ \\
\hline $\begin{array}{l}\text { Conduct Prob- } \\
\text { lems Prevention } \\
\text { Research Group } \\
2010\end{array}$ & Fast Track & School-Targeted & $\begin{array}{l}\text { Pregnancy by } \\
\text { age } 18\end{array}$ & $\begin{array}{l}\text { At age } 18 \text {, the proportion of participants report- } \\
\text { ing pregnancy were as follows: girls: urban African } \\
\text { American } 40 \% \text {, urban European American } 21 \% \text {, rur- } \\
\text { al European American } 17 \% \text {; boys: urban African } \\
\text { American } 27 \% \text {, urban European American } 12 \% \text {, rur- } \\
\text { al European American } 11 \% \text {. }\end{array}$ \\
\hline $\begin{array}{l}\text { Schweinhart } \\
1993\end{array}$ & $\begin{array}{l}\text { High/Scope Per- } \\
\text { ry Preschool Pro- } \\
\text { gram }\end{array}$ & School-Targeted & $\begin{array}{l}\text { At least } 1 \text { preg- } \\
\text { nancy by age } 19 \\
\text { (females only) }\end{array}$ & $\begin{array}{l}\text { At } 14 \text {-year follow-up, } 12 \text { of } 25 \text { ( } 48 \% \text { ) in the interven- } \\
\text { tion group had had at least } 1 \text { pregnancy by age } 19 \\
\text { compared to } 16 \text { of } 24(67 \%) \text { in the control group } \\
\text { (effect size } 0.5 \text { ). }\end{array}$ \\
\hline
\end{tabular}

\section{Health problems}

\begin{tabular}{|c|c|c|c|c|}
\hline $\begin{array}{l}\text { Schweinhart } \\
1993\end{array}$ & $\begin{array}{l}\text { High/Scope Per- } \\
\text { ry Preschool } \\
\text { Study }\end{array}$ & School-Targeted & $\begin{array}{l}\text { Long-term health } \\
\text { problems }\end{array}$ & $\begin{array}{l}22 \text { years following completion of the intervention, } \\
36 \% \text { of those in the intervention group had had } \\
\text { health problems treated in the previous } 5 \text { years } \\
\text { compared to } 38 \% \text { in the control group (effect size } \\
0.04, P=0.823 \text { ). } 30 \% \text { of those in the intervention } \\
\text { group had been hospitalised in the previous } 12 \\
\text { months compared to } 15 \% \text { of those in the control } \\
\text { group (effect size } 0.38 ; P=0.043 \text { ). }\end{array}$ \\
\hline
\end{tabular}

ASPD: antisocial personality disorder.

$\mathrm{Cl}$ : confidence interval.

COPE: Creating Opportunities for Personal Empowerment.

CPC: Community Practice Condition.

DARE: Drug Abuse and Resistance Education.

DSM: Diagnostic and Statistical Manual of Mental Disorders.

ES: Effect size.

GBG: Good Behaviour Game.

GPA: grade point average.

HLM: hierarchical linear modelling.

LIFT: Linking the Interests of Families and Teachers.

MPCP: Mother program plus child program.

OR: odds ratio.

PATHS: Promoting Alternative Thinking Strategies. 
ProSAAF: Protecting Stronger African American Families program.

ROAD: Reducing Offenses of Adolescent Drivers.

SafERteen: brief intervention aimed at reducing and preventing violence and alcohol use.

SD: standard deviation.

SE: standard error.

SES: socioeconomic status.

SMD: standardised mean difference. 


\begin{tabular}{|c|c|c|c|c|c|c|c|c|c|c|c|}
\hline $\begin{array}{l}\text { Primary } \\
\text { authors }\end{array}$ & Trial & $\begin{array}{l}\text { Study } \\
\text { type }\end{array}$ & Country & $\begin{array}{l}\text { Dura- } \\
\text { tion }\end{array}$ & Theory & $\begin{array}{l}\text { Fol- } \\
\text { low-up } \\
\text { (post in- } \\
\text { terven- } \\
\text { tion) }\end{array}$ & Components & $\begin{array}{l}\text { Age tar- } \\
\text { geted }\end{array}$ & $\begin{array}{l}\text { Behaviour } \\
\text { targeted }\end{array}$ & $\begin{array}{l}\mathrm{N} \text { be- } \\
\text { hav- } \\
\text { iours } \\
\text { target- } \\
\text { ed }\end{array}$ & $\begin{array}{l}\text { Process } \\
\text { evaluation }\end{array}$ \\
\hline \multicolumn{12}{|c|}{ Targeted individual-level interventions } \\
\hline $\begin{array}{l}\text { Bern- } \\
\text { stein } \\
2010\end{array}$ & $\begin{array}{l}\text { Reach- } \\
\text { ing Ado- } \\
\text { lescents } \\
\text { for Pre- } \\
\text { vention }\end{array}$ & IT & USA & $\begin{array}{l}<3 \\
\text { months } \\
\text { (motiva- } \\
\text { tional in- } \\
\text { terven- } \\
\text { tion, re- } \\
\text { ferral to } \\
\text { services, } \\
\text { tele- } \\
\text { phone } \\
\text { conver- } \\
\text { sation) }\end{array}$ & $\mathrm{N} / \mathrm{S}$ & $\begin{array}{l}12 \\
\text { months }\end{array}$ & $\begin{array}{l}\text { Motivational in- } \\
\text { terview, referral } \\
\text { to community re- } \\
\text { sources and drug } \\
\text { treatment ser- } \\
\text { vices }\end{array}$ & $14-21$ & $\begin{array}{l}\text { Alcohol use, } \\
\text { vehicle-re- } \\
\text { lated risk, } \\
\text { antisocial } \\
\text { behaviour, } \\
\text { sexual risk } \\
\text { behaviour }\end{array}$ & 4 & $\begin{array}{l}\text { Assessed } \\
\text { adherence } \\
\text { to interven- } \\
\text { tion }\end{array}$ \\
\hline $\begin{array}{l}\text { Berry } \\
2009\end{array}$ & $\begin{array}{l}\text { Coach- } \\
\text { ing for } \\
\text { Commu- } \\
\text { nities }\end{array}$ & IT & UK & $\begin{array}{l}10 \\
\text { months }\end{array}$ & $\begin{array}{l}\text { Distinc- } \\
\text { tion-based } \\
\text { learning }\end{array}$ & $\begin{array}{l}\text { Post in- } \\
\text { terven- } \\
\text { tion }\end{array}$ & $\begin{array}{l}\text { 1-week residen- } \\
\text { tial programme } \\
\text { and } 9 \text { months of } \\
\text { mentoring }\end{array}$ & $15-18$ & $\begin{array}{l}\text { Alcohol use, } \\
\text { drug use, } \\
\text { antisocial } \\
\text { behaviour, } \\
\text { educational } \\
\text { attainment }\end{array}$ & 4 & $\begin{array}{l}\text { Assessed } \\
\text { impact of } \\
\text { 'quality' of } \\
\text { the mentor } \\
\text { and exam- } \\
\text { ined impact } \\
\text { of dose of } \\
\text { the inter- } \\
\text { vention }\end{array}$ \\
\hline $\begin{array}{l}\text { Bodin } \\
2011\end{array}$ & $\begin{array}{l}\text { A Men- } \\
\text { toring } \\
\text { Interven- } \\
\text { tion }\end{array}$ & IT & Sweden & $\begin{array}{l}12 \\
\text { months }\end{array}$ & $\begin{array}{l}\text { Rhodes } \\
\text { model } \\
\text { regard- } \\
\text { ing role } \\
\text { model- } \\
\text { ling on } \\
\text { healthy } \\
\text { relation- } \\
\text { ships } \\
\text { with } \\
\text { adults }\end{array}$ & $\begin{array}{l}12 \\
\text { months }\end{array}$ & Mentoring & $13-18$ & $\begin{array}{l}\text { Alcohol use, } \\
\text { drug use, } \\
\text { academ- } \\
\text { ic develop- } \\
\text { ment, men- } \\
\text { tal health }\end{array}$ & 5 & $\begin{array}{l}\text { Yes (partial) } \\
\text { - assessed } \\
\text { dropout, } \\
\text { adherence, } \\
\text { meetings, } \\
\text { positive } \\
\text { views of } \\
\text { programme, } \\
\text { intensity }\end{array}$ \\
\hline
\end{tabular}




\begin{tabular}{|c|c|c|c|c|c|c|c|c|c|c|c|}
\hline $\begin{array}{l}\text { Cun- } \\
\text { ningham } \\
2012\end{array}$ & $\begin{array}{l}\text { SafER- } \\
\text { teens }\end{array}$ & IT & USA & $\begin{array}{l}<1 \\
\text { month } \\
\text { (MI) }\end{array}$ & $\begin{array}{l}\text { Tradi- } \\
\text { tional } \\
\text { motiva- } \\
\text { tional in- } \\
\text { terview- } \\
\text { ing mod- } \\
\text { el }\end{array}$ & $\begin{array}{l}12 \\
\text { months }\end{array}$ & $\begin{array}{l}\text { Traditional MI us- } \\
\text { ing computer or } \\
\text { therapist }\end{array}$ & $14-18$ & $\begin{array}{l}\text { Antisocial } \\
\text { behaviour, } \\
\text { alcohol use }\end{array}$ & 2 & No \\
\hline $\begin{array}{l}\text { Dolan } \\
2010\end{array}$ & $\begin{array}{l}\text { BBBS } \\
\text { Ireland }\end{array}$ & IT & Ireland & $\begin{array}{l}12 \\
\text { months }\end{array}$ & $\begin{array}{l}\text { Rhodes } \\
\text { model } \\
\text { of youth } \\
\text { mentor- } \\
\text { ing }\end{array}$ & 2 years & $\begin{array}{l}\text { Individual (men- } \\
\text { toring) }\end{array}$ & $\begin{array}{l}10-16 \\
(93 \%< \\
14 \text { years) }\end{array}$ & $\begin{array}{l}\text { Alcohol } \\
\text { use, to- } \\
\text { bacco use, } \\
\text { cannabis } \\
\text { use, anti- } \\
\text { social be- } \\
\text { haviour, } \\
\text { education- } \\
\text { al attain- } \\
\text { ment, men- } \\
\text { tal health }\end{array}$ & 6 & Yes - full \\
\hline $\begin{array}{l}\text { Freuden- } \\
\text { berg } \\
2010\end{array}$ & $\begin{array}{l}\text { REAL } \\
\text { MEN }\end{array}$ & IT & USA & $\begin{array}{l}1-2 \\
\text { months }\end{array}$ & --- & $\begin{array}{l}12 \\
\text { months }\end{array}$ & $\begin{array}{l}\text { Individual com- } \\
\text { ponents (jail- } \\
\text { based interven- } \\
\text { tion and inter- } \\
\text { vention within } \\
\text { community set- } \\
\text { ting) }\end{array}$ & $17-18$ & $\begin{array}{l}\text { Drug use, } \\
\text { sexual risk } \\
\text { behaviour, } \\
\text { antisocial } \\
\text { behaviour, } \\
\text { education, } \\
\text { and employ- } \\
\text { ment }\end{array}$ & 4 & No \\
\hline $\begin{array}{l}\text { Fried- } \\
\text { man } \\
2002\end{array}$ & $\begin{array}{l}\text { Botvin } \\
\text { LST and } \\
\text { Anti-vio- } \\
\text { lence }\end{array}$ & IT & USA & $\begin{array}{l}\text { 6-12 } \\
\text { months } \\
\text { (55 ses- } \\
\text { sions for } \\
\text { class- } \\
\text { room } \\
\text { pro- } \\
\text { gramme, } \\
20 \text { ses- } \\
\text { sions for } \\
\text { violence } \\
\text { pro- } \\
\text { gramme) }\end{array}$ & $\begin{array}{l}\text { Life } \\
\text { Skills } \\
\text { Training } \\
\text { model; } \\
\text { social } \\
\text { cogni- } \\
\text { tive pro- } \\
\text { cedures }\end{array}$ & $\begin{array}{l}6 \\
\text { months }\end{array}$ & $\begin{array}{l}\text { Individual (triple- } \\
\text { modality class- } \\
\text { room pro- } \\
\text { gramme) }\end{array}$ & $13-18$ & $\begin{array}{l}\text { Alcohol use, } \\
\text { drug use, } \\
\text { antisocial } \\
\text { behaviour, } \\
\text { educational } \\
\text { attainment }\end{array}$ & 4 & $\begin{array}{l}\text { Yes - partial } \\
\text { (feedback } \\
\text { from partic- } \\
\text { ipants, ad- } \\
\text { herence, in- } \\
\text { tensity) }\end{array}$ \\
\hline
\end{tabular}




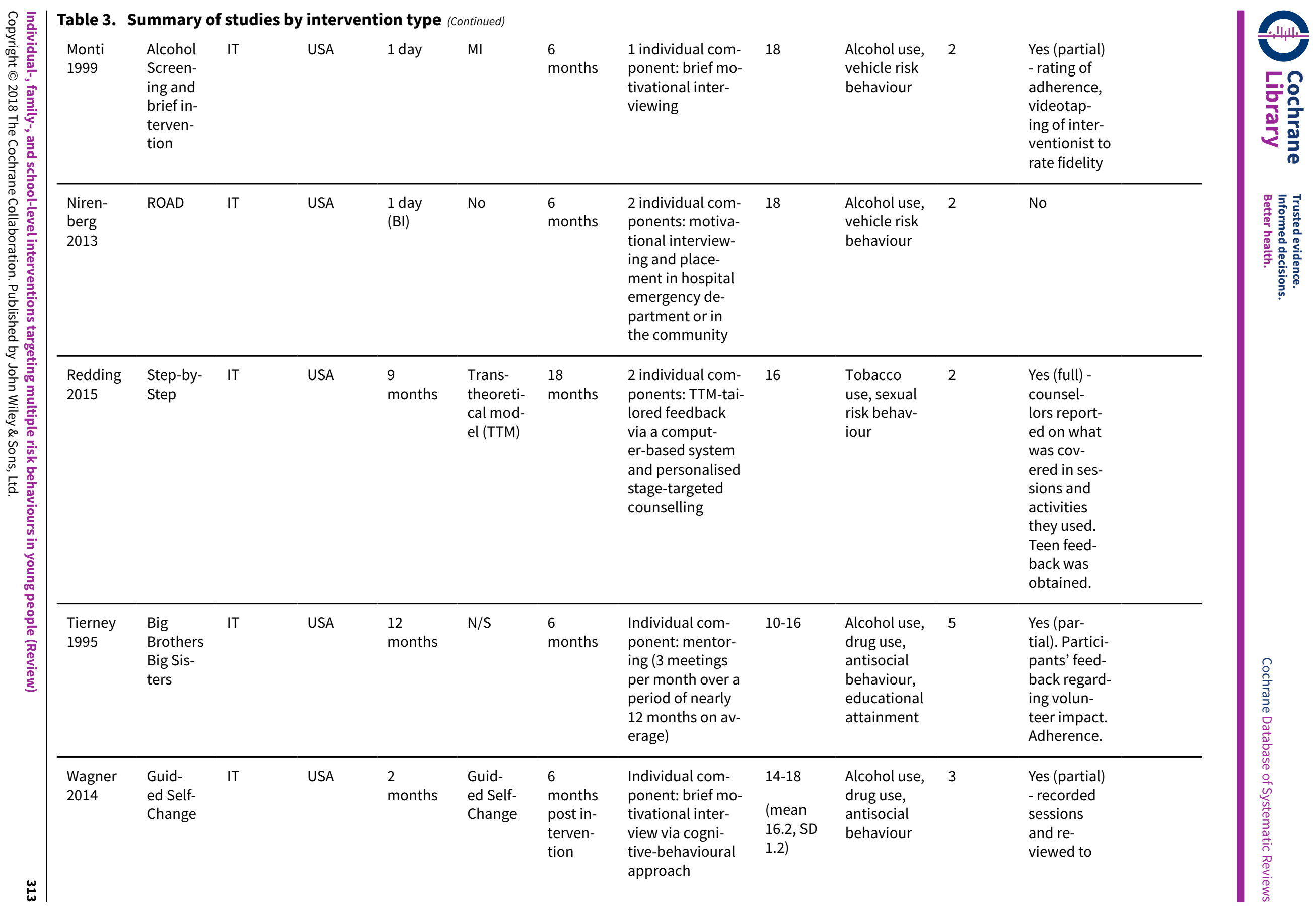




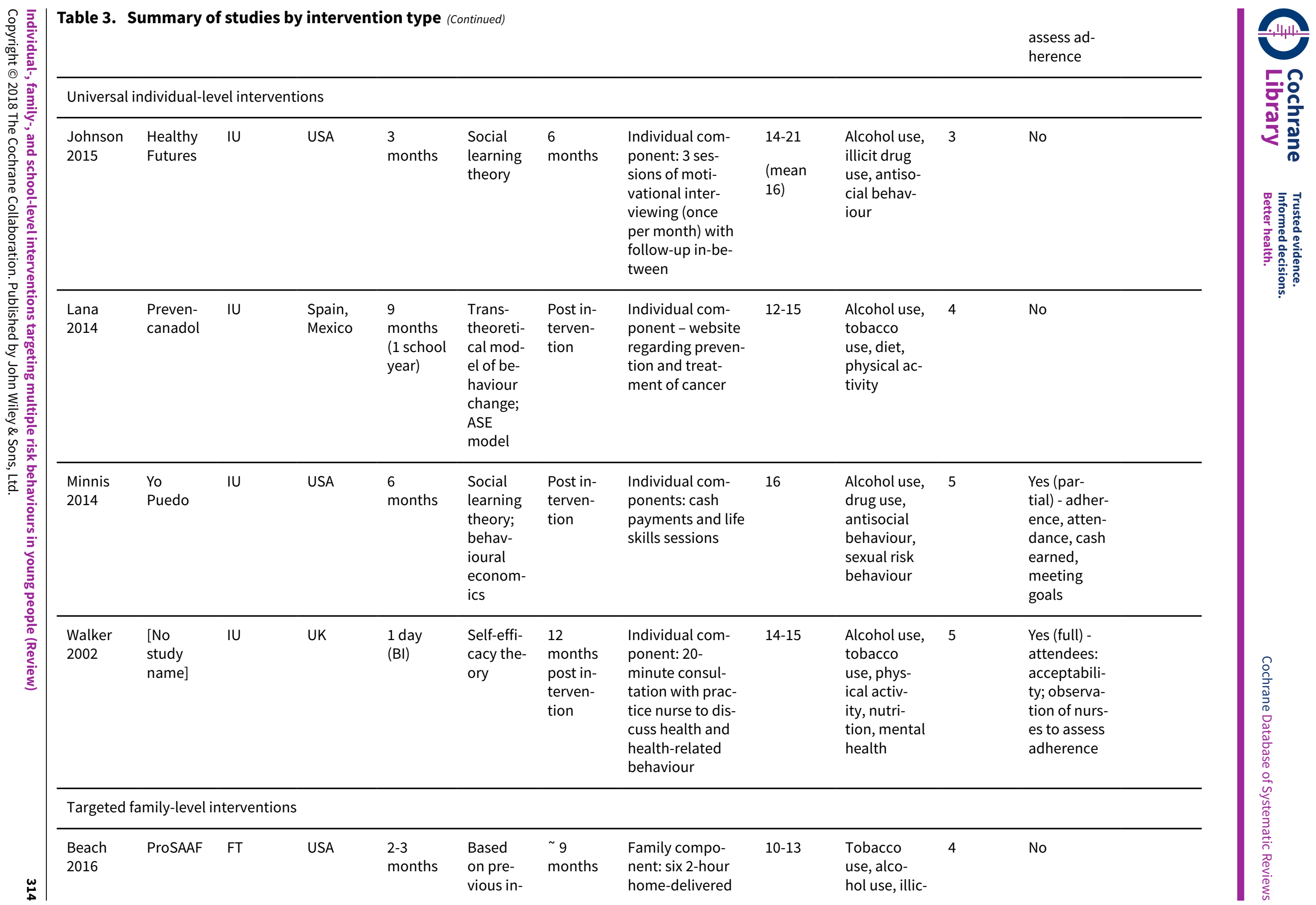




\begin{tabular}{|c|c|c|c|c|c|c|c|c|c|c|c|}
\hline & & & & & $\begin{array}{l}\text { terven- } \\
\text { tions } \\
\text { and } \\
\text { stress- } \\
\text { spillover } \\
\text { theory }\end{array}$ & & $\begin{array}{l}\text { sessions that fo- } \\
\text { cused on cou- } \\
\text { ple-based issues } \\
\text { and development } \\
\text { of protective cou- } \\
\text { ple and parenting } \\
\text { processes }\end{array}$ & & $\begin{array}{l}\text { it drug use, } \\
\text { antisocial } \\
\text { behaviour }\end{array}$ & & \\
\hline $\begin{array}{l}\text { Bonds } \\
2010\end{array}$ & $\begin{array}{l}\text { New Be- } \\
\text { ginnings }\end{array}$ & FT & USA & $\begin{array}{l}3 \\
\text { months }\end{array}$ & $\begin{array}{l}\text { Cas- } \\
\text { cading } \\
\text { pathway } \\
\text { model }\end{array}$ & $\begin{array}{l}3 \\
\text { months, } \\
6 \\
\text { months, } \\
6 \text { years, } \\
15 \text { years }\end{array}$ & $\begin{array}{l}\text { Family compo- } \\
\text { nents only (moth- } \\
\text { er-only, moth- } \\
\text { er-plus-child, and } \\
\text { child-only pro- } \\
\text { grammes) }\end{array}$ & $\begin{array}{l}9-12 \text { (av- } \\
\text { erage } \\
10.4, \text { SD } \\
1.1 \text { ) }\end{array}$ & $\begin{array}{l}\text { Substance } \\
\text { use (mari- } \\
\text { juana and } \\
\text { alcohol), } \\
\text { antisocial } \\
\text { behaviour, } \\
\text { sexual risk } \\
\text { behaviour, } \\
\text { mental } \\
\text { health }\end{array}$ & 5 & $\begin{array}{l}\text { Yes: adher- } \\
\text { ence, feed- } \\
\text { back, manu- } \\
\text { als, training, } \\
\text { supervision, } \\
\text { scores, rat- } \\
\text { ing }\end{array}$ \\
\hline $\begin{array}{l}\text { Brody } \\
2012\end{array}$ & SAAF-T & $\mathrm{FT}$ & USA & $\begin{array}{l}2-3 \\
\text { months }\end{array}$ & $\mathrm{N} / \mathrm{S}$ & $\begin{array}{l}22 \\
\text { months }\end{array}$ & $\begin{array}{l}\text { Family compo- } \\
\text { nents only (5 } \\
\text { meetings for } \\
\text { caregivers and } \\
\text { adolescents sep- } \\
\text { arately, followed } \\
\text { by a 1-hour ses- } \\
\text { sion for families } \\
\text { together) }\end{array}$ & 16 & $\begin{array}{l}\text { Drug use, } \\
\text { antisocial } \\
\text { behaviour, } \\
\text { mental } \\
\text { health }\end{array}$ & 3 & $\begin{array}{l}\text { Adherence } \\
\text { to curricu- } \\
\text { lum, video } \\
\text { of sessions } \\
\text { and feed- } \\
\text { back }\end{array}$ \\
\hline $\begin{array}{l}\text { Catalano } \\
1999\end{array}$ & $\begin{array}{l}\text { Focus on } \\
\text { Families }\end{array}$ & FT & USA & $\begin{array}{l}1 \text { year } \\
\text { (approxi- } \\
\text { mately) }\end{array}$ & $\begin{array}{l}\text { Social } \\
\text { devel- } \\
\text { opment } \\
\text { model }\end{array}$ & $\begin{array}{l}8 \\
\text { months }\end{array}$ & $\begin{array}{l}\text { Family compo- } \\
\text { nents: parent } \\
\text { skills training ses- } \\
\text { sions and case } \\
\text { management }\end{array}$ & $3-14$ & $\begin{array}{l}\text { Alcohol } \\
\text { use, to- } \\
\text { bacco use, } \\
\text { cannabis } \\
\text { use, antiso- } \\
\text { cial behav- } \\
\text { iour }\end{array}$ & 4 & $\begin{array}{l}\text { No - adher- } \\
\text { ence only }\end{array}$ \\
\hline $\begin{array}{l}\text { Estrada } \\
2016\end{array}$ & $\begin{array}{l}\text { Brief Fa- } \\
\text { milias } \\
\text { Unidas }\end{array}$ & $\mathrm{FT}$ & USA & $\begin{array}{l}2-3 \\
\text { months }\end{array}$ & NS & $\begin{array}{l}24 \\
\text { months }\end{array}$ & $\begin{array}{l}5 \text { weekly 2-hour } \\
\text { parent group ses- } \\
\text { sions, } 3 \text { home- } \\
\text { work assign- } \\
\text { ments for par- } \\
\text { ents, and one 1- } \\
\text { hour family visit }\end{array}$ & $\begin{array}{l}15 \\
\text { (mean } \\
15.3 \\
\text { years, SD } \\
0.89 \text { ) }\end{array}$ & $\begin{array}{l}\text { Tobacco } \\
\text { use, alco- } \\
\text { hol use, illic- } \\
\text { it drug use, } \\
\text { sexual risk } \\
\text { behaviour }\end{array}$ & 4 & $\begin{array}{l}\text { Yes (par- } \\
\text { tial) - atten- } \\
\text { dance data } \\
\text { only }\end{array}$ \\
\hline
\end{tabular}




\begin{tabular}{|c|c|c|c|c|c|c|c|c|c|c|c|}
\hline Gonzales & $\begin{array}{l}\text { Bridges } \\
\text { to High } \\
\text { School }\end{array}$ & FT & USA & $\begin{array}{l}3 \\
\text { months }\end{array}$ & $\begin{array}{l}\text { Social } \\
\text { devel- } \\
\text { opment } \\
\text { model }\end{array}$ & 5 years & $\begin{array}{l}\text { Family compo- } \\
\text { nents (weekly } \\
\text { group sessions } \\
\text { with separate } \\
\text { and joint sessions } \\
\text { and home visits) }\end{array}$ & $12-13$ & $\begin{array}{l}\text { Alcohol use, } \\
\text { tobacco } \\
\text { use, drug } \\
\text { use, anti- } \\
\text { social be- } \\
\text { haviour, } \\
\text { education- } \\
\text { al attain- } \\
\text { ment, men- } \\
\text { tal health }\end{array}$ & 6 & $\begin{array}{l}\text { Yes (full) - } \\
\text { post-test } \\
\text { interview } \\
\text { - rating by } \\
\text { parents; at- } \\
\text { tendance; } \\
\text { video } \\
\text { recording of } \\
\text { intervention } \\
\text { sessions - } \\
\text { adherence }\end{array}$ \\
\hline $\begin{array}{l}\text { Jalling } \\
2016\end{array}$ & $\begin{array}{l}\text { Comet } \\
12-18\end{array}$ & FT & Sweden & $\begin{array}{l}3-4 \\
\text { months }\end{array}$ & $\begin{array}{l}\text { Operant } \\
\text { learn- } \\
\text { ing and } \\
\text { social } \\
\text { learning } \\
\text { princi- } \\
\text { ples }\end{array}$ & $\begin{array}{l}6 \\
\text { months }\end{array}$ & $\begin{array}{l}\text { Family compo- } \\
\text { nent: } 9 \text { weekly } \\
\text { group sessions } \\
\text { of } 2 \text { to } 2.5 \text { hours } \\
\text { and } 1 \text { optional } \\
\text { booster session. } \\
\text { Sessions involved } \\
\text { role-play, home } \\
\text { assignments, and } \\
\text { use of video as a } \\
\text { basis for discus- } \\
\text { sion. }\end{array}$ & $\begin{array}{l}12-18 \\
\text { (mean } \\
14)\end{array}$ & $\begin{array}{l}\text { Alcohol use, } \\
\text { illicit drug } \\
\text { use, antiso- } \\
\text { cial behav- } \\
\text { iour }\end{array}$ & 3 & $\begin{array}{l}\text { Yes - atten- } \\
\text { dance and } \\
\text { self-assess- } \\
\text { ment by } \\
\text { group lead- } \\
\text { ers of extent } \\
\text { to which } \\
\text { programme } \\
\text { manual was } \\
\text { fulfilled in } \\
\text { sessions }\end{array}$ \\
\hline $\begin{array}{l}\text { Jalling } \\
2016 b\end{array}$ & $\begin{array}{l}\text { Paren- } \\
\text { tSteps }\end{array}$ & FT & Sweden & $\begin{array}{l}3-4 \\
\text { months }\end{array}$ & $\begin{array}{l}\text { Re- } \\
\text { silience } \\
\text { model }\end{array}$ & $\begin{array}{l}6 \\
\text { months }\end{array}$ & $\begin{array}{l}\text { Family compo- } \\
\text { nent: } 6 \text { weekly } \\
\text { parent sessions } \\
\text { of } 1.5 \text { to } 2 \text { hours }\end{array}$ & $\begin{array}{l}12-18 \\
\text { (mean } \\
14)\end{array}$ & $\begin{array}{l}\text { Alcohol use, } \\
\text { illicit drug } \\
\text { use, antiso- } \\
\text { cial behav- } \\
\text { iour }\end{array}$ & 3 & $\begin{array}{l}\text { Yes (par- } \\
\text { tial) - atten- } \\
\text { dance and } \\
\text { leader self- } \\
\text { assessment } \\
\text { of extent to } \\
\text { which pro- } \\
\text { gramme } \\
\text { manual was } \\
\text { fulfilled in } \\
\text { sessions }\end{array}$ \\
\hline $\begin{array}{l}\text { Kim } \\
2011\end{array}$ & $\begin{array}{l}\text { Middle } \\
\text { School } \\
\text { Success }\end{array}$ & $\mathrm{FT}$ & USA & $\begin{array}{l}3 \\
\text { months } \\
\text { with on- } \\
\text { going } \\
\text { support } \\
\text { for } 1 \\
\text { school } \\
\text { year }\end{array}$ & --- & 2 years & $\begin{array}{l}\text { Family compo- } \\
\text { nents: curricu- } \\
\text { lum to parents } \\
\text { through } 6 \text { group } \\
\text { sessions for par- } \\
\text { ents plus fol- } \\
\text { low-up sessions; } \\
6 \text { skills-based ses- }\end{array}$ & $11-12$ & $\begin{array}{l}\text { Antisocial } \\
\text { behaviour, } \\
\text { tobacco } \\
\text { use, alco- } \\
\text { hol use, } \\
\text { marijuana } \\
\text { use, mental } \\
\text { health }\end{array}$ & 5 & No \\
\hline
\end{tabular}




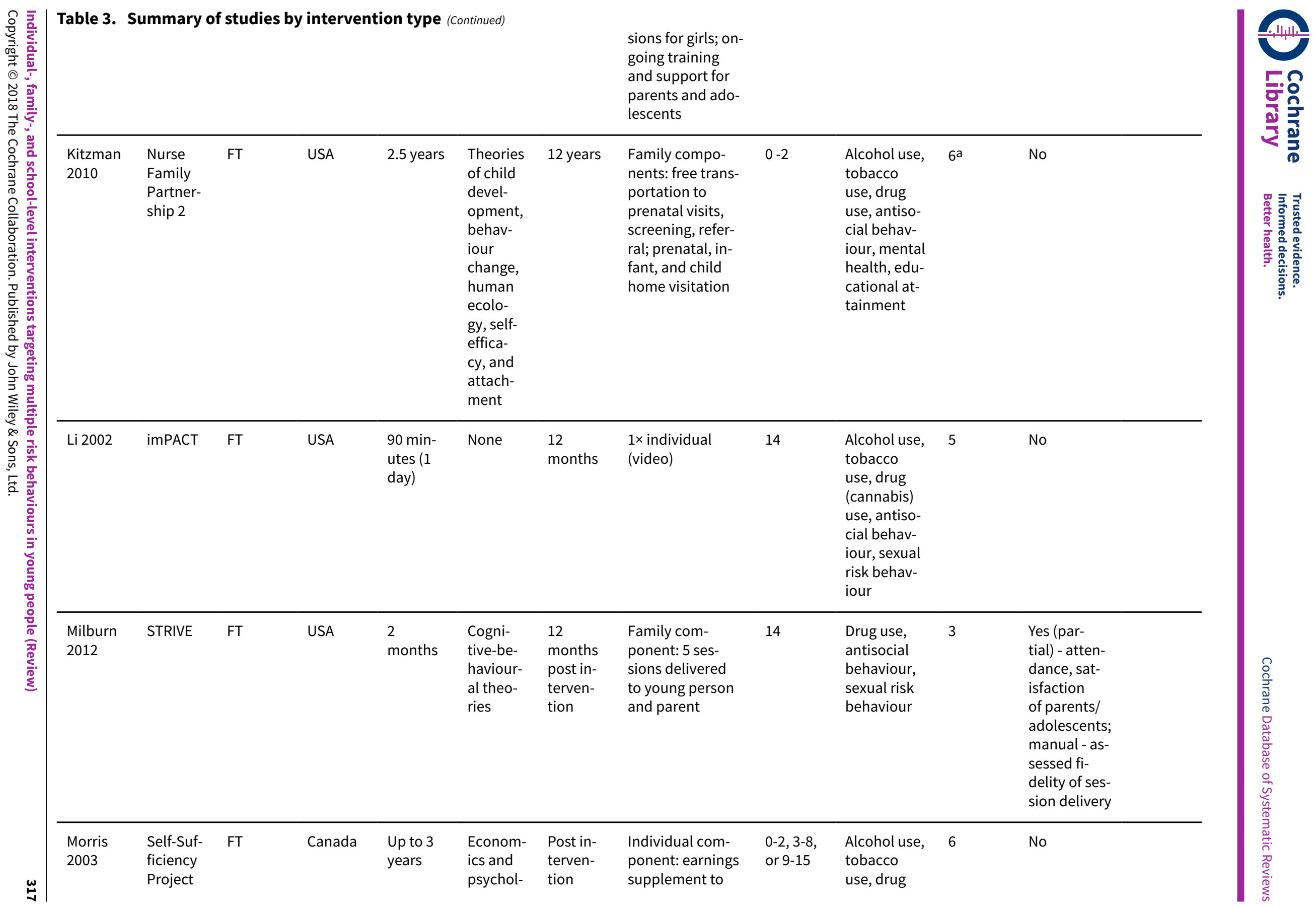




\begin{tabular}{|c|c|c|c|c|c|c|c|c|c|c|c|}
\hline & & & & & $\begin{array}{l}\text { ogy the- } \\
\text { ories }\end{array}$ & & $\begin{array}{l}\text { single parents } \\
\text { who left welfare } \\
\text { for full-time em- } \\
\text { ployment }\end{array}$ & & $\begin{array}{l}\text { use, antiso- } \\
\text { cial behav- } \\
\text { iour, mental } \\
\text { health edu- } \\
\text { cational at- } \\
\text { tainment }\end{array}$ & & \\
\hline $\begin{array}{l}\text { Murry } \\
2014\end{array}$ & $\begin{array}{l}\text { SAAF } \\
\text { (Stronger } \\
\text { African } \\
\text { Amer- } \\
\text { ican } \\
\text { Families) }\end{array}$ & FT & USA & 7 weeks & $\begin{array}{l}\text { Social } \\
\text { learn- } \\
\text { ing theo- } \\
\text { ry, prob- } \\
\text { lem be- } \\
\text { haviour } \\
\text { theory, } \\
\text { Gibbons } \\
\text { and Ger- } \\
\text { rard's } \\
\text { cog- } \\
\text { nitive } \\
\text { model of } \\
\text { adoles- } \\
\text { cent be- } \\
\text { haviour }\end{array}$ & $\begin{array}{l}65 \\
\text { months }\end{array}$ & $\begin{array}{l}1 \text { family compo- } \\
\text { nent: separate } 1- \\
\text { hour caregiver } \\
\text { and adolescent } \\
\text { session followed } \\
\text { by joint session } \\
\text { to practice skills }\end{array}$ & $\begin{array}{l}\text { Moth- } \\
\text { ers and } \\
\text { children } \\
\text { aged } 11 \\
\text { years }\end{array}$ & $\begin{array}{l}\text { Alcohol use, } \\
\text { sexual risk } \\
\text { behaviour }\end{array}$ & 2 & $\begin{array}{l}\text { Yes - atten- } \\
\text { dance mea- } \\
\text { sured, fideli- } \\
\text { ty assessed } \\
\text { using video }\end{array}$ \\
\hline $\begin{array}{l}\text { Olds } \\
1998\end{array}$ & $\begin{array}{l}\text { Nurse } \\
\text { Family } \\
\text { Partner- } \\
\text { ship }\end{array}$ & FT & USA & 2 years & $\begin{array}{l}\text { Human } \\
\text { ecology, } \\
\text { self-ef- } \\
\text { ficacy, } \\
\text { human } \\
\text { attach- } \\
\text { ment }\end{array}$ & 15 years & $\begin{array}{l}1 \text { family compo- } \\
\text { nent }\end{array}$ & $\begin{array}{l}\text { Mothers } \\
\text { aged < } \\
\text { 19, chil- } \\
\text { dren } \\
\text { aged 0-2 }\end{array}$ & $\begin{array}{l}\text { Drug use, } \\
\text { antisocial } \\
\text { behaviour, } \\
\text { sexual risk } \\
\text { behaviour, } \\
\text { educational } \\
\text { attainment }\end{array}$ & $6^{a}$ & No \\
\hline $\begin{array}{l}\text { Pantin } \\
2009\end{array}$ & $\begin{array}{l}\text { Familias } \\
\text { Unidas }\end{array}$ & FT & USA & $\mathrm{N} / \mathrm{S}$ & $\mathrm{N} / \mathrm{S}$ & $\begin{array}{l}2.5 \text { years } \\
\text { post in- } \\
\text { terven- } \\
\text { tion }\end{array}$ & $\begin{array}{l}2 \text { family compo- } \\
\text { nents: nine 2- } \\
\text { hour group ses- } \\
\text { sions, ten 1-hour } \\
\text { family visits, four } \\
\text { 1-hour booster } \\
\text { sessions. }\end{array}$ & $13-14$ & $\begin{array}{l}\text { Alcohol use, } \\
\text { tobacco } \\
\text { use, drug } \\
\text { use, antiso- } \\
\text { cial behav- } \\
\text { iour, sexual } \\
\text { risk behav- } \\
\text { iour }\end{array}$ & 5 & $\begin{array}{l}\text { Yes (par- } \\
\text { tial) - ses- } \\
\text { sions video } \\
\text { recorded } \\
\text { and rated } \\
\text { on adher- } \\
\text { ence and } \\
\text { quality }\end{array}$ \\
\hline $\begin{array}{l}\text { Schwinn } \\
2014\end{array}$ & $\begin{array}{l}\text { [No } \\
\text { study } \\
\text { name] }\end{array}$ & FT & USA & 1 month & $\mathrm{N} / \mathrm{S}$ & $\begin{array}{l}5 \\
\text { months }\end{array}$ & $\begin{array}{l}1 \text { family compo- } \\
\text { nent: 3-session } \\
\text { online health }\end{array}$ & $11-12$ & $\begin{array}{l}\text { Drug use, } \\
\text { physical ac- } \\
\text { tivity, nutri- } \\
\text { tion }\end{array}$ & 3 & $\begin{array}{l}\text { Minimal ad- } \\
\text { herence da- } \\
\text { ta only }\end{array}$ \\
\hline
\end{tabular}




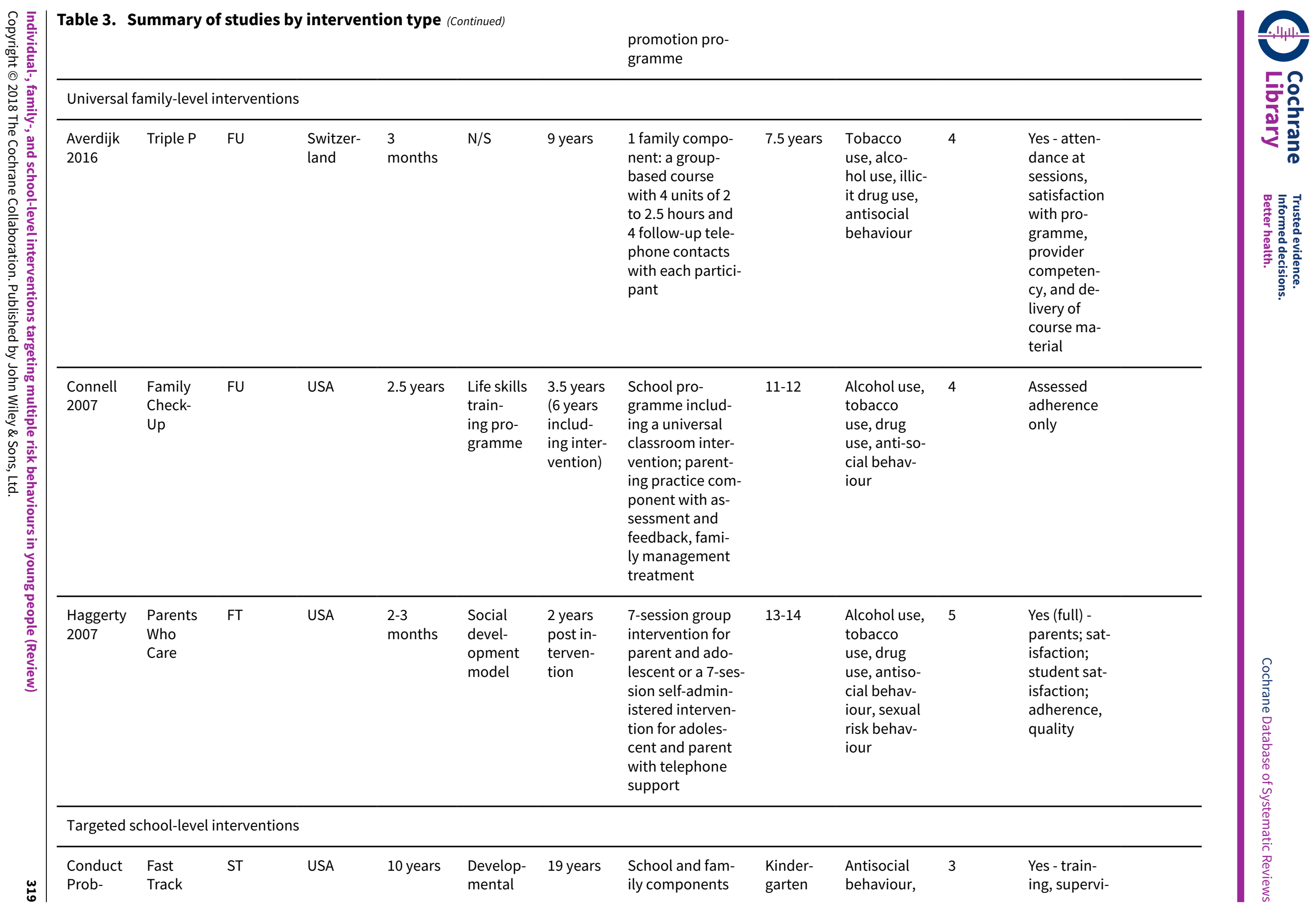




\begin{tabular}{|c|c|c|c|c|c|c|c|c|c|c|c|}
\hline $\begin{array}{l}\text { lems } \\
\text { Preven- } \\
\text { tion Re- } \\
\text { search } \\
\text { Group } \\
2014\end{array}$ & & & & & $\begin{array}{l}\text { model } \\
\text { of con- } \\
\text { duct dis- } \\
\text { orders }\end{array}$ & & $\begin{array}{l}\text { (family group } \\
\text { programme, } \\
\text { parent train- } \\
\text { ing groups, par- } \\
\text { ent-child inter- } \\
\text { action groups, } \\
\text { tutoring; school } \\
\text { curriculum; youth } \\
\text { groups, youth fo- } \\
\text { rums) }\end{array}$ & $\begin{array}{l}\text { to } 12 \text { th } \\
\text { grade }\end{array}$ & $\begin{array}{l}\text { education- } \\
\text { al attain- } \\
\text { ment, men- } \\
\text { tal health }\end{array}$ & & $\begin{array}{l}\text { sion, fideli- } \\
\text { ty - rating } \\
\text { of quality of } \\
\text { implemen- } \\
\text { tation, ob- } \\
\text { servation, } \\
\text { quality of } \\
\text { teaching, } \\
\text { quality of } \\
\text { classroom } \\
\text { manage- } \\
\text { ment }\end{array}$ \\
\hline $\begin{array}{l}\text { Lochman } \\
2003\end{array}$ & $\begin{array}{l}\text { Coping } \\
\text { Power } 1\end{array}$ & ST & USA & $\begin{array}{l}12 \\
\text { months }\end{array}$ & $\begin{array}{l}\text { Social } \\
\text { learning } \\
\text { theory }\end{array}$ & $\begin{array}{l}12 \\
\text { months } \\
\text { post in- } \\
\text { terven- } \\
\text { tion }\end{array}$ & $\begin{array}{l}\text { Parent and child } \\
\text { components: par- } \\
\text { ent group meet- } \\
\text { ings; teacher } \\
\text { meetings; group } \\
\text { school-based ses- } \\
\text { sions for children }\end{array}$ & $10-11$ & $\begin{array}{l}\text { Alcohol use, } \\
\text { tobacco } \\
\text { use, drug } \\
\text { use, antiso- } \\
\text { cial behav- } \\
\text { iour }\end{array}$ & 4 & $\begin{array}{l}\text { Yes (partial) } \\
\text { - adherence } \\
\text { to interven- } \\
\text { tion (man- } \\
\text { uals, guide- } \\
\text { lines, train- } \\
\text { ing); atten- } \\
\text { dance }\end{array}$ \\
\hline $\begin{array}{l}\text { Lochman } \\
2004\end{array}$ & $\begin{array}{l}\text { Coping } \\
\text { Power } 2\end{array}$ & ST & USA & $\begin{array}{l}15 \\
\text { months }\end{array}$ & $\begin{array}{l}\text { Social } \\
\text { learning } \\
\text { theory }\end{array}$ & $\begin{array}{l}12 \\
\text { months } \\
\text { post in- } \\
\text { terven- } \\
\text { tion }\end{array}$ & $\begin{array}{l}\text { Parent and child } \\
\text { components: par- } \\
\text { ent group ses- } \\
\text { sions and child } \\
\text { school-based } \\
\text { group sessions }\end{array}$ & $9-10$ & $\begin{array}{l}\text { Alcohol use, } \\
\text { drug use, } \\
\text { antisocial } \\
\text { behaviour }\end{array}$ & 3 & $\begin{array}{l}\text { Yes (full) - } \\
\text { meetings } \\
\text { with target } \\
\text { children; } \\
\text { staff rated } \\
\text { accomplish- } \\
\text { ment of ob- } \\
\text { jectives, au- } \\
\text { dio/video } \\
\text { taping; ob- } \\
\text { servation }\end{array}$ \\
\hline $\begin{array}{l}\text { Sanchez } \\
2007\end{array}$ & $\begin{array}{l}\text { Recon- } \\
\text { necting } \\
\text { Youth }\end{array}$ & ST & USA & 2 years & $\begin{array}{l}\text { Theo- } \\
\text { retical } \\
\text { frame- } \\
\text { work } \\
\text { based on } \\
\text { strain, } \\
\text { social } \\
\text { learning } \\
\text { and con- } \\
\text { trol the- } \\
\text { ories }\end{array}$ & $\begin{array}{l}1 \text { year } \\
\text { post in- } \\
\text { terven- } \\
\text { tion }\end{array}$ & $\begin{array}{l}1 \text { school compo- } \\
\text { nent: } 55 \text { school } \\
\text { lessons and } 24 \\
\text { booster lessons }\end{array}$ & 15 & $\begin{array}{l}\text { Drug use, } \\
\text { antisocial } \\
\text { behaviour, } \\
\text { mental } \\
\text { health, edu- } \\
\text { cational at- } \\
\text { tainment }\end{array}$ & 4 & $\begin{array}{l}\text { Yes (full) } \\
\text { - teacher } \\
\text { logs, at- } \\
\text { tendance } \\
\text { records, ob- } \\
\text { servations } \\
\text { in class- } \\
\text { room, stu- } \\
\text { dent ques- } \\
\text { tionnaires, }\end{array}$ \\
\hline
\end{tabular}




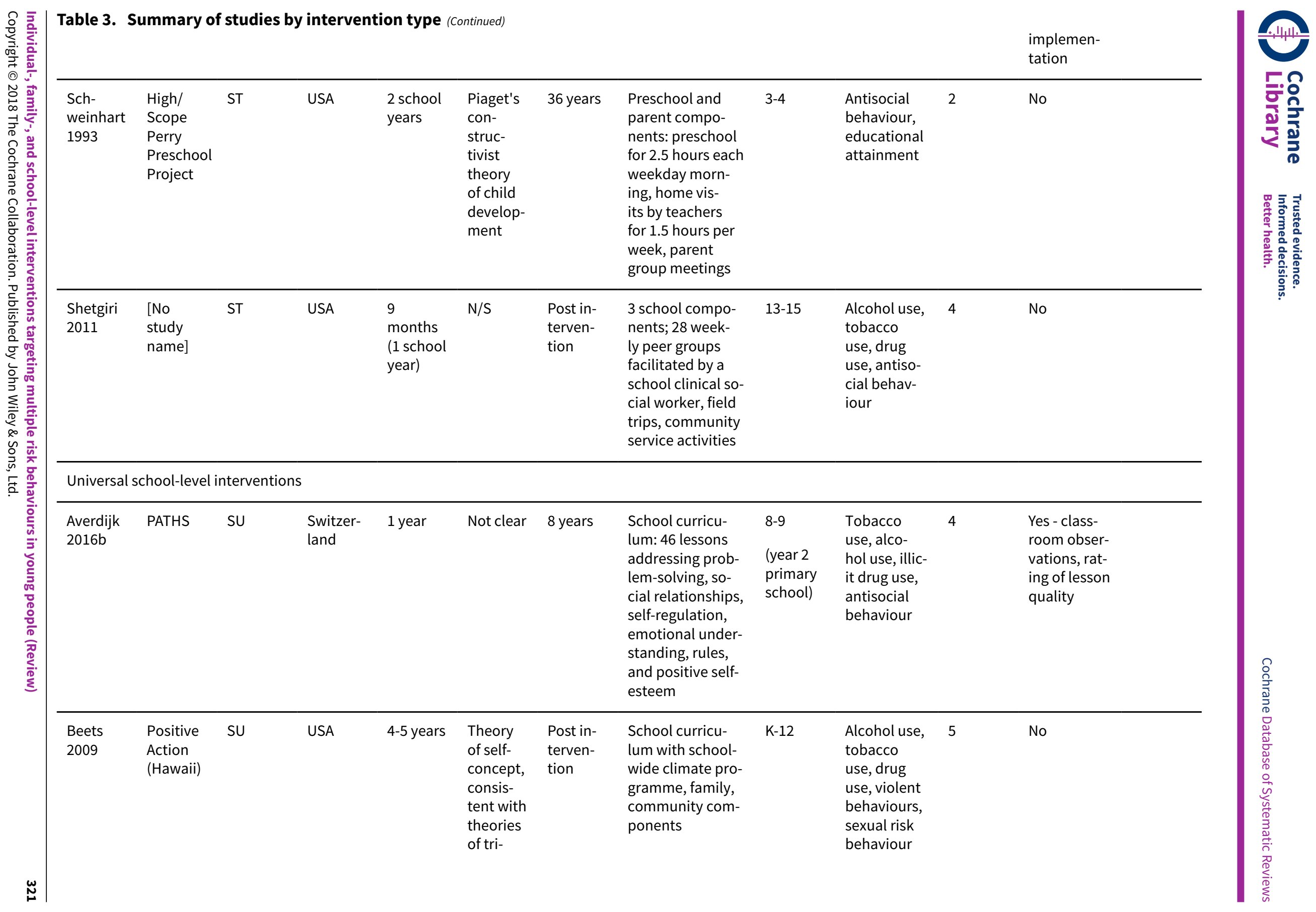




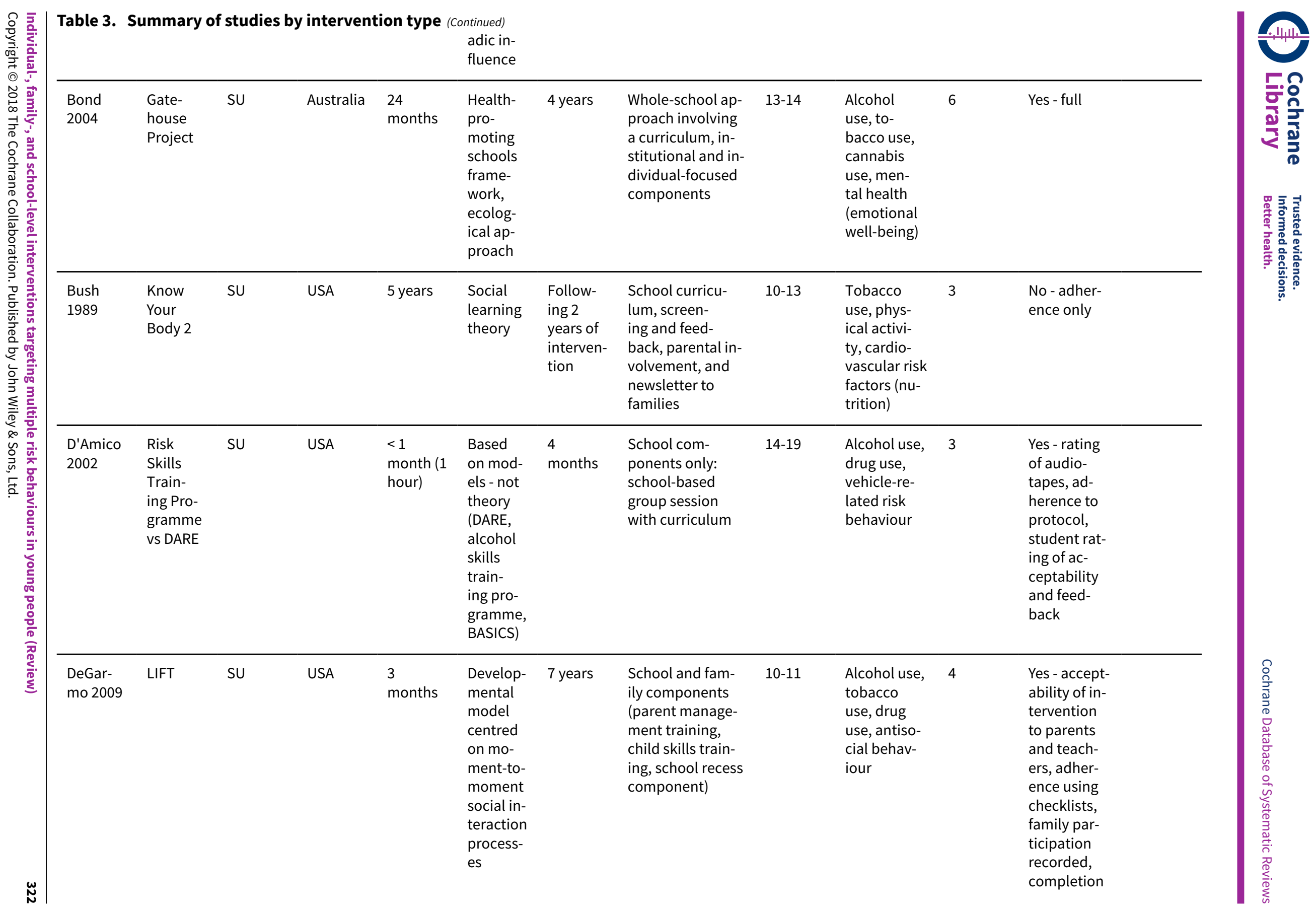




\begin{tabular}{|c|c|c|c|c|c|c|c|c|c|c|c|}
\hline & & & & & & & & & & & of critical \\
\hline $\begin{array}{l}\text { Fearnow- } \\
\text { Kenney } \\
2003\end{array}$ & $\begin{array}{l}\text { All Stars } \\
\text { Sr }\end{array}$ & SU & USA & $\begin{array}{l}9 \\
\text { months } \\
\text { (1 school } \\
\text { year) }\end{array}$ & No & $\begin{array}{l}\text { Post in- } \\
\text { terven- } \\
\text { tion }\end{array}$ & $\begin{array}{l}\text { School compo- } \\
\text { nents only (All } \\
\text { Stars activities } \\
\text { implemented by } \\
\text { teachers) }\end{array}$ & $13-19$ & $\begin{array}{l}\text { Alcohol use, } \\
\text { cannabis } \\
\text { use, tobac- } \\
\text { co use, nu- } \\
\text { trition }\end{array}$ & 4 & $\begin{array}{l}\text { Yes - full } \\
\text { (teacher and } \\
\text { student fo- } \\
\text { cus groups } \\
\text { re percep- } \\
\text { tions of pro- } \\
\text { gramme, \% } \\
\text { implement- } \\
\text { ed) }\end{array}$ \\
\hline $\begin{array}{l}\text { Flay } \\
2004\end{array}$ & Aban Aya & SU & USA & 4 years & $\begin{array}{l}\text { Theo- } \\
\text { ry of tri- } \\
\text { adic in- } \\
\text { fluence, } \\
\text { incorpo- } \\
\text { ration } \\
\text { of Ngu- } \\
\text { zo Saba } \\
\text { princi- } \\
\text { ples to } \\
\text { promote } \\
\text { African } \\
\text { Ameri- } \\
\text { can cul- } \\
\text { tural val- } \\
\text { ues }\end{array}$ & $\begin{array}{l}\text { Post in- } \\
\text { terven- } \\
\text { tion }\end{array}$ & $\begin{array}{l}\text { School curricu- } \\
\text { lum from grade } \\
5 \text { to } 8 \text { (SDC); or } \\
\text { curriculum plus } \\
\text { parental support, } \\
\text { school climate } \\
\text { and community } \\
\text { components }\end{array}$ & $10-14$ & $\begin{array}{l}\text { Antisocial } \\
\text { behaviour, } \\
\text { sexual risk } \\
\text { behaviour, } \\
\text { substance } \\
\text { use }\end{array}$ & 3 & Yes - full \\
\hline $\begin{array}{l}\text { Got- } \\
\text { tfredson } \\
2010\end{array}$ & $\begin{array}{l}\text { All Stars } \\
2\end{array}$ & SU & USA & $\begin{array}{l}32 \text { weeks } \\
\text { (1 school } \\
\text { year) }\end{array}$ & $\begin{array}{l}\text { Social } \\
\text { learning } \\
\text { theory }\end{array}$ & $\begin{array}{l}\text { Post in- } \\
\text { terven- } \\
\text { tion }\end{array}$ & $\begin{array}{l}\text { School compo- } \\
\text { nents only: class- } \\
\text { room interactive } \\
\text { sessions, home- } \\
\text { work assign- } \\
\text { ments, parental } \\
\text { attendance at } \\
\text { graduation cere- } \\
\text { monies }\end{array}$ & $11-14$ & $\begin{array}{l}\text { Alcohol use, } \\
\text { tobacco } \\
\text { use, drug } \\
\text { use, antiso- } \\
\text { cial behav- } \\
\text { iour }\end{array}$ & 4 & $\begin{array}{l}\text { Yes (full) - } \\
\text { adherence } \\
\text { - observed } \\
\text { implemen- } \\
\text { tation with } \\
\text { site vis- } \\
\text { its, fidelity } \\
\text { checklists; } \\
\text { quality rat- } \\
\text { ing and how } \\
\text { sessions } \\
\text { met objec- } \\
\text { tives; adher- } \\
\text { ence and } \\
\text { delivery }\end{array}$ \\
\hline
\end{tabular}




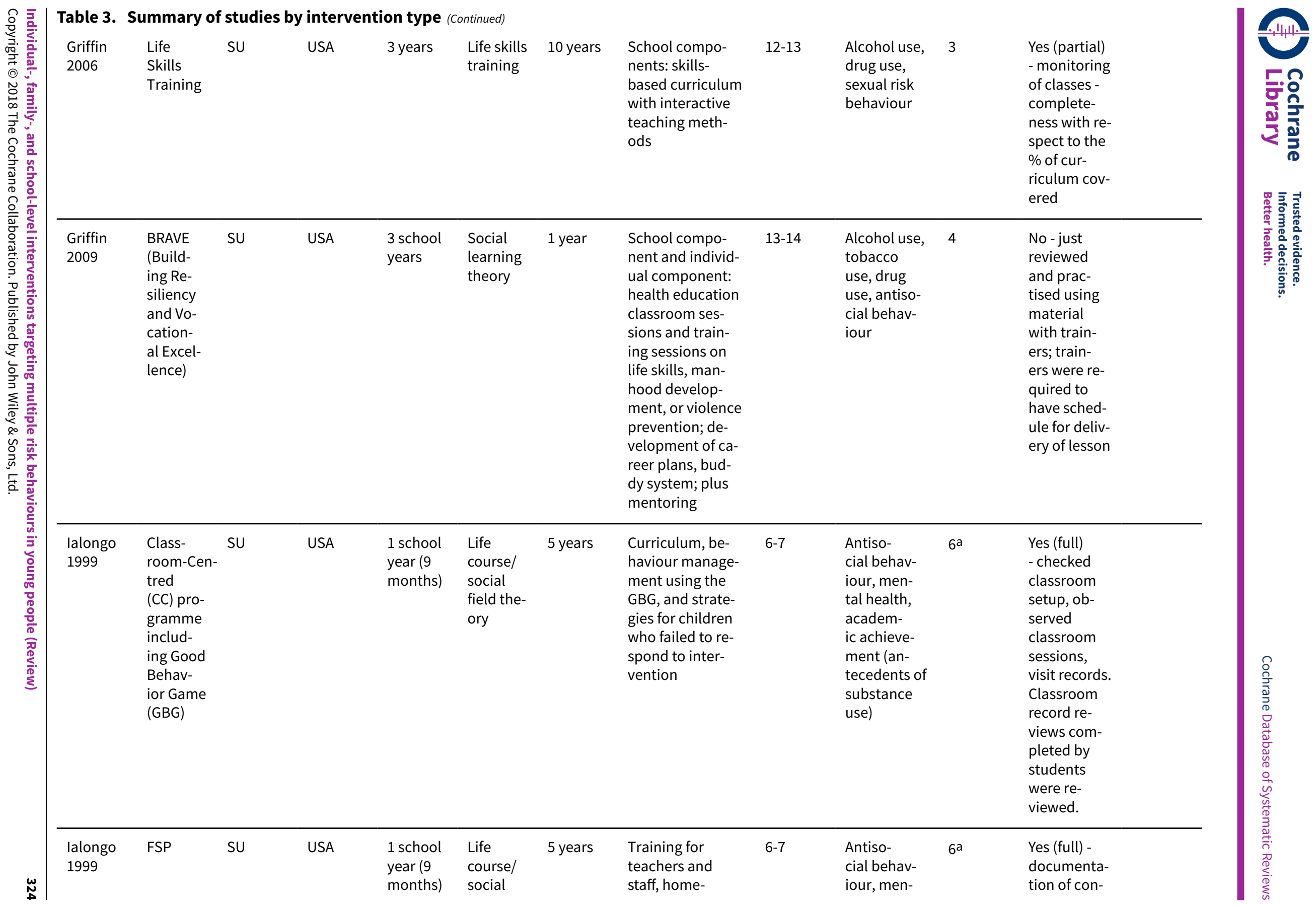




\begin{tabular}{|c|c|c|c|c|c|c|c|c|c|c|c|}
\hline & & & & & $\begin{array}{l}\text { field the- } \\
\text { ory }\end{array}$ & & $\begin{array}{l}\text { school learning } \\
\text { activities, } 9 \text { work- } \\
\text { shops for parents }\end{array}$ & & $\begin{array}{l}\text { tal health, } \\
\text { academ- } \\
\text { ic achieve- } \\
\text { ment (an- } \\
\text { tecedents of } \\
\text { substance } \\
\text { use) }\end{array}$ & & $\begin{array}{l}\text { tact with } \\
\text { parents. } \\
\text { Parents re- } \\
\text { ported on } \\
\text { implemen- } \\
\text { tation and } \\
\text { usefulness. } \\
\text { Record- } \\
\text { ed obser- } \\
\text { vations of } \\
\text { workshops }\end{array}$ \\
\hline Kellam & $\begin{array}{l}\text { Good } \\
\text { Behav- } \\
\text { iour } \\
\text { Game } \\
\text { (GBG) }\end{array}$ & SU & USA & $\begin{array}{l}2 \text { school } \\
\text { years }\end{array}$ & $\begin{array}{l}\text { Life } \\
\text { course/ } \\
\text { social } \\
\text { field the- } \\
\text { ory }\end{array}$ & $\begin{array}{l}\text { Up to } 12 \\
\text { years }\end{array}$ & $\begin{array}{l}\text { School compo- } \\
\text { nent: behaviour } \\
\text { management }\end{array}$ & $6-8$ & $\begin{array}{l}\text { Antisocial } \\
\text { behaviour, } \\
\text { education- } \\
\text { al achieve- } \\
\text { ment (an- } \\
\text { tecedents of } \\
\text { substance } \\
\text { use) }\end{array}$ & $5^{a}$ & No \\
\hline $\begin{array}{l}\text { Lewis } \\
2013\end{array}$ & $\begin{array}{l}\text { Positive } \\
\text { Action } \\
\text { (Chica- } \\
\text { go) }\end{array}$ & SU & USA & $\begin{array}{l}6 \text { school } \\
\text { years }\end{array}$ & $\begin{array}{l}\text { Self-es- } \\
\text { teem en- } \\
\text { hance- } \\
\text { ment } \\
\text { theory, } \\
\text { social } \\
\text { ecologi- } \\
\text { cal theo- } \\
\text { ry }\end{array}$ & $\begin{array}{l}\text { Post in- } \\
\text { terven- } \\
\text { tion }\end{array}$ & $\begin{array}{l}\text { School com- } \\
\text { ponents: class- } \\
\text { room curriculum; } \\
\text { school-wide cli- } \\
\text { mate develop- } \\
\text { ment; teacher, } \\
\text { family, counsel- } \\
\text { lor, and commu- } \\
\text { nity training }\end{array}$ & $\begin{array}{l}8-13 \\
\text { (grades } \\
3-8)\end{array}$ & $\begin{array}{l}\text { Alcohol use, } \\
\text { tobacco } \\
\text { use, drug } \\
\text { use, antiso- } \\
\text { cial behav- } \\
\text { iour }\end{array}$ & 6 & $\begin{array}{l}\text { Yes (par- } \\
\text { tial) - adher- } \\
\text { ence to pro- } \\
\text { gramme; } \\
\text { workshops } \\
\text { for teachers; } \\
\text { unit imple- } \\
\text { mentation } \\
\text { report at the } \\
\text { end of each } \\
\text { unit }\end{array}$ \\
\hline $\begin{array}{l}\text { LoSciuto } \\
1999\end{array}$ & $\begin{array}{l}\text { Woodrock } \\
\text { Youth } \\
\text { Devel- } \\
\text { opment } \\
\text { Project }\end{array}$ & SU & USA & $\begin{array}{l}2 \text { school } \\
\text { years }\end{array}$ & None & Post test & $\begin{array}{l}3 \text { components: } \\
\text { education } \\
\text { through semi- } \\
\text { nars, psychoso- } \\
\text { cial support } \\
\text { (mentoring, tu- } \\
\text { toring, extracur- } \\
\text { ricular activities), } \\
\text { and family and } \\
\text { community sup- } \\
\text { ports (family in- } \\
\text { volvement, coun- }\end{array}$ & $\begin{array}{l}6-14 \\
\text { (mean } \\
10, \text { SD } \\
1.7)\end{array}$ & $\begin{array}{l}\text { Alcohol use, } \\
\text { tobacco } \\
\text { use, drug } \\
\text { use, antiso- } \\
\text { cial behav- } \\
\text { iour, edu- } \\
\text { cational at- } \\
\text { tainment }\end{array}$ & 5 & No \\
\hline
\end{tabular}




\begin{tabular}{|c|c|c|c|c|c|c|c|c|c|c|c|}
\hline & & & & & & & $\begin{array}{l}\text { selling, and out- } \\
\text { reach) }\end{array}$ & & & & \\
\hline $\begin{array}{l}\text { Mathews } \\
2016\end{array}$ & $\begin{array}{l}\text { PRE- } \\
\text { PARE }\end{array}$ & SU & $\begin{array}{l}\text { South } \\
\text { Africa }\end{array}$ & $\begin{array}{l}12 \\
\text { months }\end{array}$ & $\begin{array}{l}\text { Social } \\
\text { cog- } \\
\text { nition } \\
\text { mod- } \\
\text { els in- } \\
\text { cluding } \\
\text { the rea- } \\
\text { soned } \\
\text { action } \\
\text { frame- } \\
\text { work } \\
\text { and the } \\
\text { I-Change } \\
\text { theoreti- } \\
\text { cal mod- } \\
\text { el }\end{array}$ & $\begin{array}{l}12 \\
\text { months }\end{array}$ & $\begin{array}{l}4 \text { school compo- } \\
\text { nents: } 21 \text { inter- } \\
\text { active and skills- } \\
\text { based sessions } \\
\text { of } 1-1.5 \text { hours } \\
\text { once per week; a } \\
\text { school health ser- } \\
\text { vice delivered by } \\
\text { a nurse from a lo- } \\
\text { cal public clinic; } \\
\text { safety training to } \\
\text { school personnel } \\
\text { and parent rep- } \\
\text { resentatives; and } \\
\text { a school safety } \\
\text { programme de- } \\
\text { livered to a ran- } \\
\text { domly selected } \\
\text { group of } 20 \text { vol- } \\
\text { unteers }\end{array}$ & $\begin{array}{l}\text { Grade } 8 \\
\text { (mean } \\
13 \text { years) }\end{array}$ & $\begin{array}{l}\text { Antisocial } \\
\text { behaviour, } \\
\text { sexual risk } \\
\text { behaviour }\end{array}$ & 2 & $\begin{array}{l}\text { Yes - rating } \\
\text { by partici- } \\
\text { pants of the } \\
\text { quality of } \\
\text { sessions, at- } \\
\text { tendance } \\
\text { at lessons, } \\
\text { visits to } \\
\text { the school } \\
\text { nurse, and } \\
\text { attendance } \\
\text { at the safety } \\
\text { programme. } \\
\text { Facilita- } \\
\text { tor per- } \\
\text { formance } \\
\text { scores }\end{array}$ \\
\hline $\begin{array}{l}\text { McNeal } \\
2004\end{array}$ & $\begin{array}{l}\text { All Stars } \\
1\end{array}$ & SU & USA & $\begin{array}{l}9 \\
\text { months } \\
\text { (1 school } \\
\text { year) }\end{array}$ & $\begin{array}{l}\text { Social } \\
\text { learning } \\
\text { theory }\end{array}$ & $\begin{array}{l}12 \\
\text { months } \\
\text { post in- } \\
\text { terven- } \\
\text { tion }\end{array}$ & $\begin{array}{l}\text { School-based } \\
\text { components: cur- } \\
\text { riculum includ- } \\
\text { ing classroom, } \\
\text { group, and 1-to-2 } \\
\text { sessions; home- } \\
\text { work to increase } \\
\text { parental interac- } \\
\text { tion/involvement }\end{array}$ & $11-13$ & $\begin{array}{l}\text { Alcohol use, } \\
\text { tobacco } \\
\text { use, drug } \\
\text { use, antiso- } \\
\text { cial behav- } \\
\text { iour, sexual } \\
\text { risk behav- } \\
\text { iour }\end{array}$ & 5 & $\begin{array}{l}\text { Yes - rating } \\
\text { of sessions, } \\
\text { rating of } \\
\text { objectives } \\
\text { achieved }\end{array}$ \\
\hline $\begin{array}{l}\text { Melnyk } \\
2013\end{array}$ & COPE & SU & USA & $\begin{array}{l}4 \\
\text { months }\end{array}$ & $\begin{array}{l}\text { Cogni- } \\
\text { tive-be- } \\
\text { haviour- } \\
\text { al theory }\end{array}$ & $\begin{array}{l}6 \\
\text { months }\end{array}$ & $\begin{array}{l}\text { School and par- } \\
\text { ent components: } \\
\text { educational and } \\
\text { cognitive-be- } \\
\text { havioural skills- } \\
\text { building pro- } \\
\text { gramme, includ- } \\
\text { ing physical ac- } \\
\text { tivity in each of } \\
\text { the } 15 \text { sessions, }\end{array}$ & $14-16$ & $\begin{array}{l}\text { Alcohol use, } \\
\text { drug use, } \\
\text { physical ac- } \\
\text { tivity, nutri- } \\
\text { tion, mental } \\
\text { health, edu- } \\
\text { cational at- } \\
\text { tainment }\end{array}$ & 6 & $\begin{array}{l}\text { Yes (par- } \\
\text { tial) - partic- } \\
\text { ipants com- } \\
\text { pleted ques- } \\
\text { tionnaires, } \\
\text { parents pro- } \\
\text { vided feed- } \\
\text { back. Fideli- } \\
\text { ty of teach- } \\
\text { ers mea- } \\
\text { sured }\end{array}$ \\
\hline
\end{tabular}




\begin{tabular}{|c|c|c|c|c|c|c|c|c|c|c|c|}
\hline & & & & & & & $\begin{array}{l}\text { homework, and a } \\
\text { parent newsletter }\end{array}$ & & & & \\
\hline $\begin{array}{l}\text { Nader } \\
1999\end{array}$ & CATCH 3 & SU & USA & 3 years & $\begin{array}{l}\text { Social } \\
\text { cog- } \\
\text { nitive } \\
\text { theory, } \\
\text { social } \\
\text { learning } \\
\text { theory, } \\
\text { organi- } \\
\text { sational } \\
\text { change } \\
\text { theory }\end{array}$ & $\begin{array}{l}3 \text { years } \\
\text { post in- } \\
\text { terven- } \\
\text { tion }\end{array}$ & $\begin{array}{l}\text { School and fam- } \\
\text { ily components: } \\
\text { classroom cur- } \\
\text { riculum, teacher } \\
\text { training, consul- } \\
\text { tation to teach- } \\
\text { ers, physical ac- } \\
\text { tivity sessions, } \\
\text { school policy, } \\
\text { home-based to- } \\
\text { bacco use pre- } \\
\text { vention pro- } \\
\text { gramme, family } \\
\text { activities to pro- } \\
\text { mote physical ac- } \\
\text { tivity }\end{array}$ & $8-11$ & $\begin{array}{l}\text { Tobacco } \\
\text { use, physi- } \\
\text { cal activity, } \\
\text { nutrition }\end{array}$ & 3 & Yes - full \\
\hline $\begin{array}{l}\text { O'Neill } \\
2011\end{array}$ & $\begin{array}{l}\text { Michigan } \\
\text { Mod- } \\
\text { el for } \\
\text { Health }\end{array}$ & SU & USA & 2 years & $\begin{array}{l}\text { Health } \\
\text { Belief } \\
\text { Model, } \\
\text { social } \\
\text { learning } \\
\text { theory }\end{array}$ & $\begin{array}{l}2 \\
\text { months }\end{array}$ & $\begin{array}{l}\text { School-based } \\
\text { curriculum and } \\
\text { skills-based } \\
\text { learning ( } 24 \\
\text { lessons in grade } \\
4 ; 28 \text { lessons in } \\
\text { grade } 5 \text { ) }\end{array}$ & $9-11$ & $\begin{array}{l}\text { Alcohol use, } \\
\text { tobacco } \\
\text { use, antiso- } \\
\text { cial behav- } \\
\text { iour, physi- } \\
\text { cal activity, } \\
\text { nutrition }\end{array}$ & 5 & $\begin{array}{l}\text { Yes (partial) } \\
\text { - adherence } \\
\text { to instruc- } \\
\text { tor, fidelity } \\
\text { to protocol, } \\
\text { teacher sur- } \\
\text { vey regard- } \\
\text { ing lessons } \\
\text { delivered, } \\
\text { implemen- } \\
\text { tation fideli- } \\
\text { ty assess- } \\
\text { ment }\end{array}$ \\
\hline $\begin{array}{l}\text { Perry } \\
2003\end{array}$ & $\begin{array}{l}\text { DARE vs } \\
\text { DARE+ }\end{array}$ & SU & USA & $\begin{array}{l}18 \\
\text { months }\end{array}$ & $\mathrm{N} / \mathrm{S}$ & $\begin{array}{l}\text { Post in- } \\
\text { terven- } \\
\text { tion }\end{array}$ & $\begin{array}{l}\text { DARE: } 1 \text { school } \\
\text { component in- } \\
\text { volving } 10-\text {-ses- } \\
\text { sion curricu- } \\
\text { lum delivered } \\
\text { by police offi- } \\
\text { cers. DARE-Plus: } \\
3 \text { school compo- } \\
\text { nents including } \\
4 \text {-session class- } \\
\text { room-based, }\end{array}$ & $12-13$ & $\begin{array}{l}\text { Alcohol use, } \\
\text { tobacco } \\
\text { use, drug } \\
\text { use, antiso- } \\
\text { cial behav- } \\
\text { iour }\end{array}$ & 4 & No \\
\hline
\end{tabular}




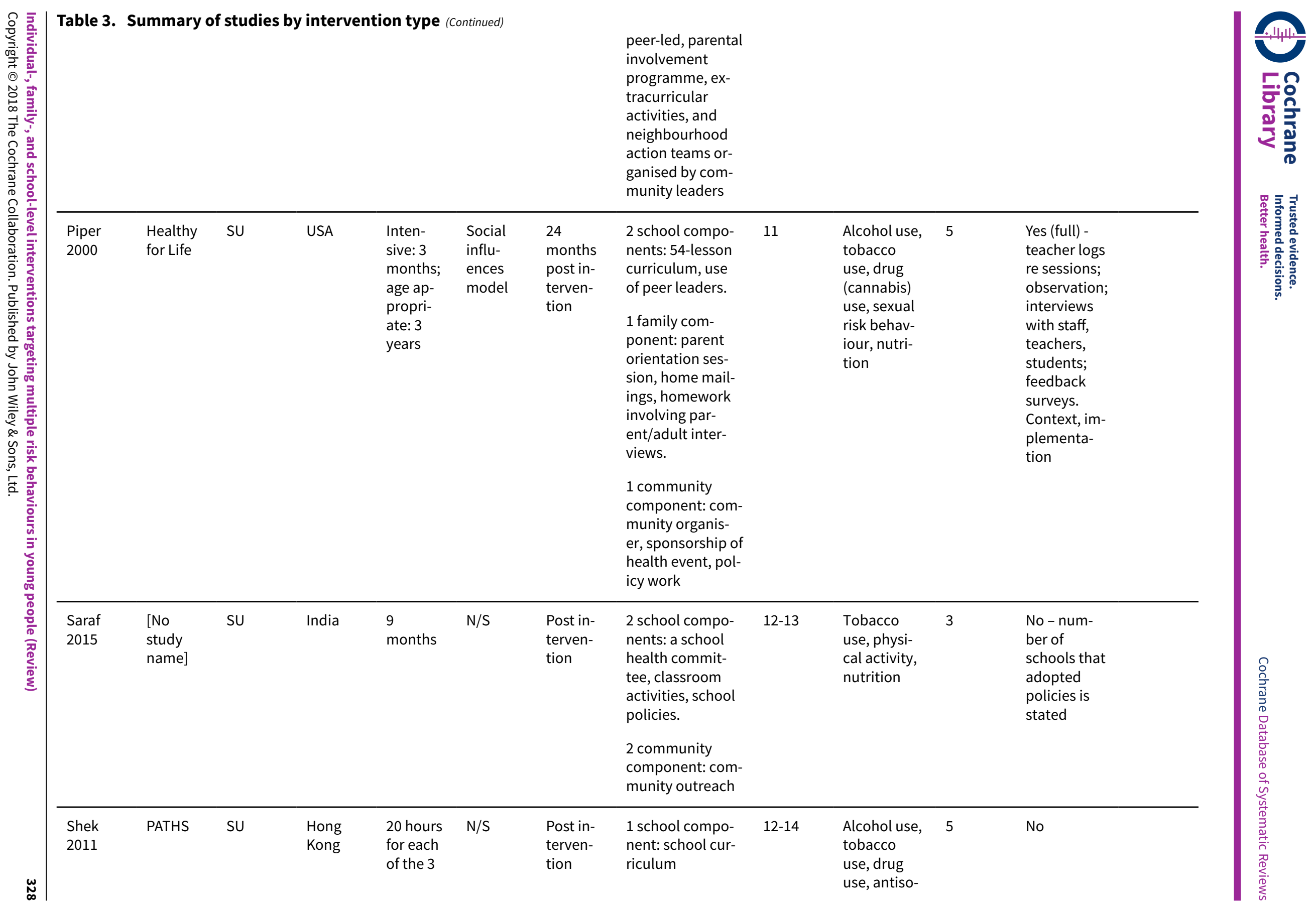




\begin{tabular}{|c|c|c|c|c|c|c|c|c|c|c|c|}
\hline & & & & $\begin{array}{l}\text { school } \\
\text { years }\end{array}$ & & & & & $\begin{array}{l}\text { cial behav- } \\
\text { iour, sexual } \\
\text { risk behav- } \\
\text { iour }\end{array}$ & & \\
\hline $\begin{array}{l}\text { Si- } \\
\text { mons-Mor- } \\
\text { ton } 2005\end{array}$ & $\begin{array}{l}\text { Going } \\
\text { Places }\end{array}$ & SU & USA & $\begin{array}{l}3 \text { school } \\
\text { years }\end{array}$ & $\begin{array}{l}\text { Social } \\
\text { devel- } \\
\text { opment } \\
\text { and so- } \\
\text { cial cog- } \\
\text { nitive } \\
\text { theory }\end{array}$ & $\begin{array}{l}3 \\
\text { months } \\
\text { post in- } \\
\text { terven- } \\
\text { tion }\end{array}$ & $\begin{array}{l}2 \text { school com- } \\
\text { ponents: social } \\
\text { skills curriculum } \\
\text { (18 sessions in } \\
6 \text { th grade, } 12 \text { in } \\
7 \text { th grade, } 8 \text { in } \\
\text { 8th grade) and } \\
\text { school environ- } \\
\text { ment enhance- } \\
\text { ment. } \\
\text { Parent compo- } \\
\text { nent: parent edu- } \\
\text { cation via video, } \\
\text { booklet, periodic } \\
\text { newsletters, and } \\
\text { involvement in } \\
\text { homework }\end{array}$ & $11-14$ & $\begin{array}{l}\text { Alcohol use, } \\
\text { tobacco } \\
\text { use, antiso- } \\
\text { cial behav- } \\
\text { iour }\end{array}$ & 3 & $\begin{array}{l}\text { Yes (full) - } \\
\text { adherence, } \\
\text { teacher rat- } \\
\text { ing of stu- } \\
\text { dents' par- } \\
\text { ticipation, } \\
\text { student } \\
\text { feedback re- } \\
\text { garding at- } \\
\text { tendance, } \\
\text { helpfulness, } \\
\text { knowledge. } \\
\text { Parent inter- } \\
\text { views. }\end{array}$ \\
\hline $\begin{array}{l}\text { Walter } \\
1989\end{array}$ & $\begin{array}{l}\text { Know } \\
\text { Your } \\
\text { Body } 1\end{array}$ & SU & USA & $5-6$ years & $\begin{array}{l}\text { PRE- } \\
\text { CEDE } \\
\text { health } \\
\text { edu- } \\
\text { cation } \\
\text { planning } \\
\text { model } \\
\text { (which } \\
\text { incorpo- } \\
\text { rates el- } \\
\text { ements } \\
\text { of social } \\
\text { learning } \\
\text { theory } \\
\text { and the } \\
\text { Health } \\
\text { Belief } \\
\text { Model) }\end{array}$ & $\begin{array}{l}\text { Post in- } \\
\text { terven- } \\
\text { tion }\end{array}$ & $\begin{array}{l}\text { School compo- } \\
\text { nent: curricu- } \\
\text { lum for } 2 \text { hours } \\
\text { per week for } \\
\text { each school year } \\
\text { (grades 4-9). } \\
\\
\text { Parent compo- } \\
\text { nent: self-assess- } \\
\text { ment, newslet- } \\
\text { ters, participa- } \\
\text { tion in activities, } \\
\text { seminars. }\end{array}$ & $\begin{array}{l}\text { 8-15 } \\
\text { (mean } 9 \\
\text { years at } \\
\text { baseline) }\end{array}$ & $\begin{array}{l}\text { Tobacco } \\
\text { use, physi- } \\
\text { cal activity, } \\
\text { nutrition }\end{array}$ & 3 & $\begin{array}{l}\text { Yes (partial) } \\
\text { - adherence } \\
\text { to proto- } \\
\text { col, visits to } \\
\text { classrooms }\end{array}$ \\
\hline
\end{tabular}


able 3. Summary of studies by intervention type (Continued)

\begin{tabular}{|c|c|c|c|c|c|c|c|c|c|c|}
\hline $\begin{array}{l}\text { Fourth } \\
\text { R-Skills } \\
\text { for Youth } \\
\text { Relation- } \\
\text { ships }\end{array}$ & SU & Canada & $\begin{array}{l}8 \\
\text { months }\end{array}$ & $\mathrm{N} / \mathrm{S}$ & 2 years & $\begin{array}{l}\text { School compo- } \\
\text { nent: } 21 \text {-lesson } \\
\text { curriculum. }\end{array}$ & $14-15$ & $\begin{array}{l}\text { Alcohol use, } \\
\text { tobacco } \\
\text { use, drug } \\
\text { use, antiso- } \\
\text { cial behav- } \\
\text { iour, sexual } \\
\text { risk behav- } \\
\text { iour }\end{array}$ & 5 & $\begin{array}{l}\text { Yes (partial) } \\
\text { - teacher } \\
\text { checklists re } \\
\text { completion }\end{array}$ \\
\hline
\end{tabular}

ASE: Attitude - social influence - self-efficacy model.

BASICS: Brief Alcohol Screening and Intervention for College Students.

BBBS: Big Brothers Big Sisters.

$\mathrm{BI}$ : Brief intervention.

BRAVE: Building Resiliency And Vocational Excellence.

CATCH: Coordinated approach to child health.

C. classroom-centred.

COPE: Creating Opportunities for Personal Empowerment.

DARE: Drug Abuse and Resistance Education.

FSP: Family Schools Partnership.

FT: family-targeted.

FU: family-universal.

GBG: Good Behaviour Game.

imPACT: Informed Parents and Children Together.

IT: individual-targeted.

IU: individual-universal.

LIFT: Linking the Interests of Families and Teachers.

LST: Life Skills Training.

MI: motivational intervention.

N/S: not stated.

PATHS: Promoting Alternative Thinking Strategies.

ProSAAF: Protecting Stronger African American Families.

ROAD: Reducing Offenses by Adolescent Drivers.

SAAF: Stronger African American Families.

SD: standard deviation.

SDC: Social Development Curriculum.

ST: school-targeted.

STRIVE: Support to Reunite, Involve and Value Each Other.

SU: school-universal.

TTM: transtheoretical model. 


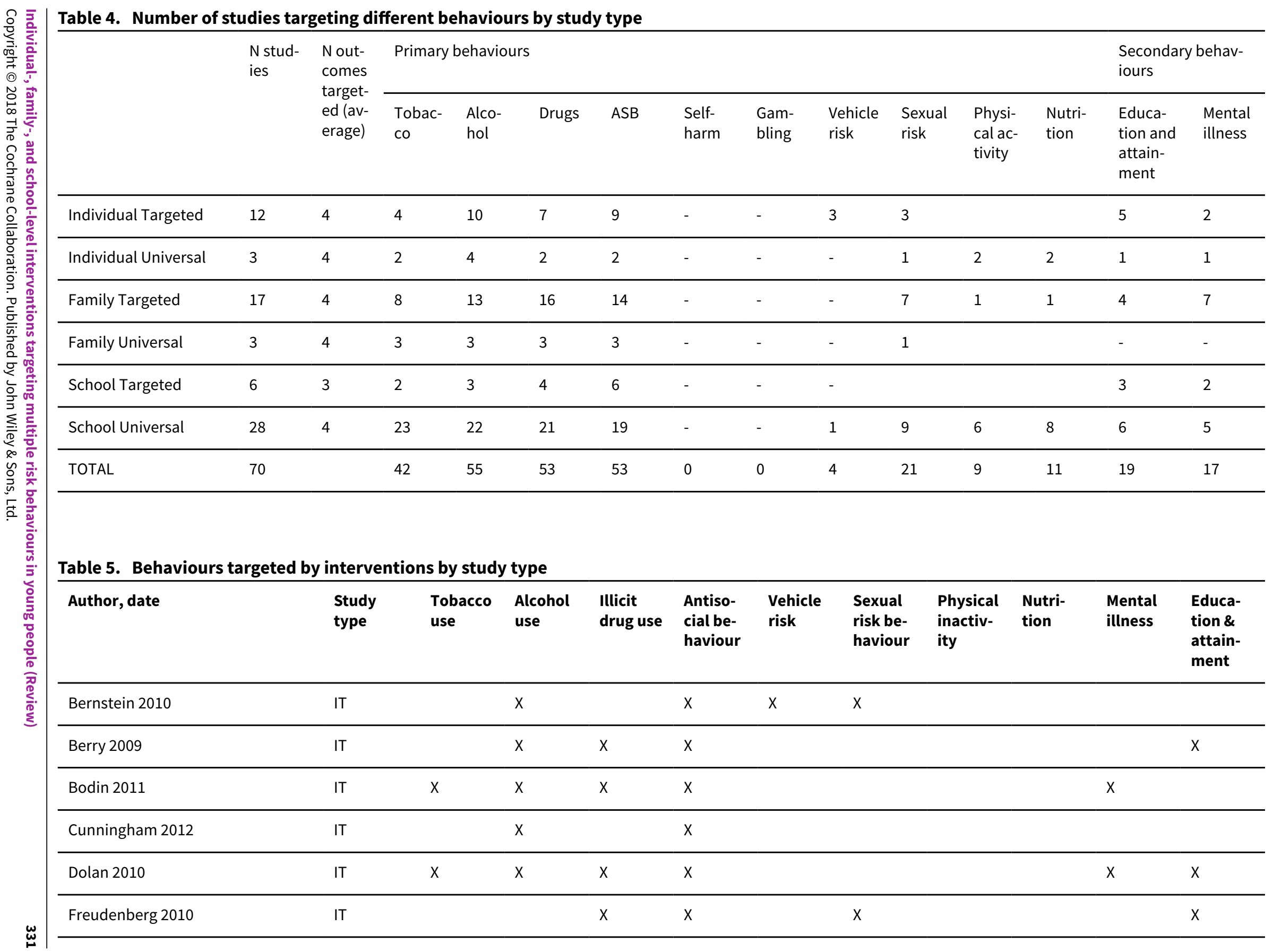




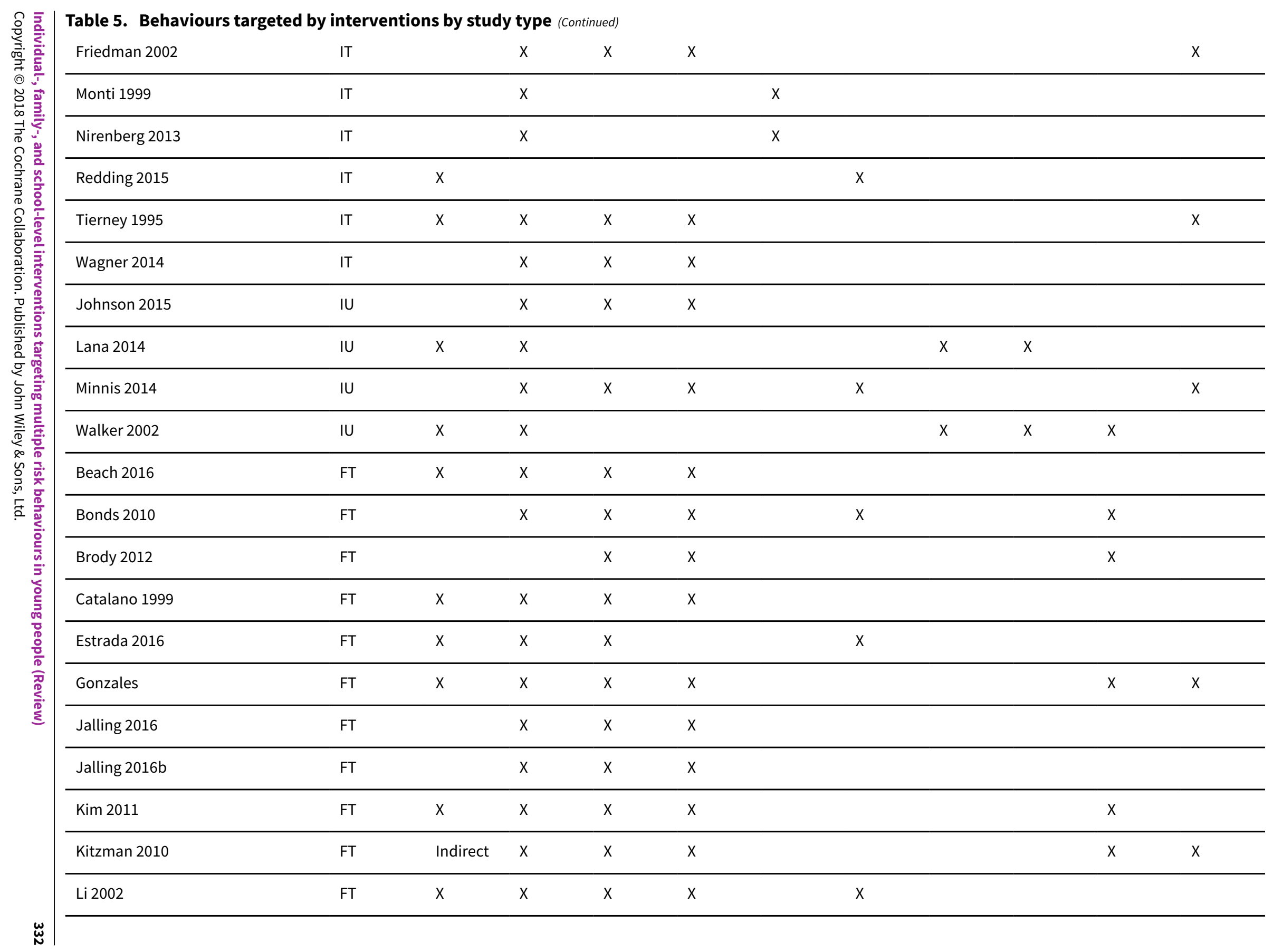




\begin{tabular}{|c|c|c|c|c|c|c|c|c|c|c|c|}
\hline Milburn 2012 & FT & & & $x$ & $x$ & & $x$ & & & & \\
\hline Morris 2003 & FT & $x$ & $x$ & $x$ & $x$ & & & & & $x$ & $x$ \\
\hline Murry 2014 & FT & & $x$ & & & & $x$ & & & & \\
\hline Olds 1998 & FT & Indirect & & $x$ & $x$ & & $x$ & & & $x$ & $x$ \\
\hline Pantin 2009 & FT & $x$ & $x$ & $x$ & $x$ & & $x$ & & & & \\
\hline Schwinn 2014 & FT & & & $x$ & & & & $x$ & $x$ & & \\
\hline Averdijk 2016 & FU & $x$ & $x$ & $x$ & $x$ & & & & & & \\
\hline Connell 2007 & FU & $x$ & $x$ & $x$ & $x$ & & & & & & \\
\hline Haggerty 2007 & FU & $x$ & $x$ & $x$ & $x$ & & $x$ & & & & \\
\hline CPRG 2014 & ST & Indirect & Indirect & Indirect & $x$ & & Indirect & & & $x$ & $x$ \\
\hline Lochman 2003 & ST & $x$ & $x$ & $x$ & $x$ & & & & & & \\
\hline Lochman 2004 & ST & & $x$ & $x$ & $x$ & & & & & & \\
\hline Sanchez 2007 & ST & & & $x$ & $x$ & & & & & $x$ & $x$ \\
\hline Schweinhart 1993 & ST & Indirect & Indirect & Indirect & $x$ & & & & & & $x$ \\
\hline Shetgiri 2011 & ST & $x$ & $\mathrm{x}$ & $x$ & $x$ & & & & & & \\
\hline Beets 2009 & SU & $x$ & $x$ & $x$ & $x$ & & $x$ & & & & \\
\hline Bond 2004 & SU & $x$ & $x$ & $x$ & $x$ & & $x$ & & & $x$ & \\
\hline Bush 1989 & SU & $x$ & & & & & & $x$ & $x$ & & \\
\hline D'Amico 2002 & SU & & $x$ & $x$ & & $x$ & & & & & \\
\hline DeGarmo 2009 & SU & $x$ & $x$ & $x$ & $x$ & & & & & & \\
\hline Fearnow-Kenney 2003 & SU & $x$ & $\mathrm{x}$ & $\mathrm{x}$ & & & & & $x$ & & \\
\hline
\end{tabular}




\begin{tabular}{|c|c|c|c|c|c|c|c|c|c|c|}
\hline \multirow{2}{*}{ 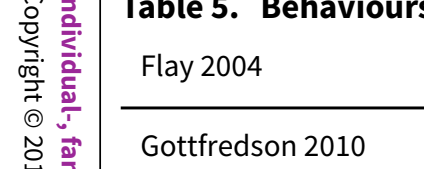 } & \multicolumn{3}{|l|}{ SU } & \multirow{2}{*}{$\frac{x}{x}$} & \multirow{2}{*}{$x$} & \multicolumn{5}{|c|}{$x$} \\
\hline & SU & $\mathrm{x}$ & $x$ & & & & & & & \\
\hline Griffin 2006 & SU & & $x$ & $x$ & & $x$ & & & & \\
\hline Griffin 2009 & SU & $x$ & $x$ & $x$ & $x$ & & & & & \\
\hline Ialongo 1999a & SU & $x$ & $X$ & $x$ & $x$ & & & & $x$ & $\mathrm{X}$ \\
\hline Ialongo 1999b & SU & $x$ & $x$ & $x$ & $x$ & & & & $x$ & $\mathrm{x}$ \\
\hline Kellam & SU & $x$ & $x$ & $x$ & $x$ & & & & & $\mathrm{x}$ \\
\hline Lewis 2013 & SU & $X$ & $x$ & $x$ & $x$ & & & & $x$ & $\mathrm{x}$ \\
\hline LoSciuto 1999 & SU & $x$ & $x$ & $x$ & $x$ & & & & & $\mathrm{X}$ \\
\hline Matthews 2016 & SU & & & & $x$ & $x$ & & & & \\
\hline McNeal 2004 & SU & $X$ & $x$ & $x$ & $x$ & $x$ & & & & \\
\hline Melnyk 2013 & SU & & $x$ & $x$ & & & $x$ & $x$ & $x$ & $\mathrm{X}$ \\
\hline Nader 1999 & SU & $x$ & & & & & $x$ & $x$ & & \\
\hline O'Neill 2011 & SU & $x$ & $x$ & & $x$ & & $x$ & $x$ & & \\
\hline Perry 2003 & SU & $x$ & $x$ & $x$ & $x$ & & & & & \\
\hline Piper 2000 & SU & $x$ & $x$ & $x$ & & $x$ & & $x$ & & \\
\hline Saraf 2015 & SU & $x$ & & & & & $x$ & $x$ & & \\
\hline Shek 2011 & SU & $x$ & $x$ & $x$ & $x$ & $x$ & & & & \\
\hline Simons-Morton 2005 & SU & $x$ & $x$ & & $x$ & & & & & \\
\hline Walter 1989 & SU & $x$ & & & & & $x$ & $x$ & & \\
\hline Wolfe 2009 & SU & $x$ & $x$ & $x$ & $x$ & $X$ & & & & \\
\hline
\end{tabular}




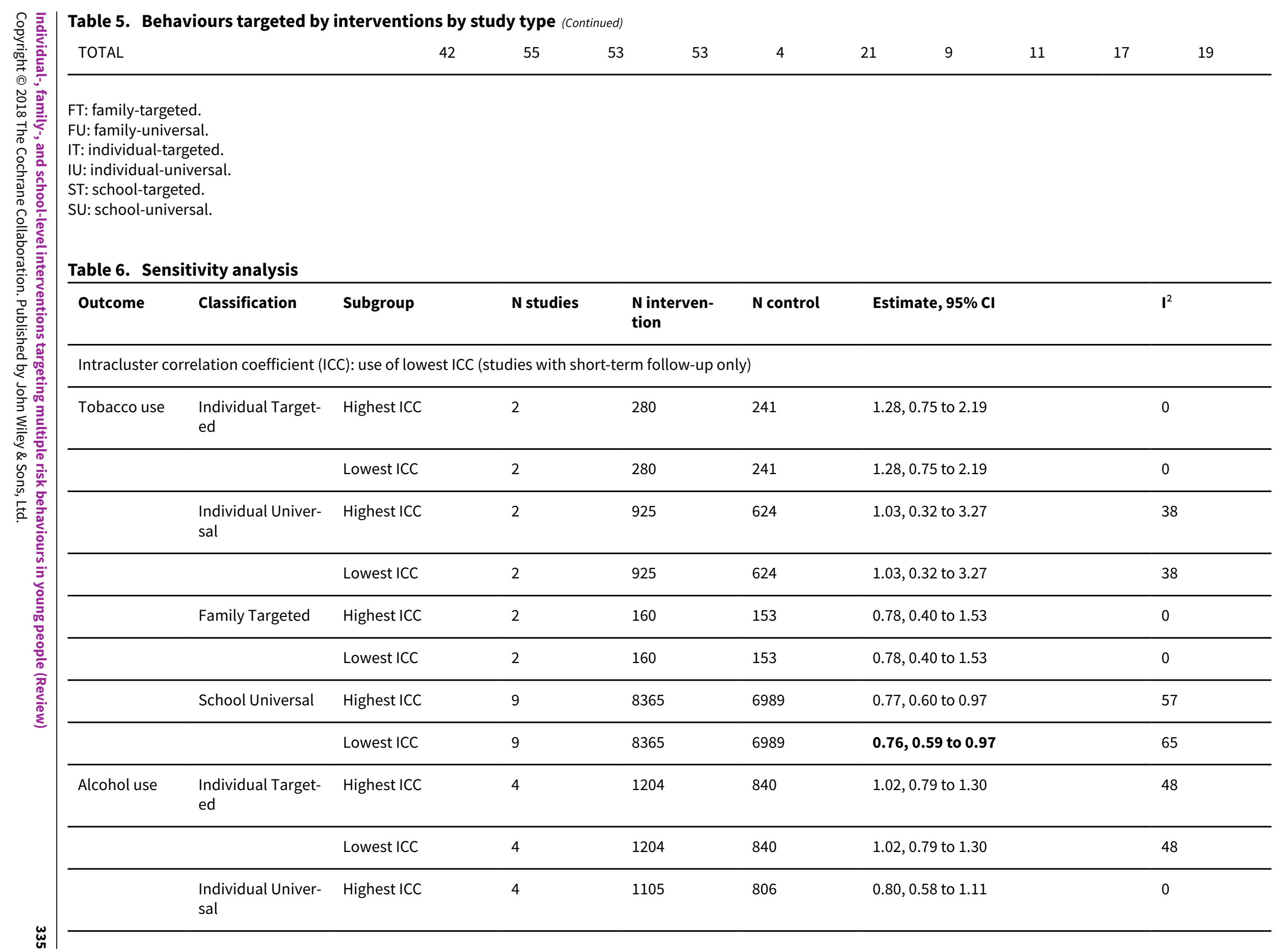




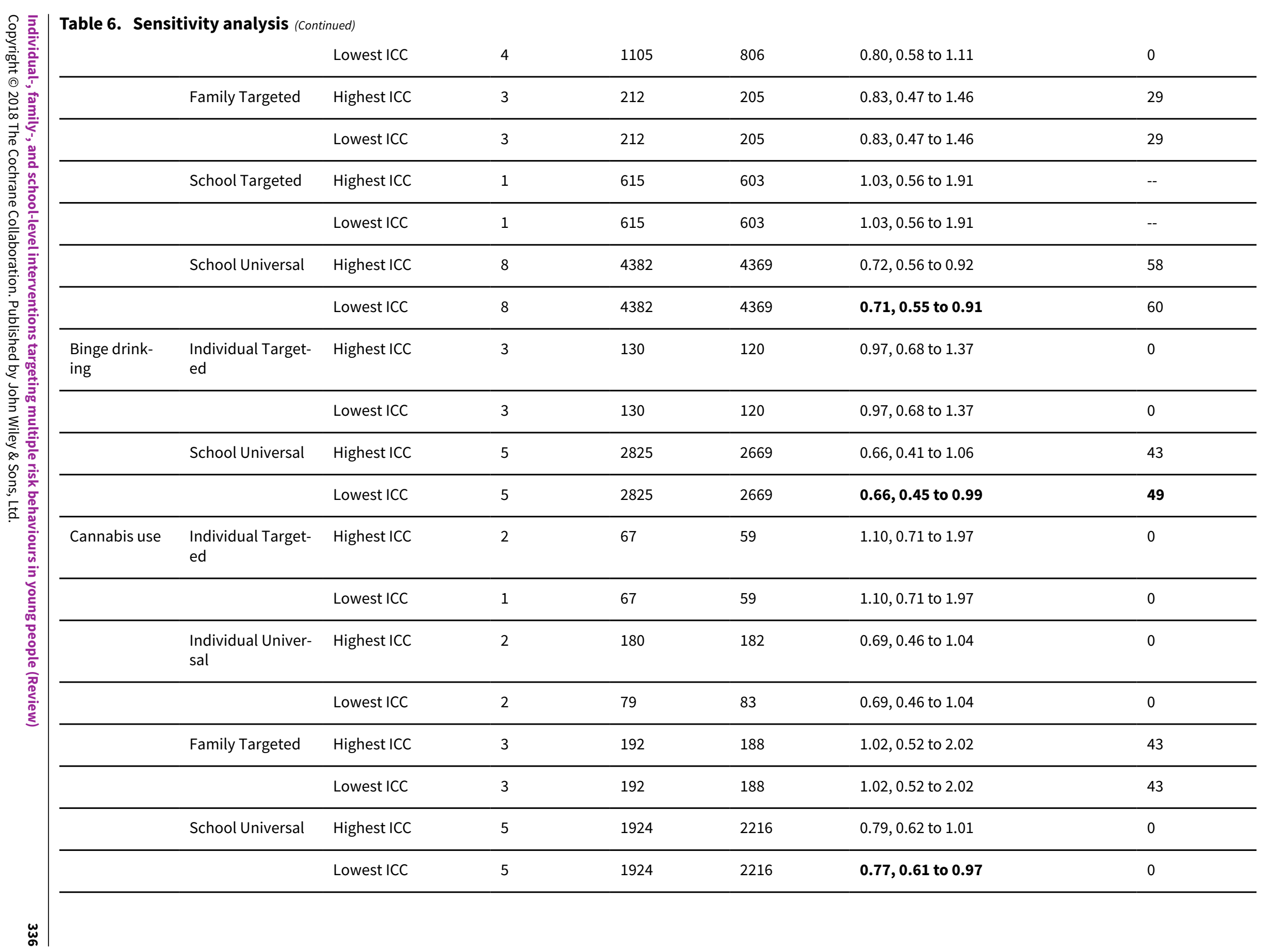




\begin{tabular}{|c|c|c|c|c|c|c|c|}
\hline Illicit drug use & $\begin{array}{l}\text { Individual Target- } \\
\text { ed }\end{array}$ & Highest ICC & 3 & 342 & 296 & $0.94,0.71$ to 1.25 & 0 \\
\hline & & Lowest ICC & 3 & 342 & 296 & $0.94,0.71$ to 1.25 & 0 \\
\hline & Family Targeted & Highest ICC & 1 & 33 & 36 & $0.74,0.42$ to 1.31 & -- \\
\hline & & Lowest ICC & 1 & 33 & 36 & $0.74,0.42$ to 1.31 & -- \\
\hline & School Targeted & Highest ICC & 4 & 1431 & 1023 & $0.75,0.53$ to 1.06 & 60 \\
\hline & & Lowest ICC & 4 & 1431 & 1023 & $0.75,0.53$ to 1.06 & 60 \\
\hline & School Universal & Highest ICC & 5 & 4745 & 6313 & $0.74,0.55$ to 1.00 & 69 \\
\hline & & Lowest ICC & 5 & 4715 & 6313 & $0.74,0.54$ to 1.03 & 82 \\
\hline \multirow[t]{6}{*}{$\begin{array}{l}\text { Tobacco, al- } \\
\text { cohol, and/or } \\
\text { illicit drug use }\end{array}$} & Family Targeted & Highest ICC & 2 & 115 & 98 & $0.81,0.50$ to 1.33 & 0 \\
\hline & & Lowest ICC & 2 & 115 & 98 & $0.81,0.50$ to 1.33 & 0 \\
\hline & School Targeted & Highest ICC & 5 & 244 & 98 & $0.55,0.24$ to 1.25 & 69 \\
\hline & & Lowest ICC & 5 & 244 & 98 & $0.54,0.24$ to 1.21 & 71 \\
\hline & School Universal & Highest ICC & 2 & 2771 & 4256 & $1.13,0.88$ to 1.44 & 0 \\
\hline & & Lowest ICC & 2 & 2771 & 4256 & $1.10,1.01$ to 1.20 & 0 \\
\hline \multirow[t]{5}{*}{$\begin{array}{l}\text { Antisocial be- } \\
\text { haviour }\end{array}$} & $\begin{array}{l}\text { Individual Target- } \\
\text { ed }\end{array}$ & Highest ICC & 4 & 409 & 355 & $1.21,0.92$ to 1.60 & 19 \\
\hline & & Lowest ICC & 4 & 409 & 355 & $1.21,0.92$ to 1.60 & 19 \\
\hline & Family Targeted & Highest ICC & 6 & 437 & 335 & $0.84,0.57$ to 1.24 & 42 \\
\hline & & Lowest ICC & 6 & 437 & 335 & $0.84,0.57$ to 1.24 & 42 \\
\hline & Family Universal & Highest ICC & 1 & 208 & 98 & $0.87,0.56$ to 1.35 & 0 \\
\hline
\end{tabular}




\begin{tabular}{|c|c|c|c|c|c|c|c|}
\hline & & Lowest ICC & 1 & 208 & 98 & $0.87,0.56$ to 1.35 & 0 \\
\hline & School Targeted & Highest ICC & 3 & 815 & 716 & $0.78,0.59$ to 1.05 & 0 \\
\hline & & Lowest ICC & 3 & 815 & 716 & $0.82,0.68$ to 0.99 & 0 \\
\hline & School Universal & Highest ICC & 12 & 9960 & 10796 & $0.81,0.66$ to 0.98 & 66 \\
\hline & & Lowest ICC & 12 & 9960 & 10796 & $0.82,0.69$ to 0.97 & 67 \\
\hline $\begin{array}{l}\text { Sexual risk } \\
\text { behaviour }\end{array}$ & $\begin{array}{l}\text { Individual Target- } \\
\text { ed }\end{array}$ & Highest ICC & 2 & 266 & 228 & $0.73,0.49$ to 1.08 & 45 \\
\hline & & Lowest ICC & 2 & 266 & 228 & $0.73,0.49$ to 1.08 & 45 \\
\hline & $\begin{array}{l}\text { Individual Univer- } \\
\text { sal }\end{array}$ & Highest ICC & 1 & 79 & 83 & $0.42,0.14$ to 1.25 & -- \\
\hline & & Lowest ICC & 1 & 79 & 83 & $0.42,0.14$ to 1.25 & -- \\
\hline & Family Targeted & Highest ICC & 3 & 188 & 183 & $0.89,0.55$ to 1.44 & 0 \\
\hline & & Lowest ICC & 3 & 188 & 183 & $0.89,0.55$ to 1.44 & 0 \\
\hline & School Universal & Highest ICC & 6 & 5757 & 6876 & $0.83,0.61$ to 1.12 & 77 \\
\hline & & Lowest ICC & 6 & 5757 & 6876 & $0.83,0.61$ to 1.12 & 77 \\
\hline $\begin{array}{l}\text { Physical activ- } \\
\text { ity }\end{array}$ & $\begin{array}{l}\text { Individual Univer- } \\
\text { sal }\end{array}$ & Highest ICC & 2 & 748 & 782 & $1.11,0.74$ to 1.67 & 0 \\
\hline & & Lowest ICC & 2 & 748 & 782 & $1.11,0.74$ to 1.67 & 0 \\
\hline & Family Targeted & Highest ICC & 1 & 31 & 30 & $0.72,0.29$ to 1.79 & -- \\
\hline & & Lowest ICC & 1 & 31 & 30 & $0.72,0.29$ to 1.79 & -- \\
\hline & School Universal & Highest ICC & 4 & 3547 & 2894 & $1.32,1.16$ to 1.50 & 0 \\
\hline & & Lowest ICC & 4 & 3547 & 2894 & $1.33,1.18$ to 1.50 & 0 \\
\hline
\end{tabular}




\begin{tabular}{|c|c|c|c|c|c|c|c|}
\hline $\begin{array}{l}\text { Nutrition } \\
\text { (BMI) }\end{array}$ & $\begin{array}{l}\text { Individual Univer- } \\
\text { sal }\end{array}$ & Highest ICC & 1 & 421 & 158 & $0.80,0.48$ to 1.31 & -- \\
\hline & & Lowest ICC & 1 & 421 & 158 & $0.80,0.48$ to 1.31 & -- \\
\hline & School Universal & Highest ICC & 3 & 2901 & 2116 & $0.84,0.60$ to 1.19 & 61 \\
\hline & & Lowest ICC & 3 & 2901 & 2116 & $0.88,0.62$ to 1.23 & 69 \\
\hline $\begin{array}{l}\text { Nutrition (un- } \\
\text { healthy diet) }\end{array}$ & $\begin{array}{l}\text { Individual Univer- } \\
\text { sal }\end{array}$ & Highest ICC & 2 & 925 & 624 & $0.76,0.42$ to 1.34 & 51 \\
\hline & & Lowest ICC & 2 & 925 & 624 & $0.76,0.42$ to 1.34 & 51 \\
\hline & School Universal & Highest ICC & 3 & 3608 & 2833 & $0.82,0.64$ to 1.06 & 49 \\
\hline & & Lowest ICC & 3 & 3608 & 2833 & $0.85,0.66$ to 1.09 & 63 \\
\hline $\begin{array}{l}\text { Educational } \\
\text { attainment } \\
\text { (academic } \\
\text { performance) }\end{array}$ & $\begin{array}{l}\text { Individual Target- } \\
\text { ed }\end{array}$ & Highest ICC & 1 & 67 & 59 & $1.34,0.71$ to 2.52 & -- \\
\hline & & Lowest ICC & 1 & 67 & 59 & $1.34,0.71$ to 2.52 & -- \\
\hline & School Targeted & Highest ICC & 3 & 619 & 628 & $0.91,0.30$ to 2.73 & 84 \\
\hline & & Lowest ICC & 3 & 619 & 628 & $0.91,0.39$ to 2.14 & 85 \\
\hline & School Universal & Highest ICC & 3 & 602 & 393 & $0.94,0.62$ to 1.44 & 0 \\
\hline & & Lowest ICC & 3 & 602 & 393 & $0.95,0.74$ to 1.22 & 0 \\
\hline Dichotomous v & continuous outcom & s (studies with & indi & follow & & & \\
\hline Tobacco use & School Universal & All & 9 & 8365 & 6989 & $0.77,0.60$ to $0.97, P=0.03$ & 57 \\
\hline & & Dichotomous & 7 & 7581 & 6275 & $0.72,0.52$ to $0.99, P=0.05$ & 60 \\
\hline & & Continuous & 2 & 784 & 714 & SMD $-0.01,-0.40$ to $0.37, P=0.95$ & 84 \\
\hline Alcohol use & School Universal & All & 8 & 4382 & 4369 & $0.72,0.56$ to $0.92, P=0.009$ & 58 \\
\hline
\end{tabular}




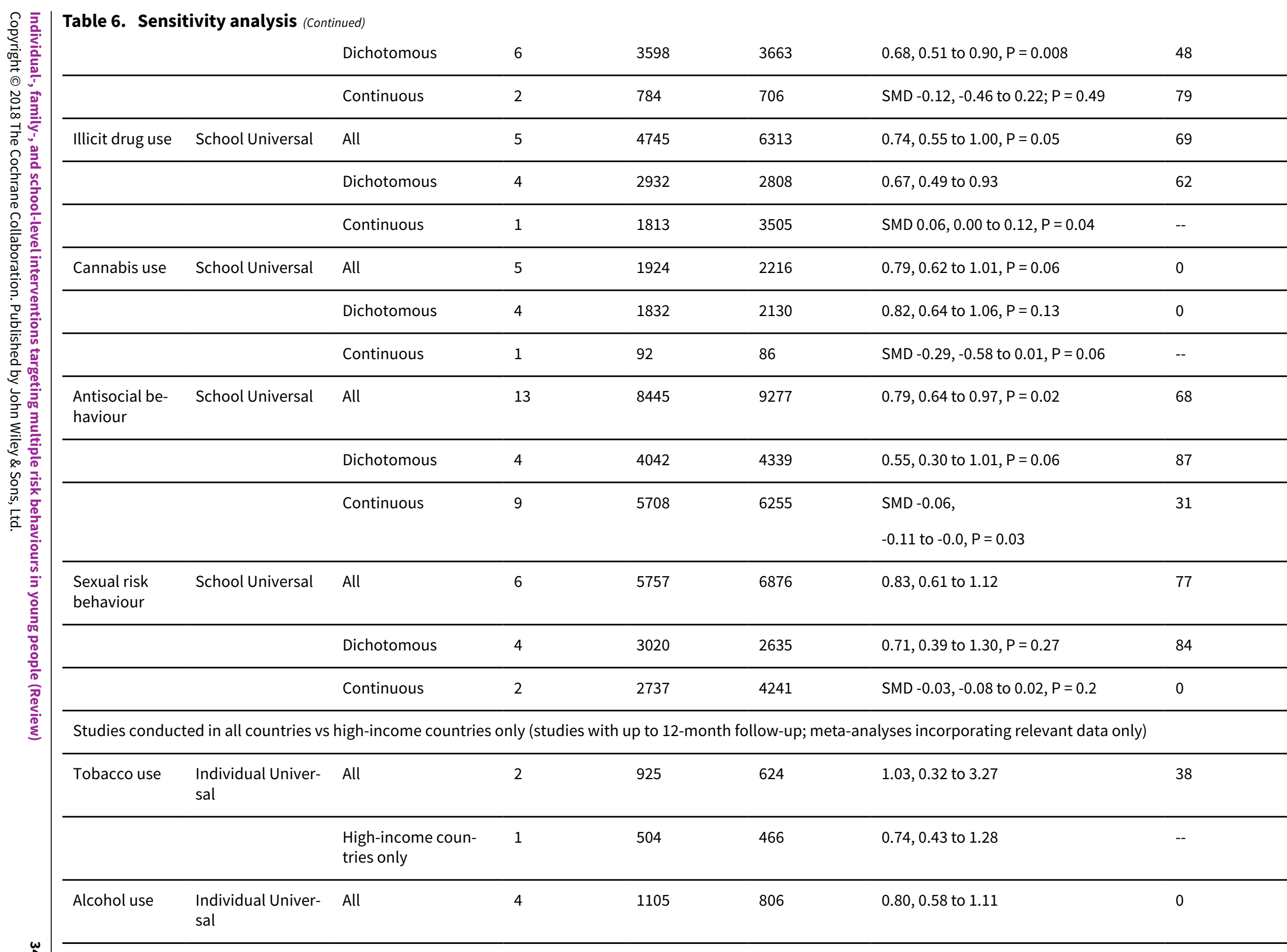




\begin{tabular}{|c|c|c|c|c|c|c|c|}
\hline & & $\begin{array}{l}\text { High-income coun- } \\
\text { tries only }\end{array}$ & 3 & 684 & 648 & $0.74,0.54$ to 1.06 & 0 \\
\hline $\begin{array}{l}\text { Antisocial be- } \\
\text { haviour }\end{array}$ & School Universal & All & 13 & 9960 & 10796 & $0.81,0.66$ to 0.98 & 66 \\
\hline & & $\begin{array}{l}\text { High-income coun- } \\
\text { tries only }\end{array}$ & 12 & 8445 & 9277 & $0.79,0.64$ to 0.97 & 68 \\
\hline $\begin{array}{l}\text { Sexual risk } \\
\text { behaviour }\end{array}$ & School Universal & All & 6 & 5757 & 6876 & $0.83,0.61$ to 1.12 & 77 \\
\hline & & $\begin{array}{l}\text { High-income coun- } \\
\text { tries only }\end{array}$ & 5 & 5654 & 6779 & $0.81,0.59$ to 1.11 & 80 \\
\hline $\begin{array}{l}\text { Physical activ- } \\
\text { ity }\end{array}$ & $\begin{array}{l}\text { Individual Univer- } \\
\text { sal }\end{array}$ & All & 2 & 748 & 782 & $1.11,0.74$ to 1.67 & 0 \\
\hline & & $\begin{array}{l}\text { High-income coun- } \\
\text { tries only }\end{array}$ & 1 & 504 & 466 & $1.40,0.67$ to 2.94 & -- \\
\hline & School Universal & All & 4 & 3547 & 2894 & $1.32,1.16$ to 1.50 & 0 \\
\hline & & $\begin{array}{l}\text { High-income coun- } \\
\text { tries only }\end{array}$ & 3 & 2533 & 1834 & $1.44,1.20$ to 1.74 & 0 \\
\hline $\begin{array}{l}\text { Unhealthy di- } \\
\text { et }\end{array}$ & $\begin{array}{l}\text { Individual Univer- } \\
\text { sal }\end{array}$ & All & 2 & 925 & 624 & $0.76,0.42$ to 1.34 & 51 \\
\hline & & $\begin{array}{l}\text { High-income coun- } \\
\text { tries only }\end{array}$ & 1 & 504 & 466 & $0.50,0.23$ to 1.08 & -- \\
\hline & School Universal & All & 3 & 3608 & 2833 & $0.82,0.64$ to 1.06 & 49 \\
\hline & & $\begin{array}{l}\text { High-income coun- } \\
\text { tries only }\end{array}$ & 2 & 2594 & 1773 & $0.95,0.76$ to 1.19 & 0 \\
\hline
\end{tabular}

$\mathrm{Cl}$ : confidence interval.

ICC: intracluster correlation coefficient. 


\section{APPENDICES}

\section{Appendix 1. Search Strategies}

Search strategy for CINA, 1950 to present. Searched 06 May 2015 and 12 November 2016.

Previously searched 31 May 2012.

\begin{tabular}{ll}
\hline S74 & (S65 and S70 and S73) \\
\hline S73 & (S71 or S72) \\
\hline S72 & "controlled clinical trial ${ }^{\star *}$ OR ( randomi?ed or placebo or randomly ) OR TI trial \\
\hline S71 & (MH "Randomized Controlled Trials") \\
\hline S70 & S66 or S67 or S68 or S69 \\
\hline S69 & $\begin{array}{l}\text { TI ( school } \\
\text { or infant }\end{array}$ \\
\hline
\end{tabular}

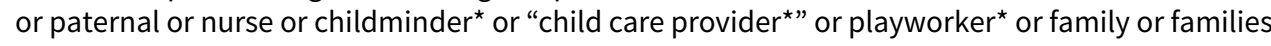
or carer* or midwife or "mid wife" or midwives or "mid wives"

(Adolescen* or teen* or "young person" or "young people" or youth* or hooligan* or "young adult" or "early adult t" or juvenile* or minor or "emerging adult" or girl or boy or apprentice* or "FE college*” or "young m?n" or "young wom?n" or "young male" or "young female" or "under $18^{\star}$ " or "sixth-form" or "secondary education" or "tertiary education" or "higher education" or "further education" or preschool* or "primary education" or infan* or kid or nurser* or playschool* or kindergarten* or prekindergarten*)

S66 (MH "Child") OR (MH "Child, Preschool") OR (MH "Infant") OR (MH "Adolescence")

( (S3 and S15) or (S3 and S18) or (S3 and S2O) or (S3 and S21) or (S3 and S26) or (S3 and S34) or (S3 and S40) or (S3 and S46) or (S3 and S52) or (S3 and S59) or (S3 and S64) ) OR ( (S15 and S18) or (S15 and S20) or (S15 and S21) or (S15 and S26) or (S15 and S34) or (S15 and S40) or (S15 and S46) or (S15 and S52) or (S15 and S59) or (S15 and S64) or (S18 and S20) or (S18 and S21) or (S18 and S26) or (S18 and S34) or (S18 and S40) or (S18 and S46) or (S18 and S52) or (S18 and S59) or (S18 and S64) or (S20 and S21) or (S20 and S26) or (S20 and S34) or (S20 and S40) or (S20 and S46) or (S20 and S52) or (S20 and S59) or (S20 and S64) or (S21 and S26) or (S21 and S34) or (S21 and S40) or (S21 and S46) or (S21 and S52) or (S21 and S59) or (S21 and S64) or (S26 and S34) or (S26 and S40) or (S26 and S46) or (S26 and S52) or (S26 and S59) or (S26 and S64) or (S34 and S40) or (S34 and S46) or (S34 and S52) or (S34 and S59) or (S34 and S64) or (S40 and S46) or (S40 and S52) or (S40 and S59) or (S40 and S64) or (S46 and S52) or (S46 and S59) or (S46 and S64) )

\begin{tabular}{|c|c|}
\hline S64 & $\mathrm{S} 60$ or $\mathrm{S} 61$ or $\mathrm{S} 62$ or $\mathrm{S} 63$ \\
\hline S63 & $\begin{array}{l}\left.\left.\text { ( (screen or sedentary or view* }) \text { N2 (time or hour }{ }^{\star} \text { or minute }{ }^{\star}\right) \text { ) OR ( (inactiv* or seden* or indoor }{ }^{\star}\right) \\
\left.\text { N2 (lifestyle } \text { or activit }^{\star}\right) \text { ) }\end{array}$ \\
\hline S62 & $\begin{array}{l}\text { gaming OR ( (view }{ }^{\star} \text { or watch } \text { or play }^{\star} \text { or game } \text { or use }^{\star} \text { or using or usage) N2 (television or tv or } \\
\text { video }^{\star} \text { or } \text { dvd }^{\star} \text { or screen or comput } \text { or laptop }^{\star} \text { or media) ) }\end{array}$ \\
\hline S61 & (MH "Video Games") \\
\hline S60 & (MH "Life Style, Sedentary") \\
\hline
\end{tabular}


(Continued)
S59
$\mathrm{S} 53$ or $\mathrm{S} 54$ or S55 or S56 or S57 or S58

\section{S58}

( (fitness or leisure) N2 (class ${ }^{\star}$ or regime ${ }^{\star}$ or program ${ }^{\star}$ or centre ${ }^{\star}$ or center $\left.^{\star}\right)$ ) OR ( (fit ${ }^{\star}$ or sport $^{\star}$ or activ $^{\star}$ or exercise or physical exer $\left.{ }^{\star}\right)$ N3 (lack* or low or no or absen $\left.{ }^{\star}\right)$ )

\section{S57}

"Physical activity" or fitness or "physical " fit" or "physical exert" or exercise or "aerobic activit" or sport* or "aerobic capacity" or "active lifestyle" or "outdoor activit" or gym" or mvpa

(MH "Sports") OR (MH "Aquatic Sports+") OR (MH "Athletic Training+") OR (MH "Caving") OR (MH "Contact Sports+") OR (MH "Cycling") OR (MH "Endurance Sports") OR (MH "Fencing") OR (MH "Golf") OR (MH "Gymnastics") OR (MH "Handball") OR (MH "Martial Arts") OR (MH "Mountaineering") OR (MH "Professional Sports") OR (MH "Race Walking") OR (MH "Racquet Sports+") OR (MH "Rock Climbing") OR (MH "Running+") OR (MH "Skating+") OR (MH "Skiing+") OR (MH "Sports Participation") OR (MH "Target Sports+") OR (MH "Team Sports+") OR (MH "Track and Field") OR (MH "Triathlon") OR (MH "Weight Lifting") OR (MH "Winter Sports+") OR (MH "Body Building") OR (MH "College Sports")

\begin{tabular}{|c|c|}
\hline S55 & (MH "Physical Endurance") \\
\hline S54 & (MH "Physical Activity") OR (MH "Physical Fitness") \\
\hline S53 & (MH "Exercise+") \\
\hline S52 & $\mathrm{S} 47$ or $\mathrm{S} 48$ or $\mathrm{S} 49$ or $\mathrm{S} 50$ or S51 \\
\hline S51 & $\begin{array}{l}\text { (poor or over* or unhealthy or health*) N3 (nutrition or diet }{ }^{\star} \text { or eat* or meal* or food }{ }^{\star} \text { or snack }{ }^{\star} \text { or } \\
\text { drink }^{\star} \text { ) }\end{array}$ \\
\hline S50 & 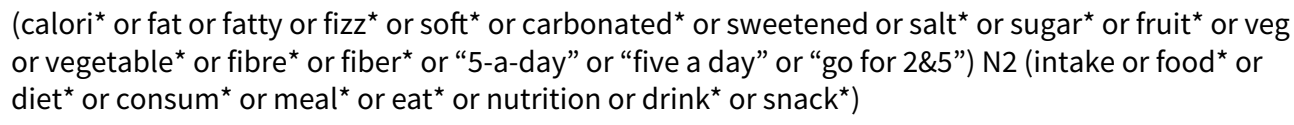 \\
\hline S49 & (MH "Dietary Fats") \\
\hline S48 & (MH "Hyperphagia") \\
\hline S47 & (MH "Diet+") \\
\hline S46 & $\mathrm{S} 41$ or $\mathrm{S} 42$ or $\mathrm{S} 43$ or $\mathrm{S} 44$ or $\mathrm{S} 45$ \\
\hline S45 & $\begin{array}{l}\text { selfinflict* or "self inflict*" or selfinjur* or "self injur" or selfharm* or selfmutilat* or "self destruc- } \\
\text { tive behavio\#r" }\end{array}$ \\
\hline S44 & $\begin{array}{l}\left.\text { ( (injur }{ }^{\star} \text { or mutil } \text { or harm }^{\star} \text { or wound } \text { or hurt }^{\star}\right) \text { N2 (self or themsel* or yoursel }{ }^{\star} \text { ) ) OR TI dsh OR AB } \\
\text { dsh }\end{array}$ \\
\hline S43 & (MH "Self-Injurious Behavior") OR (MH "Injuries, Self-Inflicted") \\
\hline S42 & 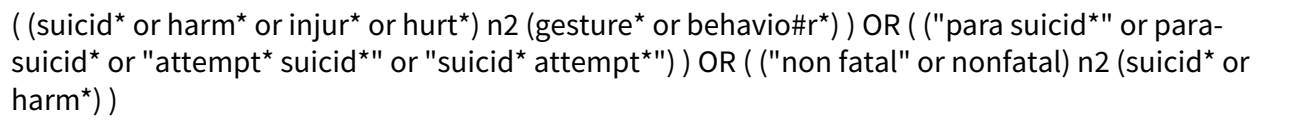 \\
\hline S41 & (MH "Suicide, Attempted") \\
\hline S40 & S35 or S36 or S37 or S38 or S39 \\
\hline
\end{tabular}


(Continued)

S39 (bicyc ${ }^{\star}$ or cycl ${ }^{\star}$ or bik ${ }^{\star}$ or motorbike* or motorcycl ${ }^{\star}$ ) N2 (helmet* or protect* or "risk reduc " or "head gear") N2 (lack or without or absen* or wear* or no or non\#)

\begin{tabular}{|c|c|}
\hline S38 & $\begin{array}{l}\text { ( (use or using or usage or wear" or wore) N2 ("seat belt*" or seatbelt* or "safety belt }{ }^{\star} \text { )) OR injur } \\
\text { N2 behav* OR ( (alcohol* or intoxica* or dr?nk*) N2 (driv* or vehicle* or motor or car\# or van\# or } \\
\text { automobile* or "auto mobile*")) }\end{array}$ \\
\hline S37 & (MH "Car Safety Devices") \\
\hline S36 & (MH "Child Safety+") \\
\hline S35 & (MH "Head Protective Devices") \\
\hline S34 & $\mathrm{S} 27$ or $\mathrm{S} 28$ or $\mathrm{S} 29$ or $\mathrm{S} 30$ or $\mathrm{S} 31$ or $\mathrm{S} 32$ or $\mathrm{S} 33$ \\
\hline S33 & 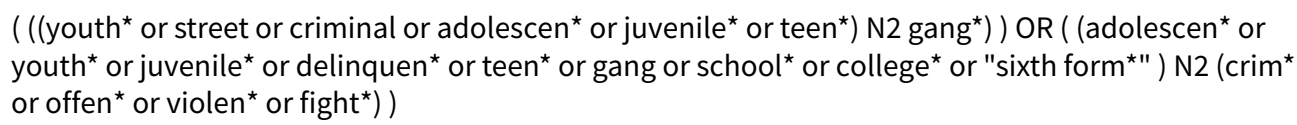 \\
\hline
\end{tabular}

( "pan handling" or panhandling or "disorderly conduct" or prostitut* or (carry* N2 weapon*) or (buy* N2 stolen) or "criminal behavio\#r" or (noisy N1 rude) or (nuisance* N2 neighbo\#r*) ) OR ( fight* or stab or stabbing or stabbed or stabwound* or wound ${ }^{\star}$ or aggress ${ }^{\star}$ or weapon* ) OR ( knife or knives or gun* or firearm* or murder*)

S31 (delinquen* or offen* or reoffend ${ }^{\star}$ or violen* or theft* or robbery or burglar* or steal ${ }^{\star}$ ) OR ( "criminal damage" or joyrid* or "joy rid" or assault* ) OR ( "sell* N1 drug" "or "devian*" or "anti social behavio\# $r^{\star}$ " or " antisocial behavio\#r*" ) OR ( graffiti or "racist abuse" or "index crime*" or (breaking N1 entering) or "strong arming" )

\begin{tabular}{|c|c|}
\hline S30 & (MH "Wounds, Stab") \\
\hline S29 & (MH "Social Behavior Disorders+") OR (MH "Aggression+") OR (MH "Violence+") \\
\hline S28 & (MH "Juvenile Delinquency") \\
\hline S27 & (MH "Juvenile Offenders+") OR (MH "Crime+") \\
\hline S26 & $\mathrm{S} 22$ or S23 or S24 or S25 \\
\hline S25 & $\begin{array}{l}\text { ( "risky sex*" or "unsafe sex*" or "safe* sex*" ) OR ( "sexual intercourse" or "reproductive be- } \\
\text { havio\#r*") OR ("sexual behavio\#r" or "sexual health" ) }\end{array}$ \\
\hline S24 & contracept ${ }^{\star}$ or condom\# or "morning after pill*" \\
\hline S23 & (MH "Contraception+") OR (MH "Contraceptive Agents+") OR (MH "Contraceptive Devices+") \\
\hline S22 & MH safe sex OR MH unsafe sex OR MH sexual abstinence OR MH sexual partners \\
\hline S21 & gambl* or betting \\
\hline S20 & $\mathrm{S} 8$ or $\mathrm{S} 9$ or $\mathrm{S} 10$ or $\mathrm{S} 11$ or $\mathrm{S} 12$ or $\mathrm{S} 13$ or $\mathrm{S} 19$ \\
\hline S19 & inhal?nt* N2 (abus or addict $^{\star}$ or depend ${ }^{\star}$ or misus ${ }^{\star}$ or sniff* or use* or usage or using) \\
\hline S18 & $\mathrm{S} 6$ or $\mathrm{S} 7$ or $\mathrm{S} 16$ or $\mathrm{S} 17$ \\
\hline
\end{tabular}




\section{$\mathrm{S} 17$ \\ ( (alcohol* or drink* or ethanol) ) N3 ( (excess* or binge* or binging or intoxicat* ${ }^{\star}$ or poison ${ }^{\star}$ or risk ${ }^{\star}$ or depend*))}

S16 ( alcohol $^{\star}$ or ethanol or beer or cider or wine or spirit or alcopop $\left.^{\star}\right)$ ) N3 ( (use or usage $^{\star}$ or using $^{*}$ or intake or consum ${ }^{\star}$ or drink or misus $^{\star}$ or abus $\left.{ }^{\star}\right)$ )
or intake or consum * or drink* or misus* or abus ${ }^{\star}$ ) )

\begin{tabular}{|c|c|}
\hline S15 & $\mathrm{S} 4$ or S5 or S14 \\
\hline S14 & ( tobacco or cigarette* or nicotine ) N3 ( addict* or use* or usage or using or intake or consum ${ }^{\star}$ ) \\
\hline S13 & 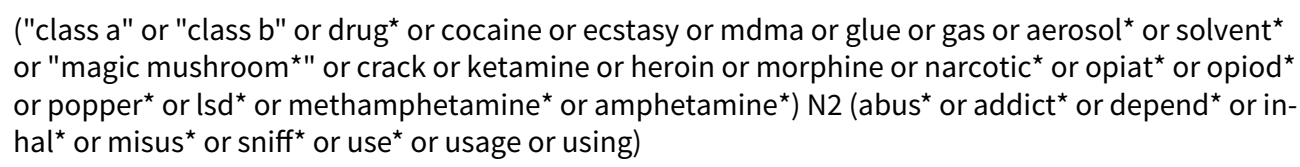 \\
\hline S12 & $\begin{array}{l}\text { substance }{ }^{\star} \text { N2 (abus }{ }^{\star} \text { or addict }{ }^{\star} \text { or depend }{ }^{\star} \text { or inject }{ }^{\star} \text { or intravenous or misus }{ }^{\star} \text { or use }{ }^{\star} \text { or usage or } \\
\text { using) }\end{array}$ \\
\hline S11 & (MH "Substance Abuse, Intravenous") \\
\hline S10 & $\begin{array}{l}\text { ( (marijuana or cannabis or "recreational drug*" or "class c"or "white widow*") ) N2 ( (abus* or use } \\
\text { or using or usage or misus" or smok* or addict* or depend") ) }\end{array}$ \\
\hline S9 & (MH "Substance Use Disorders") \\
\hline S8 & MH cannabis OR MH street drugs \\
\hline S7 & (MH "Alcohol-Related Disorders+") \\
\hline S6 & (MH "Alcohol Drinking") \\
\hline S5 & smoking \\
\hline S4 & (MH "Smoking") \\
\hline S3 & (S1 or $\mathrm{S} 2)$ \\
\hline S2 & (MH "Risk Taking Behavior+") \\
\hline S1 & 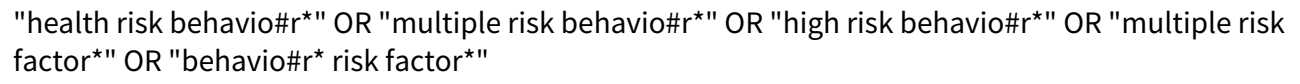 \\
\hline
\end{tabular}

Search strategy for PsycINFO (Ovid), 1806 to 2015 week 17. Searched 06 May 2015 and 10 November 2016.

Previously searched 24 May 2012.

\begin{tabular}{ll}
\hline 1 & $\begin{array}{l}\text { ("Health risk behavio? } \mathrm{r}^{\star} \text { " or "multiple risk behavio? } \mathrm{r}^{\star} \text { " or "high risk behavio? } \mathrm{r}^{\star} \text { " or "multiple risk } \\
\text { factor" or "behavio? } \mathrm{r}^{\star} \text { risk factor*").mp. [mp=title, abstract, heading word, table of contents, key } \\
\text { concepts, original title, tests \& measures] }\end{array}$ \\
\hline 2 & Dangerous Behavior/ \\
\hline 3 & Risk-Taking/
\end{tabular}




\begin{tabular}{|c|c|}
\hline 5 & "Tobacco Use Disorder"/ \\
\hline 6 & Tobacco Smoking/ \\
\hline 7 & $\begin{array}{l}\text { smoking.mp. [mp=title, abstract, heading word, table of contents, key concepts, original title, tests } \\
\text { \& measures] }\end{array}$ \\
\hline 8 & $\begin{array}{l}\text { ((tobacco or cigarette }{ }^{\star} \text { or nicotine) adj3 (addict* or use }{ }^{\star} \text { or usage or using or intake or con- } \\
\left.\left.\text { sum }{ }^{\star}\right)\right) . \mathrm{mp} .[\mathrm{mp}=\text { title, abstract, heading word, table of contents, key concepts, original title, tests \& } \\
\text { measures] }\end{array}$ \\
\hline 9 & 6 or 7 or 8 \\
\hline 10 & Drinking Behavior/ \\
\hline 11 & exp alcohol drinking patterns/ \\
\hline 12 & $\begin{array}{l}\text { ((alcohol* or ethanol or beer or cider or wine or spirit* or alcopop }{ }^{\star} \text { ) adj3 (use* or usage* or using } \\
\left.\left.\text { or intake or consum }{ }^{\star} \text { or drink* or misus }{ }^{\star} \text { or abus }{ }^{\star}\right)\right) . \mathrm{mp} .[\mathrm{mp}=\text { title, abstract, heading word, table of } \\
\text { contents, key concepts, original title, tests \& measures] }\end{array}$ \\
\hline 13 & $\begin{array}{l}\text { ((alcohol }{ }^{\star} \text { or drink }{ }^{\star} \text { or ethanol) adj3 (excess }{ }^{\star} \text { or binge* or binging or intoxicat }{ }^{\star} \text { or poison }{ }^{\star} \text { or risk }^{\star} \\
\left.\left.\text { or depend }{ }^{\star}\right)\right) . \mathrm{mp} .[\mathrm{mp}=\text { title, abstract, heading word, table of contents, key concepts, original title, } \\
\text { tests \& measures] }\end{array}$ \\
\hline 14 & 10 or 11 or 12 or 13 \\
\hline 15 & exp cannabis/ or crack cocaine/ or illegal drug distribution/ \\
\hline 16 & Drug Seeking/ \\
\hline 17 & drug abuse/ or exp drug dependency/ or inhalant abuse/ \\
\hline 18 & 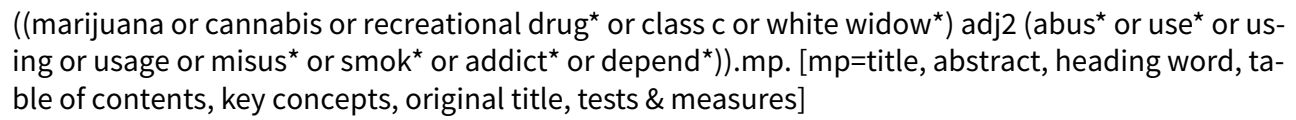 \\
\hline
\end{tabular}

19 intravenous drug usage/

20 (class c adj2 (abus ${ }^{\star}$ or addict ${ }^{\star}$ or depend ${ }^{\star}$ or misus ${ }^{\star}$ or use ${ }^{\star}$ or usage or using)).mp. [mp=title, abstract, heading word, table of contents, key concepts, original title, tests \& measures]

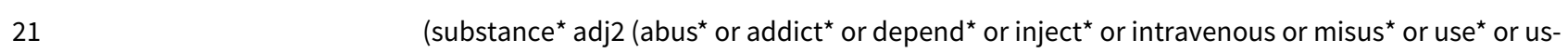
age or using)).mp. [mp=title, abstract, heading word, table of contents, key concepts, original title, tests \& measures]

((Class a or class b or drug* or cocaine or ecstasy or mdma or glue or gas or aerosol* or solvent* or magic mushroom* or crack or white widow* or ketamine or heroin or morphine or narcotic ${ }^{\star}$ or opiat $^{\star}$ or opioid* or popper $^{\star}$ or lsd or methamphetamine or amphetamine $^{\star}$ ) adj2 (abus* or addict* or depend ${ }^{\star}$ or inhal ${ }^{\star}$ or misus ${ }^{\star}$ or sniff* or use ${ }^{\star}$ or using or usage)).mp. [mp=title, abstract, heading word, table of contents, key concepts, original title, tests \& measures] 


\section{4}

25

$$
27
$$

29

\section{0}

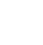

31

15 or 16 or 17 or 18 or 19 or 20 or 21 or 22 or 23

(gambl* or betting).mp. [mp=title, abstract, heading word, table of contents, key concepts, original title, tests \& measures]

Safe sex/ or sexual risk taking/or psychosexual behavior/ or sexual abstinence/ or exp birth control/ or sexual partners/

(risky sex ${ }^{\star}$ or unsafe sex $^{\star}$ or safe* sex $^{\star}$ or sexual intercourse or reproductive behavio? ${ }^{\star}$ or sexual behavio? ${ }^{\star}$ or sexual health).mp. [mp=title, abstract, heading word, table of contents, key concepts, original title, tests \& measures]

(contracept* or condom? or morning after pill ${ }^{\star}$ ).mp. [mp=title, abstract, heading word, table of contents, key concepts, original title, tests \& measures]

26 or 27 or 28

crime/ or antisocial behavior/or criminal behavior/or exp gangs/or hate crimes/or arson/or serial crime/ or exp sex offences/ or exp theft/ or vandalism/ or violent crime/ or exp juvenile delinquency/ or social behaviour disorders/

violence/ or school violence/ or aggressive behavior/ or bullying/

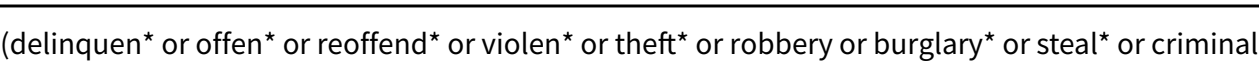
damage or joyrid ${ }^{\star}$ or joy-rid* or assault* or (sell* adj drug*) or devian* or anti-social behavio? $r^{\star}$ or antisocial behavio? $r^{\star}$ or graffiti or racist abuse or index crime* or (breaking adj entering) or strong-arming or pan-handling or panhandling or disorderly conduct or prostitut ${ }^{\star}$ or (carry ${ }^{\star}$ adj2 weapon $^{\star}$ ) or (buy* adj2 stolen) or criminal behavio? $r^{\star}$ or (noisy adj1 rude) or (nuisance* adj2 neighbour $^{\star}$ ) or fight ${ }^{\star}$ or stab? or stabbing or stabbed or stabwound ${ }^{\star}$ or wound ${ }^{\star}$ or aggress ${ }^{\star}$ or weapon* or knife* or knives or gun* or firearm* or murder $\left.{ }^{\star}\right) . m p$. [mp=title, abstract, heading word, table of contents, key concepts, original title, tests \& measures]

\section{3}

\section{4}

35 36

37

\begin{tabular}{ll}
\hline 38 & Safety belts/ \\
\hline 39 & $\begin{array}{l}\left(\left(\text { Use } e^{\star} \text { or using or usage or wear }{ }^{\star} \text { or wore) adj2 (seat-belt* or seatbelt* or safety belt*)).mp. [mp=ti- }\right.\right. \\
\text { tle, abstract, heading word, table of contents, key concepts, original title, tests \& measures] }\end{array}$ \\
\hline 40 & $\begin{array}{l}\left.\text { (injur adj2 behav }{ }^{\star}\right) . \mathrm{mp} .[\mathrm{mp}=\text { title, abstract, heading word, table of contents, key concepts, original } \\
\text { title, tests \& measures] }\end{array}$ \\
\hline
\end{tabular}

((youth^ or street or criminal ${ }^{\star}$ or adolescen ${ }^{\star}$ or juvenile* or teen ${ }^{\star}$ ) adj2 gang?).mp. [mp=title, abstract, heading word, table of contents, key concepts, original title, tests \& measures]

((adolescen* or youth* or juvenile* or delinquen* or teen* or gang? or school* or college* or sixth form $\left.{ }^{\star}\right) \operatorname{adj} 2\left(\mathrm{crim}^{\star}\right.$ or offen ${ }^{\star}$ or violen ${ }^{\star}$ or fight $\left.\left.{ }^{\star}\right)\right)$.mp. [mp=title, abstract, heading word, table of contents, key concepts, original title, tests \& measures]

\section{0 or 31 or 32 or 33 or 34}

accident prevention/

((bicyc or $^{\text {cycl }}{ }^{\star}$ or bik ${ }^{\star}$ or motorbike* or motorcycl $\left.{ }^{\star}\right)$ adj2 ((helmet ${ }^{\star}$ or protect $^{\star}$ or risk reduc or $^{*}$ head gear or head protection) adj3 (lack* or no? or without or absen* or wear $\left.\left.{ }^{\star}\right)\right)$ ).mp. [mp=title, abstract, heading word, table of contents, key concepts, original title, tests \& measures] 
driving under the influence/ or ((alcohol* or intoxica* or dr?nk ${ }^{\star}$ ) adj2 (driv* or vehicle* or motor ${ }^{\star}$ or car? or van? or automobile* or auto mobile*)).mp. [mp=title, abstract, heading word, table of contents, key concepts, original title, tests \& measures]

\begin{tabular}{ll}
\hline 42 & 36 or 37 or 38 or 39 or 40 or 41 \\
\hline $43 \quad$ attempted suicide/
\end{tabular}

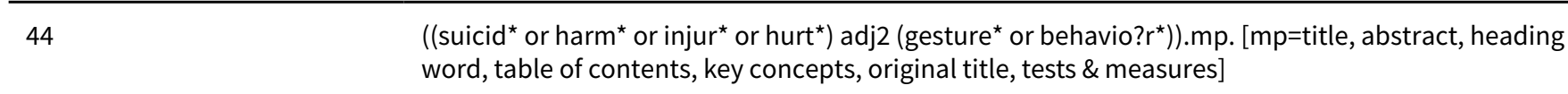

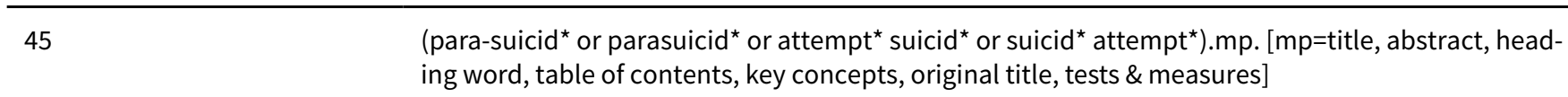
((non fatal or nonfatal) adj2 (suicid ${ }^{\star}$ or harm $\left.\left.{ }^{\star}\right)\right) . \mathrm{mp}$. [mp=title, abstract, heading word, table of con-
tents, key concepts, original title, tests \& measures] abstract, heading word, table of contents, key concepts, original title, tests \& measures]

dsh.tw. or selfinflict ${ }^{\star} . m p$. or self inflict ${ }^{\star} . m p$. or selfinjur ${ }^{\star} . m p$. or selfharm ${ }^{\star} . m p$. or selfmutilat ${ }^{\star} . m p$. or self destructive behavio? $r^{\star} . \mathrm{mp}$. [mp=title, abstract, heading word, table of contents, key concepts, original title, tests \& measures]

\begin{tabular}{|c|c|}
\hline 50 & 43 or 44 or 45 or 46 or 47 or 48 or 49 \\
\hline 51 & hyperphagia/ or binge eating/ \\
\hline 52 & 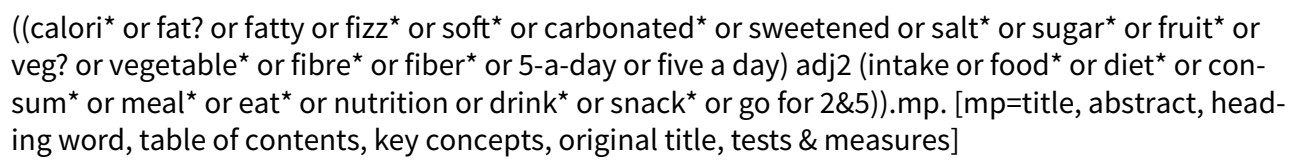 \\
\hline 53 & $\begin{array}{l}\left(\left(\text { poor or over }{ }^{\star} \text { or unhealthy or health }{ }^{\star}\right) \text { adj3 (nutrition or diet }{ }^{\star} \text { or eat }^{\star} \text { or meal }{ }^{\star} \text { or food }^{\star} \text { or snack }\right. \\
\left.\left.\text { or drink }{ }^{\star}\right)\right) . m p . \text { [mp=title, abstract, heading word, table of contents, key concepts, original title, } \\
\text { tests \& measures] }\end{array}$ \\
\hline 54 & 51 or 52 or 53 \\
\hline 55 & exp physical activity/ or physical fitness/ or physical endurance/ \\
\hline 56 & exp sports/ or walking/ \\
\hline 57 & $\begin{array}{l}\text { (Physical activity or fitness or physical } \text { fit }^{\star} \text { or physical exert }{ }^{\star} \text { or exercise or aerobic activit* or } \\
\text { sport }^{\star} \text { or aerobic capacity or active lifestyle } \text { or outdoor activit }^{\star} \text { or gym } \text { or }^{\star} \text { mpa).mp. [mp=title, } \\
\text { abstract, heading word, table of contents, key concepts, original title, tests \& measures] }\end{array}$ \\
\hline 58 & $\begin{array}{l}\left.\left.\text { ((fitness or leisure) adj2 (class* or regime* or program* or centre* or center }{ }^{\star}\right)\right) . \mathrm{mp} .[\mathrm{mp}=t i t l e, \text { ab- } \\
\text { stract, heading word, table of contents, key concepts, original title, tests \& measures] }\end{array}$ \\
\hline 59 & $\begin{array}{l}\left(\left(\text { fit }^{\star} \text { or sport* or activ* or exercise or physical exer*) adj3 (lack* or low or no or absen*)).mp. }\right.\right. \\
\text { [mp=title, abstract, heading word, table of contents, key concepts, original title, tests \& measures] }\end{array}$ \\
\hline 60 & 55 or 56 or 57 or 58 or 59 \\
\hline
\end{tabular}


61 computer games/ or television viewing/

62 gaming.mp.

63

((view* or watch* or play ${ }^{\star}$ or game* or gaming or use* or using or usage) adj2 (television or tv or

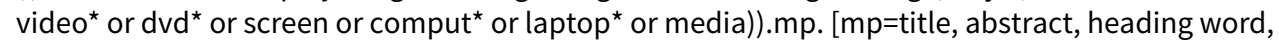
table of contents, key concepts, original title, tests \& measures]

64 ((screen or sedentary or view*) adj2 (time or hour ${ }^{\star}$ or minute*)).mp. [mp=title, abstract, heading word, table of contents, key concepts, original title, tests \& measures]

65

$\left(\left(\right.\right.$ inactiv $^{\star}$ or seden $^{\star}$ or indoor $\left.{ }^{\star}\right)$ adj3 (lifestyle* or activit $\left.\left.{ }^{\star}\right)\right) . \mathrm{mp}$. [mp=title, abstract, heading word, table of contents, key concepts, original title, tests \& measures]

$66 \quad 61$ or 62 or 63 or 64 or 65

67 (4 and 9) or (4 and 14) or ( 4 and 24) or ( 4 and 25) or ( 4 and 29) or ( 4 and 35) or (4 and 42 ) or ( 4 and $50)$ or ( 4 and 54$)$ or ( 4 and 60$)$ or ( 4 and 66$)$ or ( 9 and 14$)$ or (9 and 24$)$ or (9 and 25) or (9 and 29) or (9 and 35$)$ or ( 9 and 42$)$ or ( 9 and 50) or (9 and 54) or (9 and 60) or (9 and 66) or (14 and 24) or (14 and $25)$ or (14 and 29) or (14 and 35) or (14 and 42) or (14 and 50) or (14 and 54) or (14 and 60) or (14 and

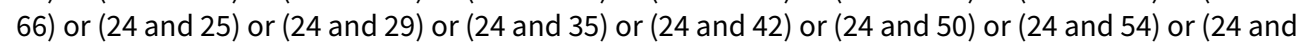
$60)$ or ( 24 and 66$)$ or ( 25 and 29 ) or ( 25 and 35 ) or ( 25 and 42$)$ or ( 25 and 50 ) or ( 25 and 54 ) or ( 25 and $60)$ or ( 25 and 66$)$ or (29 and 35) or (29 and 42) or (29 and 50) or (29 and 54) or (29 and 60) or (29 and $66)$ or ( 35 and 42$)$ or ( 35 and 50 ) or ( 35 and 54 ) or ( 35 and 60$)$ or ( 35 and 66$)$ or ( 42 and 50 ) or (42 and $54)$ or ( 42 and 60 ) or (42 and 66) or (50 and 54) or (50 and 60) or (50 and 66)

68 69 70 child/ or adolescent/ or child, preschool/ or infant/

$\left(\right.$ school $^{\star}$ or student ${ }^{\star}$ or child $^{\star}$ or pupil ${ }^{\star}$ or infant $\left.^{\star}\right)$. tw.

(Adolescen* or teen* or young person or young people or youth* or hooligan* or young adult* or early adult ${ }^{\star}$ or juvenile* or minor? or emerging adult* or girl? or boy? or apprentice* or FE college or young $\mathrm{m \# n}$ or young wom\#n or young male* or young female* or under $18^{\star}$ or sixth-form ${ }^{\star}$ or secondary education or tertiary education or higher education or further education or preschool ${ }^{\star}$ or primary education or infan* or kid? or nurser ${ }^{\star}$ or playschool $^{\star}$ or kindergarten ${ }^{\star}$ or prekindergarten*).mp.

(teacher* or parent ${ }^{\star}$ or guardian* or grandparent $^{\star}$ or mother* or father* or mum? or dad? or maternal or paternal or nurse? or childminder ${ }^{\star}$ or child care provider ${ }^{\star}$ or playworker ${ }^{\star}$ or family or families or carer ${ }^{\star}$ or midwife or mid wife or midwives or mid wives).mp. [mp=title, abstract, heading word, table of contents, key concepts, original title, tests \& measures]

\begin{tabular}{ll}
\hline 72 & 68 or 69 or 70 or 71 \\
\hline 73 & (randomized controlled trial or controlled clinical trial).pt. \\
\hline 74 & (randomi\#ed or placebo* or randomly or controlled clinical trial).ab,ti. \\
\hline 75 & trial.ti. \\
\hline 76 & 73 or 74 or 75 \\
\hline 77 & 67 and 72 and 76 \\
\hline
\end{tabular}

\section{Search strategy for Education Resources Information Centre - ERIC (1966 to current), Australian Education Index (1979 to current), and British Education Index (1975 to current) (PROQUEST). Searched 05 May 2015 and 14 November 2016.}


Previously searched 31 May 2012.

\begin{tabular}{|c|c|}
\hline S11 & gambl $^{*}$ or betting \\
\hline S12 & 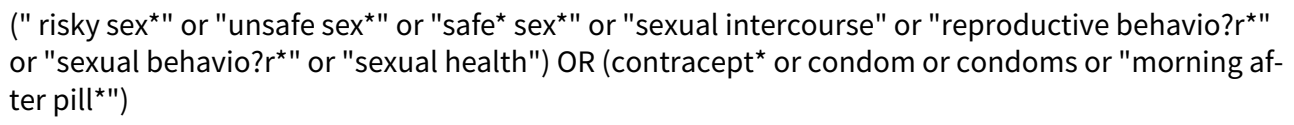 \\
\hline S13 & 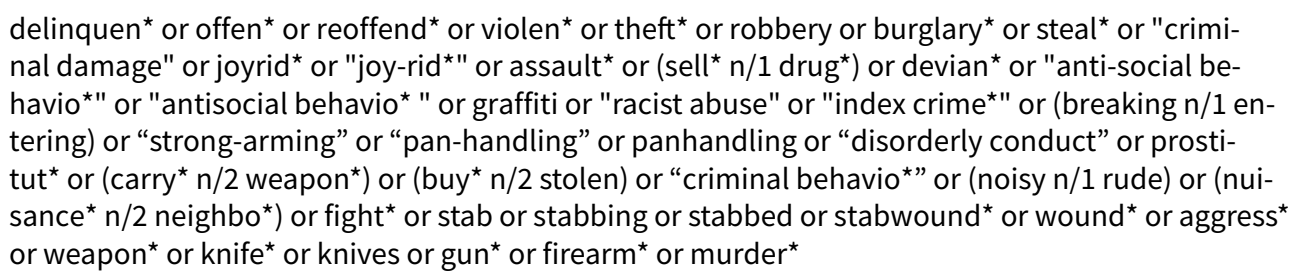 \\
\hline
\end{tabular}

\begin{tabular}{|c|c|}
\hline S14 & 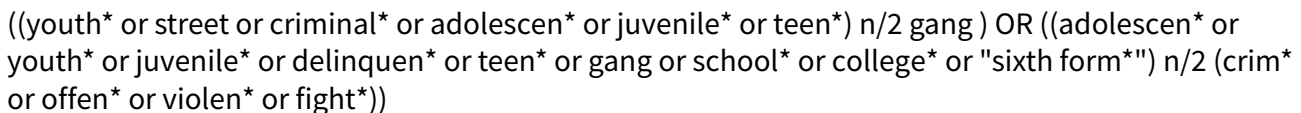 \\
\hline
\end{tabular}

\begin{tabular}{|c|c|}
\hline S15 & s13 or s14 \\
\hline S16 & 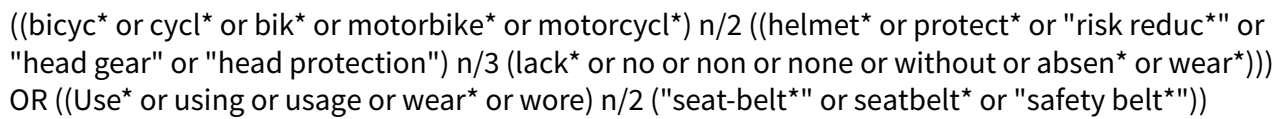 \\
\hline
\end{tabular}

(injur* n/2 behav*) OR ((alcohol* or intoxica* or drink* or drunk* $)$ n/2 (driv* or vehicle* or motor ${ }^{\star}$ or car or van or automobile* or "auto mobile*"))

\begin{tabular}{|c|c|}
\hline S18 & $\mathrm{s} 16$ or $\mathrm{s} 17$ \\
\hline S19 & 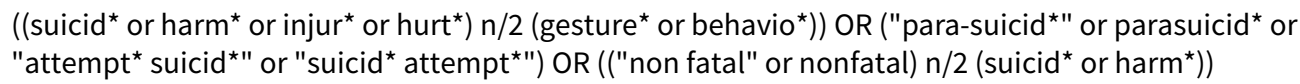 \\
\hline
\end{tabular}

S20

((injur ${ }^{\star}$ or mutil $^{\star}$ or harm ${ }^{\star}$ or wound ${ }^{*}$ or hurt $\left.^{\star}\right) \mathrm{n} / 2$ (self or themsel ${ }^{\star}$ or yoursel $\left.\left.{ }^{\star}\right)\right)$ OR (dsh or selfinflict* or "self inflict*" or selfinjur* or selfharm* or selfmutilat* or "self destructive behavio* ")

\begin{tabular}{|c|c|}
\hline S21 & s19 or s20 \\
\hline S23 & 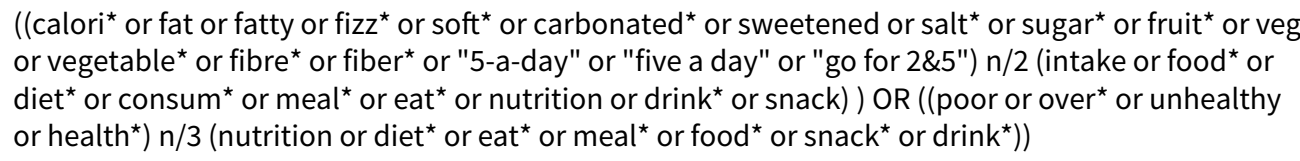 \\
\hline S24 & $\begin{array}{l}\text { (Badminton or baseball or basketball or bicycling or boxing or cycling or dancing or football or gar- } \\
\text { dening or golf or gymnastics or hockey or "martial arts" or mountaineering or rugby or running or } \\
\text { skating or skiing or snowsports or soccer or swimming or volleyball or walking or "weight lifting" } \\
\text { or wrestling) OR ("Physical activity" or fitness or "physical* fit*" or "physical exert " or exercise or } \\
\text { "aerobic activit" or sport* or "aerobic capacity" or "active lifestyle*" or "outdoor activit" or gym* } \\
\text { or mvpa) }\end{array}$ \\
\hline
\end{tabular}
activ $^{\star}$ or exercise or "physical exer ${ }^{\star}$ ) $n / 3$ (lack* or low or no or non or none or absen $\left.{ }^{\star}\right)$ ) 
S27 gaming OR ((view* or watch* or play ${ }^{\star}$ or game* or use ${ }^{\star}$ or using or usage) n/2 (television or tv or video* $^{\star}$ or dvd* or screen or comput* or laptop* or media))

S28

((screen or sedentary or view $\left.{ }^{\star}\right) \mathrm{n} / 2$ (time or hour ${ }^{\star}$ or minute $\left.\left.{ }^{\star}\right)\right)$ OR ((inactiv* or seden $^{\star}$ or indoor $\left.{ }^{\star}\right)$ $\mathrm{n} / 3$ (lifestyle* or activit*))

\begin{tabular}{ll}
\hline S29 & S27 or S28 \\
\hline S30 & $\begin{array}{l}\text { (S11 and S12) or (S11 and S15) or (S11 and S18) or (S11 and S21) or (S11 and S23) or (S11 and S26) } \\
\text { or (S11 and S29) }\end{array}$ \\
\hline S32 & (S12 and S15) or (S12 and S18) or (S12 and S21) or (S12 and S23) or (S12 and S26) or (S12 and S29) \\
\hline S33 & (S15 and S18) or (S15 and S21) or (S15 and S23) or (S15 and S26) or (S15 and S29) \\
\hline S34 & (S18 and S21) or (S18 and S23) or (S18 and S26) or (S18 and S29) \\
\hline S36 & (S21 and S23) or (S21 and S26) or (S21 and S29) \\
\hline S37 & (randomi ${ }^{\star}$ ed or randomly or placebo*) OR "clinical controlled trial" OR ti(trial) OR su(trial $\left.{ }^{*}\right)$ \\
\hline S38 & s30 and s36 \\
\hline S39 & s32 and s36 \\
\hline S40 & s33 and s36 \\
\hline S41 & S34 and s36 and s36 \\
\hline S42
\end{tabular}

((all("Health risk behavio*" OR "multiple risk behavio*" OR "high risk behavio*" OR "multiple risk factor " OR "behavio* risk factor*") AND (all((tobacco OR cigarette* OR nicotine) NEAR/3 (addict* OR use* OR usage OR using OR intake OR consum*)) OR all(smoking))) OR (all("Health risk behavio*" OR "multiple risk behavio*" OR "high risk behavio*" OR "multiple risk factor*" OR "behavio* risk factor $\left.{ }^{\star} "\right)$ AND (all((alcohol* OR ethanol OR beer OR cider OR wine OR spirit* OR alcopop*) NEAR/3 (use* OR usage* OR using OR intake OR consum* OR drink* OR misus* OR abus*)) OR all((alcohol* OR drink* OR ethanol) NEAR/3 (excess* OR binge* OR binging OR intoxicat* OR poison $^{\star}$ OR risk* OR depend*)))) OR (all("Health risk behavio*" OR "multiple risk behavio*" OR "high risk behavio*" OR "multiple risk factor*" OR "behavio* risk factor" ${ }^{\star}$ ) AND (all(substance* NEAR/2 (abus* OR addict* OR depend ${ }^{\star}$ OR misus* OR use* OR usage OR using)) OR ((inject* OR intravenous) AND (drug* OR substance*)) OR all(("class c" OR marijuana OR cannabis OR "recreational drug*" OR "white widow") NEAR/2 (abus* OR addict* OR depend* OR misus* OR smok* OR use* OR usage* OR using)) OR (all((inhalent ${ }^{\star}$ OR inhalant ${ }^{\star}$ ) NEAR/2 (abus* OR addict OR depend ${ }^{\star}$ OR misus ${ }^{\star}$ OR sniff* OR use ${ }^{\star}$ OR using OR usage)) OR all(("Class a" OR "class b" OR drug* OR cocaine OR ecstasy OR mdma OR glue OR gas OR aerosol* OR solvent* OR "magic mushroom*" OR crack OR ketamine OR heroin OR morphine OR narcotic* OR opiat* OR opioid* OR popper* OR Isd OR methamphetamine $e^{\star}$ OR amphetamine ${ }^{\star}$ ) NEAR/2 (abus ${ }^{\star}$ OR addict* OR depend ${ }^{\star}$ OR inhal ${ }^{\star}$ OR misus* OR sniff* OR use* OR using OR usage))))) OR (all("Health risk behavio*" OR "multiple risk behavio*" OR "high risk behavio*" OR "multiple risk factor*" OR "behavio* risk factor*") AND all(gambl* OR betting)) OR (alI("Health risk behavio*" OR "multiple risk behavio*" OR "high risk behavio*" OR "multiple risk factor*" OR "behavio* risk factor") AND (all("risky sex*" OR "unsafe sex" OR "safe* sex" OR "sexual intercourse" OR "reproductive behavio*" OR "sexual behavio*" OR "sexual health") OR all(contracepti* OR condom OR condoms OR "morning after pill*") OR all("sexually transmitted disease*" OR "sexually transmitted infection*" OR STD* OR STI OR STIs OR chlamydia* OR gonorrhea*OR gonorrhoea* OR HIV OR AIDS OR "human immunodeficiency virus" OR "acquired immunodeficiency syndrome"))) OR (all("Health risk behavio*" OR "multiple risk behavio*" OR "high risk behavio*" OR "multiple risk factor*" OR "behavio* risk factor*") AND (all(delinquenc* OR delinquent* OR of- 
fen ${ }^{\star}$ OR reoffend ${ }^{\star}$ OR violen* OR theft ${ }^{\star}$ OR robbery OR burglary ${ }^{\star}$ OR steal ${ }^{\star}$ OR "criminal damage" OR joyrid* OR "joy rid*" OR assault* OR (sell* NEAR/1 drug*) OR devian* OR "anti social behavio*" OR "antisocial behavio*" OR graffiti OR "racist abuse" OR "index crime*" OR (breaking NEAR/1 entering) OR "strong-arming" OR "pan-handling" OR panhandling OR "disorderly conduct" OR prostitut* OR (carry* NEAR/2 weapon*) OR (buy* N2 stolen) OR "criminal behavio*" OR (noisy NEAR/1 rude) OR (nuisance* NEAR/2 neighbo*) OR fight* OR stab? OR stabbing OR stabbed OR stabwound* OR wound ${ }^{\star}$ OR aggress ${ }^{\star}$ OR weapon* ${ }^{\star}$ OR knife ${ }^{\star}$ OR knives OR gun ${ }^{\star}$ OR firearm ${ }^{\star}$ OR murder $^{\star}$ ) OR (alI((youth* OR street OR criminal* OR adolescen* OR juvenile* OR teen $\left.{ }^{\star}\right)$ NEAR/2 (gang OR gangs)) OR all((adolescen* OR youth* OR juvenile* OR delinquen* OR teen* OR gang OR gangs) NEAR/2 (crim* OR offen* OR violen* OR fight*))))) OR (all("Health risk behavio*" OR "multiple risk behavio*" OR "high risk behavio*" OR "multiple risk factor*" OR "behavio* risk factor*") AND ((all(bicyc* OR cy$\mathrm{Cl}^{*}$ OR bik* OR motorbike* OR motorcycl*) AND all((helmet* OR protect* OR "risk reduc ${ }^{\star} "$ OR "head gear" OR "head protection") NEAR/3 (lack* OR no OR no? OR without OR absen* OR wear $\left.\left.{ }^{\star}\right)\right)$ ) OR (alI((Use ${ }^{\star}$ OR using OR usage OR wear ${ }^{\star}$ OR wore) NEAR/2 ("seat belt ${ }^{\star}$ " OR seatbelt* OR "safety belt*")) OR all(injur* NEAR/2 behavio*) OR all((alcohol* OR intoxica* OR drink* OR drunk*) NEAR/2 (driv* OR vehicle* OR motOR* OR car OR cars OR van OR vans OR automobile* OR "auto mobile*"))))) OR (all("Health risk behavio*" OR "multiple risk behavio*" OR "high risk behavio*" OR "multiple risk factor*" OR "behavio* risk factor*") AND ((all((suicid* OR harm* OR injur* OR hurt*) NEAR/2 (gesture* OR behavio $\left.{ }^{\star}\right)$ ) OR all("para suicid" " OR parasuicid* OR "attempt" suicid " OR "suicid* attempt*”) OR all(("non fatal" OR nonfatal) NEAR/2 (suicid* OR harm*))) OR (all((injur* OR mutil* OR harm* OR wound ${ }^{\star}$ OR hurt ${ }^{\star}$ ) NEAR/2 (self OR themsel ${ }^{\star}$ OR yoursel $\left.{ }^{\star}\right)$ ) OR all(dsh OR selfinflict ${ }^{\star}$ OR "self inflict*" OR selfinjur* OR selfharm* OR selfmutilat* OR "self destructive behavio*")))) OR (all("Health risk behavio*" OR "multiple risk behavio*" OR "high risk behavio*" OR "multiple risk factor" " OR "behavio* risk factor*") AND (all((calori ${ }^{\star}$ OR fat OR fats OR fatty OR fizz ${ }^{\star}$ OR soft* OR carbonated $^{\star}$ OR sweetened OR salt* OR sugar ${ }^{\star}$ OR fruit* OR veg OR vegs OR vegetable* OR fibre* OR fiber* OR "5-a-day" OR "five a day" OR "go for 2 5") NEAR/2 (intake OR food* OR diet* OR consum $^{\star}$ OR meal ${ }^{\star}$ OR eat* OR nutrition OR drink* OR snack $\left.{ }^{\star}\right)$ ) OR all((poor OR over OR unhealthy OR health*) NEAR/3 (nutrition OR diet* OR eat* OR meal ${ }^{\star}$ OR food ${ }^{\star}$ OR snack* OR drink $\left.\left.{ }^{\star}\right)\right)$ )) OR (alI("Health risk behavio*" OR "multiple risk behavio*" OR "high risk behavio*" OR "multiple risk factor*" OR "behavio* risk factor*") AND (all(Baseball OR basketball OR boxing OR cycling OR bicycling OR dancing OR football OR gardening OR golf OR gymnastics OR hockey OR "martial arts" OR mountaineering OR tennis OR badminton OR squash OR running OR jogging OR skating OR skiing OR soccer OR swimming OR volleyball OR walking OR "weight lifting" OR wrestling) OR all(("Physical activity" OR fitness OR "physical* fit*" OR "physical exert*" OR "exercise" OR "aerobic activit*" OR sport* OR "aerobic capacity" OR "active lifestyle*" OR "outdoor activit*" OR gym OR mvpa OR (fitness OR leisure) N/2 (class ${ }^{\star}$ OR regime* OR program* OR centre ${ }^{\star}$ OR center $\left.{ }^{\star}\right)$ )) OR all((fit* OR sport $^{\star}$ OR activ* OR exercise OR "physical exer*") NEAR/3 (lack* OR low OR no OR absen*)))) OR (alI("Health risk behavio*" OR "multiple risk behavio*" OR "high risk behavio*" OR "multiple risk factor " OR "behavio* risk factor $\left.{ }^{\star} "\right)$ AND (all((view* OR watch* OR play ${ }^{\star}$ OR game* OR use* OR using OR usage) NEAR/2 (television OR tv OR video* OR dvd* OR screen OR comput* OR laptop OR media)) OR all((gaming OR (screen OR sedentary OR view*) NEAR/2 (time OR hour* OR minute*))) OR all((inactiv* OR seden* OR indoor $\left.{ }^{\star}\right)$ NEAR/3 (lifestyle* OR activit*))))) AND (("randomi ${ }^{\star}$ ed controlled trial" OR "controlled clinical trial*" OR randomly OR placebo* OR randomi*ed) OR ti(trial))

Search strategy for IBSS (ProQuest, 1950 to current). Searched 07 May 2015 and 14 November 2016.

Previously searched 04 June 2012.

$$
\begin{aligned}
& \text { S1 ("randomi*ed controlled trial" or "controlled clinical trial*" or randomly or placebo* or rando- } \\
& \mathrm{mi}^{\star} \text { ed) OR ti(trial) }
\end{aligned}
$$

S2 all("Health risk behavio*" OR "multiple risk behavio*" OR "high risk behavio*" OR "multiple risk factor*" OR "behavio* risk factor*") 
(Continued)

S5

all((alcohol* OR ethanol OR beer OR cider OR wine OR spirit* OR alcopop*) NEAR/3 (use* OR usage $\left.{ }^{\star}\right)$ )OR all(substance* NEAR/2 (abus ${ }^{\star}$ OR addict ${ }^{\star}$ OR depend ${ }^{\star}$ OR misus ${ }^{\star}$ OR use ${ }^{\star}$ OR usage OR using)) (("randomi*ed controlled trial" or "controlled clinical trial*" or randomly or placebo* or randomi*ed) OR ti(trial)) AND all("Health risk behavio*" OR "multiple risk behavio*" OR "high risk behavio*" OR "multiple risk factor*" OR "behavio* risk factor*") (("randomi*ed controlled trial" or "controlled clinical trial ${ }^{\star}$ " or randomly or placebo* or randomi $^{\star}$ ed) OR ti(trial)) AND (all((tobacco OR cigarette* OR nicotine) NEAR/3 (addict* OR use* OR usage OR using OR intake OR consum*)) OR all(smoking)) mi $^{\star}$ ed) OR ti(trial)) AND (all((alcohol ${ }^{\star}$ OR ethanol OR beer OR cider OR wine OR spirit ${ }^{\star}$ OR alcopop ${ }^{\star}$ ) NEAR/3 (use* OR usage ${ }^{\star}$ )) OR all(substance ${ }^{\star}$ NEAR/2 (abus* OR addict* OR depend* OR misus ${ }^{\star}$ OR use $^{\star}$ OR usage OR using)))

S12 all(substance NEAR/2 (abus* OR addict* OR depend ${ }^{\star}$ OR misus ${ }^{\star}$ OR use ${ }^{\star}$ OR usage OR using))

S13 (inject* OR intravenous) AND (drug ${ }^{\star}$ OR substance $^{\star}$ )
OR addict* OR depend* OR misus* OR smok* OR use* OR usage* OR using))

all((inhalent ${ }^{\star}$ or inhalant $\left.{ }^{\star}\right)$ NEAR/2 (abus* or addict or depend ${ }^{\star}$ or misus $^{\star}$ or sniff ${ }^{\star}$ or use ${ }^{\star}$ or using or usage)) OR all(("Class a" OR "class b" OR drug* OR cocaine OR ecstasy OR mdma OR glue OR gas OR aerosol* OR solvent* OR "magic mushroom*" OR crack OR ketamine OR heroin OR morphine OR narcotic ${ }^{\star}$ OR opiat* OR opioid ${ }^{\star}$ OR popper* OR Isd OR methamphetamine* OR amphetamine ${ }^{\star}$ ) NEAR/2 (abus ${ }^{\star}$ OR addict* OR depend* OR inhal ${ }^{\star}$ OR misus* OR sniff* OR use* OR using OR usage))

all(substance ${ }^{\star}$ NEAR/2 (abus ${ }^{\star}$ OR addict* OR depend $^{\star}$ OR misus* OR use ${ }^{\star}$ OR usage OR using)) OR ((inject* OR intravenous) AND (drug* OR substance $\left.{ }^{\star}\right)$ ) OR all(("class C" OR marijuana OR cannabis or "recreational drug*" or "white widow") NEAR/2 (abus* OR addict* OR depend* OR misus* OR smok* OR use $^{\star}$ OR usage* OR using)) OR (all((inhalent* or inhalant $\left.{ }^{\star}\right)$ NEAR/2 (abus ${ }^{\star}$ or addict or depend $^{\star}$ or misus* or sniff* or use* or using or usage)) OR all(("Class a" OR "class b" OR drug* OR cocaine OR ecstasy OR mdma OR glue OR gas OR aerosol* OR solvent* OR "magic mushroom*" OR crack OR ketamine OR heroin OR morphine OR narcotic* OR opiat* OR opioid* OR popper* OR Isd OR methamphetamine ${ }^{\star}$ OR amphetamine ${ }^{\star}$ ) NEAR/2 (abus ${ }^{\star}$ OR $_{\text {addict }}^{\star}$ OR depend $^{\star}$ OR inhal ${ }^{\star}$ OR misus $^{\star}$ OR sniff* OR use* OR using OR usage)))

(all(substance* NEAR/2 (abus* OR addict* OR depend* OR misus* OR use* OR usage OR using)) OR ((inject* OR intravenous) AND (drug* OR substance*)) OR all(("class c" OR marijuana OR cannabis or "recreational drug*" or "white widow*") NEAR/2 (abus* OR addict* OR depend* OR misus* OR smok $^{\star}$ OR use ${ }^{\star}$ OR usage OR using)) OR (all((inhalent ${ }^{\star}$ or inhalant $^{\star}$ ) NEAR/2 (abus ${ }^{\star}$ or addict or depend $^{\star}$ or misus ${ }^{\star}$ or sniff* or use ${ }^{\star}$ or using or usage)) OR all(("Class a" OR "class b" OR drug OR cocaine OR ecstasy OR mdma OR glue OR gas OR aerosol* OR solvent* OR "magic mushroom*" OR crack OR ketamine OR heroin OR morphine OR narcotic* OR opiat* OR opioid* OR popper* OR Isd OR methamphetamine* OR amphetamine*) NEAR/2 (abus* OR addict* OR depend* OR inhal* OR misus ${ }^{\star}$ OR sniff* OR use* OR using OR usage)))) AND (("randomi*ed controlled trial" or "controlled clinical trial*" or randomly or placebo* or randomi*ed) OR ti(trial))

\begin{tabular}{ll}
\hline S18 & gambl* or betting \\
\hline S19 & $\begin{array}{l}\text { (gambl* or betting) AND (("randomi*ed controlled trial" or "controlled clinical trial }^{\star *} \text { or randomly } \\
\text { or placebo* or randomi*ed) OR ti(trial)) }\end{array}$ \\
\hline S20 & $\begin{array}{l}\text { all("risky sex*" OR "unsafe sex*" OR "safe* sex" OR "sexual intercourse" OR "reproductive be- } \\
\text { havio*" OR "sexual behavio*" OR "sexual health") OR all(contracepti* OR condom OR condoms OR } \\
\text { "morning after pill*") OR all("sexually transmitted disease*" OR "sexually transmitted infection" }\end{array}$ \\
\hline
\end{tabular}


OR STD* OR STI OR STIS OR chlamydia* OR gonorrhea*OR gonorrhoea* OR HIV OR AIDS OR "human immunodeficiency virus" OR "acquired immunodeficiency syndrome") havio*" OR "sexual behavio*" OR "sexual health") OR all(contraception* OR condom OR condoms OR "morning after pill*") OR all("sexually transmitted disease*" OR "sexually transmitted infection*" OR STD* OR STI OR stirs OR chlamydia* OR gonorrhea*OR gonorrhoea* OR HIV OR AIDS OR "human immunodeficiency virus" OR "acquired immunodeficiency syndrome")) AND (("random*ed controlled trial" OR "controlled clinical trial*" OR randomly OR placebo* OR random*ed) OR ti(trial))

all(Adolescen* or teen* or "young person" or "young people" or youth* or hooligan* or "young adult" or "early adult" or juvenile* or minor or "emerging adult" or girl or boy or apprentice* or "FE college $e^{\star}$ " or "young $\mathrm{m}^{\star} \mathrm{n}$ " or "young wom*" or "young male*"or "young female" or "under $18^{\star}$ " or "sixth-form" or "secondary education" or "tertiary education" or "higher education" or "further education" or preschool* or "primary education" or infan* or kid or nurser* or playschool* or kindergarten ${ }^{\star}$ or prekindergarten ${ }^{\star}$ ) infant $^{\star}$ ) OR su(school ${ }^{\star}$ or student ${ }^{\star}$ or child $^{\star}$ or pupil* or infant ${ }^{\star}$ ) or paternal or nurse or childminder* or "child care provider" or carer* or midwife or "mid wife" or midwives or "mid wives" adult*" OR "early adult" OR juvenile* OR minor OR "emerging adult"” OR girl OR boy OR appren-

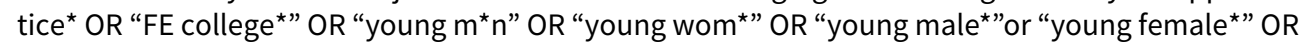
"under 18 " OR "sixth-form" OR "secondary education" OR "tertiary education" OR "higher education" OR "further education" OR preschool* OR "primary education" OR infan* OR kid OR nurser $^{\star}$ OR playschool* OR kindergarten* OR prekindergarten $\left.{ }^{\star}\right)$ OR (ab (school ${ }^{\star}$ OR student ${ }^{\star}$ OR child* OR pupil ${ }^{\star}$ OR infant ${ }^{\star}$ ) OR ti(school* OR student ${ }^{\star}$ OR child ${ }^{\star}$ OR pupil ${ }^{\star}$ OR infant ${ }^{\star}$ ) OR su(school ${ }^{\star}$ OR student ${ }^{\star}$ OR child ${ }^{\star}$ OR pupil ${ }^{\star}$ OR infant $\left.{ }^{\star}\right)$ ) OR (teacher ${ }^{\star}$ or parent ${ }^{\star}$ or guardian* or grandparent $^{\star}$ or mother ${ }^{\star}$ or father ${ }^{\star}$ or mum or dad or maternal or paternal or nurse or childminder* or "child care provider" or playworker" or family or families or carer* or midwife or "mid wife" or midwives or "mid wives") mi*ed) OR ti(trial)) AND all("Health risk behavio*" OR "multiple risk behavio*" OR "high risk behavio*" OR "multiple risk factor*" OR "behavio* risk factor*")) AND (all(Adolescen* OR teen* OR "young person" OR "young people" OR youth" OR hooligan* OR "young adult*" OR "early adult*" OR juvenile* OR minor OR "emerging adult"” OR girl OR boy OR apprentice* OR "FE college " OR "young $\mathrm{m}^{\star} \mathrm{n}$ " OR "young wom" OR “young male*”or "young female*” OR "under $18^{\star}$ ” OR "sixthform" ${ }^{\star}$ OR "secondary education" OR "tertiary education" OR "higher education" OR "further education" OR preschool* OR "primary education" OR infan* OR kid OR nurser* OR playschool* OR kindergarten ${ }^{\star}$ OR prekindergarten $\left.{ }^{\star}\right)$ OR (ab(school* OR student ${ }^{\star}$ OR child* OR pupil* OR infant ${ }^{\star}$ ) OR ti(school ${ }^{\star}$ OR student ${ }^{\star}$ OR child* OR pupil ${ }^{\star}$ OR infant ${ }^{\star}$ ) OR su(school ${ }^{\star}$ OR student ${ }^{\star}$ OR child ${ }^{\star}$ OR pupil $^{\star}$ OR infant ${ }^{\star}$ )) OR (teacher ${ }^{\star}$ or parent ${ }^{\star}$ or guardian ${ }^{\star}$ or grandparent ${ }^{\star}$ or mother ${ }^{\star}$ or father $^{\star}$ or mum or dad or maternal or paternal or nurse or childminder* or "child care provider" " or playworker* or family or families or carer* or midwife or "mid wife" or midwives or "mid wives"))

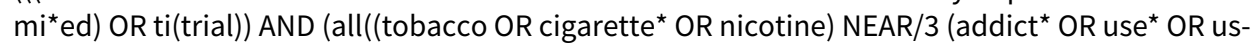
age OR using OR intake OR consum*)) OR all(smoking))) AND (all(Adolescen* OR teen* OR "young person" OR "young people" OR youth* OR hooligan* OR "young adult”” OR "early adult*” OR juvenile* OR minor OR "emerging adult*" OR girl OR boy OR apprentice* OR "FE college*" OR "young $\mathrm{m}^{\star} \mathrm{n}$ " OR "young wom" OR "young male*”or "young female " OR "under $18^{\star}$ ” OR "sixth-form" OR "secondary education" OR "tertiary education" OR "higher education" OR "further education" OR preschool* OR "primary education” OR infan* OR kid OR nurser* OR playschool* OR kindergarten* OR prekindergarten ${ }^{\star}$ ) OR (ab(school* OR student ${ }^{\star}$ OR child* OR pupil* OR infant ${ }^{\star}$ ) OR ti(school ${ }^{\star}$ 
OR student ${ }^{\star}$ OR child ${ }^{\star}$ OR pupil* OR infant* ${ }^{\star}$ OR su(school* OR student $^{\star}$ OR child* OR pupil $^{\star}$ OR infant $\left.^{\star}\right)$ ) OR (teacher ${ }^{\star}$ or parent ${ }^{\star}$ or guardian ${ }^{\star}$ or grandparent ${ }^{\star}$ or mother ${ }^{\star}$ or father ${ }^{\star}$ or mum or dad or maternal or paternal or nurse or childminder* or "child care provider" ${ }^{\star}$ or playworker ${ }^{\star}$ or family or families or carer* or midwife or "mid wife" or midwives or "mid wives"))

((("randomi*ed controlled trial" or "controlled clinical trial " or randomly or placebo* or randomi*ed) OR ti(trial)) AND (all((alcohol* OR ethanol OR beer OR cider OR wine OR spirit* OR alcopop*) NEAR/3 (use* OR usage*))OR all(substance* NEAR/2 (abus* OR addict* OR depend* OR misus* OR use* OR usage OR using)))) AND (all(Adolescen* OR teen* OR "young person" OR "young people" OR youth* OR hooligan ${ }^{\star}$ OR "young adult" OR "early adult" OR juvenile* OR minor OR "emerg-

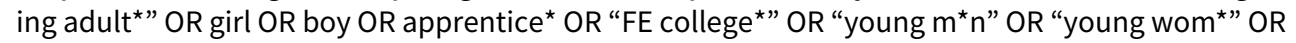
"young male*"or "young female*" OR "under $18^{\star}$ " OR "sixth-form*" OR "secondary education" OR "tertiary education" OR "higher education" OR "further education" OR preschool* OR "primary education” OR infan* OR kid OR nurser ${ }^{\star}$ OR playschool* OR kindergarten* OR prekindergarten*) OR (ab(school* OR student ${ }^{\star}$ OR child* OR pupil* OR infant ${ }^{\star}$ ) OR ti(school* OR student ${ }^{\star}$ OR child* OR pupil* OR infant $^{\star}$ ) OR su(school* OR student ${ }^{\star}$ OR child* OR pupil* OR infant $\left.{ }^{\star}\right)$ ) OR (teacher ${ }^{\star}$ or parent $^{\star}$ or guardian ${ }^{\star}$ or grandparent ${ }^{\star}$ or mother ${ }^{\star}$ or father $^{\star}$ or mum or dad or maternal or paternal or nurse or childminder* or "child care provider" or playworker* or family or families or carer ${ }^{\star}$ or midwife or "mid wife" or midwives or "mid wives"))

((all(substance* NEAR/2 (abus* OR addict* OR depend ${ }^{\star}$ OR misus ${ }^{\star}$ OR use* OR usage OR using)) OR ((inject* OR intravenous) AND (drug* OR substance*)) OR all(("class C" OR marijuana OR cannabis or "recreational drug*" or "white widow") NEAR/2 (abus* OR addict* OR depend* OR misus* OR smok $^{\star}$ OR use* OR usage* OR using)) OR (all((inhalent ${ }^{\star}$ or inhalant ${ }^{\star}$ ) NEAR/2 (abus ${ }^{\star}$ or addict or depend $^{\star}$ or misus* or sniff* or use* or using or usage)) OR all(("Class a" OR "class b" OR drug ${ }^{\star}$ OR cocaine OR ecstasy OR mdma OR glue OR gas OR aerosol* OR solvent ${ }^{\star}$ OR "magic mushroom*" OR crack OR ketamine OR heroin OR morphine OR narcotic ${ }^{\star}$ OR opiat* OR opioid* OR popper* OR Isd OR methamphetamine ${ }^{\star}$ OR amphetamine ${ }^{\star}$ ) NEAR/2 (abus ${ }^{\star}$ OR addict ${ }^{\star}$ OR depend ${ }^{\star}$ OR inhal ${ }^{*}$ OR misus* OR sniff* OR use* OR using OR usage)))) AND (("randomi*ed controlled trial" or "controlled clinical trial*" or randomly or placebo* or randomi ${ }^{\star}$ ed) OR ti(trial))) AND (all(Adolescen* OR teen* OR "young person" OR "young people" OR youth* OR hooligan* OR "young adult*" OR "early adult"” OR juvenile* OR minor OR "emerging adult"” OR girl OR boy OR apprentice* OR "FE college*” OR "young $m^{\star} n$ " OR "young wom*” OR "young male*”or "young female*" OR "under $18^{\star}$ " OR "sixth-form*” OR "secondary education" OR "tertiary education" OR "higher education" OR "further education" OR preschool* OR "primary education" OR infan* OR kid OR nurser* OR playschool* OR kindergarten* OR prekindergarten ${ }^{\star}$ ) OR (ab(school* OR student* OR child* OR pupil* OR infant $^{\star}$ ) OR ti(school* OR student ${ }^{\star}$ OR child* OR pupil* OR infant ${ }^{\star}$ ) OR su(school ${ }^{\star}$ OR student $^{\star}$ OR child ${ }^{\star}$ OR pupil OR infant $^{\star}$ )) OR (teacher ${ }^{\star}$ or parent ${ }^{\star}$ or guardian* or grandparent ${ }^{\star}$ or mother* or father* or mum or dad or maternal or paternal or nurse or childminder" or "child care provider" or playworker* or family or families or carer* or midwife or "mid wife" or midwives or "mid wives")) ly or placebo* or randomi*ed) OR ti(trial))) AND (all(Adolescen* OR teen* OR "young person" OR "young people" OR youth* OR hooligan* OR "young adult*" OR "early adult" " OR juvenile* OR minor OR "emerging adult"” OR girl OR boy OR apprentice* OR "FE college " OR "young m*n" OR "young wom" "OR "young male*"or "young female*" OR "under $18^{\star}$ " OR "sixth-form" " OR "secondary education" OR "tertiary education" OR "higher education" OR "further education" OR preschool* OR "primary education” OR infan* OR kid OR nurser* OR playschool* OR kindergarten* OR prekindergarten*) OR (ab(school* OR student* OR child* OR pupil* OR infant*) OR ti(school* OR student ${ }^{\star}$ OR child* OR pupil* OR infant $\left.{ }^{\star}\right)$ OR su(school* OR student ${ }^{\star}$ OR child* OR pupil* OR infant $\left.{ }^{\star}\right)$ ) OR (teacher ${ }^{\star}$ or parent ${ }^{\star}$ or guardian ${ }^{\star}$ or grandparent ${ }^{\star}$ or mother or father $^{\star}$ or mum or dad or maternal or paternal or nurse or childminder* or "child care provider" " or playworker* or family or families or carer* or midwife or "mid wife" or midwives or "mid wives")) havio*" OR "sexual behavio*" OR "sexual health") OR all(contraception* OR condom OR condoms OR "morning after pill*") OR all("sexually transmitted disease*" OR "sexually transmitted infection " OR STD* OR STI OR stirs OR chlamydia* OR gonorrhea*OR gonorrhoea* OR HIV OR AIDS OR "human immunodeficiency virus" OR "acquired immunodeficiency syndrome")) AND (("random*ed 
controlled trial" OR "controlled clinical trial*" OR randomly OR placebo* OR random*ed) OR ti(trial))) AND (all(Adolescen* OR teen* OR "young person" OR "young people" OR youth* OR hooligan* OR "young adult*" OR "early adult*” OR juvenile* OR minor OR "emerging adult*” OR girl OR boy OR apprentice ${ }^{\star}$ OR " $F E$ college " OR "young $m^{\star} n$ " OR "young wom" OR "young male*”or "young female*" OR "under $18^{\star}$ " OR "sixth-form" OR "secondary education" OR "tertiary education" OR "higher education" OR "further education" OR preschool* OR "primary education" OR infan* OR kid OR nurser* OR playschool* OR kindergarten* OR prekindergarten*) OR (ab(school* OR student $^{\star}$ OR child* OR pupil ${ }^{\star}$ OR infant ${ }^{\star}$ ) OR ti(school* OR student ${ }^{\star}$ OR child* OR pupil* OR infant ${ }^{\star}$ ) OR su(school* OR student ${ }^{\star}$ OR child ${ }^{\star}$ OR pupil ${ }^{\star}$ OR infant $\left.{ }^{\star}\right)$ ) OR (teacher ${ }^{\star}$ or parent $^{\star}$ or guardian* or grandparent ${ }^{\star}$ or mother ${ }^{\star}$ or father ${ }^{\star}$ or mum or dad or maternal or paternal or nurse or childminder $^{\star}$ or "child care provider" or playworker* or family or families or carer* or midwife or "mid wife" or midwives or "mid wives"))

all(delinquenc* OR delinquent ${ }^{\star}$ OR offen* OR reoffend ${ }^{\star}$ OR violen* OR theft* OR robbery OR burglary ${ }^{\star}$ OR steal ${ }^{\star}$ OR "criminal damage" OR joyrid* OR "joy rid" " OR assault* OR (sell* NEAR/1 drug*) OR devian* OR "anti social behavio*" OR "antisocial behavio*" OR graffiti OR "racist abuse" OR "index crime*" OR (breaking NEAR/1 entering) OR "strong-arming" OR "pan-handling" OR panhandling OR "disorderly conduct" OR prostitut ${ }^{\star}$ OR (carry* NEAR/2 weapon*) OR (buy ${ }^{\star}$ N2 stolen) OR "criminal behavio*" OR (noisy NEAR/1 rude) OR (nuisance* NEAR/2 neighbo*) OR fight* OR stab? OR stabbing OR stabbed OR stabwound* OR wound* OR aggress* OR weapon* OR knife* OR knives OR gun* OR firearm* OR murder ${ }^{\star}$ ) OR all((adolescen* OR youth* OR juvenile* OR delinquen* OR teen* OR gang OR gangs) NEAR/2 $\left(\right.$ crim $^{\star}$ OR offen ${ }^{\star}$ OR violen ${ }^{\star}$ OR fight $\left.\left.{ }^{\star}\right)\right)$ glary ${ }^{\star}$ OR steal ${ }^{\star}$ OR "criminal damage" OR joyrid* OR "joy rid" " OR assault* OR (sell* NEAR/1 drug*) OR devian* OR "anti social behavio*" OR "antisocial behavio*" OR graffiti OR "racist abuse" OR "index crime*" OR (breaking NEAR/1 entering) OR "strong-arming" OR "pan-handling" OR panhandling OR "disorderly conduct" OR prostitut* OR (carry* NEAR/2 weapon*) OR (buy* N2 stolen) OR "criminal behavio*" OR (noisy NEAR/1 rude) OR (nuisance* NEAR/2 neighbo*) OR fight* OR stab? OR stabbing OR stabbed OR stabwound ${ }^{\star}$ OR wound ${ }^{\star}$ OR aggress* OR weapon* OR knife* OR knives OR gun* OR firearm* OR murder ${ }^{\star}$ ) OR (all((youth* OR street OR criminal* OR adolescen* OR juvenile* OR teen $\left.{ }^{\star}\right)$ NEAR/2 (gang OR gangs)) OR all((adolescen* OR youth* OR juvenile* OR delinquen* OR teen* OR gang OR gangs) NEAR/2 (crim* OR offen* OR violen* OR fight*)))) AND (("randomi*ed controlled trial" or "controlled clinical trial*" or randomly or placebo* or randomi ${ }^{\star}$ ed) OR ti(trial))

((all(delinquenc ${ }^{\star}$ OR delinquent ${ }^{\star}$ OR offen* OR reoffend ${ }^{\star}$ OR violen ${ }^{\star} O R$ theft ${ }^{\star}$ OR robbery OR burglary* OR steal* OR "criminal damage" OR joyrid* OR "joy rid*" OR assault* OR (sell* NEAR/1 drug*) OR devian* OR "anti social behavio*" OR "antisocial behavio*" OR graffiti OR "racist abuse" OR "index crime*" OR (breaking NEAR/1 entering) OR "strong-arming" OR "pan-handling" OR panhandling OR "disorderly conduct" OR prostitut ${ }^{\star}$ OR (carry ${ }^{\star}$ NEAR/2 weapon*) OR (buy* N2 stolen) OR "criminal behavio*" OR (noisy NEAR/1 rude) OR (nuisance* NEAR/2 neighbo*) OR fight* OR stab? OR stabbing OR stabbed OR stabwound ${ }^{\star}$ OR wound ${ }^{\star}$ OR aggress ${ }^{\star}$ OR weapon* OR knife* OR knives OR gun ${ }^{\star}$ OR firearm* OR murder ${ }^{\star}$ ) OR (all((youth* OR street OR criminal* OR adolescen* OR juvenile ${ }^{\star}$ OR teen $\left.{ }^{\star}\right)$ NEAR/2 (gang OR gangs)) OR all((adolescen* OR youth* OR juvenile* OR delinquen* OR teen* OR gang OR gangs) NEAR/2 (crim* OR offen* OR violen* OR fight*)))) AND (("randomi*ed controlled trial" or "controlled clinical trial ${ }^{\star}$ " or randomly or placebo* or randomi*ed) OR ti(trial))) AND (all(Adolescen* OR teen* OR “young person” OR "young people” OR youth* OR hooligan* OR "young adult*" OR "early adult*" OR juvenile* OR minor OR "emerging adult*" OR girl OR boy OR apprentice* OR "FE college*” OR "young $\mathrm{m}^{\star} \mathrm{n}$ " OR "young wom" female " OR "under $18^{\star}$ ” OR "sixth-form" OR "secondary education" OR "tertiary education" OR "higher education" OR "further education" OR preschool* OR "primary education" OR infan* OR kid OR nurser* OR playschool* OR kindergarten* OR prekindergarten*) OR (ab(school* OR student $^{\star}$ OR child* OR pupil ${ }^{\star}$ OR infant ${ }^{\star}$ ) OR ti(school* OR student ${ }^{\star}$ OR child* OR pupil ${ }^{\star}$ OR infant ${ }^{\star}$ ) OR su(school* OR student ${ }^{\star}$ OR child* OR pupil* OR infant $\left.{ }^{\star}\right)$ ) OR (teacher ${ }^{\star}$ or parent ${ }^{\star}$ or guardian ${ }^{\star}$ or grandparent ${ }^{\star}$ or mother ${ }^{\star}$ or father ${ }^{\star}$ or mum or dad or maternal or paternal or nurse or childminder $^{\star}$ or "child care provider" or playworker* or family or families or carer* or midwife or "mid wife" or midwives or "mid wives")) 
all(bicyc ${ }^{\star}$ OR cycl ${ }^{\star}$ OR bik* OR motorbike* OR motorcycl*) AND all((helmet* OR protect ${ }^{\star}$ OR "risk reduc " OR "head gear" OR "head protection") NEAR/3 (lack* OR no OR no? OR without OR absen* OR wear $\left.\left.^{\star}\right)\right)$ belt*")) OR all(injur* NEAR/2 behavio*) OR all((alcohol* OR intoxica* OR drink* OR drunk*) NEAR/2 (driv* OR vehicle* OR motOR* OR car OR cars OR van OR vans OR automobile* OR "auto mobile*"))

((all(bicyc ${ }^{\star}$ OR cycl ${ }^{\star}$ OR bik ${ }^{\star}$ OR motorbike ${ }^{\star}$ OR motorcycl $\left.{ }^{\star}\right)$ AND all((helmet ${ }^{\star}$ OR protect ${ }^{\star}$ OR "risk reduc " OR "head gear" OR "head protection") NEAR/3 (lack* OR no OR no? OR without OR absen* OR wear $\left.\left.{ }^{\star}\right)\right)$ ) OR (all((Use* OR using OR usage OR wear* OR wore) NEAR/2 ("seat belt*" OR seatbelt* OR "safety belt*")) OR all(injur* NEAR/2 behavio*) OR all((alcohol ${ }^{\star}$ OR intoxica* OR drink* OR drunk $^{\star}$ ) NEAR/2 (driv* OR vehicle* OR motOR* OR car OR cars OR van OR vans OR automobile* OR "auto mobile*")))) AND (("randomi*ed controlled trial" or "controlled clinical trial*" or randomly or placebo* or randomi*ed) OR ti(trial))

(((all(bicyc ${ }^{\star}$ OR cycl ${ }^{\star}$ OR bik* OR motorbike* OR motorcycl $\left.{ }^{\star}\right)$ AND all((helmet ${ }^{\star}$ OR protect $^{\star}$ OR "risk reduc*" OR "head gear" OR "head protection") NEAR/3 (lack* OR no OR no? OR without OR absen $^{\star}$ OR wear $\left.\left.{ }^{\star}\right)\right)$ ) OR (all((Use* OR using OR usage OR wear ${ }^{\star}$ OR wore) NEAR/2 ("seat belt ${ }^{\star}$ " OR seatbelt* OR "safety belt*")) OR all(injur* NEAR/2 behavio*) OR all((alcohol* OR intoxica* OR drink* OR drunk $^{\star}$ ) NEAR/2 (driv* OR vehicle* OR motOR* OR car OR cars OR van OR vans OR automobile* OR "auto mobile*")))) AND (("randomi*ed controlled trial" or "controlled clinical trial*" or randomly or placebo* or randomi*ed) OR ti(trial))) AND (all(Adolescen* OR teen* OR "young person" OR "young

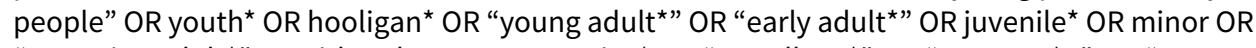
"emerging adult"” OR girl OR boy OR apprentice ${ }^{\star}$ OR "FE college " OR "young m*n" OR "young wom" "OR "young male "or "young female " OR "under $18^{\star}$ ” OR "sixth-form"” OR "secondary education" OR "tertiary education" OR "higher education" OR "further education" OR preschool* OR "primary education" OR infan* OR kid OR nurser* OR playschool* OR kindergarten* OR prekindergarten ${ }^{\star}$ ) OR (ab (school ${ }^{\star}$ OR student ${ }^{\star}$ OR child* OR pupil ${ }^{\star}$ OR infant ${ }^{\star}$ ) OR ti(school ${ }^{\star}$ OR student ${ }^{\star}$ OR child ${ }^{\star}$ OR pupil ${ }^{\star}$ OR infant ${ }^{\star}$ ) OR su(school ${ }^{\star}$ OR student ${ }^{\star}$ OR child ${ }^{\star}$ OR pupil ${ }^{\star}$ OR infant $\left.{ }^{\star}\right)$ ) OR (teacher ${ }^{\star}$ or parent ${ }^{\star}$ or guardian ${ }^{\star}$ or grandparent ${ }^{\star}$ or mother ${ }^{\star}$ or father ${ }^{\star}$ or mum or dad or maternal or paternal or nurse or childminder or "child care provider" or playworker" or family or families or carer* or midwife or "mid wife" or midwives or "mid wives")) parasuicid* OR "attempt* suicid" " OR "suicid* attempt*") OR all(("non fatal" OR nonfatal) NEAR/2 (suicid* OR harm*)) I(dsh OR selfinflict* OR "self inflict*" OR selfinjur* OR selfharm* OR selfmutilat* OR "self destructive behavio*") OR parasuicid* OR "attempt* suicid*” OR "suicid” attempt"”) OR all(("non fatal" OR nonfatal) NEAR/2 (suicid* OR harm*))) OR (all((injur ${ }^{\star}$ OR mutil* OR harm* OR wound* OR hurt*) NEAR/2 (self OR themsel* OR yoursel $\left.{ }^{\star}\right)$ ) OR all(dsh OR selfinflict* OR "self inflict*" OR selfinjur ${ }^{\star}$ OR selfharm* OR selfmutilat* OR "self destructive behavio*"))) AND (("randomi*ed controlled trial" or "controlled clinical trial ${ }^{\star}$ or randomly or placebo* or randomi*ed) OR ti(trial))

(((all((suicid* OR harm* OR injur* OR hurt*) NEAR/2 (gesture* or behavio*)) OR all("para suicid*" OR parasuicid" OR "attempt* suicid"” OR "suicid* attempt"”) OR all(("non fatal" OR nonfatal) NEAR/2 (suicid* OR harm*))) OR (all((injur ${ }^{\star}$ OR mutil* OR harm* OR wound* OR hurt*) NEAR/2 (self OR themsel ${ }^{\star}$ OR yoursel*)) OR all(dsh OR selfinflict* OR "self inflict*" OR selfinjur ${ }^{\star}$ OR selfharm* OR selfmutilat* OR "self destructive behavio*"))) AND (("randomi*ed controlled trial" or "controlled clinical trial*" or randomly or placebo* or randomi*ed) OR ti(trial))) AND (all(Adolescen* OR teen* OR “young person" OR "young people" OR youth* OR hooligan* OR "young adult" OR "early adult ${ }^{\star}$ ” OR juvenile* OR minor OR "emerging adult" " OR girl OR boy OR apprentice* OR "FE college*" OR "young $\mathrm{m}^{\star} \mathrm{n}$ " OR "young wom*" OR "young male*”or "young female*” OR "under $18^{\star}$ " OR "sixth-form " OR "secondary education" OR "tertiary education" OR "higher educa- 
tion" OR "further education" OR preschool* OR "primary education" OR infan* OR kid OR nurser* OR playschool* OR kindergarten* OR prekindergarten*) OR (ab(school* OR student* OR child* OR pupil $^{\star}$ OR infant ${ }^{\star}$ ) OR ti(school* OR student* OR child* OR pupil* OR infant ${ }^{\star}$ ) OR su(school* OR student $^{\star}$ OR child ${ }^{\star}$ OR pupil* OR infant $\left.{ }^{\star}\right)$ ) OR (teacher ${ }^{\star}$ or parent ${ }^{\star}$ or guardian* or grandparent $^{\star}$ or mother ${ }^{\star}$ or father ${ }^{\star}$ or mum or dad or maternal or paternal or nurse or childminder ${ }^{\star}$ or "child care provider" or playworker* or family or families or carer" or midwife or "mid wife" or midwives or "mid wives")) I(dsh OR selfinflict* OR "self inflict" "OR selfinjur ${ }^{\star}$ OR selfharm* OR selfmutilat* OR "self destructive behavio*") $a^{\star}$ OR fruit* OR veg OR vegs OR vegetable* OR fibre* OR fiber" OR "5-a-day" OR "five a day" or "go for $25^{\prime}$ ) NEAR/2 (intake OR food ${ }^{\star}$ OR diet* OR consum* OR meal* OR eat* OR nutrition OR drink* OR snack $\left.{ }^{\star}\right)$ ) OR all((poor OR over ${ }^{\star}$ OR unhealthy OR health*) NEAR/3 (nutrition OR diet* OR eat* OR meal $^{\star}$ OR food ${ }^{\star}$ OR snack ${ }^{\star}$ OR drink $\left.\left.{ }^{\star}\right)\right)$

(all((calori* OR fat OR fats OR fatty OR fizz ${ }^{\star}$ OR soft* OR carbonated ${ }^{\star}$ OR sweetened OR salt* OR sugar $^{\star}$ OR fruit* OR veg OR vegs OR vegetable* OR fibre* OR fiber ${ }^{\star}$ OR "5-a-day" OR "five a day" or "go for 25 ") NEAR/2 (intake OR food ${ }^{\star}$ OR diet* OR consum* OR meal* OR eat* OR nutrition OR drink* OR snack $\left.^{\star}\right)$ ) OR all((poor OR over* OR unhealthy OR health*) NEAR/3 (nutrition OR diet* OR eat ${ }^{\star}$ OR meal* OR food* OR snack* OR drink*))) AND (all(Adolescen* OR teen* OR "young person" OR "young people" OR youth* OR hooligan* OR "young adult*” OR "early adult”” OR juvenile* OR minor OR "emerging adult" " OR girl OR boy OR apprentice ${ }^{\star}$ OR "FE college ${ }^{\star}$ ” OR "young $m^{\star} n$ " OR "young wom*" OR "young male*”or "young female*” OR "under $18^{\star}$ " OR "sixth-form*” OR "secondary education" OR "tertiary education" OR "higher education" OR "further education" OR preschool* OR "primary education" OR infan* OR kid OR nurser* OR playschool* OR kindergarten* OR prekindergarten ${ }^{\star}$ ) OR (ab(school* OR student* OR child* OR pupil ${ }^{\star}$ OR infant $\left.{ }^{\star}\right)$ OR ti(school* OR student ${ }^{\star}$ OR child* OR pupil* OR infant ${ }^{\star}$ ) OR su(school* OR student ${ }^{\star}$ OR child* OR pupil* OR infant $\left.{ }^{\star}\right)$ ) OR (teacher ${ }^{\star}$ or parent ${ }^{\star}$ or guardian ${ }^{\star}$ or grandparent ${ }^{\star}$ or mother or father $^{\star}$ or mum or dad or maternal or paternal or nurse or childminder ${ }^{\star}$ or "child care provider" or playworker* or family or families or carer* or midwife or "mid wife" or midwives or "mid wives")) AND (("randomi*ed controlled trial" or "controlled clinical trial ${ }^{\star}$ " or randomly or placebo* or randomi*ed) OR ti(trial))

all(Baseball OR basketball OR boxing OR cycling OR bicycling OR dancing OR football OR gardening OR golf OR gymnastics OR hockey OR "martial arts" OR mountaineering OR tennis OR badminton OR squash OR running OR jogging OR skating OR skiing OR soccer OR swimming OR volleyball OR walking OR "weight lifting" OR wrestling) OR all(("Physical activity" OR fitness OR "physical $^{\star}$ fit $^{\star}$ " OR "physical exert*" OR "exercise" OR "aerobic activit ${ }^{\star} "$ OR sport* OR "aerobic capacity" OR "active lifestyle*" OR "outdoor activit*" OR gym* OR mvpa OR (fitness OR leisure) NEAR/2 (class* OR regime* OR program* OR centre* OR center $\left.\left.{ }^{\star}\right)\right)$ ) OR all((fit* OR sport* OR activ* OR exercise OR "physical exer*") NEAR/3 (lack* OR low OR no OR absen*)) 
mum or dad or maternal or paternal or nurse or childminder* or "child care provider" or playworker* or family or families or carer* or midwife or "mid wife" or midwives or "mid wives")) AND (("ran-

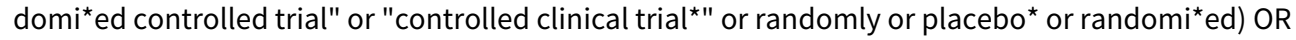
ti(trial)) video* OR dvd* OR screen OR comput* OR laptop or media)) OR all((gaming OR (screen OR sedentary OR view* ${ }^{\star}$ ) NEAR/2 (time OR hour ${ }^{\star}$ OR minute $\left.{ }^{\star}\right)$ )) OR all((inactiv* OR seden* ${ }^{\star}$ OR indoor $\left.{ }^{\star}\right)$ NEAR/3 (lifestyle* OR activit $\left.^{\star}\right)$ )

(all((view* OR watch ${ }^{\star}$ OR play ${ }^{\star}$ OR game* OR use ${ }^{\star}$ OR using OR usage) NEAR/2 (television OR tv OR video* OR dvd* OR screen OR comput* OR laptop or media)) OR all((gaming OR (screen OR sedentary OR view*) NEAR/2 (time OR hour* OR minute*))) OR all((inactiv* OR seden* OR indoor $\left.{ }^{\star}\right)$ NEAR/3 (lifestyle* OR activit*))) AND (all(Adolescen* OR teen* OR "young person" OR "young people" OR youth* OR hooligan OR "young adult"” OR "early adult" OR juvenile* OR minor OR "emerging adult"” OR girl OR boy OR apprentice* OR "FE college*” OR "young $m^{\star} n$ " OR "young wom" " OR "young male*"or "young female*" OR "under $18^{\star}$ " OR "sixth-form"” OR "secondary education" OR "tertiary education" OR "higher education" OR "further education" OR preschool* OR "primary education" OR infan* OR kid OR nurser* OR playschool* OR kindergarten* OR prekindergarten*) OR (ab (school* OR student ${ }^{\star}$ OR child* OR pupil ${ }^{\star}$ OR infant ${ }^{\star}$ ) OR ti(school* OR student ${ }^{\star}$ OR child ${ }^{\star}$ OR pupil $^{\star}$ OR infant ${ }^{\star}$ ) OR su(school ${ }^{\star}$ OR student ${ }^{\star}$ OR child ${ }^{\star}$ OR pupil ${ }^{\star}$ OR infant $\left.{ }^{\star}\right)$ ) OR (teacher ${ }^{\star}$ or parent $^{\star}$ or guardian* or grandparent ${ }^{\star}$ or mother ${ }^{\star}$ or father* or mum or dad or maternal or paternal or nurse or childminder" or "child care provider" or playworker" or family or families or carer" or midwife or "mid wife" or midwives or "mid wives")) AND (("randomi*ed controlled trial" or "controlled clinical trial*" or randomly or placebo* or randomi*ed) OR ti(trial))

(all(Baseball OR basketball OR boxing OR cycling OR bicycling OR dancing OR football OR gardening OR golf OR gymnastics OR hockey OR "martial arts" OR mountaineering OR tennis OR badminton OR squash OR running OR jogging OR skating OR skiing OR soccer OR swimming OR volleyball OR walking OR "weight lifting" OR wrestling) OR all(("Physical activity" OR fitness OR "physical $^{\star}$ fit*" OR "physical exert*" OR "exercise" OR "aerobic activit*" OR sport* OR "aerobic capacity" OR "active lifestyle*" OR "outdoor activit" OR gym* OR mvpa OR (fitness OR leisure) NEAR/2 (class* OR regime* OR program ${ }^{\star}$ OR centre* OR center $\left.\left.{ }^{\star}\right)\right)$ ) OR all((fit* OR sport* OR activ* OR exercise OR "physical exer*") NEAR/3 (lack* OR low OR no OR absen*))) AND (all(Adolescen* OR teen* OR "young person" OR "young people" OR youth* OR hooligan* OR "young adult*" OR "early adult*" OR juvenile* OR minor OR "emerging adult*” OR girl OR boy OR apprentice* OR "FE college " OR "young $\mathrm{m}^{\star} \mathrm{n}$ " OR "young wom" form" " OR "secondary education" OR "tertiary education" OR "higher education" OR "further education" OR preschool* OR "primary education" OR infan* OR kid OR nurser* OR playschool* OR kindergarten ${ }^{\star}$ OR prekindergarten $\left.{ }^{\star}\right)$ OR (ab(school* OR student ${ }^{\star}$ OR child* OR pupil ${ }^{\star}$ OR infant ${ }^{\star}$ ) OR ti(school* OR student* OR child* OR pupil* OR infant*) OR su(school* OR student* OR child* OR pupil $^{\star}$ OR infant $\left.{ }^{\star}\right)$ ) OR (teacher ${ }^{\star}$ or parent ${ }^{\star}$ or guardian* or grandparent $^{\star}$ or mother ${ }^{\star}$ or father ${ }^{\star}$ or mum or dad or maternal or paternal or nurse or childminder* or "child care provider" or playworker* or family or families or carer* or midwife or "mid wife" or midwives or "mid wives")) AND (("randomi*ed controlled trial" or "controlled clinical trial ${ }^{\star} "$ or randomly or placebo* or randomi*ed) OR ti(trial)) 
((("randomi ${ }^{\star}$ ed controlled trial" or "controlled clinical trial ${ }^{\star} "$ or randomly or placebo* or randomi ${ }^{\star}$ ed) OR ti(trial)) AND (all((tobacco OR cigarette* OR nicotine) NEAR/3 (addict* OR use* OR usage OR using OR intake OR consum*)) OR all(smoking))) AND (all(Adolescen* OR teen* OR "young person" OR "young people" OR youth* OR hooligan* OR "young adult*” OR "early adult" " OR juvenile* OR minor OR "emerging adult" OR girl OR boy OR apprentice* OR "FE college*" OR "young $\mathrm{m}^{\star} \mathrm{n}$ " OR "young wom " OR "young male*”or "young female " OR "under $18^{\star}$ " OR "sixth-form" OR "secondary education" OR "tertiary education" OR "higher education" OR "further education" OR preschool* OR "primary education" OR infan* OR kid OR nurser* OR playschool* OR kindergarten* OR prekindergarten ${ }^{\star}$ ) OR (ab(school* OR student* OR child* OR pupil* OR infant ${ }^{\star}$ ) OR ti (school* OR student ${ }^{\star}$ OR child* OR pupil* OR infant ${ }^{\star}$ ) OR su(school* OR student ${ }^{\star}$ OR child* OR pupil* OR infant $\left.{ }^{\star}\right)$ ) OR (teacher ${ }^{\star}$ or parent ${ }^{\star}$ or guardian ${ }^{\star}$ or grandparent ${ }^{\star}$ or mother or father $^{\star}$ or mum or dad or maternal or paternal or nurse or childminder or "child care provider" " or playworker" or family or families or carer* or midwife or "mid wife" or midwives or "mid wives")) ((("randomi*ed controlled trial" or "controlled clinical trial*" or randomly or placebo* or randomi*ed) OR ti(trial)) AND (all((alcohol* OR ethanol OR beer OR cider OR wine OR spirit* OR alcopop*) NEAR/3 (use* OR usage*))OR all(substance* NEAR/2 (abus* OR addict* OR depend ${ }^{\star}$ OR misus* OR use* OR usage OR using)))) AND (all(Adolescen* OR teen* OR "young person" OR "young people" OR youth* OR hooligan* OR "young adult*" OR "early adult*" OR juvenile* OR minor OR "emerg-

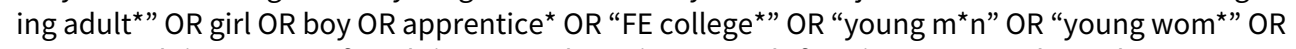
"young male*"or "young female*" OR "under $18^{\star}$ " OR "sixth-form*" OR "secondary education" OR "tertiary education" OR "higher education" OR "further education" OR preschool* OR "primary education" OR infan* OR kid OR nurser* OR playschool* OR kindergarten* OR prekindergarten*) OR (ab(school* OR student ${ }^{\star}$ OR child* OR pupil* OR infant ${ }^{\star}$ ) OR ti(school* OR student ${ }^{\star}$ OR child* OR pupil $^{\star}$ OR infant ${ }^{\star}$ ) OR su(school* OR student ${ }^{\star}$ OR child* OR pupil* OR infant $\left.{ }^{\star}\right)$ ) OR (teacher ${ }^{\star}$ or parent $^{\star}$ or guardian ${ }^{\star}$ or grandparent ${ }^{\star}$ or mother ${ }^{\star}$ or father ${ }^{\star}$ or mum or dad or maternal or paternal or nurse or childminder* or "child care provider" or playworker" or family or families or carer ${ }^{\star}$ or midwife or "mid wife" or midwives or "mid wives"))

Search strategy for Cochrane CENTRAL, searched via Wiley Online on 11 May 2015 and 10 November 2016.

Previously searched 23 May 2012.

\#1 Health risk behavio? $r^{\star *}$ or "multiple risk behavio? $r^{\star *}$ or "high risk behavio? $r^{\star *}$ or "multiple risk
factor*" or "behavio? $r^{*}$ risk factor*" in Trials

$\# 2$

(Dangerous Behavior/) or (risk taking/) in Trials

\#3 (\#1 OR \#2)

\begin{tabular}{|c|c|}
\hline \#4 & 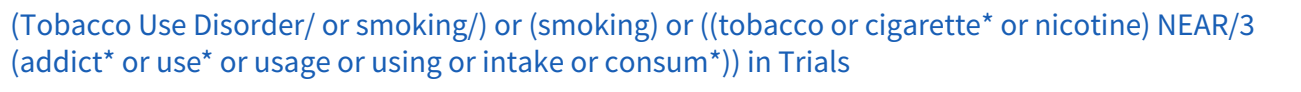 \\
\hline$\# 5$ & 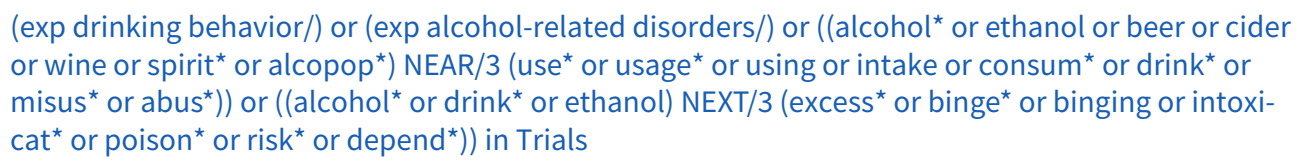 \\
\hline$\# 6$ & 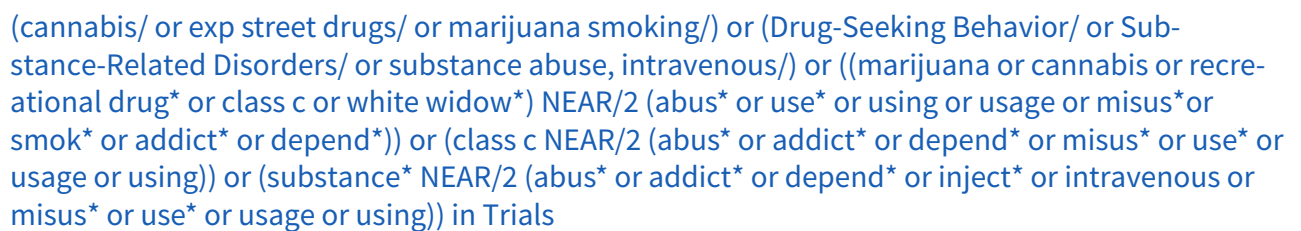 \\
\hline
\end{tabular}


((Class a or class b or drug* or cocaine or ecstasy or mdma or glue or gas or aerosol* or solvent* or magic mushroom ${ }^{\star}$ or crack or ketamine or heroin or morphine or narcotic* or opiat* or opioid* or popper $^{\star}$ or lsd or methamphetamine ${ }^{\star}$ or amphetamine ${ }^{\star}$ ) NEAR/2 (abus* or addict ${ }^{\star}$ or depend ${ }^{\star}$ or inhal* or misus* or sniff* or use* or using or usage)) in Trials

\section{\#8} \#9 $\# 10$ (inhal?nt* NEAR/2 (abus* or addict* or depend $^{*}$ or misus* ${ }^{*}$ or sniff* or use* or using or usage)) in Trials

(\#6 OR \#7 OR \#8)

(Safe sex/ or unsafe sex/ or sexual behavior/ or sexual abstinence/ or exp contraceptive devices/or contraceptive agents/ or exp contraception/ or exp reproductive behavior/ or sexual partners/) or (risky sex* or unsafe sex* or safe ${ }^{\star}$ sex $^{*}$ or sexual intercourse or reproductive behavio? $r^{\star}$ or sexual behavio? $r^{\star}$ or sexual health) or (contracept* or condom? or morning after pill*) in Trials

(exp crime/ or juvenile delinquency/ or social behaviour disorders/) or (violence/ or exp aggression/ or wounds, stab/) or (delinquen* or offen* or reoffend ${ }^{*}$ or violen* or theft* or robbery or burglary $^{\star}$ or steal ${ }^{\star}$ or criminal damage or joyrid ${ }^{\star}$ or joy-rid* or assault* or (sell ${ }^{\star}$ NEAR/ drug*) or devian* or anti-social behavio? $r^{\star}$ or antisocial behavio? $r^{\star}$ or graffiti or racist abuse or index crime* or (breaking NEAR/ entering) or strong-arming or pan-handling or panhandling or disorderly conduct or prostitut ${ }^{*}$ or (carry* NEAR/2 weapon*) or (buy* NEAR/2 stolen) or criminal behavio? $r^{\star}$ or (noisy NEAR/1 rude) or (nuisance* NEAR/2 neighbour*) or fight* or stab? or stabbing or stabbed or stabwound ${ }^{*}$ or wound ${ }^{*}$ or aggress ${ }^{\star}$ or weapon* or knife* or knives or gun* or firearm* or murder $^{\star}$ ) in Trials

((youth* or street or criminal* or adolescen* or juvenile* or teen*) NEAR/2 gang?) or ((adolescen* or youth* or juvenile* or delinquen* or teen* or gang? or school* or college* or sixth form*) NEAR/2 (crim* or offen* or violen* or fight*)) in Trials

\#14 (\#12 OR \#13)

(Head protective devices/ or exp accident prevention/ or risk reduction behavior/ or seat belts/) or ((bicyc ${ }^{\star}$ or cycl $^{*}$ or bik* or motorbike* or motorcycl $\left.{ }^{\star}\right)$ NEAR/2 ((helmet ${ }^{*}$ or protect* or risk reduc ${ }^{*}$ or

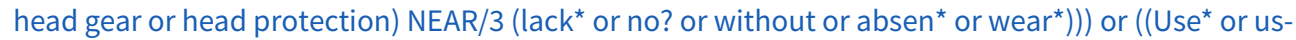
ing or usage or wear ${ }^{\star}$ or wore) NEAR/2 (seat-belt* or seatbelt* or safety belt $\left.{ }^{\star}\right)$ ) or (injur ${ }^{\star}$ NEAR/2 behav $^{\star}$ ) or ((alcohol* or intoxica* or dr?nk*) NEAR/2 (driv* or vehicle* or motor* or car? or van? or automobile* or auto mobile*)) in Trials hurt*) NEAR/2 (gesture* or behavio? r $^{\star}$ ) or (para-suicid ${ }^{\star}$ or parasuicid ${ }^{\star}$ or attempt* suicid $^{\star}$ or suicid* attempt $\left.^{\star}\right)$ or ((non fatal or nonfatal) NEAR/2 (suicid* or harm*)) in Trials

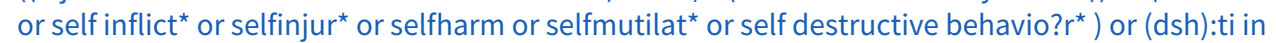
Trials

(exp diet/ or hyperphagia/ or dietary fats/) or ((calori* or fat? or fatty or fizz* or soft* or carbonated $^{\star}$ or sweetened or salt ${ }^{\star}$ or sugar ${ }^{\star}$ or fruit* or veg? or vegetable* or fibre* or fiber ${ }^{\star}$ or 5 -a-day or five a day or go for $2 \& 5$ ) NEAR/2 (intake or food ${ }^{\star}$ or diet ${ }^{*}$ or consum* or meal ${ }^{\star}$ or eat* or nutrition or drink* or snack*)) or ((poor or over ${ }^{\star}$ or unhealthy or health*) NEAR/3 (nutrition or diet ${ }^{\star}$ or eat ${ }^{\star}$ or meal $^{*}$ or food* or snack* or drink* $\left.{ }^{\star}\right)$ in Trials ball/ or basketball/ or bicycling/ or boxing/ or dancing/ or football/ or gardening/ or golf/ or gymnastics/ or hockey/ or exp martial arts/ or mountaineering/ or exp racquet sports/ or exp running/ or skating/ or snow sports/ or soccer/ or exp swimming/ or volleyball/ or walking/ or weight lifting/ or wrestling/) or (Physical activity or fitness or physical ${ }^{*}$ fit* or physical exert* or exercise or aero- 
bic activit* or sport ${ }^{\star}$ or aerobic capacity or active lifestyle* or outdoor activit* or gym or mvpa) or ((fitness or leisure) NEAR/2 (class* or regime* or program* or centre* or center $\left.{ }^{\star}\right)$ ) or ((fit* or sport* or activ* or exercise or physical exer*) NEAR/3 (lack* or low or no or absen*)) in Trials

Sedentary lifestyle/ or Video games/ or gaming or ((view* or watch* or play* or game* or gaming or use* or using or usage) NEAR/2 (television or tv or video* or $\mathrm{dvd}^{\star}$ or screen or comput* or laptop ${ }^{\star}$ or media)) or ((screen or sedentary or view*) NEAR/2 (time or hour ${ }^{\star}$ or minute*)) or ((inactiv* or seden* or indoor ${ }^{\star}$ ) NEAR/3 (lifestyle* or activit*)) in Trials

\begin{tabular}{|c|c|}
\hline$\# 21$ & (\#19 OR \#20) \\
\hline \#22 & (\#16 OR \#17) \\
\hline$\# 23$ & $\begin{array}{l}\text { (( \#3 AND \#4 ) OR ( \#3 AND \#5 ) OR ( \#3 AND \#9 ) OR ( \#3 AND \#10) OR ( \#3 AND \#11 ) OR ( \#3 AND \#14) } \\
\text { OR ( \#3 AND \#15) OR ( \#3 AND \#22 ) OR ( \#3 AND \#18 ) OR ( \#3 AND \#19) OR (\#3 AND \#20)) }\end{array}$ \\
\hline \#24 & $\begin{array}{l}\text { (( \#4 AND \#5 ) OR ( \#4 AND \#9 ) OR ( \#4 AND \#10) OR ( \#4 AND \#11 ) OR ( \#4 AND \#14) OR ( \#4 AND } \\
\text { \#15) OR (\#4 AND \#22 ) OR (\#4 AND \#18) OR (\#4 AND \#19) OR (\#4 AND \#20)) }\end{array}$ \\
\hline$\# 25$ & $\begin{array}{l}\text { (( \#5 AND \#9 ) OR ( \#5 AND \#10) OR ( \#5 AND \#11 ) OR ( \#5 AND \#14) OR (\#5 AND \#15) OR (\#5 AND } \\
\# 22 \text { ) OR ( \#5 AND \#18) OR (\#5 AND \#19) OR (\#5 AND \#20)) }\end{array}$ \\
\hline \#26 & $\begin{array}{l}\text { (( \#9 AND \#10) OR ( \#9 AND \#11) OR ( \#9 AND \#14 ) OR ( \#9 AND \#15) OR ( \#9 AND \#22 ) OR ( \#9 AND } \\
\# 18 \text { ) OR (\#9 AND \#19) OR ( \#9 AND \#20)) }\end{array}$ \\
\hline \#27 & $\begin{array}{l}\text { (( \#10 AND \#11) OR ( \#10 AND \#14) OR ( \#10 AND \#15) OR ( \#10 AND \#22 ) OR ( \#10 AND \#18) OR } \\
(\# 10 \text { AND \#19) OR (\#10 AND \#20)) }\end{array}$ \\
\hline \#28 & $\begin{array}{l}\text { (( \#11 AND \#14 ) OR ( \#11 AND \#15) OR ( \#11 AND \#22 ) OR ( \#11 AND \#18) OR ( \#11 AND \#19) OR } \\
(\# 11 \text { AND \#20)) }\end{array}$ \\
\hline \#29 & ((\#14 AND \#15) OR ( \#14 AND \#22 ) OR ( \#14 AND \#18) OR (\#14 AND \#19) OR ( \#14 AND \#20)) \\
\hline \#30 & $\begin{array}{l}\text { ((\#15 AND \#22 ) OR ( \#15 AND \#18) OR ( \#15 AND \#19) OR (\#15 AND \#20) OR ( \#22 AND \#18) OR } \\
(\# 22 \text { AND \#19) OR (\#22 AND \#20) OR (\#18 AND \#19) OR (\#18 AND \#20) OR (\#19 AND \#20)) }\end{array}$ \\
\hline \#31 & (\#23 OR \#24 OR \#25 OR \#26) \\
\hline \#32 & (\#27 OR \#28 OR \#29 OR \#30) \\
\hline \#33 & 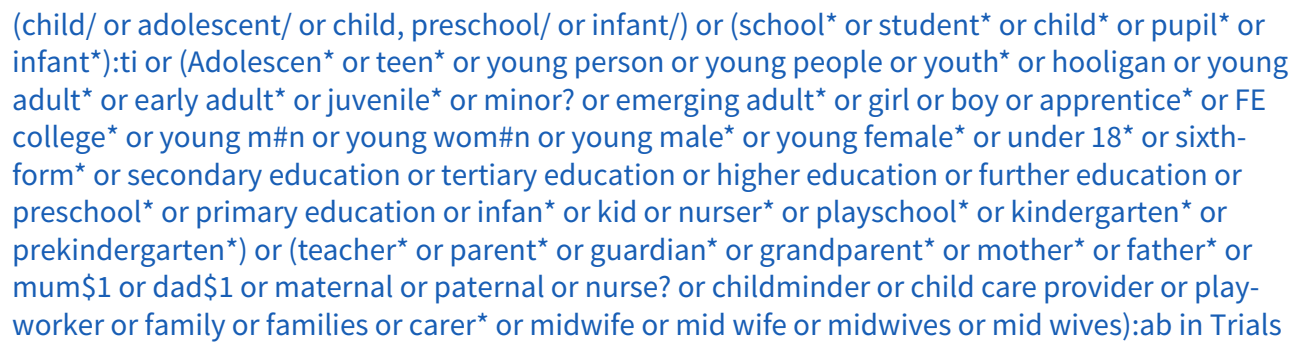 \\
\hline \#34 & (\#31 AND \#33) \\
\hline \#35 & (\#32 AND \#33) \\
\hline
\end{tabular}

Search strategy for Embase (Ovid SP), 1980 to 2015 week 16. Searched 06 May 2015 and 10 November 2016.

\section{Previously searched 24 May 2012.}




\begin{tabular}{ll}
\hline 1 & $\begin{array}{l}\text { ("Health risk behavio? } r^{\star *} \text { or "multiple risk behavio? } r^{\star *} \text { or "high risk behavio? } r^{\star *} \text { or "multiple risk } \\
\text { factor " or "behavio? } r^{\star} \text { risk factor }{ }^{\star} \text { ").mp. [mp=title, abstract, subject headings, heading word, drug } \\
\text { trade name, original title, device manufacturer, drug manufacturer, device trade name, keyword] }\end{array}$ \\
\hline 2 & Dangerous Behavior/ \\
\hline 4 & high risk behavior/ \\
\hline 5 & 1 or 3 \\
\hline 6 & tobacco dependence/ \\
\hline 7 & exp Smoking/ \\
\hline
\end{tabular}

8

((tobacco or cigarette* or nicotine) adj3 (addict ${ }^{\star}$ or use ${ }^{\star}$ or usage or using or intake or consum $\left.\left.^{\star}\right)\right) . m p$. [mp=title, abstract, subject headings, heading word, drug trade name, original title, device manufacturer, drug manufacturer, device trade name, keyword]

\begin{tabular}{|c|c|}
\hline 9 & 5 or 6 or 7 or 8 \\
\hline 10 & Drinking Behavior/ \\
\hline 11 & alcohol abuse/ or alcoholism/ \\
\hline 12 & 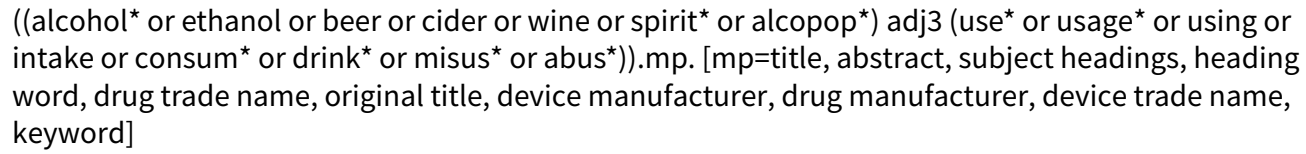 \\
\hline
\end{tabular}

((alcohol* or drink ${ }^{\star}$ or ethanol) adj3 (excess* or binge* or binging or intoxicat* or poison* or risk ${ }^{\star}$ or depend $\left.^{\star}\right)$ ).mp. [mp=title, abstract, subject headings, heading word, drug trade name, original title, device manufacturer, drug manufacturer, device trade name, keyword]

14 or 11 or 12 or 13

15 cannabis/ or cannabis addiction/or street drugs/ or cannabis smoking/

\begin{tabular}{|c|c|}
\hline 16 & Drug-Seeking Behavior/ \\
\hline 17 & addiction/ or exp drug dependence/ \\
\hline 18 & 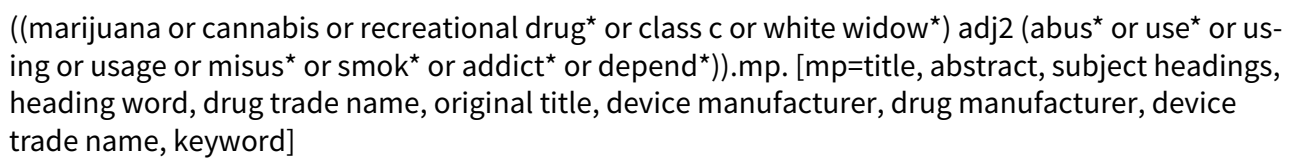 \\
\hline 19 & substance abuse/ \\
\hline 20 & $\begin{array}{l}\text { (class c adj2 (abus }{ }^{\star} \text { or addict }^{\star} \text { or depend }{ }^{\star} \text { or misus* or use }{ }^{\star} \text { or usage or using)).mp. [mp=title, ab- } \\
\text { stract, subject headings, heading word, drug trade name, original title, device manufacturer, drug } \\
\text { manufacturer, device trade name, keyword] }\end{array}$ \\
\hline
\end{tabular}


(substance adj2 (abus $^{\star}$ or addict ${ }^{\star}$ or depend ${ }^{\star}$ or inject ${ }^{\star}$ or intravenous or misus ${ }^{\star}$ or use ${ }^{\star}$ or usage or using)).mp. [mp=title, abstract, subject headings, heading word, drug trade name, original title, device manufacturer, drug manufacturer, device trade name, keyword]

((Class a or class b or drug ${ }^{\star}$ or cocaine or ecstasy or mdma or glue or gas or aerosol ${ }^{\star}$ or solvent ${ }^{\star}$ or magic mushroom ${ }^{\star}$ or crack or white widow* or ketamine or heroin or morphine or narcotic ${ }^{\star}$ or opiat* or opioid* or popper ${ }^{\star}$ or lsd or methamphetamine* or amphetamine*) adj2 (abus* or addict* or depend ${ }^{\star}$ or inhal ${ }^{\star}$ or misus ${ }^{\star}$ or sniff* or use ${ }^{\star}$ or using or usage)).mp. [mp=title, abstract, subject headings, heading word, drug trade name, original title, device manufacturer, drug manufacturer, device trade name, keyword] [mp=title, abstract, subject headings, heading word, drug trade name, original title, device manufacturer, drug manufacturer, device trade name, keyword] original title, device manufacturer, drug manufacturer, device trade name, keyword]

Safe sex/ or unsafe sex/ or sexual behavior/ or exp contraceptive agents/ or exp contraception/ or sexuality/

(risky sex ${ }^{\star}$ or unsafe sex ${ }^{\star}$ or safe ${ }^{\star}$ sex $^{\star}$ or sexual intercourse or reproductive behavio? $r^{\star}$ or sexual behavio? $\mathrm{r}^{\star}$ or sexual health).mp. [mp=title, abstract, subject headings, heading word, drug trade name, original title, device manufacturer, drug manufacturer, device trade name, keyword] word, drug trade name, original title, device manufacturer, drug manufacturer, device trade name, keyword]

26 or 27 or 28

exp crime/ or sociopathy/

(delinquen* or offen* or reoffend ${ }^{\star}$ or violen ${ }^{\star}$ or theft ${ }^{\star}$ or robbery or burglary* or steal ${ }^{*}$ or criminal damage or joyrid ${ }^{\star}$ or joy-rid* or assault* or (sell* adj drug*) or devian* or anti-social behavio? ${ }^{\star}$ or antisocial behavio? $r^{\star}$ or graffiti or racist abuse or index crime* or (breaking adj entering) or strong-arming or pan-handling or panhandling or disorderly conduct or prostitut* or (carry ${ }^{*}$ adj2 weapon $^{\star}$ ) or (buy ${ }^{\star}$ adj2 stolen) or criminal behavio? ${ }^{\star}$ or (noisy adj1 rude) or (nuisance* adj2 neighbour $^{\star}$ ) or fight* or stab? or stabbing or stabbed or stabwound ${ }^{\star}$ or wound ${ }^{\star}$ or aggress* ${ }^{\star}$ or weapon* or knife* or knives or gun ${ }^{\star}$ or firearm* or murder ${ }^{\star}$ ).mp. [mp=title, abstract, subject headings, heading word, drug trade name, original title, device manufacturer, drug manufacturer, device trade name, keyword] stract, subject headings, heading word, drug trade name, original title, device manufacturer, drug manufacturer, device trade name, keyword] form $\left.{ }^{\star}\right)$ adj2 (crim* or offen* or violen* or fight $\left.\left.{ }^{\star}\right)\right) \cdot \mathrm{mp}$. [mp=title, abstract, subject headings, heading word, drug trade name, original title, device manufacturer, drug manufacturer, device trade name, keyword] 

head gear or head protection) adj3 (lack* or no? or without or absen* or wear $\left.\left.{ }^{\star}\right)\right)$ ).mp. [mp=title, abstract, subject headings, heading word, drug trade name, original title, device manufacturer, drug manufacturer, device trade name, keyword]

\begin{tabular}{ll}
\hline 38 & seatbelt/ \\
\hline 39 & $\left(\left(\right.\right.$ Use $e^{\star}$ or using or usage or wear ${ }^{\star}$ or wore $)$ adj2 (seat-belt ${ }^{\star}$ or seatbelt ${ }^{\star}$ or safety belt $\left.\left.{ }^{\star}\right)\right) . \mathrm{mp}$. [mp=ti- \\
& tle, abstract, subject headings, heading word, drug trade name, original title, device manufacturer, \\
& drug manufacturer, device trade name, keyword]
\end{tabular}

\section{0} (injur* adj2 behav $\left.{ }^{\star}\right) . m p$. [mp=title, abstract, subject headings, heading word, drug trade name, original title, device manufacturer, drug manufacturer, device trade name, keyword]

((alcohol* or intoxica or dr?nk $\left.^{\star}\right)$ adj2 $\left(\right.$ driv* or vehicle* or motor* or car? or van? or automobile* or $^{*}$
auto mobile*)).mp. [mp=title, abstract, subject headings, heading word, drug trade name, original
title, device manufacturer, drug manufacturer, device trade name, keyword]

\section{2}

43

44

45

46

\begin{tabular}{|c|c|}
\hline 47 & automutilation/ \\
\hline 48 & $\begin{array}{l}\left(\left(\text { injur }^{\star} \text { or mutil }\right.\right. \\
\text { abstract, subject hearm } \\
\text { drug or wound } \\
\text { drug manufacturer, device trade name, keyword] }\end{array}$ \\
\hline
\end{tabular}

36 or 37 or 38 or 39 or 40 or 41

suicide attempt/ or suicidal behavior/ device trade name, keyword] turer, device trade name, keyword] keyword] ((suicid ${ }^{\star}$ or harm $^{\star}$ or injur ${ }^{\star}$ or hurt ${ }^{\star}$ ) adj2 (gesture* or behavio? $\left.{ }^{\star}\right)$ ).mp. [mp=title, abstract, subject headings, heading word, drug trade name, original title, device manufacturer, drug manufacturer,

(para-suicid $^{*}$ or parasuicid ${ }^{*}$ or attempt ${ }^{\star}$ suicid $^{\star}$ or suicid* attempt $\left.^{\star}\right) . m p$. [mp=title, abstract, subject headings, heading word, drug trade name, original title, device manufacturer, drug manufac-

((non fatal or nonfatal) adj2 (suicid* or harm*)).mp. [mp=title, abstract, subject headings, heading word, drug trade name, original title, device manufacturer, drug manufacturer, device trade name,

49 dsh.tw. or selfinflict*.mp. or self inflict*.mp. or selfinjur ${ }^{\star} . m p$. or selfharm ${ }^{\star} . m p$. or selfmutilat ${ }^{\star} . m p$. or self destructive behavio? ${ }^{\star}$.mp. [mp=title, abstract, subject headings, heading word, drug trade name, original title, device manufacturer, drug manufacturer, device trade name, keyword]

\begin{tabular}{|c|c|}
\hline 50 & 43 or 44 or 45 or 46 or 47 or 48 or 49 \\
\hline 51 & exp diet/ or hyperphagia/ or fat intake/ \\
\hline 52 & 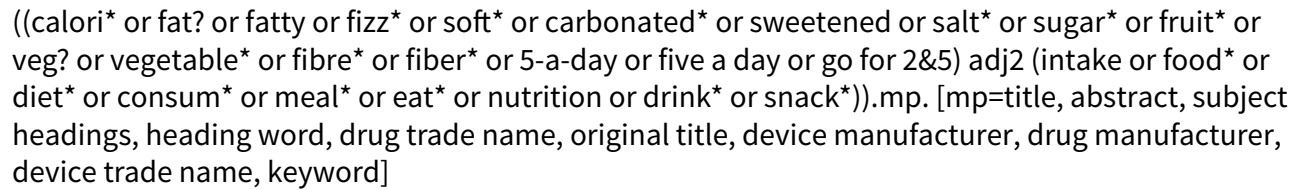 \\
\hline
\end{tabular}


((poor or over ${ }^{\star}$ or unhealthy or health ${ }^{\star}$ ) adj3 (nutrition or diet ${ }^{\star}$ or eat ${ }^{\star}$ or meal ${ }^{\star}$ or food ${ }^{\star}$ or snack or drink $\left.{ }^{\star}\right)$ ).mp. [mp=title, abstract, subject headings, heading word, drug trade name, original title, device manufacturer, drug manufacturer, device trade name, keyword]

\begin{tabular}{|c|c|}
\hline 54 & 51 or 52 or 53 \\
\hline 55 & exp exercise/ or endurance/ or fitness/ \\
\hline 56 & exp sport/ or dancing/ or walking/ \\
\hline 57 & $\begin{array}{l}\text { (Physical activity or fitness or physical }{ }^{\star} \text { fit }^{\star} \text { or physical exert } \\
\text { sport }{ }^{\star} \text { or ex aerobic capacity or active lifestyle }{ }^{\star} \text { or outdoor activit* or gym }{ }^{\star} \text { or mvpa).mp. [mp=title, } \\
\text { abstract, subject headings, heading word, drug trade name, original title, device manufacturer, } \\
\text { drug manufacturer, device trade name, keyword] }\end{array}$ \\
\hline
\end{tabular}
stract, subject headings, heading word, drug trade name, original title, device manufacturer, drug manufacturer, device trade name, keyword]

\section{9}

((fit ${ }^{\star}$ or sport $^{\star}$ or activ ${ }^{\star}$ or exercise or physical exer $\left.{ }^{\star}\right)$ adj3 (lack ${ }^{\star}$ or low or no or absen $\left.\left.{ }^{\star}\right)\right) . m p$. [ $\mathrm{mp}=$ title, abstract, subject headings, heading word, drug trade name, original title, device manufacturer, drug manufacturer, device trade name, keyword]

\begin{tabular}{|c|c|}
\hline 60 & 55 or 56 or 57 or 58 or 59 \\
\hline 61 & Sedentary lifestyle/ or television viewing/ \\
\hline 62 & gaming.mp. \\
\hline 63 & 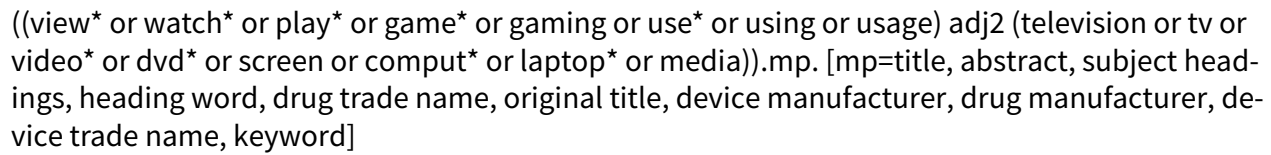 \\
\hline
\end{tabular}
64 ((screen or sedentary or view $\left.{ }^{\star}\right)$ adj2 (time or hour ${ }^{\star}$ or minute $)$ ).mp. [mp=title, abstract, subject headings, heading word, drug trade name, original title, device manufacturer, drug manufacturer, device trade name, keyword]

((inactiv ${ }^{\star}$ or seden $^{\star}$ or indoor $\left.{ }^{\star}\right)$ adj3 (lifestyle* or activit $\left.\left.{ }^{\star}\right)\right) . \mathrm{mp}$. [mp=title, abstract, subject headings, heading word, drug trade name, original title, device manufacturer, drug manufacturer, device trade name, keyword]

$66 \quad 61$ or 62 or 63 or 64 or 65

(4 and 9) or (4 and 14) or (4 and 24) or (4 and 25) or (4 and 29) or (4 and 35) or ( 4 and 42 ) or (4 and $50)$ or (4 and 54) or ( 4 and 60$)$ or ( 4 and 66) or ( 9 and 14) or (9 and 24) or (9 and 25) or (9 and 29) or (9 and 35$)$ or ( 9 and 42$)$ or (9 and 50) or (9 and 54) or (9 and 60) or (9 and 66) or (14 and 24) or (14 and $25)$ or (14 and 29) or (14 and 35) or (14 and 42) or (14 and 50) or (14 and 54) or (14 and 60) or (14 and $66)$ or (24 and 25$)$ or (24 and 29) or (24 and 35) or (24 and 42) or (24 and 50) or (24 and 54) or (24 and $60)$ or ( 24 and 66$)$ or ( 25 and 29 ) or ( 25 and 35$)$ or ( 25 and 42$)$ or (25 and 50 ) or ( 25 and 54 ) or ( 25 and $60)$ or ( 25 and 66$)$ or ( 29 and 35$)$ or (29 and 42) or (29 and 50) or (29 and 54) or (29 and 60) or (29 and $66)$ or ( 35 and 42$)$ or ( 35 and 50 ) or ( 35 and 54$)$ or ( 35 and 60$)$ or ( 35 and 66$)$ or (42 and 50$)$ or (42 and $54)$ or ( 42 and 60$)$ or (42 and 66) or (50 and 54) or (50 and 60) or (50 and 66) venile/ 
(Adolescen* or teen* or young person or young people or youth* or hooligan* or young adult* or early adult* or juvenile* or minor? or emerging adult* or girl? or boy? or apprentice* or FE college* or young m\#n or young wom\#n or young male* or young female ${ }^{\star}$ or under $18^{\star}$ or sixth-form ${ }^{\star}$ or secondary education or tertiary education or higher education or further education or preschoo ${ }^{*}$ or primary education or infan* or kid? or nurser* or playschool* or kindergarten* or prekindergarten $\left.{ }^{\star}\right) \cdot m p$.

71 (teacher ${ }^{\star}$ or parent ${ }^{\star}$ or guardian* or grandparent ${ }^{\star}$ or mother ${ }^{\star}$ or father ${ }^{\star}$ or mum? or dad? or maternal or paternal or nurse? or childminder ${ }^{\star}$ or child care provider* or playworker* or family or families or carer ${ }^{\star}$ or midwife or mid wife or midwives or mid wives).mp. [mp=title, abstract, subject headings, heading word, drug trade name, original title, device manufacturer, drug manufacturer, device trade name, keyword]

\begin{tabular}{ll}
\hline 72 & 68 or 69 or 70 or 71 \\
\hline 73 & exp controlled clinical trial/ \\
\hline 74 & (randomi\#ed or placebo* or randomly).ab. \\
\hline 75 & trial.ti. \\
\hline 76 & "clinical trial (topic)"/ \\
\hline 78 & 73 or 74 or 75 or 76 \\
\hline 79 & animal/ not human/ \\
\hline 80 & 77 not 78 \\
\hline
\end{tabular}

\section{Search strategy for Ovid MEDLINE (OvidSP), 1950 to 06 May 2015 and 10 November 2016.}

\section{Previously searched 24 May 2012.}

\begin{tabular}{|c|c|}
\hline 1 & 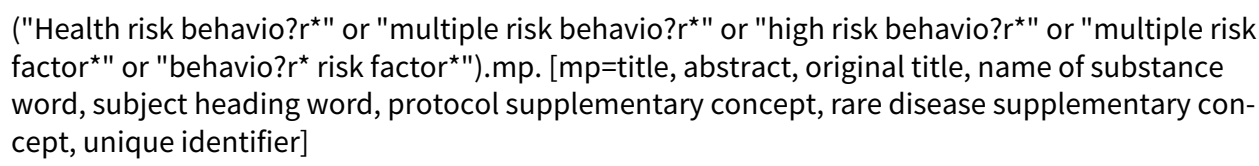 \\
\hline 2 & Dangerous Behavior/ \\
\hline 3 & Risk-Taking/ \\
\hline 4 & 1 or 2 or 3 \\
\hline 5 & "Tobacco Use Disorder"/ \\
\hline 6 & Smoking/ \\
\hline 7 & $\begin{array}{l}\text { smoking.mp. [mp=title, abstract, original title, name of substance word, subject heading word, } \\
\text { protocol supplementary concept, rare disease supplementary concept, unique identifier] }\end{array}$ \\
\hline
\end{tabular}


((tobacco or cigarette* or nicotine) adj3 (addict* or use* or usage or using or intake or consum $\left.\left.^{\star}\right)\right) . m p$. [mp=title, abstract, original title, name of substance word, subject heading word, protocol supplementary concept, rare disease supplementary concept, unique identifier]

\begin{tabular}{|c|c|}
\hline 9 & 5 or 6 or 7 or 8 \\
\hline 10 & exp Drinking Behavior/ \\
\hline 11 & exp Alcohol-Related Disorders/ \\
\hline 12 & $\begin{array}{l}\text { ((alcohol* or ethanol or beer or cider or wine or spirit* or alcopop*) adj3 (use* or usage* or using or } \\
\left.\left.\text { intake or consum }{ }^{\star} \text { or drink }{ }^{\star} \text { or misus } \text { or abus }^{\star}\right)\right) . \mathrm{mp} \text {. [mp=title, abstract, original title, name of sub- } \\
\text { stance word, subject heading word, protocol supplementary concept, rare disease supplementary } \\
\text { concept, unique identifier] }\end{array}$ \\
\hline
\end{tabular}

((alcohol* or drink $^{\star}$ or ethanol) adj3 (excess ${ }^{*}$ or binge* or binging or intoxicat* or poison $^{*}$ or risk $^{*}$ or depend $\left.{ }^{\star}\right)$ ).mp. [mp=title, abstract, original title, name of substance word, subject heading word, protocol supplementary concept, rare disease supplementary concept, unique identifier]

\begin{tabular}{|c|c|}
\hline 14 & 10 or 11 or 12 or 13 \\
\hline 15 & cannabis/ or exp street drugs/ or marijuana smoking/ \\
\hline 16 & Drug-Seeking Behavior/ \\
\hline 17 & Substance-Related Disorders/ \\
\hline 18 & $\begin{array}{l}\text { ( }\left(\text { marijuana or cannabis or recreational drug }{ }^{\star} \text { or class c or white widow }{ }^{\star}\right) \text { adj2 (abus }{ }^{\star} \text { or use }{ }^{\star} \text { or us- } \\
\left.\left.\text { ing or usage or misus* or smok }{ }^{\star} \text { or addict } \text { or depend }^{\star}\right)\right) . \mathrm{mp} \text {. [mp=title, abstract, original title, name } \\
\text { of substance word, subject heading word, protocol supplementary concept, rare disease supple- } \\
\text { mentary concept, unique identifier] }\end{array}$ \\
\hline 19 & substance abuse, intravenous/ \\
\hline 20 & $\begin{array}{l}\text { (class } \mathrm{c} \text { adj2 (abus }{ }^{\star} \text { or addict }{ }^{\star} \text { or depend }{ }^{\star} \text { or } \text { misus }^{\star} \text { or use }{ }^{\star} \text { or usage or using)).mp. [mp=title, ab- } \\
\text { stract, original title, name of substance word, subject heading word, protocol supplementary con- } \\
\text { cept, rare disease supplementary concept, unique identifier] }\end{array}$ \\
\hline 21 & $\begin{array}{l}\text { (substance* adj2 (abus } \text { or addict }^{\star} \text { or depend } \text { or inject }^{\star} \text { or intravenous or misus }{ }^{\star} \text { or use } \text { or usage }^{*} \\
\text { or using)).mp. [mp=title, abstract, original title, name of substance word, subject heading word, } \\
\text { protocol supplementary concept, rare disease supplementary concept, unique identifier] }\end{array}$ \\
\hline 22 & 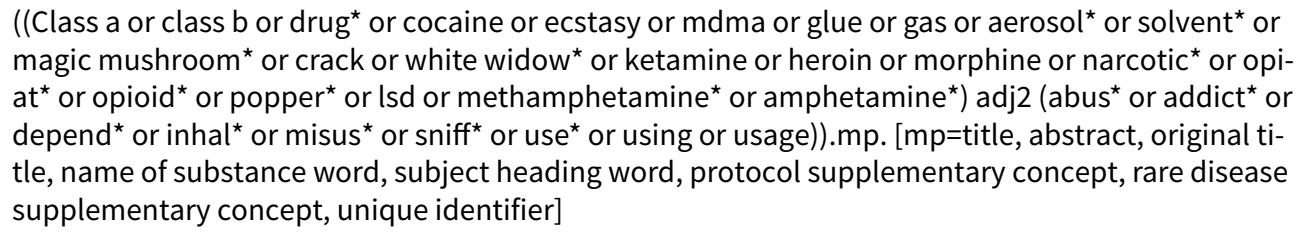 \\
\hline 23 & $\begin{array}{l}\text { (inhal?nt }{ }^{\star} \text { adj2 (abus }{ }^{\star} \text { or addict }^{\star} \text { or depend }{ }^{\star} \text { or misus* or sniff* or use* or using or usage)).mp. } \\
\text { [mp=title, abstract, original title, name of substance word, subject heading word, protocol supple- } \\
\text { mentary concept, rare disease supplementary concept, unique identifier] }\end{array}$ \\
\hline 24 & 15 or 16 or 17 or 18 or 19 or 20 or 21 or 22 or 23 \\
\hline 25 & $\begin{array}{l}\text { (gambl* or betting).mp. [mp=title, abstract, original title, name of substance word, subject heading } \\
\text { word, protocol supplementary concept, rare disease supplementary concept, unique identifier] }\end{array}$ \\
\hline
\end{tabular}


Safe sex/ or unsafe sex/ or sexual behavior/ or sexual abstinence/ or exp contraceptive devices/ or contraceptive agents/ or exp contraception/ or exp reproductive behavior/ or sexual partners/

(risky sex* or unsafe sex* or safe* sex $^{\star}$ or sexual intercourse or reproductive behavio? $r^{\star}$ or sexual behavio? $r^{\star}$ or sexual health).mp. [mp=title, abstract, original title, name of substance word, subject heading word, protocol supplementary concept, rare disease supplementary concept, unique identifier] stance word, subject heading word, protocol supplementary concept, rare disease supplementary concept, unique identifier]

\begin{tabular}{|c|c|}
\hline 29 & 26 or 27 or 28 \\
\hline 30 & exp crime/ or juvenile delinquency/ or social behaviour disorders/ \\
\hline 31 & violence/ or exp aggression/ or wounds, stab/ \\
\hline 32 & 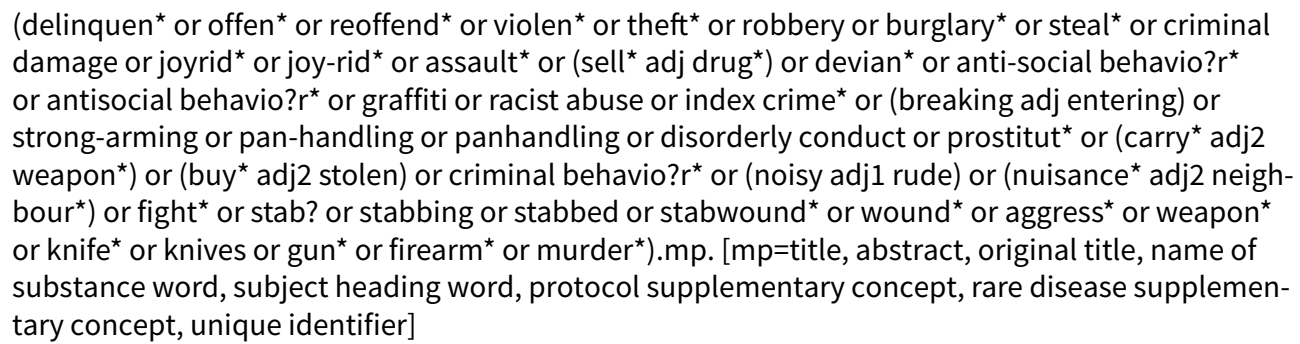 \\
\hline
\end{tabular}
stract, original title, name of substance word, subject heading word, protocol supplementary concept, rare disease supplementary concept, unique identifier] form $\left.{ }^{\star}\right)$ adj2 (crim* or offen* or violen* or fight $\left.\left.{ }^{\star}\right)\right)$.mp. [mp=title, abstract, original title, name of substance word, subject heading word, protocol supplementary concept, rare disease supplementary concept, unique identifier]

$35 \quad 30$ or 31 or 32 or 33 or 34

$36 \quad$ Head protective devices/or exp accident prevention/ or risk reduction behavior/

37 ((bicyc ${ }^{\star}$ or cycl ${ }^{\star}$ or bik ${ }^{\star}$ or motorbike* or motorcycl $\left.{ }^{\star}\right)$ adj2 ((helmet or protect $^{\star}$ or risk reduc or $^{\star}$ head gear or head protection) adj3 (lack ${ }^{\star}$ or no? or without or absen* or wear $\left.\left.{ }^{\star}\right)\right)$ ).mp. [mp=title, abstract, original title, name of substance word, subject heading word, protocol supplementary concept, rare disease supplementary concept, unique identifier]

\begin{tabular}{|c|c|}
\hline 38 & Seat Belts/ \\
\hline 39 & $\begin{array}{l}\left.\left(\left(\text { Use }^{\star} \text { or using or usage or wear }{ }^{\star} \text { or wore }\right) \text { adj2 (seat-belt* or seatbelt* or safety belt }{ }^{\star}\right)\right) \cdot \mathrm{mp} .[\mathrm{mp}=\mathrm{ti}- \\
\text { tle, abstract, original title, name of substance word, subject heading word, protocol supplementary } \\
\text { concept, rare disease supplementary concept, unique identifier] }\end{array}$ \\
\hline 40 & $\begin{array}{l}\left.\text { (injur* adj2 behav }{ }^{\star}\right) . \mathrm{mp} . \text { [mp=title, abstract, original title, name of substance word, subject head- } \\
\text { ing word, protocol supplementary concept, rare disease supplementary concept, unique identifier] }\end{array}$ \\
\hline 41 & 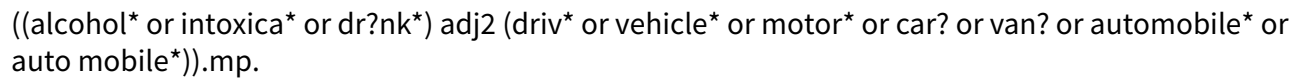 \\
\hline
\end{tabular}


42 43

\section{4} 45

46

36 or 37 or 38 or 39 or 40 or 41

43

(2)

suicide, attempted/

((suicid* or harm* or injur or hurt $^{\star}$ ) adj2 (gesture* or behavio? $\left.\mathrm{r}^{\star}\right)$ ).mp. [mp=title, abstract, original title, name of substance word, subject heading word, protocol supplementary concept, rare disease supplementary concept, unique identifier]

\begin{tabular}{|c|c|}
\hline 45 & $\begin{array}{l}\left.\text { (para-suicid }{ }^{\star} \text { or parasuicid }{ }^{\star} \text { or attempt* suicid* or suicid }{ }^{\star} \text { attempt* }\right) . m p .[\mathrm{mp}=\text { title, abstract, orig- } \\
\text { inal title, name of substance word, subject heading word, protocol supplementary concept, rare } \\
\text { disease supplementary concept, unique identifier] }\end{array}$ \\
\hline
\end{tabular}

$\begin{array}{ll}46 & \left.\left(\text { non fatal or nonfatal) adj2 (suicid* or harm }{ }^{\star}\right)\right) . m p . \text { [mp=title, abstract, original title, name of sub- } \\ \text { stance word, subject heading word, protocol supplementary concept, rare disease supplementary } \\ \text { concept, unique identifier] }\end{array}$

\begin{tabular}{|c|c|}
\hline 47 & Self-injurious behavior/ or self mutilation/ \\
\hline 48 & $\begin{array}{l}\left.\left.\left.\text { ((injur }{ }^{\star} \text { or mutil }{ }^{\star} \text { or harm }{ }^{\star} \text { or wound }{ }^{\star} \text { or hurt }{ }^{\star}\right) \text { adj2 (self or themsel }{ }^{\star} \text { or yoursel }{ }^{\star}\right)\right) \cdot \mathrm{mp} . \text { [mp=title, } \\
\text { abstract, original title, name of substance word, subject heading word, protocol supplementary } \\
\text { concept, rare disease supplementary concept, unique identifier] }\end{array}$ \\
\hline
\end{tabular}

dsh.tw. or selfinflict ${ }^{\star} . m p$. or self inflict ${ }^{\star} . m p$. or selfinjur ${ }^{\star} . m p$. or selfharm ${ }^{\star} . m p$. or selfmutilat ${ }^{\star}$.mp. or self destructive behavio? $r^{\star} . \mathrm{mp}$. [mp=title, abstract, original title, name of substance word, subject heading word, protocol supplementary concept, rare disease supplementary concept, unique identifier]

\begin{tabular}{|c|c|}
\hline 50 & 43 or 44 or 45 or 46 or 47 or 48 or 49 \\
\hline 51 & exp diet/ or hyperphagia/ or dietary fats/ \\
\hline 52 & 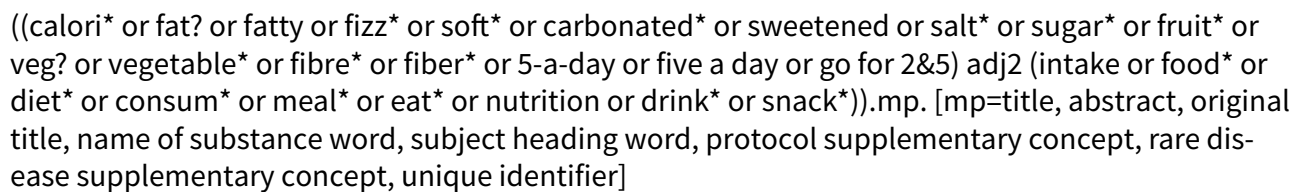 \\
\hline
\end{tabular}

((poor or over* or unhealthy or health*) adj3 (nutrition or diet* or eat* or meal ${ }^{\star}$ or food ${ }^{\star}$ or snack $^{\star}$ or drink $\left.\left.{ }^{\star}\right)\right) . m p$. [mp=title, abstract, original title, name of substance word, subject heading word, protocol supplementary concept, rare disease supplementary concept, unique identifier]

\begin{tabular}{ll}
\hline 54 & 51 or 52 or 53 \\
\hline 55 & exp exercise/ or physical exertion/ or physical fitness/ or physical endurance/ or fitness/ \\
\hline 56 & $\begin{array}{l}\text { Baseball/ or basketball/ or bicycling/ or boxing/ or dancing/ or football/ or gardening/ or golf/ or } \\
\text { ning/ or skating/ or snow sports/ or soccer/ or exp swimming/ or volleyball/ or walking/ or weight } \\
\text { lifting/ or wrestling/ }\end{array}$ \\
\hline
\end{tabular}

(Physical activity or fitness or physical ${ }^{\star}$ fit $^{\star}$ or physical exert ${ }^{\star}$ or exercise or aerobic activit ${ }^{\star}$ or sport $^{\star}$ or aerobic capacity or active lifestyle* or outdoor activit* or gym or mvpa).mp. [mp=title, abstract, original title, name of substance word, subject heading word, protocol supplementary concept, rare disease supplementary concept, unique identifier] stract, original title, name of substance word, subject heading word, protocol supplementary concept, rare disease supplementary concept, unique identifier] 
((fit ${ }^{\star}$ or sport ${ }^{\star}$ or activ* or exercise or physical exer*) adj3 (lack ${ }^{\star}$ or low or no or absen $\left.\left.{ }^{\star}\right)\right) . m p$. [mp=title, abstract, original title, name of substance word, subject heading word, protocol supplementary concept, rare disease supplementary concept, unique identifier]

\begin{tabular}{|c|c|}
\hline 60 & 55 or 56 or 57 or 58 or 59 \\
\hline 61 & Sedentary lifestyle/ or Video games/ \\
\hline 62 & gaming.mp. \\
\hline 63 & $\begin{array}{l}\text { ((view* } \text { or watch }^{\star} \text { or play }{ }^{\star} \text { or game }{ }^{\star} \text { or gaming or use* or using or usage) adj2 (television or tv or } \\
\text { video* or dvd } \text { or screen or comput }^{\star} \text { or laptop* or media)).mp. [mp=title, abstract, original title, } \\
\text { name of substance word, subject heading word, protocol supplementary concept, rare disease } \\
\text { supplementary concept, unique identifier] }\end{array}$ \\
\hline
\end{tabular}
tle, name of substance word, subject heading word, protocol supplementary concept, rare disease supplementary concept, unique identifier]

\section{5}

((inactiv* or seden $^{\star}$ or indoor $\left.{ }^{\star}\right)$ adj3 (lifestyle* or activit $\left.\left.{ }^{\star}\right)\right) . \mathrm{mp}$. [mp=title, abstract, original title, name of substance word, subject heading word, protocol supplementary concept, rare disease supplementary concept, unique identifier]

\section{6}

\section{1 or 62 or 63 or 64 or 65}

(4 and 9) or (4 and 14) or (4 and 24) or (4 and 25) or (4 and 29) or (4 and 35) or (4 and 42 ) or (4 and $50)$ or $(4$ and 54$)$ or $(4$ and 60$)$ or ( 4 and 66$)$ or ( 9 and 14$)$ or $(9$ and 24$)$ or $(9$ and 25$)$ or (9 and 29$)$ or (9 and 35$)$ or ( 9 and 42$)$ or ( 9 and 50) or (9 and 54) or ( 9 and 60$)$ or (9 and 66) or (14 and 24) or (14 and $25)$ or (14 and 29) or (14 and 35) or (14 and 42) or (14 and 50) or (14 and 54) or (14 and 60) or (14 and $66)$ or $(24$ and 25$)$ or $(24$ and 29$)$ or ( 24 and 35$)$ or ( 24 and 42$)$ or (24 and 50) or ( 24 and 54 ) or (24 and $60)$ or ( 24 and 66$)$ or ( 25 and 29$)$ or ( 25 and 35$)$ or ( 25 and 42$)$ or (25 and 50) or (25 and 54) or (25 and $60)$ or ( 25 and 66$)$ or (29 and 35$)$ or (29 and 42) or (29 and 50) or (29 and 54) or (29 and 60) or (29 and $66)$ or ( 35 and 42$)$ or ( 35 and 50$)$ or ( 35 and 54 ) or ( 35 and 60$)$ or ( 35 and 66$)$ or ( 42 and 50 ) or ( 42 and $54)$ or ( 42 and 60$)$ or ( 42 and 66$)$ or ( 50 and 54$)$ or ( 50 and 60$)$ or (50 and 66$)$

\section{8} child/ or adolescent/ or child, preschool/ or infant/

(school ${ }^{\star}$ or student ${ }^{\star}$ or child $^{\star}$ or pupil ${ }^{\star}$ or infant $\left.^{\star}\right)$.tw.

70 (Adolescen* or teen* or young person or young people or youth* or hooligan* or young adult* or early adult* or juvenile* or minor? or emerging adult* or girl ${ }^{\star}$ or boy? or apprentice* or FE college* or young $\mathrm{m \# n}$ or young wom\#n or young male* or young female* or under $18^{\star}$ or sixth-form ${ }^{\star}$ or secondary education or tertiary education or higher education or further education or preschool* or primary education or infan ${ }^{\star}$ or kid? or nurser ${ }^{\star}$ or playschool $^{\star}$ or kindergarten ${ }^{\star}$ or prekindergarten*).mp.

(teacher ${ }^{\star}$ or parent ${ }^{\star}$ or guardian ${ }^{\star}$ or grandparent ${ }^{\star}$ or mother ${ }^{\star}$ or father ${ }^{\star}$ or mum? or dad? or mater-

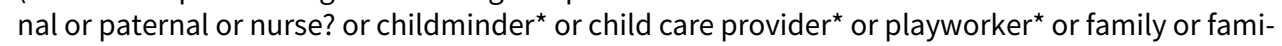
lies or carer ${ }^{\star}$ or midwife or mid wife or midwives or mid wives).mp. [mp=title, abstract, original title, name of substance word, subject heading word, protocol supplementary concept, rare disease supplementary concept, unique identifier]

72

68 or 69 or 70 or 71 
(Continued)

trial.ti.

$76 \quad$ clinical trials as topic.sh.

\begin{tabular}{ll}
\hline 77 & 73 or 74 or 75 or 76 \\
\hline 78 & exp animals/ not humans.sh. \\
\hline 79 & 77 not 78 \\
\hline 80 & 67 and 72 and 79 \\
\hline
\end{tabular}

\section{HISTORY}

Protocol first published: Issue 6, 2012

Review first published: Issue 10, 2018

\begin{tabular}{lll}
\hline Date & Event & Description \\
\hline 6September 2018 & Amended & $\begin{array}{l}\text { [Protocol] Title amended to increase clarity of intervention and } \\
\text { population under review - based on feedback at draft review } \\
\text { stage. }\end{array}$ \\
\hline 26 February 2013 & Amended & $\begin{array}{l}\text { Objectives and inclusion criteria amended to exclude interven- } \\
\text { tions that address certain combinations of behaviours to avoid } \\
\text { overlap with other published and planned reviews. These inter- } \\
\text { ventions include those that target just } 2 \text { behaviours, including } \\
\text { unhealthy diet and low levels of physical activity and/or high lev- } \\
\text { els of sedentary behaviour; and those that target a combination } \\
\text { of tobacco use, alcohol consumption, and/or drug use (see also } \\
\text { the Types of interventions section). }\end{array}$ \\
\hline
\end{tabular}

\section{CONTRIBUTIONS OF AUTHORS}

RC was responsible for primary conceptualisation of the review and its resourcing.

GJM and RK wrote the protocol. All review authors screened studies and extracted data; JR, SW, DMC, GJM, and VE assessed studies' risk of bias; GJM and DMC undertook the statistical analysis; GJM wrote the first draft of the review; and all review authors commented on and contributed to the manuscript.

\section{DECLARATIONS OF INTEREST}

RC is a scientific advisor for DECIPHer Impact, a not-for-profit company wholly owned by the Universities of Cardiff and Bristol, which licences, quality-assures, and supports the delivery of evidence-based public health promotion interventions. RC receives payment for this work.

JW has been the recipient of research funding from the National Institute for Health Research (NIHR), UK, and the National Institute for Social Care and Health Research (NISCHR), Wales.

DG has been the recipient of research funding from the NIHR, Ambulance Service and Medical Research Council (MRC), UK, and is a trustee for The Samaritans.

$\mathrm{MH}$ has been the recipient of research funding from the MRC and Economic and Social Research Council, UK, and has received payment for unrelated activity from Gilead Sciences Inc, Bristol-Myers Squibb, and Janssen, UK.

GJM, DMC, JR, SHW, RK, CC, RL, VE, RL, and KEP - no known interests. 


\section{SOURCESOF SUPPORT}

\section{Internal sources}

- No sources of support supplied

\section{External sources}

- GJM, UK.

GJM is supported by an NIHR post-doctoral fellowship award (PDF-2013-06-026). This report is independent research supported by the National Institute for Health Research (Post-Doctoral Fellowship, Dr Georgina MacArthur, PDF-2013-06-026). The views expressed in this publication are those of the author(s) and are not necessarily those of the NHS, the National Institute for Health Research, or the Department of Health. GJM has also received support from the South West Public Health Training Scheme, UK.

- $\mathrm{RK}, \mathrm{RC}, \mathrm{DG}, \mathrm{JW}, \mathrm{VE}, \mathrm{RL}, \mathrm{JR}$, SW, DMC, UK.

The work was undertaken with the support of The Centre for the Development and Evaluation of Complex Interventions for Public Health Improvement (DECIPHer), a UKCRC Public Health Research Centre of Excellence. Joint funding (MR/KO232331/1) from the British Heart Foundation, Cancer Research UK, Economic and Social Research Council, Medical Research Council, the Welsh Government, and the Wellcome Trust, under the auspices of the UK Clinical Research Collaboration, is gratefully acknowledged. This work is part of a programme of work on multiple risk behaviours in young people being undertaken within DECIPHer.

RC and DG are supported by the Higher Education Funding Council for England.

- DG, UK.

DG is an NIHR Senior Investigator.

- CC, Australia.

CC was supported by funds from an Australia Fellowship awarded to Professor John Lynch (570120) by the National Health and Medical Research Council of Australia.

- DMC, UK.

DMC was the recipient of an MRC Population Health Sciences Fellowship (G0902118).

- JR, VE, UK.

JR received support from DECIPHer, and JR and VE received support from the NIHR School for Public Health Research, of which the University of Bristol is a member.

\section{DIFFERENCES BETWEEN PROTOCOLAND REVIEW}

The original protocol for this review included studies focusing on tobacco, alcohol, and substance misuse (TAD) outcomes. It was decided that studies focusing only on two or more TAD outcomes would be excluded from this review. These studies will be covered in a separate MRB review focused on TAD-only studies. Content in the background section of the review has been updated to reflect the latest literature in the field.

Additional changes include incorporation of GRADE for judging the quality of studies and inclusion of 'Summary of findings' tables. In this review, we also more specifically outline the data synthesis and subgroup and sensitivity analyses conducted. Once we had identified eligible studies, we conducted a mapping exercise of studies to enable us to decide how best to analyse and present the data; the results of this process are reflected in the full review. This exercise demonstrated that the data could be analysed appropriately according to (1) whether the intervention was conducted at the individual, family, or school level, and (2) whether the intervention was provided universally, or whether it was targeted to particular groups. Thus, we analysed the data as per six subgroups (e.g. school-level universal interventions; family-level targeted interventions). We were not able to conduct the subgroup analyses anticipated (including analysis of the impact of population subgroups, settings, or intervention components) because data within each of the six aforementioned studytype subgroups for each behavioural outcome were insufficient to permit further analyses.

The data synthesis section of the review is more detailed compared to the protocol because more detailed description of methods was required; the review also provides detail around the sensitivity analyses conducted.

\section{IN DEX TERMS}

\section{Medical Subject Headings (MeSH)}

*Exercise; *Risk-Taking; *Smoking Prevention; Alcohol Drinking [prevention \& control]; Automobile Driving; Family Therapy; Marijuana Abuse [*prevention \& control]; Program Evaluation; Randomized Controlled Trials as Topic; Schools; Sexual Behavior; Social Behavior Disorders [ ${ }^{*}$ prevention \& control]; Substance-Related Disorders [ ${ }^{*}$ prevention \& control] 


\section{MeSH check words}

Adolescent; Child; Child, Preschool; Humans; Infant; Young Adult 